\title{
Sandwichelemente mit Deckschichten aus Hochleistungsbeton und einem Kern aus extrudiertem Polystyrol
}

\author{
Vom Fachbereich Bauingenieurwesen \\ der Technischen Universität Kaiserslautern \\ zur Verleihung des akademischen Grades
}

\section{DOKTOR-INGENIEUR (Dr.-Ing.)}

\author{
genehmigte \\ DISSERTATION
}

von

Frank Antonius Müller

aus Seck

Dekan:

Prof. Dr. rer. nat. R. Jüpner

1. Berichterstatter:

Jun.-Prof. Dr.-Ing. C. Kohlmeyer

2. Berichterstatter:

Prof. Dr.-Ing. J. Schnell

3. Berichterstatter:

Prof. Dr.-Ing. K. Berner

Tag der mündlichen Prüfung: $\quad$ 12.03.2015

Kaiserslautern 2015

(D 386) 



\section{Vorwort}

Die vorliegende Arbeit entstand in den Jahren 2009 bis 2014, während meiner Zeit als wissenschaftlicher Mitarbeiter am Fachgebiet Massivbau und Baukonstruktion im Fachbereich Bauingenieurwesen der Technischen Universität Kaiserslautern.

Meine Forschungstätigkeit wurde zum überwiegenden Teil im Rahmen des Pilotvorhabens „Innovation und Promotion“ (InnoProm) durch das Ministerium für Bildung, Wissenschaft, Weiterbildung und Kultur des Landes Rheinland-Pfalz und durch die Europäische Union aus dem Europäischen Fonds für regionale Entwicklung gefördert. Weitere finanzielle Unterstützung wurde durch die beiden Forschungspartner der BASF SE und der RETT Gitterträgerdecken $\mathrm{GmbH}$ bereitgestellt. Für die Finanzierung danke ich an dieser Stelle recht herzlich.

Mein besonderer Dank gilt dem ersten Berichterstatter Herrn Jun.-Prof. Dr.-Ing. Christian Kohlmeyer. Durch seine Förderung und Unterstützung sowie durch seine guten Anregungen in den zahlreichen Gesprächen hat er sehr zum Gelingen dieser Arbeit beigetragen.

Auch dem zweiten Berichterstatter und Fachgebietsleiter Herrn Prof. Dr.-Ing. Jürgen Schnell gilt mein Dank. Mit seiner Idee zu diesem Forschungsprojekt hat er den Grundstein zu dieser Arbeit gelegt und mich durch neue Anregungen und Anmerkungen immer wieder gefördert und gefordert.

Weiterhin danke ich dem dritten Berichterstatter Herrn Prof. Dr.-Ing. Klaus Berner für die Durchsicht dieser Arbeit. Die Übernahme eines Korreferats durch Prof. Berner, als einen Mitbegründer und Pionier der Sandwichbauweise und zudem als mein ehemaliger Professor für Statik und Holzbau, freut und ehrt mich zugleich.

Herrn Dr.-Ing. Christoph Hahn und Herrn Dipl.-Ing. Elmar Boy der BASF SE, sowie Herrn Dipl.-Ing. (FH) Matthias Ripperger der RETT Gitterträgerdecken $\mathrm{GmbH}$ danke ich, neben der finanziellen Unterstützung, auch für die technische Unterstützung und die hilfreichen Anregungen bei den zahlreichen Projekttreffen.

Meinen ehemaligen Kolleginnen und Kollegen, den wissenschaftlichen Hilfskräften, dem Laborpersonal und den Bearbeitern der Studienarbeiten danke ich ganz herzlich für die Hilfe, die unzähligen förderlichen Gespräche und die allzeit gute Zusammenarbeit.

Schließlich danke ich ganz besonders meiner Familie, meinen Freunden und meiner Lebensgefährtin dafür, dass ich mich immer auf Ihren Rückhalt und Ihr Verständnis verlassen konnte.

Stuttgart, im Mai 2015

Frank Müller 

Frank Antonius Müller

Sandwichelemente mit Deckschichten aus Hochleistungsbeton und einem Kern aus extrudiertem Polystyrol 



\section{Inhaltsverzeichnis}

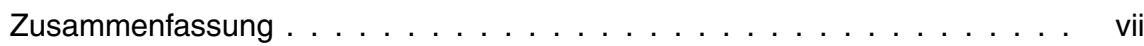

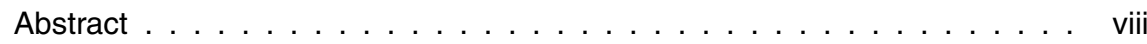

Bezeichnungen .................... ix

\begin{tabular}{lll}
\hline & Einleitung & 1
\end{tabular}

2 Erläuterungen zum Forschungsvorhaben 3

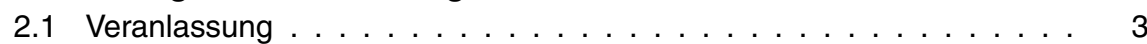

2.2 Umfang . . . . . . . . . . . . . . . . . . . . . . . . 4

2.3 Zielsetzung . . . . . . . . . . . . . . . . . . . . . . . . . . . . . . . . . 6

2.4 Vorgehensweise . . . . . . . . . . . . . . . . . . . 6

3 Zusammenstellung der Grundlagen $\quad 9$

3.1 Entwicklungen im Bereich des Hochleistungsbetons . . . . . . . . . . . 9

3.1 .1 Allgemeines. . . . . . . . . . . . . . . . . . . . . 9

3.1.2 Mikrobewehrter Hochleistungsbeton . . . . . . . . . . . . . . . . . . . . . . . . . .

3.1 .3 Textilbewehrter Beton . . . . . . . . . . . . . . . . . . . . . 13

3.2 Extrudierter Polystyrol-Hartschaum (XPS) $\ldots \ldots \ldots$. . . . . . . . . . 17

3.2 .1 Allgemeines . . . . . . . . . . . . . . . . . . . . . . . . . . . . . . . . . . . . 18

3.2 .2 Herstellung . . . . . . . . . . . . . . . . . . . . . . . . 18

3.2 .3 Materialeigenschaften . . . . . . . . . . . . . . . . . . . . . . 19

3.3 Sandwichbauweise . . . . . . . . . . . . . . . . . . . . 28

3.3 .1 Historische Entwicklung . . . . . . . . . . . . . . . . . . . . 28

3.3.2 Sandwichtheorie und Tragwirkung . . . . . . . . . . . . . . . . . 29

3.3.3 Bemessungssoftware swe2 . . . . . . . . . . . . . . . . . . 32

3.3.4 $\quad$ Bemessung von Sandwichelementen mit Stahldeckschichten . . 37

3.3.5 Untersuchungen an Sandwichelementen mit dünnen Betondeckschichten . . . . . . . . . . . . . . . . . 42

\begin{tabular}{|lll}
4 & Eigenschaften der Werkstoffe & 49
\end{tabular}

4.1 Hochleistungsbeton . . . . . . . . . . . . . . . . . . . . . . . . . . 49

4.1 .1 Allgemeines. . . . . . . . . . . . . . . . . . . . . . . 49

4.1 .2 Beschreibung der verwendeten Betonrezepturen . . . . . . . . . . 49

4.1 .3 Frisch- und Festbetoneigenschaften . . . . . . . . . . . . . . . . . . . . . . . . . . . . . . . . .

4.1 .4 Schwindverhalten . . . . . . . . . . . . . . . . . . 52

4.2 Bewehrungsmaterialien . . . . . . . . . . . . . . . . . . . . . . . . . . . . . . . . . . . . . . . 54

4.2 .1 Allgemeines . . . . . . . . . . . . . . . . . . . . . . . . . . . . . . . . . . . . . 54

4.2 .2 Stahl-Mikrobewehrung. . . . . . . . . . . . . . . . . . . . . . . . . . . . . . . . . . . . . . . 54

4.2 .3 Glasfaser-Textilbewehrung . . . . . . . . . . . . . . . . . . . . . 54

4.2 .4 Carbonfaser-Textilbewehrung . . . . . . . . . . . . . . . . . . . . . . . . . . . . 55

4.3 Extrudierter Polystyrol-Hartschaum (XPS) . . . . . . . . . . . . . . . . 56

4.3 .1 Allgemeines . . . . . . . . . . . . . . . 56 
4.3 .2 Rohdichte und Druckfestigkeit . . . . . . . . . . . . . . 56

4.3 .3 Zellstruktur und Oberflächenstruktur . . . . . . . . . . . . . . . . 56

5 Experimentelle Untersuchungen $\quad 61$

5.1 Allgemeines . . . . . . . . . . . . . . . . . . . . . . 61

5.2 Biegeversuche an Sandwichstreifen . . . . . . . . . . . . . . . . 62

5.2 .1 Allgemeines. . . . . . . . . . . . . . . . . . . . . . . . . . . . . . . . 62

5.2 .2 Versuchsprogramm . . . . . . . . . . . . . . . . . . 62

5.2 .3 Probekörper und Herstellung . . . . . . . . . . . . . . . . . . . . 64

5.2 .4 versuchsaufbau $\ldots \ldots \ldots \ldots$. . . . . . . . . . . . . 68

5.2 .5 Versuchsdurchführung . . . . . . . . . . . . . . . . . . . . . . . . . . . . . . . . .

5.2 .6 Versuchsergebnisse . . . . . . . . . . . . . . . . . . . . 70

5.3 Scherversuche an Kleinprobekörpern . . . . . . . . . . . . . . . . . 80

5.3 .1 Allgemeines. . . . . . . . . . . . . . . . . . . . . . . . . . . . . . . . . . 80

5.3 .2 Versuchsprogramm . . . . . . . . . . . . . . . . . . . . 80

5.3 .3 Probekörper und Herstellung . . . . . . . . . . . . . . . . . . . 81

5.3 .4 Versuchsaufbau . . . . . . . . . . . . . . . . . . . 82

5.3 .5 Versuchsdurchführung . . . . . . . . . . . . . . . . . . . . . . . . . . . . . . 84

5.3 .6 Versuchsergebnisse . . . . . . . . . . . . . . . . . . . . . . 84

5.4 Zug- und Biegeversuche an bewehrtem Beton . . . . . . . . . . . . . . 91

5.4 .1 Allgemeines. . . . . . . . . . . . . . . . . . . . 91

5.4 .2 Versuchsprogramm . . . . . . . . . . . . . . . . . . . . . . . . . . . . 92

5.4 .3 Probekörper und Herstellung . . . . . . . . . . . . . . . . . . . . . . . . . . . . 92

5.4 .4 Zugversuche an Dehnkörpern . . . . . . . . . . . . . . . . . . . 94

5.4 .5 Biegeversuche . . . . . . . . . . . . . . . . . . . . . 99

5.4 .6 Zusammenfassung und Erkenntnisse . . . . . . . . . . . . . . . 101

5.5 Ermüdungsversuche mit zyklischer Wechselbeanspruchung . . . . . . . 102

5.5 .1 Allgemeines. . . . . . . . . . . . . . . . . . . . 102

5.5 .2 Probekörper und Herstellung . . . . . . . . . . . . . . . . . . 103

5.5 .3 Versuchsstand . . . . . . . . . . . . . . . . . . . . . . . . . . . . . . . . . . . . . . . . . . .

5.5 .4 Versuchsdurchführung . . . . . . . . . . . . . . . . . . . . . . . . . . . . . . . . . . . . . . . . .

5.5 .5 Versuchsergebnisse . . . . . . . . . . . . . . . . . . . . . 105

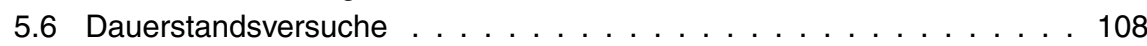

5.6 .1 Allgemeines . . . . . . . . . . . . . . . . . . . . . . . . . . . . . . . . . . . . . . . . . . . . . . . .

5.6 .2 Probekörper und Herstellung . . . . . . . . . . . . . . . . . . . . . . . . . . . . . . . . . . . . . . .

5.6 .3 Versuchsaufbau $\ldots \ldots \ldots . \ldots . \ldots . \ldots 108$

5.6 .4 Versuchsdurchführung . . . . . . . . . . . . . . . . . . . . . . . 109

5.6 .5 Versuchsergebnisse . . . . . . . . . . . . . . . . . . . . . . . . . 109

5.7 Großbauteilversuche . . . . . . . . . . . . . . . . . . . . . . . . . . 111

5.7 .1 Allgemeines. . . . . . . . . . . . . . . . . . . . . . . . . . . . . . . . . . . . . . . . .

5.7 .2 Probekörper und Herstellung . . . . . . . . . . . . . . . . . . 111

5.7 .3 Beanspruchung durch Einzellasten . . . . . . . . . . . . . . . . . 113

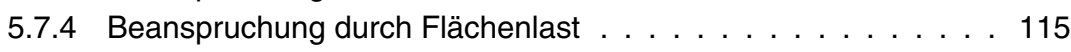

5.8 Probewände zur Ermittlung der Praxistauglichkeit und Dauerhaftigkeit . 122

5.8 .1 Allgemeines . . . . . . . . . . . . . . . . . . . . . . . . . . . . . . . . . . . . .

5.8 .2 Herstellung . . . . . . . . . . . . . . . . . . . . . . . 122

5.8 .3 Langzeitbeobachtung der Rissbildung . . . . . . . . . . . . . . . 124 
6 Rechnerische Untersuchungen 127

6.1 Allgemeines . . . . . . . . . . . . . . . . . . . . . 127

6.2 Modellierung der Deckschichten . . . . . . . . . . . . . . . 127

6.2 .1 Allgemeines. . . . . . . . . . . . . . . . . . . . . . 127

6.2 .2 Eingabewerte für den bewehrten Beton . . . . . . . . . . . 128

6.2.3 Vergleich mit den Ergebnissen der Dehnkörperversuche. . . . . 132

6.2.4 Kontrolle durch Nachrechnung der Biegeversuche an bewehrten

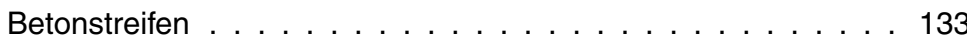

6.3 Modellierung des Kerns . . . . . . . . . . . . . . . . . . . 135

6.3 .1 Allgemeines. . . . . . . . . . . . . . . . . . . . 135

6.3 .2 Isotropes Materialgesetz „BISO $\ldots$. . . . . . . . . . . . . 135

6.3 .3 Anisotropes Materialgesetz „ANISO ${ }^{4} \ldots \ldots \ldots \ldots$. . . . . . . . 136

6.3.4 Kontrolle durch Nachrechnung der Scherversuche . . . . . . . . 138

6.4 Modellierung der Verbundfuge zwischen Deckschichten und Kern. . . . 140

6.5 Nachrechnung der Biegeversuche an Sandwichstreifen . . . . . . . 140

6.5 .1 Beschreibung des Finite-Element Modells . . . . . . . . . . 140

6.5.2 Vergleich der Berechnungsergebnisse mit den Versuchsergeb-

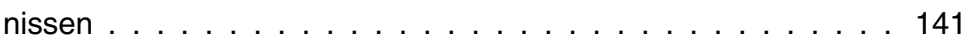

6.6 Nachrechnung der Großbauteilversuche . . . . . . . . . . . . 145

6.6 .1 Allgemeines. . . . . . . . . . . . . . . . 145

6.6 .2 Beschreibung des Finite-Element Modells . . . . . . . . . . . . 145

6.6.3 Vergleich der Berechnungsergebnisse mit den Versuchsergebnissen . . . . . . . . . . . . . . . . . . 145

7 Zusammenfassung und Diskussion der Ergebnisse 151

7.1 Generelle Beschreibung des Biegetragverhaltens der Sandwichelemente 151

7.2 Schertragverhalten des XPS $\ldots \ldots \ldots \ldots \ldots \ldots \ldots$

7.2 .1 Allgemeines. . . . . . . . . . . . . . . . . . . . . 152

7.2.2 Kritische Betrachtung der Scherversuche . . . . . . . . . . 155

7.3 Einfluss der verschiedenen Parameter auf das Biegetragverhalten der

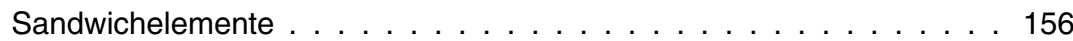

7.3 .1 Herstellungsmethode . . . . . . . . . . . . . . 156

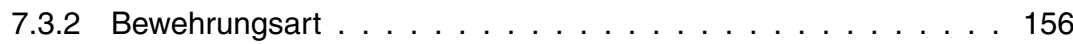

7.3 .3 Dichte des XPS. . . . . . . . . . . . . . . . . . . . . 157

7.3 .4 Schichtdicken . . . . . . . . . . . . . . . . . . . . 158

7.3 .5 Stoßausbildung . . . . . . . . . . . . . . . . . . . . . . 159

7.4 Diskussion der Versagensarten . . . . . . . . . . . . . . . . . . 160

$7.4 .1 \quad$ A: „Zugversagen der Bewehrung" . . . . . . . . . . . . . . 160

7.4 .2 B: „Druckversagen des Kern an der Lasteinleitungsstelle“ . . . 160

7.4 .3 C: „Lokales Biegeversagen der Deckschichten" $\ldots$. . . . . . . . 162

7.4 .4 D: „Lokales Querkraftversagen der Deckschichten ${ }^{7}$. . . . . . . . 163

7.4.5 E: „Querkraftversagen des Kerns und Delamination des Kerns und der Deckschichten" . . . . . . . . . . . . . . . . . 163

7.4.6 F: „Schubversagen zwischen Kern- und Deckschicht" $\ldots$. . . . 165

7.4 .7 G: "Druckversagen des Kerns an den Auflagern" $\ldots \ldots \ldots . .168$

7.5 Parameterstudien an einem Wandelement . . . . . . . . . . . . 168

7.5 .1 Allgemeines. . . . . . . . . . . . . . . . 168

7.5 .2 Beanspruchung durch Temperatur $\ldots \ldots \ldots \ldots \ldots$ 
7.5 .3 Beanspruchung durch Schwinden der Deckschichten . . . . . . 170

8 Bemessungskonzept für die untersuchten Sandwichelemente 171

8.1 Allgemeines . . . . . . . . . . . . . . . . . . . 171

8.2 Ermittlung der Schnittgrößen . . . . . . . . . . . . . . . . . . . . . 171

8.2 .1 Allgemeines. . . . . . . . . . . . . . . . . . . . . 171

8.2.2 Ansatz zur Ermittlung der Schnittgrößen unter Berücksichtigung der Rissbildung in den Deckschichten . . . . . . . . . . . . . 172

8.3 Nachweiskonzept . . . . . . . . . . . . . . . . . . . . . . . . . . . . . . . 189

8.3 .1 Nachweisführung und Sicherheitskonzept . . . . . . . . . . . . . 189

8.3.2 Nachweise im Grenzzustand der Tragfähigkeit . . . . . . . . . . . 190

8.3.3 Nachweise im Grenzzustand der Gebrauchstauglichkeit . . . . . 198

8.4 Bemessungsbeispiele . . . . . . . . . . . . . . . . . . . . . . . . . 201

8.4 .1 Allgemeines. . . . . . . . . . . . . . . . . . . . . . . . 201

8.4 .2 Wandelement mit einem Feld . . . . . . . . . . . . . . . . . . . . 201

8.4 .3 Dachelement mit zwei Feldern . . . . . . . . . . . . . . . . 206

8.5 Schwindbemessung der Sandwichelemente . . . . . . . . . . . . . . 211

8.5 .1 Ermittlung der Zwangspannungen . . . . . . . . . . . . . . . 211

8.5 .2 Einwirkungen und Widerstände . . . . . . . . . . . . . . . . . 213

8.5.3 Nachweis gegen Rissbildung der Deckschichten . . . . . . . . . 215

8.5 .4 Nachweis gegen Druckversagen des Kerns . . . . . . . . . . . . 215

8.5 .5 Nachweis gegen Versagen des Verbunds . . . . . . . . . . . . . 216

8.5 .6 Beispiel . . . . . . . . . . . . . . . . . 216

$\begin{array}{lll}9 & \text { Brandschutz } & 219\end{array}$

9.1 Klassifizierung der Baustoffe und Bauteile . . . . . . . . . . . . . . . . 219

9.1 .1 Allgemeines. . . . . . . . . . . . . . . . . . . . . 219

9.1 .2 Extrudierter Polystyrol Hartschaum. . . . . . . . . . . . . . . . . 220

9.1 .3 Hochleistungsbeton . . . . . . . . . . . . . . . . . . . . . 220

9.1 .4 Sandwichelemente . . . . . . . . . . . . . . . . . . . . . . 221

9.2 Bauaufsichtliche Anforderungen . . . . . . . . . . . . . . . . . . . . . . . . . . . . . . . . . . . . . . . . .

9.2 .1 Allgemeines. . . . . . . . . . . . . . . . . . . . . . . 221

9.2 .2 Sandwich-Wandelemente . . . . . . . . . . . . . . . . . . . . . . 221

9.2 .3 Sandwich-Dachelemente . . . . . . . . . . . . . . . . . . . 222

9.3 Feuerwiderstandsversuche . . . . . . . . . . . . . . . . . . . . . . . 222

9.3 .1 Allgemeines. . . . . . . . . . . . . . . . . . . . . 222

9.3.2 Abmessungen und Herstellung der Probekörper . . . . . . . . . 223

9.3 .3 Versuchsdurchführung . . . . . . . . . . . . . . . . . . . . . . . . . . . . . . . . . . . . . . 223

9.3 .4 Versuchsergebnisse . . . . . . . . . . . . . . . . . . 223

9.4 Zusammenfassung und Schlussfolgerungen . . . . . . . . . . . . . . . . 225

10 Zusammenfassung und Ausblick 227

10.1 Zusammenfassung . . . . . . . . . . . . . . . . . . . . . . . . . . . . . . . . . . . . . . . . . . . . . . .

10.2 Ausblick . . . . . . . . . . . . . . . . . . . . . . . . . . . . 229

\begin{tabular}{ll}
\hline Literatur & 231
\end{tabular} 
A Anhang „Experimentelle Untersuchungen" 243

A.1 Zusammenstellung zu den Biegeversuchen an Sandwichstreifen . . . . 243

A.1.1 Zusammenstellung der aufgeschnittenen Biegeprobekörper. . . 244

A.2 Zusammenstellung der Scherversuche. . . . . . . . . . . . . . 250

A.3 Zusammenstellung der Biege- und Zugversuche an bewehrtem Beton . 256

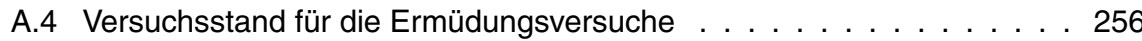

A.4.1 Zusammenstellung der aufgeschnittenen Probekörper. . . . . . 258

A.5 Zusammenstellung der Großbauteilversuche . . . . . . . . . . . . 259

A.5.1 Beanspruchung durch Einzellasten . . . . . . . . . . . . . . . . 259

A.5.2 Beanspruchung durch Flächenlasten. . . . . . . . . . . . 260

A.5.3 Zusammenstellung der aufgeschnittenen Biegeprobekörper. . . 265

B Anhang „Rechnerische Untersuchungen"

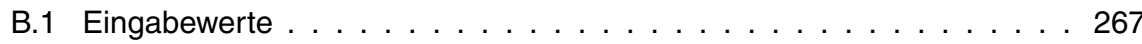

B.2 Commandfile . . . . . . . . . . . . . . . . . . . . . . . 268

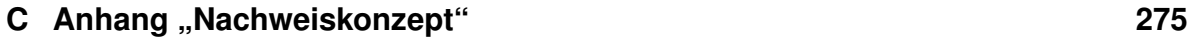

C.1 Herleitung der Gleichung zur Bestimmung des Dehnungszustandes . . 275

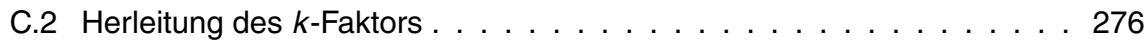

C.3 Abschätzung der Relaxation des XPS . . . . . . . . . . . . . . 277

C.4 Lastfallkombinationen eines Sandwich-Dachelements . . . . . . . . . 278

$\begin{array}{ll}\text { Lebenslauf } & 281\end{array}$ 



\section{Zusammenfassung}

Sandwichelemente vereinen aufgrund des gezielten Einsatzes verschiedener Materialien und deren schichtartigen Aufbaus sehr gute Dämmeigenschaften und ein geringes Eigengewicht mit hoher Tragfähigkeit und Biegesteifigkeit. Sie werden daher seit Beginn der 1960er-Jahre sowohl im Metallleichtbau als auch im Stahlbetonmassivbau vielfältig eingesetzt. Im Rahmen der vorliegenden Arbeit wurden Sandwichelemente mit Deckschichten aus einem mikro- oder auch textilbewehrtem Hochleistungsbeton und einem Kern aus extrudiertem Polystyrol-Hartschaum umfangreich experimentell und rechnerisch untersucht. Ziel war es, den Verbund zwischen dem Kern und den Deckschichten ohne mechanische Verbundmittel, sondern rein über die Adhäsion des profilierten Dämmkerns und den Deckschichten zu erzielen.

Das Sandwichtragverhalten wurde durch eine Vielzahl von Biege- und Scherversuchen experimentell untersucht. Die Dauerhaftigkeit des Verbunds zwischen dem Kern und den Deckschichten wurde durch eine Frost-Tau-Wechselbeanspruchung der Scherprobekörper sowie durch eine zyklische Ermüdungsbeanspruchung der Biegeprobekörper bestimmt. Anhand der Versuchsergebnisse konnte gezeigt werden, dass die Sandwichelemente bei der Verwendung als Wand- oder Dachelement den praxisüblichen Beanspruchungen standhalten und nicht vorzeitig versagen. Das Tragverhalten wurde weiterhin in Bauteilversuchen an großformatigen Sandwichelementen überprüft. Hierzu wurden in einem eigens konstruierten Unterdruckprüfstand zweifeldrig gelagerte Sandwichelemente einer realitätsnahen Beanspruchung durch eine Flächenlast ausgesetzt. Die Materialeigenschaften, die anhand der Scherversuche sowie an Dehnkörperversuchen am bewehrtem Beton ermittelt wurden, dienten zudem als Eingangsparameter zur rechnerischen Untersuchung der Sandwichelemente mit der FE-Software ANSYS. Auf Grundlage der Modellierungen konnten die Versuchsergebnisse interpretiert und das Tragverhalten in Parameterstudien vertiefend analysiert werden. Darüber hinaus wurden auch die Einwirkungen aus einer Temperatur- und Schwindbeanspruchung der Elemente ermittelt.

Über Ingenieurmodelle auf Grundlage der Sandwichtheorie konnte bisher der Einfluss der Rissbildung in den Betondeckschichten nur überschlägig und gemittelt über die Länge der Elemente berücksichtigt werden. Aus diesem Grund wurde ein bestehendes Programm zur Ermittlung der Sandwichschnittgrößen um einen Ansatz zur Berücksichtigung der Steifigkeitsreduzierung infolge der Rissbildung umfangreich erweitert. Mit dem neuen Programm swe2+ können nun erstmalig die Schnittgrößen und Verformungen von Sandwichelementen mit gerissenen Betondeckschichten zielsicher und diskret über die Länge eines Sandwichelements ermittelt werden. Durch Beispielrechnungen wurde gezeigt, dass die Umlagerung der Schnittgrößen infolge der Rissbildung in den Deckschichten zu ungünstigeren Beanspruchungen führen kann und diese Umlagerungen bei der Bemessung der Elemente entsprechend zu berücksichtigen sind. Die Bemessung der hier untersuchten Sandwichelemente kann in Anlehnung an die Norm DIN EN 14509 zur Bemessung von Sandwichelementen mit metallischen Deckschichten durchgeführt werden. Durch die Nachrechnungen der Biegeversuche wurde gezeigt, dass auf diese Weise die Elemente mit ausreichender Sicherheit und sehr guter Übereinstimmung bemessen werden können. Dies wird abschließend in zwei Bemessungsbeispielen an einem Wand- sowie einem Dachelement demonstriert. 


\section{Abstract}

Due to the carefully chosen use of materials in multiple layers, sandwich elements combine high insulation properties and a low dead weight with a high bearing capacity and high bending stiffness. For these reasons, sandwich elements have been used since the 1960s for light weight steel construction as well as for reinforced concrete construction. For this thesis, sandwich elements with facings of micro- and textile-reinforced high performance concrete and a core of extruded polystyrene foam were extensively investigated in experiments and finite-element calculations. The bond between the core and the facings was achieved without any mechanical bonding devices just by adhesion between the textured insulation core and the facings.

The sandwich bearing behaviour was investigated in a large number of bending and shear tests. The durability of the bond between the core and the facings was determined in freeze-thaw tests of the shear specimens and cyclic fatigue loading of the bending specimens. The test results show that the sandwich elements are able to transfer the anticipated loading and do not fail during the service life time when used as wall or roof elements. Furthermore, the bearing behaviour was investigated in fullscale element tests. As part of this, two-span bending specimens were exposed to a realistic distributed load in a specially constructed low-pressure test rig. The material properties, which were determined in the shear tests as well as in tensile tests on reinforced concrete, were used as input parameters for the numerical investigation of the sandwich elements with the FE-software ANSYS. On the basis of the FE-modelling, the results of the experiments were interpreted and the bearing behaviour was analysed in depth in parametric studies. As part of this, the strains resulting from shrinkage and temperature loading of the elements were determined.

With existing calculation models based on the sandwich theory, the influence of concrete cracking of the facings can only be calculated approximated and averaged over the length of the elements. For this reason, an available program for the determination of the internal forces of sandwich elements was considerably extended. By introducing an additional routine, the stiffness reduction caused by the cracking of the concrete is taken into account. With the new program swe2+, the internal forces and the deformations of sandwich elements with cracked concrete facings can now be determined accurately over the length of a sandwich element. It is shown in example calculations that the redistribution of internal forces caused by the cracking of the facings may result in more critical stress states. Therefore, this redistribution of the internal forces has to be taken into account in the structural design of the elements. The design of the elements investigated can be carried out according to the standard DIN EN 14509 for the design of sandwich elements with metal facings. By the comparison of the calculation results with the results of the bending tests, it was shown that the elements are designed with a sufficient margin of safety and correlation. Finally, the structural design of a wall and roof element is demonstrated in an example. 


\section{Bezeichnungen}

\section{Große lateinische Buchstaben}

A Querschnittsfläche, Luftporengehalt, Auflagerkraft

$B \quad$ Elementbreite, Biegesteifigkeit, Auflagerkraft

C Bemessungswert eines Kriteriums für die Gebrauchstauglichkeit, Auflagerkraft, Carbonfaser-Textilbewehrung

D $\quad$ Frischbetonrohdichte

E Elastizitätsmodul, Bemessungswert der Auswirkung einer Beanspruchung

$F \quad \quad$ Kraft, Last, Belastung

G Schubmodul, Glasfaser-Textilbewehrung

I Trägheitsmoment

L Länge

M Moment

$N \quad$ Normalkraft

$P \quad$ Aufgebrachte Last in den durchgeführten Versuchen

$R \quad$ Widerstand

$S \quad$ Schubsteifigkeit, Charakteristische Beanspruchungen, Stahl-Mikrobewehrung

T Temperatur

V Querkraft

W Widerstandsmoment

\section{Kleine lateinische Buchstaben}

$\begin{array}{ll}a & \text { Maschenweite, Abstand, Überstand } \\ b & \text { Breite } \\ d & \text { Dicke, statische Höhe } \\ e & \text { Abstand zwischen den Schwerachsen der Deckschichten } \\ f & \text { Festigkeit } \\ g & \text { ständige Last } \\ h & \text { Höhe } \\ k & \text { Faktor } \\ l & \text { Länge, Stützweite }\end{array}$




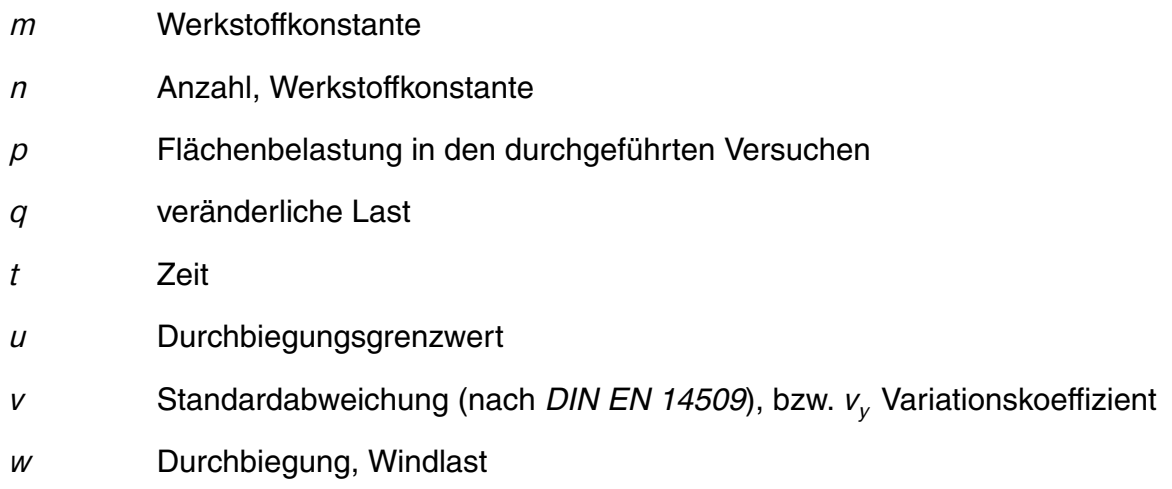

\section{Griechische Buchstaben}

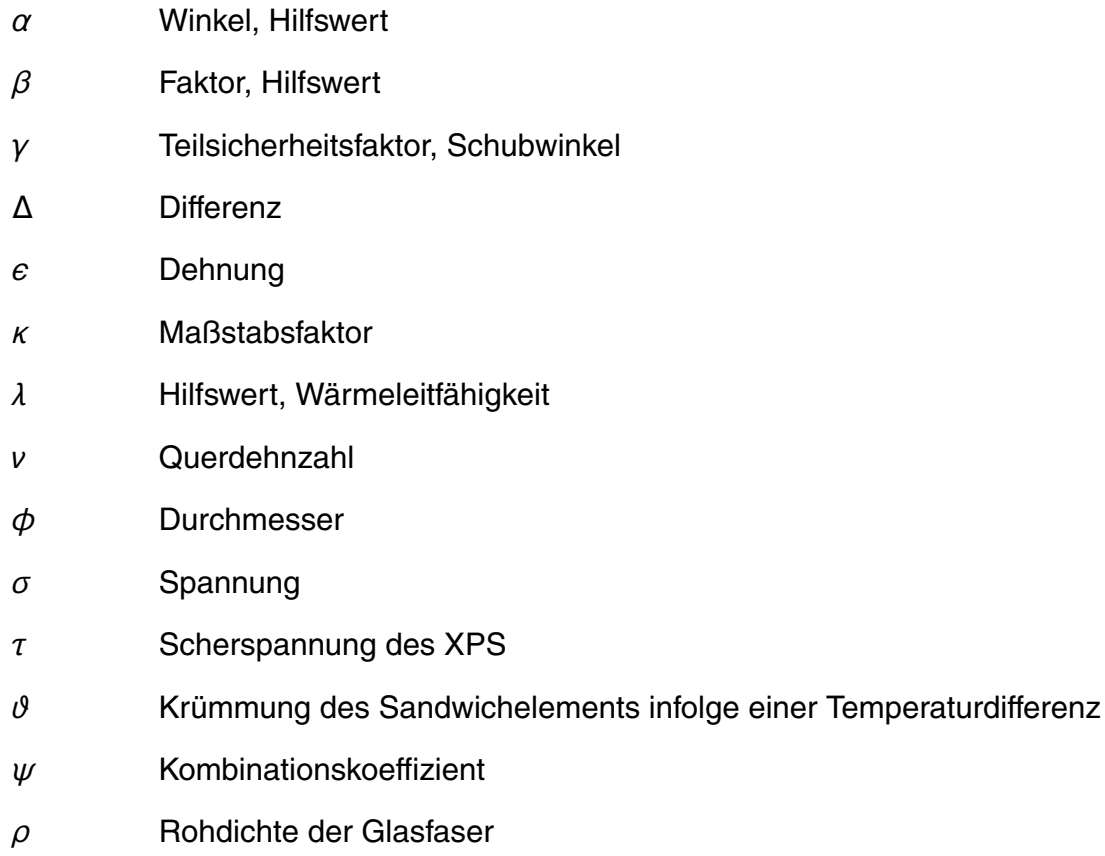

\section{Indizes}




\begin{tabular}{|c|c|}
\hline$c$ & Beton, Druckfestigkeit \\
\hline C & am Punkt C \\
\hline calc & rechnerisch ermittelter Wert \\
\hline $\mathrm{cr}$ & Erstriss (engl. crack) \\
\hline cube & Würfeldruckfestigkeit \\
\hline$d$ & Bemessungswert \\
\hline$D$ & am Punkt D, Deckschichten \\
\hline$D U C$ & $\mathrm{DUCON}^{\circledR}$ \\
\hline$e$ & im elastischen Bereich \\
\hline$E$ & am Punkt E \\
\hline eff & effektiv, wirksam \\
\hline ext & außen \\
\hline $\exp$ & experimentell ermittelter Wert \\
\hline$f$ & Lastfaktor, Faser \\
\hline fat & Ermüdung (engl. fatigue) \\
\hline$f l$ & Biegung (engl. flexural) \\
\hline fil & Filament \\
\hline$g$ & Glasfaser \\
\hline ges & gesamt \\
\hline i & Index \\
\hline I, II & Zustand I (ungerissen) bzw. Zustand II (gerissen) \\
\hline int & innen \\
\hline$k$ & charakteristischer Wert \\
\hline$l$ & Längsfaser, Längsdrähte \\
\hline$m$ & Betonmatrix,Mittelwert \\
\hline M & Material \\
\hline $\max$ & Maximalwert \\
\hline mean & Mittelwert \\
\hline$o$ & Carbonfaser, obere Deckschicht, obere Bewehrungslage \\
\hline$p$ & im plastischen Bereich \\
\hline$p l$ & an der Grenze zwischen elastischen und plastischen Bereich \\
\hline & Querfasern, Querdrähte \\
\hline
\end{tabular}


red

$S L S$

$s p$

st

(t) Zeitpunkt

tex

ULS

v

$x$

$X P S$

$x y$

$y$

$z$

zul

\section{Abkürzungen}

DMS Dehnungsmessstreifen

EPS Expandierter Polystyrol Hartschaum (engl. Expanded Polystyrene Foam)

ETK Einheitstemperaturkurve

HFKW Teilhalogenierte Fluor-Kohlenwasserstoffe

HPC Hochleistungsbeton (engl. High Performance Concrete)

PE Polyethylen (engl. Polyethylene)

PUR Polyurethan (engl. Polyurethane)

SPS Speicherprogrammierbare Steuerung

UHPC Ultrahochleistungsbeton bzw. ultrahochfester Beton (engl. Ultra High Performance Concrete)

UHPFRC Ultrahochfester Faserbeton (engl. Ultra High Performance Fibre Reinforced Concrete)

XPS Extrudierter Polystyrol Hartschaum (engl. Extruded Polystyrene Foam) 


\section{$1 \quad$ Einleitung}

Aufgrund des hohen Anteils am Gesamtenergieverbrauch in Deutschland von rund $30 \%$ (AGEB 2010), bietet die Raumheizwärme von Gebäuden ein großes Einsparpotential. Durch die Energieeinsparverordnung (EnEV) wurde die europäische Richtlinie zur Harmonisierung und Regelung der Energieeffizienz von Gebäuden (EU 2002) schrittweise in Deutschland umgesetzt. Die verschärften Anforderungen der EnEV zur Reduzierung der Transmissionswärmeverluste - d. h. der Wärmeverluste durch Übertragung über die Gebäudehülle - resultieren in steigenden Dämmstärken und Gesamtstärken von Außenbauteilen. Um diesem Umstand entgegenzuwirken, müssen die vorhandenen Bauweisen und -materialien weiter verbessert und optimiert werden.

Sandwichelemente bieten aufgrund ihres schichtartigen Aufbaus eine geringe Bauteildicke und gute Dämmeigenschaften bei gleichzeitig hoher Biegesteifigkeit und hoher Tragfähigkeit. Die verwendeten Materialien werden entsprechend ihrer Eigenschaften gezielt eingesetzt und somit effizient und ressourcenschonend genutzt. Im Vergleich zu massiven Stahlbetonfertigteilen sind Sandwichbauteile äußerst leicht und dadurch einfach zu transportieren und zu montieren.

Schon seit Beginn der 1960er-Jahre werden Sandwichelemente mit metallischen Deckschichten und Hartschaum-Kernen, aber auch Sandwichelemente mit massiven Stahlbetonschalen, verstärkt in der Bauindustrie eingesetzt. Die in den letzten Jahrzehnten vorangeschrittenen Entwicklungen im Bereich der Hochleistungsbetone ermöglichen nun auch die Herstellung von Sandwichelementen mit äußerst filigranen Betondeckschichten, so dass die herkömmlichen Bauweisen dadurch optimiert werden können.

Hochleistungsbetone (HPC) eignen sich aufgrund der hohen erzielbaren Oberflächenqualität besonders gut für die Herstellung von Deckschichten in Sichtbetonoptik. Die Betondeckschichten sind darüber hinaus besonders dauerhaft, witterungsbeständig und abriebfest. Im Vergleich zu Sandwichelementen mit Stahlblechen, neigen die Deckschichten aus Beton weniger zu Beschädigungen durch Stöße und Anprall. Eine Eigenschaft die besonders bei Industriebauten von Bedeutung ist.

Auch hinsichtlich der Architektur eröffnen die Sichtbetonoberflächen aus Hochleistungsbeton neue, umfangreiche Gestaltungsmöglichkeiten. So können beliebige Oberflächentexturen mithilfe von Strukturmatrizen hergestellt werden oder die Farbgebung durch die gezielte Auswahl von Gesteinskörnungen, Zementsorten oder die Hinzugabe von Farbpigmenten gestaltet werden.

Um eine vielfältige Anwendung dieser neuartigen Sandwichelemente in der Praxis zu ermöglichen, müssen die entsprechenden Forschungsgrundlagen geschaffen werden. Neben einem grundsätzlichen Verständnis des Tragverhaltens, ist darüber hinaus ein praxistaugliches Nachweiskonzept zur zielsicheren Bemessung der Sandwichelemente erforderlich. Diese Arbeit soll hierzu einen Beitrag leisten. 



\section{Erläuterungen zum Forschungsvorhaben}

\section{$2.1 \quad$ Veranlassung}

In der Bauindustrie kommen vorwiegend zwei Arten von Sandwichelementen zur Anwendung. Zum einen sind dies die relativ leichten Sandwichelemente des Metallleichtbaus und zum anderen die im Vergleich dazu schweren Sandwichelemente des Stahlbetonmassivbaus. Die beiden Sandwicharten unterscheiden sich vor allem in ihrem Aufbau, ihrer Herstellung und ihrer Tragweise.

Die traditionellen Sandwiche im Metallleichtbau bestehen dabei üblicherweise aus zwei Deckschichten aus Stahlfeinblech und einem Dämmkern aus Polyurethan-Hartschaum (PUR). Die Sandwiche werden vollständig automatisiert, in einer kontinuierlich arbeitenden Bandanlage gefertigt. Der Verbund zwischen den Stahlblechen und dem PURKern wird allein durch Adhäsion d. h. durch die Klebwirkung des noch frischen PURSchaums erzielt. Über die Schubsteifigkeit des Dämmkerns wird die nötige Sandwichtragwirkung erzeugt, die dem Sandwichelement mit den nur wenigen Millimeter dicken Stahlblechen die nötige Biegesteifigkeit verleiht.

Die Sandwichelemente im Massivbau bestehen in der Regel aus einer Trag- und einer Vorsatzschale aus Stahlbeton sowie einer dazwischen liegenden Dämmschicht aus einem expandierten oder extrudierten Polystyrol (EPS und XPS). Der Verbund zwischen den beiden Betonschalen wird hauptsächlich durch linien- oder punktförmige Verbundmittel sichergestellt. Die Herstellung der Elemente erfolgt in mehreren Schritten und ist aufgrund des großen Gewichts der Stahlbetonschalen sowie der erforderlichen Verbundmittel, im Vergleich zur Herstellung von Stahlblech-Sandwichen, aufwendig. Die Tragschale dient zum Abtrag von Vertikallasten und weist meist eine größere Dicke von etwa 10 bis $20 \mathrm{~cm}$ auf. Dadurch besitzt das Element bereits eine ausreichende Tragfähigkeit zur Aufnahme von Horizontallasten, so dass die Sandwichtragwirkung beim Nachweis im Grenzzustand der Tragfähigkeit in der Regel vernachlässigt wird. Aufgrund von Zwangspannungen, die unter anderem aus dem Schwinden des Betons und einer Temperaturbeanspruchung entstehen, kann sich die Sandwichtragwirkung negativ auswirken und zur Rissbildung in der Vorsatzschale führen.

Ziel der neueren Forschung im Bereich der Sandwichelemente im Massivbau ist es, in Anlehnung an den Aufbau und die Tragwirkung der Stahlblech-Sandwiche, die Grundlagen für neuartige Sandwichelemente mit dünnen Betondeckschichten zu erforschen.

Zur Gewährleistung der nötigen Biegesteifigkeit und Tragfähigkeit der Elemente unter Gebrauchslasten, muss neben der Eigenbiegesteifigkeit der Deckschichten auch die Schubsteifigkeit des Dämmkerns und somit die Sandwichtragwirkung herangezogen werden. 
Im Rahmen der Arbeit von Horstmann 2010 wurden diese neuartigen Sandwichelemente bereits umfangreich experimentell und rechnerisch untersucht. Zur Sicherstellung und Vergrößerung der Sandwichtragwirkung wurden Verbundmittel eingesetzt. Daraus ergeben sich jedoch auch Nachteile. Durch die Verwendung von Verbundmitteln wird der Herstellungsaufwand erhöht. Die Verbundmittel stellen im Vergleich zur Dämmwirkung des Kerns eine Wärmebrücke dar. Weiterhin müssen die Deckschichten die Verankerungslänge der Verbundmittel aufnehmen können und daher eine Mindestdicke aufweisen.

Aufbauend auf den Erkenntnissen aus der Arbeit von Horstmann 2010 sollen in dieser Arbeit Sandwichelemente untersucht werden, bei denen vollständig auf Verbundmittel verzichtet wird. Der nötige Verbund zwischen dem Dämmkern und den Betondeckschichten soll, wie bei den traditionellen Sandwichen des Metallleichtbaus auch, rein über Adhäsion erzielt werden. Dadurch werden Fragen hinsichtlich der Tragfähigkeit und Dauerhaftigkeit des Verbunds aufgeworfen. Diese Fragestellungen sollen in der vorliegenden Arbeit beantwortet werden.

\section{$2.2 \quad$ Umfang}

Der konstruktive Aufbau der in dieser Arbeit untersuchten Sandwichelemente gleicht dem der traditionellen Sandwichelemente mit Metalldeckschichten. Es werden zwei dünne Deckschichten mit geringer Eigenbiegesteifigkeit und einem dazwischenliegenden Kern aus einem leichten jedoch relativ schubsteifen Dämmmaterial schubfest miteinander verbunden.

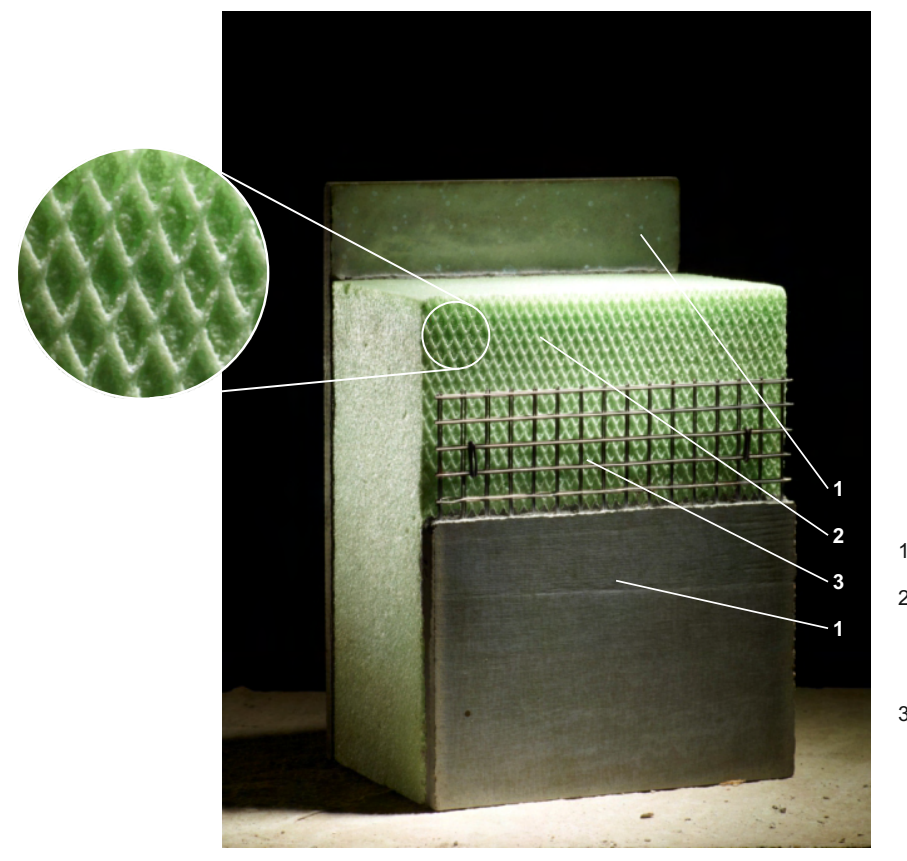

Betondeckschicht

XPS-Kern mit thermisch geprägter, rautenförmiger Oberflächenstruktur

3 Mikrobewehrung aus Stahldrahtmatten (oder Textilbewehrung aus Glasfasergewebe oder Carbonfasergelege)

Abb. 2.1: Aufbau der Sandwichelemente 
Als Kernmaterial wird ein extrudierter Polystyrol-Hartschaum (XPS) mit der Produktbezeichnung Styrodur ${ }^{\circledR} \mathrm{C}$ der Firma BASF verwendet. Zur Herstellung der Deckschichten kommt ein mikro- oder auch textilbewehrter Hochleistungsbeton zum Einsatz. Wie in Abbildung 2.1 dargestellt, wird der Verbund zwischen XPS und Beton über eine thermisch geprägte, rautenförmige Oberflächenstruktur der XPS-Dämmplatten hergestellt.

Grundsätzlich können Sandwichelemente hinsichtlich ihrer Verwendung als Wand- oder als Dachelement unterschieden werden (siehe Abbildung 2.2). Bei selbsttragenden Wandelementen treten hauptsächlich Beanspruchungen aus Wind und Temperatur auf. Es wird davon ausgegangen, dass beide Deckschichten fest gelagert oder an eine tragende Unterkonstruktion angeschlossen sind und dadurch keine Scherbeanspruchung aus der Eigenlast der Deckschichten im Kern der Elemente auftritt.

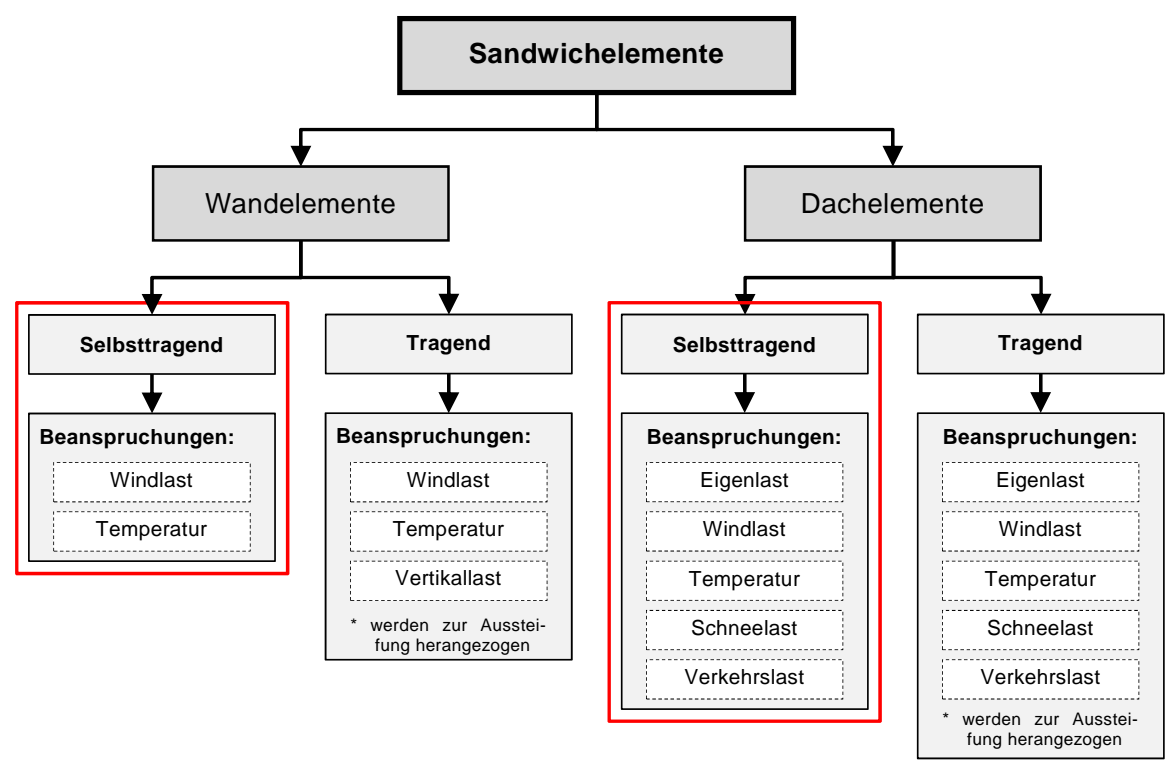

Abb. 2.2: Unterteilung der Sandwichelemente nach der Art der Einwirkungen

Bei Dachelementen treten zusätzlich Schnee- und Verkehrslasten auf. Auch die Eigenlast der Deckschichten erzeugt nun eine Biegebeanspruchung der Elemente. Durch die ständig wirkende Eigenlast der Elemente sowie durch eine längerfristig wirkende Schneelast wird im Kern eine Kriechverformung hervorgerufen, die bei den Nachweisen zu berücksichtigen ist.

Sandwichelemente können weiterhin je nach Tragfunktion in selbsttragende oder tragende Elemente unterschieden werden. Selbsttragende Elemente werden nur durch äußere Beanspruchungen aus Wind, Schnee, Temperatur etc. belastet, während bei tragenden Elementen zusätzlich lastabtragende, stabilisierende und aussteifende Funktionen hinzukommen. Diese sind beispielsweise der Abtrag von Vertikallasten bei einem Wandelement, die Stabilisierung von Wand- und Deckenriegeln gegen seitliches Ausweichen und die Ausbildung von aussteifenden Wand- und Deckenscheiben. 
Die Untersuchungen in der vorliegenden Arbeit konzentrieren sich vorwiegend auf selbsttragende Wand- und Dachelemente. Dabei werden sowohl ein- als auch zweifeldrig gelagerte Elemente untersucht.

\subsection{Zielsetzung}

Das Ziel dieser Arbeit kann wie folgt definiert werden:

Das Tragverhalten von Sandwichelementen mit dünnen Deckschichten aus Hochleistungsbeton und einem Kern aus extrudiertem Polystyrol soll experimentell und rechnerisch untersucht werden. Ziel ist es, den Verbund zwischen Beton und XPS ohne mechanische Verbundmittel, lediglich durch eine thermisch geprägte Oberfläche der Dämmplatten zu erzeugen. In den Untersuchungen sollen insbesondere die Tragfähigkeit, die Alterungsbeständigkeit und das Ermüdungsverhalten des Verbunds bestimmt werden. Das Ergebnis soll ein praxistaugliches Bemessungsmodell für diese Art von Sandwichelementen sein.

\section{$2.4 \quad$ Vorgehensweise}

In Abbildung 2.3 ist die Vorgehensweise zur Untersuchung des Tragverhaltens der Sandwichelemente sowie die Gliederung dieser Arbeit schematisch dargestellt. Zu Beginn werden in Kapitel 3 der aktuelle Stand der Forschung in den Bereichen der Hochleistungsbetone, des extrudierten Polystyrols und der Sandwichbauweise und in Kapitel 4 die generellen Materialeigenschaften der in dieser Arbeit verwendeten Werkstoffe zusammengestellt.

In Kapitel 5 sind die experimentellen Untersuchungen beschrieben, die im Rahmen dieser Arbeit durchgeführt wurden. Diese umfassten Kleinversuche, die zur Bestimmung der Materialeigenschaften durchgeführt wurden, aber auch Biegeversuche, die zur Untersuchung des generellen Tragverhaltens der Sandwichelemente dienten. Zudem wurden Versuche an großmaßstäblichen Sandwichelementen durchgeführt.

Die rechnerischen Untersuchungen sind im Kapitel6dieser Arbeit erläutert. Zunächst werden die verwendeten Materialgesetze und Eingangsparameter beschrieben und im Anschluss daran die Nachrechnungen der Biege- und Großbauteilversuche. Weitere Erkenntnisse konnten anhand von Parameterstudien gewonnen werden. Die Ergebnisse der experimentellen und rechnerischen Untersuchungen werden in Kapitel 7 zusammengefasst und diskutiert.

In Kapitel 8 wird schließlich das Nachweiskonzept zur Bemessung der Sandwichelemente vorgestellt. Zum einen wird die Schnittgrößenermittlung von Sandwichelementen mit gerissenen Deckschichten mithilfe des erstellten Programms swe2+ beschrieben. Und zum anderen wird die Nachweisführung in Anlehnung an die bestehende Norm zur Bemessung von Sandwichelementen mit metallischen Deckschichten erläutert. 
Weiterhin wurden Brandversuche zur Untersuchung des Brandwiderstands der Elemente durchgeführt. Diese sind in Kapitel 9 beschrieben. Abschließend werden die Erkenntnisse dieser Arbeit im Kapitel 10 zusammengefasst und es wird ein Ausblick auf weiterführende Forschung und Anwendungsgebiete gegeben.

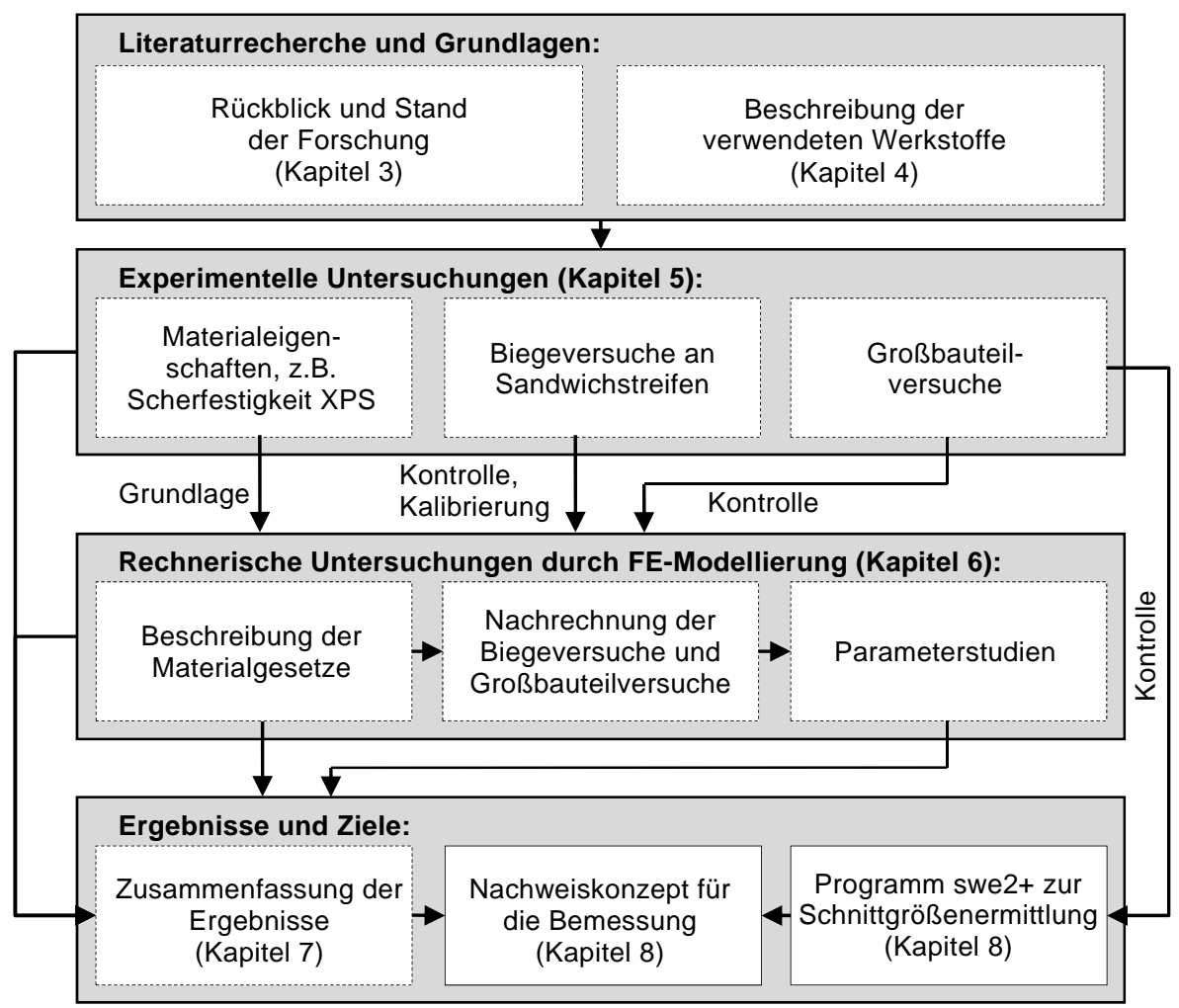

Abb. 2.3: Vorgehensweise zur Untersuchung des Tragverhaltens und Gliederung der Arbeit 



\section{$3 \quad$ Zusammenstellung der Grundlagen}

\subsection{Entwicklungen im Bereich des Hochleistungsbetons}

\subsubsection{Allgemeines}

Durch betontechnologische Entwicklungen in den 1970er-Jahren wurde die Anwendung von Hochleistungsbeton in der Praxis ermöglicht. Zum einen konnte, bei gleichzeitiger Aufrechterhaltung der Verarbeitbarkeit, durch die Verwendung von Fließmitteln das Wasserzementverhältnis (w/z-Wert) des Betons reduziert werden. Zum anderen wurde durch die Verwendung von Silikastaub die Packungsdichte des Betons verbessert. Somit wurde das klassische Drei-Komponenten-Material Beton zu dem heute bekannten modernen Fünf-Komponenten-System aus Wasser, Zement, Zuschlag, Zusatzstoffen und Zusatzmitteln weiter entwickelt.

Die hohen Festigkeiten und Steifigkeiten, die mit Hochleistungsbetonen erzielt werden können, erlauben das Herstellen von sehr schlanken und filigranen Bauteilen, die jedoch aus Gründen von zu geringer Betondeckung und Verbundwirkung nicht mit konventioneller Bewehrung bewehrt werden können. Es ist daher erforderlich andere Bewehrungsarten wie Textilbewehrung und Mikrobewehrung zu verwenden. Als Grundlage für die weiteren Untersuchungen sind die Entwicklungen in diesem Bereich in den folgenden Abschnitten kurz zusammengefasst.

\subsubsection{Mikrobewehrter Hochleistungsbeton}

\subsubsection{Allgemeines}

Obwohl der Gedanke, Beton mit feinmaschigen Drahtgeweben zu bewehren, schon seit Beginn der Geschichte des Stahlbetons verfolgt wurde, beispielsweise durch Joseph Louis Lambot und Joseph Monier Ramm 2012), hat dieser sich wohl aus Wirtschaftlichkeitsgründen in der Praxis nie richtig durchsetzen können. Erst in den 1990erJahren wurde die Idee durch Hauser 1999 wieder aufgegriffen. Die Effektivität und Wirtschaftlichkeit der Stahlfaserbeton-Herstellverfahren SIFCON (Slurry Infiltrated Fiber CONcrete) und SIMCON (Slurry Infiltrated Mat CONcrete) wurde verbessert, indem die Faserpakete mit zwei engmaschigen Drahtmatten zusammengeschnürt wurden. Dieses Verfahren wurde dann als SIMCON NEU bezeichnet.

Die Effektivität konnte weiter gesteigert werden, indem der gesamte Querschnitt vollständig mit mehreren Lagen der Drahtgittermatten bewehrt wurde. Die Problematik der variierenden Faserverteilung und Faserorientierung wurde somit umgangen. Durch die Verwendung der Bewehrung in Verbindung mit einem Hochleistungsbeton konnte eine 
deutliche Leistungssteigerung und ein äußerst duktiles Werkstoffverhalten erzielt werden. Der neue Werkstoff wurde als mikrobewehrter Hochleistungsbeton bezeichnet und $\mathrm{DUCON}^{\circledR}(D U$ ctile CONcrete) genannt, welcher nun von der gleichnamigen Firma vertrieben wird. DUCON ${ }^{\circledR}$ wird mittlerweile für eine Vielzahl unterschiedlicher Anwendungen eingesetzt, u.a. für die Sanierung und Herstellung von Industriefußböden, zur Herstellung von schuß- und explosionssicheren Wänden und Fassaden, zur Verstärkung von Decken und Stützen gegen außergewöhnliche Einwirkungen, für die Herstellung von gestalterischen und architektonischen Elementen. Als tragende Bauteile wurden bisher filigrane Treppen, Rahmentragwerke und Vordächer hergestellt .

Die Mikrobewehrung besteht üblicherweise aus glattem Stahldraht mit einem Durchmesser von ca. 0,3 bis $2 \mathrm{~mm}$. Die Quer- und Längsdrähte werden mit Maschenweiten von 10 bis $20 \mathrm{~mm}$ in einer kontinuierlich arbeitenden Anlage zu einer endlosen Matte zusammengeschweißt, die dann abgelängt und auf einer Rolle transportiert werden kann.

\subsubsection{Experimentelle Untersuchungen}

Das Tragverhalten von mikrobewehrtem Hochleistungsbeton wurde bisher hauptsächlich durch Hauser 1999 sowie durch Schnellenbach-Held u. Prager 2011 untersucht. Hauser 1999 untersuchte in einer Vielzahl von Tastversuchen das Biegetragverhalten an plattenartigen Probekörpern mit einer $90^{\circ}$ und einer $45^{\circ}$-Anordnung der Drahtgittermatten, das Drucktragverhalten an zylindrigen Probekörpern mit horizontaler und vertikaler Anordnung der Matten, sowie das Zugtragverhalten an Prismen mit verschieden Abmessungen. Die Versuche wurden mit verschieden Bewehrungsgehalten, Stahlsorten und Mattentypen durchgeführt.

Durch Schnellenbach-Held $u$. Prager 2011 wurde ebenfalls das Drucktragverhalten an Zylindern mit horizontaler Anordnung der Bewehrung untersucht. Das Zugtragverhalten wurde an kleinen Prismen und das Biegetragverhalten an Balken mit unterschiedlichem Bewehrungsgehalt und stehender und liegender Anordnung der Drahtgittermatten untersucht. In Ausziehversuchen wurde das Verbundverhalten zwischen Beton und Mikrobewehrung ermittelt. Die Versuche dienten als Grundlage für eine FEModellierung mit diskret modellierter Mikrobewehrung.

An der TU Kaiserslautern wurde als Demonstrationsprojekt ein Weinberghaus vollständig aus $30 \mathrm{~mm}$ dünnen Fertigteilelementen aus mikrobewehrtem Hochleistungsbeton hergestellt. Zur Bemessung der Tragfähigkeit der dünnwandigen Elemente, sowie deren Fügung durch eine Nut- und Federverbindung, wurden Biegeversuche an Platten mit einer Dicke von 25 und 30 mm durchgeführt (vgl. Müller|2009, Keil u. Schmitt2010). Zurzeit werden weiterhin zugbeanspruchte Klebverbindungen durch Oster 2012 untersucht. Als Bewehrung kommen auch hier Drahtgittermatten zum Einsatz.

\subsubsection{Tragverhalten}

Über die glatte Oberfläche des Drahtes kann kein ausreichender Verbund mit dem Beton erzielt werden. Jedoch wird durch die angeschweißten Querstäbe Schlupf zwischen Draht und Beton verhindert und der Verbund kann als starr angenommen wer- 
den. Dies wurde in Ausziehversuchen durch Schnellenbach-Held u. Prager 2011 bestätigt.

Die Tragmechanismen sind vergleichbar mit denen des konventionellen Stahlbetons. Betrachtet man die Lastverformungskurve eines 4-Punkt-Biegeversuchs an einer mikrobewehrten Betonplatte mit einer Dicke von $25 \mathrm{~mm}$ (siehe Abbildung 3.1), lassen sich drei Bereiche identifizieren. Zuerst bildet sich ein kurzer, steil ansteigender Teil der Lastverformungskurve im Zustand I aus. Daran schließt ein flacherer Ast an, der durch die Rissbildung im Zustand II bedingt ist. Und letztendlich entsteht ein ausgeprägtes, leicht ansteigendes Fließplateau (Zustand III).

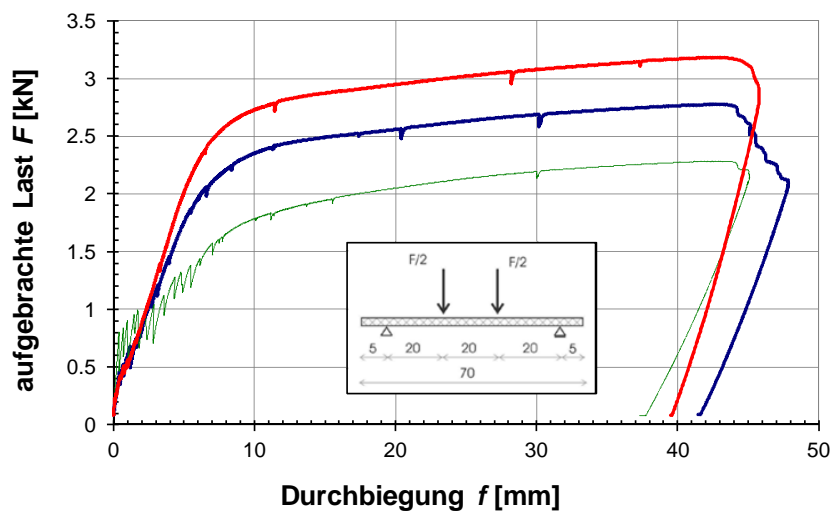

Abb. 3.1: Beispiel für ein typisches Lastverformungsdiagramm eines 4-Punkt-Biegeversuchs an einem mikrobewehrten Betonstreifen mit einer Dicke von $25 \mathrm{~mm}$ [Müller|2009]

Im Zustand II kommt es zu einer ausgeprägten Mehrfachrissbildung mit Rissabständen, die denen der Maschenweite der Drahtgittermatten entsprechen. Die Rissbreiten liegen im Bereich von weniger als 0,04 bis $0,08 \mathrm{~mm}$. Erst bei Erreichen des Fließplateaus, bei dem Drahtgitterlagen nacheinander zu plastizieren beginnen, steigen die Rissbreiten an und die Druckzone der Platte wird eingeschnürt. Schließlich kommt es zum Versagen der Bewehrungslagen oder einem Versagen der Druckzone.

\subsubsection{Bemessung}

Für die praxistaugliche Biegebemessung wurde von Hauser 1999 ein Bemessungsansatz aufgestellt, der dem klassischen $k_{d}$-Verfahren im Stahlbetonbau entspricht. Aus einer Bemessungstabelle kann mithilfe des „aufzunehmenden Moments in Abhängigkeit der Bauteilgeometrie" (Faktors $k$ ) ein Bewehrungsgehalt in Belastungsrichtung $V_{f, \text { eff }}$ in Prozent abgelesen werden, der über den Querschnitt „verschmiert“ angenommen wird. Diesem ingenieurmäßigen Ansatz liegen eine Reihe von Vereinfachungen zugrunde. Infolge der angesetzten Materialeigenschaften, beschränkt sich eine Verwendung der Bemessungstabellen auf die in der Arbeit von Hauser 1999 untersuchte DUCON $^{\circledR}$-Betonrezeptur.

Für eine entsprechende Dehnungsebene, mit der Betondehnung $\epsilon_{D}$ und einer Stahldehnung $\epsilon_{Z}$, kann der Bewehrungsgehalt in Belastungsrichtung $V_{f, \text { eff }}$ wie folgt berech- 
net werden:

$$
V_{f, \text { eff }}=\frac{\sigma_{c k} \cdot\left(\epsilon_{D}-\frac{\sigma_{c k}}{2 \cdot E_{D U C}}\right)}{\frac{1}{2} \cdot\left(f_{y k}+f_{t k}\right) \cdot\left(\epsilon_{Z}-\frac{f_{y k}}{2 \cdot E_{S}}\right)}
$$

Hierbei bedeuten:

$\begin{array}{llll}\sigma_{c k} & : & \text { charakteristische Druckspannung des Betons in } \mathrm{N} / \mathrm{mm}^{2} \\ f_{y k} & : & \text { charakteristische Streckgrenze des Stahls in } \mathrm{N} / \mathrm{mm}^{2} \\ f_{t k} & : & \text { charakteristische Zuggrenze des Stahls in } \mathrm{N} / \mathrm{mm}^{2} \\ E_{D U C} & : & \text { Elastizitätsmodul des bewehrten Betons in } \mathrm{N} / \mathrm{mm}^{2} \text { nach Gl. } \\ & & 3.2 \\ E_{S} & : & \text { Elastizitätsmodul des Stahls in } \mathrm{N} / \mathrm{mm}^{2}\end{array}$

Dabei wird $E_{D U C}$ vereinfachend mit der folgenden empirischen Beziehung berechnet:

$$
E_{D U C}=\left(1-V_{f}\right) \cdot E_{C}+V_{f} \cdot E_{S}
$$

Mit:

$$
\begin{array}{lll}
V_{f} & : & \text { Gesamtbewehrungsgehalt mit } V_{f}=2 \cdot V_{f, \text { eff }} \\
E_{C} & : & \text { Elastizitätsmodul des Betons in } \mathrm{N} / \mathrm{mm}^{2}
\end{array}
$$

Die beiden Gleichungen 3.1 und 3.2 sind voneinander abhängig. Eine explizite Lösung ist sehr komplex. Durch Iteration kann jedoch der Bewehrungsgehalt $V_{f \text {, eff }}$ in Abhängigkeit vom Elastizitätsmodul $E_{D U C}$ für eine bestimmte Dehnungsebene gefunden werden.

Das aufnehmbare Moment $M_{z u l}$ berechnet sich aus dem dem Faktor $k$ wie folgt:

$$
M_{z u l}=\frac{2 \cdot k}{b \cdot h^{2}}
$$

Dabei sind die Höhe $h$ und die Breite $b$ die Bauteilabmessungen. Der Faktor k errechnet sich zu:

$$
k=\sigma_{c k} \cdot\left(T_{D 1}{ }^{2}-\frac{1}{3} T_{D 2}{ }^{2}\right)+V_{f, \text { eff }} \cdot \frac{1}{2}\left(f_{y k}+f_{t k}\right) \cdot\left(T_{D 1}{ }^{2}-\frac{1}{3} T_{Z}{ }^{2}-2 T_{D 1}+1\right)
$$

Mit den Hilfstermen:

$$
\begin{aligned}
& T_{D 1}=\frac{\epsilon_{D}}{\epsilon_{Z}+\epsilon_{D}} \\
& T_{D 2}=\frac{1}{\epsilon_{Z}+\epsilon_{D}} \cdot \frac{\sigma_{c k}}{E_{D U C}} \\
& T_{Z}=\frac{1}{\epsilon_{Z}+\epsilon_{D}} \cdot \frac{f_{y k}}{E_{S}}
\end{aligned}
$$


Der Faktor $k$ kann in Abhängigkeit von $V_{f \text {, eff }}$ und $E_{D U C}$ für die jeweilige Dehnungsebene berechnet werden. Stellt man die Werte in einer Tabelle zusammen, ist eine Bemessung einfach möglich. Dabei kann ausgehend von einem vorhandenen Bewehrungsgehalt $V_{f, \text { eff }}$ das zulässige Moment $M_{z u l}$ oder ausgehend von einem aufzunehmenden Moment, der erforderliche Bewehrungsgehalt bestimmt werden.

In Hauser 1999 wird nicht angegeben, auf welche Weise Teilsicherheitsbeiwerte bei der Erstellung der Bemessungstabellen eingeflossen sind. Aus den Versuchsserien ergaben sich für den mikrobewehrten Beton, Teilsicherheitsbeiwerte $\gamma_{D U C}$ im Bereich von 1,06 bis 1,18 (Hauser $u$. Wörner 1999).

\subsubsection{Textilbewehrter Beton}

\subsubsection{Allgemeines}

Die Gründe für die Entwicklung des Textilbetons sind vergleichbar mit den Gründen für die Entwicklung des mikrobewehrten Hochleistungsbetons. Durch die Verwendung von in Beanspruchungsrichtung orientierten Endlosfasern sollte die Effektivität von Faserbeton aus nichtmetallischen Fasern verbessert werden. Verfahren aus der Textiltechnik ermöglichen das Herstellen von großflächigen Textilien aus den Hochleistungsfasern und erlauben einen wirtschaftlichen Einsatz in der Bauindustrie.

Textilbewehrter Beton ist in den 1990er-Jahren erstmalig verwendet und untersucht worden. In den beiden Sonderforschungsbereichen (SFB) der Deutschen Forschungsgemeinschaft an der TU Dresden und der RWTH Aachen (SFB 528 „Textile Bewehrungen zur bautechnischen Verstärkung und Instandsetzung“ und SFB 532 „Textilbewehrter Beton - Grundlagen für die Entwicklung einer neuartigen Technologie“) wurde Textilbeton umfassend untersucht. In den Forschungsbereichen wurden vor allem alkaliresistende (AR) Glasfaser-Textilien und später auch verstärkt Carbonfaser-Textilien eingesetzt. Einen guten Überblick über die Ergebnisse und einen Einstieg in die Thematik bietet vor allem Jesse $u$. Curbach 2010. Äußerst umfangreiche Informationen finden sich in den Tagungsbänden der Kolloquien, die im Rahmen der Sonderforschungsbereiche stattfanden Hegger 2001. Curbach 2003 Hegger u. a. 2006 Curbach u. Jesse 2009), sowie in den entsprechenden Dissertationen (bspw. Jesse 2004 Molter 2005 Voss 2008.

\subsubsection{Grundlagen und Begrifflichkeiten}

Da Textilbeton zurzeit noch eine sehr junge Entwicklung in der Bauindustrie darstellt, werden die in der vorliegenden Arbeit verwendeten Fachbegriffe aus der Textiltechnik zur besseren Verständlichkeit im Folgenden kurz erläutert:

- Filament - bezeichnet eine einzelne Elementarfaser mit unbegrenzter oder großer Länge.

- Multifilamentgarn (auch Filamentgarn, Roving) - sind Begriffe die ein Bündel aus zahlreichen parallelen Filamenten, d. h. ohne Drehung der einzelnen Filamente, bezeichnen. 
- Faden - ist ein Oberbegriff von Garn und Zwirn.

- Faser, Garn, Zwirn - sind Oberbegriffe für Elemente, die zur Herstellung von textilen Strukturen, z. B. Gewebe, Gelege, geeignet sind. Je nach Kontext kann der Begriff „Faser" auch ein Filament, ein Multifilamentgarn oder eine Kurzfaser bezeichnen. Der Begriff „Garn“ wird verwendet, wenn die einzelnen Filamente einen gewissen Zusammenhalt aufweisen, z. B. durch eine Drehung der Filamente (gedrehtes Garn) oder durch eine Verdrehung mehrerer Garne (Zwirn), oder durch eine Schlichte.

- Schlichte - ist eine während der Garnherstellung aufgetragene Beschichtung.

- Sekundärbeschichtung - ist eine zweite Beschichtung, die neben anderen Funktionen vor allem zur Verstärkung und Stabilität, sowie zur Verbesserung der Verbundeigenschaften des Garns dient.

- Feinheit - ist ein Maß zur Beschreibung der linearen Dichte eines Filaments oder einer Faser. Die Maßeinheit der Feinheit $f$ ist tex. Dabei entspricht 1 tex einem Gramm je $1000 \mathrm{~m}$ Länge der Textilfaser. Der Querschnitt einer Faser berechnet sich zu:

$$
A[\mathrm{~mm}]=\frac{f}{\rho}=\frac{f[\mathrm{~g} / 1000 \mathrm{~m}]}{\rho\left[\mathrm{g} / \mathrm{cm}^{3}\right] \cdot 10^{3}}
$$

- Textil - ist ein Sammelbegriff für ein durch unterschiedliche Textilverfahren hergestelltes Gebilde, z. B. Gelege, Gewebe, Gestrick etc.

- Gelege - sind Textilien die durch das Aufeinanderlegen paralleler Fäden entstehen. Zur Verwendung des Geleges als Bewehrung müssen die Fäden an den Knotenpunkten durch eine Bindung mit einem Nähfaden fixiert werden. Mit dem Nähverfahren können unterschiedliche Bindungsarten, z. B. Trikot- und Fransebindung, hergestellt werden.

- Gewebe - sind Textilien die durch Fachbildung aus rechtwinklig verkreuzten Kettund Schußfäden hergestellt werden.

- Kettfaden - ist der Faden, der in Herstellungsrichtung, d. h. Kettrichtung, des Textils verläuft.

- Schussfaden - ist der Faden, der rechtwinklig zur Herstellungsrichtung, d. h. Schussrichtung, des Textils verläuft.

Die Begriffserläuterungen stammen hauptsächlich aus Jesse $u$. Curbach 2010 Für eine detaillierte Beschreibung der Begriffe sowie der Herstellungsverfahren sei hierauf verwiesen.

\subsubsection{Experimentelle Untersuchungen}

Das Tragverhalten von textilbewehrtem Beton wurde ausführlich durch Jesse 2004 Molter 2005 und Voss 2008 experimentell untersucht. In allen drei Arbeiten wurde das Zugtragverhalten in Dehnkörperversuchen ermittelt. Durch Molter 2005 und Voss 2008 wurden weiterhin Biege-, und Querkraftversuche durchgeführt. 
Die Dehnkörperversuche wurden an Probekörpern durchgeführt, die sich hinsichtlich inrer Herstellungsweise, der Form, den Gesamtabmessungen, dem Lasteinleitungsverfahren und der Lasteinleitungslänge unterschieden. In den Versuchen durch Jesse 2004 zeigte sich, dass sich liegend hergestellte Probekörper nur bedingt eignen, da diese eine ungleichmäßige Schal- und Betonierseite aufweisen, die sich im Hinblick auf ihre Oberflächenqualitäten, Nachbehandlung, Schwindverhalten und Zusammensetzung des Betons unterscheiden. Im Rahmen der Arbeiten von Molter 2005 und Voss 2008 wurden daher stehend betonierte Probekörper verwendet. Zur Sicherstellung einer exakten Lage und eines geraden Verlaufs der Textilbewehrung wurde diese gegen die Schalung leicht angespannt.

Weiterhin zeigte sich, dass sich Probekörper, die im Messbereich in der Dicke oder der Breite tailliert sind, am besten eignen. Durch die Taillierung kann sichergestellt werden, dass die Rissbildung des Betons und das Versagen der Textilbewehrung im Messbereich auftreten.

Als Lasteinleitungskonstruktion wurde durch Jesse 2004 eine Keilklemmverankerung verwendet. Da bei dieser Konstruktion jedoch eine ungleichmäßige Pressung der Probekörper im Bereich der Lasteinleitung auftrat, wurden im Rahmen der Arbeiten von Molter 2005 und Voss 2008 Lochbleche mit einer angeschweißten Hülse einbetoniert, die gelenkig an die Lasteinleitungs- bzw. Auflagerkonstruktion angeschlossen werden konnte.

\subsubsection{Tragverhalten}

Das Lastverformungsverhalten von textilbewehrtem Beton gleicht dem Verhalten des konventionellen Stahlbetons (siehe Abbildung 3.2). Im ungerissenen Zustand des Betons werden die Spannungen überwiegend über die steife Betonmatrix übertragen. Beim Überschreiten der Betonzugfestigkeit geht das Werkstoffverhalten in den Zustand II a über. Die Lastverformungskurve flacht hier deutlich ab. Erst wenn ein abgeschlossenes Rissbild entstanden ist, steigt die Lastverformungskurve wieder an (Zustand II b) bis schließlich die Bewehrungsfasern spröde und ohne Vorankündigung reiBen.

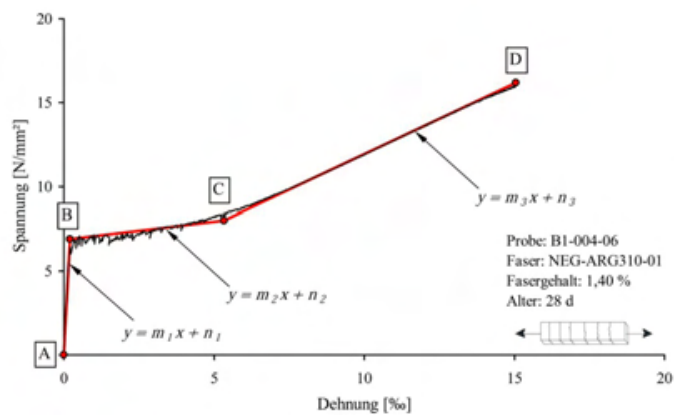

Abb. 3.2: Beispiel für ein typisches Spannungsdehnungsdiagramm eines Dehnkörperversuchs an Textilbeton Jesse 2004 
Im Unterschied zum Stahlbeton, kann bei Textilbeton kein voller Verbund zwischen Beton und den Filamenten der Bewehrung angenommen werden. Die Randfasern des Rovings, die im vollen oder teilweisen Verbund mit der Betonmatrix stehen, werden stärker beansprucht, als die Kernfasern, die keinen direkten Kontakt zur Betonmatrix aufweisen, und dadurch nur zum Teil oder gar nicht beansprucht werden. Der Faserquerschnitt der Textilien erreicht daher nicht die theoretische mögliche Festigkeit. Die praktisch erreichbaren Festigkeiten sind von einer Vielzahl unterschiedlicher Parameter abhängig. In Jesse u. Curbach 2010 sind einige dieser Parameter beispielhaft angegeben: Garnsorte, Anzahl der Filamente, Parameter der textilen Verarbeitung, Typ und Qualität der Sekundärbeschichtung, etc. Die theoretisch erreichbare Festigkeit wird daher zur Bemessung um einen Effektivitätsfaktor abgemindert.

\subsubsection{Bemessung}

\section{Zugtragfähigkeit:}

Die Zugtragfähigkeit kann vereinfachend nach Hegger $u$. Bornefeld 2012 berechnet werden:

$$
F_{t}=A_{t} \cdot f_{\text {fil }} \cdot k_{1}
$$

Dabei bedeuten:

$\begin{array}{lll}A_{t} & : & \text { Querschnittsfläche der Bewehrung in } \mathrm{mm}^{2} \\ f_{\text {fil }} & : & \text { Filamentzugfestigkeit in } \mathrm{N} / \mathrm{mm}^{2} \\ k_{1} & : & \text { Effektivitätsfaktor der Bewehrung }\end{array}$

Der Effektivitätsfaktor $k_{1}$ beschreibt das Verhältnis der experimentell ermittelten Bruchspannung der Filamente im eingebauten Zustand zur Filamentzugfestigkeit. Der Effektivitätsfaktor gibt an in wieweit das Roving beansprucht bzw. aktiviert werden kann und ist von den zuvor genannten Faktoren abhängig. Aufgrund der Vielzahl der unterschiedlichen Parameter gibt es bisher keine Ingenieurmodelle zur Abschätzung des Effektivitätsfaktors, so dass dieser experimentell in Dehnkörperversuchen bestimmt werden muss. Die bereits in Versuchen bestimmten Effektivitätsfaktoren liegen im Bereich von 0,18 bis 0,51 für AR-Glasfaser und 0,15 bis 0,23 für Carbonfaser-Textilien Hegger $u$. Bornefeld 2012, Hegger u. a. 2007).

Durch die Abminderungsfaktoren $k_{0, \alpha}, k_{2}, k_{3}$ und $k_{\rho}$ können weitere negative Einflüsse durch schiefwinklige Beanspruchung, zweiaxiale Beanspruchung, Herstellung durch Laminieren und erhöhte Bewehrungsgrade berücksichtigt werden.

\section{Biegetragfähigkeit:}

Die Biegetragfähigkeit errechnet sich aus der Zugtragfähigkeit $F_{t}$ wie folgt:

$$
M_{u}=k_{f l} \cdot F_{t} \cdot z
$$

Dabei ist $z$ der innere Hebelarm und $k_{f l}$ ein Beiwert zur Berücksichtigung der Biegetragfähigkeit. Der Beiwert wurde aus Versuchen an I-Profilen und Rechtquerschnitten 
mit unterschiedlichen Textilarten bestimmt. Der Beiwert für Rechteckquerschnitte (und Textilien mit Fransebindung und Teilschuss) ist in Hegger $u$. Bornefeld 2012 wie folgt angegeben:

$$
k_{f l}=1,0+0,47 \cdot \rho_{\text {geom }}
$$

Dabei ist $\rho_{\text {geom }}$ der geometrische Längsbewehrungsgrad in Prozent.

\section{Querkrafttragfähigkeit:}

Die Ansätze zur Bemessung der Querkrafttragfähigkeit von textilbewehrtem Beton mit und ohne Querkraftbewehrung wurden durch Voss 2008 aufgestellt. Für die Bemessung der Querkrafttragfähigkeit ohne Querkraftbewehrung wurden ein empirischer als auch ein modellbasierter Ansatz aufgestellt. Nach dem empirischen Ansatz in Anlehnung an Eurocode 2 (DIN EN 1992 2011) errechnet sich die Querkrafttragfähigkeit wie folgt:

$$
V_{c}=0,158 \cdot \beta \cdot \kappa \cdot\left(100 \cdot \rho_{l} \cdot \frac{\sigma_{t e x}}{f_{y k}} \cdot f_{c m}\right)^{1 / 3} \cdot b_{s, e f f} \cdot d
$$

Mit:

$$
\begin{array}{lll}
\beta=\frac{3}{a / d}>1,0 & : & \begin{array}{l}
\text { Faktor zur Berücksichtigung der Sprengtragwir- } \\
\text { kung, mit a des Auflagerabstands und } d \text { der } \\
\text { statischen Höhe }
\end{array} \\
\kappa=1+\sqrt{\frac{200}{d} \leq 2,0} & : & \text { Maßstabsfaktor mit } d \text { in mm } \\
\rho_{l}=\frac{A_{t}}{b_{w} \cdot d} & : & \text { Längsbewehrungsgrad } \\
\sigma_{t e x} & : & \text { Textilspannung in Längsrichtung } \\
f_{y k} & : & \begin{array}{l}
\text { charakteristische Zugfestigkeit des Betonstahls } \\
\text { an der Streckgrenze }
\end{array} \\
f_{c m} & : & \begin{array}{l}
\text { mittlere Betondruckfestigkeit } \\
\text { effektive äquivalente Stegbreite, bei Rechteck- } \\
b_{s, \text { eff }}
\end{array} \\
& \text { querschnitten } b_{s, \text { eff }}=b
\end{array}
$$

Weiterhin wurden bereits Ansätze für die Bemessung von textilbewehrten Bauteilen unter punktförmiger Zug- und Druckbeanspruchung aufgestellt. Durch Hegger $u$. Bornefeld 2012 wird für alle Nachweise im Zustand der Tragfähigkeit ein einheitlicher Materialsicherheitsbeiwert $\gamma_{\text {tex }}$ von 1,5 vorgeschlagen.

\subsection{Extrudierter Polystyrol-Hartschaum (XPS)}

\subsubsection{Allgemeines}

Extrudierter Polystyrol-Hartschaum (XPS), auch Polystyrol-Extruderschaum genannt, wurde 1941 von der Firma Dow Chemical Company im Auftrag des US-Verteidigungs- 
ministeriums entwickelt. Im 2. Weltkrieg sollten hiermit schnell installierbare und belastbare Schwimm- und Auftriebskörper für militärische Landemanöver und Flussüberquerungen hergestellt werden (FPX 2012). Nach dem Krieg ab 1948 wurde das Material aufgrund seiner guten Dämmeigenschaften und seiner hohen Feuchtebeständigkeit unter anderem im Schiffsbau und zum Bau von Kühlhäusern eingesetzt. Vor allem wurde das XPS aber im Baubereich zur Wärmedämmung von Wohn- und Industriegebäuden verwendet. Das Produkt wurde von der Firma Dow unter dem Namen „Styrofoam ${ }^{\circledR}$ “ vermarktet. 1963 begann erstmalig die Vermarktung von Styrofoam ${ }^{\circledR}$ in Europa. Zeitgleich erfolgte auch die Entwicklung eines XPS-Dämmmaterials in Europa durch die Firma BASF. Die hellgrünen Hartschaumplatten sind auf dem deutschen Markt unter dem Namen „Styrodur ${ }^{\circledR}$ “" bekannt und weit verbreitet.

Im Vergleich zu anderen Dämmstoffen weist XPS eine hohe Druckfestigkeit sowie eine hohe Feuchte- und Verrottungsbeständigkeit auf. Aus diesen Gründen wird XPS überwiegend zur Dämmung von druck- und feuchtebeanspruchten Bauteilen und Konstruktionen eingesetzt, beispielsweise als Perimeterdämmung von erdberührten Kellerwänden und Bodenplatten, oder für die Dämmung von Flachdächern in Umkehrbauweise. Weiterhin wird XPS für die Konstruktion von druckbeanspruchten Flächen eingesetzt, z. B. Parkdecks, Industriefußböden, Frostschutzschichten im Verkehrswegebau etc. (Boy 2005).

Die Angaben in der Literatur zum Tragverhalten von XPS beschränken sich aufgrund der genannten Anwendungen überwiegend auf das Tragverhaltens unter einer Druckbeanspruchung. Durch Krollmann 1995 wurde das Langzeitverhalten von extrudiertem Polystyrol-Hartschaum unter konstanter und zyklisch wechselnder Druckbeanspruchung untersucht. Das Kurzzeit- und Langzeittragverhalten unter Schubbeanspruchung wurde im Auftrag der BASF durch die Leibniz Universität Hannover (Fouad 2010b Fouad 2008) ermittelt.

\subsubsection{Herstellung}

Ausgangsstoff für die Herstellung von extrudiertem Polystyrol-Hartschaum ist Polystyrolgranulat. Polystyrolgranulat wird aus den beiden, aus Erdöl gewonnenen, Grundstoffen Benzol und Ethylen hergestellt. Diese werden unter Verwendung eines Katalysators zu Ethylenbenzol alkyliert, welches anschließend zu monomerem Styrol dehydriert wird. Unter Zugabe von Treibmitteln, Additiven und Stabilisatoren wird das Styrol polymerisiert. Es ensteht ein perlenförmiges, glasähnliches Granulat mit einem Durchmesser von 1 bis $3 \mathrm{~mm}$ (Fouad 2010a). Vereinfachend kann der chemische Prozess auch als eine Polymerisation von Monostyrol zusammengefasst werden (Störi 1979). Daher resultiert der Name „Polystyrol“.

Zur Herstellung des Schaumstoffs wird das Granulat aufgeschmolzen und mit einem Treibmittel in einem Extruder vermischt. Als Treibmittel werden $\mathrm{CO}_{2}$ (Kohlendioxid, ca. 7 Gew.-\%) oder HFKW (teilfluorierte Kohlenwasserstoffe) hinzugegeben. Weiterhin werden 1 bis 2 Gew.-\% Bromverbindungen als Flammschutzmittel hinzugefügt (Fouad 2010a). Das extrudierte Gemisch aus aufgeschmolzenem Polystyrolgranulat, Treibund Zusatzmitteln, wird aus dem Extruder über eine sogenannte Breitschlitzdüse in einem endlosen Schaumstoffband auf ein Förderband ausgetragen. Dabei lassen sich 
Dicken von 20 bis $200 \mathrm{~mm}$ herstellen. Im Anschluss durchläuft der Schaumstoff eine Kühlzone und wird zu Dämmplatten mit unterschiedlichen Abmessungen und Kantenausbildungen konfektioniert (siehe Abbildung 3.3. Abschließend werden die Platten bis zur Massenkonstanz gelagert [Fouad 2010a, Boy 2005). Bei mit $\mathrm{CO}_{2}$ geschäumtem XPS entweicht das Zellgas schon kurz nach der Herstellung und Luft diffundiert in das Zellgefüge ein (Boy 2005).

Aufschmelzen des Polystyrols und Zugabe des Treibmittels

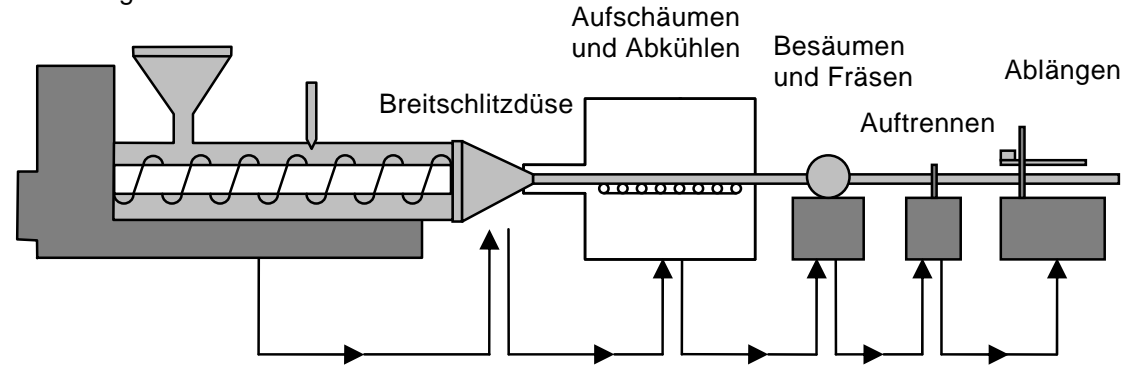

Abb. 3.3: Schematische Darstellung der Herstellung von XPS (Fouad 2010a)

Produktionstechnisch bedingt wird der noch warme, extrudierte Schaumstoff beim Austragen aus der Breitschlitzdüse im Randbereich verdichtet. Dadurch entsteht die charakteristische glatte Schäumhaut der Dämmplatten. Um bei speziellen Anwendungen des XPS einen besseren Haftverbund mit beispielsweise Beton und Mörtel zu erzielen, kann die Schäumhaut abgehobelt werden oder eine Struktur thermisch eingeprägt werden (Boy 2005). Bei den für dieses Forschungsprojekt verwendeten Styrodur ${ }^{\circledR}$-Platten wird eine rautenförmige Waffelstruktur mithilfe einer auf ca. $200^{\circ} \mathrm{C}$ erhitzen Walze eingeprägt.

\subsubsection{Materialeigenschaften}

\subsubsection{Zellstruktur und Rohdichte}

Durch den Extrusionsprozess entsteht ein geschlossenzelliger Schaumstoff. Die Wände der Zellen sind ebene, polygonale Flächen. Diese formen eine Struktur aus unregelmäßigen Polyedern (siehe Abbildung 3.4 und 3.5. Die Zellwände weisen über ihre Länge eine veränderliche Dicke auf. Durch die Oberflächenspannung wird das flüssige Polystyrol beim Extrusionsprozess zum Teil in die Randbereiche der Zellwände gezogen. Im Vergleich zu den Zellwänden, weisen die Zellkanten aufgrund ihrer gröBeren Dicke eine höhere Steifigkeit auf und neigen durch die aussteifenden Zellwände weniger zum Ausknicken.

Durch Variation der verschiedenen Verfahrensparameter kann die Schaumstoffrohdichte in gewissen Grenzen gesteuert werden. So ist es möglich XPS im Rohdichtebereich von ca. 25 bis $50 \mathrm{~kg} / \mathrm{m}^{3}$ herzustellen. Abhängig von der Rohdichte ändert sich auch die Zellgröße des Schaumstoffs. Ein XPS mit geringer Rohdichte weist größere Zellen auf, als ein XPS mit großer Rohdichte. 


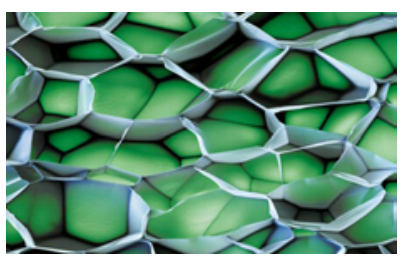

Abb. 3.4: Darstellung der geschlossenen, Polyeder-förmigen Zellen eines XPS „Styrodur ${ }^{\circledR}$ C“ Chemiereport.at 2008

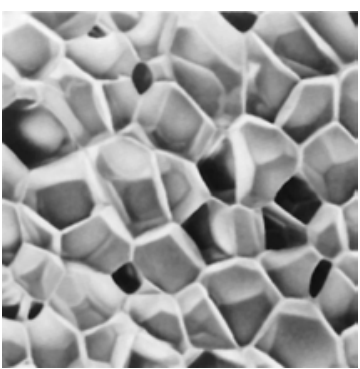

Abb. 3.5: Darstellung der geschlossenen, Polyeder-förmigen Zellen eines XPS (FPX 2013

Bedingt durch den Extrusionsprozess ist die Rohdichte über die Dickenrichtung der Platten nicht konstant. Zum Rand der Platte hin nimmt die Rohdichte ab und die Zellgröße zu. In der Mitte der Platten ist die Rohdichte am größten und die Zellgröße am kleinsten. Dieser Effekt ist bei dickeren Platten deutlicher ausgeprägt als bei dünnen Platten.

Auch die Zellform wird durch den Extrusionsprozess beeinflusst. Die Zellen sind in der Regel nicht gedrungen, sondern länglich und senkrecht zur Extrusionsrichtung ausgerichtet, d.h senkrecht zur Extrusionsrichtung sind die Zellen im Durchschnitt am längsten, quer zur Extrusionsrichtung nimmt die Breite und Höhe der Zellen ab Gausepohl u. Gellert 1996). Da das Materialverhalten eines Schaums grundsätzlich von der Zellgeometrie abhängig ist, kann XPS aufgrund des Einflusses des Extrusionsprozesses als anisotrop charakterisiert werden.

Die Zellstruktur des XPS wurde im Rahmen dieser Arbeit durch Auflichtmikroskop- und Computertomographie-Aufnahmen näher untersucht (siehe Abschnitt 4.3.3, Zellstruktur und Oberflächenstruktur ").

\subsubsection{Materialverhalten unter Druckbeanspruchung}

Das Materialverhalten von Schaumstoffen unter Druckbeanspruchung kann mit denen in Gibson $u$. Ashby 2001 zusammengefassten Theorien zum Tragverhalten von offen- und geschlossenzelligen Schäumen beschrieben werden. Nach deren Lastverformungsverhalten können Schaumstoffe in die drei folgenden Kategorien unterteilt werden:

- „elastomeric foam“ - elastischer Schaumstoff

- „elastic-plastic foam“ - elastisch-plastischer Schaumstoff

- „elastic-brittle foam“ - elastisch-spröder Schaumstoff

Die Lastverformungskurven aller drei Kategorien weisen im Bereich geringer Belastung ein linear-elastisches Verhalten auf (siehe Abbildung 3.6. Im Anschluss bildet sich ein Fließplateau aus. Im Falle des elastischen Schaumstoffs steigt das Fließplateau leicht an. Wobei das Plateau eines elastisch-plastischen Schaumstoffs nahezu 
horizontal verläuft. Bei einem elastisch-spröden Schaumstoff ist das Fließplateau charakterisiert durch eine gezackte Lastverformungskurve. Bei allen drei Schaumstoffen steigen im Anschluss an das Fließplateau aufgrund der Verdichtung des Schaumstoffs die Lastverformungskurven wieder steil an.
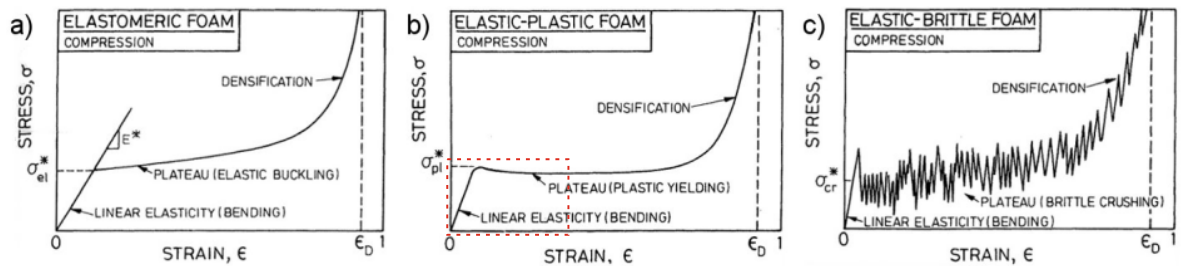

Abb. 3.6: Spannungsdehnungsdiagramm unter Druckbeanspruchung eines a) elastischen Schaumstoffs, b) elastisch-plastischen Schaumstoffs, c) elastisch-spröden Schaumstoffs (Gibson u. Ashby|2001)

Das Lastverformungsverhalten ist abhängig vom Ausgangsmaterial des Schaumstoffs. Ist das Material elastisch, so ist auch das Lastverformungsverhalten des Schaumstoffs elastisch. Besitzt das Material eine Fließgrenze, wie zum Beispiel Polymere oder Metalle, verhält sich der daraus bestehende Schaumstoff elastisch-plastisch. Bei spröden Materialien, wie beispielsweise Keramik oder Glas, entsteht ein elastisch-spröder Schaumstoff. Polystyrol ist ein elastisch-plastischer bzw. thermoplastischer Werkstoff. Somit kann XPS als elastisch-plastischer Schaumstoff kategorisiert werden. Vergleicht man die in Druckversuchen ermittelten Spannungsdehnungskurven eines XPS der Marke Styrodur $^{\circledR}$ (siehe Abbildung 3.7) mit denen in Gibson u. Ashby 2001 (siehe Abbildung 3.6 b) markierter Bereich) ist eine gute Übereinstimmung im Verlauf der Kurven zu erkennen.

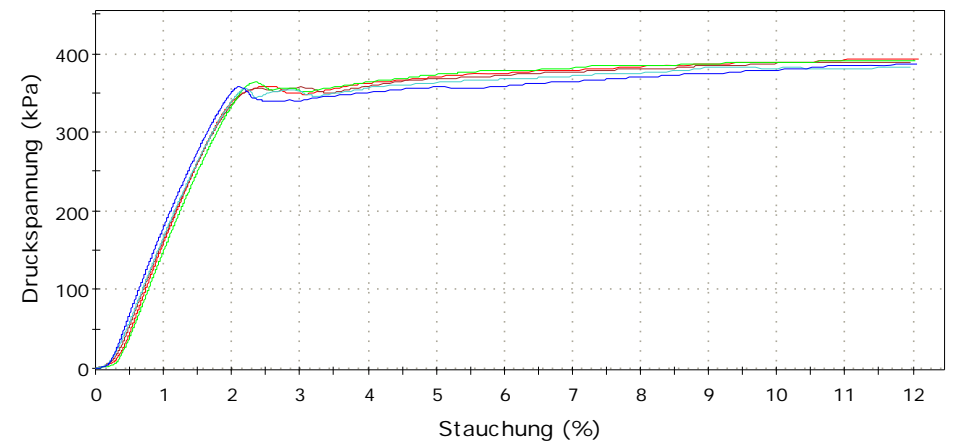

Abb. 3.7: Beispielhaftes Druckspannungsdehnungsdiagramm eines XPS (Styrodur ${ }^{\circledR} 3035$ CS, $d=100 \mathrm{~mm}$ )

Im linear-elastischen Bereich des Materialverhaltens setzen sich die Verformungsmechanismen eines elastisch-plastischen Schaumstoffs aus mehreren Anteilen zusammen (siehe Abbildung 3.8. Die auf die Zellstruktur wirkende Druckbeanspruchung wird überwiegend über die steifen Kanten der Zellen übertragen. Diese bilden ein räumliches Fachwerk aus biegesteif miteinander verbundenen Zug- und Druckstäben. Somit 
setzt sich die Verformung aus einer Biegeverformung, sowie einer Zug- und Druckdehnung der Zellkanten zusammen. Die weniger steifen Zellwände neigen unter Druckbeanspruchung in Belastungsrichtung zum Ausknicken bzw. Beulen. Rechtwinklig zur Belastungsrichtung tragen sie jedoch als Zugbänder zur Erhöhung der Steifigkeit des räumlichen Tragwerks bei. Weiterhin trägt das Zellgas in den geschlossenen Zellen zur Erhöhung der Steifigkeit bei.

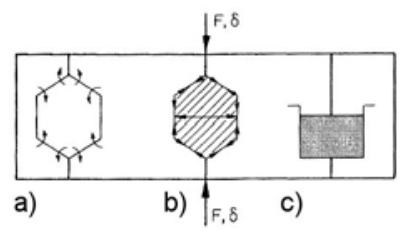

Abb. 3.8: Verformungsmechanismen im linear-elastischen Lastverformungsbereich eines geschlossenzelligen Schaumstoffs unter Druckbeanspruchung: a) Biegung der Zellkanten, b) Dehnung der Zellkanten und Zugdehnung der Zellwände, c) Kompression des Zellgases Gibson u. Ashby 2001)

Wird der Schaumstoff über den linear-elastischen Bereich hinaus beansprucht, beginnen die als Zugbänder wirkenden Zellwände zu plastizieren. An den Knotenpunkten der Zellkanten bilden sich Fließgelenke und es kommt zum plastischen Versagen der Zellen. Bei weiterer Belastung werden die Zellen aufeinander gedrückt und verdichtet, so dass eine Kraftübertragung verbessert wird und die Lastverformungskurve wieder steil ansteigt.

Bei einem XPS mit einer geringen Rohdichte muss das Polystyrol im Extrusionsprozess stärker expandiert werden. Dadurch nehmen die Abmessungen der Zellen zu. Die Zellwände und -kanten werden insgesamt schlanker und neigen schneller zum Ausknicken. Durch die geringere Dicke der Zellwände nehmen auch die Biege- und Dehnverformungen erheblich zu, so dass generell ein XPS mit geringer Rohdichte einen kleineren Elastizitätsmodul und eine geringere Festigkeit aufweist, als ein XPS mit größerer Rohdichte (siehe Tabelle 3.1. . Der Zusammenhang zwischen Rohdichte und Druckfestigkeit ist ungefähr linear (Boy 2005). Nach Tabelle 3.1]entspricht der Elastizitätsmodul etwa dem 60 bis 75-fachen der Druckfestigkeit.

Tab. 3.1: Anhaltswerte der Druckfestigkeit und des Kurzzeit-Druckelastizitätsmoduls von XPS in Dickenrichtung in Abhängigkeit der Rohdichte Boy 2005

\begin{tabular}{|ccc|}
\hline $\begin{array}{c}\text { Rohdichte } \\
{\left[\mathrm{kg} / \mathrm{m}^{3}\right]}\end{array}$ & $\begin{array}{c}\text { Druckfestigkeit } \\
{\left[\mathrm{kN} / \mathrm{m}^{2}\right]}\end{array}$ & $\begin{array}{c}\text { Kurzzeit-Druckelastizitätsmodul } \\
{\left[\mathrm{kN} / \mathrm{m}^{2}\right]}\end{array}$ \\
\hline$<30$ & 200 & 15.000 \\
$30-35$ & 300 & 20.000 \\
$35-45$ & 500 & 30.000 \\
$>45$ & 700 & 40.000 \\
\hline
\end{tabular}

Die Bestimmung des Verhalten von Wärmedämmstoffen unter Druckbeanspruchung ist in DIN EN 826 geregelt. Um eine Druckfestigkeit des Wärmedämmstoffs definieren zu können, werden die Druckspannungen üblicherweise bei einer Stauchung von $10 \%$ angegeben. Falls erforderlich, kann die Druckfestigkeit auch bei niedrigeren Stau- 
chungen definiert werden. Nach DIN EN 826 wird der Elastizitätsmodul für den „exakt geraden Abschnitt“ der Kraftverformungskurve berechnet.

\subsubsection{Materialverhalten unter Zugbeanspruchung}

Das Materialverhalten von XPS unter Zugbeanspruchung ist linear-elastisch. Wie unter Druckbeanspruchung auch, setzen sich die Dehnungen unter einer Zugbeanspruchung aus der Biegeverformung der Zellkanten, der Dehnung der Zellwände und des Gasdrucks im Inneren der Zellen zusammen. Daher weisen die Druckfließspannung und die Zugfließspannung eines elastisch-plastischen Schaumstoffs in etwa die gleichen Werte auf.

Das Versagen bei Zugbeanspruchung ist hingegen spröde. Eine kleine Beschädigung in der Zellstruktur verursacht eine Spannungskonzentration und das Reißen einer Zellwand. Der entstandene Riss setzt sich in der Zellstruktur weiter fort und führt dann zu einem schlagartigen Versagen des Schaumstoffs (Ashby 1983).

Die Bestimmung der Zugfestigkeit in Plattenebene erfolgt nach DIN EN 1608 und die Bestimmung der Zugfestigkeit senkrecht zur Plattenebene nach DIN EN 1607. Zur Berechnung der Zugfestigkeit wird die größte aufgezeichnete Zugkraft verwendet. Eine Angabe zur Berechnung des Zug-Elastizitätsmoduls wird nicht gemacht.

\subsubsection{Materialverhalten unter Scherbeanspruchung}

Für das Materialverhalten von Schaumstoffen unter einer Scherbeanspruchung wird in der Literatur kein Modell angegeben an dem die Verformungsmechanismen erläutert und begründet werden könnten. Transformiert man jedoch eine unter Scherbeanspruchung stehende Zelle in Richtung der Hauptspannungen, so wird diese durch einen zweiaxialen Spannungszustand aus Zug- und Druckkräften beansprucht. Somit lassen sich, die aus einer Scherbeanspruchung resultierenden Verformungen, wieder auf die bereits zuvor beschriebenen Mechanismen zurückführen, d.h. die Biegeverformung der Zellkanten, die Dehnung der Zellwände und der Gasdruck im Inneren der Zellen.

Das Scherverhalten von XPS wird nach DIN EN 12090 bestimmt. Für die Bestimmung der Schereigenschaften werden zwei Versuchsaufbauten empfohlen. Zum einen ist dies ein einhüftiger Aufbau für die Prüfung von Einzelprobekörpern (Abbildung 3.9). Und zum anderen werden zwei Varianten eines zweihüftigen Aufbaus für die Prüfung von Doppelprobekörpern vorgeschlagen. Die Versuchsaufbauten sind in der Norm beispielhaft dargestellt.

Die Abmessungen der Einzelprobekörper des einhüftigen Aufbaus sind in der entsprechenden Zeichnung (siehe Abbildung 3.9 mit einer Länge $l$ von $250 \mathrm{~mm}$ und einer Breite $b$ von $50 \mathrm{~mm}$ angegeben. In Abschnitt 6.1.2 der Norm ist weiterhin definiert, dass die Dicke $d$ der Probekörper der Lieferdicke des Produkts entsprechen, dabei aber $50 \mathrm{~mm}$ nicht überschreiten sollte. 


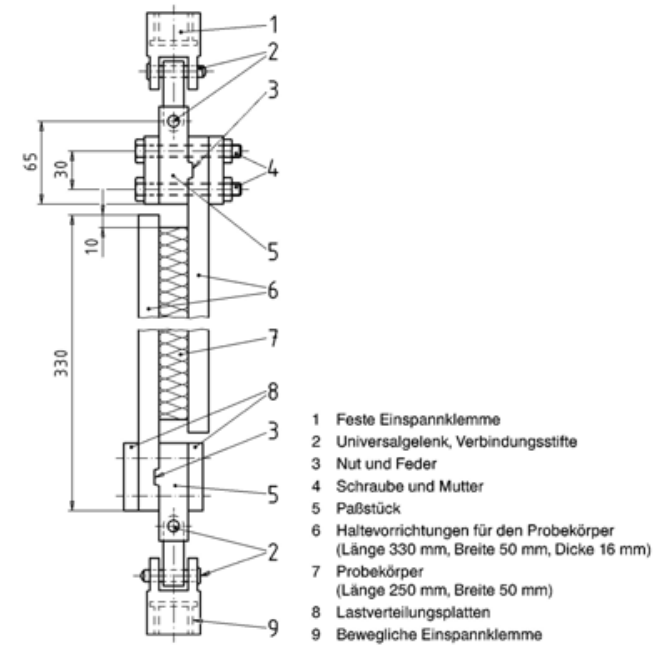

Abb. 3.9: Beispiel für den Aufbau einer Prüfung mit einem Einzelprobekörper nach DIN EN 12090 (Abmessungen in $\mathrm{mm}$ )

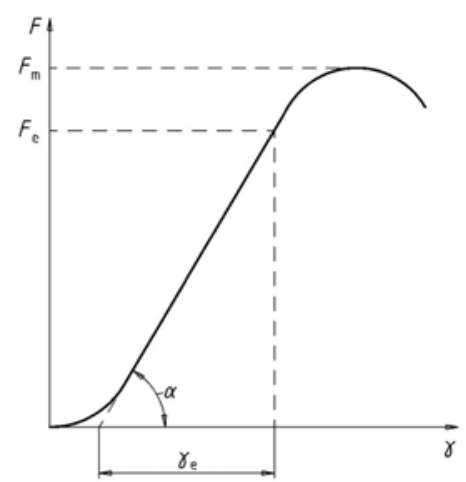

Abb. 3.10: Beispiel einer KraftVerformungskurve nach DIN EN 12090

Die Scherfestigkeit errechnet sich aus der maximal aufgebrachten Kraft $F_{m}$ und der Fläche $A=l \cdot b$ wie folgt:

$$
\tau=\frac{F_{m}}{A}
$$

Der Schubmodul $G$ ist nach folgender Gleichung zu berechnen:

$$
G=\frac{d \cdot \tan \alpha}{A}
$$

Dabei ist $d$ die Dicke des Probekörpers und $\alpha$ der Neigungswinkel des linearen Teils der Kraft-Verformungskurve nach Gleichung 3.15:

$$
\tan \alpha=\frac{F_{e}}{\gamma_{e}}
$$

$\gamma_{e}$ ist hierbei die Gesamtverformung im elastischen Bereich, d. h. nach DIN EN 12090 „genau abgegrenzter geradliniger Teil der Kraft-Verformungs-Kurve“, mit $F_{e}$ der dazu gehörigen Kraft (siehe auch Abbildung 3.10.

\subsubsection{Materialverhalten unter dauerhafter Beanspruchung}

XPS weist unter einer dauerhaften Beanspruchung ein ausgeprägtes Kriechverhalten auf. Durch Krollmann 1995 wurde das Langzeitverhalten von XPS unter konstanter und zyklisch wechselnder Druckbeanspruchung untersucht. Bei einer dauerhaften konstanten Druckbeanspruchung nimmt die Stauchung mit zunehmender Zeit zu. Je größer die Beanspruchung ist, desto mehr nehmen diese Stauchungen zu. Auch bei einer dauerhaften zyklischen Be- und Entlastung nehmen die Stauchungen mit zunehmender 
Zeit zu, jedoch ist die Zunahme geringer als bei einer konstanten Druckbeanspruchung. Die Bestimmung des Langzeit-Kriechverhaltens bei Druckbeanspruchung ist in DIN EN 1606 genormt. Die Prüfung soll mindestens 90 Tage dauern. Mithilfe der sogenannten Findley-Gleichung (Findley 1944) ist es zulässig die Stauchung auf die bis zu dreißigfache Prüfdauer zu extrapolieren, wenn ein Bestimmtheitsmaß $r^{2}$ von $90 \%$ nicht unterschritten wird.

Die Gesamtstauchung $\epsilon$ wird hierbei wie folgt berechnet:

$$
\epsilon=\epsilon_{0}+m \cdot t^{n}
$$

Die Gleichung 3.16) kann zu einer linearen Beziehung umgeformt werden:

$$
\log \left(\epsilon-\epsilon_{0}\right)=\log m+n \cdot \log t
$$

Hierbei ist $\epsilon_{0}$ die Stauchung unmittelbar nach dem Aufbringen der Belastung. Der Kriechanteil wird mit dem Ausdruck $m \cdot t^{n}$ beschrieben. $t$ ist die Belastungszeit, $m$ und $n$ sind von der Prüfspannung abhängige Werkstoffkonstanten. Trägt man die Stauchung und die Zeit in logarithmierter Form auf, kann mittels linearer Regression eine Kurvenanpassung von Gleichung 3.17) an die Versuchsergebnisse vorgenommen und somit $m$ und $n$ bestimmt werden.

Auch unter einer dauerhaften Schubbeanspruchung kommt es zu einem Schubkriechen des XPS. Im Auftrag der BASF wurde durch Fouad 2008 das Langzeittragverhalten unter Schub- und kombinierter Schub-Druck-Beanspruchung von XPS untersucht. Ebenso wie beim Langzeitverhalten unter Druckbeanspruchung, steigen die Schubverformungen mit zunehmender Zeit sowie mit zunehmender Schubbeanspruchung an. Die voraussichtlichen Verformungen zu einem bestimmten Zeitpunkt können ebenfalls mithilfe des Findley. Ansatzes extrapoliert werden. Eine Norm zur Bestimmung des Langzeittragverhaltens unter Schubbeanspruchung existiert bisher noch nicht.

\subsubsection{Materialverhalten unter Temperaturbeanspruchung}

Polystyrol gehört zu den thermoplastischen Kunststoffen und weist aus diesem Grund ein temperaturabhängiges Werkstoffverhalten auf. Bei hohen Temperaturen verliert XPS die Steifigkeit und Festigkeit. Bei niedrigen Temperaturen wird XPS hingegen spröde. Die Anwendungsgrenztemperaturen von gebräuchlichem XPS liegen daher zwischen $+75^{\circ} \mathrm{C}$ bis $+85^{\circ} \mathrm{C}$ und $-180^{\circ} \mathrm{C}$ (Boy 2005). Neuere Entwicklungen ermöglichen die Anwendung von XPS bis zu einer Temperatur von $105^{\circ} \mathrm{C}$ (BASF SE 2011a).

Durch die Firma BASF wurde die Druckfestigkeit bei einer erhöhten Temperaturbeanspruchung untersucht. Dazu wurden Druckversuche an XPS-Proben unter einer bestimmten Temperaturbeanspruchung durchgeführt. Mit jedem Versuch wurde die Temperatur um $20^{\circ} \mathrm{K}$ auf bis zu $100^{\circ} \mathrm{C}$ erhöht. Die Versuchsergebnisse zeigen, dass bei $80^{\circ} \mathrm{C}$ die Druckfestigkeit $82 \%$ der Druckfestigkeit bei $23^{\circ} \mathrm{C}$ entspricht, während bei einer Temperatur von $100^{\circ} \mathrm{C}$ die Druckfestigkeit lediglich $59 \%$ der Druckfestigkeit bei $23^{\circ} \mathrm{C}$ beträgt.

In der Norm DIN EN 1605, Wärmedämmstoffe für das Bauwesen - Bestimmung der Verformung bei definierter Druck- und Temperaturbeanspruchung“ ist eine Möglichkeit 


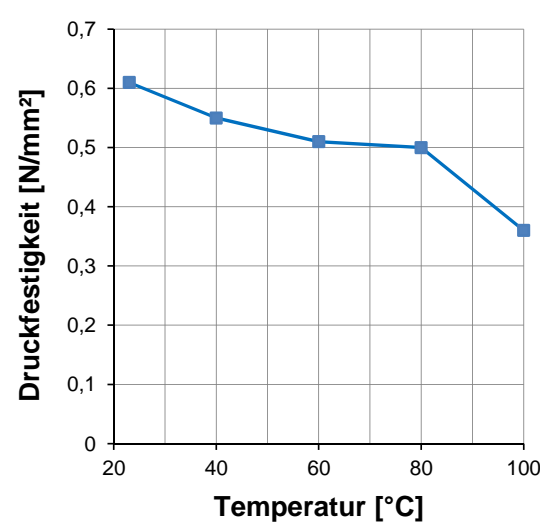

Abb. 3.11: Druckfestigkeit bei Temperaturbeanspruchung

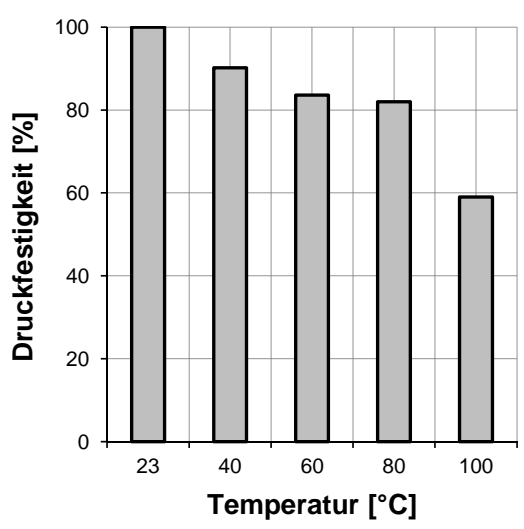

Abb. 3.12: Verbleibende Druckfestigkeit bei Temperaturbeanspruchung in \%

gegeben, den Verlust der Steifigkeit unter einer Temperaturbeanspruchung zu quantifizieren. Dabei werden die Probekörper einer zweistufigen Druckprüfung unterzogen. In der ersten Prüfstufe A wird über eine Dauer von $48 \mathrm{~h}$ eine Druckspannung je nach vorgeschriebener Prüfbedingung von 20, 40 oder $80 \mathrm{kN} / \mathrm{m}^{2}$ bei einer Temperatur von $23^{\circ} \mathrm{C}$ aufgebracht. In Prüfstufe B wird der Probekörper dann in Abhängigkeit der Prüfbedingung zusätzlich einer definierten Temperaturbeanspruchung mit unterschiedlicher Dauer ausgesetzt. Anschließend wird die Stauchung des Probekörpers nach der Prüfstufe A und die Gesamtstauchung nach der zweiten Prüfstufe B berechnet.

\subsubsection{Materialverhalten bei Feuchteeinwirkung und unter Frost-Tau-Wechselbeanspruchung}

Aufgrund der hohen Geschlossenzelligkeit und dem relativ homogenen Gefüge ist die Wasseraufnahme von XPS sehr gering. Die Wasseraufnahme von Styrodur ${ }^{\circledR} \mathrm{C}$ bei langzeitigem Eintauchen nach DIN EN 12087 liegt bei weniger als 0,2 Vol.-\%. Die Wasseraufnahme in einem Diffusionsversuch nach DIN EN 12088 von XPS-Platten mit einer Dicke von über $50 \mathrm{~mm}$ Dicke beträgt weniger als 3 Vol.- $\%$. Im Vergleich dazu liegt die Wasseraufnahme von EPS beim Eintauchen bei ca. $5 \mathrm{Vol} . \%$ und in einem Diffusionsversuch bei 30 Vol.-\% (Boy 2005).

Resultierend aus der geringen Wasseraufnahme, ist das Verhalten von XPS unter Frost-Tau-Wechselbeanspruchung eher unproblematisch. Zur Bestimmung des Verhaltens unter dieser Beanspruchung (DIN EN 12091) werden die Probekörper zuerst einer der beiden Wasseraufnahme-Prüfungen nach DIN EN 12087 oder DIN EN 12088 unterzogen. Anschließend werden die Probekörper 300 aufeinander folgenden Zyklen von trockenen Umgebungsbedingungen bei $-20^{\circ} \mathrm{C}$ und feuchten Umgebungsbedingungen bei $+20^{\circ} \mathrm{C}$ ausgesetzt.

Dazu werden die Probekörper eine Stunde lang bei einer Temperatur von $-20^{\circ} \mathrm{C}$ im trockenen und anschließend bei $+20^{\circ} \mathrm{C}$ im eingetauchten Zustand gelagert. Im Anschluss wird das Wasser abgelassen und der Zyklus entsprechend wiederholt. Die 


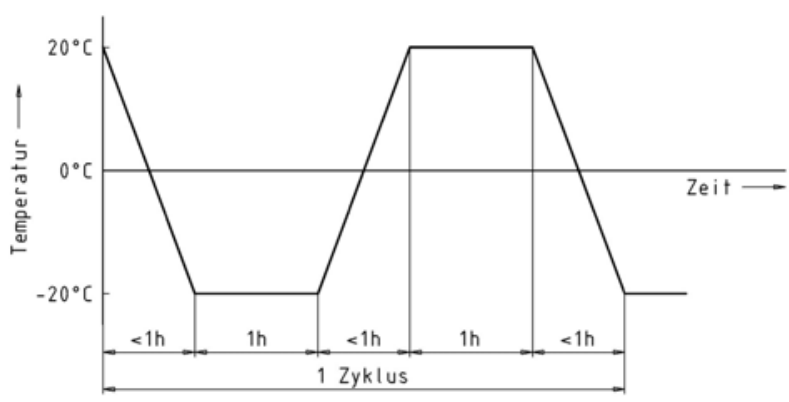

Abb. 3.13: Dauer eines Prüfzyklus nach DIN EN 12091

Phasen des Abkühlens und Erwärmens dürfen nach Norm nicht länger als eine Stunde dauern (siehe Abbildung 3.13.

Nach der Durchführung der Frost-Tau-Beanspruchung wird erneut die Masse der Probekörper bestimmt. Zusammen mit den Massen, die durch die zuvor durchgeführten Wasseraufnahme-Prüfungen bestimmt wurden, kann daraus die massebezogene oder volumenbezogene Wasseraufnahme durch Frost-Tau-Wechselbeanspruchung ermittelt werden. Diese soll nach der bauaufsichtlichen Zulassung von XPS bei einer Plattendicke von mehr als $50 \mathrm{~mm}$, weniger als $1 \mathrm{Vol} .-\%$ betragen. Weiterhin kann nach der Frost-Tau-Beanspruchung eine Druckprüfung durchgeführt werden und zusammen mit der Druckfestigkeit des unbehandelten Produkts, die Änderung des Verhaltens bei Druckbeanspruchung ermittelt werden. Die Abnahme der Druckfestigkeit von XPS durch eine Frost-Tau-Beanspruchung darf nach der Zulassung von XPS (z. B. DIBt 2014 nicht mehr als $10 \%$ betragen.

\subsubsection{Querdehnzahl}

Aufgrund der Anisotropie von XPS kann die Querdehnzahl nur schwer definiert werden. Die Angaben in der Literatur sind nur unzureichend und variieren zum Teil beträchtlich. Durch Gibson u. Ashby 2001 wurden die Querdehnzahlen von geschlossenzelligen Schäumen in Abhängigkeit ihrer relativen Dichte zusammengestellt. Dabei lagen die Werte zwischen 0,2 und 0,55. Es lässt sich feststellen, dass die geschlossenzelligen Schäume mit einer geringeren relativen Dichte, höhere Querdehnzahlen aufweisen, als die Schäume mit einer höheren Dichte.

In Rinde 1970 werden die Querdehnzahlen für XPS unter Zugbeanspruchung in Abhängigkeit der Extrusionsrichtung angegeben. Diese betragen für ein XPS mit einer Rohdichte von $65 \mathrm{~kg} / \mathrm{m}^{2}, 0,46$ in Dickenrichtung, 0,53 in Breitenrichtung und 0,4 in Längs- bzw. Extrusionsrichtung. Bei hohen Dehnungen im plastischen Bereich des Materialverhaltens nimmt die Querdehnzahl stark ab. Bei einem XPS unter Druckbeanspruchung und Dehnungen von 10-50\% beträgt die Querdehnzahl nur noch 0,03 (Shaw u. Sata 1966). 


\subsubsection{Alterungsbeständigkeit}

Kurzwellige UV-Strahlung verursacht bei längerer Einwirkungsdauer eine Versprödung des XPS. Daher sollte XPS vor UV-Strahlung durch entsprechende Maßnahmen wie Anstriche, Deckschichten etc. geschützt werden.

Bei mit HFKW extrudiertem XPS verringert sich durch das Auspermeieren des Zellgases die Wärmeleitfähigkeit. Nach Boy 2005 treten ansonsten keine weiteren Alterungsprozesse auf.

Durch die in Fouad 2008 zusammengetragenen Untersuchungen des Druckverhaltens an natürlich gealtertem XPS aus bestehenden Umkehrdächern konnte dies bestätigt werden. Die Druckfestigkeiten der untersuchten XPS-Dämmplatten mit einem Alter von 5 bis 22 Jahren wiesen gegenüber dem durch den Hersteller angegebenen Festigkeiten ausreichende Sicherheiten auf.

\subsection{Sandwichbauweise}

\subsubsection{Historische Entwicklung}

Durch die Verbundbauweise werden mehrere Werkstoffe miteinander verbunden, so dass sich deren Schwächen ausgleichen und ein Verbundwerkstoff mit verbesserten Eigenschaften entsteht. Bei der Sandwichbauweise, die ein Teilgebiet der Verbundbauweise darstellt, werden meist zwei Werkstoffe, zwei Deckschichten aus einem besonders dichten und festen Werkstoff mit einem Kern aus einem leichten und weniger dichten Werkstoff verbunden, so dass ein Verbundwerkstoff mit einer sehr hohen Tragfähigkeit und Steifigkeit bei gleichzeitig geringem Gewicht entsteht. Dieses Prinzip findet evolutionsbedingt in der Natur seit Millionen von Jahren häufig Anwendung und es lassen sich unzählige Beispiele finden. So besteht beispielsweise der menschliche Schädel oder die Flügel eines Vogels aus einer Art Sandwichkonstruktion.

Die Entwicklungen im Bereich der Sandwichbauweise in technischen Anwendungen gehen nahezu alle auf den Flugzeugbau zurück. Diese beginnen Ende des 19. Jahrhunderts bei den ersten Sandwich-Doppeldecker-Strukturen und reichen bis zu den heutigen, modernen Sandwichkonstruktionen aus faserverstärktem Kunststoff, die zum Bau von Flugzeugen wie dem A380 oder für die Produktion von Rotorblättern für Windkraftanlagen eingesetzt werden.

In der Bauindustrie wurden Sandwichkonstruktionen erst seit Beginn der 1960er-Jahre im Rahmen des Metalleichtbaus untersucht und entwickelt. Üblicherweise werden diese Sandwichelemente in kontinuierlich arbeitenden Fertigungsanlagen mit einem Kern aus Polyurethan-Hartschaum und Deckschichten aus Stahlblech hergestellt Pöter 2009). Diese traditionellen Sandwichelemente mit metallischen Deckschichten werden in der Regel vielseitig für Dacheindeckungen und Wandverkleidungen verwendet. Die Bemessung dieser selbsttragenden Sandwichelemente erfolgt nach DIN EN 14509 In der jüngeren Forschung Käpplein u. Ummenhofer 2010 Lange u. a. 2011 Berner 2009, werden unter anderem tragende Sandwichelemente untersucht, die zum Abtrag von Vertikallasten und zur Aussteifung und Stabilisierung dienen können. 
Auch auf dem Gebiet des Massivbaus wurden in den 1960er-Jahren mehrschichtige, vorgefertigte Wandelemente aus Beton entwickelt. Diese finden heutzutage ebenfalls häufige Verwendung. Die Elemente bestehen in der Regel aus einem dreischichtigen Aufbau, d. h. einer Tragschale, einer Dämmschicht und einer Vorsatzschale. Trag- und Vorsatzschale werden in der Regel durch linien- oder punktförmige Verbindungsmittel, beispielsweise Gitterträger oder Verbundnadeln, miteinander verbunden. Bei der Bemessung der Wandelemente im Grenzustand der Tragfähigkeit wird üblicherweise die Sandwichtragwirkung nicht berücksichtigt. Es wird angenommen, dass alle Lasten von der Tragschale übertragen werden. Lediglich im Grenzzustand der Gebrauchstauglichkeit werden die Schnitt- und Verformungsgrößen, die durch die Kopplung der beiden Betonschalen entstehen, berücksichtigt. Die Bemessung der Sandwiche erfolgt nach Eurocode 2 (DIN EN 1992). Nach dessen Nationalem Anhang DIN EN 1992/NA sind hierbei die „Einflüsse von Temperatur, Feuchtigkeit, Austrocknen und Schwinden in ihrem zeitlichen Verlauf" zu berücksichtigen. Zur Ermittlung der Schnitt- und Verformungsgrößen bei dieser Art von Sandwichen sei auf Pahn 2011 verwiesen.

\subsubsection{Sandwichtheorie und Tragwirkung}

Durch die Querkraftbeanspruchung des schubweichen Kerns eines Sandwichelements entsteht eine Schubverformung, wodurch die Bernoullische Hypothese des „Ebenbleiben der Querschnitte“ außer Kraft gesetzt wird. Die Schnittgrößen über den Querschnitt eines Sandwichelements können daher nicht mit der Balkentheorie berechnet werden. Hierzu muss die Sandwichtheorie angewendet werden. Die Sandwichtheorie basiert auf der Theorie des elastischen bzw. verschieblichen Verbunds. Der elastische Verbund der beiden Deckschichten wird hierbei über eine dritte Kernschicht mit der Dicke $d_{s}$ und dem Schubmodul $G$ beschrieben.

Grundsätzlich muss zwischen Sandwichelementen mit biegeschlaffen und biegesteifen Deckschichten unterschieden werden (siehe Abbildung 3.14):

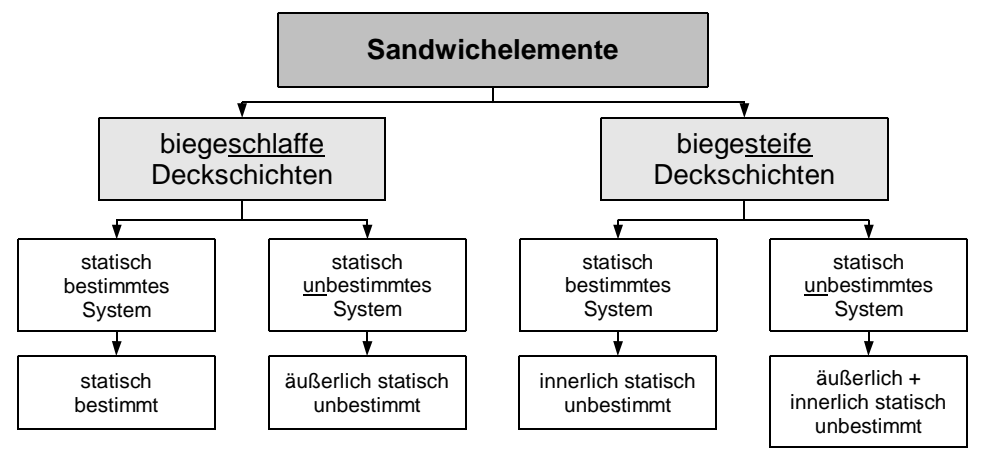

Abb. 3.14: Unterteilung der Sandwichelemente nach der Steifigkeit der Deckschichten

Sandwichelemente mit biegeschlaffen Deckschichten sind innerlich statisch bestimmt. Das aufgebrachte Moment wird als Kräftepaar über die Deckschichten und die Querkraft vollständig über den Kern abgetragen. Die Gesamtverformung eines Sandwichelements mit biegeschlaffen Deckschichten besteht aus der Biegeverformung, die 
durch die Dehnung der Deckschichten hervorgerufen wird und der Querkraftverformung, die durch Schubverzerrung des Kerns verursacht wird. Die Biegeverformung wird mit der Biegesteifigkeit des Sandwichquerschnitts $B_{S}$ berechnet. Diese setzt sich aus den Steiner'schen Anteilen der Deckschichten mit den Flächen $A_{o}$ und $A_{u}$ und dem Abstand der Schwerachsen $e$, sowie den Elastizitätsmodulen $E_{o}$ und $E_{u}$ nach Gleichung (3.18) zusammen (siehe Abbildung 3.17):

$$
B_{S}=\frac{E_{o} A_{o} \cdot E_{u} A_{u} \cdot e^{2}}{E_{o} A_{o}+E_{u} A_{u}}
$$

Die Schubverformung wird mit der Schubsteifigkeit des Kerns $S$ ermittelt. In die Gleichung (3.19) zur Berechnung von $S$ geht der Schubmodul des Kernmaterials $G$, der Abstand der Schwerachsen der Deckschichten $e$, die Querschnittsbreite $b$ und die Kernhöhe $d_{S}$ ein:

$$
S=\frac{G \cdot e^{2} \cdot b}{d_{S}}
$$

Bei statisch bestimmten Systemen können die Schnittkräfte noch leicht mit den üblichen statischen Verfahren, bspw. dem Kraftgrößenverfahren, unter Berücksichtigung der Schubverformung des Kerns bestimmt werden. Bei statisch unbestimmten Systemen müssen zur Lösung des Systems bereits Annahmen und Vereinfachungen getroffen werden oder die entsprechenden Differentialgleichungen gelöst werden.

Sandwichelemente mit biegesteifen Deckschichten sind innerlich statisch unbestimmt. Neben der Biegesteifigkeit des Sandwichquerschnitts $B_{S}$ nach GI. (3.18) und der Schubsteifigkeit des Kerns $S$ nach Gl. 3.19) müssen nun noch die Eigenbiegesteifigkeiten der Deckschichten $B_{o}$ und $B_{u}$ berücksichtigt werden.

$$
B_{\text {obzw. } u}=E_{o \text { bzw. } u} \cdot l_{o \text { bzw. } u}
$$

Die Summe der Deckschichtbiegesteifigkeiten entspricht $B_{D}$ :

$$
B_{D}=B_{o}+B_{u}
$$

Die Verteilung der Schnittgrößen wird zum einen durch das Verhältnis der Eigenbiegesteifigkeit der Deckschichten $B_{D}$ zur Biegesteifigkeit des Sandwichquerschnitts $B_{S}$ und zum anderen durch das Verhältnis der Biegesteifigkeit des Sandwichquerschnitts zur Schubsteifigkeit $S$ und Länge des Elements $l$ in Form des Faktors $k$ beeinflusst (Berner 1994).

$$
k=\frac{B_{S}}{S \cdot l^{2}}
$$

Mit zunehmender Eigenbiegesteifigkeit der Deckschichten und abnehmender Schubsteifigkeit des Kerns, wird das Verhältnis der Deckschichtmomente $M_{o}$ und $M_{u}$ zum Gesamtmoment $M$ größer. Mit abnehmender Eigenbiegesteifigkeit der Deckschichten und zunehmender Schubsteifigkeit des Kerns, wird hingegen der Anteil des Sandwichmoments $M_{s}$ am Gesamtmoment $M$ größer (siehe bspw. Abbildung 3.15. 


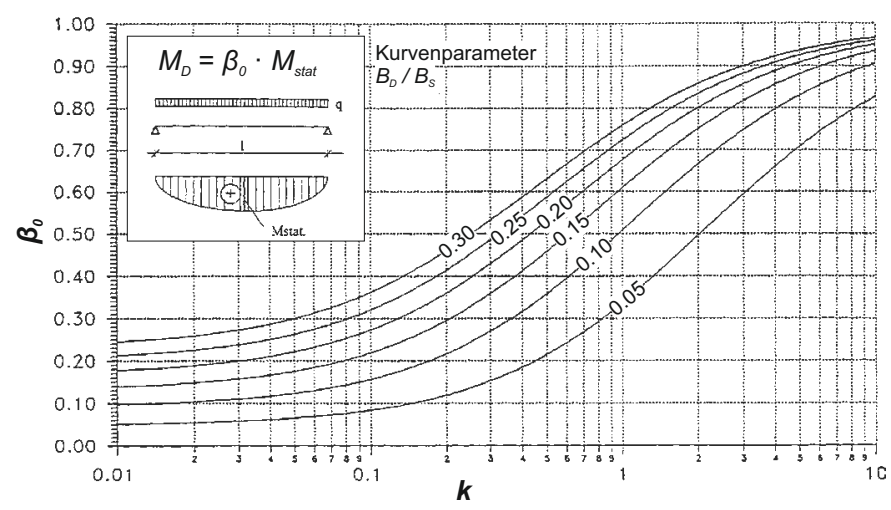

Abb. 3.15: Ermittlung des Momentanteils der Deckschichten $M_{D}$ von Einfeld-Sandwichelementen mit biegesteifen Deckschichten (Berner 1994)

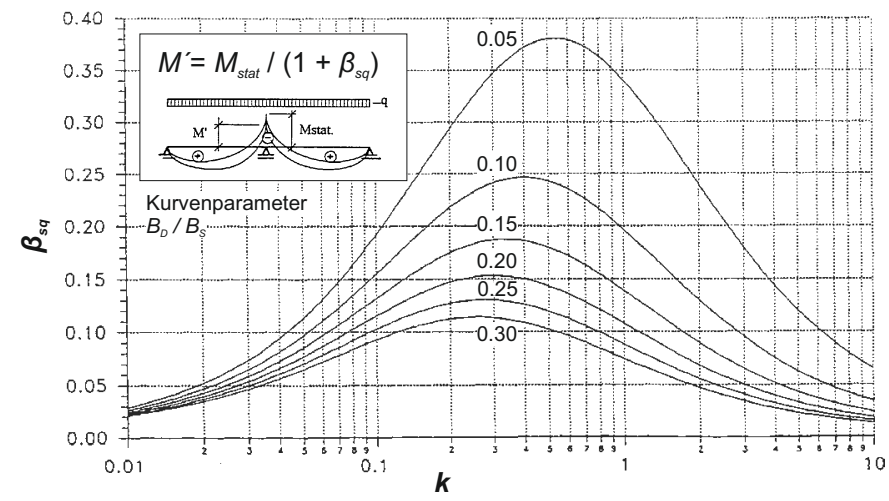

Abb. 3.16: Ermittlung des Stützmomentes $M^{\prime}$ von Zweifeld-Sandwichelementen mit gleichen Stützweiten und biegesteifen Deckschichten Berner 1994

Die Verhältnisse der Steifigkeiten beeinflussen nicht nur die Verteilung der SchnittgröBen über den Querschnitt, sondern bei statisch unbestimmten Systemen auch die Verteilung der Schnittgrößen über die Länge eines Sandwichelements. Durch die Schubverformung des Kerns werden bei Mehrfeldsystemen die Stützmomente im Vergleich zu den Momenten $M_{\text {stat }}$, die sich bei starrem Verbund einstellen würden, abgemindert und die Feldmomente entsprechend vergrößert (siehe bspw. Abbildung 3.16.

Zur Ermittlung der Schnittgrößenverteilung eines Sandwichelements mit biegesteifen Deckschichten müssen aufgrund der innerlich statischen Unbestimmheit die entsprechenden Differentialgleichungen gelöst werden. Für die meisten der gebräuchlichen Fälle sind die exakten Lösungen durch Stamm u. Witte 1974 hergeleitet worden. Weitere Herleitungen und Lösungen zur Sandwichtheorie für Sandwichelemente mit biegesteifen Deckschichten wurden u.a. durch Schwarze 1984, Jungbluth u. Berner 1986 Wölfel 1987 Berner 1994, Berner 1996 etc. veröffentlicht.

Aus den Schnittgrößen des gesamten Sandwichquerschnitt (siehe Abbildung 3.17) können dann mit den folgenden Gleichungen die Normalspannungen der Deckschich- 
ten und die Schubspannungen des Kerns errechnet werden:

$$
\begin{aligned}
& \sigma_{o, 1 \text { bzw. } o, 2}=-\frac{N_{s}}{A_{o}} \pm \frac{M_{o}}{W_{o}} \\
& \sigma_{u, 1 \text { bzw. } u, 2}=\frac{N_{s}}{A_{u}} \pm \frac{M_{u}}{W_{u}} \\
& \tau=\frac{V_{s}}{e \cdot b}
\end{aligned}
$$

In der Sandwichtheorie werden üblicherweise aufgrund des geringen Elastizitätsmoduls des Dämmmaterials die auf den Kern wirkenden Normalspannungen vernachlässigt.

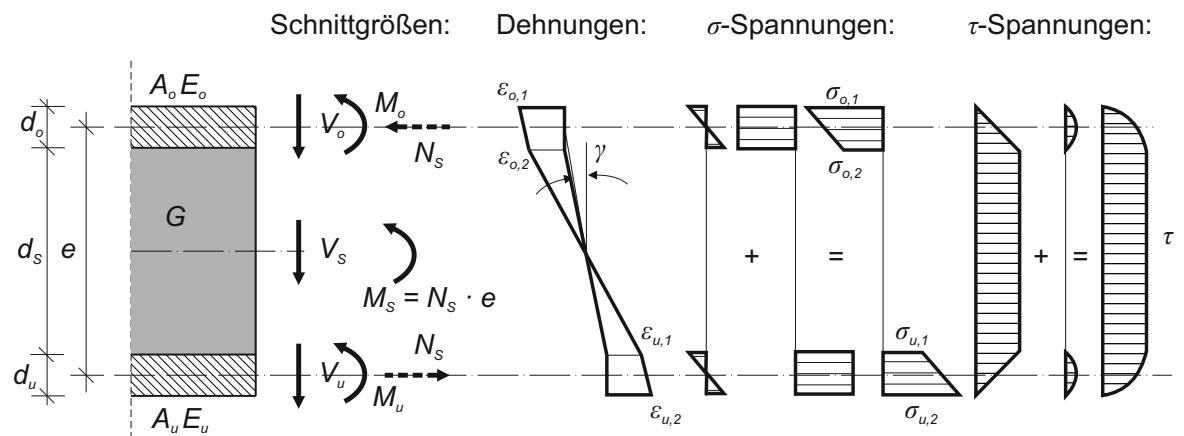

Abb. 3.17: Schnittgrößen- und Spannungsverteilung eines Sandwichquerschnitts mit biegesteifen Deckschichten

Die Herleitungen zur Sandwichtheorie wurden zum Teil in benutzerfreundliche SoftwareLösungen umgesetzt. Das Institut für Sandwichtechnik in Mainz bietet EDV-Programme auf Basis des Tabellenkalkulationsprogramms EXCEL ${ }^{\circledR}$ von Microsoft ${ }^{\circledR}$ zum Download an (Raabe 2003). Das Bemessungsprogramm SandStat kann von der iS-engineering $\mathrm{GmbH} 2007$ erworben werden. Ein weiteres Bemessungsprogramm ist die freiverfügbare Software swe2 von Urbanek 2011.

\subsubsection{Bemessungssoftware swe2}

\subsubsection{Allgemeines}

Zur Bemessung von Sandwichelementen mit Betondeckschichten wurde im Rahmen dieser Arbeit das Programm swe2 entsprechend umfangreich erweitert. Daher sei das Programm und dessen Funktionsweise in diesem Abschnitt kurz näher erläutert.

Das Programm swe2 wurde unter den GNU General Public License-Bedingungen (FSF 2007) für „Freie Software“ erstellt und ist aus diesem Grund freiverfügbar. Unter der Voraussetzung, dass die Software weiter unter diesen Lizenzbedingungen bereitgestellt wird, darf diese beliebig verändert und erweitert werden. Das Programm wurde auf Basis der Programmiersprache $\mathrm{C}++$ als Windows ${ }^{\circledR}$-Anwendung mit grafischer Benutzeroberfläche erstellt. 


\subsubsection{Ansatz von Schwarze zur Lösung der Differentialgleichung}

Das Programm beruht auf dem Ansatz nach Schwarze 1984. Unter Voraussetzung linear-elastischen Materialverhaltens können aus allgemeinen Beziehungen zwischen den Schnittgrößen und den Verformungen der einzelnen Schichten eines Sandwichelements mit biegesteifen Deckschichten (siehe Schwarze 1984), die beiden folgenden Differentialgleichungen hergeleitet werden:

$$
\begin{aligned}
& S \cdot \gamma-B_{D} \cdot w^{\prime \prime \prime}=V \\
& B_{S} \cdot\left(\gamma^{\prime}+\vartheta\right)-B \cdot w^{\prime \prime}=M
\end{aligned}
$$

Dabei sind:

$$
\begin{array}{llll}
\gamma & : & \text { Schubwinkel der Kernschicht } \\
w & : & \text { Gesamtdurchbiegung } \\
\vartheta & : & \text { Krümmung des Sandwichelements infolge der Temperatur- } \\
& & \text { differenz der Deckschichten mit } \vartheta=\left(\alpha_{t o} T_{o}-\alpha_{t u} T_{u}\right) / e . \text { Da- } \\
& & \text { bei sind } \alpha_{t o} \text { und } \alpha_{t u} \text { die Temperaturausdehnungskoeffizien- } \\
& & \text { ten der jeweiligen Deckschichten. } \\
\text { B } & : \quad & \text { Gesamtbiegesteifigkeit mit } B=B_{S}+B_{D}
\end{array}
$$

Mithilfe der Beziehung $V^{\prime}=-q$ kann aus den beiden Gleichungen 3.26 und 3.27 der Schubwinkel $\gamma$ eliminiert und eine inhomogene Differentialgleichung 4. Ordnung hergeleitet werden:

$$
w_{I V}-\left(\frac{\lambda}{l}\right)^{2} w^{\prime \prime}=\left(\frac{\lambda}{l}\right)^{2} \frac{M}{B}+\frac{1+\alpha}{\alpha} \frac{q}{B}-\left(\frac{\lambda}{l}\right)^{2} \frac{\vartheta}{1+\alpha}
$$

Mit den folgenden Abkürzungen:

$$
\lambda^{2}=\frac{1+\alpha}{\alpha \beta} ; \quad \alpha=\frac{B_{D}}{B_{S}} ; \quad \beta=\frac{B_{S}}{S l^{2}}
$$

Die allgemeine Form der Differentialgleichung 3.30 lautet:

$$
w=w_{h}+w_{p}
$$

Diese allgemeine Form wird für einen beliebigen stetigen Teilbereich eines Sandwichelements gelöst. Dabei ist $w_{h}$ der homogene Teil der Gleichung, der sich u.a. aus den Integrationskonstanten $C_{1}$ bis $C_{4}$ zusammensetzt. Für den inhomogenen Teil wurde durch einen Potenzreihenansatz und Einsetzen einer allgemeinen Funktion für $M$ in Abhängigkeit des linken und rechten Randmomentes $M_{L}$ und $M_{R}$ das Partikularintegral $w_{p}$ gebildet.

Die allgemeine Lösung für $w$ aus $w_{h}$ und $w_{p}$ wird dann dreimal abgeleitet. Mit $w^{\prime \prime \prime}$ und einer allgemeinen Funktion für die Querkraft $V$, die wiederum abhängig ist von Randmomenten $M_{L}$ und $M_{R}$, kann aus Gleichung [3.26 der Schubwinkel $\gamma$ bestimmt werden. Der Schubwinkel $\gamma$ wird ebenfalls abgeleitet, um dann mit den Differentialgleichungen 3.26) und 3.27) die Querkraft $V$ und das Moment $M$ bestimmen zu können. 
Zur Ermittlung der einzelnen Werte $w, w^{\prime}, w^{\prime \prime}, w^{\prime \prime \prime}, \gamma, \gamma^{\prime}, M$ und $V$, die in Schwarze 1984 auch Zustandsgrößen genannt werden, können die Gleichungen für einen Teilbereich $k$ in Matrizenschreibweise wie folgt dargestellt werden:

$$
Z_{k}=F_{k} \cdot K_{k}+L_{k}
$$

Dabei sind:

$$
\begin{array}{lll}
Z_{k} & : & \text { der Vektor der Zustandsgrößen } \\
F_{k} & : & \begin{array}{l}
\text { die Funktionsmatrix bestehend aus den Koeffizienten der } \\
\text { hergeleiteten Gleichungen }
\end{array} \\
K_{k} & : \quad \begin{array}{l}
\text { der Vektor der Integrationskonstanten und der Randmomen- } \\
\text { te }
\end{array} \\
L_{k} & : \quad \begin{array}{l}
\text { der Vektor der Belastung bestehend aus den Konstanten der } \\
\text { hergleiteten Gleichungen }
\end{array}
\end{array}
$$

Die Matrizengleichung mit den entsprechenden Beziehungen ist in Abbildung 3.18 dargestellt.

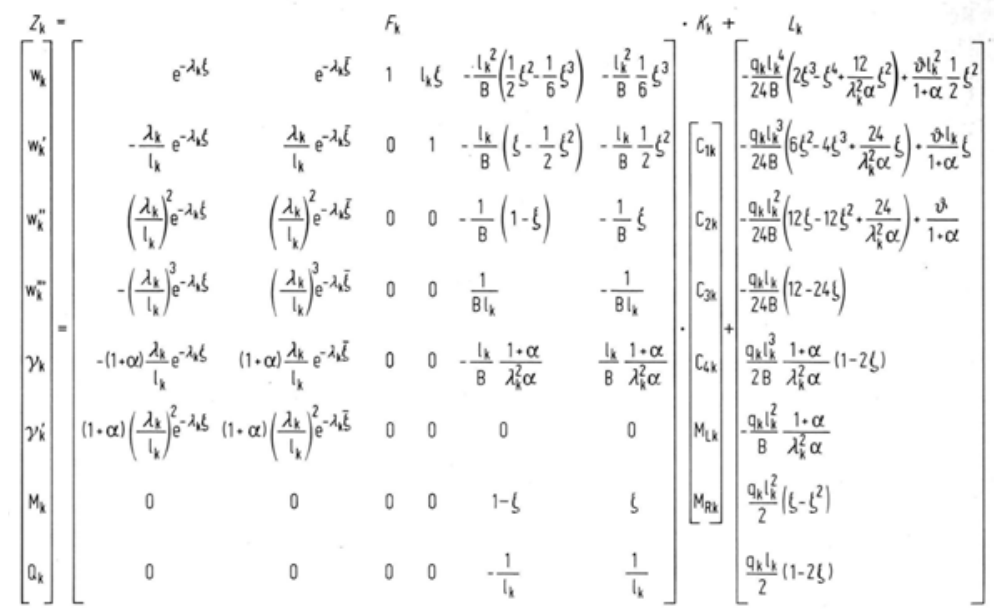

Abb. 3.18: Matrizengleichung für die Berechnung der Zustandsgrößen für einen Teilbereich $k$ nach Schwarze 1984

Für die rechnerische Ermittlung der Durchbiegung $w$ und des Schubwinkels $\gamma$, fehlen lediglich die Integrationskonstanten $C_{1}$ bis $C_{4}$ sowie die Randmomente $M_{L}$ und $M_{R}$. Dazu müssen nun die allgemeinen Lösungen aller Teilbereiche des Sandwichelements über Rand- und Übergangsbedingungen (siehe Tabelle 3.2 zu einem linearen Gleichungssystem mit dem Lösungsvektor $K$ der unbekannten Integrationskonstanten und Randmomente zusammengesetzt werden. Das Gleichungssystem in Matrizenschreibweise sieht wie folgt aus:

$$
\boldsymbol{A} \cdot \boldsymbol{K}+\boldsymbol{R}=0
$$

Die Lösung erfolgt zu:

$$
K=-R \cdot A^{-1}
$$


Dabei sind:

A : die Koeffizientenmatrix. Diese besteht entsprechend der Rand- und Übergangsbedingungen aus den jeweils benötigten Zeilen der Funktionsmatrizen $F_{\boldsymbol{k}}$ der einzelnen Teilbereiche und setzt sich wie in Abbildung 3.19 beispielhaft für ein Sandwichelement mit drei Teilbereichen dargestellt ist zusammen.

K : $\quad$ der Lösungsvektor mit den aneinander gereihten Integrationskonstanten und Randmomenten der einzelnen Teilbereiche.

$\boldsymbol{R} \quad$ : $\quad$ der Vektor der Belastung. Dieser enthält die den Zeilen der Funktionsmatrix dazugehörigen Belastungswerte der Belastungsvektoren $L_{k}$ der einzelnen Teilbereiche, wie in Abbildung 3.19 beispielhaft dargestellt.

Tab. 3.2: Rand- und Übergangsbedingungen nach Schwarze 1984 (weitere Übergangsbedingungen siehe Urbanek2011

\begin{tabular}{|c|c|c|c|}
\hline $\begin{array}{l}\text { Freies Stobende } \\
\qquad F\end{array}$ & $\begin{array}{l}\text { Orehbor ouf- } \\
\text { liegendes } \\
\text { Stobende }\end{array}$ & Zwischenouflager & Lostsprung in Feld \\
\hline & $\leftarrow$ & $k^{\wedge} k+1$ & k $k+1$ \\
\hline$w^{\prime \prime}=0 ;\left(M_{0}=0\right)$ & $w=0$ & $w_{k}=0$ & $w_{k}-w_{k+1}=0$ \\
\hline$M=0$ & $W^{\prime \prime}=0 ;\left(M_{0}=0\right)$ & $w_{k+1}=0$ & $w_{k}^{\prime}-w_{k+1}^{\prime}=0$ \\
\hline \multirow[t]{4}{*}{$0=-F$} & $M=0$ & $w_{k}^{i}-w_{k+1}^{i}=0$ & $w_{k}^{\prime \prime}-w_{k+1}^{\prime \prime}=0$ \\
\hline & & $w_{k}^{*}-w_{k+1}^{\prime \prime}=0$ & $\gamma_{k}-\gamma_{k+1}=0$ \\
\hline & & $\gamma_{k}-\gamma_{k+1}=0$ & $M_{k}-M_{k+1}=0$ \\
\hline & & $M_{k}-M_{k+1}=0$ & $a_{k}-a_{k+1}=F$ \\
\hline
\end{tabular}

Analog zu Gleichung 3.33) wird dann global der Lösungsvektor der Integrationswerte und Randmomente $K$ mit der Funktionsmatrix $F$, bestehend aus den einzelnen Funktionsmatrizen $F_{k}$ der Teilbereiche, multipliziert und um den Vektor der Belastungen $L$, bestehend aus den einzelnen Belastungsvektoren $L_{k}$, vergrößert. Das Ergebnis ist der Vektor der Zustandsgrößen $Z$ der aneinander gereihten Teilbereiche des gesamten Sandwichelements.

$$
Z=F \cdot K+L
$$

Mit dem Lösungsvektor $Z$ sind die Verformungen sowie die globalen Momente und Querkräfte über die Länge des Sandwichelements bekannt. Daraus lassen sich dann die Teilschnittgrößen wie folgt berechnen:

$$
\begin{aligned}
M_{o} & =-B_{o} \cdot w^{\prime \prime} \\
M_{u} & =-B_{u} \cdot w^{\prime \prime} \\
M_{s} & =M-M_{o}-M_{u} \\
V_{o} & =-B_{o} \cdot w^{\prime \prime \prime} \\
V_{u} & =-B_{u} \cdot w^{\prime \prime \prime} \\
V_{s} & =S \cdot \gamma
\end{aligned}
$$




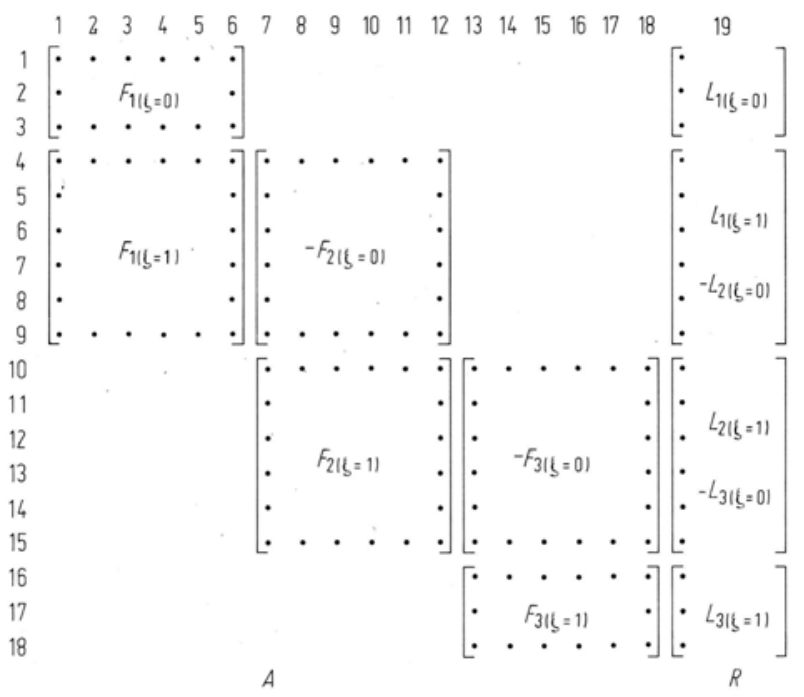

Abb. 3.19: Beispiel eines Gleichungssystems nach Gl. 3.34 für ein Sandwichelement mit drei Teilabschnitten

Mit den Teilschnittgrößen können nach GI. 3.23 bis 3.25 die Normal- und Scherspannungen über den Sandwichquerschnitt berechnet werden.

\subsubsection{Programmtechnische Umsetzung des Ansatzes}

Die Umsetzung des Programms ist ausführlich in Urbanek 2011 beschrieben. Als Modifikation zu Schwarze 1984 wird das zu berechnende Sandwichelement nicht nur an den Unstetigkeitsstellen in Teilbereiche, sondern in sehr viel kleinere Teilbereiche aufgetrennt (siehe Abbildung 3.20. Aufgrund der heutzutage zur Verfügung stehenden Rechenleistung ist dies problemlos möglich und bietet insbesondere bei der Umsetzung des Programms Vorteile.
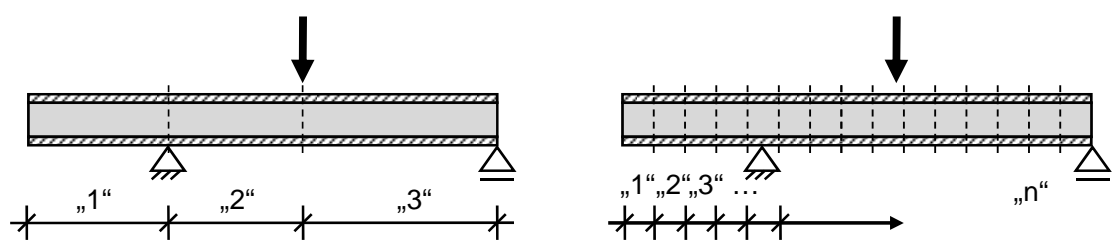

Abb. 3.20: Beispiel zur Unterteilung eines Sandwichelements in Teilbereiche

Für die Einteilung in Teilbereiche, d. h. die Diskretisierung des Sandwichelements, können drei verschiedene Auflösungen $n=5.120,10.240$ oder 20.480 ausgewählt werden. Die Gesamtlänge des Sandwichelements $l$ wird dann in $n$ Teilbereiche mit der Länge $l_{k}=l / n$ aufgeteilt. Fallen die Unstetigkeitsstellen infolge eines Auflagers oder einer Last nicht exakt auf einer der Grenzen zwischen den Teilbereichen, wie in Abbildung 3.20 (rechts) angedeutet, so wird die Unstetigkeitsstelle der jeweiligen nächsten Grenze zugewiesen. Durch diese Annahme entsteht eine geringe Ungenauigkeit, die 
im ungünstigsten Fall, bei der geringsten Auflösung $n$ von 5.120, zu einer Abweichung von $0,7 \%$ zur mathematisch exakten Lösung führen kann (siehe auch Urbanek 2011).

Das Programm swe2 kann als Windows ${ }^{\circledR}$-Anwendung installiert werden. Über eine grafische Benutzeroberfläche können die Geometrie, die Lasten und die Querschnittseigenschaften benutzerfreundlich eingegeben werden. Die Ausgabe der Berechnungsergebnisse erfolgt in grafischer oder tabellarischer Form.

Die Schnittgrößenberechnung mit dem Programm swe2 beschränkt sich auf Sandwichelemente mit metallischen Deckschichten, da der Elastizitätsmodul der Deckschichten von $210.000 \mathrm{~N} / \mathrm{mm}^{2}$ in der Eingabemaske nicht geändert werden kann. Weiterhin ist die Berechnung auf Sandwichelemente mit nur einer biegesteifen Deckschicht begrenzt. Durch Umrechnung der Berechnungsergebnisse könnten mit dem Programm trotzdem die Schnittgrößen eines Sandwichelements mit zwei biegesteifen Betondeckschichten im Zustand I berechnet werden, jedoch wäre dies für den Einsatz in der Praxis zeitaufwendig und fehleranfällig.

\subsubsection{Bemessung von Sandwichelementen mit Stahldeckschichten}

\subsubsection{Allgemeines}

Seit 2010 sind selbsttragende Sandwichelemente mit metallischen Deckschichten in Deutschland durch die europaweit harmonisierte Norm DIN EN 14509 geregelt. Das Bemessungsverfahren der Elemente ist dort in Anhang $E$ beschrieben.

Die Bemessung erfolgt üblicherweise durch einen „Vergleich der Bemessungswerte der Beanspruchungen und der Bemessungswerte des Widerstandes (...) in Form von Spannungen“ (DIN EN 14509). Die einwirkenden Spannungen werden dabei aus den mit der Sandwichtheorie ermittelten Schnittgrößen berechnet.

\subsubsection{Versagensarten in den Grenzzuständen}

In der Norm werden für die Grenzzustände der Tragfähigkeit (ULS, engl. Ultimate Limit State) und der Gebrauchstauglichkeit (SLS, engl. Serviceability Limit State) die in Tabelle 3.3 zusammengestellten Versagensarten bzw. Zustände unterschieden. Zu beachten ist hier, dass sich im Grenzzustand der Tragfähigkeit, durch das Fließen oder Knittern (d.h. dem örtlichen Beulen) der Deckschichten, Fließgelenke ausbilden dürfen, jedoch unter der Voraussetzung, dass diese nicht zu einem Versagen des Elements führen. Im Grenzzustand der Gebrauchstauglichkeit unter Gebrauchslasten muss dann weiterhin nachgewiesen werden, dass mit ausreichender Sicherheit kein Fließen und Knittern der Deckschichten, sowie kein Schubversagen des Kerns und des Verbunds auftreten.

\subsubsection{Sicherheitskonzept}

Das Sicherheitskonzept der DIN EN 14509 basiert auf dem semiprobabilistischem Teilsicherheitssystem des Eurocode 0 (DIN EN 1990). Die Bemessungwerte für die Aus- 
Tab. 3.3: Grenzzustände der Tragfähigkeit und der Gebrauchstauglichkeit für Sandwichelemente mit metallischen Deckschichten nach DIN EN 14509

\begin{tabular}{|l|c|c|}
\hline & $\begin{array}{c}\text { Grenzzustand der } \\
\text { Tragfähigkeit (ULS) }\end{array}$ & $\begin{array}{c}\text { Grenzzustand der } \\
\text { Gebrauchstauglichkeit (SLS) }\end{array}$ \\
\hline Deckschichten & $\begin{array}{c}\text { Fließen mit result. Versagen } \\
\text { Knittern mit result. Versagen } \\
\text { Schubversagen }\end{array}$ & $\begin{array}{c}\text { Fließen ohne Versagen } \\
\text { Knittern ohne Versagen }\end{array}$ \\
\hline Kern & $\begin{array}{c}\text { Schubversagen } \\
\text { Druckversagen }\end{array}$ & - \\
\hline Verbund & Versagen & Schubversagen \\
Verbindungsmittel & Versagen & - \\
\hline Durchbiegung & - & Versagen \\
\hline
\end{tabular}

wirkungen der Einwirkungen $E_{d}$ müssen kleiner sein, als die Bemessungswerte des Widerstands $R_{d}$ bzw. die Kriterien für die Gebrauchstauglichkeit $C_{d}$ :

$$
\begin{aligned}
& E_{U L S ; d} \leq R_{d} \\
& E_{S L S ; d} \leq C_{d}
\end{aligned}
$$

Die Bemessungswerte der Auswirkungen $E_{d}$ berechnen sich aus den charakteristischen Beanspruchungen $S_{k i}$ wie folgt:

$$
E_{d}=\sum \gamma_{f} \cdot \psi_{i} \cdot S_{k i}
$$

Und der Bemessungswert des Widerstands $R_{d}$ zu:

$$
R_{d}=\frac{R_{k}}{\gamma_{M}}
$$

\subsubsection{Lastfaktoren}

Die Lastfaktoren $\gamma_{f}$ in den beiden Grenzzuständen entsprechen denen des Eurocode 0 bzw. dessen Nationalen Anhängen. In Deutschland gelten dementsprechend die Lastfaktoren, die in Tabelle 3.4 zusammengestellt sind.

Tab. 3.4: Lastfaktoren $\gamma_{f}$ nach Eurocode 0 mit Nationalem Anhang DIN EN 1990 DIN EN $1990 / N A$

\begin{tabular}{|l|c|c|}
\hline Beanspruchung & $\begin{array}{c}\text { Grenzzustand der } \\
\text { Tragfähigkeit (ULS) }\end{array}$ & $\begin{array}{c}\text { Grenzzustand der } \\
\text { Gebrauchstauglichkeit (SLS) }\end{array}$ \\
\hline Dauerhaft G: & 1,35 & 1,00 \\
$\quad$ ungünstig & 1,00 & 1,00 \\
$\quad$ günstig & 1,50 & 1,00 \\
Veränderlich Q: & - & - \\
$\quad$ ungünstig & 1,50 & 1,00 \\
günstig & 1,00 & 1,00 \\
\hline Temperatur & & \\
\hline Kriechen: & & \\
\hline
\end{tabular}




\subsubsection{Kombinationsregeln}

Die Bemessungswerte der Beanspruchungen im Grenzzustand der Tragfähigkeit berechnen sich wie folgt:

$$
S_{d}=\gamma_{G} G_{k}+\gamma_{Q 1} Q_{k 1}+\sum_{i>1} \gamma_{Q i} \psi_{0 i} Q_{k i}
$$

Für die Bemessungswerte im Grenzzustand der Gebrauchstauglichkeit werden die selten auftretende und die häufig auftretende Kombination unterschieden:

$$
\begin{array}{ll}
S_{d}=\sum_{j>1} G_{k j}+Q_{k 1}+\sum_{i>1} \psi_{0 i} Q_{k i} & \text { (selten auftretende Kombination) } \\
S_{d}=\sum_{j>1} G_{k j}+\psi_{11} Q_{k 1}+\sum_{i>1} \psi_{0 i} \psi_{1 i} Q_{k i} & \text { (häufig auftretende Kombination) }
\end{array}
$$

Die selten auftretende Kombination wird verwendet um nachzuweisen, dass im Grenzzustand der Gebrauchstauglichkeit keine sichtbaren Schäden am Element auftreten. Die häufig auftretende Kombination wird angewendet um die Durchbiegungen zu überprüfen.

Die Werte für die Kombinationskoeffizienten $\psi_{i}$ wurden speziell an die besonderen Merkmale des Sandwichtragverhaltens, d. h. des großen Einfluss von Temperatureinwirkungen, angepasst. Die Kombinationskoeffizienten $\psi_{i}$ können der Norm DIN EN

\begin{tabular}{|c|c|c|}
\hline Beanspruchung & $\Psi_{0}$ & $\Psi_{1}$ \\
\hline Schnee & 0,60 & $0,75^{a} / 1,00^{b}$ \\
\hline Wind & 0,60 & $0,75^{a} / 1,00^{b}$ \\
\hline Temperatur & 0,60 & 1,00 \\
\hline Temperatur + Schnee & 1,00 & 1,00 \\
\hline
\end{tabular}
14509 entnommen werden (siehe Tabelle 3.5.

Tab. 3.5: $\quad$ Kombinationskoeffizienten $\psi_{0}$ und $\psi_{1}$ nach DIN EN 14509

\subsubsection{Beanspruchungen}

Es sind die ständigen und veränderlichen Beanspruchungen nach Eurocode 1 [DINEN 1991, DIN EN 1991/NA anzuwenden. Als eine weitere veränderliche Beanspruchung ist eine Temperaturdifferenz zwischen den beiden Deckschichten zu berücksichtigen. Es wird zwischen einem Sommer- und einem Winterlastfall unterschieden.

Im Regelfall ist die Temperatur der inneren Deckschicht $T_{2}$ im Winter mit $20^{\circ} \mathrm{C}$ und im Sommer mit $25^{\circ} \mathrm{C}$ anzunehmen. Die Temperatur der äußeren Deckschicht $T_{1}$ ist abhängig von weiteren Faktoren. Beispielsweise wird im Grenzzustand der Gebrauchstauglichkeit im Sommerlastfall zwischen drei Farbgruppen unterschieden (siehe Tabelle 3.6. 
Tab. 3.6: Temperaturen der äußeren Deckschicht $T_{1}$ nach DIN EN 14509 und Teil 2 der LTB

\begin{tabular}{|l|c|ccc|}
\hline Jahreszeit & ULS & \multicolumn{3}{c|}{ SLS } \\
Reflex.
\end{tabular}

Farbgruppe I: sehr helle Farben, Farbgruppe II: helle Farben, Farbgruppe III: dunkle Farben

${ }^{2}$ Reflextionsgrad in Bezug auf Magnesiumoxid $=100 \%$

Die betragsmäßigen maximalen Temperaturdifferenzen betragen in den jeweiligen Lastfällen, im Winter (ohne Schneelast, im ULS und SLS) $-40^{\circ} \mathrm{K}$, und im Sommer (im ULS und im SLS bei dunklen Oberflächenfarben) $+55^{\circ} \mathrm{K}$.

Weiterhin muss bei Sandwich-Dachelementen das Kriechen der Kernschicht als ständige Beanspruchung berücksichtigt werden. Das Kriechverhalten von Kunststoffdämmmaterialien ist zeitlich nicht begrenzt und führt bei Sandwichelementen zu einer Vergrößerung der Durchbiegungen und zu einer Schnittgrößenumlagerung. Das Kriechen des Kerns wird durch eine Abminderung des Schubmoduls berücksichtigt:

$$
G_{t}=\frac{G}{1+\varphi_{t}}
$$

Der Kriechfaktor $\varphi_{t}$ kann in einem entsprechenden Versuch ermittelt oder für PUR, EPS und XPS-Hartschaumstoffe wie folgt angenommen werden:

$$
\begin{array}{ll}
\varphi_{t}=2,4 & \text { für } t=2.000 \mathrm{~h} \text { bei Beanspruchung durch eine Schneelast } \\
\varphi_{t}=7,0 & \text { für } t=100.000 \mathrm{~h} \text { bei dauerhaften Beanspruchungen }
\end{array}
$$

\subsubsection{Materialfaktoren}

Die Materialfaktoren $\gamma_{M}$ können nach DIN EN 14509 mit denen in Anlehnung an Eurocode 0 (DIN EN 1990) angegebenen Gleichungen berechnet werden. Für den Grenzzustand der Tragfähigkeit wird angegeben:

$$
\gamma_{M}=1,05 \cdot e^{2,115 \cdot v}
$$

Und für den Grenzzustand der Gebrauchstauglichkeit:

$$
\gamma_{M}=1,0 \cdot e^{0,755 \cdot v}
$$

Nach DIN EN 14509 ist vorgesehen, die Standardabweichung $v$ aus werkseigenen Produktionskontrollen zu ermitteln. Zusätzlich sind in Tabelle E.9 der Norm, Materialfaktoren als Beispielwerte angegeben, die bei einem Produkt mit geringen Streuungen erreicht werden können. 
In Deutschland gelten die nationalen Reglen, d. h. die Materialfaktoren müssen aus der jeweiligen Zulassung des Sandwichelements entnommen werden. Bei Sandwichelementen ohne Zulassung muss Anlage 5/23 nach Teil 2 der Liste der technischen Baubestimmungen (Teil 2 der LTB) verwendet werden. Die Beispielwerte aus DIN EN 14509 Anlage 5/23 des Teil 2 der $L T B$ sowie ein Wertebereich aus den Zulassungen (Berner 2012) sind in Tabelle 3.7 gegenübergestellt.

Tab. 3.7: Materialfaktoren $\gamma_{M}$ nach DIN EN 14509 Tab. E.9, Anlage 5/23 aus Teil 2 der LTB und Wertebereich aus Zulassungen (Berner 2012)

\begin{tabular}{|c|c|c|c|c|c|c|}
\hline \multirow{2}{*}{ Versagen } & \multicolumn{2}{|c|}{ DIN EN 14509} & \multicolumn{2}{|c|}{ Teil 2 der LTB } & \multicolumn{2}{|c|}{ Zulassungen } \\
\hline & ULS & SLS & ULS & SLS & ULS & SLS \\
\hline \multicolumn{7}{|l|}{ Deckschichten: } \\
\hline Fließen & 1,10 & 1,00 & 1,10 & 1,00 & 1,10 & 1,00 \\
\hline Knittern & $1,25^{1}$ & $1,10^{1}$ & 2,80 & 1,40 & $1,12-1,51$ & $1,02-1,24$ \\
\hline Schubversagen & 1,10 & 1,00 & 1,10 & 1,00 & NA & NA \\
\hline \multicolumn{7}{|l|}{ Kern: } \\
\hline Schubversagen & $1,50^{2}$ & $1,10^{2}$ & 2,40 & 1,30 & $1,18-1,65$ & $1,04-1,17$ \\
\hline Druckversagen & $1,40^{3}$ & $1,10^{3}$ & 2,40 & 1,30 & $1,15-1,67$ & $1,03-1,18$ \\
\hline Verbund: & NA & NA & NA & NA & NA & NA \\
\hline Verbindungsmittel: & 1,33 & 1,00 & NA & NA & 1,33 & NA \\
\hline
\end{tabular}

\subsubsection{Widerstandswerte und Verformungsgrenzen}

Die Widerstandswerte $R_{d}$ werden nach Anhang A „Verfahren zur Prüfung von Werkstoffeigenschaften“ der DIN EN 14509 ermittelt. Dabei entsprechen die charakteristischen Widerstandswerte einem $5 \%$-Fraktilwert unter Annahme eines $75 \%$-Vertrauensniveaus. Die Widerstandswerte müssen auf dem CE-Kennzeichen, das auf der Verpackung der Sandwichelemente angebracht ist, abgedruckt werden.

Für Dachelemente unter Kurzzeit-Belastung ist die Verformung auf $1 / 200$ und für Dachelemente unter Langzeit-Belastung sowie Wandelemente auf 1/100 der Stützweite $l$ begrenzt.

\subsubsection{Prüfung der Dauerhaftigkeit von Sandwichelementen}

In Anhang B der DIN EN 14509 werden verschiedene Verfahren zur Bestimmung der Dauerhaftigkeit eines Sandwichelements beschrieben:

- Die Prüfung DUR1 ist eine Zugfestigkeitsprüfung nach DIN EN 1607 bei vorheriger Lagerung der Prüfkörper bei einer Prüftemperatur von $65^{\circ} \mathrm{C}, 75^{\circ} \mathrm{C}$ oder $95^{\circ} \mathrm{C}$, die je nach Farbe der äußeren Deckschicht gewählt wird. Dauerhaftigkeitskriterium ist unter anderem die Abnahme der Zugfestigkeit gegenüber der Festigkeit an Referenzproben, die nicht mehr als $50 \%$ betragen darf. 
- Die Prüfung DUR2 ist ebenfalls eine Zugfestigkeitsprüfung nach DIN EN 1607 jedoch bei vorheriger Lagerung der Prüfkörper in einem Prüfbehälter bei einer Lufttemperatur von $65^{\circ} \mathrm{C}$ und einer Luftfeuchte von $100 \%$. Neben weiteren Kriterien, darf die Abnahme der Zugfestigkeit gegenüber der Festigkeit an Referenzproben nicht mehr als $40 \%$ betragen.

- Bei der Keilprüfung werden die beiden Deckschichten nach einem festgelegten Verfahren zuerst zusammengeklebt. Anschließend wird ein genormter Keil mit einer Kraft von $3 \mathrm{~N}$ zwischen die Deckschichten gedrückt. Der entstehende Riss darf nicht länger als $30 \mathrm{~mm}$ sein und nach 24-stündiger Lagerung in $70^{\circ} \mathrm{C}$ warmen Wasser nicht um mehr als $20 \mathrm{~mm}$ anwachsen.

- Bei der Prüfung mit wiederholter Belastung wird ein Sandwichelement in einem Biegeversuch 5.000 Lastzyklen zwischen einem oberen und einem unteren Grenzwert ausgesetzt. Wobei der untere Grenzwert nicht mehr als der Masse des Probekörpers $+0,5 \mathrm{kN}$ betragen darf. Als oberer Grenzwert wird eine Last aufgebracht, die eine Knitterspannung auf Gebrauchslastniveau, d.h. dividiert durch $\gamma_{M}$ und $\gamma_{F}$, hervorruft. Anschließend wird das Sandwichelement bis zum Versagen belastet. Durch die Wechselbeanspruchung darf die Abnahme der Knitterfestigkeit einen gewissen Grenzwert nicht überschreiten. Weiterhin dürfen die maximalen Durchbiegungen infolge der Wechselbeanspruchung um nicht mehr als $5 \%$ zunehmen.

- Zur Prüfung mit plötzlichem Temperaturwechsel werden die Sandwichelemente in ein genormtes, vertikales Rahmengestell eingebaut und dann in einem definierten Ablauf einer zyklischen Temperaturbeanspruchung ausgesetzt. Bei der maximalen Temperaturdifferenz von $60^{\circ} \mathrm{K}$ wird das Element mit Wasser besprüht und so innerhalb von 10 Minuten abgekühlt. Während des gesamten Verfahrens werden die Sandwichelemente auf mögliche Schäden und optische Beeinträchtigungen geprüft.

\subsubsection{Untersuchungen an Sandwichelementen mit dünnen Betondeckschichten}

\subsubsection{Allgemeines}

Im Rahmen der Arbeit von Horstmann 2010 wurden bereits selbsttragende Sandwichelemente mit textilbewehrten, dünnen Betondeckschichten umfangreich untersucht. In den Versuchen wurden unterschiedliche Parameterkombinationen der Sandwichelemente untersucht. Dazu wurden sowohl die Schichtdicken, als auch die Bewehrungsund Kernmaterialien variiert.

Die Deckschichtdicken der untersuchten Sandwichquerschnitte betrugen dabei zwischen 15 und $85 \mathrm{~mm}$, und die Kernschichtdicke zwischen 150 und $160 \mathrm{~mm}$. Für die Deckschichten wurden Feinkornbetone mit mittleren Würfeldruckfestigkeiten von 70 bis $75 \mathrm{~N} / \mathrm{mm}^{2}$ verwendet. Als Bewehrung kamen hauptsächlich AR-Glasfasergelege, aber auch Carbonfasergewebe und GFK-Stäbe (Glasfaserverstärkte Kunststoffe) zum Einsatz. Zum Teil wurde der Beton zusätzlich mit 1-2 M.-\% Kurzfasern aus AR-Glas bewehrt. 
Als Kernmaterial kamen EPS, XPS und PUR-Hartschäume zum Einsatz. Dabei wiesen die Dämmstoffe eine genutete, eine gewaffelte oder eine aufgeraute bzw. gehobelte Oberfläche auf. Als punktförmige Verbundmittel wurden sogenannte TM-Anker (Thermomass ${ }^{\circledR}$-Anker der Firma CSM Construction Systems Marketing Ltd.) eingesetzt. Als linienförmige Verbundmittel kamen, unter einem Winkel von $45^{\circ}$ ausgerichtete, Schubgitter aus epoxidharzbeschichtetem Carbonfasergewebe zum Einsatz. Zur Sicherstellung der Verbundtiefe wurden die Deckschichten im Bereich der Schubgitter aufgedickt.

\subsubsection{Experimentelle Untersuchungen}

Die Herstellung der Sandwich-Probekörper erfolgte durch unterschiedliche Herstellungsmethoden (HSM) (siehe Abbildung 3.21):

- HSM1 - durch Eindrücken des Dämmkerns (mit genuteter Oberfläche) in den frischen Beton

- HSM2 - durch Eindrücken des Dämmkerns und Betonieren auf dem Dämmkern

- HSM2* - wie HSM2, jedoch mit zusätzlichem Abziehen der ersten Deckschicht mit einer Rüttelbohle und Einrütteln des Dämmkerns

- HSM3 - durch Verkleben von vorgefertigten Deckschichten mit dem Kern.

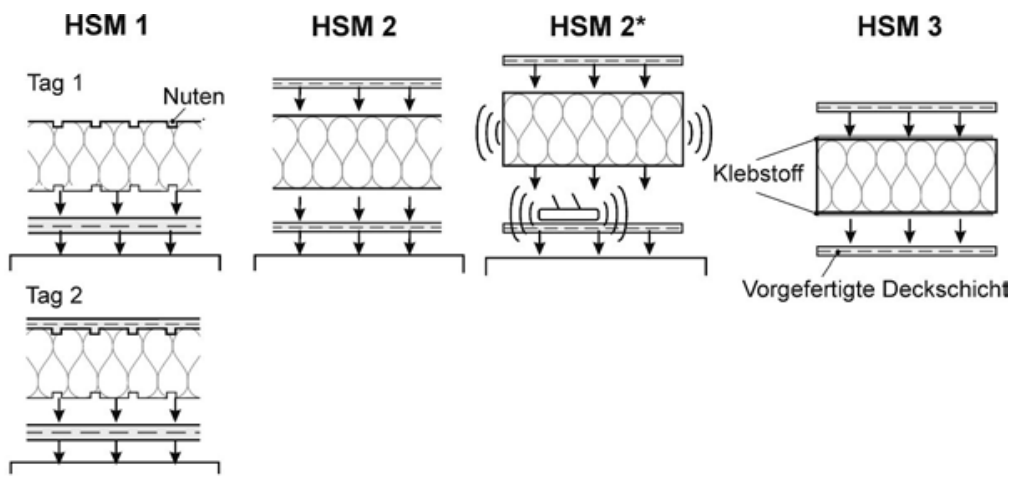

Abb. 3.21: In Horstmann 2010 angewendete Herstellungsmethoden der Sandwichelemente

In den experimentellen Untersuchungen an den Sandwichelementquerschnitten wurden Zugversuche an Probekörpern mit quadratischem Grundriss, Scherversuche an Pushout-Probekörpern sowie 3-Punkt und 4-Punkt-Biegeversuchen an kurzen und langen Sandwichstreifen durchgeführt. Alle Probekörperarten wurden sowohl mit als auch ohne Verbundmittel getestet.

Generell kann zusammengefasst werden, dass das Tragverhalten der Sandwichelemente ohne Verbundmittel hauptsächlich von der Steifigkeit und Festigkeit des Dämmstoffs sowie dessen Verbundfestigkeit zum Beton abhängt. Bei sorgfältiger Herstellung konnten bei dem Herstellverfahren HSM1 bei genuteter Oberfläche des Kerns, aber 
auch bei nicht profilierter Oberfläche mit dem Verfahren HSM2* hohe Verbundfestigkeiten erzielt werden und die Tragfähigkeit des Dämmstoffs ausgeschöpft werden.

Durch den Einsatz der Schubgitter konnte die Steifigkeit, Tragfähigkeit und Dukilität der Sandwichelemente deutlich gesteigert werden. Aufgrund ihrer geringen Biegesteifigkeit war der Einfluss der TM-Anker auf das Tragverhalten der Sandwiche unter Schubund Biegebeanspruchung gering.

\subsubsection{Rechnerische Untersuchungen}

Das Tragverhalten wurde numerisch durch FE-Modellierungen mit den beiden Programmen InfoCAD und Abaqus, sowie analytisch durch Vergleichsberechnungen mithilfe der Sandwichtheorie untersucht. Durch die FE-Modellierung mit Abaqus konnten die Biegeversuche an den Sandwichstreifen zutreffend abgebildet werden. Durch ein erweitertes Versagensmodell konnte auch das Versagen mit guter Übereinstimmung nachgerechnet werden.

In den Berechnungen auf Grundlage der Sandwichtheorie wurden verschiedene Ansätze von Aicher $u$. Roth 1987, Allen 1969 Holmberg u. Plem 1965 Stamm u. Witte 1974 und Wölfel 1987 verglichen. Alle Ansätze erzielten gute Ergebnisse im Vergleich zur FE-Berechnung mit InfoCAD, bei der zur Bestimmung der Schnittgrößen, die Deckschichten mit Schalenelementen abgebildet wurden.

\subsubsection{Ingenieurmodelle}

Zur Beschreibung des Tragverhaltens der Sandwichelemente wurden verschiedene Ingenieurmodelle ausgearbeitet. Zunächst wurden Bemessungsmodelle zur Beschreibung des Tragverhaltens der Verbundmittel unter Zug- und Druckbeanspruchung, sowie unter Biege- und Querkraftbeanspruchung ausgearbeitet. Anschließend wurde ein Ingenieurmodell zur Beschreibung des Zug- und Schubtragverhaltens von Sandwichquerschnitten mit Verbundmitteln erstellt.

Zur Berechnung des Biegetragverhaltens der Sandwichquerschnitte wurde der Ansatz von Stamm u. Witte 1974 angewendet. Dazu wurden die Schubfedersteifigkeiten $c_{i}$ des Dämmkerns und der Verbundmittel in Schubsteifigkeiten $S_{i}$ transformiert und aufsummiert. Mit der resultierenden Schubsteifigkeit $S$ können dann wieder die Gleichungen zur Ermittlung der Schnittgrößen und Verformungen nach Stamm U. Witte 1974 angewendet werden. Im Vergleich zu den FE-Berechnungen mit der Software Abaqus lieferte das Ingenieurmodell bereits gute Ergebnisse. Hierzu wurden jeweils die Schubspannungen im Dämmkern von Querschnitten mit und ohne Verbundmitteln sowie die Spannungen in den Schubgittern bei verschiedenen Laststufen verglichen.

Zur Abbildung der Rissbildung in den Deckschichten wurde das Ingenieurmodell entsprechend erweitert. Hierzu wurde ein „vereinfachtes Rissbildungsmodell“ aufgestellt mit dem die Steifigkeit im Zustand II der Deckschichten in Abhängigkeit von Moment und Normalkraft für Biege- und Trennrisse berechnet werden kann.

Zur Beschreibung der Lastverformungskurve wird die Last durch ein Lastschrittverfahren schrittweise auf das Sandwichelement aufgebracht. Dabei werden bei jedem Last- 
schritt die Schnittgrößen und Verformungen berechnet. Über die Deckschichtlänge wird abschnittsweise geprüft, ob die aus den Schnittgrößen resultierenden Spannungen die Zugfestigkeit des Betons überschreiten. Kommt es zu Biege- oder Trennrissen werden die Steifigkeiten der jeweiligen Abschnitte über das Rissbildungsmodell berechnet und mit einem Wichtungsansatz über die gesamte Deckschichtlänge gemittelt. Die dadurch ermittelte Steifigkeit der Deckschicht wird dann beim nächsten Lastschritt angesetzt.

Für die Wichtung der Steifigkeiten wurden drei verschiedene Ansätze vorgeschlagen:

- Ansatz A - „Arithmetische Mittelwertbildung“

- Ansatz B - „Wichtung über die Durchbiegungslinie“

- Ansatz C - „Wichtung über die Schnittkraftverläufe“

Der Berechnungsalgorithmus für das Lastschrittverfahren mit den drei Wichtungsansätzen wurde mit der Software MATLAB erstellt. Durch die Nachrechnung der durchgeführten Biegeversuche zeigte sich, dass durch die Wichtung über die Durchbiegungslinie (Ansatz B) die besten Übereinstimmungen erzielt wurden. Durch die Verwendung des Ansatz A wurde der Einfluss der Rissbildung unterschätzt. Durch Ansatz $\mathrm{C}$ wurde der Einfluss der Rissbildung überschätzt.

Die Berechnungen mit dem Lastschrittverfahren beschränkten sich überwiegend auf die durchgeführten einfeldrig gelagerten 4-Punkt-Biegeversuche. Im Falle von zweifeldrig gelagerten Sandwichelementen würde die Wichtung über die Durchbiegungslinie aufgrund des Mittelauflagers zu Ungenauigkeiten führen.

Eine weitere Vergleichsberechnung wurde an einem Sandwichelement mit einer Spannweite von 8,1 m unter Wind- und Temperaturbeanspruchung durchgeführt. Die Durchbiegungen, Schubspannungen und Schnittgrößen wurde mithilfe des Ingenieurmodells ohne Berücksichtigung der Rissbildung und mit einer linear-elastischen FE-Berechnung mit InfoCAD ermittelt. Dabei waren die Abweichungen des Ingenieurmodells gegenüber der FE-Berechnung meist gering und auf der sicheren Seite.

\subsubsection{Vorschläge für Bemessungskonzepte}

In Horstmann 2010 wurden auf Grundlage der Erkenntnisse aus den experimentellen und rechnerischen Untersuchungen einige Vorschläge für die Bemessung der Sandwichelemente gegeben, die im Folgenden kurz zusammengefasst werden.

Allgemeines:

- „Sandwichelemente mit Deckschichten aus Beton sind aufgrund von Verformungen und der schälenden Beanspruchung aus hygrothermischen Einwirkungen und zur Sicherstellung einer dauerhaften Verbundtragwirkung stets mit Verbundmitteln auszuführen.“

Zwangbeanspruchungen:

- Zur Ermittlung der Temperaturbeanspruchung wird auf DIBt 1995 verwiesen. Falls kein genauer Nachweis über das Temperaturgefälle in den Betondeckschich- 
ten durchgeführt wird, muss hierzu ein Temperaturgradient von $\Delta T=5^{\circ} \mathrm{C}$ angenommen werden.

- Zur Berücksichtigung der Beanspruchung infolge Schwinden wird auf die Zulassungen von Verbundsystemen von mehrschichtigen Stahlbeton-Wandtafeln verwiesen (bspw. DIBt 2008). Hier wird vereinfachend empfohlen, die Temperatur der Außenschale um $10^{\circ} \mathrm{K}$ zu erhöhen. Dies entspricht bei einem Temperaturausdehnungskoeffizienten von Normalbeton einem Schwindmaß von etwa $0,1 \%$. Horstmann 2010 empfiehlt diesen Wert entsprechend den höheren Schwindmaßen von Feinkornbetonen zu skalieren. Dabei könne berücksichtigt werden, dass ein Teil des Zwangs durch das Kriechen des Betons wieder abgebaut wird.

Grenzzustand der Gebrauchstauglichkeit:

- „Sandwichbauteile (...) sollten aus optischen Gesichtspunkten und zur Sicherstellung einer dauerhaften Funktionsfähigkeit der Wärmedämmung im Grenzzustand der Gebrauchstauglichkeit ungerissen bleiben.“

- Zur Bestimmung der Verformungen unter kurzzeitigen Beanspruchungen für ungerissene Bauteile können elastische Berechnungen durchgeführt werden. Ansonsten ist die Rissbildung mit dem erweiterten Ingenieurmodell zu berücksichtigen.

Grenzzustand der Tragfähigkeit:

- „Unter Voraussetzung einer hohen Verbundfestigkeit zwischen Dämmung und Beton (...) kann die Schubtragwirkung des Dämmstoffs für kurzzeitig wirkende Belastungen angesetzt werden."

- „Analog zum Nachweisformat der DIN EN 14509 (...) wird die Zugtragfähigkeit des Dämmkerns in den Nachweisen nicht berücksichtigt." Der charakteristische Wert der Zugfestigkeit soll jedoch größer als $0,018 \mathrm{~N} / \mathrm{mm}^{2}$ sein.

- Die Bemessungswerte der kurzzeitigen Verbund- bzw. Zug- / Schubfestigkeit sollen als 5\%-Quantilwerte berechnet werden. Es wird vorgeschlagen kurze Sandwichbalken aus Musterplatten, die in der vorgesehenen Herstelltechnik produziert wurden, herauszulösen und dann zur Bestimmung der „ansetzbaren Schubfestigkeit“" in Biegeversuchen zu prüfen.

- Dabei soll der Teilsicherheitsbeiwert statistisch ermittelt werden und zwischen 1,5 (nach DIN EN 14509) und 1,8 (für unbewehrten Beton) liegen.

- Einflüsse auf das kurzzeitige Materialverhalten durch Temperatureinwirkungen, Bewitterung und Dämmstoffalterung sind zu berücksichtigen und zu untersuchen.

- Weiterhin werden Konzepte für die Bemessung von Sandwichelementen mit punktund linienförmigen Verbundmitteln angegeben, auf die an dieser Stelle nicht weiter eingegangen wird. 


\subsubsection{Anwendungsbeispiele}

In dem durch die EU geförderten Projekt INSUSHELL (Hegger u. a. 2009) wurden bereits selbsttragende Sandwichelemente zum Neubau des Instituts für Textiltechnik in Aachen eingesetzt. Auch eine tragende Variante der Elemente wurde für den Bau eines Demonstratorgebäudes verwendet (Schneider u. a.|2009). Zur Sicherstellung und Verbesserung des Verbunds wurden in beiden Projekten Verbundmittel aus Edelstahl verwendet.

\subsubsection{Weitere Untersuchungen an Sandwichelementen mit dünnen Betondeckschichten}

Weitere experimentelle und rechnerische Untersuchungen an Sandwichelementen mit dünnen Deckschichten aus Textilbeton wurden an der TU Dresden durchgeführt Curbach $u$. Häußler-Combe 2010, Curbach u. Häußler-Combe 2011, Finzel u. HäußlerCombe 2010). Die experimentell untersuchten Sandwichelemente bestanden aus einem Kern aus Polyurethan-Hartschaum oder Porenbeton mit einer Dicke von $80 \mathrm{~mm}$ und zwei Deckschichten aus Textilbeton mit einer Dicke von je $8 \mathrm{~mm}$. Der Kern und die Deckschichten wurden mit Epoxidharzkleber miteinander verbunden.

Zur rechnerischen Untersuchung wurden verschiedene FE-Programme eingesetzt, $d$. $h$. Abaqus, ANSYS and nmk (Numerische Methode Karlsruhe). Dabei wurden verschiedene Materialgesetze getestet und untersucht. Neben der Berechnung des Lastverformungsverhalten und der Verformungsfiguren der Sandwichelemente, soll die Schubrissbildung im Dämmkern möglichst exakt simuliert werden.

Durch Di Prisco u. Zani 2012 wurde ebenfalls das Tragverhalten von Sandwichelementen mit dünnen Betondeckschichten experimentell und rechnerisch untersucht. Die Elemente bestehen aus einer Deckschicht aus ultrahochfestem Faserbeton (UHPFRC) mit $25 \mathrm{~mm}$ Dicke, einer Deckschicht aus Textilbeton mit $8 \mathrm{~mm}$ Dicke und einem Kern aus Polystyrol-Hartschau mit $80 \mathrm{~mm}$. Die rechnerischen Untersuchungen wurde mit der FE-Software DIANA durchgeführt. 



\section{$4 \quad$ Eigenschaften der Werkstoffe}

\subsection{Hochleistungsbeton}

\subsubsection{Allgemeines}

Für die Herstellung der Probekörper wurden verschiedene Rezepturen eines sehr gefügedichten, selbstverdichtenden Feinkornbetons mit einer Druckfestigkeit von 130 bis $150 \mathrm{~N} / \mathrm{mm}^{2}$ verwendet. Das Größtkorn betrug maximal $2 \mathrm{~mm}$. Die Betonrezepturen glichen aufgrund ihrer Zusammensetzung denen von ultrahochfesten Betonen (UHPC, engl. Ultra High Performance Concrete). Nach Definition betragen die Druckfestigkeiten von ultrahochfesten Betonen jedoch mindestens $150 \mathrm{~N} / \mathrm{mm}^{2}$ (Schmidt 2008). Daher wird die in dieser Arbeit verwendete Matrix lediglich als Hochleistungsbeton (HPC, engl. High Performance Concrete) bezeichnet. Der Begriff „Hochleistungsbeton“ spiegelt zusätzlich wieder, dass der Beton neben der hohen Druckfestigkeit auch weiteren Anforderungen, z. B. hohe Dauerhaftigkeit, hohe Fließfähigkeit, möglichst geringes Schwindverhalten, entsprechen muss.

\subsubsection{Beschreibung der verwendeten Betonrezepturen}

Im Laufe des Projekts wurden vier unterschiedliche HPC-Rezepturen (siehe Tabelle 4.1 verwendet und hinsichtlich ihrer Frisch- und Festbetoneigenschaften untersucht und optimiert. Die Rezepturen HPC-1 und HPC-2 unterschieden sich lediglich in der Art des verwendeten Zements. Für die Rezeptur HPC-1 wurde ein CEM I 42,5 R verwendet, wobei für die Rezeptur HPC-2 ein Zement CEM I 42,5 R SR mit erhöhtem Sulfatwiderstand verwendet wurde. Die Rezeptur HPC-3 glich der Rezeptur HPC-2, jedoch wurde zusätzlich ein Schwindreduzierer hinzugegeben. Um eine homogenere Verteilung des Betonmörtels um die Bewehrung zu erzielen und einen Siebeffekt der Bewehrung beim Betonieren zu vermeiden, wurde im Falle von Rezeptur HPC-4 das Größtkorn auf 0,5 mm verringert. Die feinkörnigere Rezeptur HPC-4 wurde im Rahmen der Dissertation von Heese 2014 hinsichtlich ihrer rheologischen Eigenschaften optimiert.

\subsubsection{Frisch- und Festbetoneigenschaften}

Grundsätzlich wurden die Festbetoneigenschaften der verwendeten Betone im Zuge der Bauteilversuchsreihen ermittelt. Dabei wurden meist auch die Frischbetoneigenschaften bestimmt. Jedoch variierten aus verschiedenen Gründen die Herstellungsund Verarbeitungsbedingungen der Betone, so dass ein direkter Vergleich der Ergebnisse nur bedingt möglich war. 
Tab. 4.1: Zusammensetzung der verwendeten HPC-Rezepturen

\begin{tabular}{|lcccc|}
\hline Ausgangsstoffe & $\begin{array}{c}\text { HPC-1 } \\
{\left[\mathrm{kg} / \mathrm{m}^{3}\right]}\end{array}$ & $\begin{array}{c}\text { HPC-2 } \\
{\left[\mathrm{kg} / \mathrm{m}^{3}\right]}\end{array}$ & $\begin{array}{c}\text { HPC-3 } \\
{\left[\mathrm{kg} / \mathrm{m}^{3}\right]}\end{array}$ & $\begin{array}{c}\text { HPC-4 } \\
{\left[\mathrm{kg} / \mathrm{m}^{3}\right]}\end{array}$ \\
\hline Zement CEM I 42,5 R SR & $831,6^{1}$ & 831.6 & 831.6 & 695 \\
Wasser & 73.8 & 73.8 & 73.8 & 101 \\
Sand 0/2 & 694 & 694 & 694 & - \\
Sand 0,125/0,5 & 223.9 & 223.9 & 223.9 & 779 \\
Quarzmehl QM 1600 & 205.4 & 205.4 & 205.4 & 487 \\
Silikasuspension (incl. 50\% Wasser) & 249.5 & 249.5 & 249.5 & 216 \\
Fließmittel (PCE) & 35.8 & 35.8 & 35.8 & 29.89 \\
Schwindreduzierer & - & - & 6.2 & \\
'Zement CEMI 42,5 Rohne SR & & &
\end{tabular}

${ }^{1}$ Zement CEM I 42,5 R ohne SR

Zum direkten Vergleich unter konstanten Bedingungen wurde daher eine zusätzliche Versuchsreihe zur Untersuchung der Fest- und Frischbetoneigenschaften durchgeführt. Die Verfahren zur Herstellung der Betone und zur Ermittlung der Materialeigenschaften waren identisch mit denen die im Rahmen der Bauteilversuche verwendet wurden. Im Folgenden sind diese daher kurz exemplarisch erläutert.

Die Ausgangsstoffe wurden vor dem Mischen auf $7^{\circ} \mathrm{C}$ herab gekühlt und in einem Einwellen-Zwangsmischer in einer definierten Mischreihenfolge gemischt. Im Anschluss wurden das Setzfließmaß, die Zeit $t_{500}$, sowie der Luftporengehalt nach DIN EN 12350 ermittelt. Die ermittelten Eigenschaften sind in Tabelle 4.2 zusammengefasst.

Tab. 4.2: Frischbetoneigenschaften der verwendeten HPC-Rezepturen

\begin{tabular}{|llcccc|}
\hline & & HPC-1 & HPC-2 & HPC-3 & HPC-4 \\
\hline Setzfließmaß SF & {$[\mathrm{mm}]$} & 960 & 940 & 930 & 1030 \\
Zeit $t_{500}$ & {$[\mathrm{~s}]$} & 4 & 3,3 & 3,8 & 4,1 \\
Frischbetonrohdichte $D$ & {$\left[\mathrm{~kg} / \mathrm{m}^{3}\right]$} & 2301 & 2287 & 2315 & 2310 \\
Luftporengehalt $A_{c}$ & {$[\%]$} & 2,5 & 3,2 & 2,2 & 1,8 \\
\hline
\end{tabular}

Die Probekörper wurden generell, wie die Bauteilversuchskörper auch, trocken gelagert und lediglich mit Folien abgedeckt. Die Festbetoneigenschaften wurden nach DIN EN 12390 ermittelt. Dabei wurde die Druckfestigkeit der Betone an Würfeln mit $150 \mathrm{~mm}$ Kantenlänge bestimmt. Der Elastizitätsmodul wurde an Zylindern mit einem Durchmesser von $150 \mathrm{~mm}$ und einer Höhe von $300 \mathrm{~mm}$ ermittelt. Die Zylinder wurden anschließend zur Bestimmung der Spaltzugfestigkeit verwendet. Weiterhin wurden die Biegezugfestigkeit an kleinen Mörtelprismen mit einer Länge von $160 \mathrm{~mm}$ und einem quadratischen Querschnitt von $40 \mathrm{~mm}$ ermittelt.

Die Ergebnisse weisen eindeutige Tendenzen auf (siehe Tabelle 4.3. Die Verwendung des Zements mit erhöhtem Sulfatwiderstand bewirkte eine Steigerung der Druckfestigkeit und des Elastizitätsmoduls. Obwohl für die Rezeptur HPC-4 rund 16\% weniger Zement und rund $13 \%$ weniger Silikasuspension verwendet wurde, weißt die Druckfestigkeit vergleichbare Werte zu Rezeptur HPC-2 auf. Der Elastizitätsmodul des Betons HPC-4 ist im Vergleich zu den anderen Betonen deutlich größer. Was mit einer sehr gu- 
te Homogenität und Dichte des Gefüges der Betonmatrix begründet werden kann. Dies zeigten auch die Rissflächen der Spaltzugfestigkeitsprüfkörper. Bei den Prüfkörpern der Rezepturen HPC-1 bis HPC-3 zeigten sich vereinzelt Entmischungserscheinungen des Größtkorns (siehe Abbildung 4.1), während bei den Prüfkörpern von Beton HPC-4 eine sehr gute Verteilung der Gesteinskörnung und ein geringer Luftporengehalt zu beobachten war.

Tab. 4.3: Festbetoneigenschaften der verwendeten HPC-Rezepturen

\begin{tabular}{|c|c|c|c|c|c|}
\hline & & HPC-1 & HPC-2 & HPC-3 & HPC-4 \\
\hline Mittlere Druckfestigkeit $f_{c m, \text { cube }}{ }^{1}$ & {$\left[\mathrm{~N} / \mathrm{mm}^{2}\right]$} & 128,0 & 152,6 & 142,3 & 151,6 \\
\hline Mittlerer Elastizitätsmodul $E_{c m}{ }^{2}$ & {$\left[\mathrm{~N} / \mathrm{mm}^{2}\right]$} & 35418 & 39902 & 39135 & 43247 \\
\hline Mittlere Spaltzugfestigkeit $f_{c t m, s p}^{c m} 2$ & {$\left[\mathrm{~N} / \mathrm{mm}^{2}\right]$} & 3,1 & 3,5 & 5,1 & 3,7 \\
\hline
\end{tabular}

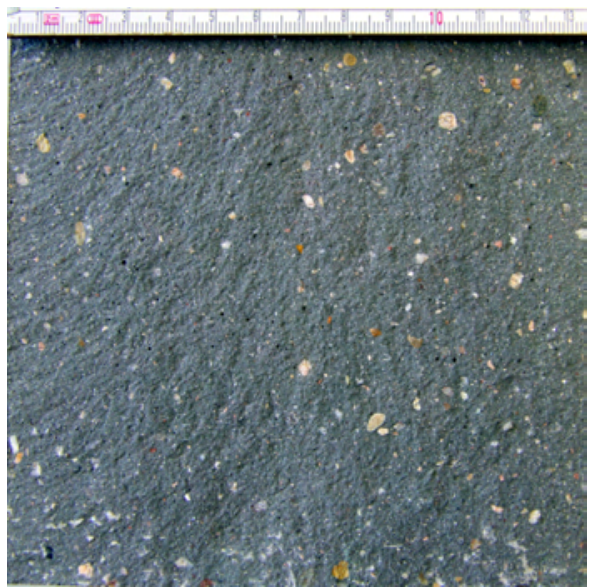

Abb. 4.1: Rissfläche eines Spaltzugkörpers des Betons HPC-1

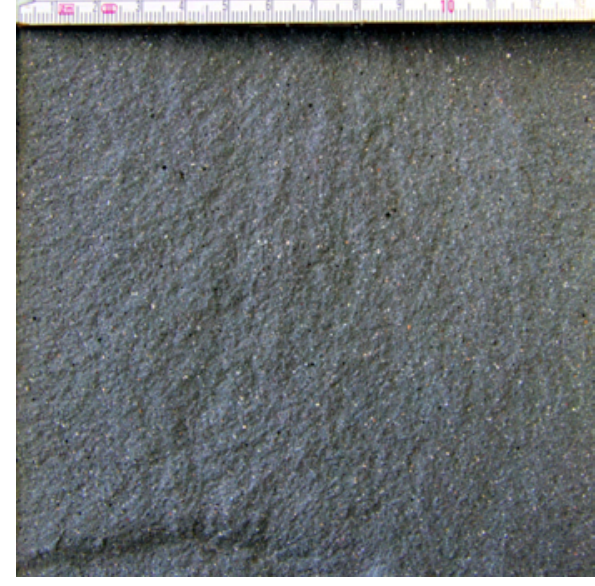

Abb. 4.2: Rissfläche eines Spaltzugkörpers des Betons HPC-4

Für hochfeste Normalbetone (ab C55/67) darf nach DIN 1045-2 aus der Druckfestigkeit $f_{c, \text { dry }}$ von Probekörpern die nach DIN EN 12390-2 Ber 1 gelagert wurden mit Gleichung 4.1 die Druckfestigkeit $f_{c \text {, cube }}$ bei Lagerung nach dem Referenzverfahren (DIN EN 12390-2) ermittelt werden. Diese Gleichung wird hier vereinfachend angewendet.

$$
f_{c, \text { cube }}=0,95 \cdot f_{c, \text { dry }}
$$

Die Zylinderdruckfestigkeit kann aus der Würfeldruckfestigkeit an Probekörpern mit einer Kantenlänge von 150 mm für höherfeste Betone nach folgender Gleichung ermittelt werden (Schnell 2011):

$$
f_{c, c y l}=0,84 \cdot f_{c, \text { cube }}
$$

Die charakteristische Druckfestigkeit des Betons ist dann:

$$
f_{c k}=f_{c, c y l}-8 \mathrm{~N} / \mathrm{mm}^{2}
$$


Nach den Gleichungen (4.1) bis 4.3 ergibt sich für die Rezeptur HPC-4 eine charakteristische Druckfestigkeit $f_{c k}$ von $110 \mathrm{~N} / \mathrm{mm}^{2}$. Dies entspricht in etwa einer Druckfestigkeitsklasse $\mathrm{C} 100 / 115$.

Die zentrische Zugfestigkeit $f_{c t m}$ entspricht bei einem UHPC in etwa der Spaltzugfestigkeit $f_{c t m, s p}$. Alternativ kann $f_{c t m}$ auch nach Gleichung 4.4 aus der Biegezugfestigkeit $f_{c t m, f l}$ ermittelt werden (Schmidt 2008, König u. a. 2001).

$$
f_{c t m}=f_{c t m, f l} \cdot \frac{2 \cdot\left(h / h_{0}\right)^{0,7}}{1+2 \cdot\left(h / h_{0}\right)^{0,7}}
$$

Mit:

$$
\begin{array}{lll}
h_{0} & : & 100 \mathrm{~mm} \\
h & : & 40 \mathrm{~mm} \text { (Prismenhöhe in } \mathrm{mm} \text { ) }
\end{array}
$$

Ergibt sich:

$$
f_{c t m} \approx 0,5 \cdot f_{c t m, f l}
$$

Bei der Auswertung der eigenen Versuche wurde die zentrische Zugfestigkeit jeweils aus der Spalt- und der Biegezugfestigkeit ermittelt. Die Spaltzugfestigkeit lieferte grundsätzlich geringere Werte mit geringerer Streuung. Die Werte die aus der Biegezugfestigkeit errechnet wurden wiesen zum Teil deutlich größere Werte mit größerer Streuung auf. Für die Nachrechnung der Bauteilversuche wurde daher die Spaltzugfestigkeit als zentrische Zugfestigkeit angenommen.

\subsubsection{Schwindverhalten}

Im Zuge der Versuchsreihe zur Bestimmung der Frisch- und Festbetoneigenschaften wurde auch das Schwindverhalten der vier verwendeten Hochleistungsbetone untersucht. Die Schwinduntersuchungen wurde an kleinen Mörtelprismen mit den Abmessungen von $40 \mathrm{~mm} \cdot 40 \mathrm{~mm} \cdot 160 \mathrm{~mm}$ durchgeführt. Zur Bestimmung des Anteils der Schwinddehnung, der infolge des Trocknungsschwindens auftritt, wurden je drei der insgesamt sechs Probekörper eines jeden Betons mit Epoxidharz versiegelt. Die Dehnungsmessung der Versuche wurde mit einem Setzdehnmessgerät durchgeführt. Um Streuungen der Messwerte zu vermeiden, wurde jede Messung dreimal wiederholt und daraus ein Mittelwert gebildet.

Die Dehnungswerte sind als Punktwolke über der Zeit in den Abbildungen 4.3 und 4.4 dargestellt. Durch die gestrichelten Linien werden der Verläufe der Schwinddehnungen über der Zeit vereinfachend dargestellt. Trotz der Mittelwertbildung der Messwerte, weisen die gemessenen Schwinddehnungen deutliche Streuungen auf. Diese sind neben der Ungenauigkeit der Messung mit dem Setzdehnmessgerät auch auf Schwankungen der Luftfeuchte und der Temperatur im klimatisierten Messraum zurückzuführen.

Dennoch kann anhand der Messergebnisse das Schwindverhalten der vier verschiedenen Betonrezepturen qualitativ beurteilt und verglichen werden. Durch die Verwendung 


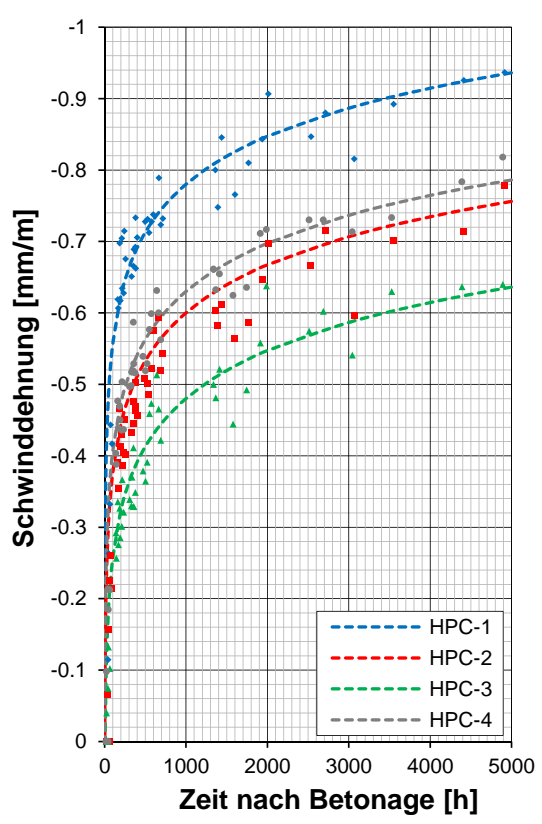

Abb. 4.3: Schwinddehnung der unversiegelten Probekörper

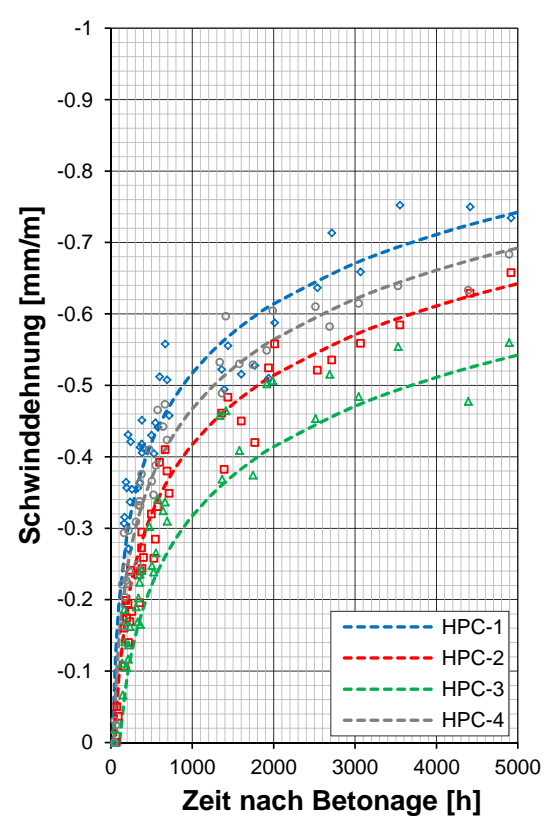

Abb. 4.4: Schwinddehnung der versiegelten Probekörper

eines Zements mit hohem Sulfatwiderstand und durch die Zugabe eines Schwindreduzierers konnten die Schwinddehnungen verringert werden.

Im Fall der versiegelten Primsen sind die ermittelten Schwinddehnungen rein auf das autogene bzw. chemische Schwinden des Betons zurückzuführen. Im Fall der unversiegelten Primsen kann neben dem autogenen Schwinden auch Trocknungsschwinden auftreten, was zu entsprechend größeren Schwinddehnungen führt.

Die Messung der Schwinddehnungen an den unversiegelten Prismen wurde erst nach circa 24 Stunden und die Messung an den versiegelten Primsen erst nach 2 bzw. 3 Tagen begonnen. Dadurch konnte in den Versuchen lediglich ein Teil des Schwindens bestimmt werden. Jedoch tritt bereits ein großer Anteil der Gesamtschwinddehnung schon in Form des plastischen Schwindens und des frühen autogenen Schwindens in diesen ersten Stunden auf.

Durch Fontana 2013 und Pirskawetz u. a. 2011 wurden das frühe autogene Schwinden von verschiedenen Hochleistungsbetonen untersucht. Dabei traten in den ersten 6 bis 10 Stunden nach der Erstarrung des Betons bereits Verformungen von etwa 0,7 bis $1,5 \mathrm{~mm} / \mathrm{m}$ auf. Diese wurden neben dem autogenen Schwinden jedoch auch auf das Abfließen der Hydratationswärme zurückgeführt. Es muss daher davon ausgegangen werden, dass die in den Schwindversuchen gemessenen Werte um bis zu $1,5 \mathrm{~mm} / \mathrm{m}$ vergrößert werden müssen. Die Gesamtschwinddehnung der untersuchten Betone beträgt somit etwa bis zu $2,5 \mathrm{~mm} / \mathrm{m}$. 


\subsection{Bewehrungsmaterialien}

\subsubsection{Allgemeines}

Für die vorliegende Arbeit wurden Stahl-Mikrobewehrung, Glasfaser-Textilbewehrung und Carbon-Textilbewehrung verwendet (siehe Abbildung 4.5 bis 4.7). Die drei Bewehrungsarten werden im folgenden kurz erläutert.

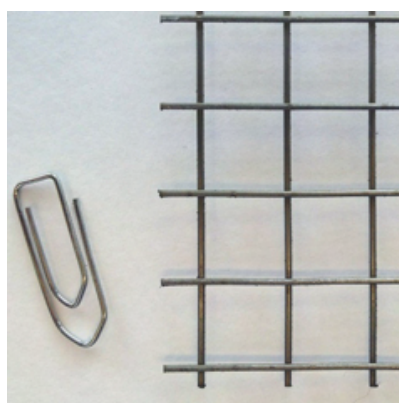

Abb. 4.5: StahlMikrobewehrung

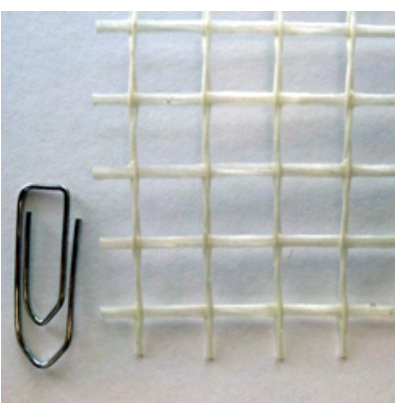

Abb. 4.6: GlasfaserTextilbewehrung

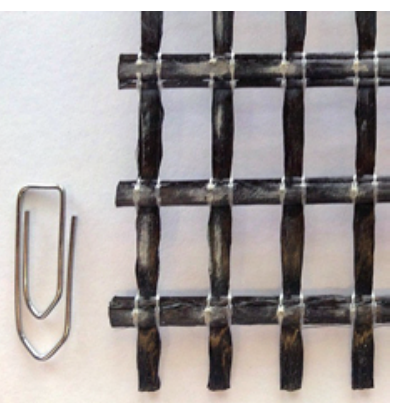

Abb. 4.7: CarbonfaserTextilbewehrung

\subsubsection{Stahl-Mikrobewehrung}

Als Mikrobewehrung wurden durch die Firma DUCON ${ }^{\circledR}$ Drahgittermatten zur Verfügung gestellt. Die Matten bestehen aus normalfestem, glattem Stahldraht. Im Zugversuch weist der Draht das charakteristische Spannungsdehnungsverhalten eines kaltverformten Stahls mit einem ausgeprägten Fließplateau mit maximalen Dehnungen von etwa $300 \%$ auf (DUCON2010). Die Materialeigenschaften der Drahtgittermatten sind in Tabelle 4.4 zusammengefasst.

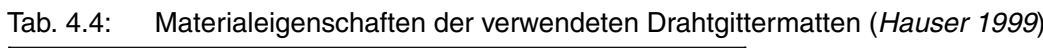

\begin{tabular}{|llc|}
\hline & & S \\
\hline Drahtdurchmesser $d$ & {$[\mathrm{~mm}]$} & 1,0 \\
Maschenweite $a$ & {$[\mathrm{~mm}]$} & 12,7 \\
Mattenquerschnitt $A$ & {$\left[\mathrm{~mm}^{2} / \mathrm{m}\right]$} & 61,8 \\
Mittlere Zugfestigkeit $f_{s t m}$ & {$\left[\mathrm{~N} / \mathrm{mm}^{2}\right]$} & $350-400$ \\
Bruchdehnung $\varepsilon_{\text {su }}$ & {$[\% 0]$} & $170-220$ \\
Elastizitätsmodul $E_{s}$ & {$\left[\mathrm{~N} / \mathrm{mm}^{2}\right]$} & 205.000 \\
\hline
\end{tabular}

\subsubsection{Glasfaser-Textilbewehrung}

Die Glasfaserbewehrung wurde von der Firma NOVACRET Faserbaustofftechnik GmbH zur Verfügung gestellt. Es wurde das alkaliresistente biaxiale Glasfasergewebe cretex ${ }^{\circledR}$ 
M124-10 eingesetzt. Das Textil ist ein Halbdrehergewebe mit Kettfäden bestehend aus jeweils zwei AR310S-800 Fasern und Schussfäden bestehend aus jeweils einer AR620S-800 Faser der Firma Nippon Eletric Glass (NEG). Die Filamentanzahl der beiden Fasern beträgt 800 bzw. 1600. Der Filamentdurchmesser beträgt 13,5 $\mu \mathrm{m}$. Anhaltswerte für die Materialeigenschaften des Gewebes sind in Tabelle 4.5 zusammengestellt.

Tab. 4.5: Materialeigenschaften des verwendeten Glaserfasergewebes cretex ${ }^{\circledR}$ M124-10 |NOVACRET 2000a NOVACRET 2000b NOVACRET 2006 NOVACRET 2012 Jesse u. Curbach 2010)

\begin{tabular}{|llc|}
\hline & & G \\
\hline Faserfeinheit in Längsrichtung $f_{l}$ & {$[\mathrm{tex}]$} & $2 \times 310$ \\
Faserfeinheit in Querrichtung $f_{q}$ & {$[\mathrm{tex}]$} & 620 \\
Maschenweite $a$ & {$[\mathrm{~mm}]$} & 10,0 \\
Rohdichte der Faser $\rho_{g}$ & {$\left[\mathrm{~g} / \mathrm{cm}^{3}\right]$} & 2,7 \\
Gewebequerschnitt $A$ & {$\left[\mathrm{~mm}^{2} / \mathrm{m}\right]$} & 23,0 \\
Faser-Zugfestigkeit $f_{g t}$ & {$\left[\mathrm{~N} / \mathrm{mm}^{2}\right]$} & $1.000-1.400$ \\
Faser-Bruchdehnung $\varepsilon_{g u}$ & {$[\% \circ]$} & $15-20$ \\
Faser-Elastizitätsmodul $E_{g}$ & {$\left[\mathrm{~N} / \mathrm{mm}^{2}\right]$} & $74.000-80.000$ \\
\hline
\end{tabular}

\subsubsection{Carbonfaser-Textilbewehrung}

Von der Firma SGL Carbon SE wurde die Carbonfaserbewehrung zur Verfügung gestellt. Hier kam das biaxiale Carbonfasergelege SIGRATEX ${ }^{\circledR}$ Grid 600 mit gegenlegiger Trikotbindung zum Einsatz. Für Quer- und Längsfasern wurde die Faser Carbon C30 T050 EPY eingesetzt. Diese Faser wird aufgrund der hohen Anzahl von 50.000 Filamente als „heavy-tow“ bezeichnet. Der Filamentdurchmesser des Rovings beträgt $6,5 \mu \mathrm{m}$. Die weiteren Materialeigenschaften sind in Tabelle 4.6 zusammengefasst.

Tab. 4.6: Materialeigenschaften des verwendeten Carbonfasergeleges SIGRATEX ${ }^{\circledR}$ Grid 600 SGL Carbon 2012a, SGL Carbon 2012b Jesse u. Curbach2010,

\begin{tabular}{|llc|}
\hline & & C \\
\hline Faserfeinheit in Längsrichtung $f_{l}$ & {$[\mathrm{tex}]$} & 3.500 \\
Faserfeinheit in Querrichtung $f_{q}$ & {$[\mathrm{tex}]$} & 3.500 \\
Abstand der Längsfasern $a_{l}$ & {$[\mathrm{~mm}]$} & 10,8 \\
Abstand der Querfasern $a_{q}$ & {$[\mathrm{~mm}]$} & 18,0 \\
Rohdichte der Faser $\rho_{c}$ & {$\left[\mathrm{~g} / \mathrm{cm}^{3}\right]$} & 1,8 \\
Gelegequerschnitt längs $A_{l}$ & {$\left[\mathrm{~mm}^{2} / \mathrm{m}\right]$} & 180,0 \\
Gelegequerschnitt quer $A_{q}$ & {$\left[\mathrm{~mm}^{2} / \mathrm{m}\right]$} & 108,0 \\
Faser-Zugfestigkeit $f_{c t}$ & {$\left[\mathrm{~N} / \mathrm{mm}^{2}\right]$} & 4.000 \\
Faser-Bruchdehnung $\varepsilon_{c u}$ & {$[\% 0]$} & 17 \\
Faser-Elastizitätsmodul $E_{c}$ & {$\left[\mathrm{~N} / \mathrm{mm}^{2}\right]$} & 240.000 \\
\hline
\end{tabular}




\subsection{Extrudierter Polystyrol-Hartschaum (XPS)}

\subsubsection{Allgemeines}

Für die Versuche wurden drei verschiedene XPS-Sorten mit den Produktbezeichnungen „Styrodur ${ }^{\circledR}$ “ 2800 C, 4000 CS und 5000 CS der Firma BASF verwendet. Styrodur ${ }^{\circledR}$ $2800 \mathrm{C}$ weist handelsüblich eine thermisch geprägte, rautenförmige Oberflächenstruktur auf. Die Rauten sind in Extrusionsrichtung ausgerichtet und ca. 11,5 mm lang und ca. $5,5 \mathrm{~mm}$ breit. Das Styrodur ${ }^{\circledR} 4000 \mathrm{CS}$ und $5000 \mathrm{CS}$ wird üblicherweise nur mit glatter Schäumhaut hergestellt. Jedoch wurden auch in diese Dämmplatten eine identische Oberflächenstruktur nachträglich durch die BASF eingeprägt.

\subsubsection{Rohdichte und Druckfestigkeit}

Die Rohdichten und Druckfestigkeiten des verwendeten Styrodurs ${ }^{\circledR}$ sind in Tabelle 4.7 zusammengestellt. Die Styrodur $^{\circledR}$-Sorten 4000 CS und 5000 CS weisen eine höhere Rohdichte auf als die des $2800 \mathrm{C}$. Jedoch variieren die Rohdichten und damit auch die Materialeigenschaften des Styrodurs ${ }^{\circledR}$ mit jeder Charge. Zur besseren Unterscheidung werden die sieben verwendeten Chargen daher mit X0 bis $\mathrm{X} 7$ nummeriert.

Tab. 4.7: Rohdichte und Druckfestigkeiten der verwendeten XPS-Sorten

\begin{tabular}{|llccc|}
\hline Nr. & XPS-Produkt & $\begin{array}{c}\text { Dicke } \\
{[\mathrm{mm}]}\end{array}$ & $\begin{array}{c}\text { Rohdichte } \\
{\left[\mathrm{kg} / \mathrm{m}^{3}\right]}\end{array}$ & $\begin{array}{c}\text { Druckfestigkeit } \\
{\left[\mathrm{kN} / \mathrm{m}^{2}\right]}\end{array}$ \\
\hline X0 & Styrodur $^{\circledR}$ 2800 C & 120 & 32,0 & 311 \\
X1 & Styrodur $^{\circledR} 2800 \mathrm{C}$ & 120 & 36,7 & 469 \\
X2 & Styrodur $^{\circledR}$ 4000 CS & 120 & $\mathbf{3 7 , 7}$ & N.V. \\
X3 & Styrodur $^{\circledR}$ 5000 CS & 120 & $\mathbf{4 6 , 9}$ & 746 \\
X4 & Styrodur $^{\circledR}$ 2800 C & 140 & 36,0 & N.V. \\
X5 & Styrodur $^{\circledR} 2800 \mathrm{C}$ & 120 & 31,4 & 331 \\
X6 & Styrodur $^{\circledR} 2800 \mathrm{C}$ & 120 & 34,0 & 416 \\
X7 & Styrodur $^{\circledR}$ 2800 C & 120 & 33,4 & 478 \\
\hline
\end{tabular}

\subsubsection{Zellstruktur und Oberflächenstruktur}

\subsubsection{Allgemeines}

Um die Aussagen über die Zellstruktur des XPS aus der Literatur zu bestätigen (siehe Abschnitt 3.2, Extrudierter Polystyrol-Hartschaum (XPS) ]') wurden eine Reihe von Mikroskopaufnahmen und Computertomographie-Visualisierungen erstellt. Die Darstellungen dienen weiterhin zur Interpretation der Verformungsmechanismen und der Versuchsergebnisse aus Kapitel 5, Experimentelle Untersuchungen]: 


\subsubsection{Mikroskopaufnahmen}

Die Mikroskopaufnahmen wurden mit einem Auflichtmikroskop erstellt. Hierzu wurden aus den verschiedenen XPS-Chargen mit einem Skalpell über die Höhe der jeweiligen Dämmplatte, wie in Abbildung 4.8 dargestellt, drei etwa $1 \mathrm{~mm}$ dünne Proben mit quadratischem Grundriss herausgeschnitten.

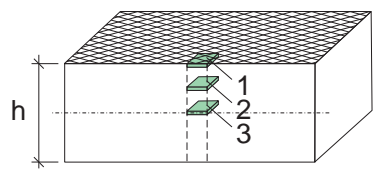

Abb. 4.8: Lage der entnommen XPS-Proben über die Höhe der Dämmplatte

Im Vergleich der Aufnahmen in Abbildung 4.9 und 4.10 zeigt sich, dass die Zellen des XPS X4 mit einer geringeren Rohdichte $\left(\rho=36,0 \mathrm{~kg} / \mathrm{m}^{3}\right)$ deutlicher größer sind als die Zellen des XPS X2 mit größerer Rohdichte $\left(\rho=37,7 \mathrm{~kg} / \mathrm{m}^{3}\right)$. Weiterhin lässt sich durch den Vergleich der drei Aufnahmen einer jeweiligen Dämmplatte erkennen, dass die Zellgröße der Proben in Dickenrichtung von der Mitte zum Rand der Dämmplatte zunimmt.
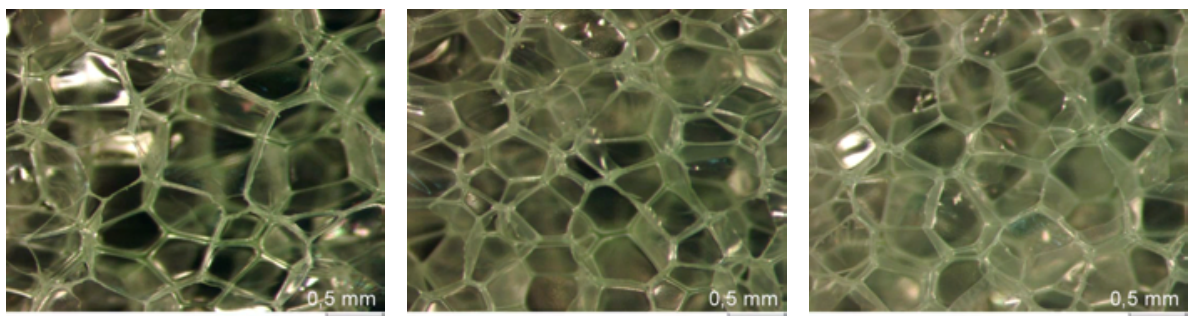

Abb. 4.9: Mikroskopaufnahmen der XPS-Charge X4 in den Lagen 1, 2 und 3 (von links nach rechts)
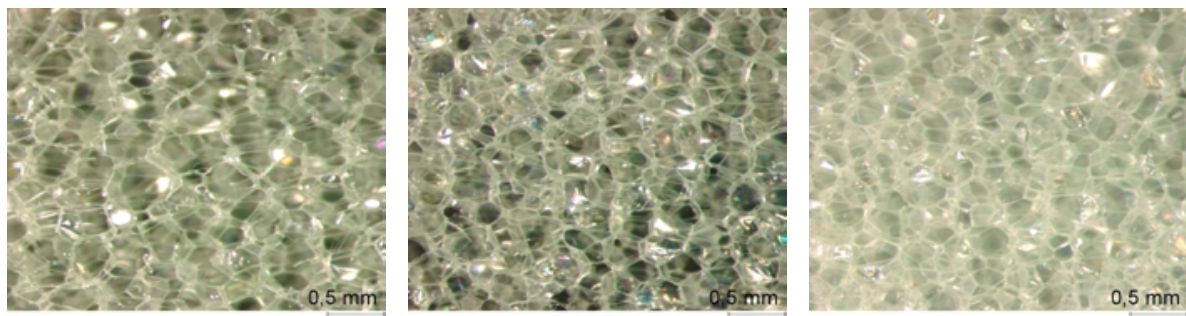

Abb. 4.10: Mikroskopaufnahmen der XPS-Charge X2 in den Lagen 1, 2 und 3 (von links nach rechts)

In den Mikroskopaufnahmen (Abbildung 4.11 und 4.12) des XPS konnten teilweise verformte Zellwände festgestellt werden. Es wird angenommen, dass die Zellstruktur bereits durch das Heraus- und Zuschneiden mit dem Skalpell gestört und verformt wurden. 


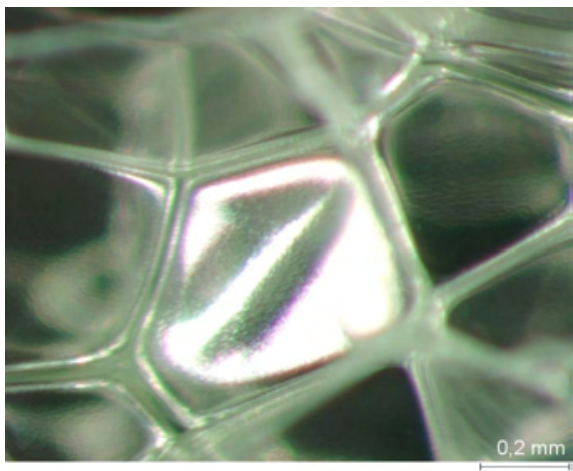

Abb. 4.11: Mikroskopaufnahme einer verformten Zellwand des XPS X5

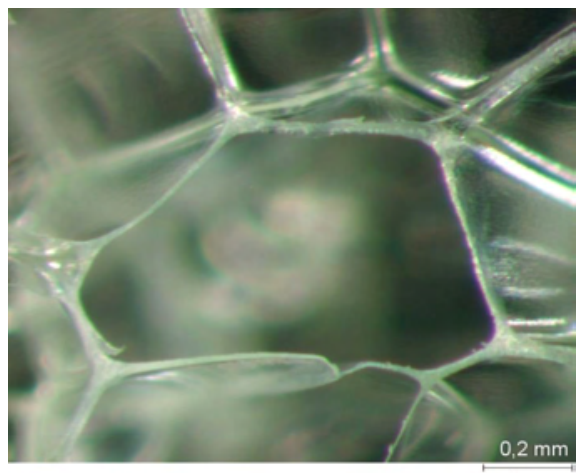

Abb. 4.12: Mikroskopaufnahme einer geschnittenen Zelle des XPS X5

\subsubsection{Computertomographie-Aufnahmen}

In Zusammenarbeit des Fachgebiets Werkstoffe im Bauwesen der TU Kaiserslautern sowie dem Fraunhofer-Institut für Techno- und Wirtschaftsmathematik in Kaiserslautern wurden 3D-Visualisierungen des verwendeten XPS auf Grundlage von Computertomographie-Aufnahmen erstellt.

Dazu wurden die untersuchten XPS-Proben mit einem Skalpell großzügig aus den Dämmplatten herausgeschnitten und dann zu Würfeln mit einer Kantenlänge von etwa $10 \mathrm{~mm}$ zugeschnitten. Mit dem Röntgentomograph des Fraunhofer-Instituts wurden die Probewürfel auf einem drehbaren Teller schrittweise aus unterschiedlichen Winkelpositionen durchstrahlt. Die dadurch erzeugten Projektionsbilder bzw. Röntgenaufnahmen der einzelnen Winkelpositionen wurden dann mit einem Computer zu einem räumlichen Bild weiterverarbeitet, einer sogenannten Computertomographie (CT)-Aufnahme.

Aufgrund des Röntgenverfahrens bestanden die CT-Aufnahmen, wie übliche Röntgenaufnahmen auch, nur aus Graustufen. Dabei wiesen die Bestandteile der Proben mit einer großen Dichte, d. h. das Polystyrol der Zellwände und -kanten, hellere Graustufen auf, als die Bestandteile mit einer geringen Dichte, d. h. die Luft bzw. das Zellgas im Inneren der Zellen.

Um die beiden Bestandteile, Polystyrol und Luft bzw. Zellgas, klar von einander zu trennen bzw. zu „segmentieren“, wurde mit einer Analysesoftware ein sogenanntes Schwellwertverfahren durchgeführt. Dabei wird ein Grauwert definiert, der einen Grenzwert oder auch „Schwellwert“ zwischen Polystyrol und Luft bzw. Zellgas darstellt. Allen Komponenten mit einem helleren Grauwert als dem Schwellwert wird der Wert "1“ (d. h. Polystrol) und allen dunkleren Komponenten der Wert „0“ (d. h. Luft bzw. Zellgas) zugewiesen. Deshalb nennt man dieses Verfahren auch „Binarisierung“ SSchnell u.a. 2010).

Mit der Analysesoftware kann dieses binarisierte räumliche Bild leicht weiter bearbeitet und ausgewertet werden. Aus der tomographierten XPS-Probe wurden mit der Software Bereiche „herausgeschnitten“. Dies bietet den Vorteil, dass das ungestörte XPS untersucht werden kann. 
In den Abbildungen 4.13 und 4.14 sind die Visualisierungen zweier XPS-Würfel, die mit einer Kantenlänge von 3,8 mm aus der Probe herausgeschnitten wurden dargestellt. Auch hier lässt sich deutlich der Unterschied in der Zellgröße der beiden XPS-Sorten erkennen.

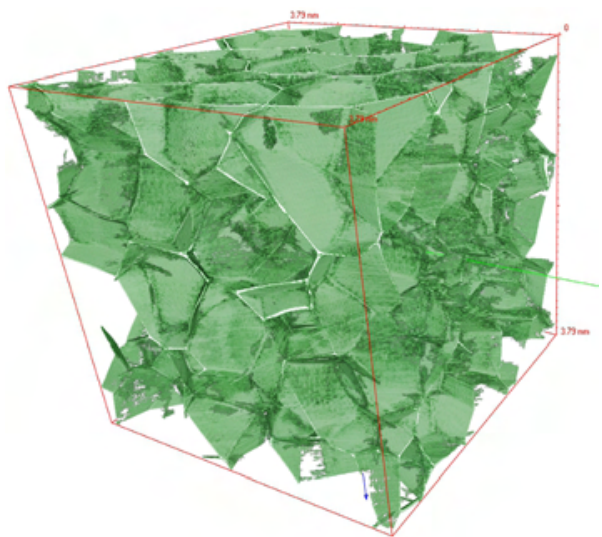

Abb. 4.13: Mit Computertomographie erstellte 3D-Visualisierung von XPS mit geringer Rohdichte

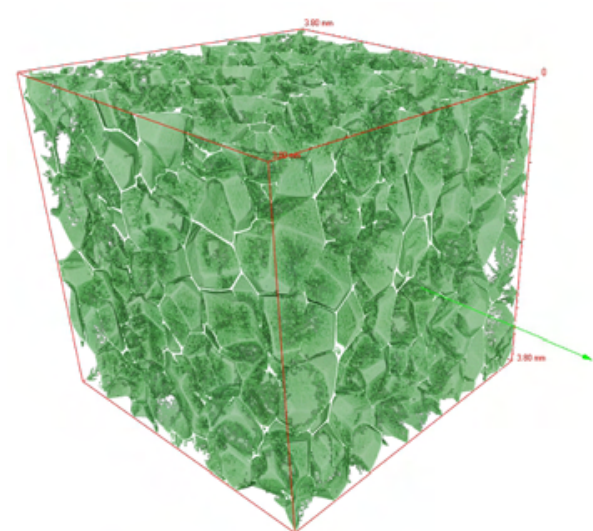

Abb. 4.14: Mit Computertomographie erstellte 3D-Visualisierungvon von XPS mit großer Rohdichte 



\section{$5 \quad$ Experimentelle Untersuchungen}

\subsection{Allgemeines}

Die im Rahmen dieses Arbeit durchgeführten Versuche können in drei Gruppen gegliedert werden (siehe Abbildung 5.1).
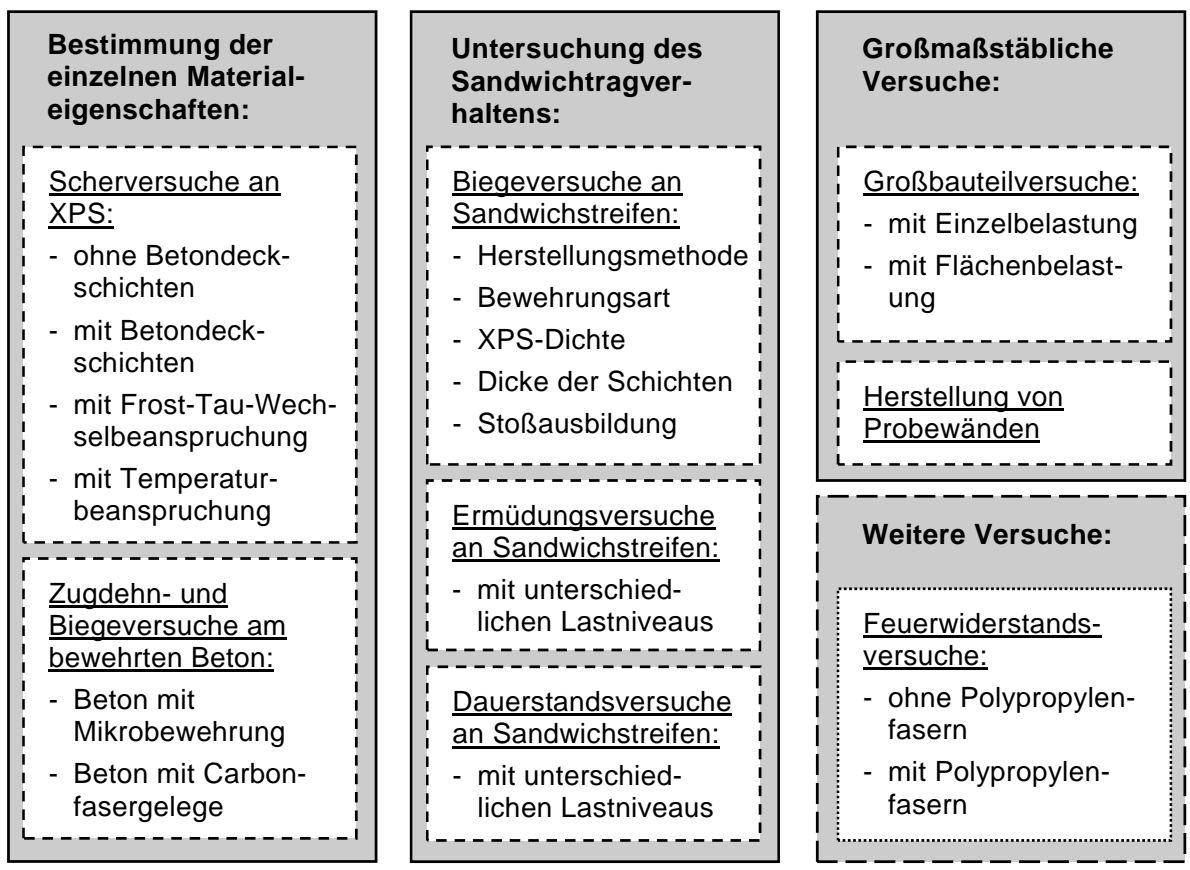

Abb. 5.1: Gliederung der experimentellen Untersuchungen

In Kleinteilversuchen wurden einzelne Materialeigenschaften bestimmt. Diese beinhalteten 85 Scherversuche zur Bestimmung der Schereigenschaften des XPS und des Verbunds zwischen XPS und Beton, sowie insgesamt 21 Zug- und Biegeversuche an bewehrtem Hochleistungsbeton. Ziel war es, das generelle Tragverhalten der verwendeten Materialien getrennt voneinander zu untersuchen sowie deren wichtigsten Werkstoffeigenschaften zu bestimmen. Die ermittelten Werkstoffeigenschaften dienten weiterhin als Eingangsparameter für die rechnerischen Untersuchungen und zur Kontrolle des Nachweiskonzepts. Mithilfe von Scherversuchen wurden die Scherfestigkeit und der Schubmodul des XPS sowie die Scherfestigkeit der Verbundfuge zwischen XPS und Beton ermittelt. Anhand von Zug- und Biegeversuchen wurden die Zugfestigkeiten, sowie die Elastizitätsmoduln der Bewehrung und des bewehrten Betons bestimmt. 
Das generelle Sandwichtragverhalten und somit das Zusammenwirken der beiden Materialien XPS und bewehrter Beton wurde in Biegeversuchen an Sandwichstreifen untersucht. Dazu wurden insgesamt 46 Versuche durchgeführt. Aufgrund der Verwendung von relativ kurzen und schmalen Sandwichstreifen mit einer Spannweite von $1,15 \mathrm{~m}$ und einer Breite von 0,2 $\mathrm{m}$ konnte mit verhältnismäßig geringem Aufwand eine Vielzahl verschiedener Parameter untersucht werden. Ziel war es, durch die Änderung jeweils eines Parameters der Sandwichstreifen dessen Einfluss auf das Lastverformungsverhalten zu untersuchen. Weiterhin sollte durch die Variation der Parameter eine möglichst große Anzahl an verschiedenen Versagensarten erzielt werden. Die Ergebnisse aus den Biegeversuchen an Sandwichstreifen dienten weiterhin als Grundlage für die rechnerischen Untersuchungen, insbesondere zur Kalibrierung des FE-Modells und zur Kontrolle des Bemessungskonzepts. Durch Variation der Belastungsart und des Aufbaus der Biegeversuche konnte zusätzlich das Ermüdungs- und das Dauerstandsverhalten der Sandwichelemente untersucht werden. Dies erfolgte durch eine zyklische Biegebeanspruchung von elf Sandwichstreifen mit einer positiven und negativen Momentenbeanspruchung sowie einer Langzeitbelastung in drei Dauerstandsversuchen.

Abschließend wurde in sechs Großbauteilversuchen die Tragfähigkeit von großformatigen Sandwich-Wandelementen unter möglichst realitätsnaher Belastung untersucht. Dabei erfolgte die Belastung in drei der Versuche mit einem hydraulischen Prüfzylinder durch vier gleichmäßig verteilte Einzellasten. In drei weiteren Versuchen wurde eine flächige Belastung mithilfe eines eigens konstruierten Vakuum-Prüfstands aufgebracht. Ziel der Versuche war es, mögliche Maßstabseffekte zu bestimmen, die in den Biegeversuchen an Sandwichstreifen nicht erfasst wurden. Die Versuchsergebnisse der Großbauteilversuche dienten weiterhin zur Kontrolle des Bemessungskonzepts. Schließlich wurde durch die Konstruktion von zwei Probewänden die praktische Herstellbarkeit erprobt und demonstriert.

Neben dem Tragverhalten wurde auch das Brandverhalten der Sandwichelemente in zwei Tastversuchen experimentell untersucht. Diese sind im Abschnitt 9.3, Feuerwiderstandsversuche beschrieben.

\subsection{Biegeversuche an Sandwichstreifen}

\subsubsection{Allgemeines}

Zur Bestimmung des Biegetragverhaltens und der Versagensarten wurde in Anlehnung an DIN EN 14509 ein Versuchsaufbau zur Prüfung von kurzen Sandwichstreifen mit einer Stützweite von $1.150 \mathrm{~mm}$ und einer Breite von $200 \mathrm{~mm}$ verwendet.

\subsubsection{Versuchsprogramm}

Im Versuchsprogramm wurde in fünf Versuchsreihen jeweils nur eine begrenzte Anzahl von Parametern bzw. Eigenschaften mit dem zu erwartenden größten Einfluss auf das Tragverhalten der Sandwichstreifen variiert: 


\section{- Herstellungsmethode:}

In der ersten Versuchsreihe wurden der Einfluss von zwei verschiedenen Herstellungsvarianten auf das Lastverformungsverhalten untersucht. Bei der ersten Variante wurde der XPS-Kern in den frischen Beton eingedrückt. Bei der zweiten Variante wurde direkt auf den Kern aufbetoniert. Die Vorgehensweise wird ausführlich in Abschnitt 5.2.3, Probekörper und Herstellung' erläutert.

\section{- Bewehrungsart:}

Zur Untersuchung des Einflusses des Bewehrungsmaterials, wurden in einer Versuchsreihe drei verschiedene Arten der Bewehrung verwendet. Es kamen hierzu ein Glasfasergewebe, ein Carbonfasergelege und eine Mikrobewehrung aus Stahldrahtmatten zum Einsatz. In die Deckschichten der Probekörper mit Glasfaser- und Mikrobewehrung wurden jeweils drei Lagen eingebracht. Aufgrund der hohen Zugfestigkeit und des großen Querschnitts, wurden die Deckschichten mit Carbongelege mit je nur einer Lage bewehrt.

\section{- Dichte des XPS:}

Die Schereigenschaften der Kernschicht sind abhängig von der Dichte des verwendeten XPS. Um den Einfluss der Schereigenschaften auf das Tragverhalten zu bestimmen, wurden verschiedene XPS-Chargen mit unterschiedlicher Dichte verwendet. Da die ersten acht mikrobewehrten Probekörper zum Teil durch ein Zugversagen der Bewehrung versagten, wurden zusätzlich sechs weitere Probekörper mit Carbonbewehrung hergestellt, um somit ein Zugversagen der Bewehrung auszuschließen.

\section{- Schichtdicken:}

Die Schichtdicke ist ein weiterer Einfluss, der das Tragverhalten maßgeblich beeinflusst. Daher wurden jeweils drei Probekörper mit dem Dickenverhältnis von Deckschicht zu Kernschicht von $10 \mathrm{~mm}$ zu $140 \mathrm{~mm}, 15 \mathrm{~mm}$ zu $120 \mathrm{~mm}$ und $20 \mathrm{~mm}$ zu $120 \mathrm{~mm}$ hergestellt. Die Deckschichten aller Probekörper wurden mit Mikrobewehrung und mit gleichem Bewehrungsgrad bewehrt. Anstatt mit 3 Bewehrungslagen, wurden die Deckschichten mit einer Dicke von $15 \mathrm{~mm}$ daher mit 4 Lagen und einer zusätzlichen fünften Lage, die nur den halben Querschnitt aufwies, bewehrt. Die Deckschichten mit einer Dicke von 20 mm wurden mit 6 Lagen bewehrt.

\section{- Stoßausbildung des XPS:}

Aufgrund der verfügbaren Abmessungen der Dämmplatten von 1,25 m x 0,60 mm, müssen diese zur Herstellung von größeren Sandwichelementen miteinander an den Stößen verklebt werden. Zur Untersuchung dieser Stoßverklebung wurden die Kerne der Probekörper dieser Versuchsreihe zwischen der rechten Lasteinleistungsstelle und dem rechten Auflagerpunkt an jeweils drei Stellen auseinander geschnitten und anschließend mit einem PUR-Klebstoff wieder zusammengefügt (siehe Abschnitt 5.2.3, Probekörper und Herstellung').

Die einzelnen Versuchsreihen mit den jeweiligen Versuchen sind in den Tabellen 5.1 und 5.2, Versuchsprogramm Biegeversuche' zusammengestellt. Die Bezeichnung der Probekörper ist in Abbildung 5.2 erläutert. Diese ist in drei Teile gegliedert, die durch Bindestriche voneinander getrennt sind. Der erste Teil der Bezeichnung gibt die Art der Bewehrung und der XPS-Charge wieder. Der zweite Teil entspricht den Dicken 
der einzelnen Schichten und der letzte Teil ist eine fortlaufende Nummerierung der jeweiligen Parameterkonstellation.

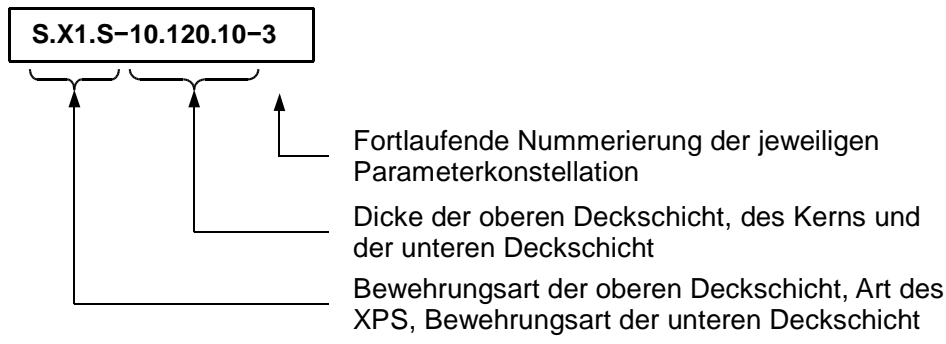

Abb. 5.2: Probekörperbezeichnung

\subsubsection{Probekörper und Herstellung}

Vor der Betonage der Elemente wurden die XPS-Platten mithilfe eines GlühdrahtSchneidegeräts in Streifen von $200 \mathrm{~mm}$ Breite zugeschnitten. Die Waffelstruktur war somit in Längsrichtung der Streifen ausgerichtet. Zur Bestimmung ihrer Rohdichte, wurden die Streifen im Anschluss gewogen und vermessen. Die Zuschläge des Hochleistungsbetons wurden ebenfalls abgewogen und in einer Kühlkammer auf $7^{\circ} \mathrm{C}$ herabgekühlt. Durch das Herabkühlen konnte eine längere Verarbeitungszeit und reproduzierbare Frischbetoneigenschaften gewährleistet werden.

Die XPS-Streifen der Versuchsreihe „Stoßausbildung des XPS“ wurden an drei Stellen (siehe Abbildung 5.3) auseinander geschnitten. Anschließend wurden die Streifen mit dem Polyurethan (PUR)-Schmelzklebstoff 1387 der Firma Bühnen zusammengefügt. Der Klebstoff wurde mit einer pneumatischen Schmelzklebstoff-Auftragsspistole aufgetragen. Hierbei wurde der Klebstoff im Auftragsgerät auf $140^{\circ} \mathrm{C}$ erhitzt und dann mithilfe einer Auftragsdüse und einem über Kompressor aufgebrachten Druck von 6 bis 7 bar auf die Stoßfläche aufgesprüht (siehe Abbildung 5.4). Die einzelnen Teilstücke wurden dann aneinander gelegt, ausgerichtet und etwa eine Minute leicht zusammengedrückt (vgl. Abbildung 5.5.

Die Herstellung und Betonage der Sandwichstreifen erfolgte dann in den beiden folgenden Varianten:

\section{- Variante „Eindrücken des XPS-Kerns in den Beton“:}

Als horizontale Schalung diente eine Schaltafel. Als vertikale Schalung wurden Holzleisten mit der Höhe der Deckschichtdicke verwendet, die dann auf die Schaltafel aufgeschraubt wurden. Die einzelnen Lagen der Mikrobewehrung und des Glasfasergewebes wurden zu Bündeln zusammengebunden und in die Schalung gelegt (siehe Abbildung 5.6. Das Carbongelege wurde stets einlagig verwendet. Um die Bewehrungslagen mittig in der Deckschicht anzuordnen wurden zum Teil Abstandhalter verwendet. Plastikverbinder, die zum Verschluss von PERohrisolierungen verwendet werden, haben sich hierbei als hilfreich erwiesen. 
Tab. 5.1: Versuchsprogramm Biegeversuche

\begin{tabular}{|c|c|c|c|c|c|c|c|}
\hline Parameter & Probekörper & HPC & VA $^{1}$ & {$\left[\begin{array}{c}a^{2} \\
{[\mathrm{~mm}]}\end{array}\right]$} & $\left.\begin{array}{c}\boldsymbol{b}^{3} \\
{[\mathrm{~mm}]}\end{array}\right]$ & $\begin{array}{c}c^{4} \\
{[\mathrm{~mm}]}\end{array}$ & $\mathrm{BA}^{5}$ \\
\hline \multicolumn{8}{|l|}{ Herstellungsmethode: } \\
\hline \multirow{6}{*}{$\begin{array}{l}\text { Eindrücken des XPS-Kerns } \\
\text { in den Beton }\end{array}$} & S.X0.S-10-120.10-1 $1^{6}$ & 1 & 4-PBV & 100 & 350 & 50 & $Q S+L$ \\
\hline & S.X0.S-10.120.10-2 & 1 & 4-PBV & 50 & 400 & 50 & $Q S+L$ \\
\hline & S.X0.S-10.120.10-3 & 1 & 4-PBV & 50 & 400 & 50 & $Q S+L$ \\
\hline & S.X0.S-10.120.10-4 & 1 & 4-PBV & 50 & 400 & 50 & $Q S+L$ \\
\hline & $0.120 .10-5$ & 1 & 3-BPV & 50 & 575 & 50 & $Q S+L$ \\
\hline & $0-6$ & 1 & 3-BPV & 50 & 575 & 50 & $Q S+L$ \\
\hline \multirow{3}{*}{$\begin{array}{l}\text { Direktes Betonieren auf dem } \\
\text { XPS-Kern }\end{array}$} & S.X0.S & 1 & 4-PBV & 50 & 400 & 50 & $Q S+L$ \\
\hline & S.X0.S- & 1 & 4-PBV & 50 & 400 & 50 & $Q S+L$ \\
\hline & S.X0. & 1 & 4-PBV & 50 & 400 & 50 & z \\
\hline \multicolumn{8}{|l|}{ Bewehrungsart: } \\
\hline \multirow{4}{*}{3 Lagen Glasfasergewebe } & G.X1.G-10.120.10-1 & 1 & 3-BPV & 50 & 575 & 60 & $Q S+L$ \\
\hline & $X 1 . G-10.120 .10-2$ & 1 & 3-BPV & 50 & 575 & 60 & $Q S+L$ \\
\hline & G.X1.G-10.120.10-3 & 1 & 3-BPV & 50 & 575 & 150 & Z \\
\hline & G.X1.G-10.120.10-4 & 1 & 3-BPV & 50 & 575 & 150 & $Q S+L$ \\
\hline \multirow{4}{*}{1 Lage Carbonfasergelege } & X1.C-10.120.10-1 & 1 & 3-BPV & 50 & 575 & 60 & $Q S+L$ \\
\hline & & 1 & 3-BPV & 50 & 575 & 60 & $Q S+L$ \\
\hline & C.X1.C-10.120.10-3 & 1 & 3-BPV & 50 & 575 & 150 & $Q S+L$ \\
\hline & C.X1.C-10.120.10-4 & 1 & 3-BPV & 50 & 575 & 150 & $Q S+L$ \\
\hline \multirow{3}{*}{3 Lagen Mikrobewehrung } & S.X1.S-10.120.10-1 & 1 & 3-BPV & 50 & 575 & 60 & $Q S+L$ \\
\hline & S.X1.S-10.120.10-2 & 1 & 3-BPV & 50 & 575 & 60 & z \\
\hline & S.X1.S-10. & 1 & 3-BPV & 50 & 575 & 60 & $Q S+L$ \\
\hline \multicolumn{8}{|l|}{ XPS-Dichte: } \\
\hline \multirow{5}{*}{$\begin{array}{l}\left.\text { X2 (Dichte }=36,6 \mathrm{~kg} / \mathrm{m}^{3}\right) \\
3 \text { Lagen Mikrobewehrung }\end{array}$} & S.X2.S-10.120.10-1 & 1 & 4-PBV & 35 & 400 & 50 & $Q S+L$ \\
\hline & S.X2.S-10.120.10-2 & 1 & 4-PBV & 85 & 350 & 50 & $Q S+L$ \\
\hline & S.X2.S-10.120.10-3 & 1 & 4-PBV & 35 & 200 & 50 & $Q S+L$ \\
\hline & S.X2.S-10.120.10-4 & 1 & 4-PBV & 35 & 200 & 50 & $Q S+L$ \\
\hline & S.X2.S-10.120.10-5 & 1 & 4-PBV & 35 & 200 & 50 & $Q S+L$ \\
\hline \multirow{3}{*}{$\begin{array}{l}\text { X3 }\left(\text { Dichte }=45,8 \mathrm{~kg} / \mathrm{m}^{3}\right) \\
3 \text { Lagen Mikrobewehrung }\end{array}$} & S.X3.S-10.120.10-1 & 1 & 4-PBV & 35 & 200 & 50 & $Q S+L$ \\
\hline & S.X3.S-10.120.10-2 & 1 & 4-PBV & 35 & 200 & 50 & $Q S+L$ \\
\hline & S.X3.S-10.120.10-3 & 1 & 4-PBV & 35 & 200 & 50 & $Q S+L$ \\
\hline \multirow{3}{*}{$\begin{array}{l}\left.\text { X2 (Dichte }=36.6 \mathrm{~kg} / \mathrm{m}^{3}\right) \\
1 \text { Lage Carbonfasergelege }\end{array}$} & C.X2.C-10.120.10-1 & 2 & 4-PBV & 35 & 400 & 50 & QS \\
\hline & C.X2.C-10.120.10-2 & 2 & 4-PBV & 35 & 400 & 50 & QS \\
\hline & C.X2.C-10.120.10-3 & 2 & 4-PBV & 35 & 400 & 50 & QS \\
\hline \multirow{3}{*}{$\begin{array}{l}X 3\left(\text { Dichte }=45,8 \mathrm{~kg} / \mathrm{m}^{3}\right), \\
1 \text { Lage Carbonfasergelege }\end{array}$} & C.X3.C-10.120.10-1 & 2 & 4-PBV & 35 & 400 & 50 & QS \\
\hline & C.X3.C-10.120.10-2 & 2 & 4-PBV & 35 & 400 & 50 & QS \\
\hline & C.X3.C-10.120.10-3 & 2 & 4-PBV & 35 & 400 & 50 & QS \\
\hline
\end{tabular}

${ }^{1}$ Versuchsaufbau: 3-PBV = 3-Punkt-Biegeversuch, 4-PBV = 4-Punkt-Biegeversuch (siehe Abbildung 5.3)

2 Überstand an den Auflagerpunkten (siehe Abbildung 5.3)

${ }^{3}$ Abstand zwischen Auflager- und Lasteinleitungspunkten (siehe Abbildung 5.3)

${ }^{4}$ Breite der Lasteinleitungsplatte (siehe Abbildung 5.3)

${ }^{5}$ Belastungsart: $\mathrm{QS}=$ quasi-statisch, $\mathrm{QS}+\mathrm{L}=$ quasi-statisch mit Lastwechseln, $\mathrm{Z}=$ zyklisch

${ }^{6}$ Stützweite $=1.050 \mathrm{~mm}$ 
Tab. 5.2: Versuchsprogramm Biegeversuche (Fortsetzung)

\begin{tabular}{|c|c|c|c|c|c|c|c|}
\hline Parameter & Probekörper & HPC & VA $^{1}$ & $\begin{array}{c}a^{2} \\
{[\mathrm{~mm}]}\end{array}$ & $\begin{array}{c}\boldsymbol{b}^{3} \\
{[\mathrm{~mm}]}\end{array}$ & {$\left[\begin{array}{c}c^{4} \\
{[\mathrm{~mm}]}\end{array}\right]$} & $\mathrm{BA}^{5}$ \\
\hline \multicolumn{8}{|l|}{ Schichtdicken: } \\
\hline \multirow{3}{*}{$\begin{array}{l}\text { Deckschichtdicke }=10 \mathrm{~mm} \text {, } \\
\text { Kerndicke }=140 \mathrm{~mm}\end{array}$} & S.X4.S-10.140.10-1 & 1 & 4-PBV & 50 & 400 & 50 & QS \\
\hline & S.X4.S-10.140.10-2 & 1 & 4-PBV & 50 & 400 & 50 & QS \\
\hline & S.X4.S-10.140.10-3 & 1 & 4-PBV & 50 & 400 & 50 & QS \\
\hline \multirow{3}{*}{$\begin{array}{l}\text { Deckschichtdicke }=15 \mathrm{~mm} \text {, } \\
\text { Kerndicke }=120 \mathrm{~mm}\end{array}$} & S.X5.S-15.120.15-1 & 1 & 4-PBV & 50 & 400 & 50 & QS \\
\hline & S.X5.S-15.120.15-2 & 1 & 4-PBV & 50 & 400 & 50 & QS \\
\hline & S.X5.S-15.120.15-3 & 1 & 4-PBV & 50 & 400 & 50 & QS \\
\hline \multirow{3}{*}{$\begin{array}{l}\text { Deckschichtdicke }=20 \mathrm{~mm} \text {, } \\
\text { Kerndicke }=120 \mathrm{~mm}\end{array}$} & S.X5.S-20.120.20-1 & 1 & 4-PBV & 50 & 400 & 50 & QS \\
\hline & S.X5.S-20.120.20-2 & 1 & 4-PBV & 50 & 400 & 50 & QS \\
\hline & S.X5.S-20.120.20-3 & 1 & 4-PBV & 50 & 400 & 50 & QS \\
\hline \multicolumn{8}{|l|}{ Stoßausbildung: } \\
\hline \multirow{3}{*}{$\begin{array}{l}\text { Stumpfstoß mit } \\
\text { PUR-Klebstoff }\end{array}$} & S.X5.S-10.120.10-1 & 2 & 4-PBV & 50 & 400 & 50 & $Q S+L$ \\
\hline & S.X5.S-10.120.10-2 & 2 & 4-PBV & 50 & 400 & 50 & $Q S+L$ \\
\hline & S.X5.S-10.120.10-3 & 2 & 4-PBV & 50 & 400 & 50 & $\mathrm{QS}+\mathrm{L}$ \\
\hline
\end{tabular}
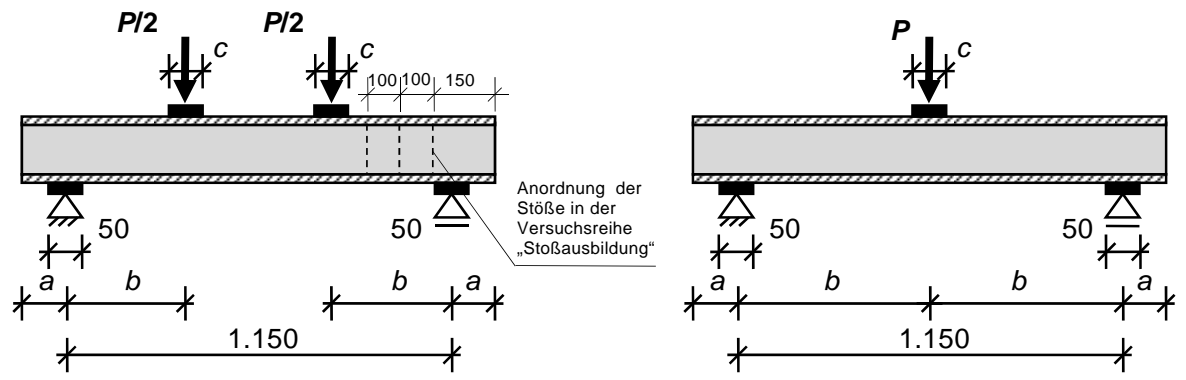

Abb. 5.3: Schematische Darstellung eines 4-Punkt-Biegeversuchs (links) und eines 3-PunktBiegeversuchs (rechts) mit Darstellung der Abmessungen aus Tab. 5.1] und 5.2

Der Beton wurde anschließend mithilfe eines Eimers in die Schalung gegossen und gegebenenfalls mit einer Kelle grob verteilt. Durch die sehr fließfähige, selbstverdichtende Konsistenz des Betons, wurden eine ausreichende Nivellierung des Betonspiegels sowie eine gute Verdichtung ohne zusätzlich erforderliche Maßnahmen erzielt.

Im Anschluss wurde der XPS-Kern in den frischen Beton eingedrückt. Indem der Kern mit einer Längsseite zuerst in den Beton eingebracht und dann flächig aufgelegt wurde, sollten Lufteinschlüsse im Verbund verhindert werden. Zur Gewährleistung eines guten Verbunds zwischen Beton und der Oberflächenstruktur des XPS, wurde der Kern von Hand durch vibrationsartiges Hin- und Herbewegen in den Beton eingedrückt und anschließend ausgerichtet (siehe Abbildung 5.7). Der Kern wurde nun mit Stahlgewichten beschwert um ein Aufschwimmen 


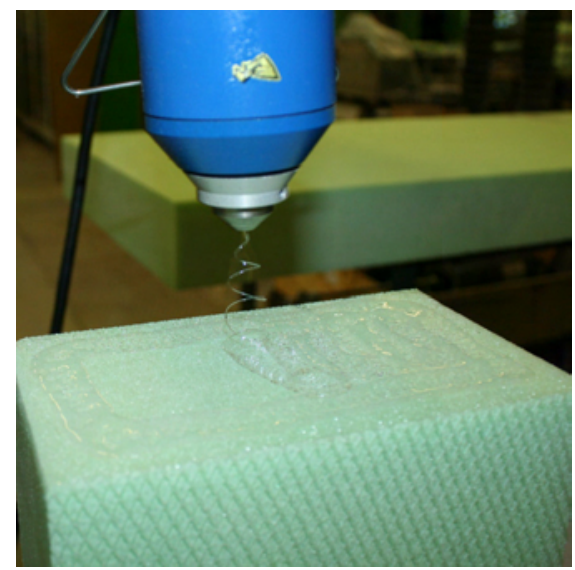

Abb. 5.4: Auftragen des PUR-Klebstoffs mit einer Auftragspistole

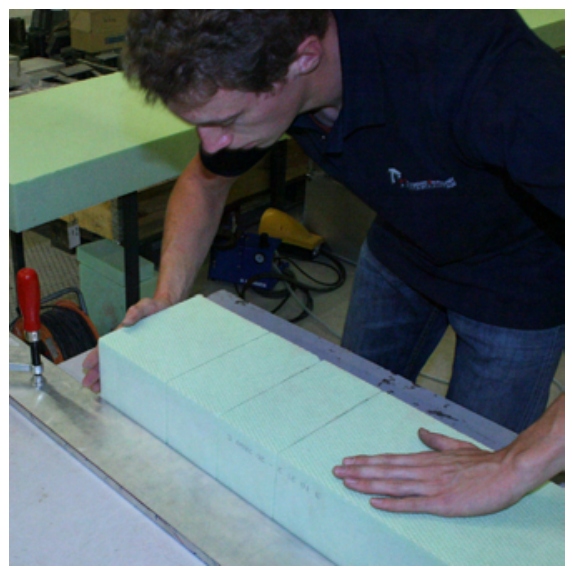

Abb. 5.5: Ausrichten und Andrücken des geklebten XPS-Kerns

des Kerns zu verhindern (siehe Abbildung 5.8). Als Nachbehandlung wurden die Probekörper lediglich mit einer Folie abgedeckt.

Bereits nach einem Tag konnten die Probekörper ausgeschalt und die Schalung für die Betonage der zweiten Deckschicht vorbereitet werden. Die Herstellung erfolgte analog zur Vorgehensweise der ersten Deckschicht (siehe Abbildung 5.9).

\section{- Variante „Direktes Betonieren auf dem XPS-Kern“:}

Um den XPS-Kern wurde eine kastenförmige Schalung mit den Abmessungen der Probekörper konstruiert. Die Bewehrung wurde in Form eines Bündels auf den Kern gelegt (siehe Abbildung 5.10. Anschließend wurde der Beton in die Schalung gegossen und grob verteilt (siehe Abbildung 5.11. Die Nachbehandlung erfolgte erneut lediglich über Abdecken der Probekörper mit Folie. Die Vorgehensweise wurde zur Herstellung der zweiten Deckschicht wiederholt.

Das Eindrücken des XPS-Kerns in den Beton weist einige Vorteile gegenüber dem direktes Aufbetonieren auf dem Kern auf. Mittels der ersten Variante können Probekörper mit beidseitiger Sichtbetonoberfläche hergestellt werden. Wobei hingegen die Oberflächen der Probekörper der zweiten Variante rau und ungleichmäßig beschaffen sind. In Teilbereichen schaute die Mikrobewehrung, die aufgrund des Transports auf einer Rolle eine gewisse Welligkeit aufwies, aus der Betonoberfläche heraus (siehe Abbildung 5.11). Durch Eindrücken des XPS-Kerns in den frischen Beton wird gleichzeitig auch die Bewehrung herunter gedrückt und ein Herausschauen der Bewehrung verhindert.

Weiterhin kann auf die Nachbehandlung der Probekörper bei der ersten Variante nahezu verzichtet werden. Die Schalung und der XPS-Kern schützen den frischen Beton vor Austrocknung und verhindern somit plastisches Schwinden und Schrumpfrisse. Zusätzlich wird durch die wärmedämmende Wirkung des Kerns ein langsames Abfließen der Hydratationswärme begünstigt und eine Rissbildung durch schnelles Abkühlen verhindert. 
Aufgrund der genannten Vorteile bei der Herstellung der Elemente, wurde für alle weiteren Versuchsreihen die erste Herstellungsvariante angewendet. Die Unterschiede in der Tragfähigkeit der Probekörper werden im Abschnitt 5.2.6, Versuchsergebnisse; erläutert.

Nach dem Ausschalen der Probekörper wurden diese mindestens 28 Tage zusammen mit den Probekörpern zur Bestimmung der Festbetoneigenschaften unter Folien abdeckt in der Prüfhalle gelagert.

\subsubsection{Versuchsaufbau}

Die Prüfung der Biegeprobekörper erfolgte im Labor für konstruktiven Ingenieurbau der TU Kaiserslautern. Für die Prüfung wurden, je nach Verfügbarkeit, zwei verschiedene Prüfmaschinen, ein Vier-Säulen-Prüfrahmen mit einem servohydraulischen Prüfzylinder und einer maximalen Zylinderkraft von $630 \mathrm{kN}$ oder ein Zwei-Säulen-Prüfrahmen mit 250 kN Zylinderkraft, eingesetzt. Die Zylinderkraft wurde mithilfe einer Lasteinleitungskonstruktion zentrisch auf den Probekörper aufgebracht. Diese Konstruktion bestand aus einer Zylinderverlängerung, einer Kraftmessdose, einer Kalotte zur Zentrierung der Last auf die Kraftmessdose, Rollenlagern, sowie aus Lastverteilungsplatten. Im Falle der 4-Punkt-Biegeversuche wurden zusätzlich Traversen mit unterschiedlichen Längen verwendet.

Die Lagerung der Probekörper erfolgte ebenfalls auf Lastverteilungsplatten sowie auf einem Rollen- und einem Festlager. Um Raum für die Installation von Messtechnik und zur Begutachtung der Unterseite der Probekörper zu schaffen, wurden diese auf Abstandhaltern auf einem Lagerklotz gelagert.

Zur Variation des Moment-Querkraft-Verhältnisses, wurden sowohl 3-Punkt- als auch 4-Punkt-Biegeversuche durchgeführt (siehe Abbildung 5.3. Zudem wurden in den 4Punkt-Biegeversuchen die Abstände zwischen den Lasteinleitungs- und Auflagerpunkten variiert. Die Lastverteilungsplatten wurden verwendet um ein lokales Versagen der Deckschichten durch eine punktförmige Belastung im Bereich der Lasteinleitungs- und Auflagerpunkte zu verhindern. Die Breite der Lasteinleitungsplatten wurde zum Teil vergrößert.

\subsubsection{Versuchsdurchführung}

Die Belastung aller Probekörper erfolgte weggesteuert. Zu Beginn der quasi-statischen Versuche betrug die Belastungsgeschwindigkeit 0,2 bzw. 0,3 $\mathrm{mm} / \mathrm{min}$. Im Laufe der Versuche wurden die Belastungsgeschwindigkeiten in Abhängigkeit der Lastverformungskurve gesteigert. Gegen Ende der Versuche, beim Erreichen eines Fließplateaus, wurde die Geschwindigkeit zum Teil bis auf $5,0 \mathrm{~mm} / \mathrm{min}$ gesteigert.

Zur Messung der Verformungen der Probekörper wurden induktive Wegaufnehmer mit unterschiedlichen Messlängen eingesetzt. In allen Versuchen wurde die Durchbiegungen in Feldmitte an den unteren und oberen Deckschichten gemessen. Weiterhin wurden die Verformungen an den oberen Deckschichten an den beiden Auflagern gemessen. Zusätzlich wurden die Dehnungen in Längsrichtung der Probekör- 


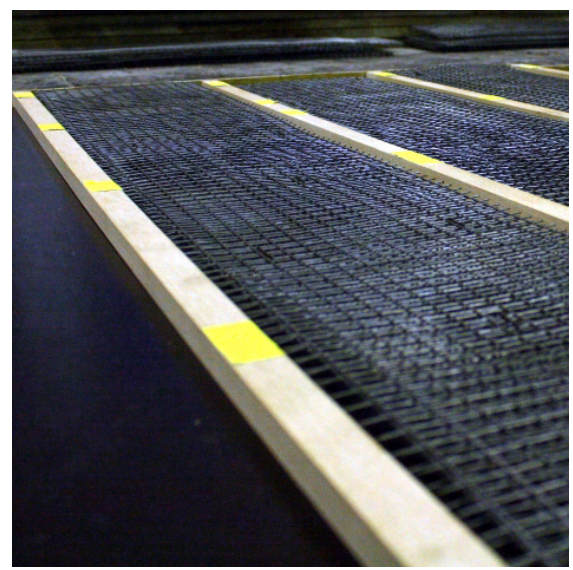

Abb. 5.6: Vorbereitete Schalung mit Mikrobewehrung

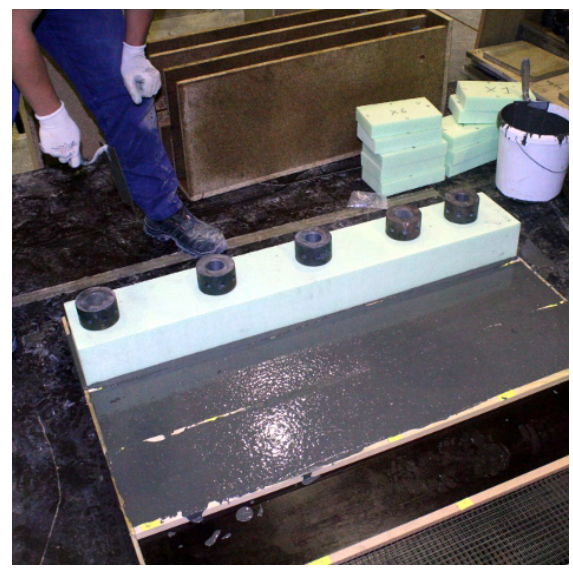

Abb. 5.8: Beschweren des Kerns mit Gewichten

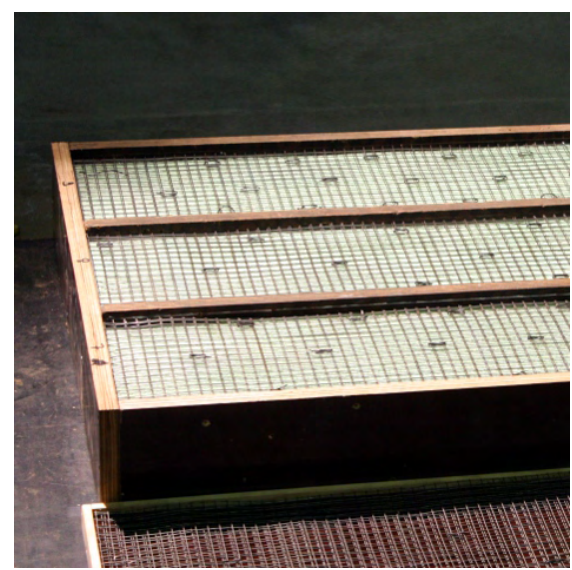

Abb. 5.10: Vorbereitete Schalung zum Aufbetonieren auf den Kern

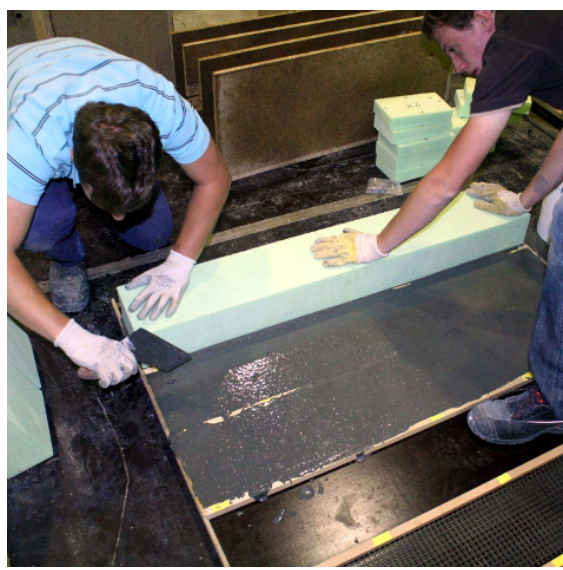

Abb. 5.7: Eindrücken und Ausrichten des XPS-Kerns

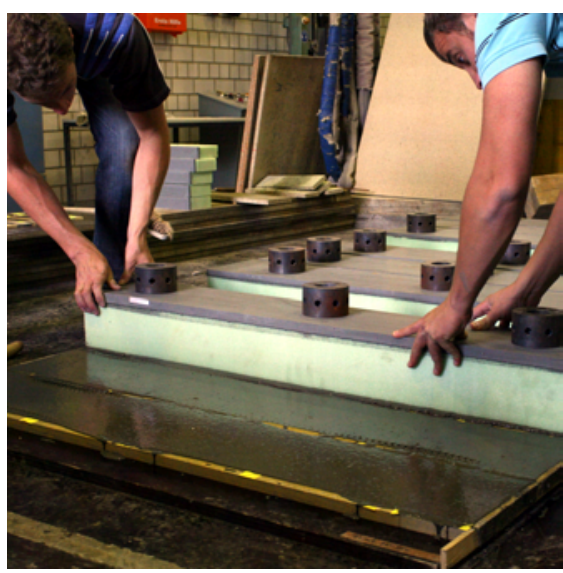

Abb. 5.9: Eindrücken des XPS-Kerns in den Beton am 2. Herstellungstag

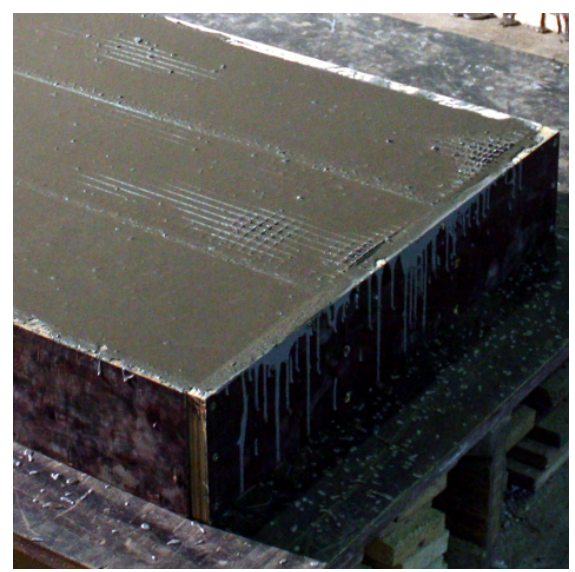

Abb. 5.11: Oberfläche mit herauschauender Mikrobewehrung 


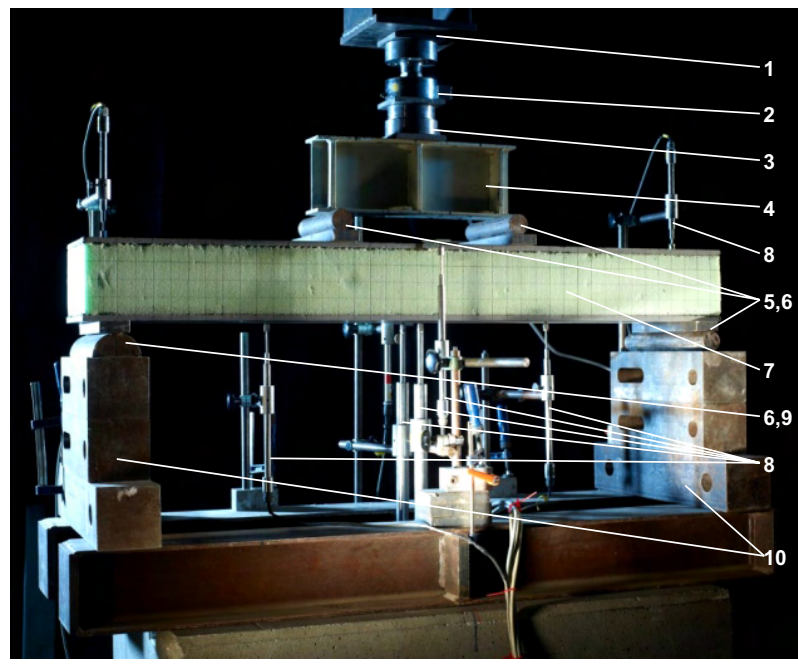

$1 \quad$ Lasteinleitung über Verlängerung des Zylinders

2 Kraftmessdose

3 Kalotte

4 Traverse

5 Rollenlager

Lastverteilungsplatte

Probekörper

Induktive Wegaufnehme

Festlager

Abstandhalter

Abb. 5.12: Exemplarischer Aufbau eines 4-Punkt-Biegeversuchs

per in Feldmitte an der Oberfläche der oberen und unteren Deckschichten mit Dehnungsmessstreifen (DMS) gemessen. Die Messwerte der quasi-statischen Versuche wurden mit einer Messfrequenz von $1 \mathrm{~Hz}$ mittels eines PCs aufgezeichnet. In den Versuchen C.X2.C-10.120.10-3 und C.X3.C-10.120.10-2 wurde zusätzlich ein optisches 3D-Messverfahren eingesetzt. Während der Versuche wurden weiterhin die Risse auf dem Probekörper nachgezeichnet und der Zeitpunkt der Entstehung protokolliert.

Mit Ausnahme der Versuchsreihe „Schichtdicken“ und den letzten sechs Versuchen der Reihe „Dichte“ (siehe Tabelle 5.1), wurden in allen quasi-statischen Versuchen, in einem regelmäßigem Abstand von 2,5 kN Laststufen angefahren. Beim Erreichen der jeweiligen Laststufe wurden dann 20 Lastwechsel zwischen $0,5 \mathrm{kN}$ und der jeweiligen Laststufe gefahren. Die Lastwechsel wurden mit deutlich höheren Geschwindigkeiten von bis zu $200 \mathrm{~mm} / \mathrm{min}$ gefahren.

Anstelle von Lastwechseln wurden in den drei Versuchen S.X0.S-10.120.10-9, G.X1.G10.120.10-3 und S.X1.S-10.120.10-2 eine zyklische Belastung aufgebracht. Hierzu wurde ein Durchbiegungswert $u$ am Übergang vom Zustand II zum plastischen Bereich der Lastverformungskurve definiert. In Anlehnung an DIBt 1995 wurde dann 20.000mal der Durchbiegungswert $u$ zu 4/7, 2.000-mal zu 6/7 und 100-mal vollständig angefahren.

\subsubsection{Versuchsergebnisse}

\subsubsection{Versagensarten}

Im Rahmen der Biegeversuche konnten sieben verschiedene Versagensarten festgestellt werden, die in Tabelle 5.3 zusammengestellt sind. Die jeweilige Versagensart der Probekörper ist in den Abbildungen 5.13 bis 5.16 sowie in den Lastverformungsdiagrammen in den Abbildungen 5.17 bis 5.20 durch einen Buchstaben gekennzeichnet. 
Tab. 5.3: Versagensarten

\begin{tabular}{|c|c|c|c|}
\hline Art & Beschreibung & Schematische Darstellung & Beispielfoto \\
\hline A & $\begin{array}{l}\text { Zugversagen der } \\
\text { unteren Bewehrung }\end{array}$ & & \\
\hline B & $\begin{array}{l}\text { Druckversagen des } \\
\text { Kerns an der Lastein- } \\
\text { leitung }\end{array}$ & & \\
\hline C & $\begin{array}{l}\text { Lokales Biegever- } \\
\text { sagen der oberen } \\
\text { Deckschicht }\end{array}$ & 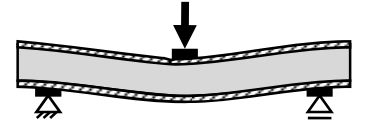 & \\
\hline D & $\begin{array}{l}\text { Lokales Querkraft- } \\
\text { versagen der oberen } \\
\text { Deckschicht }\end{array}$ & & \\
\hline $\mathbf{E}$ & $\begin{array}{l}\text { Querkraftversagen im } \\
\text { Kern und Delamina- } \\
\text { tion des Kerns und } \\
\text { den Deckschichten }\end{array}$ & & \\
\hline $\mathbf{F}$ & $\begin{array}{l}\text { Schubversagen } \\
\text { zwischen Kern und } \\
\text { Deckschicht }\end{array}$ & & \\
\hline G & $\begin{array}{l}\text { Druckversagen des } \\
\text { Kerns an den } \\
\text { Auflagern }\end{array}$ & 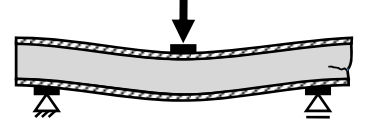 & \\
\hline
\end{tabular}

\section{- A: „Zugversagen der unteren Bewehrung“}

Bei dieser Versagensart riss die Bewehrung der unteren Deckschicht im mittleren Drittel der Stützweite. Durch das Versagen der Bewehrung riss auch der XPSKern über die volle Höhe auf. Lediglich die obere Deckschicht blieb bei dieser Versagensart intakt. Das Versagen erfolgte schlagartig, d. h. die aufgebrachte Last fiel abrupt ab und die Verformungen nahmen schlagartig zu. Bei den mikrobewehrten Sandwichstreifen deutete sich das Versagen durch ein Aufweiten des entsprechenden Risses im Beton an. Bei den glasfaserbewehrten Sandwichelementen konnten keine Versagensvorankündigung beobachtet werden. In den 
Versuchen mit Carbonfaserbewehrung konnte kein Versagen der Textilbewehrung erzielt werden.

\section{- B: „Druckversagen im Kern an der Lasteinleitung“}

Unter der Lasteinleitungsplatte wurde bei dieser Versagensart das XPS lokal stark zusammengedrückt. In einem Abstand von ca. 5-10 cm zur Lasteinleitungskonstruktion konnten Verformungen in den auf dem Dämmkern aufgezeichneten Linien wahrgenommen werden (siehe Tabelle 5.3. Weiterhin wurde die obere Deckschicht im Bereich der Lasteinleitung einer großen Biegeverformung ausgesetzt. In zwei der Versuche führte diese Verformung in der Deckschicht zu einem sekundären Biege- oder Querkraftversagen der oberen Deckschicht (siehe Versagen $C$ und D).

- C: „Lokales Biegeversagen der oberen Deckschicht“

Bei zwei der carbonbewehrten Sandwichstreifen versagte die obere Deckschicht aufgrund einer Biegeverformung. Dabei platzten zum Teil mehrere Zentimeter große Betonbruchstücke von der Deckschicht ab. In Versuch C.X1.C-10.120.10-1 versagte die Deckschicht etwa in $10 \mathrm{~cm}$ Abstand zur Lasteinleitungskonstruktion. Dabei platzten die Betonstücke auf der obere Seite der Deckschicht ab, so dass zum Teil das Carbongelege freilag. In Versuch C.X1.C-10.120.10-3 versagte die Deckschicht direkt unter einer Kante der Lasteinleitungsplatte. Die Bruchstücke platzten hier an der Unterseite der Deckschicht ab und drückten sich dabei in den XPS-Kern.

- D: „Lokales Querkraftversagen der oberen Deckschicht“

Das Versagen des Probekörpers C.X1.C-10.120.10-2 kann als lokales Querkraftversagen der oberen Deckschicht klassifiziert werden. Dabei bildete sich ein diagonaler Riss, der beginnend von der linken Kante der Lasteinleitungsplatte zur Unterseite der Deckschicht verlief.

- E: „Querkraftversagen im Kern und Delamination des Kerns und der Deckschichten"

In sechs Versuchen bildete sich ein diagonal verlaufender Schubriss über die vollständige Höhe des Dämmkerns aus. Zusätzlich löste sich dabei im Bereich zwischen Auflager und Schubriss die untere Deckschicht vom Dämmkern. Im Bereich zwischen Schubriss und Lasteinleitungsplatte löste sich die obere Deckschicht vom Dämmkern. Das Versagen trat schlagartig auf. Dabei konnte nicht genau festgestellt werden, in welcher Abfolge das Versagen auftrat.

\section{- F: „Schubversagen zwischen Kern und Deckschicht“}

In einigen der Versuchen versagte die Verbundfuge zwischen der oberen Deckschicht und dem Kern am linken oder rechten, in manchen Fällen auch an beiden Enden des Probekörpers. Dabei wurde die obere Deckschicht über den Kern hinweg geschoben. Eine Relativverschiebung zwischen dem Kern und der Deckschicht konnte vor allem am äußeren Ende der Probekörper beobachtet werden. Das Lastniveau konnte bei zunehmender Verformung gehalten werden. Das Lastverformungsverhalten der Probekörper, die auf diese Weise versagten, kann als duktil bezeichnet werden. 


\section{- G: „Druckversagen im Kern am Auflager“}

Bei dieser Versagensart wurde das XPS an den Probekörperenden an den Auflagern zusammengedrückt. Meist bildete sich hier ein charakteristischer Knick im XPS aus (siehe Abbildung in Tabelle 5.3. Das Versagen trat, wie bei Versagensart F, zum Teil am linken oder rechten Ende, oder auch an beiden Enden der Probekörper auf. Die Last konnte auch hier auf dem Lastniveau gehalten werden. Die Versuche wurden meist aufgrund zu großer Verformungen abgebrochen.

Eine Bewertung der Versagensarten erfolgt im Abschnitt 7.4, Diskussion der Versagensarten' (siehe S. 160.

\subsubsection{Allgemeine Beschreibung des Biegetragverhaltens}

Die Abbildungen 5.17 bis 5.20 zeigen die Verformungskurven aller Versuchsreihen. Zur besseren Vergleichbarkeit der Ergebnisse, wurden aus der aufgebrachten Last das Moment in Feldmitte und die Querkraft zwischen den Lasteinleitungs- und Auflagerpunkten errechnet. Das Moment und die Querkraft wurden über der Durchbiegung in Feldmitte auf der linken bzw. rechten Ordinate aufgetragen.

Generell lassen sich die Lastverformungskurven in drei unterschiedliche Bereiche unterteilen:

- Bereich I: Einen ersten, meist kurzen linear-elastischen Bereich im ungerissen Zustand I der unteren Deckschicht.

- Bereich II: Einen zweiten Bereich, in dem sich die Risse in der unteren Deckschicht und teilweise an dem linken und rechten Ende der oberen Deckschicht bilden. Durch die Rissbildung und den damit verbundenen Steifigkeitsabfall flachen die Lastverformungskurven dabei ab.

- Bereich III: Und gegebenenfalls einen dritten Bereich, in dem durch Plastizierungen im XPS oder in der Bewehrung die Lastverformungskurven nahezu horizontal verlaufen oder auch leicht abfallen.

In den Abbildungen 5.13 bis 5.16 sind die Lasten $P_{c r}$ beim Übergang der Probekörper vom Bereich I nach II, $P_{p l}$ beim Übergang der Probekörper vom Bereich II nach III und $P_{\max }$ beim Versagen der Probekörper angegeben. Die Versagensart eines jeden Probekörpers ist mit dem entsprechenden Buchstaben nach Tabelle 5.3 gekennzeichnet.

Eine detaillierte Erläuterung des Biegetragverhaltens erfolgt in Abschnitt 7.1, Generelle Beschreibung des Biegetragverhaltens der Sandwichelemente' (siehe S. 151). Die nachfolgend beschriebenen Versuchsergebnisse der einzelnen Versuchsreihen werden weiterhin in Abschnitt 7.3, Einfluss der verschiedenen Parameter auf das Biegetragverhalten der Sandwichelemente ' (siehe S. $156 \mathrm{ff}$.) bewertet und diskutiert.

\subsubsection{Versuchsreihe „Herstellungsmethode“}

In der ersten Versuchsreihe zum Biegetragverhalten der Sandwichelemente wurde der Einfluss des Herstellungsverfahrens untersucht. Generell wiesen alle Probekörper, die 


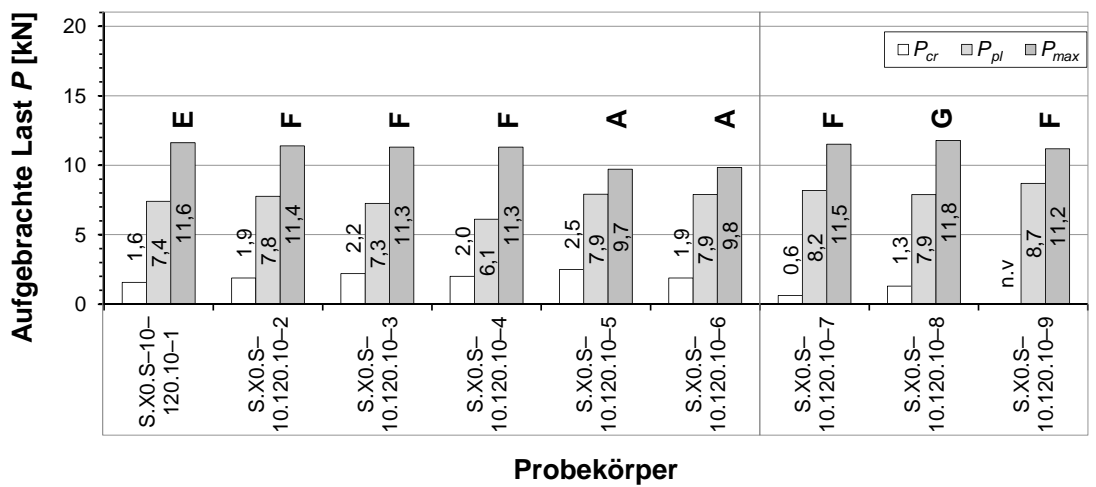

Abb. 5.13: Versagensarten und aufgebrachte Lasten beim Erstriss, beim Übergang in den plastischen Bereich und beim Versagen der Versuchsreihe „Herstellungsmethode“

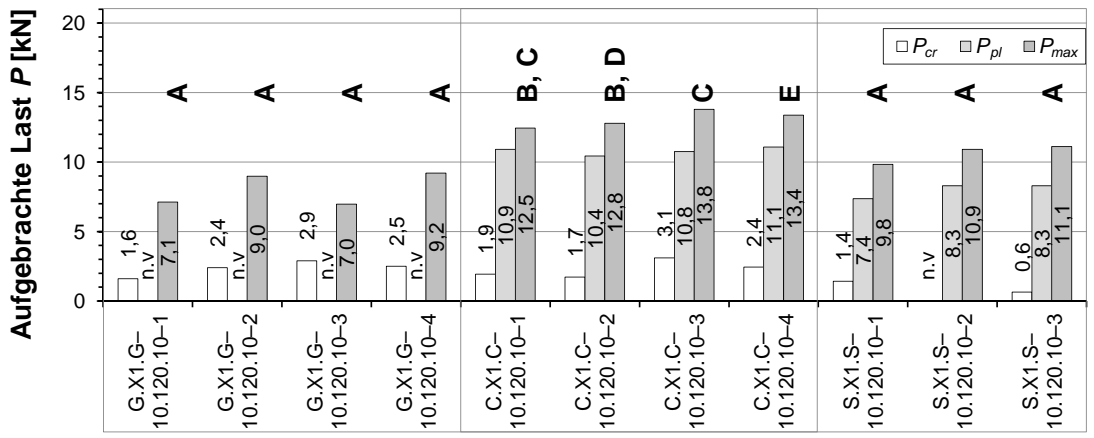

Probekörper

Abb. 5.14: Versagensarten und aufgebrachte Lasten beim Erstriss, beim Übergang in den plastischen Bereich und beim Versagen der Versuchsreihe "Bewehrungsart“

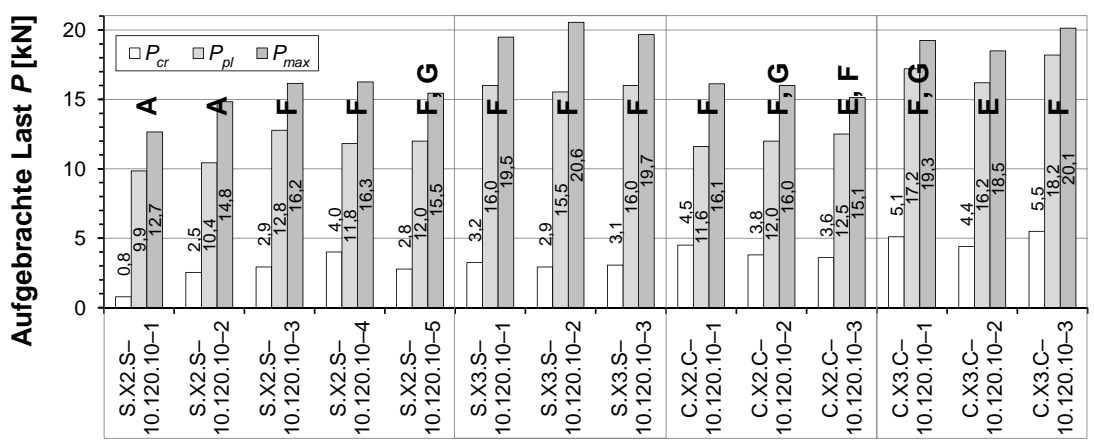

Probekörper

Abb. 5.15: Versagensarten und aufgebrachte Lasten beim Erstriss, beim Übergang in den plastischen Bereich und beim Versagen der Versuchsreihe „XPS-Dichte“ 


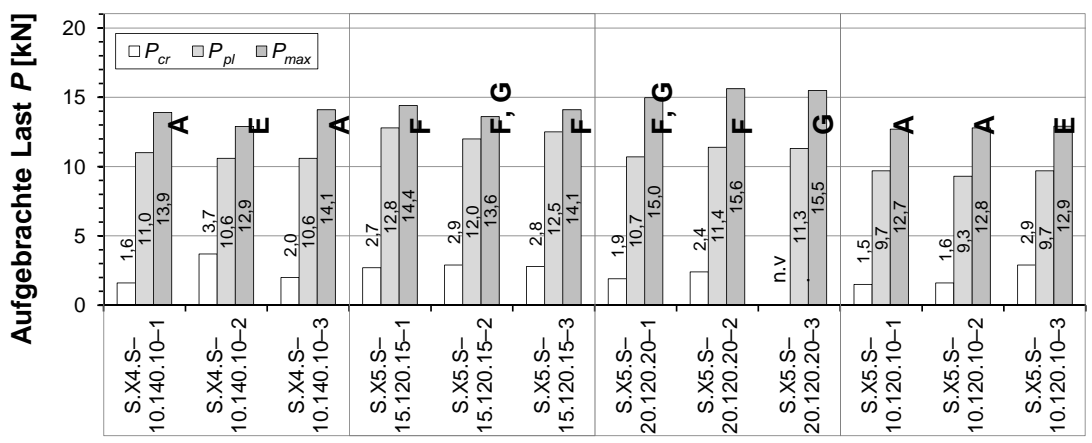

Probekörper

Abb. 5.16: Versagensarten und aufgebrachte Lasten beim Erstriss, beim Übergang in den plastischen Bereich und beim Versagen der Versuchsreihe „Schichtdicken“ und „Stoßausbildung“

in einem 4-Punkt-Biegeversuch (blaue und grüne Kurven in Abbildung 5.17) geprüft wurden, ein ausgeprägtes Fließplateau mit Verformungen bis zu $95 \mathrm{~mm}$ auf. Die beiden im 3-Punkt-Biegeversuch geprüften Probekörper (rote Kurve) versagten bereits bei einer Verformung von $30 \mathrm{~mm}$, wiesen dabei jedoch auch ein plastisches Verformungsverhalten auf.

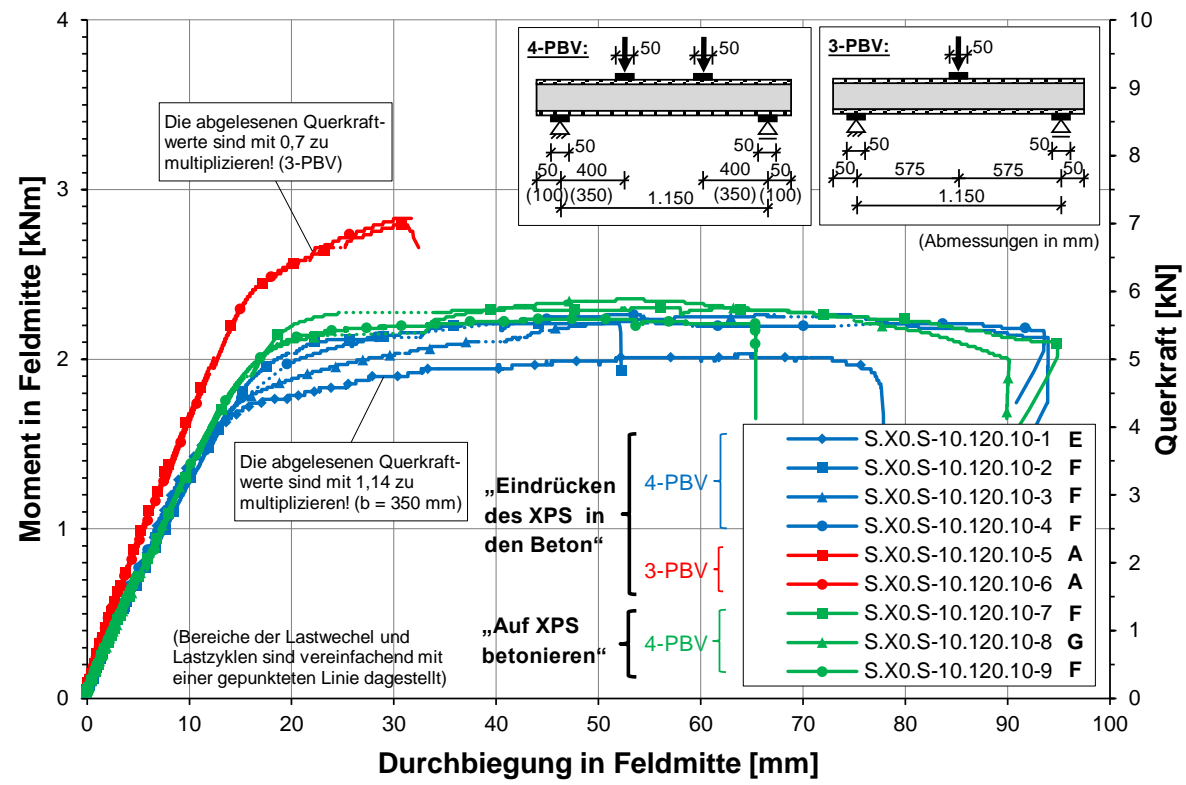

Abb. 5.17: Lastverformungsverhalten der Versuchsreihe „Herstellungsmethode“

Die Lastverformungskurven der Probekörper der beiden Herstellungsmethoden, die im 4-Punkt-Biegeversuch geprüft wurden, verliefen nahezu identisch (siehe Abbildung 5.17). Dabei gingen die Kurven der Probekörper der ersten Herstellmethode „Ein- 
drücken des XPS-Kerns in den Beton" schon bei etwa $14 \%$ geringerer Belastung in das Fließplateau über. Dabei erreichten die Probekörper mit einer Abweichung von rund $2 \%$ in etwa die gleichen Versagenslasten.

Die Prüfkörper die im 3-Punkt-Biegeversuch geprüft wurden, versagten alle durch ein Reißen der Bewehrung der unteren Deckschicht (Versagensart A). Die Prüfkörper der 4-Punkt-Biegeversuche versagten überwiegend durch ein Versagen des Verbunds zwischen oberer Deckschicht und Kern (Versagenart F). In einem Versuch trat ein Schubriss (Versagensart E) und in einem weiteren Versuch ein Druckversagen des XPS im Auflagerbereich (Versagensart G) auf.

\subsubsection{Versuchsreihe „Bewehrungsart“}

In der Versuchsreihe „Bewehrungsart“ wurde der Einfluss der drei unterschiedlichen Bewehrungsmaterialien auf das Tragverhalten und die Tragfähigkeit untersucht und verglichen. Im Zustand I war das Lastverformungsverhalten der drei Probekörperarten nahezu identisch. Auch die Erstrisslasten waren in etwa gleich. Im Zustand II wiesen die mikrobewehrten Probekörper (grüne Kurven in Abbildung 5.18 das steifste Lastverformungsverhalten auf. Danach folgen die carbonfaserbewehrten Probekörper (blaue Kurven). Die Steifigkeit der glasfaserbewehrten Probekörper (rote Kurven) im Zustand II war am geringsten.

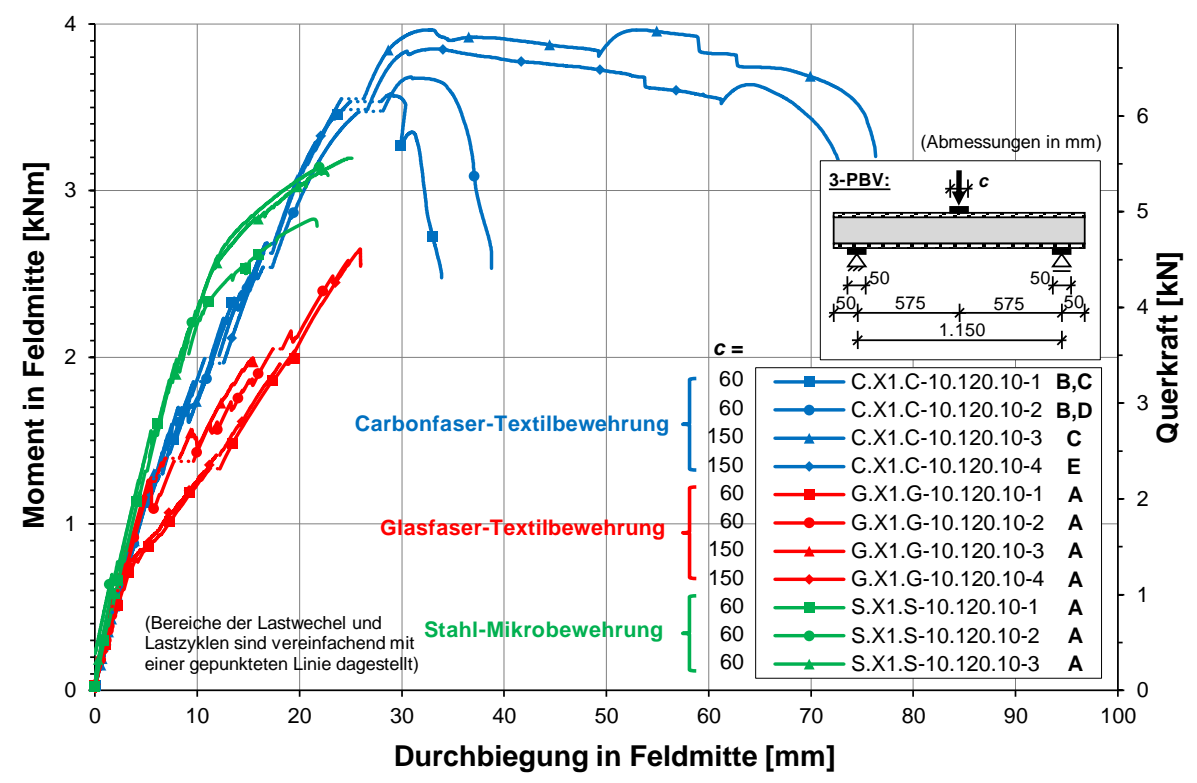

Abb. 5.18: Lastverformungsverhalten der Versuchsreihe „Bewehrungsart“

Durch das Fließen der Mikrobewehrung und durch Plastizierungen im XPS bei den Versuchen mit carbonfaserbewehrten Probekörper deutete sich deren Versagen durch ein Zunahme der Verformungen an. Die glasfaserbewehrten Probekörper wiesen kein duktiles Bauteilverhalten auf. 
Alle mikrobewehrten und glasfaserbewehrten Probekörper versagten aufgrund des Reißens der Bewehrung in der unteren Deckschicht. Jeweils ein Probekörper mit Mikrobewehrung und ein Probekörper mit Glasfasergewebe wurde nach DIBt 1995 zyklisch belastet. Dabei konnte bei dem mikrobewehrten Probekörper im Vergleich zu den durchgeführten quasi-statischen Versuchen kein nachteiliger Einfluss auf die Versagenslast festgestellt werden. Jedoch versagte der zyklische belastete, glasfaserbewehrte Probekörper G.X1.G-10.120.10-3 vorzeitig bei der 60. Wiederholung der Verformung von 6/7u. Weiterhin versagte der Probekörper G.X1.G-10.120.10-1 bei der 20. Wiederholung des Lastwechsel bei einer Laststufe von $7,5 \mathrm{kN}$, woraus auf ein unzureichendes Ermüdungsverhalten geschlossen werden kann.

Ein Zugversagen der carbonfaserbewehrten Probekörper konnte nicht hervorgerufen werden. Einer der Probekörper versagte aufgrund eines Schubrisses im XPS (Versagensart E). Die weiteren drei Probekörper versagten durch ein lokales Biege- oder Querkraftversagen der oberen Deckschicht an den Lasteinleitungspunkten (Versagensart $C$ und D), welches einherging mit einem Druckversagen des XPS in diesem Bereich (Versagensart B).

\subsubsection{Versuchsreihe „XPS-Dichte“}

In der Versuchsreihe „XPS-Dichte“ wurden die beiden XPS-Chargen X2 mit einer Rohdichte von $37,7 \mathrm{~kg} / \mathrm{m}^{2}$ (rote Kurven in Abbildung 5.19 und X3 mit einer rund $25 \%$ höheren Rohdichte von $46,9 \mathrm{~kg} / \mathrm{m}^{2}$ (blaue Kurven) verwendet. Beide Probekörperarten mit X2 und X3-Kern wurden sowohl mit Mikrobewehrung (durchgezogene Kurven) als auch mit Carbonfaserbewehrung (gestrichelte Kurven) geprüft.

In den ersten beiden Versuchen an den Probekörpern mit X2-Kern und mikrobewehrten Deckschichten versagte die Bewehrung durch Reißen der Bewehrung. Um eine höhere Querkraftbeanspruchung der mikrobewehrten Probekörper zu erreichen und ein Zugversagen auszuschließen, wurden die Lasteinleitungspunkte für die weiteren Versuche bis auf $200 \mathrm{~mm}$ an die Auflagerpunkte herangerückt. Aufgrund der höheren Zugtragfähigkeit des Carbonfasergeleges war dies bei den carbonfaserbewehrten Probekörpern nicht erforderlich. In diesen Versuchen wurde der übliche Abstand $b$ von $400 \mathrm{~mm}$ zwischen Lasteinleitungs- und Auflagerpunkten verwendet. Durch die Darstellung der einwirkenden Momente und der Querkräfte in Abhängigkeit der Durchbiegung (siehe Abbildung 5.19) ist jedoch ein direkter Vergleich möglich.

Für die carbonfaserbewehrten Probekörper wurde die Betonrezeptur HPC-2 mit HSZement eingesetzt, für alle mikrobewehrten Probekörper wurde die Rezeptur HPC-1 mit gewöhnlichem Zement verwendet. Durch die Verwendung des HS-Zements und den daraus resultierenden höheren Betonzugfestigkeiten konnten die Erstrisslasten um rund $40 \%$ gesteigert werden (vgl. Abbildung 5.15 .

Das Lastverformungsverhalten der Probekörper mit XPS-Charge X3 war im Zustand II geringfügig steifer als das der Probekörper mit XPS-Charge X2. Die beiden ersten Probekörper S.X2.S-10.120.10-1 und S.X2.S-10.120.10-2, die durch Reißen der Mikrobewehrung versagten, wiesen eine leicht ansteigende Kurve im plastischen Bereich auf. Dieser Anstieg konnte auch bei den 3-Punkt-Biegeversuchen der anderen mikrobewehrten Probekörper der Versuchsreihen „Herstellungsmethode“ und „Bewehrungsart“ 


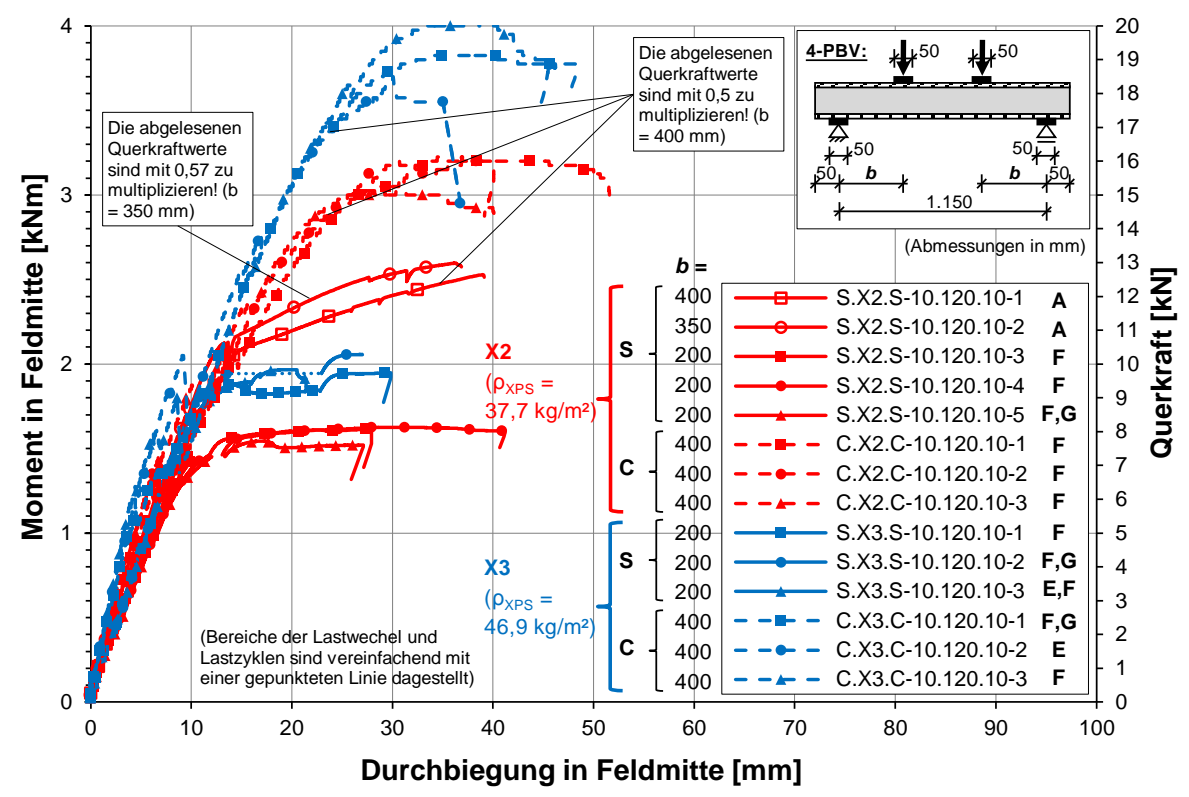

Abb. 5.19: Lastverformungsverhalten der Versuchsreihe „XPS-Dichte“

beobachtet werden. Alle anderen Kurven der Probekörper wiesen ein eher horizontal verlaufendes Plateau im plastischen Bereich auf. Die unterschiedlichen Bewehrungsarten und die unterschiedliche Anordnung der Lasteinleitungstelle hatten keinen nennenswerten Einfluss auf die Höhe der Querkräfte oder die aufgebrachten Lasten (siehe Abbildung 5.15bzw. Abbildung 5.19).

Die Probekörper versagten in den meisten Fällen im Verbund zwischen Kern und Deckschicht (Versagensart F). In drei Fällen konnte zusätzlich ein Druckversagen des XPS am Auflager beobachtet werden. In zwei Fällen trat ein Schubriss im Kern auf.

\subsubsection{Versuchsreihen „Schichtdicken“ und „Stoßausbildung“}

In Abbildung 5.20 sind die Verformungskurven der Versuchsreihen zur Untersuchung des Einflusses der Schichtdicken dargestellt. Die direkte Vergleichbarkeit der Versuche mit unterschiedlichen Schichtdicken ist nur bedingt möglich. Für die Versuche mit $140 \mathrm{~mm}$ Kerndicke musste die XPS-Charge X4 verwendet werden. Diese wies eine ca. $15 \%$ höhere Rohdichte auf, als die XPS-Charge X5 der übrigen Probekörper.

Durch die Vergrößerung der Deckschichtdicke wurde gleichzeitig auch der innere Hebelarm zwischen den Deckschichten vergrößert. Somit betrug der Abstand der Schwerachsen der Deckschichten $150 \mathrm{~mm}, 135 \mathrm{~mm}$ und $140 \mathrm{~mm}$ bei den Probekörpern mit den Abmessungen 10/140/10 mm (Dicke der oberen Deckschicht / Dicke des Kerns / Dicke der unteren Deckschicht), 15/120/15 mm und 20/120/20 mm. Generell konnte jedoch festgestellt werden, dass die Probekörper mit den Schichtdicken 20/120/20 mm (grüne Kurven) das steifste Lastverformungsverhalten aufwiesen. Die Kurven der Probekörper mit den Abmessungen 20/120/20 mm (grüne Kurven) und 15/120/15 mm (ro- 
te Kurven) wiesen ein ausgeprägtes Plateau im plastischen Bereich mit Verformungen von bis zu $75 \mathrm{~mm}$ auf. Die Probekörper versagten dann zwischen dem Kern und den Deckschichten und teilweise auch durch ein Druckversagen des Kerns am Auflager. Zwei der Probekörper mit 10/140/10 mm Schichtdicken (blaue Kurven) versagten durch Reißen der Bewehrung und ein Probekörper durch einen Schubriss im XPS. Die Verformungen erreichten dabei maximal $30 \mathrm{~mm}$.

Die Probekörper deren XPS-Kern auf einer Seite zwischen den Lasteinleitungs- und Auflagerpunkten verklebt wurden sind ebenfalls in Abbildung 5.20 dargestellt (schwarze Kurven). Bei den Versuchen konnte kein Versagen der Klebstöße beobachtet werden. Zwei der Probekörper versagten durch Reißen der Mikrobewehrung. Beim dritten Probekörper trat ein Schubriss im XPS auf, jedoch auf der linken, ungestörten Seite des Probekörpers.

Das Lastverformungsverhalten der Versuche mit Stoßverklebung kann auch hier nicht direkt mit den übrigen Versuchen verglichen werden, da zwar das XPS der selben Charge X5 verwendet wurde, jedoch hier die Deckschichtdicke nur $10 \mathrm{~mm}$ betrug. Zum besseren Vergleich der Versuche wurden zusätzliche FE-Berechnungen durchgeführt. Diese sind im Kapitel 6, Rechnerische Untersuchungen' beschrieben.

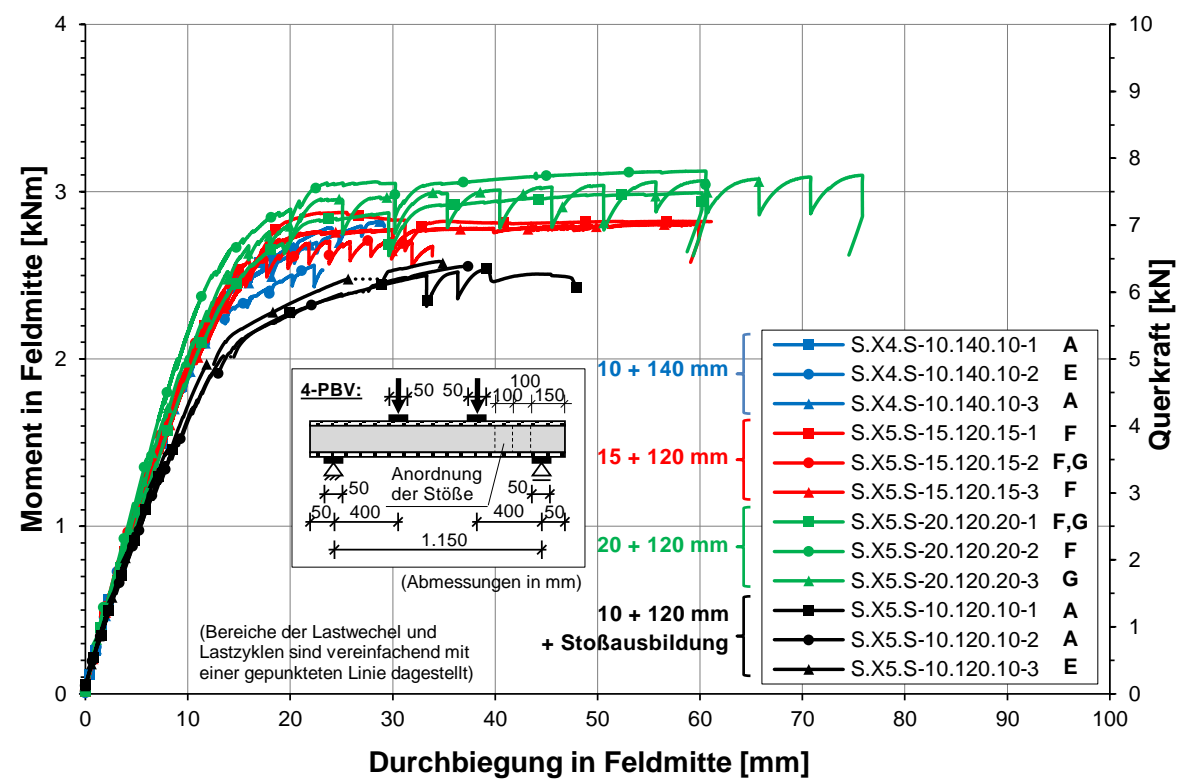

Abb. 5.20: Lastverformungsverhalten der Versuchsreihen „Schichtdicken“ und „Stoßausbildung“ 


\subsection{Scherversuche an Kleinprobekörpern}

\subsubsection{Allgemeines}

Um die Schereigenschaften des XPS zu bestimmen, wurden Scherversuche in Anlehnung an DIN EN 12090 durchgeführt. Es wurde der bereits im Abschnitt 3.2.3.4 ,Materialverhalten unter Scherbeanspruchung erwähnte einhüftige Versuchsaufbau verwendet.

\subsubsection{Versuchsprogramm}

In der folgenden Tabelle 5.4 sind alle durchgeführten Versuchsreihen zusammengestellt. In jeder der 17 Versuchsreihen wurden jeweils 5 Probekörper geprüft.

Tab. 5.4: Versuchsprogramm der Scherversuche

\begin{tabular}{|c|c|c|c|c|c|}
\hline & Nr. & XPS-Produkt & $\begin{array}{c}\text { Dicke XPS } \\
d[\mathrm{~mm}]\end{array}$ & $\begin{array}{l}\text { Betondeck- } \\
\text { schichten }\end{array}$ & $\begin{array}{l}\text { Rohdichte XPS } \\
\rho_{\text {XPS, mean }}\left[\mathrm{kg} / \mathrm{m}^{3}\right]\end{array}$ \\
\hline \multirow{2}{*}{$\mathrm{X} 1$} & - & \multirow{2}{*}{$2800 \mathrm{C}$} & \multirow{2}{*}{120} & ohne & 36,7 \\
\hline & B & & & mit & $36,7^{\#}$ \\
\hline \multirow{2}{*}{$\mathrm{X} 2$} & - & \multirow{2}{*}{$4000 \mathrm{CS}$} & \multirow{2}{*}{120} & ohne & 37,7 \\
\hline & B & & & mit & 37,1 \\
\hline \multirow{2}{*}{$\mathrm{X} 3$} & - & \multirow{2}{*}{$5000 \mathrm{CS}$} & \multirow{2}{*}{120} & ohne & 46,9 \\
\hline & B & & & mit & 46,2 \\
\hline$X 4$ & - & $2800 \mathrm{C}$ & 140 & ohne & 36,0 \\
\hline \multirow{6}{*}{$x 5$} & - & \multirow{6}{*}{$2800 \mathrm{C}$} & \multirow{6}{*}{120} & ohne & 32,4 \\
\hline & B1 & & & mit & 31,2 \\
\hline & B2 & & & mit & 31,9 \\
\hline & $\mathrm{B} 2-\mathrm{F}^{*}$ & & & mit & 31,7 \\
\hline & $\mathrm{KL}^{* *}$ & & & ohne & 32,3 \\
\hline & $K Q^{\star \star \star}$ & & & ohne & 32,1 \\
\hline \multirow{2}{*}{$\mathrm{X} 6$} & - & \multirow{2}{*}{$2800 \mathrm{C}$} & \multirow{2}{*}{120} & ohne & 33,6 \\
\hline & $\mathrm{B}$ & & & mit & 33,4 \\
\hline \multirow{2}{*}{$\mathrm{X} 7$} & - & \multirow{2}{*}{$2800 \mathrm{C}$} & \multirow{2}{*}{120} & ohne & 32,1 \\
\hline & $\mathrm{B}$ & & & mit & 32,0 \\
\hline
\end{tabular}

${ }^{\star} \mathrm{F}=$ Frost-Tau-Wechselbeanspruchung; ${ }^{* \star} \mathrm{KL}=$ Verklebung längs; ${ }^{* \star} \mathrm{KQ}=$ Verklebung quer "Annahme

Es wurden die folgenden Versuchsreihen durchgeführt:

\section{- Scherversuche ohne Betondeckschichten:}

Es wurden die Schereigenschaften aller sieben, der in dieser Arbeit verwendeten XPS-Chargen, bestimmt. Die Bezeichnung jeder Versuchsreihe ist identisch mit der Bezeichnung der jeweiligen Charge, z. B. X5.

- Scherversuche mit Betondeckschichten:

Mit Ausnahme von Charge X4 wurde jede XPS-Charge auch mit beidseitig hergestellten Betondeckschichten getestet. Dadurch konnten Rückschlüsse auf das 
Scherverhalten der Verbundfuge zwischen Beton und XPS gezogen werden. Die Versuchsreihen an Probekörpern mit Betondeckschichten sind mit dem Buchstaben „B“ gekennzeichnet, z. B. X5 B.

\section{- Scherversuche mit geklebtem Stoß:}

In den Versuchsreihen $\mathrm{X} 5 \mathrm{KL}$ und $\mathrm{X} 5 \mathrm{KQ}$ wurde der Effekt eines geklebten StoBes, längs $(\mathrm{KL})$ und quer $(\mathrm{KQ})$ zur Beanspruchungsrichtung, auf das Schertragverhalten untersucht.

\section{- Scherversuche mit Forst-Tau-Wechselbeanspruchung:}

Um die Dauerhaftigkeit der Verbundfuge zwischen Beton und XPS zu untersuchen, wurden weiterhin die Probekörper der Versuchsreihe X5 B2-F einer FrostTau-Wechselbeanspruchung ausgesetzt. Die Versuchreihe X5B2 diente hierzu als Referenz.

\subsubsection{Probekörper und Herstellung}

\subsubsection{Allgemeines}

Die für die Probekörper benötigten XPS-Streifen wurden alle mit einer Länge von $250 \mathrm{~mm}$ und einer Breite von $50 \mathrm{~mm}$ mithilfe eines Glühdraht-Schneidegeräts aus den XPS-Dämmplatten geschnitten. Die Dicke $d$ der Streifen entsprach damit folglich immer denen der XPS-Platten, d. h. 120 oder $140 \mathrm{~mm}$ (siehe Abbildung 5.21). Weiterhin wiesen alle XPS-Streifen auch die eingeprägte, rautenförmige Waffelstruktur auf. Die Längsrichtung aller XPS-Streifen war grundsätzlich in Extrusionsrichtung der Dämmplatten ausgerichtet.

a)

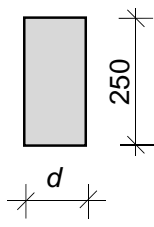

b)

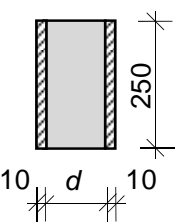

c)

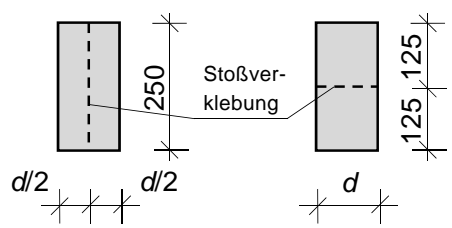

Abb. 5.21: Abmessungen der Scherversuchskörper a) ohne Betondeckschichten, $b$ ) mit Betondeckschichten, $c$ ) mit Verklebung eines Längsstoßes und d) mit Verklebung eines Querstoßes in mm

\subsubsection{Probekörper ohne Betondeckschichten}

Für die Scherversuche an Probekörpern ohne Betondeckschichten mussten die aus den XPS-Dämmplatten herausgeschnittenen Streifen nicht weiter bearbeitet werden und konnten direkt geprüft werden. 


\subsubsection{Probekörper mit Betondeckschichten}

In den Versuchsreihen an Probekörpern mit Betondeckschichten wurden beidseitig Betondeckschichten mit einer Dicke von $10 \mathrm{~mm}$ hergestellt. Es kam die in Abschnitt5.2.3 beschriebene Herstellungsvariante „Eindrücken des XPS-Kerns in den Beton“ zum Einsatz, d. h. der Hochleistungsbeton wurde dazu in eine entsprechende Schalung gegossen und der XPS-Kern im Anschluss in den frischen Beton eingedrückt. Das justieren und fixieren des XPS-Streifens auf dem frischen Beton stellte sich teilweise als schwierig heraus, da die Streifen zum Umkippen neigten. Auf diese Problematik wird im Zuge der Auswertung der Versuchsergebnisse noch eingegangen.

\subsubsection{Probekörper mit geklebtem Stoß}

In der Versuchsreihe $\mathrm{X} 5 \mathrm{KL}$ und $\mathrm{X} 5 \mathrm{KQ}$ wurden die Probekörper mittig, entsprechend in Längs- oder Querrichtung, mit dem Glühdraht-Schneidegerät auseinander geschnitten und mit dem zuvor in Abschnitt 5.2.3, Probekörper und Herstellung (Seite 64) beschriebenen Verfahren wieder zusammengefügt (siehe Abbildung 5.21).

\subsubsection{Probekörper mit Frost-Tau-Wechselbeanspruchung}

Die Probekörper der Versuchsreihe X5 B2-F wurden in Anlehnung an DIN EN 12091 einer Frost-Tau-Wechselbeanspruchung von 300 aufeinanderfolgenden Zyklen von trockenen Frost-Umgebungsbedingungen und feuchten Tau-Umgebungsbedingungen ausgesetzt (siehe auch Abschnitt 3.2.3.7, Materialverhalten bei Feuchteeinwirkung und unter Frost-Tau-Wechselbeanspruchung]', S. 26. Dazu wurden die Probekörper in einer temperaturkontrollierten Prüftruhe (siehe Abbildung 5.22) dreihundertmal im Wechsel einer einstündigen Temperaturbeanspruchung von $-20^{\circ} \mathrm{C}$ im nicht eingetauchten Zustand und einer Temperatur von $+20^{\circ} \mathrm{C}$ im eingetauchten Zustand ausgesetzt. Die Probekörper wurden dabei mit einer Halterung gegen Aufschwimmen gesichert (siehe Abbildung 5.23.

Abgesehen von weißen, salzartigen Ablagerungen auf den Oberflächen der Probekörper sowie deren Halterungen, konnten nach Beendigung der 300 Zyklen keine weiteren Veränderungen der Probekörper festgestellt werden.

Die Ablagerungen resultierten vermutlich aus Bestandteilen, die aus dem Hochleistungsbeton gelöst wurden und sich dann in der Frostphase auf den Probekörpern ablagerten.

\subsubsection{Versuchsaufbau}

Die Scherversuche wurden im Materialprüflabor der BASF durchgeführt. Dort konnten der Prüfrahmen und die bereits bestehende Prüfeinrichtung zur Bestimmung der Schereigenschaften nach DIN EN 12090 verwendet werden (siehe Abbildung 5.24.

Die Prüfeinrichtung besteht im wesentlichen aus zwei steifen Stahlplatten, die als Haltevorrichtungen dienen. An die Stahlplatten kann jeweils ein in der Länge variables 


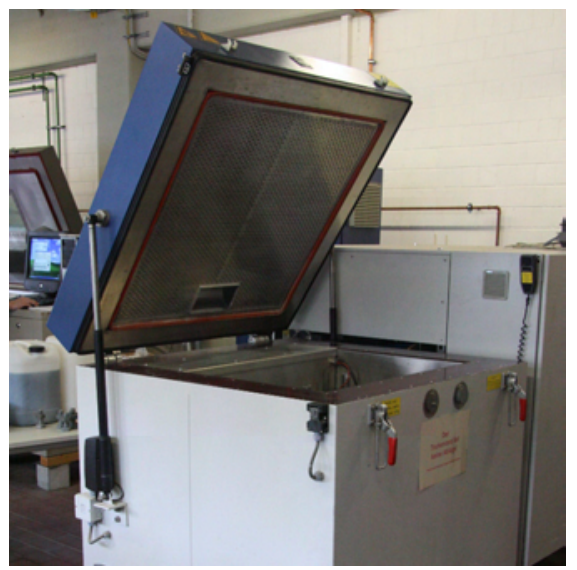

Abb. 5.22: Temperaturkontrollierte Prüftruhe zur Frost-Tau-Prüfung

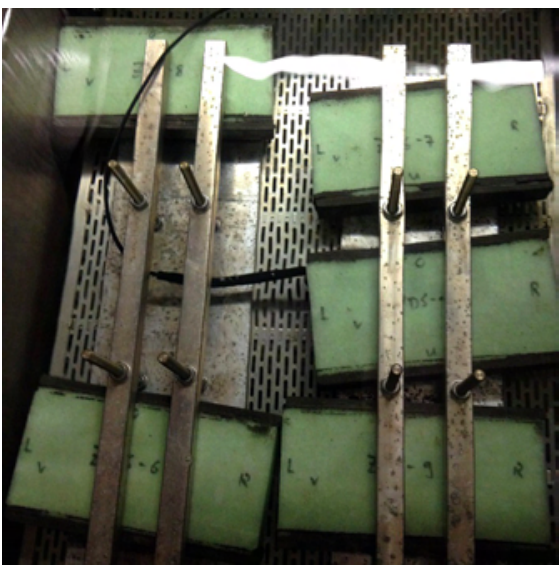

Abb. 5.23: Wasser gelagerte Probekörper während der Tau-Phase

Passstück biegesteif angeschlossen werden, welches dann zugfest und gelenkig gelagert mit dem Festpunkt des Prüfrahmens und dem Lasteinleitungspunkt des Zylinder verbunden wird.

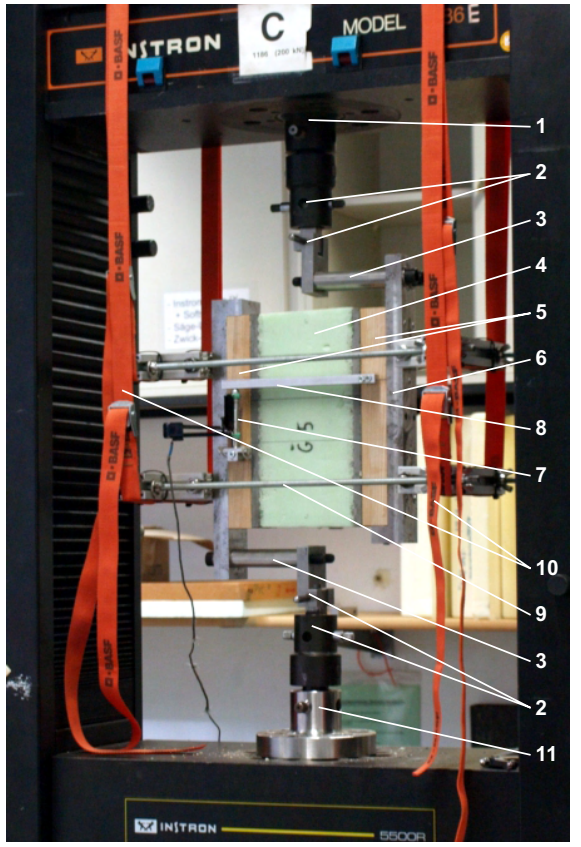

$1 \quad$ Lasteinleitungspunkt am Zylinder

Gelenk

Passstück mit Schraube

Probekörper mit Betondeckschichten

$5 \quad$ Holzleiste mit Probekörper verklebt

$6 \quad$ Haltevorrichtung mit angeschraubter Holzleiste

$7 \quad$ Wegaufnehmer an linker Holzleiste befestigt

8 Quertraverse an rechter Holzleiste befestigt

$9 \quad$ Querhalterung mit Gewindestäben

10 Spanngurt zur Lagesicherung der

Querverspannung

11 Festpunkt am Prüfrahmen

Abb. 5.24: Aufbau zur Prüfung eines Scherprobekörpers mit Betondeckschichten

Vor den Versuchen wurden Linien rechtwinklig zur Belastungsrichtung auf die Probekörper gezeichnet. Dadurch konnten die während der Versuche auftretenden Verformungen innerhalb des XPS und, im Fall der Scherversuche mit Betondeckschichten, die Relativverschiebungen zwischen XPS und Beton besser wahrgenommen werden. 
Um die Probekörper leicht ein- und ausbauen zu können, wurden beidseitig Holzleisten auf die Waffelstruktur bzw. auf die Betondeckschichten der Probekörper aufgeklebt und diese dann mit den Stahlplatten der Haltevorrichtung verschraubt.

Die Probekörper mussten exakt mittig in einer Flucht zwischen dem Festpunkt und dem Lasteinleitungspunkt des Prüfrahmens ausgerichtet werden, um ungewollte Exzentrizitäten zu vermeiden. Mithilfe der Passstücke wurde der Abstand zwischen der Haltevorrichtung und der Mittelachse des Probekörpers exakt eingestellt.

Gegen seitliches Auseinanderspreizen wurden die beiden Haltevorrichtungen zusätzlich durch eine Querhalterung mit Gewindestangen gesichert. Diese wurden nur von Hand leicht angezogen und dann zusätzlich durch Gurte in der Lage gesichert. Als Messtechnik wurden ein Wegaufnehmer und eine dazu gehörige Quertraverse auf der linken bzw. rechten Holzleiste befestigt.

\subsubsection{Versuchsdurchführung}

Mithilfe des Zylinders wurde die Prüfeinrichtung weggesteuert mit einer konstanten Belastungsgeschwindigkeit von $3 \mathrm{~mm} / \mathrm{min}$ gezogen und die Probekörper somit einer Scherbeanspruchung ausgesetzt. Während der Versuche wurden die Zylinderkraft, der Zylinderweg sowie die Verformung des Wegaufnehmers mit einem PC aufgezeichnet.

\subsubsection{Versuchsergebnisse}

\subsubsection{Allgemeines}

Die aufgebrachte Scherspannung wurde aus der Zylinderkraft $F$ sowie der Länge $l$ und Breite $b$ der Prüfkörper nach Gleichung 3.13 berechnet. Die mittlere Scherfestigkeit wurde aus den maximalen Werten der Scherspannungen einer jeden Versuchsreihe errechnet.

Der Schubmodul $G$ wurde nach Gleichung (3.14 berechnet (siehe S. 24). Dazu musste der Neigungswinkel $\alpha$ des linearen Teils der Kraft-Verformungskurve nach Gleichung 3.15 bestimmt werden. Dieser lineare Teil der Kurve lässt sich jedoch nicht eindeutig festlegen, da die Kurve in nahezu allen Versuchen mit zunehmender Verformung kontinuierlich abflacht und teilweise in ein Fließplateau übergeht. Aus diesem Grund wurden zwei verschiedene Schubmoduln bestimmt: ein Tangenten-Schubmodul und ein Sekanten-Schubmodul. Der Tangenten-Schubmodul beschreibt die Steigung der Lastverformungskurve zu Beginn des Versuchs. Der Sekanten-Schubmodul beschreibt die Steigung der Sekante vom Nullpunkt bis zu dem Erreichen des Fließplateau, bzw. falls kein ausgeprägtes Fließplateau besteht, bis zu einem merklichen Lastabfall der Kurve.

In den Abbildungen 5.25 und 5.26 sind die Scherfestigkeiten sowie die TangentenSchubmoduln eines jeden einzelnen Versuchs über der XPS-Rohdichte des jeweiligen Probekörpers als Punktwolke dargestellt. Dabei kennzeichnen die rechteckigen Markierungspunkte die Versuche an Probekörpern ohne Betondeckschichten. Die dreieckigen Markierungspunkte kennzeichnen die Probekörper mit Betondeckschichten. In den Abbildungen 5.27 und 5.28 sind weiterhin die mittleren Scherfestigkeiten sowie 
die mittleren Tangenten- und Sekanten-Schubmoduln der einzelnen Versuchsreihen zusammengestellt.

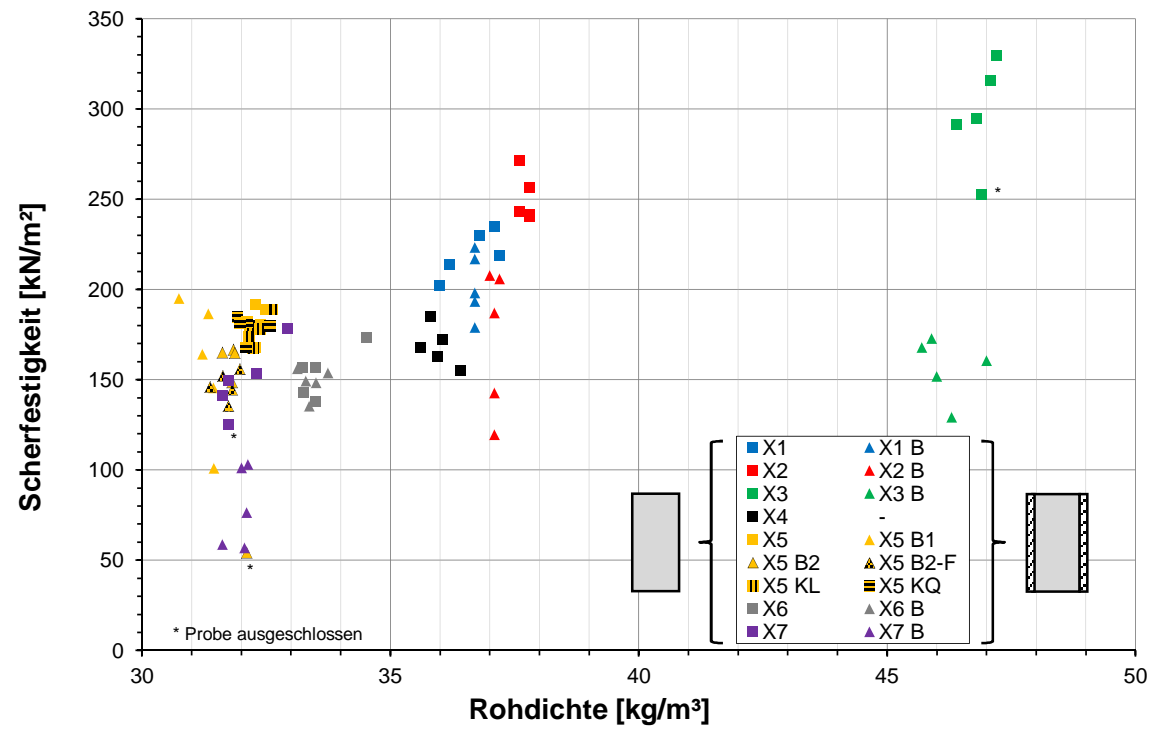

Abb. 5.25: Scherfestigkeit in Abhängigkeit der Dichte

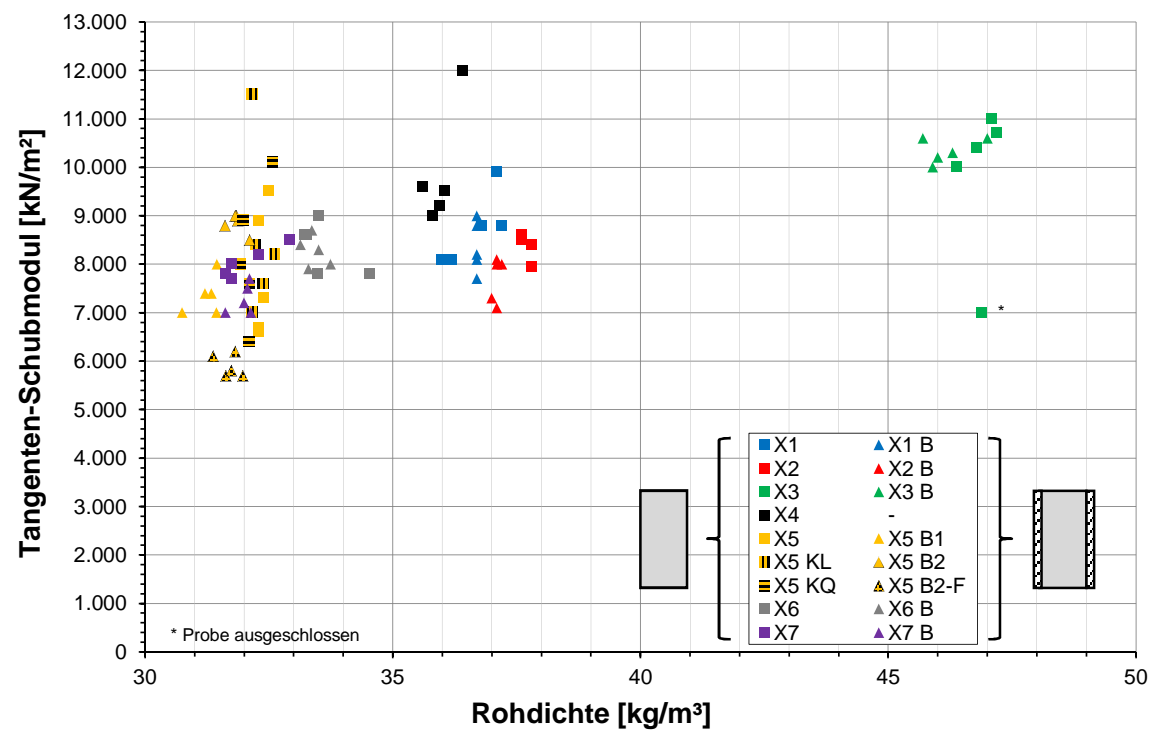

Abb. 5.26: Tangenten-Schubmodul in Abhängigkeit der Dichte 


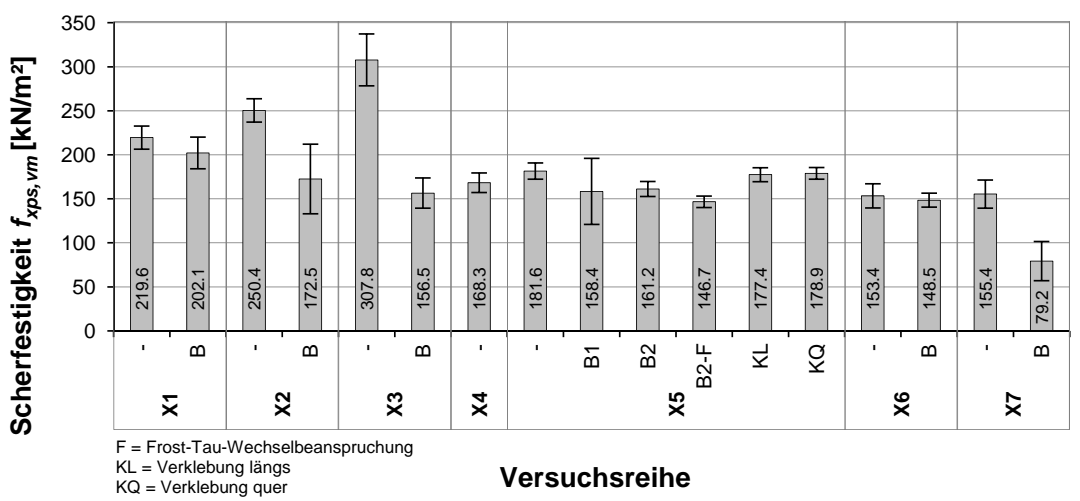

Abb. 5.27: Mittlere Scherfestigkeiten der Versuchsreihen mit Darstellung der empirischen Standardabweichung

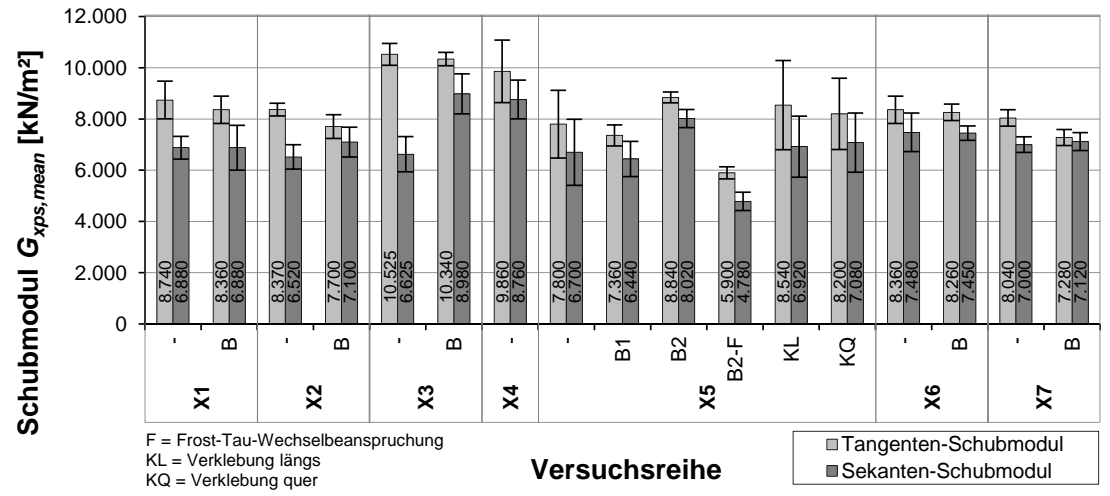

Abb. 5.28: Mittlere Tangenten- und Sekanten-Schubmodule der Versuchsreihen mit Darstellung der empirischen Standardabweichung

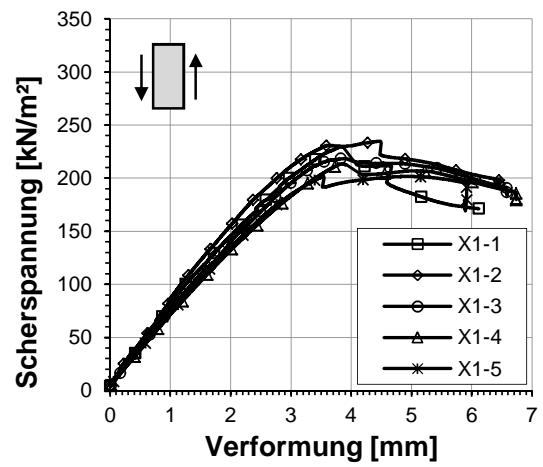

Abb. 5.29: Scherspannungs-VerformungsDiagramm der Versuchsreihe $\mathbf{X} \mathbf{1}$ ohne Betondeckschichten

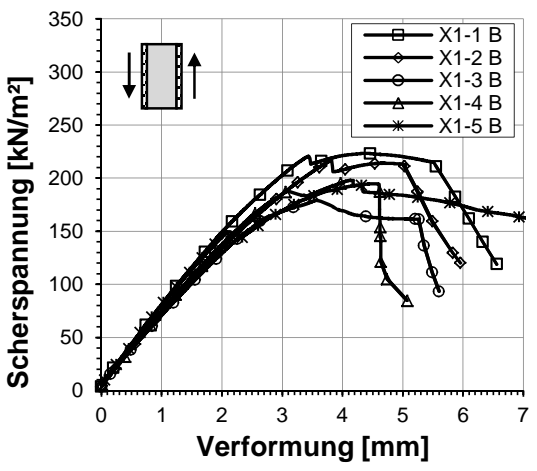

Abb. 5.30: Scherspannungs-VerformungsDiagramm der Versuchsreihe X1 B mit Betondeckschichten 


\subsubsection{Scherprobekörper ohne Betondeckschichten}

Das Scherspannungs-Verformungs-Diagramm der Versuchsreihe $\mathrm{X} 1$ ist beispielhaft in Abbildung 5.29 dargestellt. Die Diagramme aller weiteren Versuchsreihen sind dieser Arbeit im Anhang A.2 (siehe S. 250 angefügt.

Die Scherspannungs-Verformungs-Linie stieg zu Beginn eines Versuchs nahezu linear an. Bei steigender Belastung flachte die Kurve jedoch stetig ab. Die gemessene Verformung ließ sich auch visuell durch Neigung der aufgezeichneten Linien in Belastungsrichtung erkennen.

Vor dem Erreichen der Maximallast traten zum Teil plastische Verformungen im XPS auf (siehe Abbildung 5.31. Diese konnten durch Verformungen der aufgezeichneten Linien abgelesen werden. Die Verformungen traten lokal in einem Bereich auf, der diagonal von der unteren, linken bis zur oberen, rechten Ecke des Probekörpers verlief. Bei weiterer Laststeigerung bildete sich beginnend an diesen Eckbereichen eine Fuge aus, die sich dann über die Länge des Probekörpers weiter fortsetzte (siehe Abbildung 5.31.
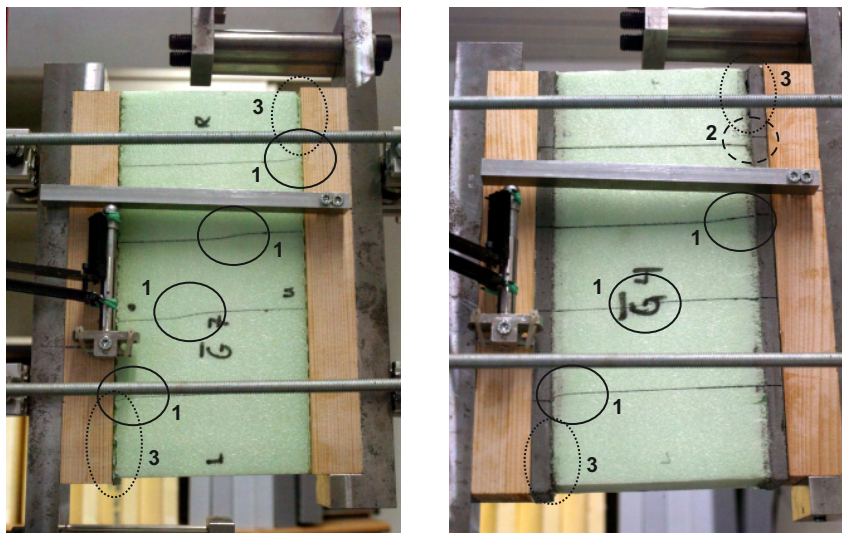

1 Plastische Verformungen im XPS

2 Relativverschiebungen zwischen Beton und XPS

$3 \quad$ Klaffende Fuge

Abb. 5.31: Probekörper X1-2 (links) und X1-4 B (rechts) unmittelbar vor dem Versagen

Die Fugen traten generell in der Grenzschicht zwischen der Waffelstruktur und dem ungestörten XPS auf. Das Versagen der Probekörper ohne Betondeckschichten trat ebenfalls in dieser Grenzschicht auf. In den meisten Versuchen trat dieses Versagen schlagartig entweder auf der linken oder auf der rechten Seite über die gesamte Länge des Probekörpers auf. Dabei fiel die aufgebrachte Last abrupt ab. In manchen Versuchen versagte die Grenzschicht erst über eine Teillänge und anschließend über die verbleibende Länge, so dass die Last vor dem vollständigen Lastabfall noch auf einem niedrigeren Niveau gehalten werden konnte.

Generell besteht die Tendenz, dass die Probekörper mit einer höheren Rohdichte auch eine höhere Scherfestigkeit aufweisen (siehe Abbildung 5.25. Die mittleren Scherfestigkeiten der Chargen X2 $\left(\rho_{X P S, \text { mean }}=37,7 \mathrm{~kg} / \mathrm{m}^{3}\right)$ und X3 $\left(\rho_{X P S, \text { mean }}=46,9 \mathrm{~kg} / \mathrm{m}^{3}\right)$ sind um rund $61 \%$ bzw. $98 \%$ größer als die Scherfestigkeit der Charge mit der niedrigsten Rohdiche X7 $\left(\rho_{X P S, \text { mean }}=32,1 \mathrm{~kg} / \mathrm{m}^{3}\right)$. 
Jedoch lässt sich keine eindeutige Beziehung zwischen der Rohdichte und der Scherfestigkeit erkennen. Die Charge X6 mit einer Rohdichte von $33,6 \mathrm{~kg} / \mathrm{m}^{3}$ weist beispielsweise eine um $16 \%$ geringere Scherfestigkeit auf als die Charge X5 mit einer Rohdichte von lediglich $32,4 \mathrm{~kg} / \mathrm{m}^{3}$.

Mit steigender Rohdichte des XPS nimmt auch dessen Schubmodul zu. Der TangentenSchubmodul der Chargen X2 $\left(\rho_{X P S \text {, mean }}=37,7 \mathrm{~kg} / \mathrm{m}^{3}\right)$ und X3 $\left(\rho_{X P S \text {, mean }}=46,9 \mathrm{~kg} / \mathrm{m}^{3}\right)$ sind um 4,1\% bzw. 30,9\% größer als Charge X7 $\left(\rho_{X P S \text {, mean }}=32,1 \mathrm{~kg} / \mathrm{m}^{3}\right)$.

Es ist davon auszugehen, dass neben der Rohdichte weitere Faktoren die Schereigenschaften des XPS beeinflussen. Die Zellstruktur sowie die Zellgröße und Zellwandstärke dürften auf die Schereigenschaften ebenfalls einen gravierenden Einfluss haben.

\subsubsection{Scherprobekörper mit Betondeckschichten}

Das Scherspannungs-Verformungsverhalten der Probekörper mit Betondeckschichten ist vergleichbar mit dem Verhalten der Probekörper ohne Betondeckschichten (Abbildung 5.29 und 5.30). Die Scherspannung-Verformungs-Kurven der Probekörper mit Betondeckschichten wiesen zu Beginn einen annähernd linearen Verlauf auf und flachten mit zunehmender Belastung ab.

Die plastischen Verformungen im XPS, die durch Krümmung der aufgezeichneten Linien wahrgenommen werden konnten, traten auch bei den Probekörpern mit Betondeckschichten auf (siehe Abbildung 5.31). In den Eckbereichen konnten ebenfalls klaffende Fugen in den Eckbereichen beobachtet werden. Diese traten jedoch im Bereich zwischen XPS-Kern und den Betondeckschichten auf, und nicht wie bei den Versuchen ohne Betondeckschichten zwischen dem XPS-Kern und den aufgeklebten Holzleisten.

Die Probekörper mit Betondeckschichten versagten durch das Ablösen der Deckschichten vom Kern. Mit Ausnahme von Versuchsreihe X1B, in der die Kurven einiger Versuche ein kurzes plastisches Plateau ausbildeten, wiesen die Kurven aller anderen Versuchsreihen beim Erreichen der maximalen Scherspannung einen abrupten Lastabfall auf.

Betrachtet man die versagten Grenzschichten zwischen Beton und XPS aller Probekörper (siehe Anhang A.2, kann man zwei unterschiedliche Versagensarten unterscheiden:

\section{- Versagen des XPS (Typ A):}

Das XPS versagt in unmittelbarer Nähe zur Waffelstruktur (siehe Abbildung 5.32). Die Waffelstruktur löst sich dabei nahezu vollständig ab und bleibt an der abgescherten Betondeckschicht haften.

\section{- Versagen der Verbundfuge (Typ B):}

In Teilbereichen lösen sich XPS und Beton (siehe Abbildung 5.33. Es bleiben Betonreste in der Waffelstruktur des XPS haften.

Die Scherflächen der meisten Probekörper weisen nur eine der beiden Versagensarten A oder B auf. In manchen Fällen können jedoch verteilt über die Scherflächen beide Versagenstypen A und B festgestellt werden (z. B. Probekörper X2-5 B, siehe Abbildung A.64, S. 253). Weiterhin lassen sich bei einigen wenigen Probekörpern auch 


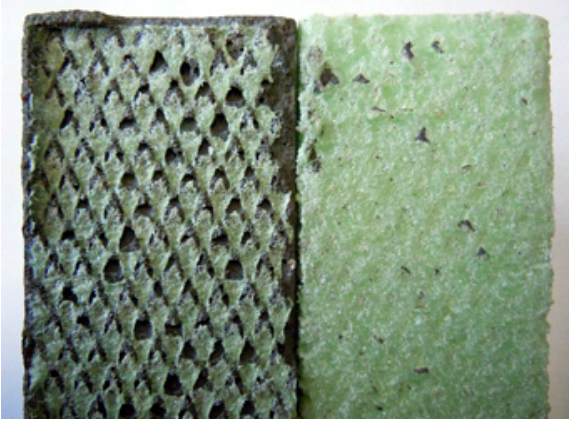

Abb. 5.32: Ansicht der Scherflächen des Probekörpers X1-1 B, Beton (links), XPS (rechts)

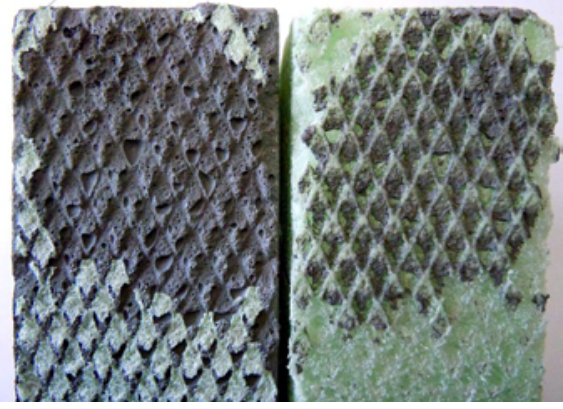

Abb. 5.33: Ansicht der Scherflächen des Probekörpers X1-5 B, Beton (links), XPS (rechts)

Teilbereiche feststellen in denen gar kein Verbund zwischen Beton und XPS vorhanden war. Das XPS hatte beim Herstellen der Probekörper den frischen Beton nicht berührt. Dadurch sind in der Grenzschicht teilweise größere Luftblasen festzustellen.

Die Scherfestigkeiten der Probekörper mit Betondeckschichten streuen generell stärker als die der Probekörper ohne (siehe Abbildung 5.27 und 5.25). In den Versuchsreihen X1 B, X5B1 und X6B sind die Scherfestigkeiten im Mittel lediglich um 8,0\%, $12,8 \%$ und $3,2 \%$ geringer, als in den dazugehörigen Versuchsreihen $X 1, X 5$ und $X 6$ ohne Betondeckschichten. In den Versuchsreihen X2 B, X3 B und X7 B sind die Scherfestigkeiten um $31,1 \%, 49,2 \%$ und $49,0 \%$ jedoch deutlich geringer, als die entsprechenden Versuchsreihen X2, X3 und X7 ohne Betondeckschichten.

In Abbildung 5.34 sind die Scherfestigkeiten aller Probekörper mit Betondeckschichten und deren über die Scherfläche vorwiegend auftretende Versagensart dargestellt. Probekörper, die beide Versagenstypen A und B flächenmäßig gleich aufwiesen, sind mit A/B gekennzeichnet. Durch die Darstellung in Abbildung 5.34 lässt sich erkennen, dass die Probekörper einer Versuchsreihe, die durch Versagensart B versagten, geringere Festigkeiten erreichten, als die Probekörper der gleichen Versuchsreihe der Versagensart A. In den Versuchsreihen X2 B, X3 B und X7 B, sowie die Versuche X5$2 \mathrm{~B} 1$ und $\mathrm{X} 5-2 \mathrm{~B} 2$ wird dies besonders deutlich.

Die Tangenten-Schubmoduln der Probekörper mit Betondeckschichten sind nur zwischen 1,2\% und 9,5\% geringer als die Schubmoduln der Probekörper ohne Betondeckschichten. Die Streuung der Schubmoduln der Probekörper mit Betondeckschichten ist gegenüber der Probekörper ohne Betondeckschichten nicht angestiegen (siehe Abbildung 5.28.

\subsubsection{Scherprobekörper mit verklebtem Stoß}

Im Vergleich zu den ungestörten Probekörpern (X5), weisen die Probekörper mit einem verklebtem Längs- und Querstoß (X5 KL und X5KQ) im Scherverhalten und den Versagensarten keinen nennenswerten Unterschied auf. Es trat kein Versagen in einem Längs- oder Querstoß auf. Die Probekörper versagten, wie alle anderen Probekörper 

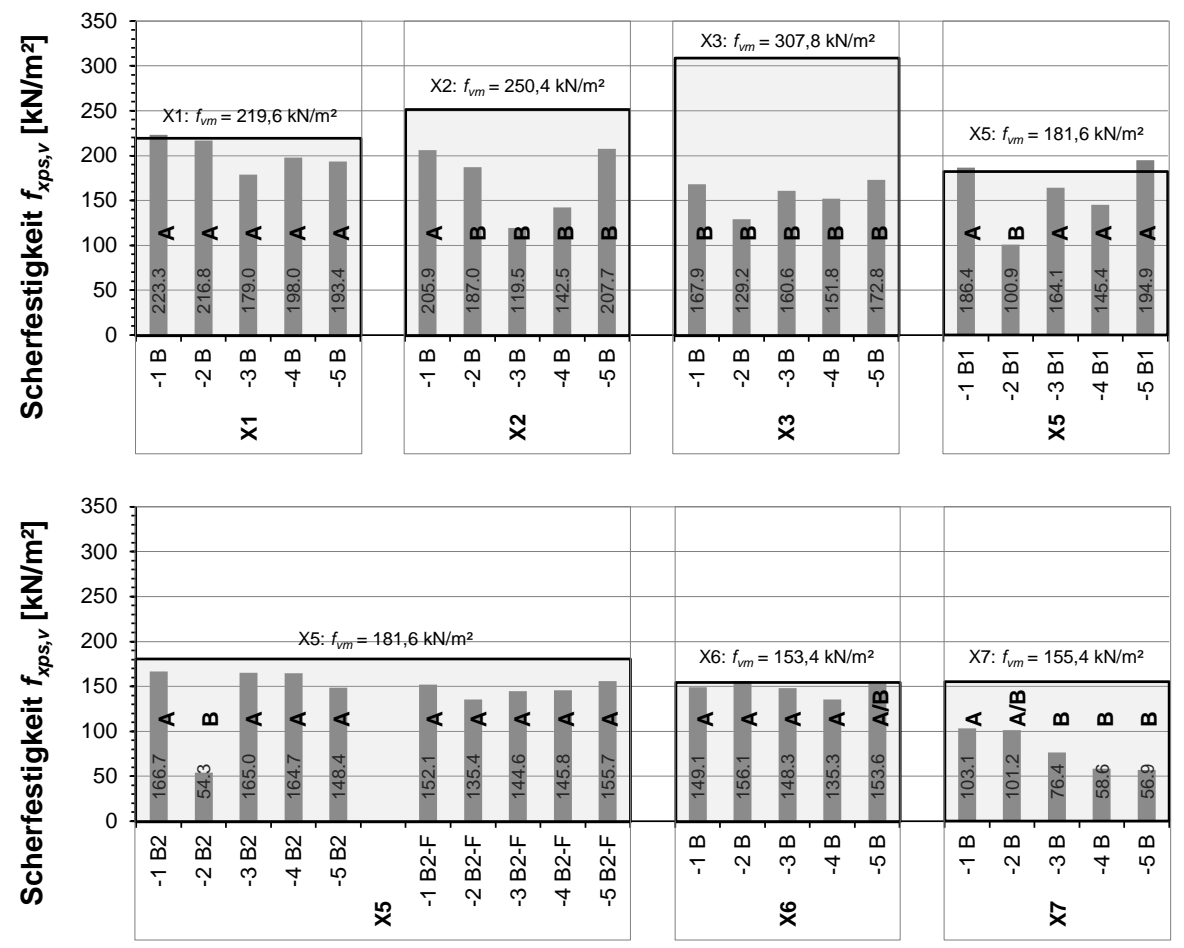

\section{Probekörper}

Abb. 5.34: Scherfestigkeiten und Versagensarten der Probekörper mit Betondeckschichten im Vergleich zu den mittleren Scherfestigkeiten der Probekörper ohne Betondeckschichten

ohne Betondeckschicht auch, in der Grenzschicht zwischen ungestörtem XPS und der Waffelstruktur.

Die mittleren Scherfestigkeiten der Versuchsreihe X5KL und X5KQ sind um 2,3\% bzw. 1,5\% geringer als die Scherfestigkeit der vergleichbaren Versuchsreihe X5. Die Tangentenschubmoduln sind um 9,5\% bzw. 5,1\% größer.

\subsubsection{Scherprobekörper mit Frost-Tau-Wechselbeanspruchung}

Im Vergleich zur Referenz Versuchsreihe X5 B2, weisen die Probekörper der Versuchsreihe X5B2-F eine wesentlich geringere Steifigkeit auf. Der Tangenten- sowie der Sekanten-Schubmodul sind um 33,3\% und 40,4\% geringer als die Werte der Versuchsreihe X5 B2 (siehe Abbildungen 5.35 und 5.36.

Die mittlere Scherfestikeit der Versuchsreihe X5 B2-F ist lediglich um 9,0\% geringer als die der Versuchsreihe X5 B2. Alle Probekörper der Versuchsreihe X5 B2-F versagten im XPS (Versagensart A). Die Scherflächen der verschiedenen Probekörper (siehe Abbildung A.67 und A.68, lassen keine Unterschiede erkennen. 


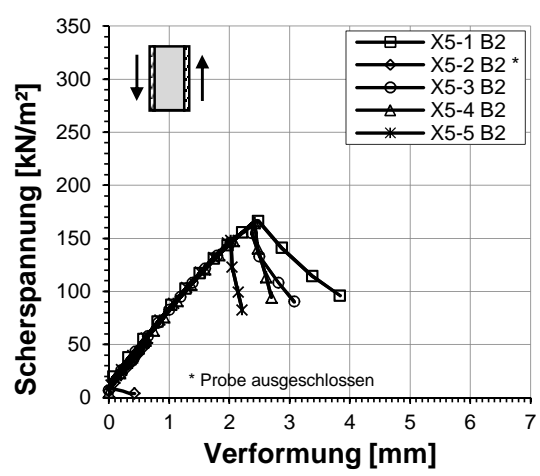

Abb. 5.35: Scherspannungs-VerformungsDiagramm der Versuchsreihe X5 B2 mit Betondeckschichten

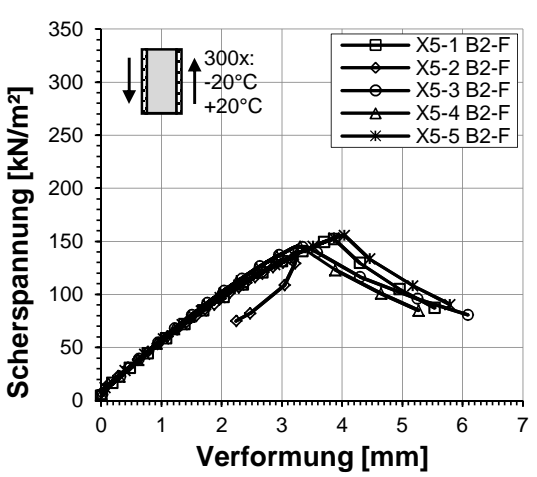

Abb. 5.36: Scherspannungs-VerformungsDiagramm der Versuchsreihe X5 B2-F mit Betondeckschichten und Frost-Tau-Beanspruchung

\subsection{Zug- und Biegeversuche an bewehrtem Beton}

\subsubsection{Allgemeines}

Das Zug- und Biegetragverhalten des bewehrten Betons beeinflusst entscheidend das Tragverhalten der Sandwichelemente. Es gibt bisher jedoch keine ausreichenden Angaben in der Literatur, um gesicherte Voraussagen über das Tragverhalten des mikround textilbewehrten Betons zu treffen. Daher sollten in Versuchen die Elastizitätsmoduln, die Zugfestigkeiten, Tension-Stiffening-Effekte, die Versagensarten und der Effektivitätsparamters $k_{1}$ der Textilbewehrung, insbesondere bei Verwendung der hier eingesetzten Hochleistungsbetonrezeptur, genau ermittelt werden. Die ermittelten Werte dienten vor allem zu einem besseren Vergleich der Bewehrungseigenschaften, zur Interpretation der Ergebnisse der Biegeversuche an den Sandwichelementen, als Eingangswerte für die Materialgesetze der FE-Modellierung sowie zur Kontrolle des erstellten Nachweiskonzepts.

Zur Untersuchung des Zugtragverhaltens wurden Dehnkörperversuche und zur Untersuchung der Biegetragverhalten 4-Punkt-Biegeversuche durchgeführt. Die Versuche wurden basierend auf den Erfahrungen aus vorangegangen Forschungsprojekten konzipiert und entsprechend optimiert. Es wurden Versuche an stahlmikrobewehrten und carbonfaserbewehrten Probekörpern durchgeführt. Aufgrund des unvorteilhaften Tragverhaltens, das bereits in den Biegeversuchen an Sandwichstreifen beobachtet werden konnte, wurde eine Verwendung der Glasfaser-Textilbewehrung bereits im Vorfeld ausgeschlossen.

Die nachfolgend beschriebenen experimentellen Untersuchungen wurden durch Joas u. Weber 2012 im Rahmen einer Studienarbeit durchgeführt. 


\subsubsection{Versuchsprogramm}

In den Zugversuchen wurden zwei verschiedene Dehnkörpertypen (Typ I und II) verwendet. Je Dehnkörper- und Bewehrungstyp wurden drei Probekörper geprüft. Im Falle der carbonbewehrten Dehnkörper Typ II wurden sechs Dehnkörper geprüft. Zur Untersuchung des Biegetragverhaltens wurden ebenfalls jeweils drei Probekörper eines weiteren Typ III geprüft (siehe Tabelle 5.5.

Die Bezeichnung der Probekörper setzt sich zusammen aus einem ersten Buchstaben, $D$ oder $B$ für Dehnkörper oder Biegeprobekörper, und aus einem zweiten Buchstaben, $S$ oder $C$ für Stahl-Mikrobewehrung oder Carbon-Textilbewehrung. Im Anschluss folgt eine fortlaufende Nummer und im Fall der Dehnkörper die römischen Zahlen I oder II um den jeweiligen Probekörpertyp zu kennzeichnen.

Tab. 5.5: Versuchsprogramm der Zug- und Biegeversuche an bewehrtem Beton

\begin{tabular}{|c|c|c|c|}
\hline Versuchsart & Probekörper & Form & Bewehrung \\
\hline \multirow{15}{*}{ Zugversuche } & DS1-I & & \multirow{6}{*}{$\begin{array}{c}\text { Stahl-Mikrobewehrung } \\
\text { (3 Lagen mit je } \\
16 \text { Längsdrähten) }\end{array}$} \\
\hline & DS2-I & $\begin{array}{c}\text { Typ I } \\
\text { (tailliert) }\end{array}$ & \\
\hline & DS3-I & & \\
\hline & DS1-II & \multirow{3}{*}{$\begin{array}{c}\text { Typ II } \\
\text { (abgestellt) }\end{array}$} & \\
\hline & DS2-II & & \\
\hline & DS3-II & & \\
\hline & DC1-I & \multirow{3}{*}{$\begin{array}{c}\text { Typ I } \\
\text { (tailliert) }\end{array}$} & \multirow{9}{*}{$\begin{array}{c}\text { Carbon-Textilbewehrung } \\
\text { (1 Lage mit je } \\
18 \text { Längsfasern) }\end{array}$} \\
\hline & DC2-I & & \\
\hline & DC3-I & & \\
\hline & DC1-II & \multirow{6}{*}{$\begin{array}{c}\text { Typ II } \\
\text { (abgestellt) }\end{array}$} & \\
\hline & DC2-II & & \\
\hline & DC3-II & & \\
\hline & DC4-II & & \\
\hline & DC5-II & & \\
\hline & DC6-II & & \\
\hline \multirow{6}{*}{ Biegeversuche } & BS1 & \multirow{3}{*}{$\begin{array}{c}\text { Typ III } \\
\text { (durchgehend) }\end{array}$} & \multirow{3}{*}{$\begin{array}{l}\text { Stahl-Mikrobewehrung } \\
\text { (10 Lagen mit je } \\
12 \text { Längsdrähten) }\end{array}$} \\
\hline & BS2 & & \\
\hline & BS3 & & \\
\hline & $\mathrm{BC} 1$ & \multirow{3}{*}{$\begin{array}{c}\text { Typ III } \\
\text { (durchgehend) }\end{array}$} & \multirow{3}{*}{$\begin{array}{c}\text { Carbon-Textilbewehrung } \\
\text { (3 Lagen mit je } \\
13 \text { Längsdrähten) }\end{array}$} \\
\hline & $\mathrm{BC} 2$ & & \\
\hline & $\mathrm{BC} 3$ & & \\
\hline
\end{tabular}

\subsubsection{Probekörper und Herstellung}

Die Länge, Dicke und Breite der beiden Dehnkörpertypen I und II betrugen $800 \mathrm{~mm}$, $30 \mathrm{~mm}$ und $200 \mathrm{~mm}$. Typ I wies eine in Dickenrichtung taillierte Form auf. Hierzu wurde die Dicke des Probekörpers im Messbereich auf $10 \mathrm{~mm}$ reduziert. Im Fall von Typ II wurde die Bewehrung über den Messbereich abgestellt, so dass diese nach der Betonage freilag. Die Lasteinleitungslänge beider Probekörper betrug $200 \mathrm{~mm}$ (siehe Ab- 
bildung 5.37). Die Dicke der Biegeprobekörper (Typ III) betrug ebenfalls $30 \mathrm{~mm}$. Die Länge und Breite betrugen $700 \mathrm{~mm}$ bzw. $150 \mathrm{~mm}$.
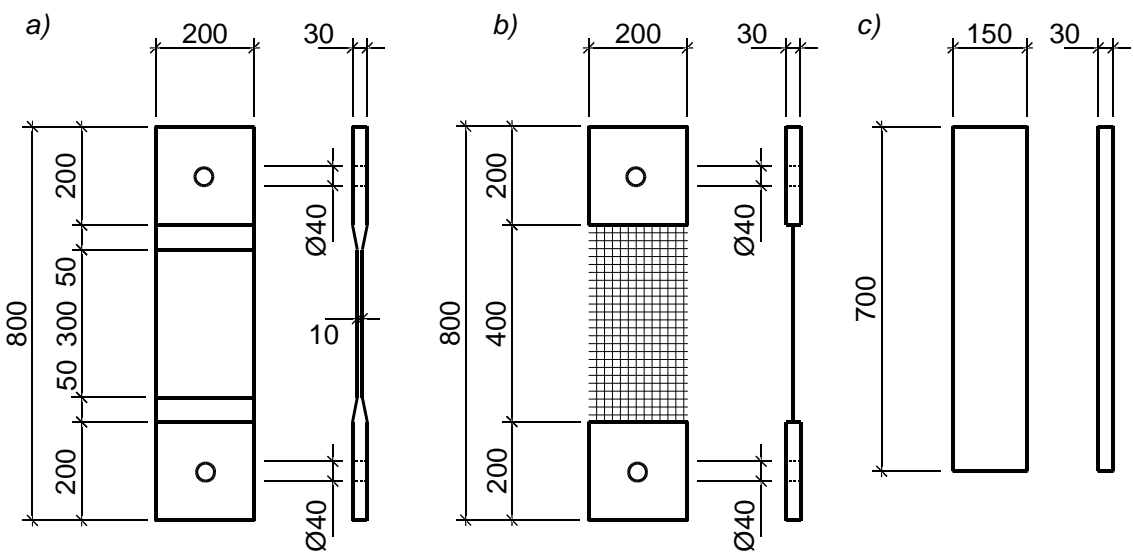

Abb. 5.37: Abmessungen der Dehnprobekörper a) Typ I (tailliert) und b) Typ II (abgestellt), und der Biegeprobekörper c) Typ III (durchgehend) in $\mathrm{mm}$

Alle Probekörper wurden in einer stehenden Schalung betoniert. Die Bewehrung wurde in der Lage durch entsprechende Abstandhalter aus PVC- und Stahlbleche mittig in der Schalung ausgerichtet, gegen die Schalung leicht angespannt und mit Schrauben fixiert (siehe Abbildung 5.38.

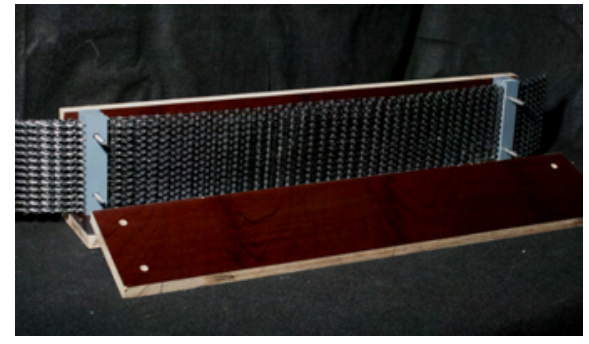

Abb. 5.38: Schalung mit vorbereiteter Bewehrung für Probekörper Typ III

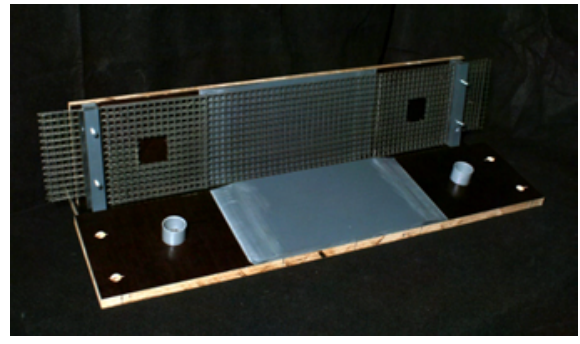

Abb. 5.39: Schalung mit vorbereiteter Bewehrung für Probekörper Typ I

Bei den beiden Dehnkörpern (Typ I und II) wurde mittels einer PVC-Rohrhülse eine Öffnung für die Lasteinleitungskonstruktion vorgesehen. Im Fall von Typ I wurde die Taillierung durch zwei an den Enden abgeschrägte PVC-Platten erzeugt (siehe Abbildung 5.39. Für Typ II wurde die Bewehrung mithilfe von XPS-Streifen abgestellt.

Im Fall der Dehnkörper (Typ I und II) wurden drei Lagen Mikrobewehrung mit je 16 Längsdrähten bzw. eine Lage Textilbewehrung mit je 18 Rovings durchgehend eingebaut. Dadurch waren die Bewehrungsquerschnitte der beiden Bewehrungsarten mit $A_{S}=37,7 \mathrm{~mm}^{2}$ und $A_{C}=35,0 \mathrm{~mm}^{2}$ in etwa gleich groß. Als Verstärkung der durch die Öffnung geschwächten Lasteinleitungsbereiche wurden hier zusätzlich zwei Lagen Mikrobewehrung bzw. eine Lage Textilbewehrung je Vorder- und Rückseite verlegt. Die Bewehrung wurde im Bereich der Öffnung ausgespart. Die Biegeprobekörper Typ III 
wurden mit 10 Lagen Drahtgittermatten bzw. drei Lagen Carbonfasergelege bewehrt. Die Betondeckung der äußeren Bewehrungslagen betrug dabei etwa 3 bis $4 \mathrm{~mm}$. Die inneren Bewehrungslagen wurde gleichmäßig verteilt über die Querschnittshöhe angeordnet.

Für die Probekörper wurde die Rezeptur HPC-4 verwendet. Der Beton wurde in die Schalung gegossen. Eine Verdichtung mithilfe eines Rütteltischs war aufgrund der sehr fließfähigen Konsistenz der Rezeptur nicht erforderlich. Nach einem Tag wurden die Probekörper ausgeschalt und mit feuchten Tüchern und Folien abgedeckt. Nach 28tägiger Lagerung wurden die Probekörper geprüft.

\subsubsection{Zugversuche an Dehnkörpern}

\subsubsection{Versuchsaufbau}

Die Last wurde in einem Vier-Säulen-Prüfrahmen durch einen servohydraulischen Prüfzylinder mit einer maximalen Zylinderkraft von $630 \mathrm{kN}$ aufgebracht (siehe Abbildung 5.40. Die Probekörper wurden mittels der beiden Lasteinleitungskonstruktionen zugfest und frei drehbar durch Kugelgelenke an der Verlängerung des Prüfzylinders sowie der Grundplatte des Prüfrahmens befestigt.
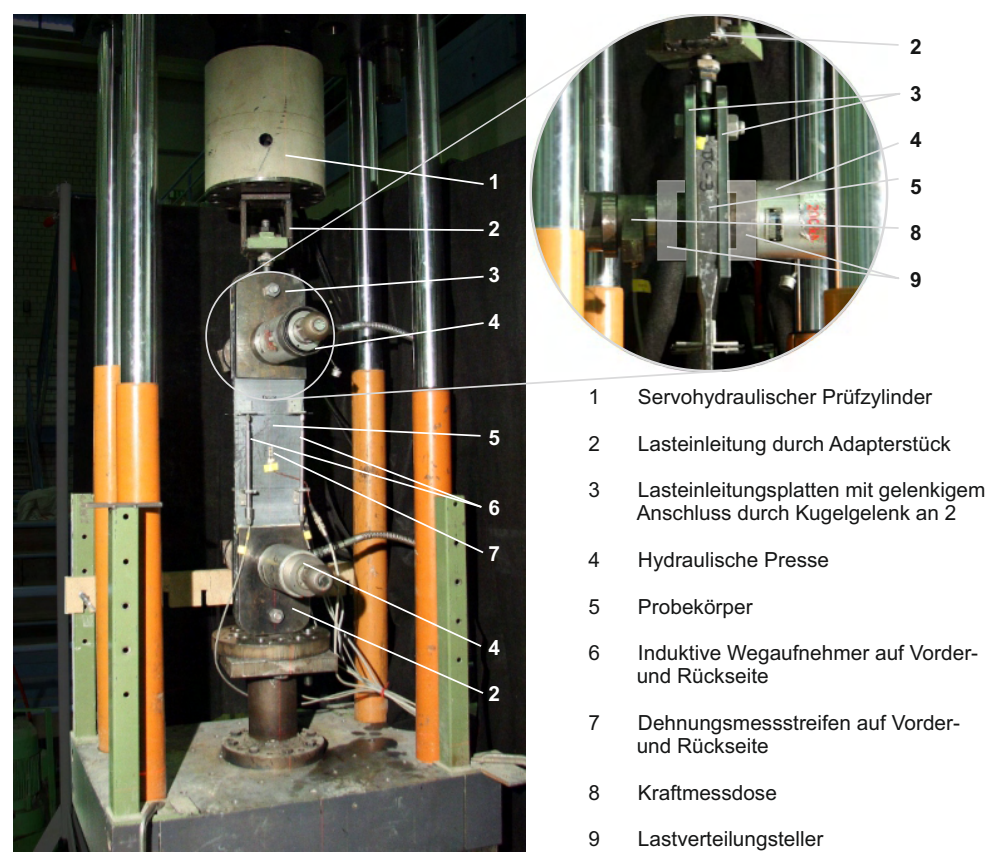

Abb. 5.40: Aufbau zur Zugprüfung eines taillierten Dehnprüfkörpers (Typ I)

Die Lasteinleitungskonstruktion wurde in Zusammenarbeit mit Oster 2012 entwickelt. Die Probekörper wurden mittels zweier hydraulischer Pressen zwischen zwei Stahlplatten gespannt. Unebenheiten zwischen den Stahlplatten und den Probekörpern wurden 
mit Elastomermatten ausgeglichen. Über eigens konstruierte Lasteinleitungsteller wurde die Last gleichmäßig auf die Stahlplatten aufgebracht. Die gewählte Anpresslast von $130 \mathrm{kN}$ konnte jeweils mit einer Kraftmessdose überprüft werden. Durch Stahlgewichte wurde eine Schiefstellung durch das einseitig wirkende Gewicht der hydraulischen Pressen ausgeglichen und die Lasteinleitungskonstruktionen in der Waage gehalten.

Als Messtechnik kamen sowohl induktive Wegaufnehmer als auch Dehnungsmessstreifen zum Einsatz. Im Fall der Dehnkörper Typ I wurden jeweils zwei Wegaufnehmer je Seite des Probekörpers mit Winkelhalterungen und Klebstoff auf der Betonoberfläche befestigt. Als Messlänge wurde der Abstand der Mittelachsen der beiden Winkelhalterungen definiert. Diese betrug im Mittel $250 \mathrm{~mm}$. Im Fall der Dehnkörper Typ II wurde je ein Wegaufnehmer seitlich auf die beiden Seitenflächen der Lasteinleitungsbereiche aufgeklebt. Die Messlänge betrug hier $400 \mathrm{~mm}$ und wurde als lichter Abstand der aufgeklebten Halterungen definiert. Dehnungsmessstreifen wurden nur auf die Dehnkörper Typ I, jeweils mittig auf Vorder- und Rückseite aufgeklebt.

Anstelle von Wegaufnehmern, wurden zur Messungen der Verformungen des Probekörpers DC2-I, das fotogrammetrische Messystem ARAMIS der Firma GOM mbH eingesetzt.

\subsubsection{Versuchsdurchführung}

Die Steuerung der Dehnkörperversuche erfolgte weggeregelt mit einer Belastungsgeschwindigkeit von 0,1 bis $0,2 \mathrm{~mm} / \mathrm{min}$. Bei den Versuchen mit mikrobewehrten Probekörpern wurde bei Abflachen der Last-Verformungskurve die Geschwindigkeit dann auf $0,4 \mathrm{~mm} / \mathrm{min}$ (Typ I) bzw. auf $1,0 \mathrm{~mm} / \mathrm{min}$ (Typ II) erhöht.

Die Messwerte der aufgebrachten Zylinderkraft, des Zylinderwegs, der Pressenkraft, der Wegaufnehmer und der Dehnmessstreifen wurde mit einer Messfrequenz von $2 \mathrm{~Hz}$ mit einem Messrechner aufgezeichnet.

\subsubsection{Versuchsergebnisse}

In Diagramm 5.41 sind die Spannungsdehnungskurven der 15 Versuche dargestellt. Zur Berechnung der Spannungen der taillierten Probekörper (Typ I), wurde die Zugkraft auf die wirkliche Brutto-Betonquerschnittsfläche der Probekörper bezogen. Im Fall der abgestellten Probekörper (Typ II) wurde eine bezogene Spannung errechnet, indem die Zugkraft durch einen fiktiven Betonquerschnitt mit der Fläche von $10 \mathrm{~mm}$ und $200 \mathrm{~mm}$ geteilt wurde. Diese fiktive Spannung erlaubt einen einfacheren Vergleich der Kurven der beiden Probekörper Typ I und II.

Die Dehnungen errechneten sich aus dem Mittelwert der Wegaufnehmer bezogen auf die jeweilige Messlänge. Aufgrund von Rissen, lösten sich in den Versuchen DC1-II bis DC3-II zum Teil mehrere Halterungen der Wegaufnehmer von den Probekörpern. Die Dehnungen konnten jedoch aus den Messwerten der übrigen Wegaufnehmer und des Zylinderwegs errechnet werden (Joas u. Weber|2012). 


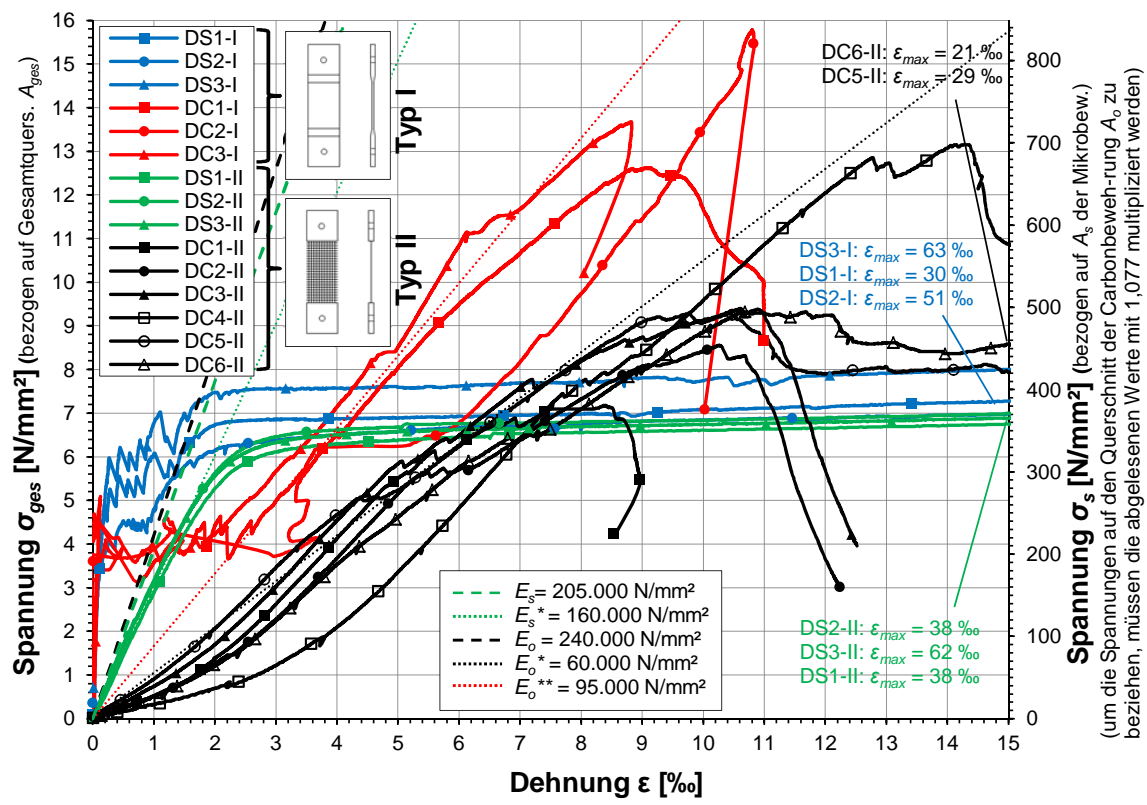

Abb. 5.41: Spannungs-Dehnungs-Diagramm der Dehnkörperversuche

Tab. 5.6: Ergebnisse der Dehnkörperversuche

\begin{tabular}{|c|c|c|c|c|c|c|c|c|}
\hline \multirow[b]{2}{*}{ Bez. } & \multicolumn{2}{|c|}{ Erstriss } & \multicolumn{3}{|c|}{ Dehngrenze } & \multicolumn{3}{|c|}{ Zugfestigkeit } \\
\hline & $\begin{array}{c}\sigma_{c r} \\
{\left[\mathrm{~N} / \mathrm{mm}^{2}\right]}\end{array}$ & $\begin{array}{c}\boldsymbol{\varepsilon}_{c r} \\
{[\% o]}\end{array}$ & $\begin{array}{c}\sigma_{p l, c} \\
{\left[\mathrm{~N} / \mathrm{mm}^{2}\right]}\end{array}$ & $\begin{array}{r}\sigma_{p l, s} \\
{\left[\mathrm{~N} / \mathrm{mm}^{2}\right]}\end{array}$ & $\begin{array}{c}\varepsilon_{p l} \\
{[\% \circ]}\end{array}$ & $\begin{array}{r}\sigma_{\max , c} \\
{\left[\mathrm{~N} / \mathrm{mm}^{2}\right]}\end{array}$ & $\begin{array}{l}\sigma_{\max , \text { s bzw. o }} \\
{\left[\mathrm{N} / \mathrm{mm}^{2}\right]}\end{array}$ & $\begin{array}{l}\varepsilon_{\max } \\
{[\% 0]}\end{array}$ \\
\hline DS1-I & 4,9 & 0,20 & 6,8 & 388 & 2,3 & 7,6 & 434 & 30 \\
\hline DS2-I & 3,5 & 0,12 & 6,3 & 357 & 2,6 & 7,7 & 437 & 51 \\
\hline DS3-I & 5,9 & 0,25 & 7,5 & 377 & 2,1 & 8,7 & 438 & 63 \\
\hline DS1-II & - & - & 6,3 & 335 & 4 & 7,4 & 393 & 38 \\
\hline DS2-II & - & - & 6,6 & 352 & 4,2 & 7,6 & 404 & 38 \\
\hline DS3-II & - & - & 6,5 & 347 & 4,1 & 8 & 425 & 62 \\
\hline DC1-I & 4,5 & 0,08 & - & - & - & 12,6 & 610 & 9 \\
\hline DC2-I & 3,8 & 0,05 & - & - & - & 15,8 & 874 & 11 \\
\hline DC3-I & 5 & 0,12 & - & - & - & 13,7 & 772 & 9 \\
\hline DC1-II & - & - & - & - & - & 7,2 & 410 & 8 \\
\hline DC2-II & - & - & - & - & - & 8,6 & 489 & 10 \\
\hline DC3-II & - & - & - & - & - & 9,4 & 537 & 11 \\
\hline DC4-II & - & - & - & - & - & 13,2 & 752 & 14 \\
\hline DC5-II & - & - & - & - & - & 9,3 & 531 & 10 \\
\hline DC6-II & - & - & - & - & - & 9,5 & 545 & 21 \\
\hline
\end{tabular}




\section{Mikrobewehrte Probekörper (Typ DS-I und DS-II):}

Die taillierten, mikrobewehrten Probekörper (Typ DS-I) wiesen im ungerissenen Zustand ein nahezu linear-elastisches Verformungsverhalten auf. Bei einer Spannung von 3,5 bis $7,0 \mathrm{~N} / \mathrm{mm}^{2}$ bildeten sich horizontale Trennrisse in der Betonmatrix. Aus den Risse resultierten mehrere aufeinanderfolgende, kurze Lastabfälle. Nach jedem Lastabfall stieg die Spannung wieder über das zuvor erreichte Lastniveau an und es bildeten sich weitere Risse.

Bei einer Dehnung von ca. 1,4\%。 wurde ein abgeschlossenes Rissbild erreicht. Im Durchschnitt bildeten sich über die Messlänge von $250 \mathrm{~mm} 13$ Risse. Die Risse traten meist mit einem Abstand von ein bis zweimal der Maschenweiten an den angeschweißten Querdrähten auf. Dies kann damit begründet werden, dass an diesen Stellen der Betonquerschnitt geschwächt war und die Kraft über die angeschweißten Querstäbe besonders gut in den Beton eingeleitet werden konnte.

Die Spannung stieg dann noch weiter bis zum Erreichen der Dehngrenze der Drahtgittermatten an. Im Anschluss folgte ein leicht ansteigendes, ausgedehntes Plateau von Dehnungen bis zu 63\% und Spannungen, die im Mittel etwa $17 \%$ über der Dehngrenze lagen. Das Versagen der Probekörper wurde durch das Reißen einzelner Drähte eingeleitet, welches sich dann über die Breite des Probekörpers fortsetzte.

Die abgestellen, mikrobewehrten Probekörper (Typ DS-II) wiesen zu Beginn ein annähernd linear-elastischen Verhalten auf. Die Steigung der Kurve in diesem Bereich ist jedoch flacher, als die theoretisch erreichbare Steigung bei einem Elastizitätsmodul von $205.000 \mathrm{~N} / \mathrm{mm}^{2}$ (siehe Abbildung 5.41 strichlierte grüne Linie). Der rückgerechnete Elastizitätsmodul $E_{s}^{*}$ beträgt hier etwa $160.000 \mathrm{~N} / \mathrm{mm}^{2}$. Die Dehnungen der abgestellten Probekörper wurden mit der Länge der abgestellten Bewehrung von $400 \mathrm{~mm}$ berechnet. Es werden jedoch bereits Verformungen durch Schlupf zwischen Beton und Bewehrung außerhalb des abgestellten Bereichs verursacht, die zur Vergrößerung der Dehnungen und somit zur Veringerung des Elastizitätsmodul führen. Weiterhin wird vermutet, dass der geringe Elastizitätsmodul zum Teil durch Querschnittsschwächungen an den Schweißstellen der Drähte hervorgerufen wurde.

Der linear-elastische Bereich ist bei etwa $2 \%$ zu Ende. Ab etwa 4\% zeigt sich ein ausgeprägtes plastischen Verhalten. Generell weist die Mikrobewehrung das typische Spannungsdehnungsverhalten eines kaltverformten Stahls auf. Im Übergang zwischen dem elastischen und plastischen Bereich, kann kein ausgeprägter Fließbereich festgestellt werden. Dennoch kann eine Dehngrenze bei 0,2\% definiert werden.

Die maximalen Dehnungen betrugen ebenfalls Werte von bis zu $62 \%$. Die maximale Zugspannung erreichte im Mittel rund $400 \mathrm{~N} / \mathrm{mm}^{2}$ und lag etwa $18 \%$ über der Dehngrenze von $0,2 \%$. Das Versagen der Probekörper Typ DS-II wurde ebenfalls durch das Reißen einzelner Drähte eingeleitet.

\section{Textilbewehrte Probekörper (Typ DC-I und DC-II):}

Die taillierten, carbonfaserbewehrten Probekörper (Typ DC-I) wiesen bis zum Erstriss ebenfalls ein linear-elastisches Materialverhalten auf. Die Erstrissspannungen entsprachen in etwa denen der mikrobewehrten Probekörpern (siehe Tabelle 5.6. Im Bereich 
der Rissbildung weist das Spannungsdehungsdiagramm (siehe Abbildung 5.41) ein nahezu horizontales Plateau auf.

Erst bei einer Dehnung von etwa 2,5\% wird ein nahezu abgeschlossenes Rissbild erreicht. Es bildeten sich etwa 6 bis 8 Risse über den Messbereich von $250 \mathrm{~mm}$. Die Kurven steigen dann anschließend wieder linear bis zum Versagen der Probekörper bei einer Spannung zwischen 610 bis $880 \mathrm{~N} / \mathrm{mm}^{2}$ an. Dies entspricht einer Ausnutzung von rund $20 \%$ bezogen auf die Faserzugfestigkeit von $4.000 \mathrm{~N} / \mathrm{mm}^{2}$. Der Effektivitätsfaktor $k_{1}$ beträgt somit 0,2 .

Der lineare Anstieg im Zustand II weist eine Steigung auf, die einem Elastizitätsmodul $E_{o}^{*}$ von etwa $95.000 \mathrm{~N} / \mathrm{mm}^{2}$ entspricht und ist wesentlich flacher als der theoretisch erreichbare Anstieg bei einem Elastizitätsmodul von $240.000 \mathrm{~N} / \mathrm{mm}^{2}$. Aufgrund des Tension-Stiffening-Effekts, ist der lineare Ast im Zustand II gegenüber der Kurven der abgestellten Probekörper (Typ II) um einen annähernd gleichbleibenden Betrag verschoben.

Das Versagen der Bewehrung aller drei Probekörpern erfolgte zwischen dem Ende der Taillierung und der Lasteinleitungskonstruktion. Dabei kündigte sich das Versagen bereits durch das Aufweiten des entsprechenden Risses in diesem Bereich an. Die aufgebrachte Last viel abrupt ab und der Versagensriss klaffte weit auf.

Die genaue Abfolge des Versagens ließ sich abschließend nicht feststellen. Es versagten jedoch nur ein Teil der Filamente eines Rovings, die in direktem Verbund mit der Betonmatrix standen und daher am meisten beansprucht wurden. Die inneren Kernfilamente wurden beim Versagen nicht durchtrennt, sondern aufgrund des Verbundverlusts zu den Randfilamenten aus dem Roving herausgezogen.

Durch die Verformungsmessung mit dem 3D-Fotogrammetrie-Messsystems konnte eine Wölbung des Probekörpers DC2-I in z-Richtung, d. h. senkrecht zur Probekörperebene, von bis zu $2 \mathrm{~mm}$ festgestellt werden (siehe Abbildung 5.43). Diese Verformung wurde durch eine außermittige Anordnung der Bewehrungslage verursacht. Obwohl das Carbongelege vor der Betonage leicht angespannt und mittig ausgerichtet wurde, konnte durch das Aufschneiden der geprüften Probekörper festgestellt werden, dass die Bewehrung unplanmäßig seitlich verschoben war. Es wird vermutet, dass diese Verschiebung durch das Einfüllen des Betons verursacht wurde. Die Wölbung der Probekörper fand vor allem bei Verwendung der einlagigen Carbonfaserbewehrung statt. Bei den mikrobewehrten Probekörpern wurde eine außerplanmäßige Anordnung durch den dreilagigen Aufbau und die damit verbundene größere Biegesteifigkeit behindert.

Bei den abgestellten, carbonfaserbewehrten Probekörpern (Typ DC-II) stiegen die Spannungsdehnungskurven zu Beginn der Belastung nur langsam an und verliefen dann zunehmend steiler. Gegen Ende der Versuche flachten die Kurven etwas ab und erreichten Bruchspannungen zwischen 410 und $750 \mathrm{~N} / \mathrm{mm}^{2}$. Der Elastizitätsmodul der abgestellten Bewehrung $E_{o}^{* *}$ betrug im Mittel rund $60.000 \mathrm{~N} / \mathrm{mm}^{2}$.

Das Versagen der Probekörper erfolgte im Übergang zwischen dem Lasteinleitungsbereich und der abgestellten Bewehrung. Hier ließ sich deutlich erkennen, dass die äußeren Filamente versagten, und die inneren Filamente aus dem Rovingkern herausgezogen wurden. Wie bereits durch Molter 2005 beschrieben, ließ sich dabei nicht abschließend feststellen, in welcher Reihenfolge das Versagen erfolgte, d.h. ob die 
Randfilamente zuerst versagten und sich dann der Verbund zwischen Rand- und Kernfilamenten löste, oder ob sich zuerst der Verbund zwischen den Filamenten löste und daraus ein Versagen der Randfilamente resultierte.

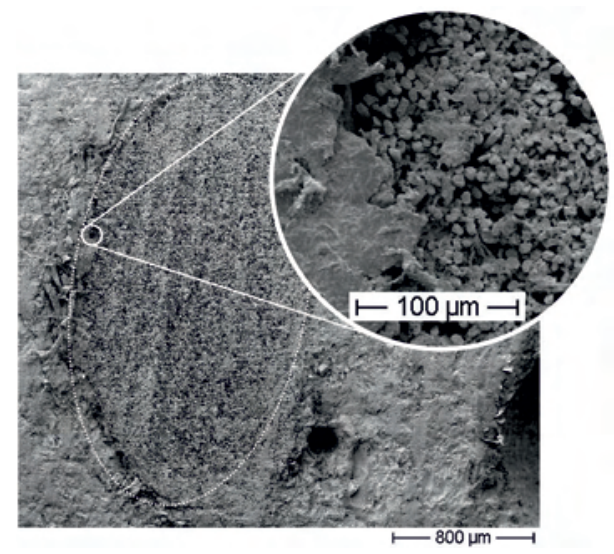

Abb. 5.42: REM-Aufnahme einer Carbonfaser eines Biegeprobekörpers

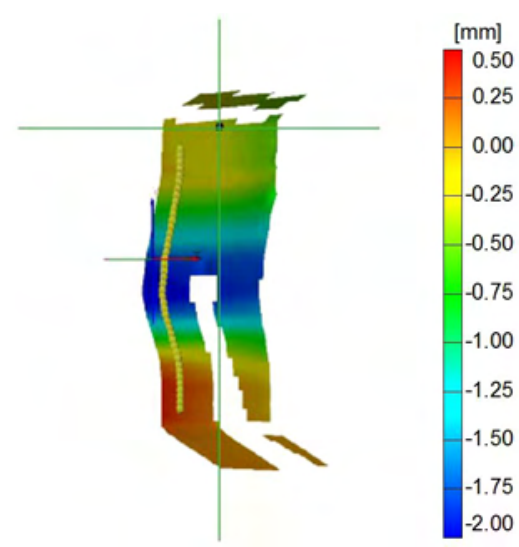

Abb. 5.43: Mit 3D-Fotogrammetrie bestimmte Verschiebung rechtwinklig zur Probekörperebene (Probekörper DC2-I bei Last von $20 \mathrm{kN}$ )

\subsubsection{Biegeversuche}

\subsubsection{Versuchsaufbau}

Die Last wurde mithilfe eines servohydraulischen Prüfzylinders mit einer maximalen Prüflast von $250 \mathrm{kN}$ wie in Abbildung 5.44 dargestellt auf die Probekörper aufgebracht. Die Probekörper wurden in einem 4-Punkt-Biegeversuch mit einer Spannweite von $600 \mathrm{~mm}$ geprüft. Die Last wurde dabei in den beiden Drittelspunkten mit Rollenlagern auf die Probekörper aufgebracht.

\subsubsection{Versuchsdurchführung}

Die Versuche wurden weggesteuert mit einer Anfangsbelastungsgeschwindigkeit von $0,2 \mathrm{~mm} / \mathrm{min}$ durchgeführt. Beim Abflachen der Lastverformungskurve wurde die Belastungsgeschwindgkeit schrittweise auf $1,0 \mathrm{~mm} / \mathrm{min}$ gesteigert. Die aufgebrachte Last, die Dehnungen und Verformungen in Feldmitte wurden mithilfe einer Kraftmessdose, Dehnungsmessstreifen und induktiven Wegaufnehmern gemessen und mit einem Messrechner aufgezeichnet.

\subsubsection{Versuchsergebnisse}

Die mikrobewehrten Probekörper wiesen, wie die carbonfaserbewehrten Probekörper auch, bis zum Erstriss ein linear-elastischen Verformungsverhalten auf (siehe Abbil- 


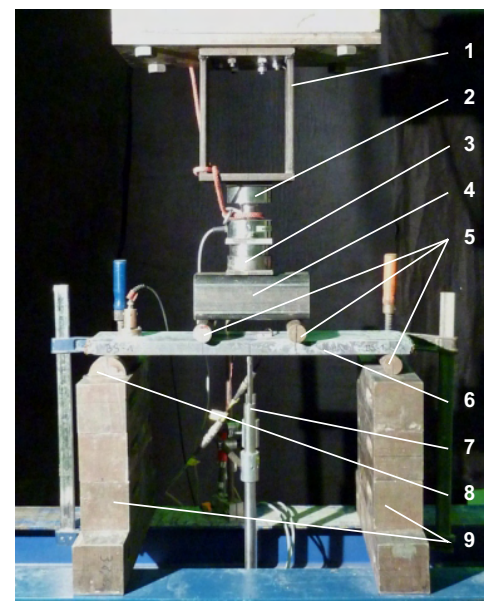

Lasteinleitung über Verlängerung des Zylinders

2 Kraftmessdose

$3 \quad$ Kalotte

4 Traverse

5 Rollenlager

6 Probekörper

$\dddot{i}$ Induktiver Wegauf-

nehmer

Festlager

Abstandhalter

Abb. 5.44: Aufbau eines Biegeversuchs

dung 5.45). Die Last beim Erstriss war für beide Probekörperarten in etwa gleich (siehe Tabelle 5.7). Die Kurven der mikrobewehrten Probekörper flachten dann etwas ab und gingen dann bei einer Verformung von 6 bis $10 \mathrm{~mm}$ in ein langes Plateau über. Die einzelnen Bewehrungslagen begannen nacheinander zu plastizieren. Dabei weiteten sich die Risse auf. Die Risslänge entsprach bis zu $80 \%$ der Querschnittshöhe. Dann rissen einzelne Drähte der Bewehrung und die Last fiel bei einer Verformung von rund $50 \mathrm{~mm}$ ab.

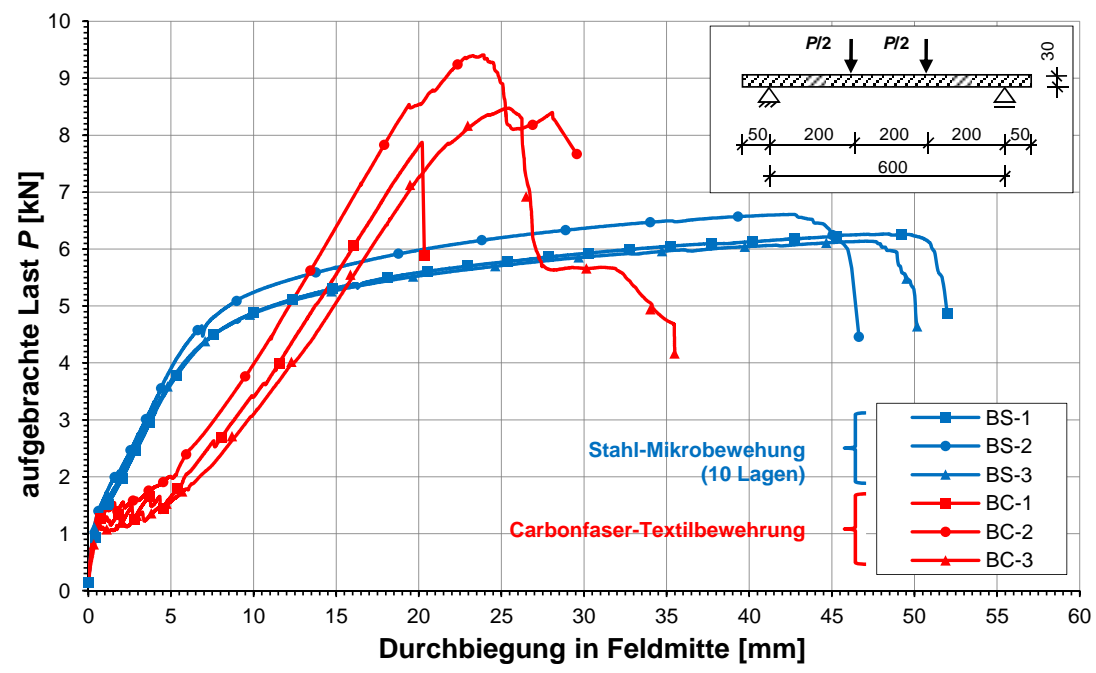

Abb. 5.45: Lastverformungs-Diagramm der Biegeversuche

Bei den carbonfaserbewehrten Probekörper fiel während der Rissbildung im Zustand Il a die Last bei jedem Riss ab. Die Lastverformungskurven flachten in diesem Bereich deutlich ab. Ab einer Verformung von etwa $6 \mathrm{~mm}$ stiegen die Kurven wieder an. Dieser lineare Anstieg im Zustand II a war flacher als der Anstieg der mikrobewehrten Pro- 
Tab. 5.7: Ergebnisse der Biegeversuche

\begin{tabular}{|c|cccc|c|}
\hline \multirow{2}{*}{ Bez. } & \multicolumn{2}{|c|}{ Erstriss } & \multicolumn{2}{c|}{ Zugfestigkeit } & Versagensart \\
& $\boldsymbol{P}_{c r}$ & $\boldsymbol{f}_{c r}$ & $\boldsymbol{P}_{\max }$ & $\boldsymbol{f}_{\max }$ & \\
\hline BS1 & 2,4 & 2,5 & 6,3 & 48,2 & Zugversagen Bewehrung \\
BS2 & 2,1 & 1,8 & 6,6 & 41,9 & Zugversagen Bewehrung \\
BS3 & 1,7 & 1,4 & 6,1 & 47,4 & Zugversagen Bewehrung \\
BC1 & 1,2 & 1,2 & 7,9 & 20,2 & Querkraftversagen \\
BC2 & 1,5 & 1,1 & 9,4 & 24,2 & Querkraftversagen \\
BC3 & 1,1 & 0,9 & 8,5 & 25,3 & Zugversagen Bewehrung \\
\hline
\end{tabular}

bekörper. Dabei stiegen die aufgebrachten Lasten noch bis $9,4 \mathrm{kN}$ an, bis dann die Probekörper abrupt aufgrund des Reißens der Carbonfasern oder infolge eines Querkraftrisses versagten.

\subsubsection{Zusammenfassung und Erkenntnisse}

Die mikro- und carbonfaserbewehrten Dehn- und Biegeprobekörper erlauben aufgrund ihres ähnlichen Bewehrungsgehaltes eine gute Vergleichbarkeit. Generell sind die Erstrisslasten in etwa gleich. Es kann daher daraus geschlossen werden, dass die Bewehrungsart auf das Tragverhalten des bewehrten Betons im Zustand I nur einen geringen Einfluss hat.

Im Bereich der Rissbildung im Zustand II a unterscheidet sich das Lastverformungsverhalten jedoch. Die taillierten, carbonfaserbewehrten Dehnkörper DC-I weisen bei der Rissbildung im Vergleich zu den mikrobewehrten Probekörpern eine stärkere Zunahme der Dehnungen auf. Auch die carbonfaserbewehrten Biegeprobekörper weisen im Zustand II a diese Zunahme der Verformungen auf. Die Steifigkeitsreduzierung wird durch Jesse 2004 damit begründet, dass bei der Rissbildung die äußeren Filamente zum Teil reißen und dadurch die Verformungen zunehmen und die Effektivität entsprechend vermindert wird.

Es ist jedoch zu vermuten, dass die Zunahme der Dehnungen durch die Welligkeit der Filamente verursacht wird. Die abgestellten Probekörper DC-Il weisen zu Beginn eine geringe Steigung auf. Erst im weiteren Versuchsverlauf nimmt die Steigung der Kurven zu. Zu Beginn werden die wenigen Filamente, die bereits einen geraden, gestreckten Verlauf aufweisen, stärker beansprucht als die übrigen. Erst bei zunehmender Dehnung nehmen die weiteren Filamente nennenswert Last auf und tragen somit zur Erhöhung der Dehnsteifigkeit des Probekörpers bei. Bei den taillierten Probekörpern ist die Welligkeit der Filamente ebenfalls vorhanden. Jedoch können sich die Filamente erst dann gerade ziehen, wenn es zur Rissbildung in der Betonmatrix kommt. Infolge dessen, nehmen die Verformungen während der Rissbildung stark zu.

Im Zustand II b, bei abgeschlossenem Rissbild der Dehnkörperversuche werden mit beiden Bewehrungsarten nicht die theoretisch erreichbaren Steifigkeiten erzielt. Bei den mikrobewehrten Probekörpern kann dies damit begründet werden, dass der Drahtquerschnitt an den Schweißstellen etwas geschwächt ist. Bei den carbonfaserbewehr- 
ten Dehnkörpern liegt die Ausnutzung der Steifigkeit lediglich bei $25 \%$. Hier wird aufgrund der schlechten Verbundeigenschaften der Filamente untereinander nicht der gesamte Faserquerschnitt aktiviert. Trotz eines etwas geringeren Elastizitätsmoduls des Bewehrungsmaterials, weisen im Zustand II b die Spannungsdehnungkurven der mikrobewehrten Dehnkörper eine größere Steigung auf als die carbonfaserbewehrten Dehnkörper.

Es lässt sich weiterhin vermuten, dass die Verbundlänge in der Betonmatrix einen Einfluss auf die Zugfestigkeit der textilbewehrten Probekörper hat. Die taillierten Probekörper DC-I wiesen höhere Bruchspannungen auf, als nahezu alle abgestellten Probekörper. Dies kann damit begründet werden, dass die Fasern über eine größere Länge in der Betonmatrix eingebettet sind und dadurch besser aktiviert werden können.

Generell weißt das Carbonfasergelege mit einer Ausnutzung der theoretischen Zugfestigkeit von $20 \%$ und des Elastizitätsmoduls von etwa $25 \%$ eine geringe Effektivität auf. Dies liegt an der Verwendung der 50 k-Faser mit einer sehr großen Filamentanzahl von 50.000 (siehe Abbildung 5.42). Durch die Verwendung einer feineren Faser würde sich das Verhältnis der Anzahl an Rand- zu Kernfilamenten verbessern. Eine weitere Möglichkeit, die Ausnutzung der Kernfilamente zu verbessern, wäre eine Imprägnierung der Rovings, beispielsweise mit einem Epoxidharz.

\subsection{Ermüdungsversuche mit zyklischer Wechselbeanspruchung}

\subsubsection{Allgemeines}

In den Biegeversuchen in Abschnitt 5.2, Biegeversuche an Sandwichstreifen wurden bereits einige der Sandwichelemente zyklisch beansprucht. Jedoch erlaubte der Versuchsaufbau nur eine Belastung der Probekörper durch eine über den Zylinder aufgebrachte Druckkraft. Durch diese Belastungart wurde in den Probekörper lediglich eine Beanspruchung in einer Richtung hervorgerufen, d. h. eine positive Momentenbeanspruchung mit den einhergehenden Scherbeanspruchungen im Kern und im Verbund. Eine Beanspruchung im späteren Einsatz als raumabschließendes Element, aus Windoder Temperatureinwirkung durch Winddruck und Windsog bzw. durch Erwärmung und Abkühlung der Deckschichten, wirkt jedoch in beide Richtungen.

Damit sich der Versuchsaufbau bei den zuvor durchgeführten zyklischen Versuchen nicht verschieben konnte, wurden diese dabei nicht vollständig entlastet. Sollte durch die zyklische Belastung des Probekörpers die Haftwirkung zwischen Beton und XPS ausgefallen sein, so konnte weiterhin bei senkrecht zum Verbund wirkender Belastung über Reibwirkung eine Schubkraft übertragen werden.

Ziel war es daher, in weiteren Ermüdungsversuchen durch eine realitätsnahe zyklische Wechselbeanspruchung mögliche Schädigungen des XPS-Kerns und des Verbunds zwischen Beton und XPS zu erzeugen. Hierzu wurde ein Prüfstand konstruiert, bei dem durch eine druck- und zugfeste Lager- und Lasteinleitungskonstruktion der Probekörper, diese sowohl durch positive als auch negative Momente beansprucht werden konnten. 


\subsubsection{Probekörper und Herstellung}

Die untersuchten Probekörper wiesen mit einer Länge von $1.250 \mathrm{~mm}$ und einer Breite von $200 \mathrm{~mm}$ die selben Abmessungen auf, wie die Probekörper der Biegeversuche (siehe Abschnitt5.2). Die Kernschicht- und Deckschichtdicken betrugen $120 \mathrm{~mm}$ bzw. $10 \mathrm{~mm}$. Es wurde die Betonrezeptur HPC-4 verwendet. Als Bewehrung wurden 3 Lagen Mikrobewehrung je Deckschicht und als Kernmaterial die XPS-Charge X5 verwendet. Die Herstellung erfolgte über die Variante „Eindrücken des XPS in den Beton“.

\subsubsection{Versuchsstand}

Der Versuchsstand für die Ermüdungsversuche ist in Abbildung 5.46 und in einer detaillierteren Zeichnung im Anhang A.4 dargestellt. Die Versuchsanordnung glich dabei den 4-Punkt-Biegeversuchen in Abschnitt 5.2 , Biegeversuche an Sandwichstreifen: Die Spannweite betrug ebenfalls $1.150 \mathrm{~mm}$. Der Versuchsstand selbst bestand aus einer Lager- und einer Lasteinleitungskonstruktion.
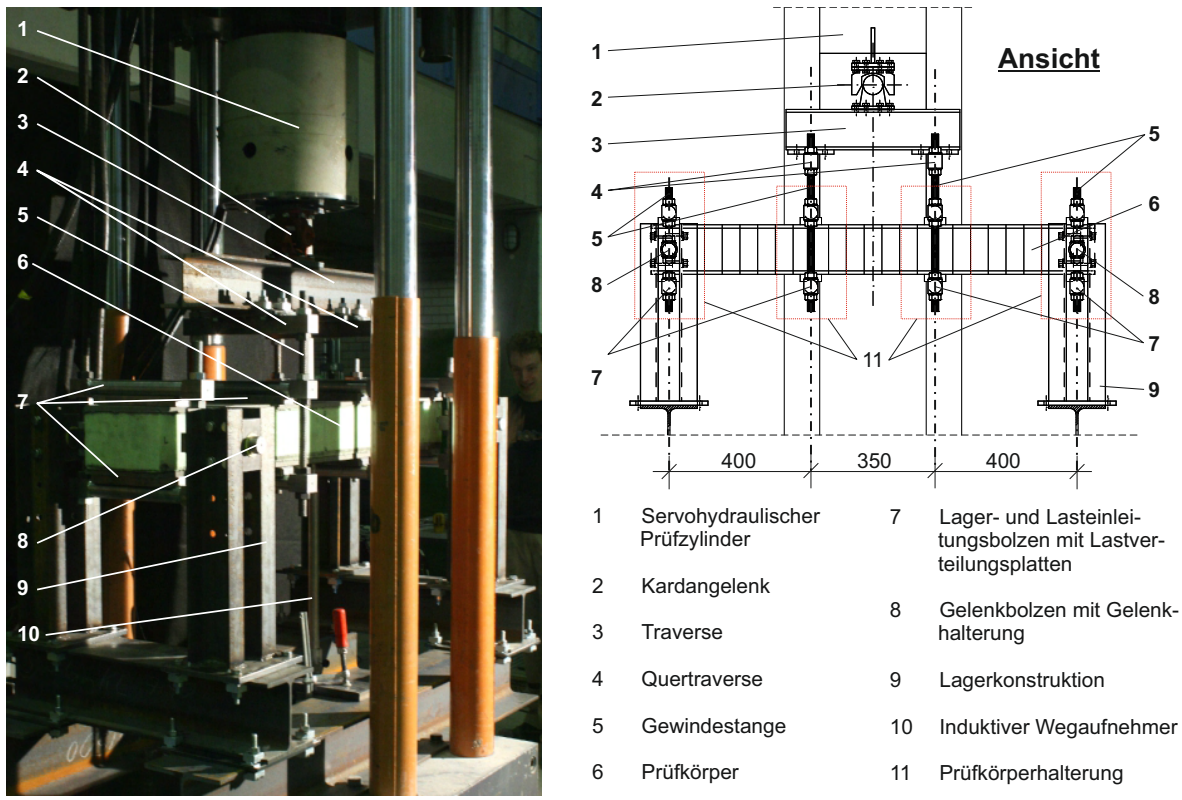

Abb. 5.46: Versuchsstand zum Aufbringen positiver und negativer Momente

Die Haltekonstruktion bestand aus zwei um $180^{\circ}$-gedrehten Stahlrahmen, die zug- und druckfest über Stahlträger an die Bodenplatte des Prüfrahmen angeschlossen waren. Zwischen den Stielen eines jeden Stahlrahmens war eine Probekörperhalterung freidrehbar angeordnet.

Die Probekörperhalterungen bestanden aus je zwei Lastverteilungsplatten, zwei knochenförmigen Lager- bzw. Lastverteilungsbolzen und zwei Gewindestangen. Die Probekörper wurden mittels der Gewindestangen und der Bolzen zwischen die Lastverteilungsplatten gespannt. Die Einspannung der Probekörper erfolgte mit einer gerin- 
gen Vorspannung, die mit Federunterlegscheiben und Drehmomentenschlüssel leicht eingestellt werden konnte. Das Vorspannen war erforderlich, um Verschiebungen des Probekörpers während der zyklischen Belastung zu minimieren.

Die Lasteinleitungskonstruktion bestand im Wesentlichen aus einem stählernen IProfil, das als Traverse zug- und druckfest sowie gelenkig über ein Kardangelenk mit dem Prüfzylinder verbunden war. An das Stahlprofil waren ebenfalls zwei Probekörperhalterungen zur Lasteinleitung angeschlossen, zwischen die der Probekörper eingespannt wurde.

\subsubsection{Versuchsdurchführung}

Es wurden insgesamt elf Versuche durchgeführt (siehe Tabelle 5.8). Bei drei der Versuche wurde eine quasi-statische Belastung bis zum Versagen der Probekörper aufgebracht um eine Referenztraglast zu ermitteln.

Tab. 5.8: Versuchsprogramm Ermüdungsversuche, Versagensarten vgl. Tab. 5.3 und 5.9

\begin{tabular}{|c|c|c|c|c|c|c|}
\hline Probekörper & Versuch & $\begin{array}{c}\text { Amp. } \\
{[\mathrm{kN}]}\end{array}$ & $\begin{array}{c}\text { Freq. } \\
{[\mathrm{Hz}]}\end{array}$ & $\begin{array}{c}\text { Zyklen } \\
{[-]}\end{array}$ & $\begin{array}{c}\text { Last } \\
{[\mathrm{kN}]}\end{array}$ & Versagensart \\
\hline S.X5.S-10.120.10-1 & stat. & - & - & - & 14,0 & Zugversagen (A) \\
S.X5.S-10.120.10-2 & stat. & - & - & - & 14,0 & (nicht eindeutig) \\
S.X5.S-10.120.10-3 & stat. & - & - & - & 14,2 & Zugversagen (A) \\
S.X5.S-10.120.10-4 & zykl. / stat. & \pm 3 & 1,0 & 1.160 .748 & 12,4 & Zugversagen (A) \\
S.X5.S-10.120.10-5 & zykl. & \pm 4 & $0,5 / 0,3$ & 219.552 & - & Schubriss (E) \\
S.X5.S-10.120.10-6 & zykl. & \pm 5 & 0,7 & 85.251 & - & Verbundvers. (F) \\
S.X5.S-10.120.10-7 & zykl. & \pm 6 & 1,0 & 23.711 & - & Schubriss (E, F) \\
S.X5.S-10.120.10-8 & zykl. & \pm 7 & $0,7 / 0,4$ & 6.670 & - & Schubriss (E, F) \\
S.X5.S-10.120.10-9 & zykl. & \pm 8 & 0,5 & 2.260 & - & Schubriss (E, F) \\
S.X5.S-10.120.10-10 & zykl. & \pm 9 & 0,5 & 1.111 & - & Schubriss (E, F) \\
S.X5.S-10.120.10-11 & zykl. & \pm 10 & 0,5 & 606 & - & Schubriss (E) \\
\hline
\end{tabular}

In den weiteren acht Versuchen wurde eine Wechselbeanspruchung mit Lastamplituden von $\pm 3 \mathrm{kN}$ bis $\pm 10 \mathrm{kN}$ aufgebracht. Jeder Lastzyklus umfasste eine positive und eine negative Lastamplitude. Die beiden Lastamplituden wurden vor der eigentlichen zyklischen Belastung zuerst weggesteuert, quasi-statisch angefahren. Dadurch wurde der erreichte Zylinderweg bei der jeweiligen Last bestimmt, der für die Steuerung der zyklischen Belastung angegeben werden musste. Weiterhin wurde durch diese Vorbelastung der Großteil der Rissbildung in den Deckschichten vorweggenommen.

Im Anschluss wurde die zyklische Beanspruchung gestartet und die Lastamplituden mit einem sinusförmigen Verlauf und einer definierten Schwingfrequenz kraftgeregelt auf die Probekörper aufgebracht. Um eine exakte Ansteuerung der Lastamplituden zu gewährleisten, musste die Schwingfrequenz für jeden Versuch entsprechend angepasst werden. Aufgrund der im Vergleich zu gewöhnlichen Stahlbetonprobekörpern geringen aufgebrachten Kräfte und der geringen Bauteilsteifigkeiten der Sandwichstreifen, mussten die Versuche mit relativ geringen Schwingfrequenzen von 0,3 und $1,0 \mathrm{~Hz}$ belastet werden. 
Zu Beginn der zyklischen Belastung wurde die Lastamplitude über die Steuerung des Zylinders automatisch langsam gesteigert, bis die gewünschten Werte erreicht wurden. Die Dauer dieser Einschwingphase variierte zum Teil, war jedoch in allen Versuchen nach höchstens 200 Zyklen beendet.

Die Wechselbeanspruchung wurde bis zum Ermüdungsversagen, d. h. der überproportionalen Zunahme der Verformungen, aufrechterhalten. Mit der Steuerung des Prüfzylinders wurden Verformungsgrenzen definiert, bei deren Überschreiten der Versuch automatisch gestoppt wurde.

Es wurden die aufgebrachte Zylinderkraft, der Zylinderweg und die Verformung in Feldmitte mit Wegaufnehmern gemessen. Zum Teil wurden die Dehnungen in Feldmitte auf den Deckschichtaußenflächen mit Dehnungsmessstreifen gemessen. Die Messung erfolgte periodisch mit einem Messintervall von 5 Minuten. Die Messungen wurden mit einer Messrate von $2 \mathrm{~Hz}$ und einer Messdauer von 3 Sekunden durchgeführt. Durch das Messverfahren konnten die sinusförmigen Lastwechsel mit hoher Genauigkeit abgebildet werden (siehe Abbildung 5.47). Weiterhin wurden die erreichten Lastzyklen aufgezeichnet.

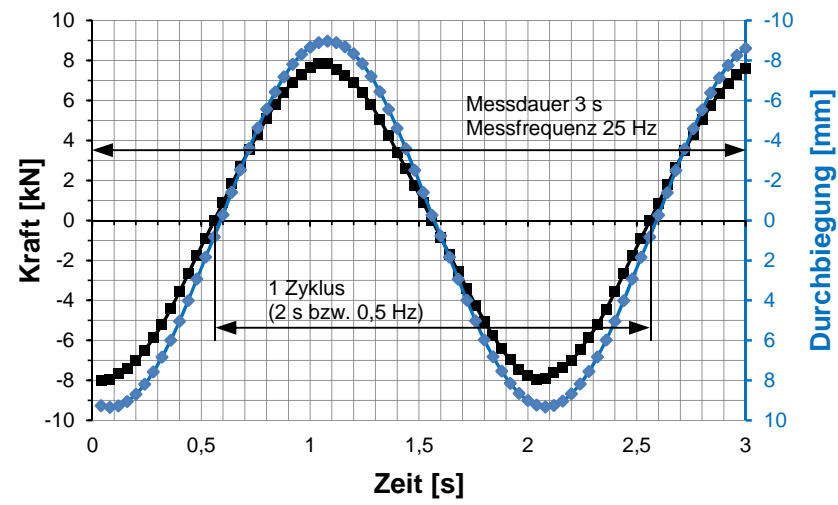

Abb. 5.47: Darstellung der Lastwechsel am Beispiel des Versuchs S.X5.S-10.120.10-9 bei einer Kraftamplitude von $\pm 8 \mathrm{kN}$

\subsubsection{Versuchsergebnisse}

Die Lastwechsel konnten über die Steuerung des Zylinders mit einem relativ exakten sinusförmigen Verlauf aufgebracht werden. Die angegebenen Kraftamplituden wurden erreicht (siehe beispielhaft Abbildung 5.47.

In Abbildung 5.48 sind die Wegamplituden der Versuche über der Anzahl der Zyklen aufgetragen. Während der überwiegenden Anzahl der Lastzyklen bleibt die Wegamplitude eines jeden Versuchs nahezu konstant. Während der letzten etwas $10 \%$ der Zyklen wachsen die Wegamplituden schließlich exponentiell an.

Das Versagen der Probekörper wurde in allen Versuchen durch ein lokales Reißen des XPS in unmittelbarem Grenzbereich zum Verbund eingleitet. Es bildete sich dabei ein horizontaler Riss, der sich bereits frühzeitig durch Relativverschiebungen der 


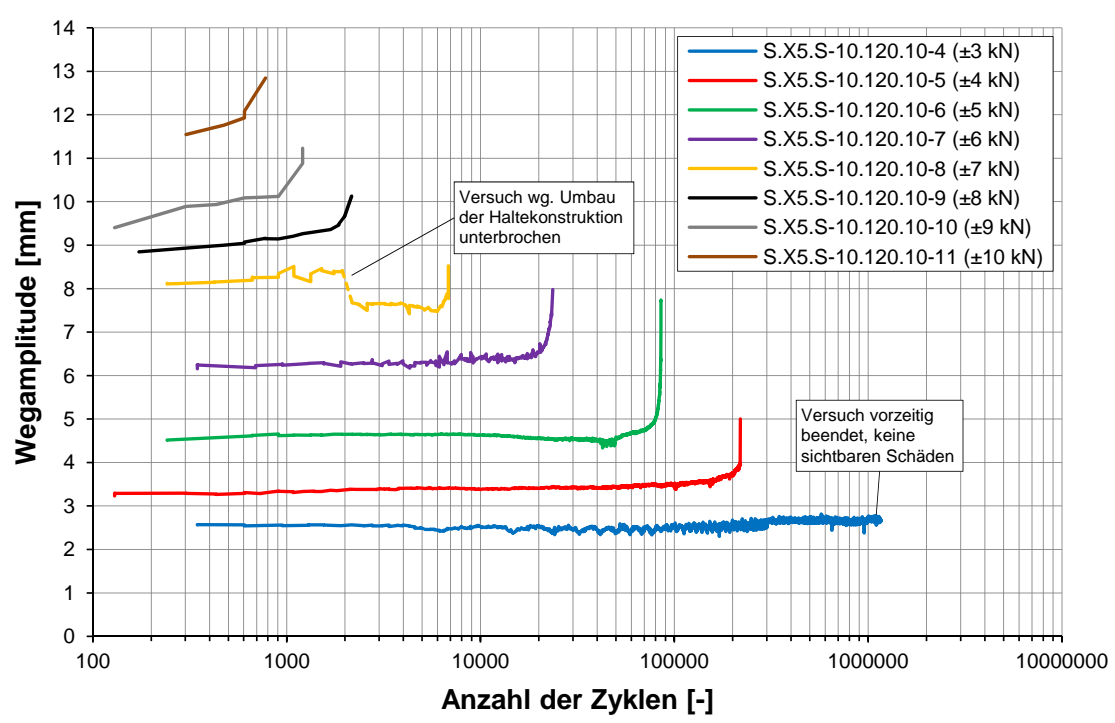

Abb. 5.48: gemessene Wegamplitude in Feldmitte in Abhängigkeit der gefahrenen Lastzyklen

Tab. 5.9: Versagensbilder

\begin{tabular}{|c|c|}
\hline Probekörper & Versagensbild \\
\hline S.X5.S-10.120.10-5 & $\begin{array}{lllll} & & 1 & 1\end{array}$ \\
\hline S.X5.S-10.120.10-6 & i \\
\hline S.X5.S-10.120.10-7 & 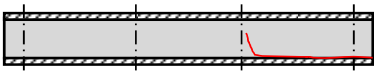 \\
\hline S.X5.S-10.120.10-8 & \begin{tabular}{|l|lll}
$i$ & $i$ & $i$ & $i$ \\
\end{tabular} \\
\hline S.X5.S-10.120.10-9 & i \\
\hline S.X5.S-10.120.10-10 & i) $\quad !$ \\
\hline S.X5.S-10.120.10-11 & 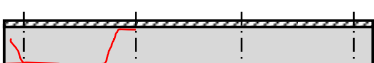 \\
\hline
\end{tabular}


Rissufer während der zyklischen Belastung zeigte (siehe Tabelle 5.9). Im weiteren Verlauf, begannen sich in nahezu allen Versuchen die Risse mit zunehmender Anzahl an Zyklen zur Mitte des XPS-Kerns (bezogen auf die Bauteilhöhe) hin fortzusetzen. Der Ablauf des Versagens glich dabei den Beobachtungen in anderen Untersuchungen an Sandwichquerschnitten mit Schaumstoffkernen (vgl. Kulkarni u. a. 2004).

Schließlich riss in einigen Fällen das XPS über die volle Höhe des Kerns auf. In manchen Fällen vergrößerten sich durch die Rissbildung im XPS-Kern die Verformungen des Probekörpers bereits so stark, dass die festgelegten Durchbiegungsgrenzen erreicht und der Versuch gestoppt wurde. In Versuch S.X5.S-10.120.10-6 setzte sich der horizontale Riss bereits soweit fort, dass ein vorzeitiges Versagen des Probekörpers ohne weitere Schubrisse erreicht wurde.

In Versuch S.X5.S-10.120.10-4 wurde nach rund 1,16 Millionen Zyklen und 14-tägiger Versuchsdauer kein vorzeitiges Versagen erreicht. Der Versuch wurde an dieser Stelle abgebrochen und die Resttragfähigkeit des Probekörpers ermittelt. Dieser versagte, wie die Referenzprobekörper auch, durch das Reißen der Bewehrung in der unteren Deckschicht. Die Bruchlast betrug 12,4 kN und war damit rund $10 \%$ geringer als die der Referenzprobekörper.

Durch die Belastung der Probekörper wird zwischen den Lasteinleitungspunkten und den Auflagerpunkten eine Gesamtquerkraft erzeugt, die der Hälfte der aufgebrachten Last entspricht. Durch Vergleichsberechnungen wurde ermittelt, dass in diesem Bereich, bei den vorhandenen Abmessungen des Probekörperquerschnitts und den vorhandenen Dehn- und Schubsteifigkeiten des Betons bzw. des Kerns, rund 10\% der Gesamtquerkraft über die Deckschichten abgetragen wird. Die verbleibenden $90 \%$ der Querkraft, die über den Sandwichquerschnitt übertragen werden, erzeugen Scherspannungen im Kern bzw. im Verbund. Diese können nach Gleichung 3.25 (siehe Seite 32 aus der verbleibenden Querkraft des Sandwichquerschnitts $V_{s}$ berechnet werden.

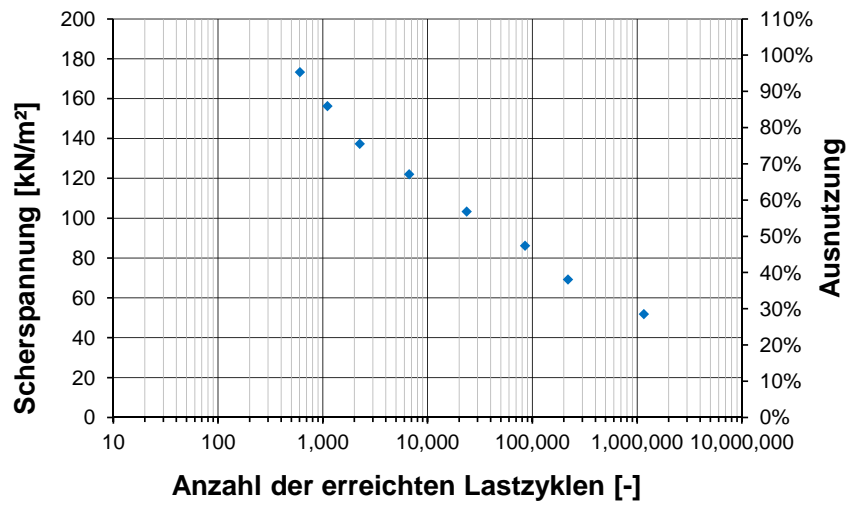

Abb. 5.49: Erreichte Lastzyklen bei den jeweiligen Lastamplituden (Wöhler-Linie)

Die berechneten Scherspannungen wurden dann über der erreichten Anzahl an Zyklen in einem Diagramm in Abbildung 5.49 als Wöhler-Linie dargestellt. Die Ausnutzung des XPS als Verhältnis der berechneten Scherspannungen zur mittleren Scherfestigkeit der 
XPS-Charge $\mathrm{X} 5 f_{X P S, v m}$ von $181,6 \mathrm{~N} / \mathrm{mm}^{2}$ ist im Diagramm auf der rechten Ordinate aufgetragen. Bei einer Ausnutzung des XPS von $95 \%$ können etwa 600 Zyklen übertragen werden, bei rund $55 \%$ sind es entsprechend etwa 24.000 und bei rund $30 \%$ sind es etwa 1,1 Millionen.

Mit dieser Wöhler-Linie kann ein vereinfachender Ermüdungsnachweis der Scherfestigkeit des XPS bzw. des Verbunds zwischen XPS und Beton auf Grundlage der linearen Schadensakkumulation nach der Palmgren-Miner-Regel durchgeführt werden. Dieser vereinfachte Ermüdungsnachweis wird im Abschnitt 8.3.3.3, Ermüdungsnachweis' (siehe S. 199) näher beschrieben.

\subsection{Dauerstandsversuche}

\subsubsection{Allgemeines}

Um das Tragverhalten der Sandwichelemente unter dauerhafter Beanspruchung zu untersuchen, wurden drei Sandwichstreifen einer rund anderthalbjährigen Biegebeanspruchung in einem Dauerstandsversuch ausgesetzt.

\subsubsection{Probekörper und Herstellung}

Die Länge und Breite der Probekörper betrug $2.500 \mathrm{~mm}$ bzw. $200 \mathrm{~mm}$. Wie in den überwiegenden Biegeversuchen auch, betrug die Dicke der Betondeckschichten $10 \mathrm{~mm}$ und die Dicke des Kerns betrug $120 \mathrm{~mm}$. Als Kernmaterial wurde die XPS-Charge X5 verwendet.

Für die Herstellung der Probekörper wurden jeweils zwei XPS-Streifen mithilfe des PUR-Heißklebstoffs zusammengefügt. Als Bewehrung wurden je Deckschicht, 3 Lagen Drahtgittermatten verwendet. Für die Betonage wurde die Herstellungsvariante „Eindrücken des XPS-Kerns in den Beton,, angewendet.

\subsubsection{Versuchsaufbau}

Zur Belastung der Probekörper wurde ein 4-Punkt-Biegeversuchsaufbau gewählt. Über eine Lasteinleitungskonstruktion wurde die Last bestehend aus Stahlgewichten in den Drittelspunkten des jeweiligen Probekörpers eingeleitet. Das Gesamtgewicht des Aufbaus aus den Stahlgewichten und der Lasteinleitungskonstruktion betrug in den drei Versuchen $50 \mathrm{~kg}, 100 \mathrm{~kg}$ bzw. $200 \mathrm{~kg}$. Umgerechnet auf die Oberfläche der Probekörper ergibt sich aus dem Gewicht eine Flächenlast von $1 \mathrm{kN} / \mathrm{m}^{2}, 2 \mathrm{kN} / \mathrm{m}^{2}$ und $4 \mathrm{kN} / \mathrm{m}^{2}$.

Zur Bestimmungen der Durchbiegungen $u$ wurde je ein induktiver Wegaufnehmer und eine analoge Messuhr mittig unter den unteren Deckschichten installiert. Die Messwerte der Wegaufnehmer wurden mit einem PC aufgezeichnet. Die Messuhren dienten dabei lediglich als redundantes Messsystem. 


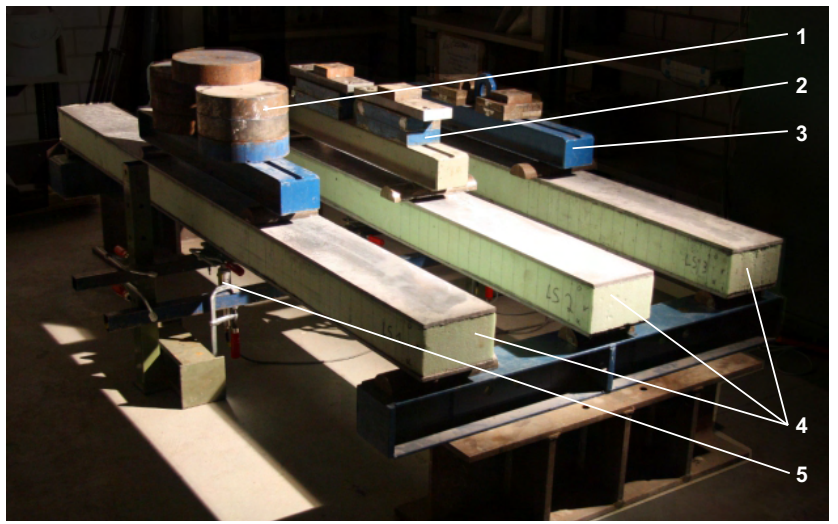

Belastung $200 \mathrm{~kg}$

Belastung $100 \mathrm{~kg}$

Belastung $50 \mathrm{~kg}$

Probekörper

Wegaufnehmer und Messuhren

Abb. 5.50: Versuchsaufbau der Dauerstandsversuche

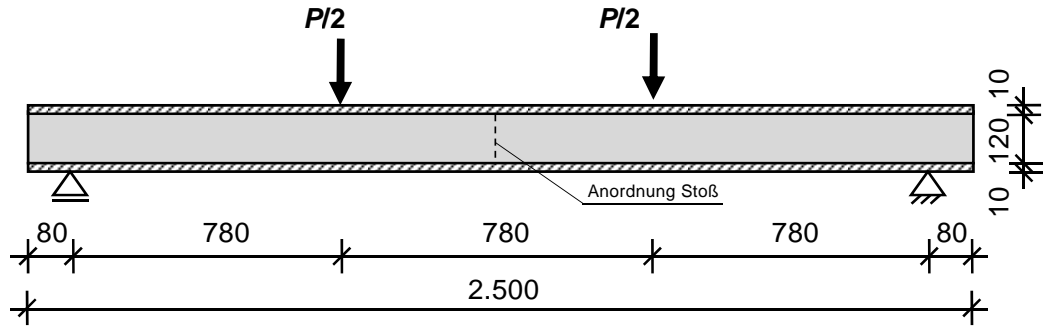

Abb. 5.51: Schematische Darstellung eines Dauerstandsversuchs

\subsubsection{Versuchsdurchführung}

Zu Beginn des Versuche wurden die Wegaufnehmer und Messuhren zu Null gesetzt um im Anschluss daran die Lasteinleitungskonstruktion und die Gewichte auf den Probekörpern aufzubringen. Die Werte wurden zu Beginn der Messdauer alle 2 Stunden und später alle 4 Stunden automatisch mittels des PCs aufgezeichnet. Aufgrund eines mehrmaligen Ausfalls der automatisierten Messung wurden im weiteren Verlauf der Versuche die Messwerte über einen Remote-Zugriff manuell abgelesen. Das Messintervall wurden gegen Ende der Versuche auf eine Woche und schließlich auf einen Monat vergrößert.

\subsubsection{Versuchsergebnisse}

Nach dem Aufbringen der Last betrug die Anfangsverformung $u_{0}$ der Probekörper mit $50 \mathrm{~kg}$-Belastung $1,0 \mathrm{~mm}$, mit $100 \mathrm{~kg}$-Belastung 2,0 mm und mit $200 \mathrm{~kg}$-Belastung 4,1 $\mathrm{mm}$. Nach einer Versuchsdauer von 12.500 Stunden bzw. einem Jahr und rund fünf Monaten betrugen die Verformungen der jeweiligen Probekörper dann 1,8 mm, $3,2 \mathrm{~mm}$ und $6,1 \mathrm{~mm}$.

Zur Extrapolation der Durchbiegungswerte mithilfe des Findley-Ansatzes, wurde wie in Abschnitt 3.2.3.5, "Materialverhalten unter dauerhafter Beanspruchung]' (siehe S. 24 
beschrieben, der Kriechanteil der Durchbiegungswerte $u-u_{0}$ über der Zeit in logarithmierter Darstellung aufgetragen (siehe Abbildung 5.52). Durch lineare Regression können die Konstanten $m$ und $n$ für den jeweiligen Versuch gefunden werden. Die Beschreibung der Kriechverformung mithilfe der Findley-Gleichung stimmt sehr gut mit den experimentell ermittelten Werten überein. Die Bestimmtheitsmaße liegen dabei zwischen 98,5 und 99,5\%. Lediglich zu Beginn und gegen Ende der Versuchsdauer sind größere Abweichungen festzustellen.

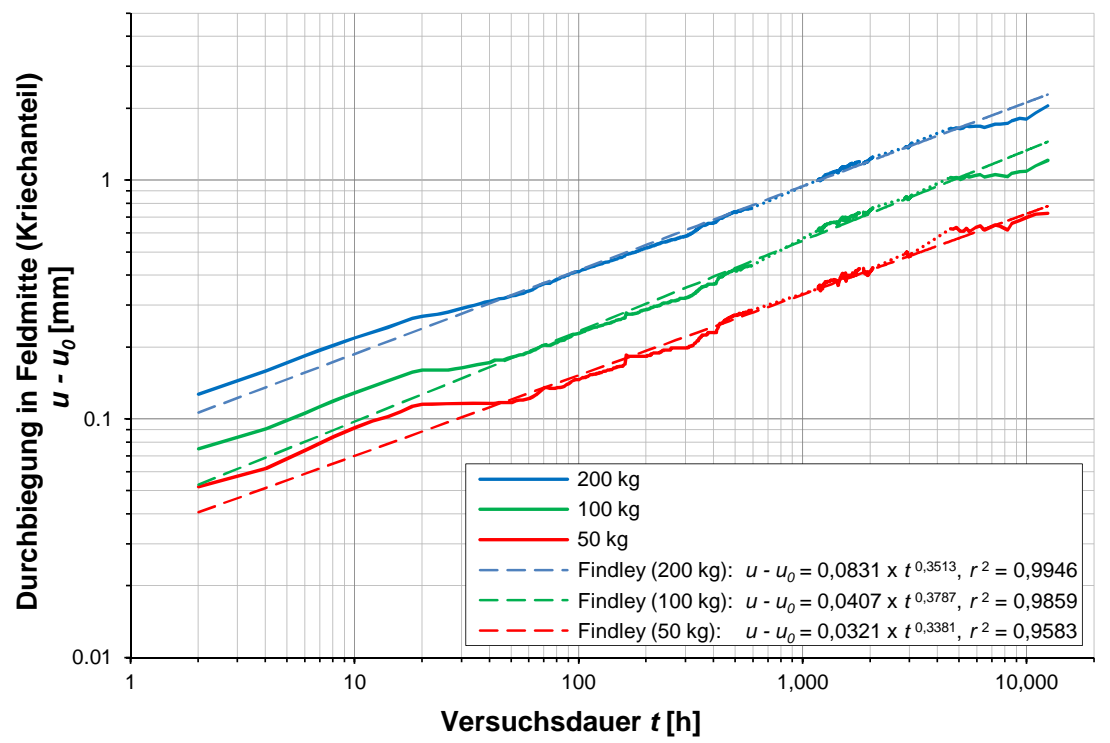

Abb. 5.52: Verformungskurven der Dauerstandsversuche

In Tabelle 5.10 sind die Anfangsdurchbiegungen $u_{0}$, die Konstanten $m$ und $n$ sowie die Bestimmtgeitsmaße $r^{2}$ zusammengestellt. Weiterhin sind in der Tabelle die experimentell ermittelten Durchbiegungswerte bei einer Dauer von $2.000 \mathrm{~h}$ und $12.500 \mathrm{~h}$, sowie die durch Extrapolation mit dem Findley-Ansatz berechneten Werte bei einer Dauer von $100.000 \mathrm{~h}$ und $375.000 \mathrm{~h}$ angegeben.

Tab. 5.10: Berechnungsergebnisse der Dauerstandsversuche

\begin{tabular}{|ccccccccc|}
\hline \multirow{2}{*}{ Last } & $\begin{array}{c}\text { Anfangsd. } \\
\boldsymbol{u}_{\boldsymbol{o}}[\mathrm{mm}]\end{array}$ & $\boldsymbol{m}$ & $\boldsymbol{n}$ & \multirow{2}{*}{$\boldsymbol{r}^{2}$} & \multicolumn{4}{c|}{ Durchbiegung $\boldsymbol{u}_{\mathrm{e}}[\mathrm{mm}]$ bei $\boldsymbol{t}=$} \\
\hline $200 \mathrm{~kg}$ & 4,09 & 0,083 & 0,351 & 0,995 & 5,32 & 6,14 & 8,83 & 11,64 \\
$100 \mathrm{~kg}$ & 2,02 & 0,041 & 0,379 & 0,986 & 2,76 & 3,22 & 5,20 & 7,27 \\
$50 \mathrm{~kg}$ & 1,03 & 0,032 & 0,338 & 0,985 & 1,44 & 1,75 & 2,60 & 3,49 \\
\hline
\end{tabular}

aus den Versuchsergebnissen

${ }^{2}$ mit Findley-Gleichung extrapolierte Werte

Die Werte bei einer Versuchsdauer von $2.000 \mathrm{~h}$ und $100.000 \mathrm{~h}$ werden bei der Bemessung von Sandwichelementen unter dauerhafter Beanspruchung zur Berechnung des Kriechfaktors $\varphi_{t}$ benötigt. Mithilfe von Gleichung 3.50 (siehe S. 40) wurde mittels der Verformung und einem reduzierten Schubmodul jeweils ein Kriechfaktors $\varphi_{t}$ 
zurückgerechnet. Die Ergebnisse sind in Abschnitt 8.3.3.2, Begrenzung der Durchbiegungen' (siehe S. 198) erläutert. Die Durchbiegung bei einer Dauer von 375.000 h ist die Gesamtverformung nach der extrapolierten 30-fachen Prüfdauer und entspricht der Gesamtverformung nach rund 43 Jahren.

\subsection{Großbauteilversuche}

\subsubsection{Allgemeines}

Es wurden insgesamt sechs Probekörper in Biegeversuchen geprüft. Drei der Probekörper wurden als Einfeldträger in einem quasi-statischen Versuch mit einem hydraulischen Prüfzylinder durch vier Einzellasten belastet. Die anderen drei Probekörper wurden als Zweifeldträger in einem eigens konstruierten Unterdruck-Prüfstand durch eine flächige Belastung beansprucht (siehe Tabelle 5.11).

Tab. 5.11: Versuchsprogramm der Großbauteilversuche

\begin{tabular}{|c|c|c|c|}
\hline Probekörper & System & Beanspruchung & $\begin{array}{c}\text { Materialien - } \\
\text { Schichtdicken }\end{array}$ \\
\hline E1 & Einfeldträger & Einzellasten & \\
E2 & & & \\
E3 & & C.X6.C-10.120.10 \\
F1 & Zweifeldträger & Flächenlasten & \\
F2 & & & \\
F3 & & \\
\hline
\end{tabular}

\subsubsection{Probekörper und Herstellung}

Die Probekörper wiesen eine Breite von 0,60 m und eine Länge von 5,00 m auf. Die Dicke der Deckschichten betrug $10 \mathrm{~mm}$ und die Dicke des Kern $120 \mathrm{~mm}$. Als Kernmaterial wurde die XPS-Charge X6 verwendet. Bei allen sechs Probekörpern wurde die Betonrezeptur HPC-4 und Carbonfasergelege als Bewehrung eingesetzt. Die Herstellung der Großbauteil-Probekörper erfolgte analog zur Herstellung der Probekörper der Biegeversuche an Sandwichstreifen. Es wurde die Herstellungsvariante „Eindrücken des XPS-Kerns in den Beton“ angewendet.

Zur Vorbereitung der Betonage wurden jeweils drei XPS-Platten zu Streifen mit einer Länge von 5,0 m und einer Breite von 0,6 m zusammengeklebt. Die Klebung erfolgte auch hier durch Aufsprühen eines PUR-Klebstoffs mittels einer Auftragspistole. Weiterhin wurde das Carbonfasergelege zu entsprechenden Streifen zugeschnitten. Die Enden der Streifen wurden anschließend mit Epoxidharz zwischen zwei Stahlbleche geklebt. Mit zwei an den Blechen angeschweißten Gewindestäben und einer Haltevorrichtung konnte die Bewehrung mit einem Drehmomentenschlüssel kontrolliert angespannt werden. Auf diese Weise konnte die Bewehrung nahezu gerade und mittig in den Deckschichten ausgerichtet werden (siehe Abbildung 5.53). 


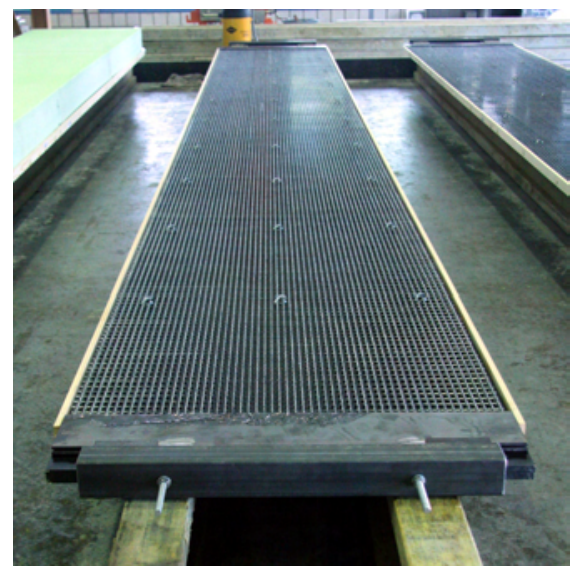

Abb. 5.53: Vorbereitete Schalung mit Carbonbewehrung

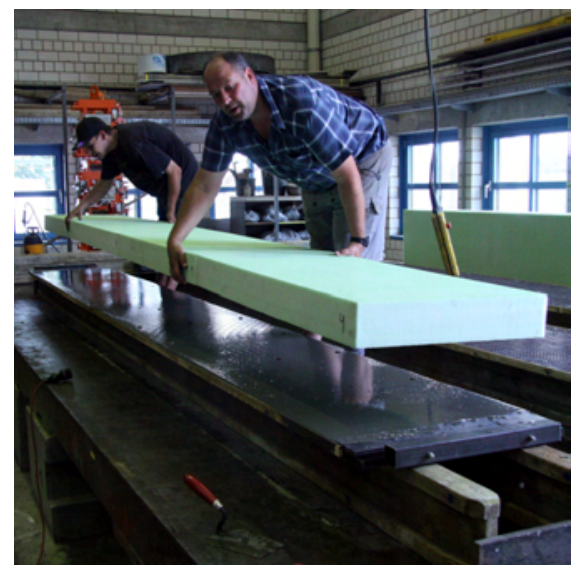

Abb. 5.55: Auflegen des XPS-Streifens auf den Frischbeton

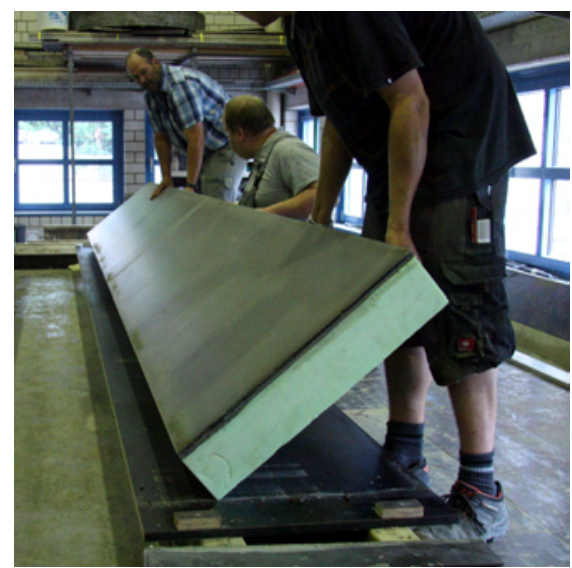

Abb. 5.57: Wenden des Probekörpers nach dem ersten Herstellungstag

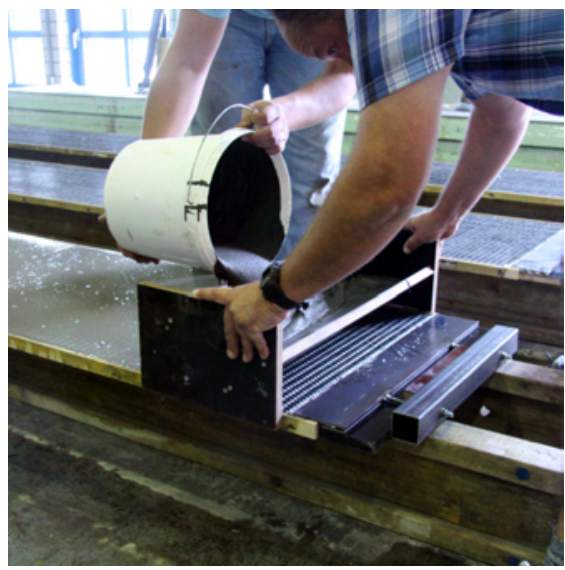

Abb. 5.54: Einbringen des Betons in die Schalung

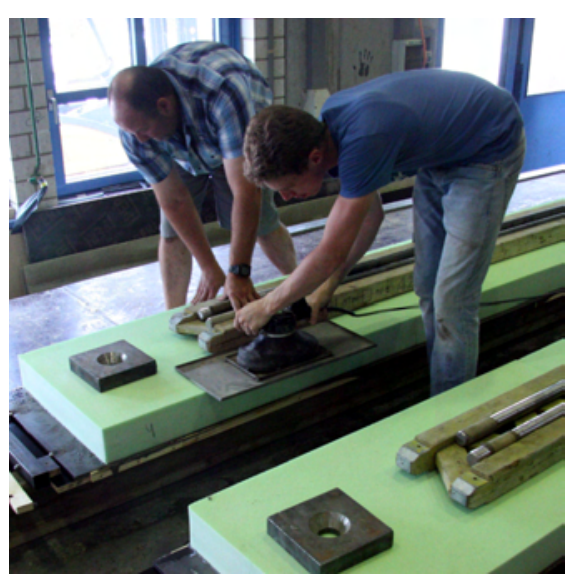

Abb. 5.56: Eindrücken des XPS-Kerns mithilfe des Flächenrüttlers

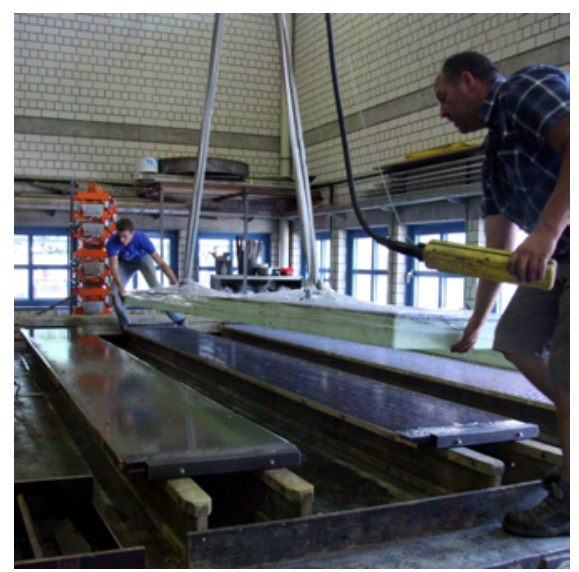

Abb. 5.58: Einheben des Probekörpers am zweiten Herstellungstag 
Am ersten Herstellungstag wurde der Beton mit einem Einfüllschlitten kontinuierlich in die Schalung eingebracht (siehe Abbildung 5.54. Direkt im Anschluss wurden die XPS-Streifen von Hand auf den frischen Beton aufgelegt (siehe Abbildung 5.55, ausgerichtet und dann mit Gewichten beschwert, um ein Aufschwimmen des XPS zu verhindern. Mithilfe eines Klein-Flächenrüttlers wurden die XPS-Streifen zusätzlich in den Beton eingedrückt (siehe Abbildung 5.56). Weitere Nachbehandlungsmaßnahmen wurden nicht durchgeführt.

Am zweiten Herstellungstag wurden die Probekörper ausgeschalt und von Hand, wie in Abbildung 5.57 dargestellt, gewendet. Mithilfe von zwei Vakuumsaugern konnte die Probekörper mit einem Kran einfach transportiert werden. Um die Deckschichten vor Austrocknung zu schützen, wurden diese mit PE-Folie abgedeckt. Im Anschluss wurden die Schalungen für die zweite Betonage vorbereitet. Die Bewehrung wurde wieder wie am Vortag mithilfe angeklebter Stahlbleche und daran angeschweißter Gewindestäbe angespannt. Nachdem der Beton mit dem Einfüllschlitten eingebracht wurde, konnte der XPS-Streifen mit einer Deckschicht mithilfe des Krans und der Vakuumsauger eingehoben werden (siehe Abbildung 5.58). Anschließend wurden die Probekörper wieder mit Gewichten beschwert und mit dem Flächenrüttler in den Frischbeton eingerüttelt. Am Folgetag konnten die Probekörper bereits ausgeschalt werden und die zweite Deckschicht ebenfalls mit PE-Folie abgedeckt werden.

In allen Deckschichten der Probekörper waren weder nach dem Ausschalen noch vor der Versuchsdurchführung Schwindrisse zu beobachten. In einer Deckschicht des Probekörpers E1 konnte eine großflächige Lunkerbildung festgestellt werden, welche durch unzureichende Fließeigenschaften des Frischbetons verursacht wurde.

\subsubsection{Beanspruchung durch Einzellasten}

\subsubsection{Versuchsaufbau}

Die Versuche mit Beanspruchung durch Einzellasten wurden in einem Zwei-SäulenPrüfrahmen durch einen servohydraulischen Prüfzylinder mit einer maximalen Prüflast von $250 \mathrm{kN}$ durchgeführt. Die Last wurde über eine Lasteinleitungskonstruktion an vier Stellen in den Probekörper eingeleitet. Dazu wurde eine Stahltraverse mit einer Länge von $2 \mathrm{~m}$ mit einem Kardangelenk gelenkig an den Prüfzylinder angeschlossen. Auf diese Weise wurde verhindert, dass die Probekörper bereits zu stark durch das Gewicht des Aufbaus belastet wurden. Über die Stahltraverse wurde die Last je über eine Kraftmessdose und eine Kalotte an zwei weitere kurze Stahltraversen weitergeleitet und dann über vier Rollenlager und vier Holzbalken gleichmäßig über die Breite des Probekörpers auf die obere Deckschicht der Probekörper aufgebracht. Die Probekörper wurden über ein Fest- und ein Rollenlager auf zwei Auflagerböcken auf dem Aufspannfeld des Labors gelagert (siehe Abbildung 5.59. 


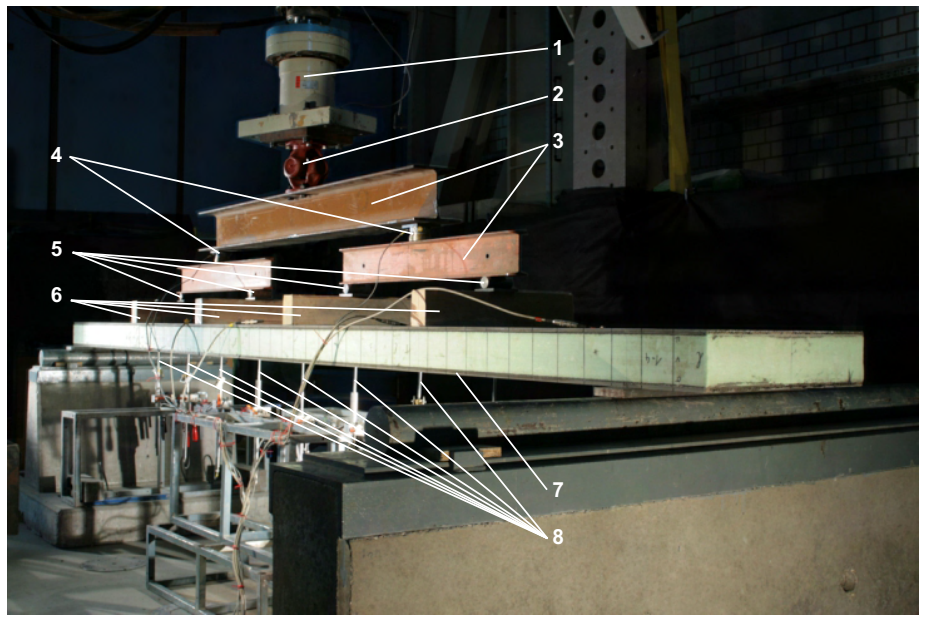

\author{
hydraulischer \\ Zylinder \\ Kardangelenk \\ Traverse \\ Kraftmessdose und \\ Kalotte \\ Rollenlager \\ Lasteinleitungs- \\ balken \\ Probekörper \\ Induktive \\ Wegaufnehmer
}

Abb. 5.59: Aufbau eines Versuchs mit Einzellasten

\title{
5.7.3.2 Versuchsdurchführung
}

Die Steuerung der drei quasi-statischen Biegeversuche erfolgte weggesteuert. Die Belastungsgeschwindigkeit betrug zu Beginn der Versuche $0,5 \mathrm{~mm} / \mathrm{min}$ und wurde im weiteren Verlauf auf $1,0 \mathrm{~mm} / \mathrm{min}$ und schließlich auf $2,0 \mathrm{~mm} / \mathrm{min}$ gesteigert.

Neben der aufgebrachten Kraft des Zylinders, sowie dessen gefahrenen Weg, wurden die Kraft der zwei Kraftmessdosen, die Dehnungen in Längsrichtung an bis zu 5 Stellen auf der Oberseite der oberen Deckschicht mittels Dehnungsmessstreifen und die Durchbiegungen an neun Stellen an der unteren Deckschicht mittels Wegaufnehmern gemessen und mit einer Messfrequenz von einem $1 \mathrm{~Hz}$ durch einen Messrechner aufgezeichnet (siehe Abbildung 5.60). Während des Versuchs E2 wurden weiterhin die Rissbreiten zweier Risse gemessen und notiert.

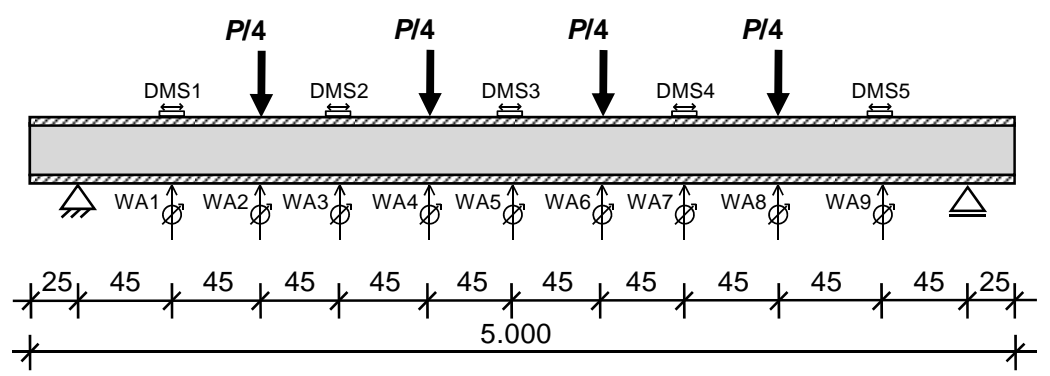

Abb. 5.60: Schematische Darstellung eines Versuchs mit Einzellasten

\subsubsection{Versuchsauswertung}

In Abbildung 5.61 ist die aufgebrachte Last $P$ über der Durchbiegung in Feldmitte der Probekörper aufgetragen. Im Zustand I der Elemente stieg die Lastverformungskurve 
linear an. Ab einer Durchbiegung von ca. $5 \mathrm{~mm}$ bildeten sich verstärkt Trennrisse in der unteren Deckschicht der Probekörper. Diese verursachten kurze Abfälle der aufgebrachten Last und resultieren in einem gezackten, flacheren Ast der Lastverformungskurven. Ab einer Durchbiegung von etwa $100 \mathrm{~mm}$ bildeten sich nur noch vereinzelnd Risse und die Lastverformungskurve stieg hier wieder linear an.

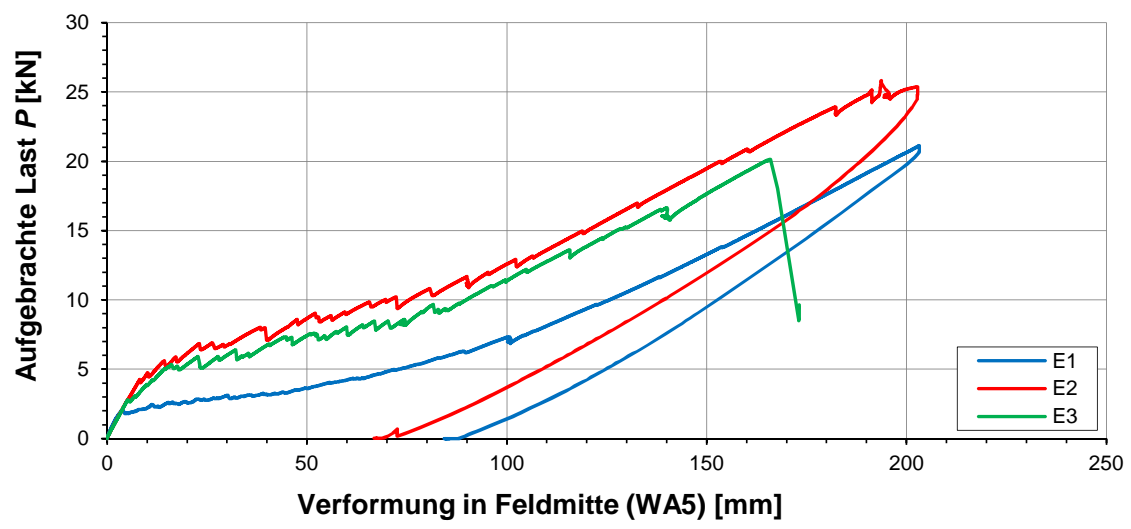

Abb. 5.61: Lastverformungsverhalten der Versuche mit Einzellasten

Die Lastverformungskurve des Versuchs E1 verlief parallel zu den Kurven der Versuche E2 und E3, jedoch auf einem insgesamt niedrigerem Lastniveau. Aufgrund der Lunkerbildung in der unteren Deckschicht war der Betonquerschnitt bereits geschwächt. Aus diesem Grund ging der Probekörper E1 bei einer geringeren Last in den Zustand II über. Aufgrund des schlechteren Verbunds zwischen der Bewehrung und dem Beton ist der Tension-Stiffening-Effekt geringer, was zu einer Zunahme der Dehnungen im Zustand II des Probekörpers führt.

Aufgrund zu großer Verformungen wurden die beiden Versuche E1 und E2 bei einer Durchbiegung von ca. $205 \mathrm{~mm}$ aus Sicherheitsgründen beendet und die Probekörper wieder entlastet. Der Probekörper E3 versagte durch Reißen der Carbonbewehrung der unteren Deckschicht bei einer aufgebrachten Last von 20,1 kN.

In den Abbildungen 5.62 und 5.63 sind beispielhaft die Durchbiegungen und die Dehnungen über die Länge des Probekörpers E1 bei verschiedenen Laststufen dargestellt. Die Diagramme der beiden Versuche E2 und E3 sind im Anhang A.83 bis A.86 (Seite 259 bis 260 angefügt.

\subsubsection{Beanspruchung durch Flächenlast}

\subsubsection{Versuchsaufbau}

Um eine flächige Belastung auf die Probekörper aufzubringen wurde ein UnterdruckPrüfstand konstruiert, dessen Prinzip bereits in zwei weiteren Forschungsanwendungen an der TU Kaiserslautern (Chopradub 2010 und Kohlmeyer 2011) eingesetzt und erprobt wurde. 


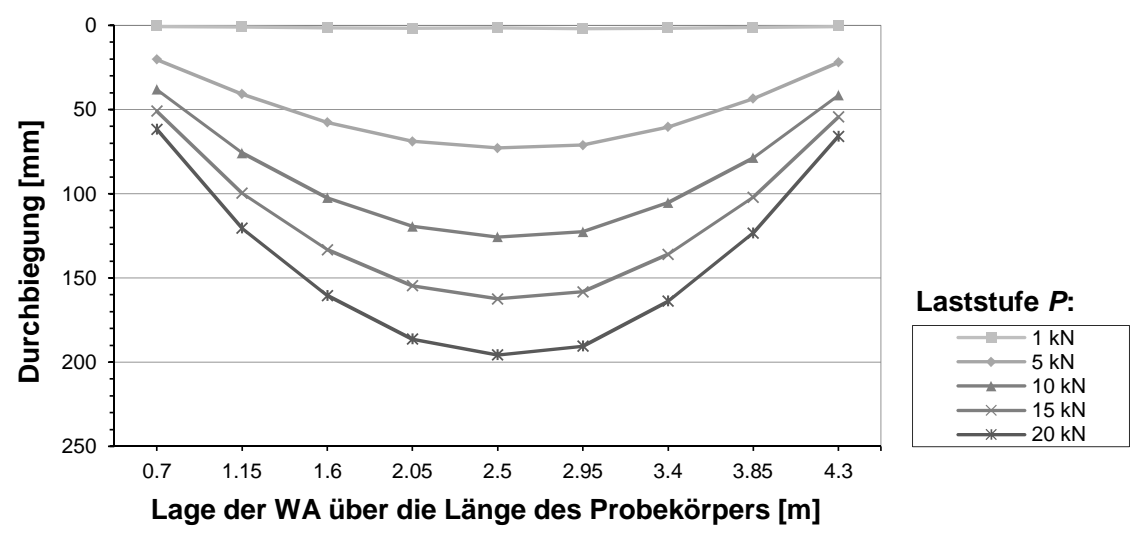

Abb. 5.62: Durchbiegungen der unteren Deckschicht über Länge des Probekörpers E1

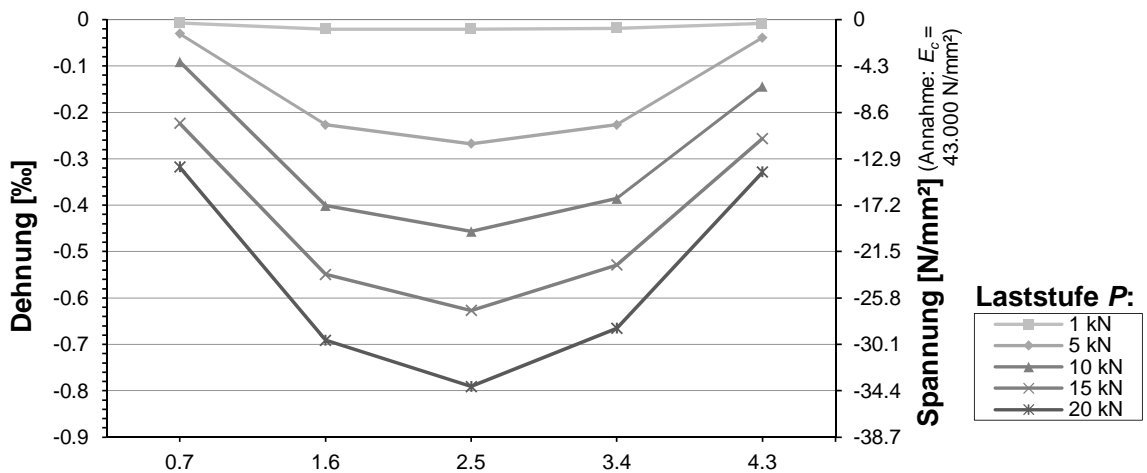

Lage der DMS über die Länge des Probekörpers [m]

Abb. 5.63: Dehnungen und Spannungen auf der Oberseite der oberen Deckschicht über Länge des Probekörpers E1

Das Prinzip des Unterdruck-Prüfstands besteht darin, im Inneren eines luftdicht abgeschlossenen Raums, dessen obere Begrenzungsfläche der zu prüfende Probekörper darstellt, mithilfe einer Vakuumpumpe einen Unterdruck zu erzeugen. Aus der Differenz des Luftdrucks im Inneren des Raums $p_{\text {int }}$ in mbar und des Umgebungsluftdrucks $p_{\text {ext }}$ in mbar kann die auf den Probekörper wirkende Flächenlast $p_{\text {baro }}$ in $\mathrm{kN} / \mathrm{m}^{2}$ nach Gleichung 5.1 berechnet werden:

$$
p_{\text {baro }}=\frac{p_{\text {ext }}-p_{\text {int }}}{10}
$$

Der Innenraum des Unterdruck-Prüfstands entsprach mit einer Grundfläche von 5,0 m auf 0,6 m der Größe eines Probekörpers. Die Höhe betrug 0,3 m. Als Boden des Prüfstands diente der Stahl-Schaltisch des Labors. Die Seitenwände des Prüfstandes bildeten Furnier-Sperrholzplatten, die mit winkelförmigen Stahl-Schalungsträgern und Haftmagneten auf dem Schaltisch befestigt wurden (siehe Abbildung 5.64). Die StöBe zwischen den einzelnen Furnier-Sperrholzplatten, sowie den Sperrholzplatten und dem Schaltisch wurden mit Vakuumdichtband abgedichtet. 
An die Seitenwände des Prüfstands wurden weiterhin vier Anschlussadapter zur Befestigung der erforderlichen $\mathrm{Zu}$ - und Abluftschläufe zum Absaugen und Befüllen mittels der Vakuumpumpe, sowie zum Anschluss zweier elektronischer Barometer montiert. Die Barometer dienten jeweils zur Steuerung des Versuchs und zur Erfassung der Messwerte. Weiterhin wurden zwei Barometer zur Erfassung des Umgebungsluftdrucks verwendet.

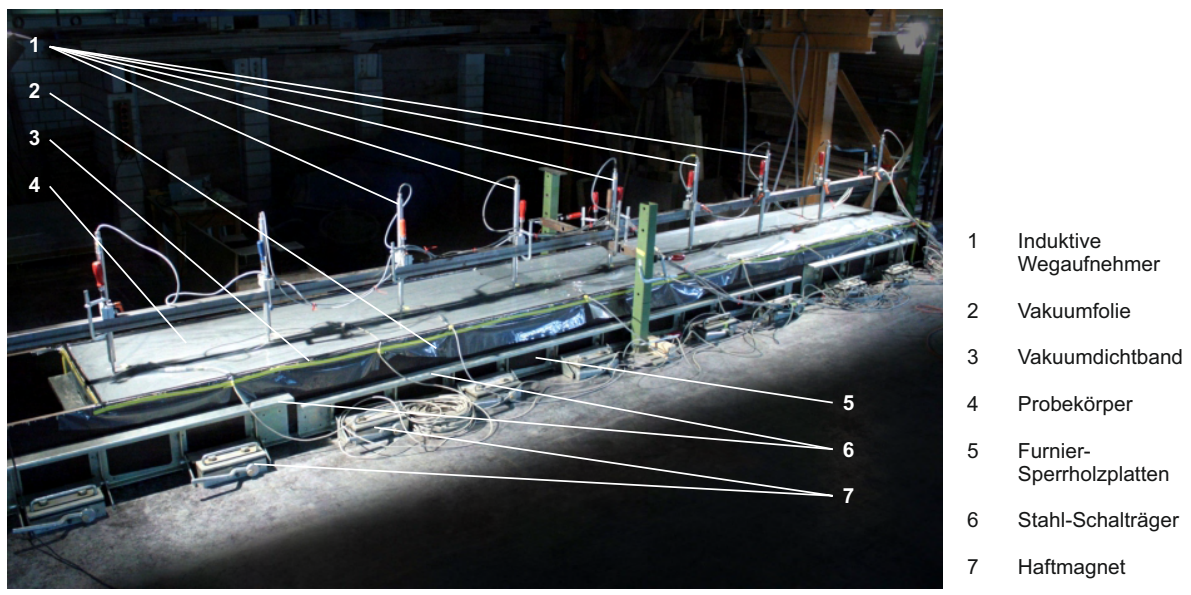

Abb. 5.64: Unterdruckprüfstand vor der Versuchsdurchführung

Im Inneren des Prüfstands dienten drei Stahlbleche mit einer Länge von 0,6 m als Auflagerstreifen. Jedes dieser Stahlbleche war, wie in Abbildung 5.65dargestellt, auf zwei punktförmigen Auflagerkonstruktionen auf dem Schaltisch aufgelagert. Diese Konstruktionen bestanden je nach der gewünschten Lagerungsart aus einem zylinderförmigen Rollenlager oder einem halbzylinderförmigen Festlager, sowie aus Stahlblechen und einer Kalotte. Zur Bestimmung der Auflagerkraft wurde bei einem der beiden Punkte eines Auflagerstreifens eine Kraftmessdose angeordnet.

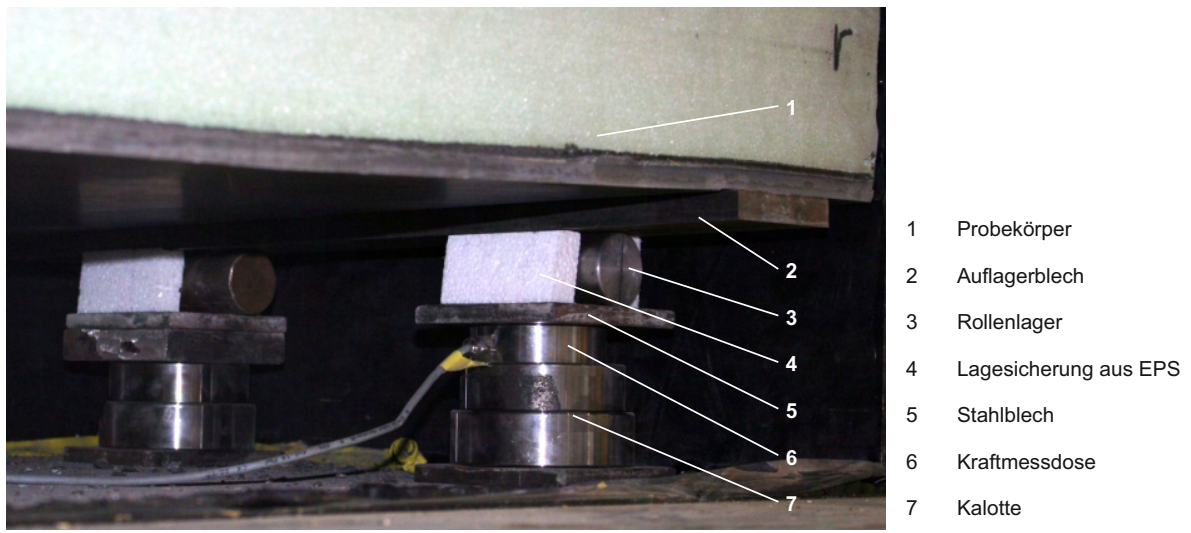

Abb. 5.65: Auflagerkonstruktion im Inneren des Unterdruck-Prüfstands (links ohne und rechts mit Kraftmessdose) 
Die Probekörper wurden in den Prüfstand eingehoben und bildeten mit der oberen Deckschicht einen bündigen Abschluss mit den Seitenwänden des Prüfstandes. Um auch diese Fugen luftdicht abzudichten, wurde eine dehnfähige Vakuumfolie vollflächig über den Probekörper ausgelegt und mit den Stirn- und Seitenflächen der FurnierSperrholzplatten mit Vakuumdichtband luftdicht verklebt.

\subsubsection{Versuchsdurchführung}

Die Steuerung des Versuchs erfolgte über eine speicherprogrammierbare Steuerung (SPS), welche das Absaugen und Befüllen des Prüfstands mittels Ansteuern von Sperrventilen regulierte. Durch die SPS konnten gezielt Unterdruck-Laststufen angefahren und durch die automatische Steuerung der Sperrventile gehalten werden. Eine Regulierung der Belastungsgeschwindigkeit war nur manuell mittels eines Druckregelventils möglich.

Die Belastungsgeschwindigkeit und die erreichbare Maximallast waren stark von der Luftdichtigkeit des Prüfstands abhängig. Bereits kleine nadelstich große Undichtigkeiten zwischen den Seitenwänden und dem Boden des Prüfstands oder zwischen der Folie und den Seitenwänden führten dazu, dass der Unterdruck kaum oder gar nicht erzeugt werden konnte bzw. während des Versuchs stark abfiel.

Zu Beginn des ersten Versuchs F3 mussten diese Undichtigkeiten erst ausfindig gemacht werden, so dass nur sehr niedrige Belastungsgeschwindigkeiten erreicht wurden. In den anschließenden Versuchen F2 und F1 konnten dann sehr hohe Belastungsgeschwindigkeiten von bis zu $0,12 \mathrm{kN} / \mathrm{m}^{2} \mathrm{~s}$ erreicht werden. Aufgrund der hohen Lasten und der großen Verformungen der Probekörper gegen Ende der Versuche, konnte der Prüfstand nur sehr schwer dicht gehalten werden, so dass die Versuche dann beendet werden mussten.

Neben den Luftdruckwerten und den Auflagerkräften, wurden die Durchbiegungen und Dehnungen auf der Oberseite der oberen Deckschicht mit neun induktiven Wegaufnehmern und neun Dehnungsmessstreifen gemessen (siehe Abbildung 5.66). Alle Messwerte wurden mit einem PC und einer Messfrequenz von $2 \mathrm{~Hz}$ aufgezeichnet.

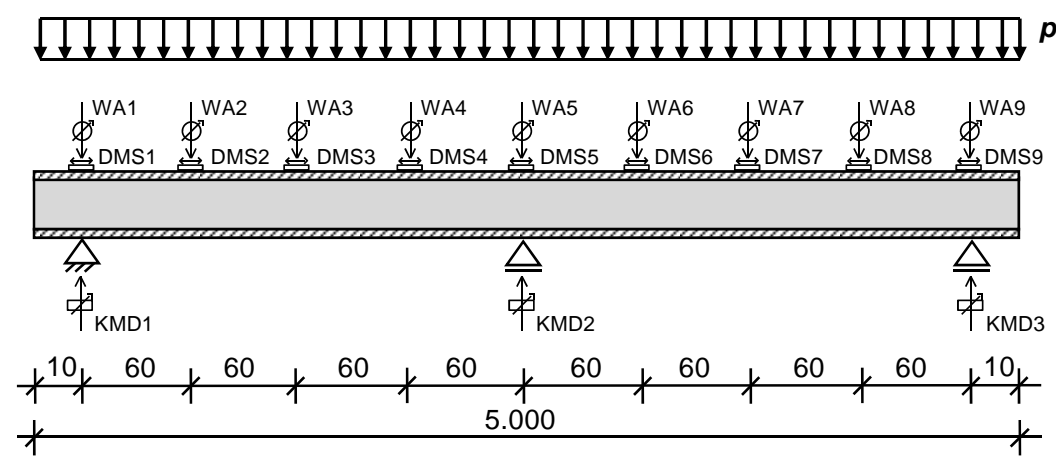

Abb. 5.66: Schematische Darstellung des Unterdruck-Prüfstands 


\subsubsection{Versuchsergebnisse}

Für die Auswertung der Unterdruck-Versuche wurde die aufgebrachte Flächenlast $p_{\text {baro }}$ aus den Messwerten der Barometer nach Gleichung (5.1) berechnet. Weiterhin wurde die aufgebrachte Flächenlast $p_{k m d}$ aus den Messwerten der Kraftmessdosen ermittelt. Die Werte einer Kraftmessdose wurden verdoppelt um die jeweilige Auflagerkraft zu ermitteln. Die Summe der Auflagerkräfte geteilt durch die Gesamtfläche des Probekörpers von 5,0 m auf 0,6 m ergibt die Flächenlast $p_{k m d}$.

Exemplarisch sind die beiden Flächenlasten $p_{\text {baro }}$ und $p_{k m d}$ über der Verformung des Wegaufnehmers WA3 als Lastverformungsdiagramm des Versuchs F2 in Abbildung 5.67 dargestellt. Weiterhin ist auf der rechten Ordinate das Verhältnis von $p_{k m d} \mathrm{zu} p_{\text {baro }}$ aufgetragen.

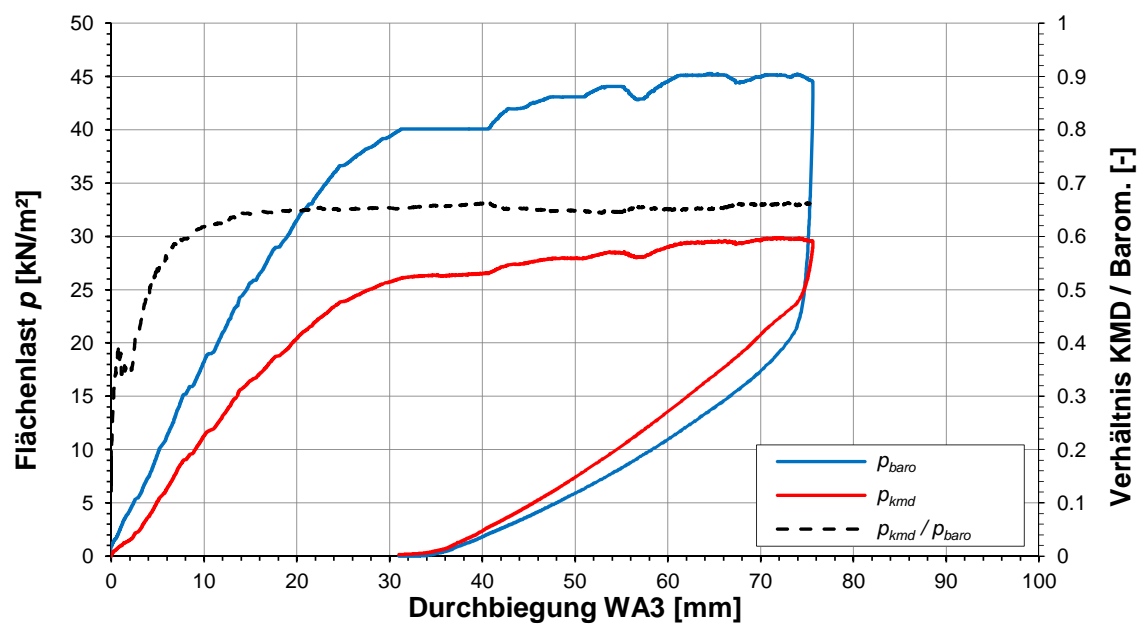

Abb. 5.67: Flächenlast-Durchbiegungs-Diagramm des Versuchs F2

Generell verliefen die beiden Lastverformungskurven $p_{\text {baro }}$ und $p_{k m d}$ bis zu einer Verformung von rund $30 \mathrm{~mm}$ in etwa linear. Dann begannen beide Kurven abzuflachen. Es bildet sich ein leicht ansteigendes Plateau aus. Bei einer Verformung von $78 \mathrm{~mm}$ wurde der Versuch abgebrochen.

Die Werte der beiden Flächenlasten $p_{k m d}$ und $p_{\text {baro }}$ unterschieden sich stark. Generell war die über die Barometer ermittelte Flächenlast $p_{\text {baro }}$ größer, als die Flächenlast $p_{k m d}$, die über die Werte der Kraftmessdosen ermittelt wurde. Das Verhältnis von $p_{k m d}$ zu $p_{\text {baro }}$ stieg bis zu einer Verformung von $10 \mathrm{~mm}$ stark an. Dann stellte sich ein etwa konstantes Verhältnis von 0,65 ein.

Es wird vermutet, dass der Unterschied in den Flächenlasten durch die Reibung zwischen den Seitenwände und dem Prüfkörper verursacht wurde. Die Seitenwänden des Prüfstandes konnten sich aufgrund ihrer geringen Steifigkeit infolge des Unterdrucks verformen und somit gegen die Seitenflächen des Probekörpers abstützen. Durch Schleifspuren auf der Oberflächen der Seitenwände wird dies bestätigt. Aufgrund der entstehenden Reibung, wurde ein Teil der aufgebrachten Flächenlast direkt über die 
Seitenwände des Prüfstands, und somit nicht über die vorgesehenen Auflagerstreifen und Kraftmessdosen, zum Boden des Prüfstands hin abgetragen.

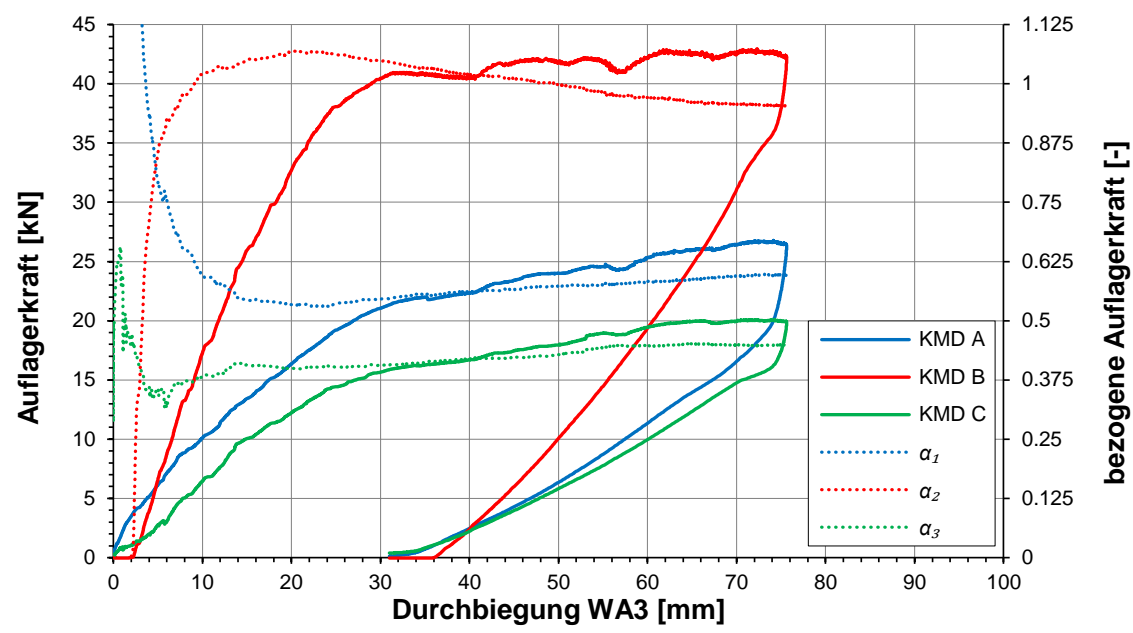

Abb. 5.68: Auflagerkräfte-Durchbiegungs-Diagramm des Versuchs F2

Im Diagramm in Abbildung 5.68 sind auf der linken Ordinate die Auflagerkräfte der beiden Endauflager (KMD1 und KMD3) und des Mittelauflagers (KMD2) über der Verformung des Wegaufnehmers WA3 für den Versuch F2 dargestellt. Auf der rechten Ordinate sind die Faktoren $\alpha_{1}$ bis $\alpha_{3}$ aufgetragen. Die Faktoren $\alpha$ wurden nach Gleichung 5.2 aus der dazugehörigen Auflagerkraft $F_{k m d, i}$, der aufgebrachten Flächenlast $p_{k m d}$, der Stützweite $l$ und der Breite $b$ berechnet. Die Faktoren beschreiben die Lasteinzugsfläche bezogen auf die Stützweite $l$.

$$
\alpha_{i}=\frac{F_{k m d, i}}{p_{k m d} \cdot l \cdot b}
$$

Bei einem Zweifeld-Biegeträger mit konstanter Steifigkeit und gleichen Stützweiten entsprechen die Faktoren $\alpha_{1}$ und $\alpha_{3}=0,375$. Am Mittelauflager beträgt der Faktor $\alpha_{2}=1,25$. Im Versuch hingegen betrug der $\alpha_{2}$-Faktor bei einer Verformung von $22 \mathrm{~mm}$ jedoch maximal 1,07. Bei weiterer Verformung nahm der Wert stetig ab und betrug am Ende des Versuchs 0,95. Die Abnahme des Faktors $\alpha_{2}$ kann auf eine Plastizierung am Mittelauflager zurückgeführt werden. Während des Versuchs nahmen die Rissbreiten der oberen Deckschicht über dem Mittelauflager stark zu. Dort wurde die Dehngrenze der Mikrobewehrung überschritten. Weiterhin wurde der XPS-Kern durch die hohen Auflagerkräfte senkrecht zur Proberkörperebene plastisch gestaucht. Durch diese plastischen Verformungen bildete sich am Mittelauflager ein Fließgelenk aus, so dass sich die Lasteinzugsfläche aus der linken und rechten Hälfte der Spannweite zusammensetzte und sich der Faktor $\alpha_{2}$ dem Wert 1,0 annäherte.

Weiterhin sind die Durchbiegungen, gemessen auf der oberen Deckschicht, über die Länge des Probekörpers F2 bei verschiedenen Laststufen in Abbildung 5.69 dargestellt. Am Mittelauflager entstanden Stauchungen von bis zu $20 \mathrm{~mm}$. Die maximalen Verformungen in den beiden Feldern betrugen bis zu $80 \mathrm{~mm}$. Die maximalen Durchbiegungswerte lagen in etwa mittig in den beiden Feldern. Dies bestätigt, dass sich 
am Mittelauflager ein Fließgelenk ausbildete und der Probekörper wie zwei gelenkig miteinander verbundene Einfeldträger wirkte.

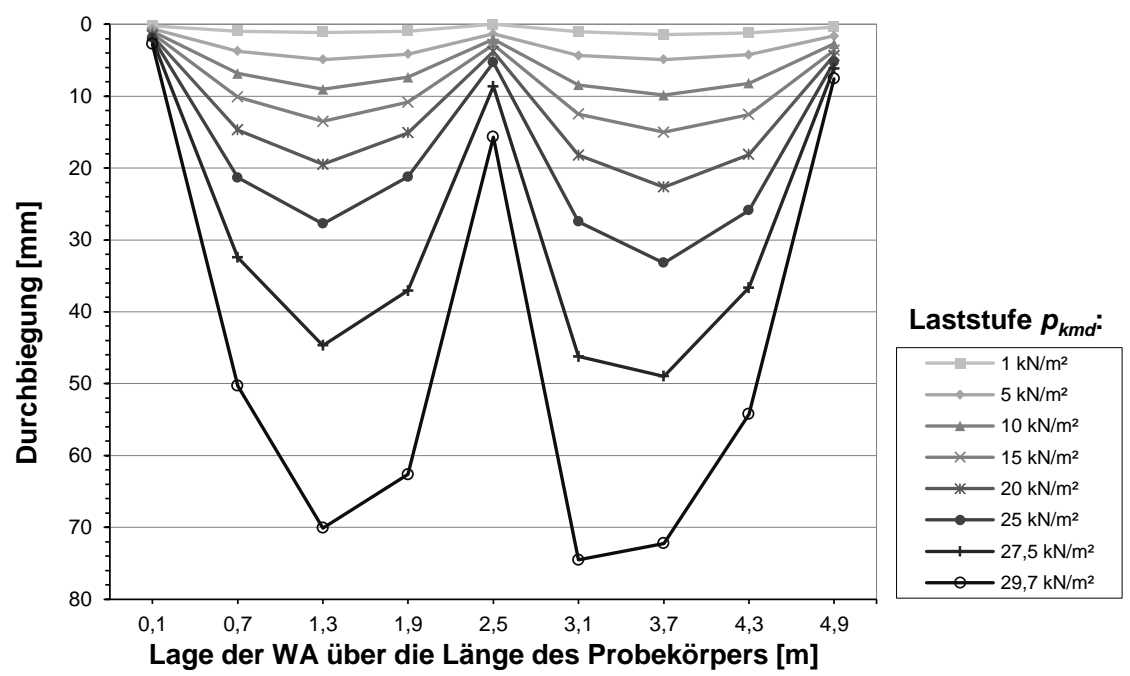

Abb. 5.69: Durchbiegungen der oberen Deckschicht über die Länge des Probekörpers F2

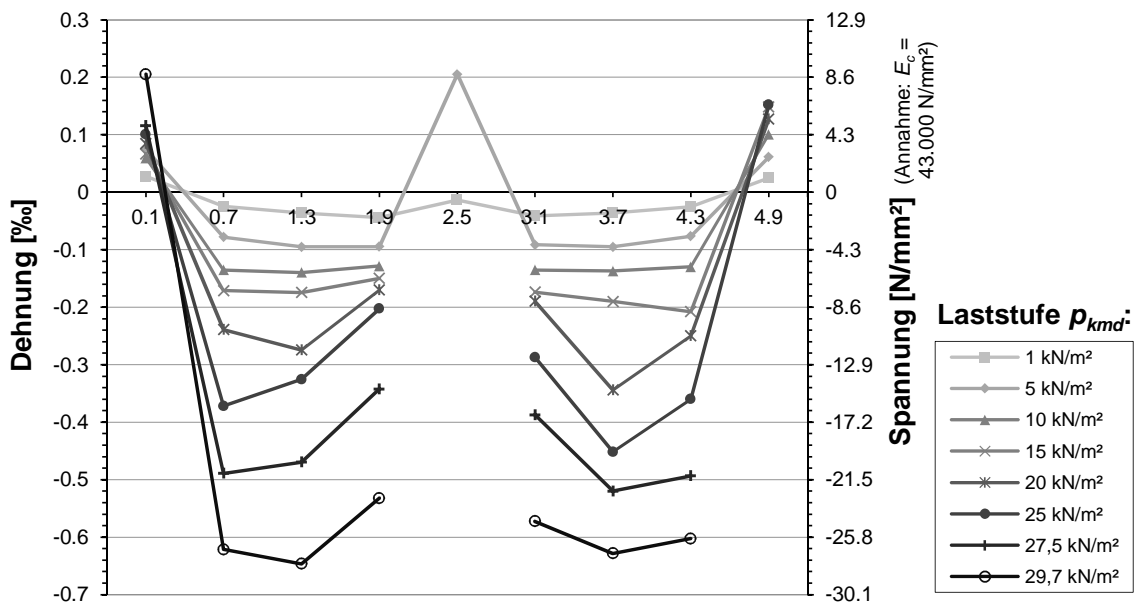

Lage der DMS über die Länge des Probekörpers [m]

Abb. 5.70: Dehnungen und Spannungen auf der Oberseite der oberen Deckschicht über die Länge des Probekörpers F2

In Abbildung 5.70 sind die auf der oberen Deckschicht gemessenen Dehnungswerte über die Länge des Probekörpers aufgetragen. Über dem Mittel- sowie über den beiden Endauflagern bildeten sich Zugdehnungen von bis zu 0,2\%. Bei einem Elastizitätsmodul von $43.000 \mathrm{~N} / \mathrm{mm}^{2}$ entspricht dies einer Zugspannung von 8,6 N/mm $\mathrm{mm}^{2}$. Bei zunehmender Belastung waren die DMS-Streifen am Mittelauflager durch Rissbildung 
ausgefallen. In den Feldern des Probekörpers betrugen die Druckdehnungen maximal $0,65 \%$. Dies entspricht einer Druckspannung von $28,0 \mathrm{~N} / \mathrm{mm}^{2}$.

Die Diagramme der Versuche F1 und F3 können Anhang A.87 bis A.94 (Seite 261 bis 264) entnommen werden.

\subsection{Probewände zur Ermittlung der Praxistauglichkeit und Dauerhaftigkeit}

\subsubsection{Allgemeines}

Durch die Konstruktion von zwei Probewänden sollten Fragestellungen hinsichtlich der Herstellbarkeit von großformatigen Sandwichelementen mit dünnen Betondeckschichten untersucht werden. Beide Elemente wurden mithilfe des Verfahrens „Eindrücken in den Beton" hergestellt. Das Wenden der Elemente erfolgte von Hand unter Zuhilfenahme eines Vakuumsaugers. Es wurde überprüft, ob durch das Wenden der Elemente Risse in den noch jungen Deckschichten entstehen und ob plastische Verformungen im XPS auftreten können.

Weiterhin wurde durch die Herstellung der Elemente überprüft, ob durch das Schwinden des Betons Schwindrisse entstehen. Ferner wurden festgestellt, in wieweit sich Witterungseinflüsse durch Sonneneinstrahlung, Wind und Beregnung auf die Sandwichelemente auswirken und ob Alterungserscheinungen auftreten.

\subsubsection{Herstellung}

Die Breite und Länge der Elemente betrug 1,80 m und 4,75 m. Die Abmessungen wurden so festgelegt, dass diese noch mit den im Labor der Universität zur Verfügung stehenden technischen Mittel hergestellt und mithilfe eines gewöhnlichen LKWs transportiert werden konnten. Die beiden Deckschichten einer Wand wurden mit Carbonfasergelege (Wand C), die der anderen mit Mikrobewehrung bewehrt (Wand S). Die Dicke der Deckschichten betrug $10 \mathrm{~mm}$ und die des Kerns $120 \mathrm{~mm}$.

Die Herstellung der Probewände erfolgte analog zu den bereits zuvor hergestellten Großbauteilprobekörpern. In einem ersten Schritt wurden die XPS-Platten mit PURKlebstoff zu einer großformatigen Platte zusammengefügt (siehe Abbildung 5.71. Dabei wurden die Querstöße der Platten versetzt angeordnet. Ein späteres Zuschneiden der Platte auf die genauen Abmessungen der Wandelemente konnte problemlos mit einer Handkreissäge durchgeführt werden.

Zur Vorbereitung des ersten Tags der Betonage wurde eine Lage des Carbonfasergewebes, wie bereits zuvor erläutert, zwischen zwei Stahlblechen mit Epoxidharz eingeklebt. Bei der Probewand mit Mikrobewehrung wurden drei Lagen eingeklebt. Da die Breite der Probewände die Breite der verfügbaren Drahtmatten und des Carbongeleges überschritt, mussten die Bewehrungslagen in Querrichtung gestoßen werden. Die Carbonfaserlagen wurden mit einer Länge von $30 \mathrm{~cm}$ übergriffen. Die drei Lagen der Mikrobewehrung wurden stumpf gestoßen. Dabei wurden die drei Stöße versetzt ange- 


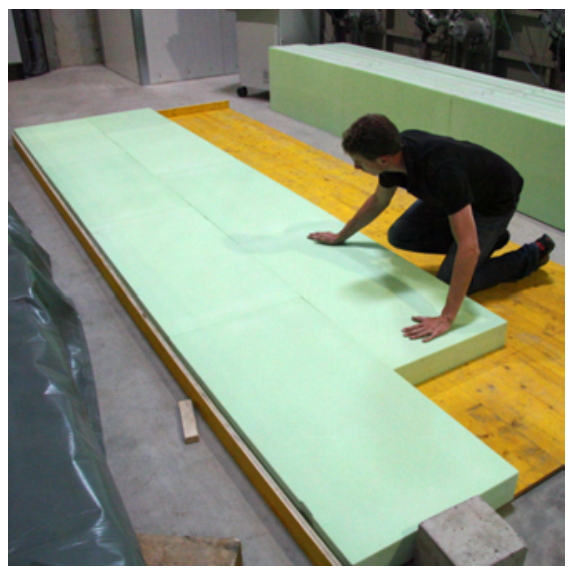

Abb. 5.71: Zusammenkleben und Ausrichten der XPS-Platten

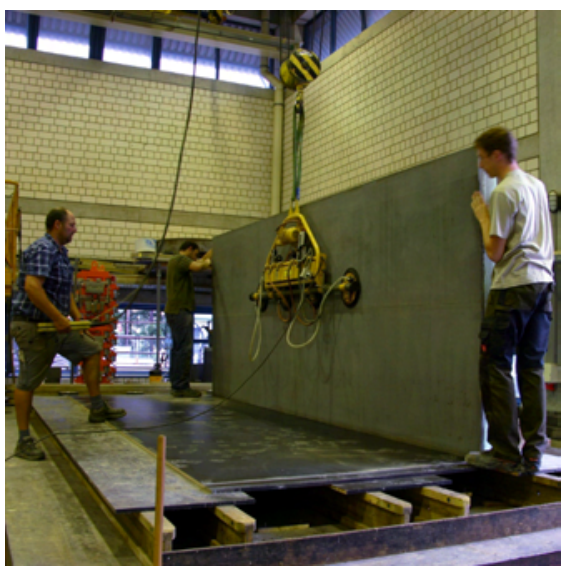

Abb. 5.73: Anheben des Wandelements mithilfe des Vakum-Glassaugers

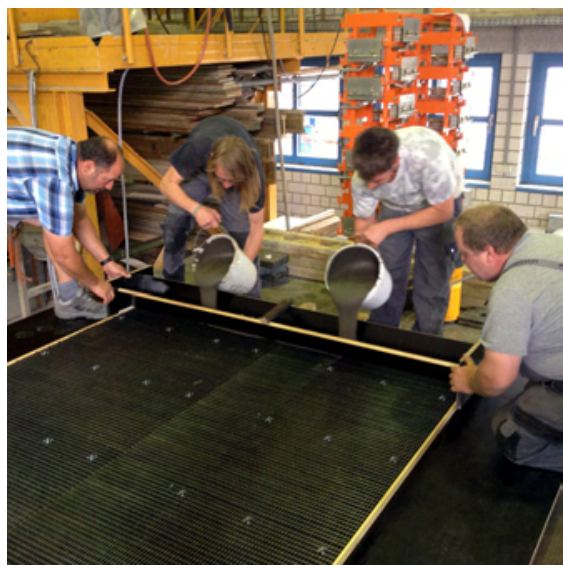

Abb. 5.72: Einbringen des Betons mithilfe des Einfüllschlittens

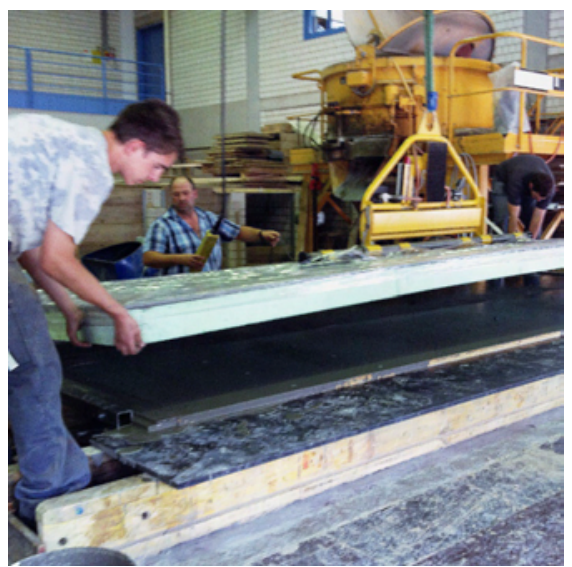

Abb. 5.74: Einheben des XPS-Kerns in den Beton am 2. Herstellungstag

ordnet. Durch Anspannen der Bewehrung mittels Gewindestäbe konnte diese nahezu gerade, mittig in der Schalung ausgerichtet werden.

Die Schalung wurde vor der Betonage gründlich gereinigt und mit Schalöl behandelt um optimale Oberflächenqualitäten zu erzielen. Die Ausgangstoffe wurden vor der Betonage auf ca. $7-8^{\circ} \mathrm{C}$ herabgekühlt. Zum Herstellen des Betons wurde eine optimierte Mischreihenfolge angewendet, mit der eine Lunkerbildung auf den Deckschichtoberflächen vollständig vermieden werden konnten.

Das Einbringen des Hochleistungsbetons in die Schalung erfolgte wiederum mittels eines Einfüllschlittens, der kontinuierlich über die Randschalung gezogen und dabei befüllt wurde (siehe Abbildung 5.72). Längere Einfüllpausen von über fünf Minuten sollten unbedingt vermieden werden, da sich hierdurch eine Betonierfuge auf der Sichtbetonoberfläche abzeichnet. Nach dem Betonieren wurde die XPS-Platte auf den frischen Beton aufgelegt und anschließend mit Gewichten gleichmäßig beschwert. Mithilfe ei- 
nes Flächenrüttlers wurde die Platte zusätzlich in den Frischbeton eingedrückt. Eine weitere Nachbehandlung wurde nicht durchgeführt.

Bereits am nächsten Morgen konnte die XPS-Platte mit einer Deckschicht mit mehreren Personen aufgestellt und mittels eines auf der Sichtbetonfläche angebrachten Vakuum-Glassaugers angehoben werden (siehe Abbildung 5.73). Die Deckschicht wurde direkt im Anschluss mit einer PE-Folie abgedeckt um vorzeitiges Austrocknen des Betons zu verhindern. Die mit Carbonfasergelege bewehrte Deckschicht wies zu diesem Zeitpunkt keine erkennbaren Schwindrisse und -verformungen auf. Bei den mikrobewehrten Deckschichten konnten auf der Längsseite mehrere etwa $50 \mathrm{~cm}$ lange feine Haarrisse festgestellt werden. Verformungen konnten nicht festgestellt werden.

Die Betonage der zweiten Deckschicht erfolgte analog zur Herstellung der ersten Deckschicht. Hier konnte die Platten nun mithilfe des Glassaugers eingehoben werden. Die Platte wurde ebenfalls mit Gewichten beschwert und mithilfe des Rüttlers eingedrückt. Am Folgetag wurden die fertigen Probewände mittels des Glassaugers angehoben und auf ein Transportgestell für Glasscheiben abgestellt und dann auf dem Hof des Labors gelagert.

\subsubsection{Langzeitbeobachtung der Rissbildung}

Alle Deckschichten waren mit PE-Folien abgedeckt. Jedoch lösten sich diese zum Teil, so dass die Deckschichten ungleichmäßig abgedeckt waren. Durch die ungleichmäBige Nachbehandlung konnten bereits nach wenigen Tagen eine Fleckenbildung festgestellt werden (siehe Abbildung 5.75 und 5.76). Diese Flecken zeichneten sich nach einigen Wochen immer deutlicher ab. Ebenfalls zeichneten sich die Abdrücke des Vakuumsaugers auf der Oberfläche ab. Es ist zu empfehlen, den Vakuumsauger nur kurz zum Transport einzusetzen und anschließend direkt wieder zu entfernen.

Abgesehen von den bereits beschriebenen feinen Haarrissen in den Deckschichten der mikrobewehrten Probewand, konnten keine Risse nach dem Ausschalen festgestellt werden. Erst nach wenigen Tagen konnten erste Risse auf den Oberflächen der Deckschichten beobachtet werden. In den carbonfaserbewehrten Deckschichten waren zu Beginn nur drei Risse festzustellen (siehe Abbildung 5.75). Erst nach drei Wochen zeichnete sich ein feineres netzartiges Rissbild ab (siehe Abbildung 5.79). Im Vergleich dazu bildete sich im Fall der mikrobewehrten Probewand bereits nach wenigen Tagen ein netzartiges Rissbild (siehe Abbildung 5.76) aus. Dabei waren die Risse meist horizontal und vertikal ausgerichtet, während bei den carbonfaserbewehrten Deckschichten die Risse zum Teil auch diagonal verliefen.

Im Vergleich zu den Probewänden, waren im Fall der Großbauteilprobekörper keine Risse festzustellen. Diese wurden auf die gleiche Art und Weise hergestellt, jedoch wurden diese in der Laborhalle gelagert. Es wird daher angenommen, dass die Rissbildung der Probewände vor allem auf die Lagerung im Freien zurückzuführen ist. Das Trocknungsschwinden der Deckschichten wird im Freien begünstigt und verstärkt.

Weiterhin ist zu vermuten, dass die Rissbildung stark durch Temperatureinwirkungen beeinflusst wurde. Die Herstellung erfolgte im Sommer. Durch die Sonneneinstrahlung erhitzten sich die der Sonne zugewandten Deckschichten auf bis zu $65^{\circ} \mathrm{C}$. Durch die 


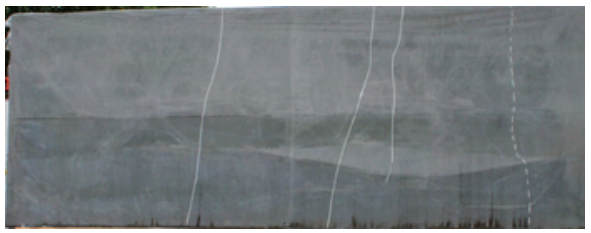

Abb. 5.75: Wand C nach 6 Tagen

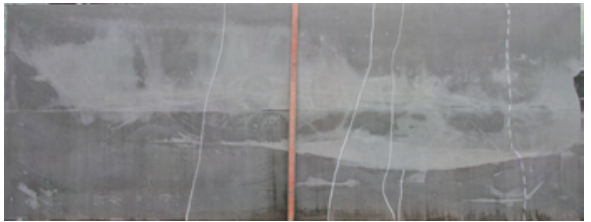

Abb. 5.77: Wand $C$ nach 13 Tagen

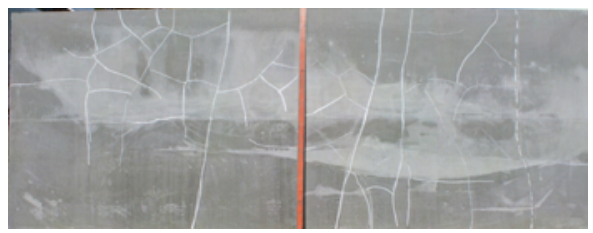

Abb. 5.79: Wand C nach 22 Tagen

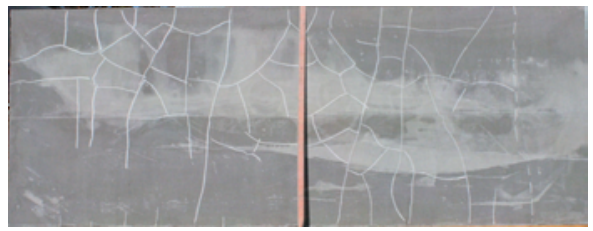

Abb. 5.81: Wand C nach 49 Tagen

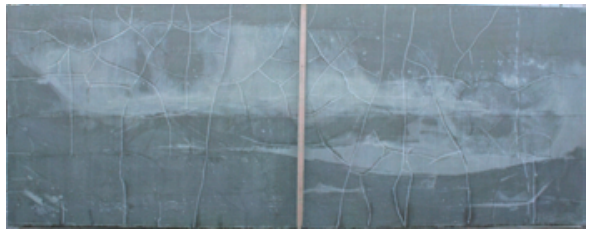

Abb. 5.83: Wand C nach 593 Tagen

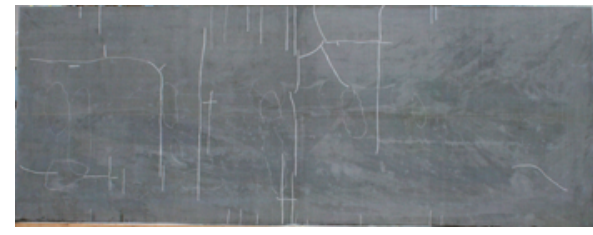

Abb. 5.76: Wand S nach 2 Tagen

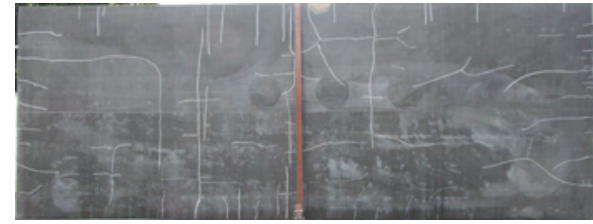

Abb. 5.78: Wand S nach 9 Tagen

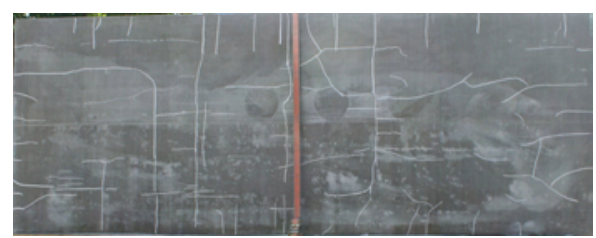

Abb. 5.80: Wand S nach 18 Tagen

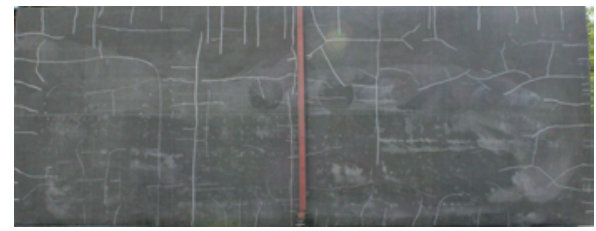

Abb. 5.82: Wand S nach 45 Tagen

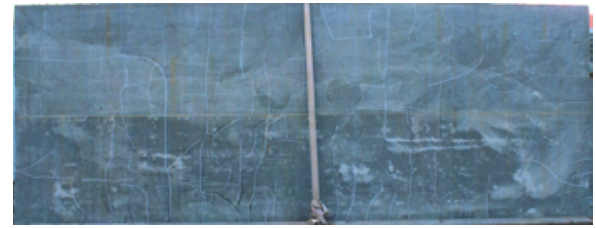

Abb. 5.84: Wand S nach 593 Tagen 
Temperaturdifferenz zwischen den Deckschichten resultierten Zwangzugspannungen, die zur Rissbildung beitrugen. Weiterhin wird vermutet, dass eine schlagartige Abkühlung der Deckschichten durch Beregnung zu weiteren Rissen geführt hat.

Die Rissbreiten wurden jeweils an drei Stellen einer Wand gemessen. In den beiden Abbildungen 5.85 und 5.86 sind die Messwerte über der Zeit nach der Herstellung aufgetragen. Generell sind die Rissbreiten der carbonfaserbewehrten Wand etwa um den Faktor 3 bis 4 größer, als die der mikrobewehrten Wand. Dies liegt zum einen an der etwas größeren Dehnsteifigkeit der Mikrobewehrung. Zum anderen wird der Querschnitt der Betonmatrix durch die einlagige Carbonfaserbewehrung weniger stark reduziert, als im Fall der dreilagigen Mikrobewehrung. Dies führt zu einer höheren Risslast und somit zu größeren Rissabständen und Rissbreiten.

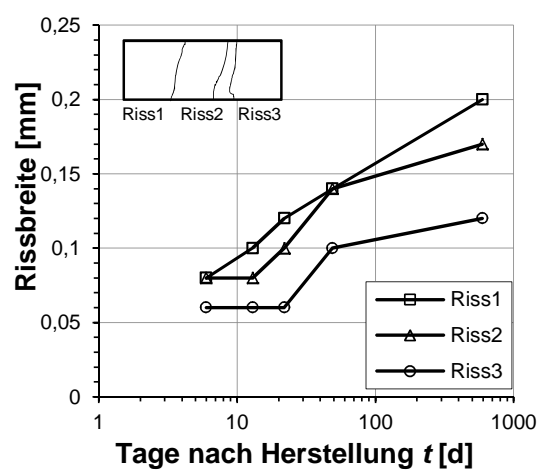

Abb. 5.85: Rissbreite über der Zeit nach Herstellung der Wand $\mathrm{C}$

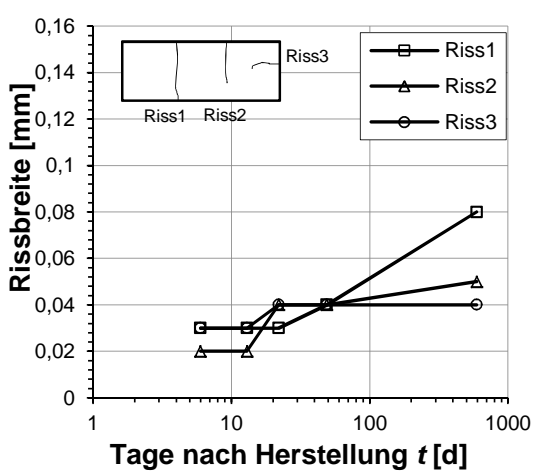

Abb. 5.86: Rissbreite über der Zeit nach Herstellung der Wand $S$ 


\section{Rechnerische Untersuchungen}

\subsection{Allgemeines}

Neben den experimentellen Untersuchungen wurde das Tragverhalten auch rechnerisch untersucht. Die Finite-Elemente Modellierung der Sandwichelemente erfolgte vor allem zur Interpretation der in den Versuchen beobachteten Versagens- und Verformungsmechanismen. Durch die Modellierung konnten Spannungs- und Dehnungswerte ermittelt werden, die in den Versuchen messtechnisch nicht erfasst werden konnten. Mit dem kalibrierten Modell konnten die Auswirkungen aus Schwind- und Temperatureinwirkungen detailliert untersucht werden. Weiterhin wurden Parameterstudien durchgeführt, um den Einfluss von verschiedenen Parameter auf das Lastverformungsund Tragverhalten der Sandwichelemente zu bestimmen.

Zur Modellierung wurde die Software ANSYS ${ }^{\circledR}$ Classic in den Versionen 12.1 und 14.0 der Firma ANSYS Inc. verwendet. Die Eingabe erfolgte mit der Skriptsprache APDL (Ansys Parametric Design Language). Zur Reduzierung der Rechenzeit wurden alle hier untersuchten Sandwichelemente lediglich zweidimensional modelliert. Die Effekte des räumlichen Materialverhaltens auf das Tragverhalten der Sandwichelemente schien, im Vergleich zu anderen Parametern, vernachlässigbar.

\subsection{Modellierung der Deckschichten}

\subsubsection{Allgemeines}

Zur Modellierung der Deckschichten wurde die Bewehrung „verschmiert“ über den Betonquerschnitt angenommen. Der bewehrte Beton wurde mit dem Vier-Knoten-Element PLANE182 mit ebenem Verzerrungszustand (plane strain) abgebildet.

Als Materialgesetz kam das isotrope Werkstoffmodell CAST (d.h. cast iron plasticity model) zum Einsatz. Das Modell wurde für die Modellierung von Grauguss entwickelt. Grauguss weist aufgrund des spröden Werkstoffverhaltens unter Zugbeanspruchung eine gewisse Analogie zum Tragverhalten des Betons auf.

Im Fall des Werkstoffmodels CAST wird im linear-elastischen Bereich das Materialverhalten unter Druck- und Zugbeanspruchung identisch angenommen, d. h. es kann für beide Beanspruchungsrichtungen nur ein Elastizitätsmodul angegeben werden. Für die daran anschließenden plastischen Bereiche unter Zug- und Druckbeanspruchung können über die Angabe von Spannungs- und Dehnungswerten, jeweils unabhängig voneinander zwei multilineare Kurven definiert werden. 
Für die Modellierung des bewehrten Betons der Deckschichten wurde das Materialverhalten unter Druckbeanspruchung als linear-elastisch angenommen, was für einen hochfesten Beton bis zu einem Spannungsniveau von $80 \%$ der Festigkeit zutreffend ist (Schmidt 2008). Für das Materialverhalten unter Zugbeanspruchung wurde eine idealisierte Spannungsdehnungsbeziehung angegeben. Die einzelnen Zustände wurde durch die Angabe von Punkten auf der Spannungsdehnungslinie definiert:

- Zustand I (Punkt A-B): ungerissener Zustand

- Zustand II a (Punkt B-C): Bereich der Rissbildung im Beton

- Zustand II b (Punkt C-D): abgeschlossenes Rissbild

- Zustand III (Punkt D-E): Fließen der Bewehrung bei mikrobewehrtem Beton

Die einzelnen Spannungs- und Dehnungswerte können mit den nachfolgenden Gleichungen aus den Eigenschaften der Bewehrung und denen des Betons ermittelt werden. Mit einem Tabellenkalkulationsprogramm können die Werte leicht für ein Sandwichelement mit beliebigen Deckschichteigenschaften berechnet werden.

\subsubsection{Eingabewerte für den bewehrten Beton}

\subsubsection{Zustand I}

\section{Elastizitätsmodul:}

Für den linear-elastischen Bereich unter Zug- und Druckbeanspruchung wird der Elastizitätsmodul $E_{c}$ angenommen. $E_{c}$ errechnet sich vereinfachend aus $E_{c, \text { red }}$ bezogen auf den Querschnitt der Betonmatrix $A_{m}$ und der Bewehrung $A_{f}$ :

$$
E_{c, \text { red }}=E_{f} \cdot V_{f}+E_{m} \cdot V_{m}
$$

Dabei bedeuten:

$\begin{array}{lll}E_{f} & : \quad \begin{array}{l}\text { Elastizitätsmodul der Bewehrung in } \mathrm{N} / \mathrm{mm}^{2}, \text { mit } \\ \end{array} & E_{f, s}=205.000 \mathrm{~N} / \mathrm{mm}^{2} \text { (siehe Tab. 4.4 und vereinfachend } \\ & E_{f, o}=240.000 \mathrm{~N} / \mathrm{mm}^{2} \text { (siehe Tab. 4.6 }\end{array}$

$V_{f}$ und $V_{m}$ können aus den Querschnittsflächen berechnet werden:

$$
\begin{aligned}
V_{f} & =\frac{A_{f}}{A_{f}+A_{m}} \\
V_{m} & =\frac{A_{m}}{A_{f}+A_{m}}
\end{aligned}
$$


Dabei bedeuten:

$A_{f} \quad: \quad$ Querschnittsfläche der Bewehrung in $\mathrm{mm}^{2}$

$A_{m} \quad$ : Querschnittsfläche der Betonmatrix $\mathrm{mm}^{2}$

Die Querschnittsfläche der Betonmatrix $A_{m}$ errechnet sich aus der Gesamtquerschnittsfläche abzüglich der Querschnittsfläche der Längsdrähte bzw. Längsfasern und der Fläche der Querdrähte bzw. Querfasern:

$$
A_{m}=A_{g e s}-A_{f}-A_{f, q}
$$

$$
\begin{array}{lll}
A_{\text {ges }} \quad: \quad & \text { Fläche des gesamten Querschnitts in } \mathrm{mm}^{2} \\
A_{f, q} & : & \begin{array}{l}
\text { Ansichtsfläche der Querdrähte bzw. Querfasern, die die Flä- } \\
\text { che der Betonmatrix reduzieren in } \mathrm{mm}^{2}
\end{array}
\end{array}
$$

Die Höhe des ellipsenförmigen Querschnitts der Schussfäden wurde mit 0,5 mm angenommen. Daher beträgt $A_{f, q}$ bei einlagiger Verwendung des Carbonfasergeleges $0,5 \cdot b$ bzw. $500 \mathrm{~mm}^{2} / \mathrm{m}$. Der Drahtdurchmesser der Mikrobewehrung beträgt $1 \mathrm{~mm}$. Vorausgesetzt, dass bei mehrlagiger Verwendung der Mikrobewehrung, die Drahtmatten gegeneinander versetzt angeordnet werden, beträgt $A_{f, q}=1,0 \cdot b \mathrm{bzw} .1000 \mathrm{~mm}^{2} / \mathrm{m}$.

Bezogen auf den Gesamtbetonquerschnitt ergibt sich $E_{c}$ zu:

$$
E_{c}=E_{c, \text { red }} \cdot \frac{A_{f}+A_{m}}{A_{\text {ges }}}
$$

Die Berechnung des Elastizitätsmoduls nach den obigen Gleichungen stellt eine Vereinfachung dar. Der Einfluss der Querfasern bzw. der Querdrähte wird über die volle Länge angenommen. Allerdings wird der Elastizitätsmodul nur in einem lokalen Bereich durch Querfasern bzw. Querdrähte beeinflusst. Bei einem geringen Abstand der Querfasern bzw. Querdrähte sowie bei der mehrlagigen Verwendung der Bewehrung ist die Annahme einer konstanten Querschnittsschwächung jedoch zutreffend.

\section{Punkt B:}

Die Zugspannung $\sigma_{c, B}$ am Punkt B der idealisierten Spannungsdehnungskurve, bei dem der Beton vom Zustand I in den Zustand II wechselt, berechnet sich aus der Spannung $\sigma_{c, B, \text { red }}$ bezogen auf den Querschnitt von $A_{m}+A_{f}$ :

$$
\sigma_{c, B, r e d}=\sigma_{f, B} \cdot V_{f}+f_{c t m} \cdot V_{m}
$$

Dabei ist $\sigma_{f, B}$ die Spannung der Bewehrung am Punkt B:

$$
\sigma_{f, B}=\frac{f_{c t m}}{E_{m}} \cdot E_{f}
$$

Und $f_{c t m}$ die mittlere zentrische Zugfestigkeit der Betonmatrix. Diese entspricht in etwa der Spaltzugfestigkeit $f_{c t m, s p}$ Schmidt 2008. 
Aus den Gleichungen 6.6 mit 6.7 folgt dann:

$$
\sigma_{c, B, r e d}=f_{c t m} \cdot\left(\frac{E_{f}}{E_{m}} \cdot V_{f}+V_{m}\right)
$$

Die Spannung sowie alle nachfolgend berechneten Spannungen können dann wieder auf den Gesamtquerschnitt bezogen werden:

$$
\sigma_{c, B}=\sigma_{c, \text { red }} \cdot \frac{A_{f}+A_{m}}{A_{\text {ges }}}
$$

Die Dehnung am Punkt B errechnet sich dann zu:

$$
\epsilon_{c, B}=\frac{\sigma_{c, B, r e d}}{E_{c, \text { red }}}
$$

\subsubsection{Zustand II und III des mikrobewehrten Betons}

\section{Punkt C:}

Am Punkt $\mathrm{C}$ der idealisierten Spannungsdehnungskurve stellt sich ein abgeschlossenes Rissbild ein. Bei der Auswertung der durchgeführten Zugversuche am mikrobewehrten Beton konnte ein Verhältnis der Spannungen beim Erstriss $\sigma_{c, B, \text { red }} z u$ den Spannungen bei abgeschlossenem Rissbild $\sigma_{c, C, \text { red }}$ von etwa 1,2 festgestellt werden.

$$
\frac{\sigma_{c, C, \text { red }}}{\sigma_{c, B, \text { red }}}=1,2 \quad \sigma_{c, C, \text { red }}=1,2 \cdot \sigma_{c, B, \text { red }}
$$

Die Dehnung $\epsilon_{c, C}$ am Punkt $C$ errechnet sich aus der reinen Stahldehnung $\epsilon_{f, C}$ an dieser Stelle, abzüglich einer Reduzierung $\Delta \epsilon_{s m}$, die durch den Tension-Stiffening-Effekt, d. h. des Mitwirkens des Betons zwischen den Rissen, hervorgerufen wird.

$$
\epsilon_{c, C}=\epsilon_{f, C}-\Delta \epsilon_{s m}
$$

Die Reduzierung $\Delta \epsilon_{s m}$ wird hier auf Grundlage des Eurocode DIN EN 19922011 (vgl. auch Schnell 2013) wie folgt angenommen:

$$
\Delta \epsilon_{s m}=0,4 \cdot \frac{f_{c t m}}{E_{f} \cdot \rho}
$$

Dabei ist:

$$
\rho=\frac{A_{f}}{A_{m}}
$$

Und:

$$
\epsilon_{f, C}=\frac{\sigma_{c, C, r e d}}{E_{f} \cdot V_{f}}
$$




\section{Punkt D:}

Im Punkt $D$ des Spannungsdehnungsdiagramms erreicht der Bewehrungsstahl die Streckgrenze $f_{s y}$ und beginnt zu plastizieren.

$$
\begin{aligned}
& \sigma_{c, D, \text { red }}=f_{s y} \cdot V_{f} \\
& \epsilon_{c, D}=\epsilon_{f, D}-\Delta \epsilon_{s m}
\end{aligned}
$$

Mit:

$$
\epsilon_{f, D}=\frac{f_{s y}}{E_{f}}
$$

Die Streckgrenze der Mikrobewehrung wird mit $f_{s y}=380 \mathrm{~N} / \mathrm{mm}^{2}$ angenommen.

\section{Punkt E:}

Die Bruchspannung des mikrobewehrten Betons errechnet sich aus der Zugfestigkeit $f_{s u}$ der Bewehrung:

$$
\sigma_{c, E, r e d}=f_{s u} \cdot V_{f}
$$

Dazu wird die Zugfestigkeit der Mikrobewehrung mit $f_{s u}=440 \mathrm{~N} / \mathrm{mm}^{2}$ angenommen. Die Dehnung am Punkt E wird mit $\epsilon_{c, E}=50 \%$ ongenommen.

\section{Querdehnzahl:}

Die Querdehnzahl des mikrobewehrten Betons $v_{c}$ wird mit 0,2 angenommen.

\subsubsection{Zustand II des textilbewehrten Betons}

\section{Punkt C:}

Das Verhältnis der Spannungen bei abgeschlossenem Rissbild zur den Spannungen beim Erstriss kann zwischen 1,0 und 1,3 variieren (Jesse 2004). In den hier durchgeführten Versuchen an den mit Carbongelege bewehrten Dehnkörpern wurde ein Faktor von 1,05 festgestellt. Damit gilt:

$$
\sigma_{c, C, r e d}=1,05 \cdot \sigma_{c, B, r e d}
$$

Zur Berechnung der Dehnungen im Zustand II wurde der Ansatz von SchießI 1989 zuerst von Molter 2005 und anschließend von Voss 2008 wie folgt erweitert:

$$
\epsilon_{t e x, C}=\frac{\sigma_{t e x, C}}{E_{f i l} \cdot k_{s t}} \cdot\left(1-\beta_{1} \cdot\left[\frac{\sigma_{t e x, B}}{\sigma_{t e x, C}}\right]^{2}\right)
$$

Mit den Spannungen bezogen auf den Querschnitt $A_{m}+A_{f}$ gilt:

$$
\epsilon_{c, C}=\frac{\sigma_{c, C, \text { red }}}{E_{f} \cdot V_{f} \cdot k_{s t}} \cdot\left(1-\beta_{1} \cdot\left[\frac{\sigma_{c, B, r e d}}{\sigma_{c, C, r e d}}\right]^{2}\right)
$$


Mit:

$k_{s t} \quad: \quad$ Faktor zur Berücksichtigung des Steifigkeitsabfalls

$\beta_{1} \quad$ : Faktor zur Erfassung der Verbundeigenschaften

Mit den beiden Faktoren $k_{s t}$ und $\beta_{1}$ kann die theoretische Spannungsdehnungskurve an die in den Versuchen ermittelten Kurven angepasst werden. Nach Voss 2008 werden mit $k_{s t}=0,9$ und $\beta_{1}=0,85$ gute Übereinstimmungen erreicht. Molter 2005 erreichte für carbonfaserbewehrte Dehnkörper mit $k_{s t}=k_{1}$ (Effektivitätsbeiwert) und $\beta_{1}$ $=0,5$ gute Übereinstimmungen.

Für die in dieser Arbeit durchgeführten Versuche ergaben sich die besten Übereinstimmungen mit $k_{s t}=0,35$ und $\beta_{1}=0,25$. Der $\beta_{1}$-Faktor ist mit 0,25 sehr gering (vgl. SchießI 1989, $\beta_{1}=1,0$ gerippter Bewehrungsstahl und $\beta_{1}=0,5$ glatter Bewehrungsstahl). Dies begründet sich durch die schlechte Aktivierung der Fasern. Dieser Effekt ist durch das verwendete Gelege mit 50 k-Fasern (d. h. 50.000 Filamenten pro Faser) deutlicher ausgeprägt, als in den Versuchen nach Molter 2005 und Voss 2008 mit 24 kFasern. Der Grund hierfür ist, dass dickere Fasern ein größeres Verhältnis von Kernzu Randfilamenten aufweisen, als dünnere Fasern.

\section{Punkt D:}

Die Bruchspannung kann analog zur Bemessung der Zugtragfähigkeit (siehe GI. (3.9), S. 16 mit dem Effektivitätsfaktor $k_{1}$ und der Faserzugfestigkeit $f_{t}$ ermittelt werden:

$$
\sigma_{c, D, \text { red }}=f_{t} \cdot k_{1} \cdot V_{f}
$$

Der Effektivitätsfaktor wurde entsprechend in den Dehnkörperversuchen mit $k_{1}=0,2$ ermittelt. Die Faserzugfestigkeit wurde nach den Herstellerangaben mit $f_{t}=4.000 \mathrm{~N} / \mathrm{mm}^{2}$ gewählt.

Die Dehnung errechnet sich analog zu Gleichung 6.22:

$$
\epsilon_{c, D}=\frac{\sigma_{c, D, r e d}}{E_{f} \cdot V_{f} \cdot k_{s t}} \cdot\left(1-\beta_{1} \cdot\left[\frac{\sigma_{c, B, r e d}}{\sigma_{c, D, r e d}}\right]^{2}\right)
$$

Durch die Berechnung der Dehnungen im Zustand II mit dem Ansatz nach SchießI 1989 würde sich eine leicht parabelförmige Spannungsdehnungskurve einstellen. Zur Eingabe des Materialgesetzes wurden jedoch lediglich die Dehnungen an den Punkten C und D berechnet. Somit stellt sich ein linearer Verlauf der Kurve ein. Jedoch kann mit dem linearen Verlauf das in den Versuchen ermittelte Spannungsdehnungsverhalten hinreichend genau beschrieben werden (siehe Abbildung 6.2

\section{Querdehnzahl:}

Die Querdehnzahl des textilbewehrten Betons $v_{c}$ wird ebenfalls mit 0,2 angenommen.

\subsubsection{Vergleich mit den Ergebnissen der Dehnkörperversuche}

Anhand der Ergebnisse der Dehnkörperversuche konnten die getroffenen Annahmen überprüft werden. Dazu wurden die entsprechenden Abmessungen und Materialeigen- 
schaften der Dehnprobekörper (siehe Anhang A.3 in die Gleichungen eingesetzt. Die resultierenden idealisierten Spannungsdehnunglinien wurden dann in die Spannungsdehnungsdiagramme der Versuche eingezeichnet (siehe Abbildungen 6.1 und 6.2). Die idealisierten Kurven stimmen sehr gut mit den in den Versuchen ermittelten überein.

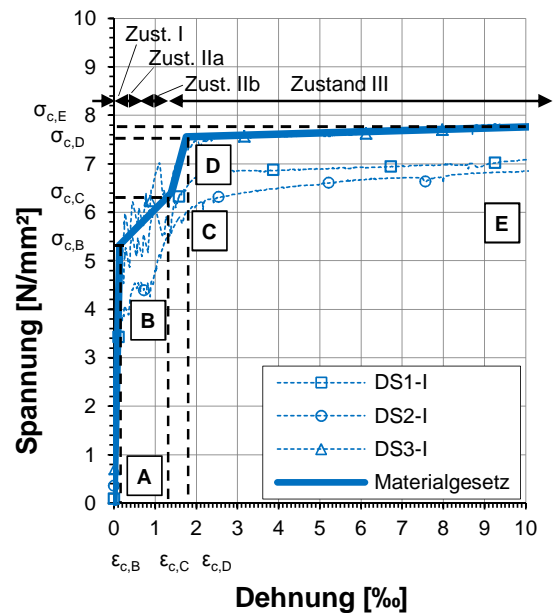

Abb. 6.1: Idealisierte und experimentell ermittelte Zugspannungsdehnungskurven des mikrobewehrten Betons

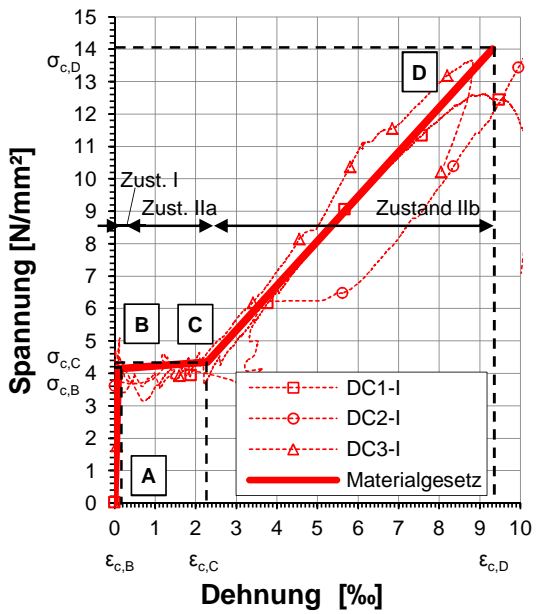

Abb. 6.2: Idealisierte und experimentell ermittelte Zugspannungsdehnungskurven des carbonfaserbewehrten Betons

\subsubsection{Kontrolle durch Nachrechnung der Biegeversuche an bewehrten Betonstreifen}

\subsubsection{Beschreibung des Finite-Element Modells}

Zur Kontrolle der Materialgesetze des bewehrten Betons wurden die Biegeversuche an den bewehrten Betonstreifen nachgerechnet. Die Materialgesetze wurden mit den Gleichungen aus Abschnitt 6.2.2, Eingabewerte für den bewehrten Beton ' auf Grundlage der Probekörpereigenschaften (siehe Anhang A.3) berechnet. Die FE-Netzgröße entsprach $2,5 \mathrm{~mm}$.

\subsubsection{Berechnungsergebnisse}

In Abbildung 6.3 sind die rechnerisch und experimentell ermittelten Lastverformungskurven dargestellt. Die blaue, gepunktete Kurve stellt das nachgerechnete Lastverformungsverhalten der 4-Punkt-Biegeversuche an mikrobewehrten Probekörpern und die rote Kurve das Verhalten an carbonfaserbewehrten Probekörpern dar.

Tendenziell gleichen sich die Verläufe der rechnerisch ermittelten Kurven und der jeweiligen gemessenen Kurven. Beide rechnerisch ermittelten Kurven weisen einen linearelastischen Verlauf im Zustand I auf und flachen dann stetig bei einer Last von rund 


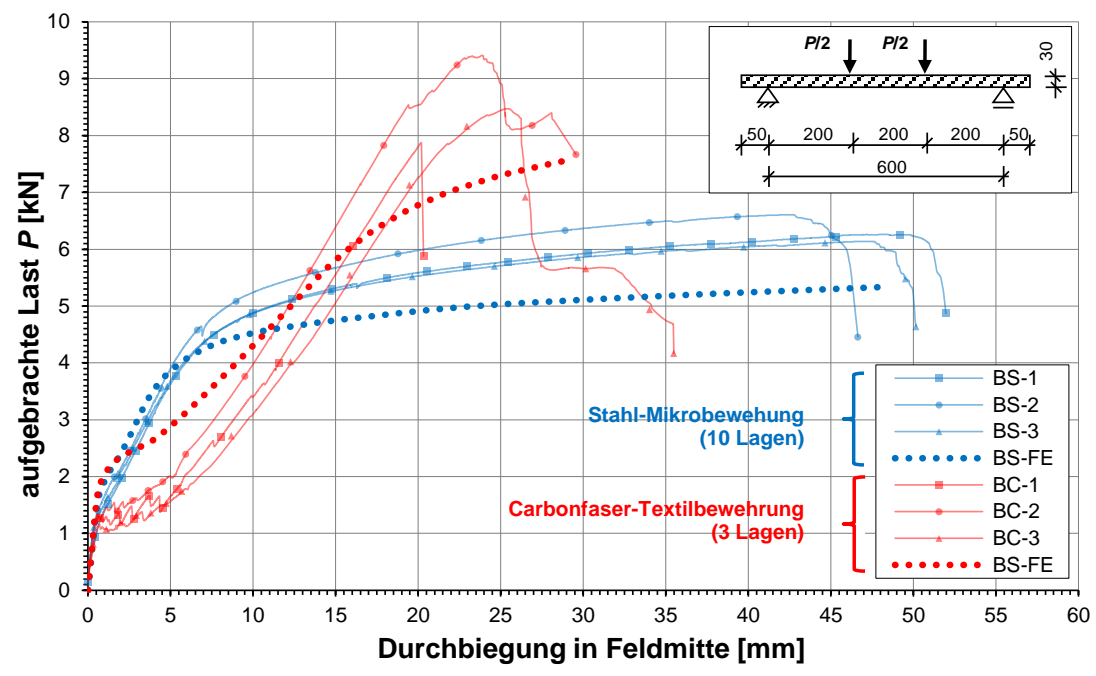

Abb. 6.3: Vergleich der rechnerisch und experimentell ermittelten Lastverformungskurven der Biegeversuche an bewehrten Betonstreifen

$2 \mathrm{kN}$ ab. Die experimentell ermittelten Kurven verlaufen ebenfalls linear-elastisch, flachen jedoch bereits bei einer Last von etwa $1,3 \mathrm{kN}$ relativ stark ab.

Die Vermutung liegt nahe, dass die Betonzugfestigkeit durch die Materialmodelle überschätzt wird. Bei der Auswertung der Spannungsverteilung über den Querschnitt des Modells zeigt sich jedoch, dass bereits im linear-elastischen Bereich der Kurven, vor dem Erreichen des Lastniveaus von $2 \mathrm{kN}$, die auftretenden Spannungen die Zugfestigkeit des Betons überschreiten. Im Gegensatz zu den Versuchen, führen diese Überschreitungen jedoch nicht zum Reißen des Betons und zu einem abruptem Steifigkeitsabfall. Vielmehr führt das Plastizieren des Werkstoffes durch Überschreiten der Betonzugfestigkeit am Punkt B der idealisierten Spannungsdehnungsbeziehung zu einem langsamen Übergang in den Zustand II a.

Im Zustand II b weisen die rechnerisch und experimentell ermittelten Kurven der mikrobewehrten Probekörper etwa die gleiche Steigung auf. Im Fall der carbonfaserbewehrten Probekörper verlaufen die rechnerisch ermittelten Kurven etwas flacher als die gemessenen Kurven. Dieser Unterschied resultiert aus der Tatsache, dass die Materialeigenschaften des carbonfaserbewehrten Modells in Dehnkörperversuchen bestimmt wurden. Wie jedoch bereits durch Voss 2008 festgestellt wurde, werden die Fasern in Biegeversuchen besser ausgenutzt. Durch die Bauteilkrümmung werden die Fasern an der Risskante umgelenkt. Infolge der entstehenden Querpressungen werden die Verbundspannungen zwischen den Filamenten verbessert und die Kernfilamente stärker aktiviert, wodurch auch der effektive Elastizitätsmodul ansteigt.

In beiden Modellierungen werden geringere maximale Lasten errechnet, als in den Versuchen ermittelt wurden. Die berechneten Kurven der mikrobewehrten Probekörper weisen das in den Versuchen beobachte charakteristische Fließplateau auf. Die Probekörper gehen jedoch bei einem niedrigeren Lastniveau in den Zustand III über. Im Fall der carbonfaserbewehrten Probekörper flachen die berechneten Kurven vor dem 
Erreichen der maximalen Belastung ab. Dies wurde durch zusätzliche Plastizierungen in $y$-Richtung im Bereich zwischen den Auflager- und den Lasteinleitungspunkten verursacht. Durch diese deutet sich in der Modellierung das Querkraftversagen an, das auch in den Versuchen beobachtet werden konnte.

Mit dem gewählten Werkstoffgesetz und der Annahme einer über den Querschnitt „,verschmierten“ Bewehrung, kann das Lastverformungsverhalten eines 4-Punkt-Biegeversuchs gut abgebildet werden. Der Übergang vom Zustand I in den Zustand II a sowie die erreichten Maximallasten werden in den Modellierungen nicht exakt abgebildet. Da die Beanspruchung der Deckschichten in den Sandwichversuchen überwiegend aus Normalkräften, die aus dem Sandwichmoment resultieren, erfolgt, wurden die Materialmodelle des bewehrten Betons jedoch nicht weiter angepasst.

\subsection{Modellierung des Kerns}

\subsubsection{Allgemeines}

Zur Modellierung des Kerns wurde das Vier-Knoten-Element PLANE42 mit ebenem Spannungszustand (plane stress) verwendet. Es wurden zwei alternative Materialgesetze verwendet. Mit der Materialoption BISo wurde ein isotropes, bilineares Materialgesetz erstellt und mit der Materialoption ANISO ein anisotropes, bilineares Materialgesetz.

\subsubsection{Isotropes Materialgesetz „BISO“}

Das isotrope Materialmodell BISO ist ein relativ einfaches elastoplastisches Werkstoffgesetz. Es gilt die Plastizitätstheorie nach von Mises in Verbindung mit isotroper Verfestigung. Das Materialmodell BISO wird lediglich über die Angabe des Elastizitätsmoduls $E$ im linear-elastischen Bereich des Werkstoffverhaltens, der Fließspannung $\sigma_{y}$ beim Übergang in den plastischen Bereich, dem Tangentenmodul im plastischen Bereich $E_{y}$ und der Querdehnzahl $v$ definiert.

Um die Schereigenschaften der XPS-Chargen exakt abbilden zu können, wurde der Elastizitätsmodul $E$ und die Fließspannung $\sigma_{y}$ auf Grundlage des Tangenten-Schubmoduls $G$ und der Scherfestigkeit $\tau_{y}$ aus den Scherversuchen berechnet.

$$
\begin{aligned}
& E=2 \cdot G \cdot(1+v) \\
& \sigma_{y}=\sqrt{3} \cdot \tau_{y}
\end{aligned}
$$

Die Querdehnzahl wurde nach den Angaben in der Literatur (siehe auch Abschnitt 3.2.3.8 S. 27 mit 0,45 gewählt. Der Tangentenmodul wurde mit $E_{y}=0,5$ festgelegt. Die Eingabeparameter für das XPS-Materialmodell BISO sind für die einzelnen Chargen in Tabelle 6.1 zusammengefasst.

Weiterhin bietet das Materialmodell BISO die Möglichkeit, weitere Fließspannungen und Tangentenmoduln in Abhängigkeit der Werkstofftemperatur zu definieren. Auf diese Weise kann die Entfestigung des XPS unter Temperaturbeanspruchung modelliert werden. 
Tab. 6.1: Eingabeparameter zur Modellierung der verschiedenen XPS-Chargen mit dem Materialgesetz BISO

\begin{tabular}{|c|c|c|c|c|c|c|c|c|}
\hline \multicolumn{2}{|c|}{$\begin{array}{l}\text { XPS-Charge } \\
\text { Rohdichte }\left(\mathrm{kg} / \mathrm{m}^{3}\right) \\
\text { Parameter }\end{array}$} & $\begin{array}{c}\mathbf{X 1} \\
36,7 \\
{\left[\mathrm{~N} / \mathrm{mm}^{2}\right]}\end{array}$ & $\begin{array}{c}\mathrm{X} 2 \\
37,7 \\
{\left[\mathrm{~N} / \mathrm{mm}^{2}\right]}\end{array}$ & $\begin{array}{c}\mathrm{X} 3 \\
46,9 \\
{\left[\mathrm{~N} / \mathrm{mm}^{2}\right]}\end{array}$ & $\begin{array}{c}\text { X4 } \\
36 \\
{\left[\mathrm{~N} / \mathrm{mm}^{2}\right]}\end{array}$ & $\begin{array}{c}\mathbf{X 5} \\
31,4 \\
{\left[\mathrm{~N} / \mathrm{mm}^{2}\right]}\end{array}$ & $\begin{array}{c}\mathbf{X 6} \\
34 \\
{\left[\mathrm{~N} / \mathrm{mm}^{2}\right]}\end{array}$ & $\begin{array}{c}\mathbf{X 7} \\
33,4 \\
{\left[\mathrm{~N} / \mathrm{mm}^{2}\right]}\end{array}$ \\
\hline$G^{1}$ & Schubmodul & 6,88 & 6,52 & $8,62^{2}$ & 8,76 & 6,70 & 7,48 & 7,00 \\
\hline$\tau^{1}$ & Schubspannung & 0,22 & 0,25 & 0,31 & 0,17 & 0,18 & 0,15 & 0,16 \\
\hline$E$ & Elastizitätsmodul & 19,95 & 18,91 & 25,00 & 25,40 & 19,43 & 21,69 & 20,30 \\
\hline$\sigma$ & Fließspannung & 0,38 & 0,43 & 0,54 & 0,29 & 0,31 & 0,26 & 0,28 \\
\hline & Querdehnzahl & \multicolumn{7}{|c|}{0,45} \\
\hline & Fließ-E-Modul & \multicolumn{7}{|c|}{0,05} \\
\hline
\end{tabular}

${ }^{1}$ Werte aus Scherversuchen (siehe Abbildungen 5.27 und 5.28)

${ }^{2}$ um $30 \%$ von 6,63 auf $8,62 \mathrm{~N} / \mathrm{mm}^{2}$ erhöht

\subsubsection{Anisotropes Materialgesetz „ANISO“}

Mit der Materialoption AnIso wurde zusätzlich ein anisotropes, bilineares Werkstoffgesetz erstellt. Hierzu wird das Materialverhalten durch die Eingabe von bilinearen Spannungsdehnungslinien für alle drei Materialachsen unter Zug-, Druck,- und Schubbeanspruchung definiert.

Das Materialmodell ANISo basiert auf der „generalized Hill potenial theory“. Durch die Hill'sche Fließbedingung wird die Plastizitätsgrenze bei unterschiedlichen Fließspannungen in den drei orthogonalen Richtungen definiert (Hill 1983). Eine Erweiterung von Shih und Lee (Shih $u$. Lee 1978) ermöglicht dies auch bei unterschiedlichen Fließspannungen unter Druck- und Zugbeanspruchung.

Das Materialverhalten wird dabei im linear-elastischen Bereich unter Druck- und Zugbeanspruchung durch die Eingabe der Elastizitätsmoduln $E_{x}, E_{y}$ und $E_{z}$ in den drei Materialachsen $x, y$ und $z$ definiert. Die Schubsteifigkeit in den Materialebenen $x y, x z$ und $y z$ wird analog dazu durch die Angabe von drei Schubmoduln $G$ beschrieben. Der plastische Bereich des Materialverhaltens für die jeweilige Beanspruchungsart und die jeweilige Richtung wird durch die Angabe von Fließspannungen $\sigma_{c}, \sigma_{t}$ und $\tau$, sowie von Tangentenmoduln $E_{p, c}, E_{p, t}$ und $G$ beschrieben. Weiterhin werden die Querdehnzahlen in den drei Raumachsen definiert.

Insgesamt müssen folglich, neben den drei Querdehnzahlen, 24 Parameter zur Beschreibung des Materialverhaltens des XPS gewählt werden. Davon wirken sich bei einer zwei-dimensionalen Modellierung jedoch nur die Materialeigenschaften in $x-, y-$, und $x y$-Richtung auf das Tragverhalten aus. Von diesen 13 verbleibenden Parametern sind die Werte $\sigma_{c, y}, G_{x y}$ und $\tau_{x y}$ jeder einzelnen Charge bekannt. Für alle weiteren Parameter wurden Annahmen getroffen oder anhand von vergleichbaren Versuchsergebnissen der BASF sinnvolle Werte gewählt.

Die Annahme einer großen Anzahl an Eingangsparameter scheint bei der Nachrechnung der Versuche wenig zielführend. Jedoch bietet das ANISO-Materialmodell gegenüber dem BISo-Modell den Vorteil, dass die Schub- und die Elastizitätsmoduln, sowie 
die Fließspannungen unabhängig voneinander gewählt werden können und somit die Anisotropie des XPS modelliert werden kann.

Die Eingabeparameter für das XPS-Materialgesetz ANISO sind für die einzelnen Chargen in Tabelle 6.2 zusammengefasst. Dabei entspricht die $x$-Richtung der Extrusionsrichtung und die $y$-Richtung der Dickenrichtung des XPS. Bei der Modellierung müssen die Werte je nach Ausrichtung der XPS-Platten entsprechend getauscht werden.

Tab. 6.2: Eingabeparameter zur Modellierung der verschiedenen XPS-Chargen mit dem Materialgesetz ANISO

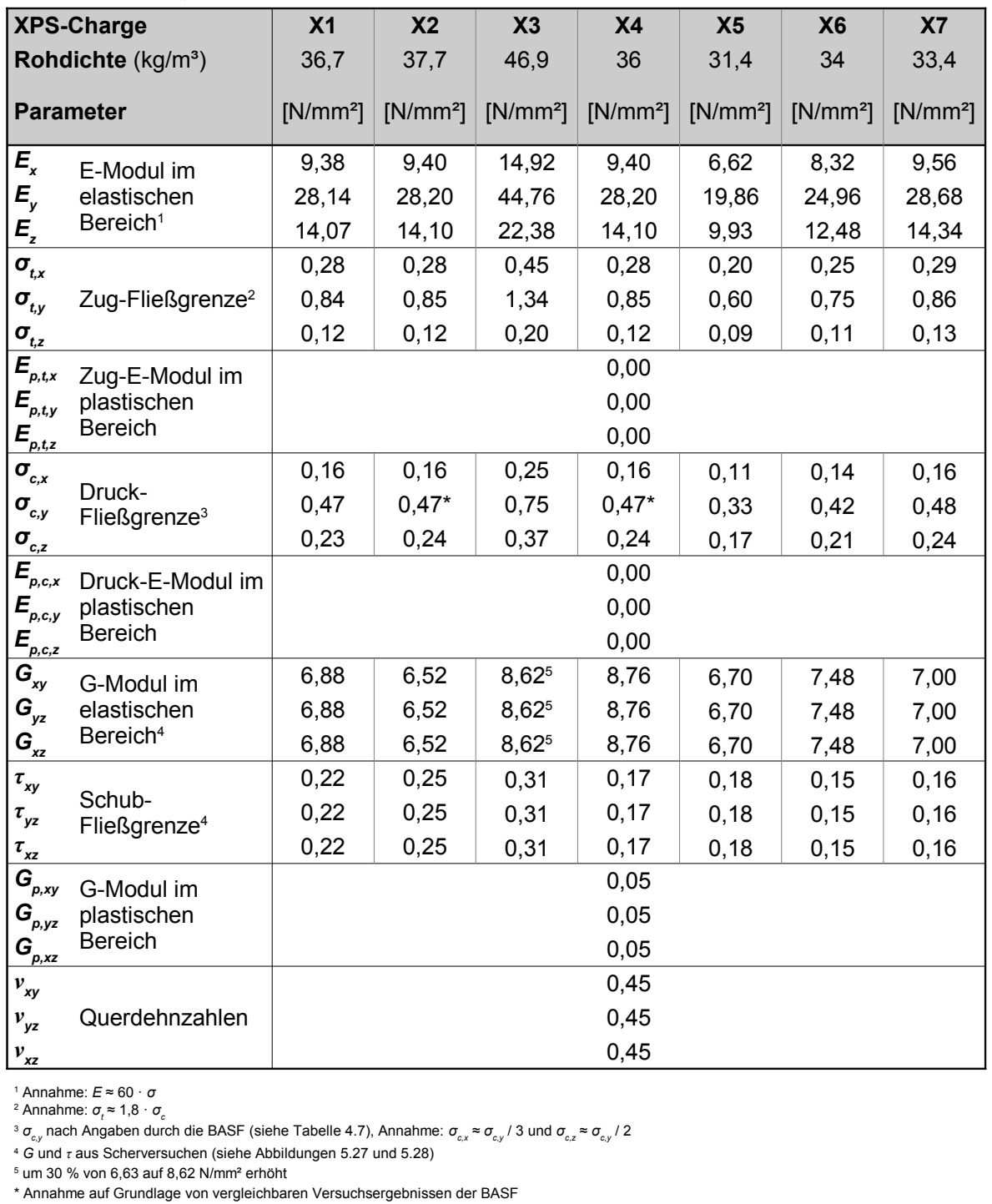




\subsubsection{Kontrolle durch Nachrechnung der Scherversuche}

\subsubsection{Beschreibung des Finite-Element Modells}

Die Annahmen zum Materialverhalten des XPS wurden durch Nachrechnung der Scherversuche überprüft. Weiterhin dienten die Berechnungen zur Interpretation und Bewertung der Versuchsergebnisse.

Die Länge $l$ der modellierten Probekörper betrug grundsätzlich $250 \mathrm{~mm}$. Für die Dicke $d$ wurden jeweils die wahren Abmessungen der getesteten XPS-Chargen übernommen. Diese konnten wenige Millimeter von der angestrebten Dicke abweichen. Die Netzgröße des Modells wurde mit $10 \mathrm{~mm}$ vorgegeben.

Die Scherversuche wurden in den ersten Berechnungen sowohl mit als auch ohne die Lasteinleitungskonstruktion aus Stahlwinkeln und Holzleisten modelliert. Dabei war der Einfluss der Lasteinleitungskonstruktion auf das Tragverhalten gering. Alle weiteren Scherversuche wurden daher ohne die Lasteinleitungskonstruktion modelliert. Dazu wurde der Probekörper in Analogie zu den Versuchen an der rechten Seite über die komplette Höhe fest gelagert. An der linken Seite wurde die Verformung in $y$-Richtung über die komplette Höhe aufgebracht und zusätzlich eine Verschiebung in $x$-Richtung, wie in den Versuchen mittels der seitlichen Halterung, behindert (siehe Abbildung 6.4).

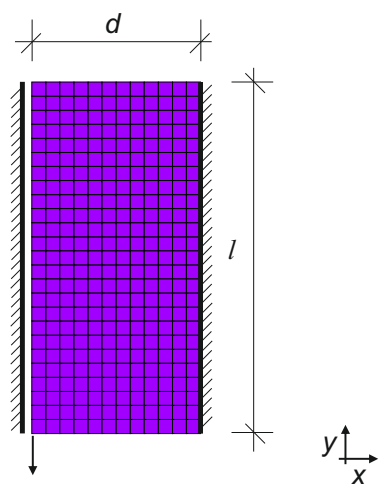

Abb. 6.4: FE-Modell des Scherversuchs an Probekörpern ohne Deckschichten

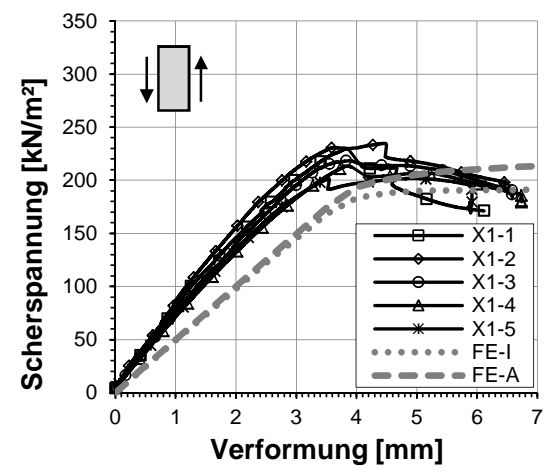

Abb. 6.5: Vergleich der rechnerisch und experimentell ermittelten Scherspannungsverformungskurven der Versuchsreihe $\mathrm{X} 1$

\subsubsection{Berechnungsergebnisse}

Aus den Auflagerkräften des Modells können bezogen auf die Scherfläche $l \cdot b$ die Scherspannungen berechnet werden. In Abbildung 6.5 sind diese über der berechneten Verformungen des Versuchs $\mathrm{X} 1$ und mit den dazugehörigen experimentell ermittelten Scherspannungsverformungslinien dargestellt. Im Diagramm sowie im weiteren Verlauf dieser Arbeit werden die Berechnungsergebnisse, die mit dem Materialgesetz 
BISO erstellt wurden, grundsätzlich als gepunktete Linie und die Ergebnisse, die mit dem Materialgesetz ANSIo erstellt wurden, als gestrichelte Linie dargestellt.

Mit den beiden Materialmodellen kann das Lastverformungsverhalten der Scherprobekörper gut abgebildet werden. Im linear-elastischen Bereich ist das Materialverhalten der beiden Materialgesetze in etwa identisch und im Vergleich zu den Versuchen zu weich. Die daran anschließenden Fließplateaus resultieren aus der Eingabe der Fließspannungen. Das Fließplateau des BISo-Modells ist nahezu horizontal, wobei hingegen das Plateau des ANiso-Modells leicht ansteigt. Obwohl in beiden Berechnungen die gleichen Fließspannungen $\tau$ definiert wurden, unterscheiden sich die Werte bei denen die plastischen Verformungen einsetzen. Im Fall des BISO-Modells ist die berechnete Scherspannung geringer, als in den Versuchen. Das Niveau der Berechnungen mit dem ANISO-Materialgesetz stimmt hier genauer mit den Versuchen überein.

In der Abbildung 6.6 sind die Hauptdehnungen der beiden Modelle bei $8 \mathrm{~mm}$ Verformung vektoriell dargestellt. In den Eckbereichen der Modelle bilden sich die größten Dehnungen aus. In den unteren linken und den oberen rechten Ecken bilden sich Zugdehnungen (schwarze Pfeile) aus. Auch bei den Versuchskörpern konnten in diesem Bereich klaffende Fugen infolge von Zugspannungen festgestellt werden (siehe Abbildung 6.7). Spiegelbildlich zu den Zugdehnungen treten in der oberen linken und der unteren rechten Ecken der Modelle Druckstauchungen (blaue Pfeile) auf.

Die Hauptdehnungen verlaufen im mittleren Bereich der beiden Schermodelle großflächig in einem Winkel von $45^{\circ}$. Die Fläche mit gleichen Hauptdehnungen ist bei der Modellierung mit dem Materialgesetz ANISO größer und grenzt nah an den oberen bzw. unteren Rand des Modells an.

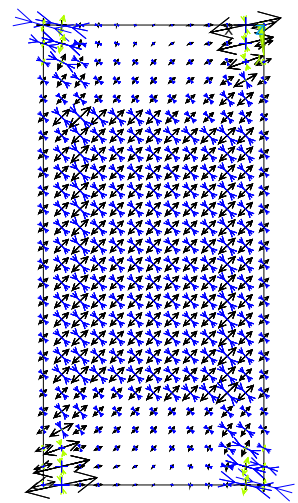

Abb. 6.6

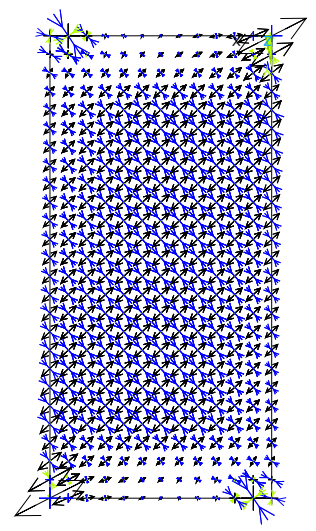

Vektorielle Darstellung der Hauptdehnungen der Scherversuchsreihe $\mathrm{X} 1$ ermittelt mit dem Materialgesetz BISO (links) und ANISO (rechts) bei $8 \mathrm{~mm}$ Verformung

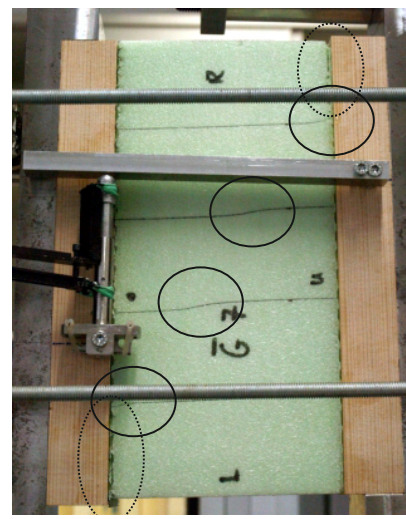

Abb. 6.7: Darstellung der Verformungen in Versuch X1-2

In einer Parameterstudie wurde ein Einfluss der Länge $l$ auf den Verlauf der Scherspannungsverformungskurve festgestellt. Bei der Modellierung von Probekörpern mit einer größeren Länge $l$ lagen die Berechnungsergebnisse generell näher an den eingegebenen Scherparametern. Auf diesen Effekt wird bei der Bewertung der Scherversuche in Abschnitt 7.2.2, 'Kritische Betrachtung der Scherversuche' (siehe S. 155 näher einge- 
gangen. Da in den Biegeversuchen die Länge des Kerns, die durch Scherspannungen beansprucht wird, jedoch grundsätzlich größer als $250 \mathrm{~mm}$ war, wurden die Materialgesetze des XPS nicht weiter angepasst.

\subsection{Modellierung der Verbundfuge zwischen Deckschichten und Kern}

Die Verbundfugen zwischen dem Kern und der oberen und unteren Deckschicht wurden über Kontaktelemente modelliert. Dafür wurden die beiden 2D-Kontaktelemente CONTA171 und TARGE169 verwendet. Der Verbund wurde für die Nachrechnung der Versuche und für die Parameterstudien als starr angenommen.

\subsection{Nachrechnung der Biegeversuche an Sandwichstreifen}

\subsubsection{Beschreibung des Finite-Element Modells}

Die Eingangswerte für die Modellierung des Kerns wurden entsprechend der jeweiligen XPS-Charge aus den Tabellen 6.1 und 6.2 entnommen. Die Schereigenschaften der XPS-Charge X0 der Versuchsreihe „Herstellung“ wurden im Rahmen der Untersuchungen nicht ermittelt. Da deren Rohdichte in etwa der Rohdichte der Charge X5 entsprach, wurden die Materialeigenschaften der XPS-Charge X5 angenommen.

Für die Modellierung der Deckschichten wurden die Punkte der idealisierten Spannungsdehnungkurven nach den Formeln aus Abschnitten 6.2.2, Eingabewerte für den bewehrten Beton' berechnet. Hierzu wurden die in der jeweiligen Versuchsreihe ermittelten Elastizitätsmoduln und Zugfestigkeiten zu Grunde gelegt.

Für die Dicke der Deckschichten und des Kerns wurden die wahren Abmessungen als Mittelwerte der jeweiligen Versuche übernommen. Die Deckschichten der Modelle wurden mit einer FE-Netzweite von rund 2,5 mm und die Kernschichten mit einer Netzweite von rund $10 \mathrm{~mm}$ erstellt. Um Singularitäten an der Lasteinleitung und an den Auflagern zu verhindern, wurden auch die stählernen Lastverteilungsplatten modelliert und ebenfalls mit einer Elementgröße von 2,5 mm vernetzt. Die Deckschichten und Kernschichten, sowie die Deckschichten und die Lastverteilungsplatten wurden mit Kontaktelementen starr miteinander verbunden. Die Kontaktelemente wurden ebenfalls mit rund 2,5 mm Elementgröße vernetzt.

Die Anordnung der Auflager- und Lasteinleitungspunkte, sowie die Lage und Breite der Lastverteilungsplatten wurde aus den Versuchen übernommen (siehe Tabelle 5.1 und 5.2. Wie im Versuch, wurde jeweils die Lastverteilungsplatte am linken Auflager in $x$ - und in $y$-Richtung unverschieblich gelagert. Am rechten Auflager wurden die Lastverteilungsplatten nur in $y$-Richtung unverschieblich gelagert. Die Belastung wurde als Verformung in $y$-Richtung mittig auf die modellierten Lastverteilungsplatten aufgebracht. Die Summe, der durch die Verformung resultierenden Auflagerreaktionen an den beiden Lagerpunkten, entsprach der aufgebrachten Belastung. In den Abbildun- 
gen 6.8 und 6.9 sind die Modelle für einen 3-Punkt und einen 4-Punkt-Biegeversuch beispielhaft dargestellt.

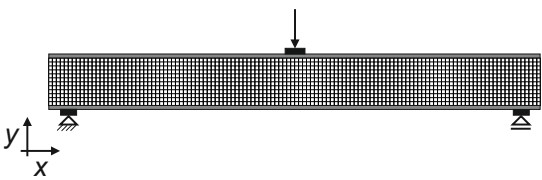

Abb. 6.8: FE-Modell eines 3-PunktBiegeversuchs

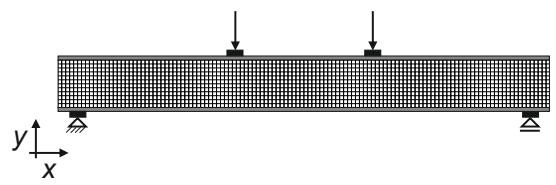

Abb. 6.9: FE-Modell eines 4-PunktBiegeversuchs

Alle Eingangswerte der Berechnungen sind in den Tabellen B.1 und B.2 im Anhang zusammengestellt. Die Eingabedatei, d. h. „Commandfile“, zur Modellierung eines 3Punkt-Biegeversuchs ist exemplarisch dieser Arbeit im Anhang B.1 angehängt.

\subsubsection{Vergleich der Berechnungsergebnisse mit den Versuchsergebnissen}

Aus den Auflagerkräften des FE-Modells wurden die Momente und Querkräfte errechnet und mit den experimentell ermittelten Momenten und Querkräften über der Verformung in den Abbildungen 6.10 bis 6.13 als Lastverformungsdiagramme dargestellt. Zur Unterscheidung sind die Lastverformungskurven als gepunktete Linien für die Modellierung mit dem Materialgesetz BISO und als gestrichelte Linien für die Modellierung mit dem Materialgesetz ANISO dargestellt.

Grundsätzlich weisen die Kurvenverläufe der Berechnungsergebnisse ein sehr gute Übereinstimmung mit denen der Versuchsergebnisse auf. Die berechneten Kurven weisen die selben charakteristischen Stellen der Versuchskurven auf:

Die Kurven flachen beim Übergang vom Zustand I in den Zustand II durch das Reißen des Betons ab. Aufgrund der Wahl der Materialgesetze führt dies nicht zu einem abruptem Steifigkeitsabfall, wie in den Versuchen beobachtet werden konnte, jedoch zu einem Knick in den Lastverformungskurven. Dies lässt sich besonders deutlich bei den Ergebnissen der carbonfaserbewehrten Probekörper (siehe Abbildung 6.11 und 6.12 feststellen.

Weiterhin weisen alle berechneten Lastverformungskurven, wie auch die experimentell ermittelten Kurven, ein ausgeprägtes Fließplateau auf. Die Plastizitätsgrenzen $P_{p l, c a l c}$, bei denen dieses Fließplateau erreicht wird, weichen nur maximal $13 \%$ von denen in den Versuchen ermittelten mittleren Lasten $P_{p l, \text { exp }}$ ab. Das Verhältnis von $P_{p l, c a l c} \mathrm{zu}$ $P_{p l, \text { exp }}$ ist in Abbildung 6.14 dargestellt. $P_{p l}$ wurde dazu am Ende des linear-elastischen Anstiegs der Kurven abgelesen (Schnittpunkt zwischen der Kurve und einer Parallelen zum linear-elastischen Anstieg, die um einen Abszissenwert von $1 \mathrm{~mm}$ verschoben ist). Weiterhin lassen sich die Ursachen für die Plastizierungen und den Übergang in das Fließplateau durch das Auswerten der einzelnen Spannungs- und Dehnungsbilder der Modellierung bestimmen. Diese sind ebenfalls in Tabelle 6.3 angegeben.

Der Kurvenverlauf der 3-Punkt-Biegeversuche, die mit den beiden Werkstoffgesetzen BISO und ANISO ermittelt wurden (siehe Abbildung 6.10 und 6.11), sind bis zum Errei- 


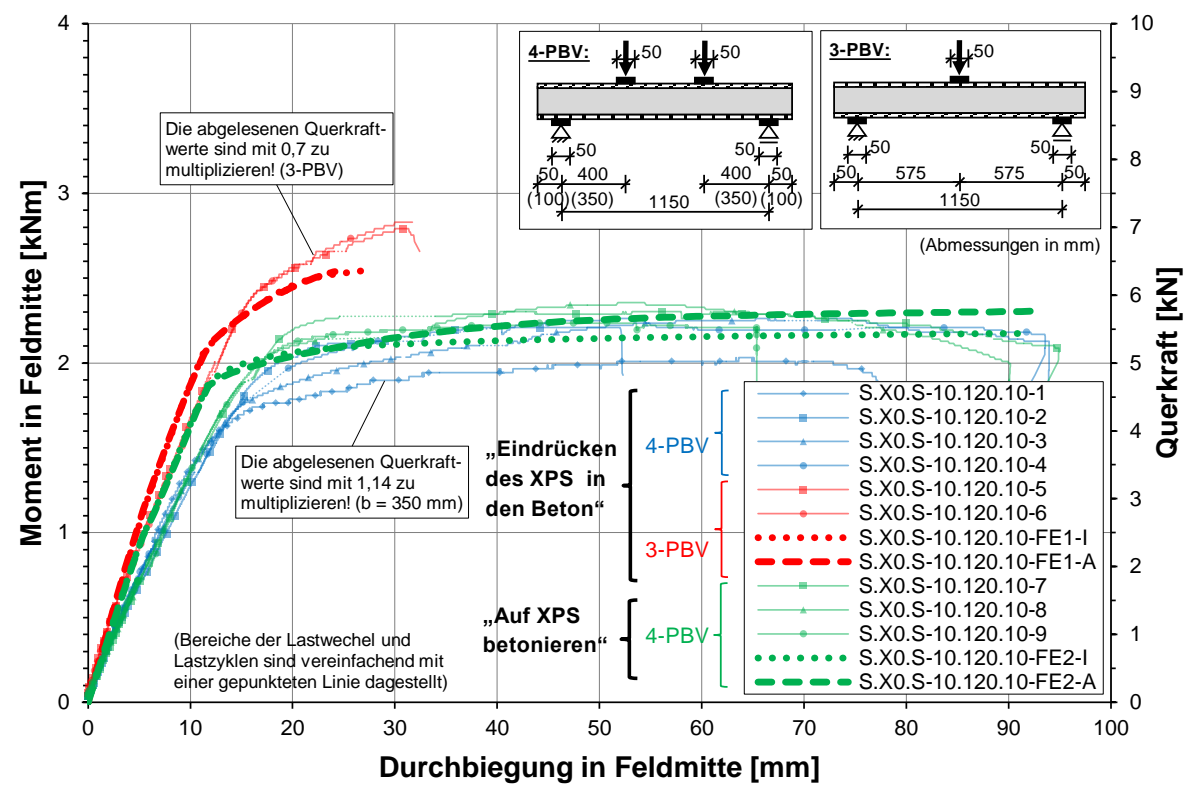

Abb. 6.10: Nachrechnung der Versuchsreihe „Herstellungsmethode“

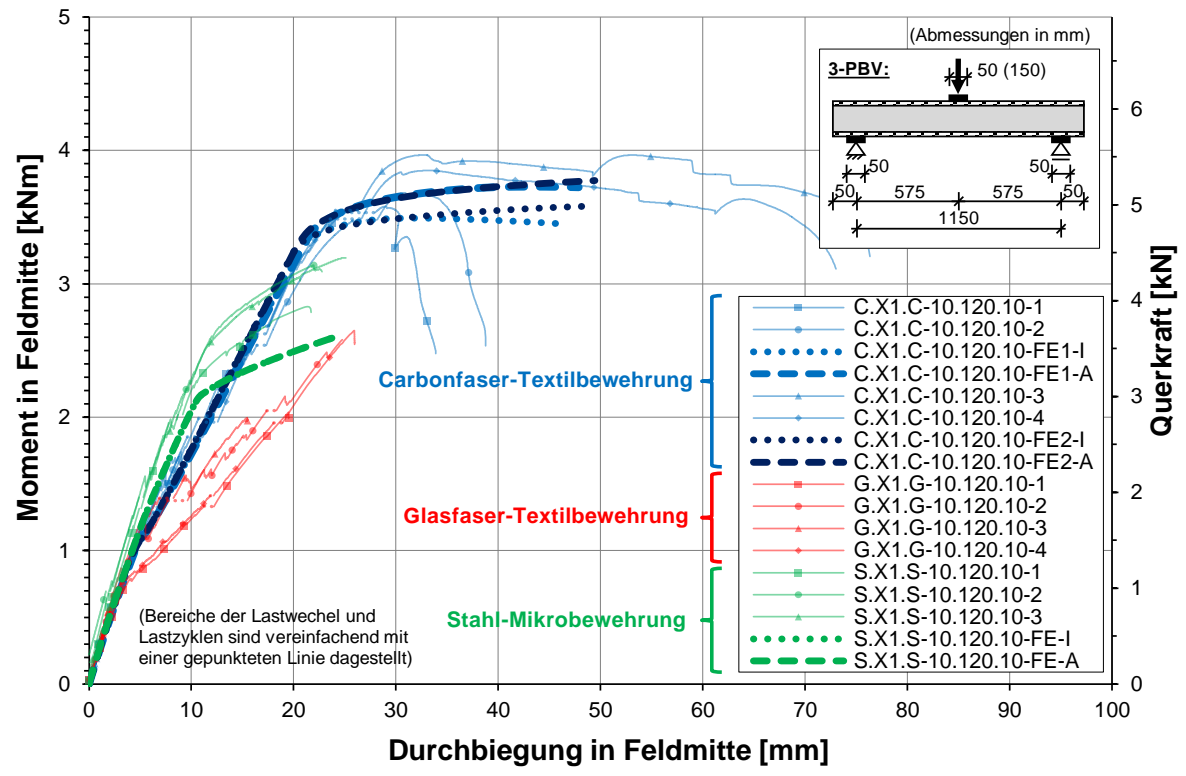

Abb. 6.11: Nachrechnung der Versuchsreihe „Bewehrungsart“ 


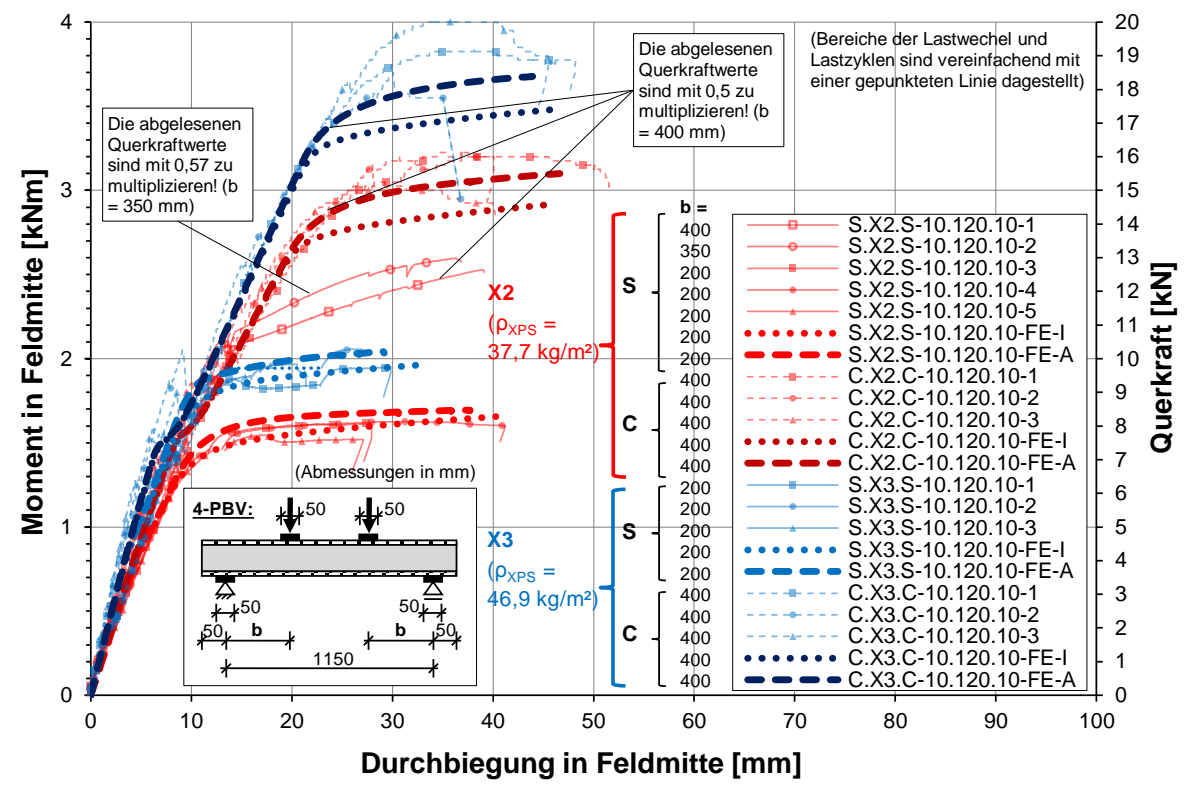

Abb. 6.12: Nachrechnung der Versuchsreihe „Dichte“

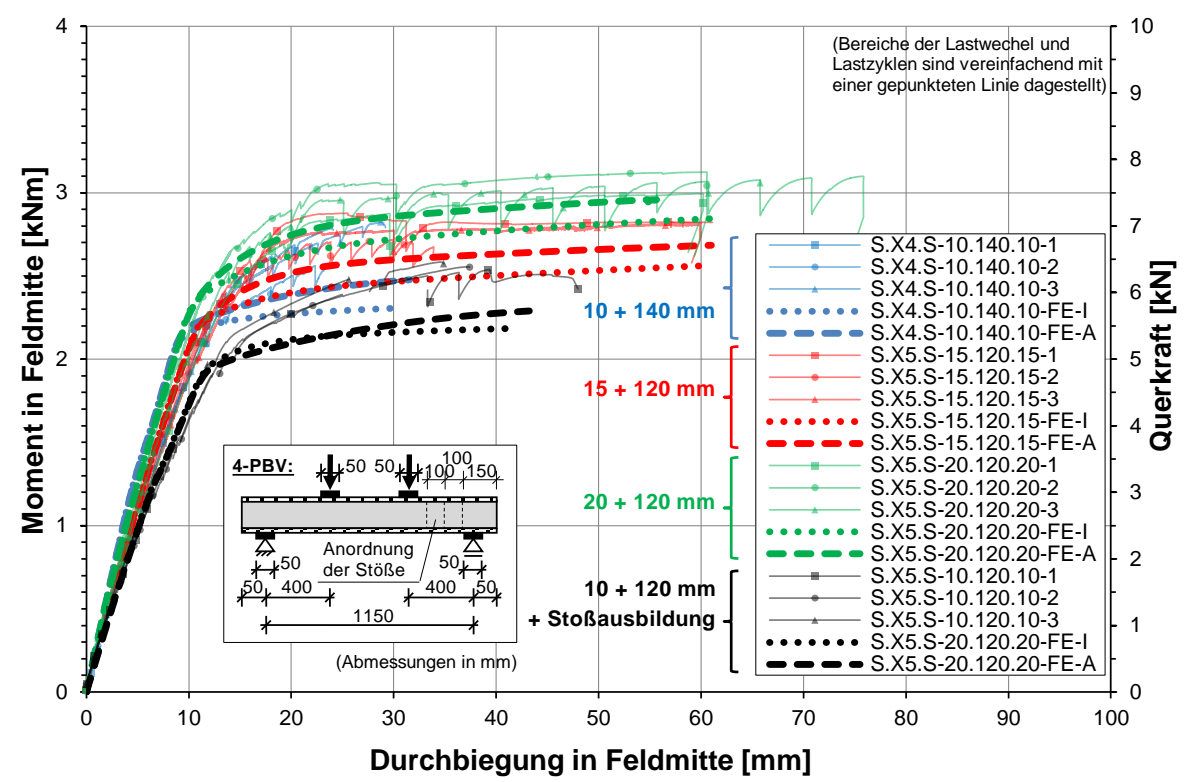

Abb. 6.13: Nachrechnung der Versuchsreihen „Schichtdicken“ und „Stoßausbildung“ 


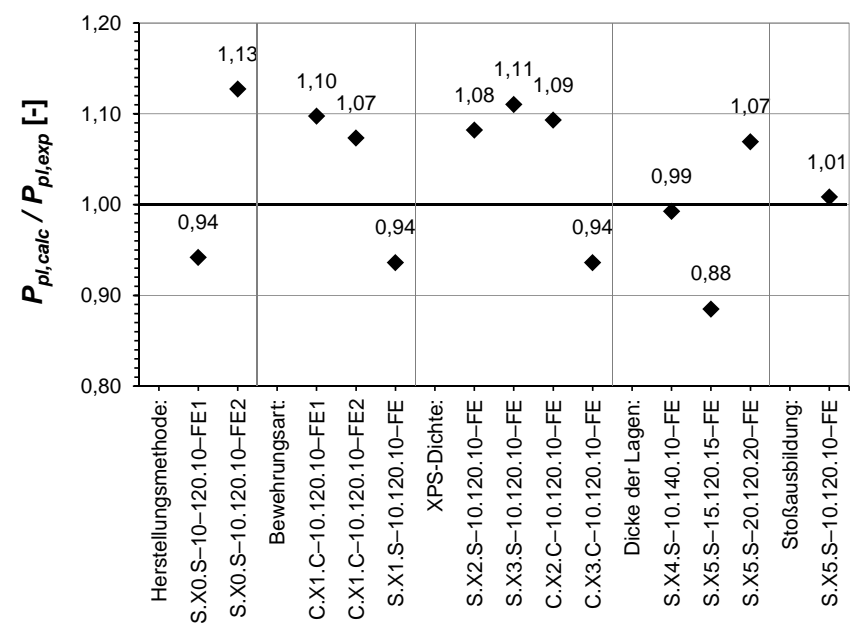

Abb. 6.14: Verhältnis der rechnerisch ermittelten Last bei Erreichen der Plastizitätsgrenze zu dem Mittelwert der Lasten in den entsprechenden Versuchen

Tab. 6.3: Berechnete Lasten bei Erreichen der Plastizitätsgrenze und Beschreibung der in den FE-Modellen auftretenden plastischen Verformungen

\begin{tabular}{|ccl|}
\hline Berechnung & $\begin{array}{c}\boldsymbol{P}_{p l, c a l c} \\
{[\mathrm{kN}]}\end{array}$ & \multicolumn{1}{c|}{ Plastizierung } \\
\hline Herstellungsmethode: & & \\
S.X0.S-10.120.10-FE1 & 7,5 & Fließen der Bewehrung \\
S.X0.S-10.120.10-FE2 & 9,3 & Schubfließen des XPS, Fließen der Bewehrung \\
\hline Bewehrungsart: & & \\
C.X1.C-10.120.10-FE1 & 11,7 & Schubfließen des XPS und lokale Plastizierungen des \\
C.X1.C-10.120.10-FE2 & 11,7 & XPS im Lasteinleitungsbereich \\
S.X1.S-10.120.10-FE & 7,5 & Fließen der Bewehrung \\
\hline XPS-Dichte: & & \\
S.X2.S-10.120.10-FE & 13,2 & \\
S.X3.S-10.120.10-FE & 17,6 & Schubfließen des XPS und lokale Plastizierungen des \\
C.X2.C-10.120.10-FE & 13,2 & XPS im Auflagerbereich \\
C.X3.C-10.120.10-FE & 16,1 & \\
\hline Schichtdicken: & & \\
S.X4.S-10.140.10-FE & 10,7 & Schubfließen des XPS, Fließen der Bewehrung \\
S.X5.S-15.120.15-FE & 11,0 & Schubfließen des XPS und lokale Plastizierungen des \\
S.X5.S-20.120.20-FE & 11,9 & XPS im Auflagerbereich \\
\hline Stoßausbildung: & & \\
S.X5.S-10.120.10-FE & 9,7 & Schubfließen des XPS, Fließen der Bewehrung \\
\hline
\end{tabular}


chen der Plastizitätsgrenze nahezu identisch. Wenn der Übergang in den plastischen Bereich durch das Fließen der Bewehrung verursacht wird, wie in der Modellierung S.X0.S-10.120.10-FE1 und S.X1.S-10.120.10-FE, verlaufen die Kurven des BISO und ANISO-Berechnungen weiterhin in Übereinstimmung. Treten jedoch plastische Verformungen im XPS auf, so verzweigen sich die Kurven der Berechnungen mit dem BISO und ANISO-Materialgesetz. Die mit dem ANISO-Werkstoffgesetz ermittelten Lasten sind im Bereich des Fließplateaus größer als die mit dem BISo-Werkstoffgesetz ermittelten Werte und liegen damit generell näher an den wirklichen Lasten, die in den Versuchen ermittelt wurden.

\subsection{Nachrechnung der Großbauteilversuche}

\subsubsection{Allgemeines}

Um die Ergebnisse der Großbauteilversuche zu interpretieren, wurde diese mithilfe von FE-Modellierungen nachgerechnet. Die Modellierungen wurden zum Teil im Rahmen der Studienarbeit von Ngoga 2014 unter Anleitung durchgeführt.

\subsubsection{Beschreibung des Finite-Element Modells}

Wie bei der Modellierung der Biegeversuche an den Sandwichstreifen, wurden die Parameter für die Materialgesetze der XPS-Charge X6 aus den Tabellen 6.1 und 6.2 entnommen. Die Eingangswerte für das Materialgesetz des carbonfaserbewehrten Betons wurden nach den Gleichungen aus Abschnitt 6.2.2, Eingabewerte für den bewehrten Beton' berechnet. Alle Parameter sind in Tabelle B.3 im Anhang zusammengestellt.

Die Deckschichten der Probekörper wurden mit einer Netzweite von 2,5 mm modelliert. Die Kernschicht wurde bei der Modellierung der Versuche mit Einzellasten mit einer Netzweite von $10 \mathrm{~mm}$ und bei den Versuchen mit Flächenlast mit einer Netzweite von $5 \mathrm{~mm}$ erstellt. Zur Vermeidung von Singularitäten wurden die Lasteinleitungsplatten des Versuchsaufbaus ebenfalls modelliert. Zur Berechnung der Versuche mit Einzellasten wurden zur Lasteinleitung ebenfalls die hölzernen Lastverteilungsbalken und zwei der stählnernen Traversen durch PLANE-Elemente und daran gelenkig angeschlossene BEAM-Elemente modelliert. Die Beanspruchung des Modells erfolgte über die Definition von mittig auf die BEAM-Elemente angesetzte Verformungswerte in $y$-Richtung. Die Beanspruchung der Probekörper mit flächiger Belastung erfolgte über die Eingabe einer Linienlast auf der oberen Deckschicht des Modells (siehe Abbildung6.15.

\subsubsection{Vergleich der Berechnungsergebnisse mit den Versuchsergebnissen}

\subsubsection{Großbauteilversuche mit Beanspruchung durch Einzellasten}

Die nachgerechnete Lastverformungskurve der Versuche mit Einzellasten stimmt sehr gut mit den experimentell ermittelten Kurven überein (siehe Abbildung 6.16. Lediglich 


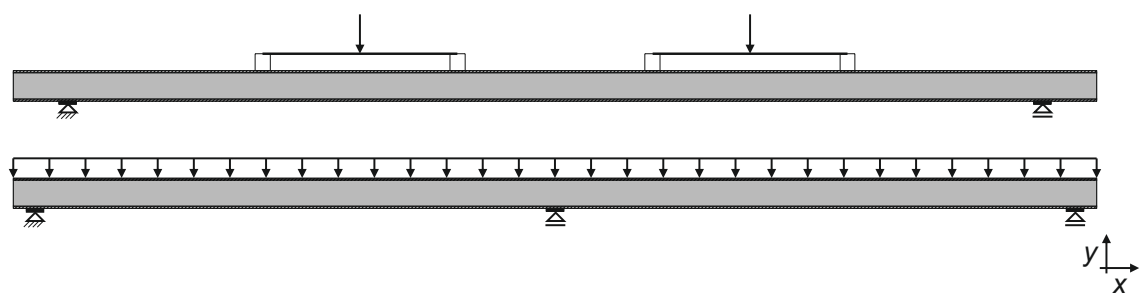

Abb. 6.15: FE-Modelle der Großbauteilversuche mit Beanspruchung durch Einzellasten (oben) und durch eine Flächenlast (unten)

im Bereich der maximalen Belastung sind die berechneten Kurven etwas flacher als die der Versuche. Die Berechnung bricht bei einer maximalen Belastung von 17,2 kN aufgrund des Erreichens der maximal definierten Spannungsdehnungswerte des Materialgesetzes des bewehrten Betons ab. Das Materialgesetz, das anhand der Dehnkörperversuche erstellt wurde, weist im Vergleich zu den vorhandenen Eigenschaften des eingebauten Geleges in den Großbauteilversuchen eine etwas geringere Steifigkeit und eine geringere Zugfestigkeit auf.

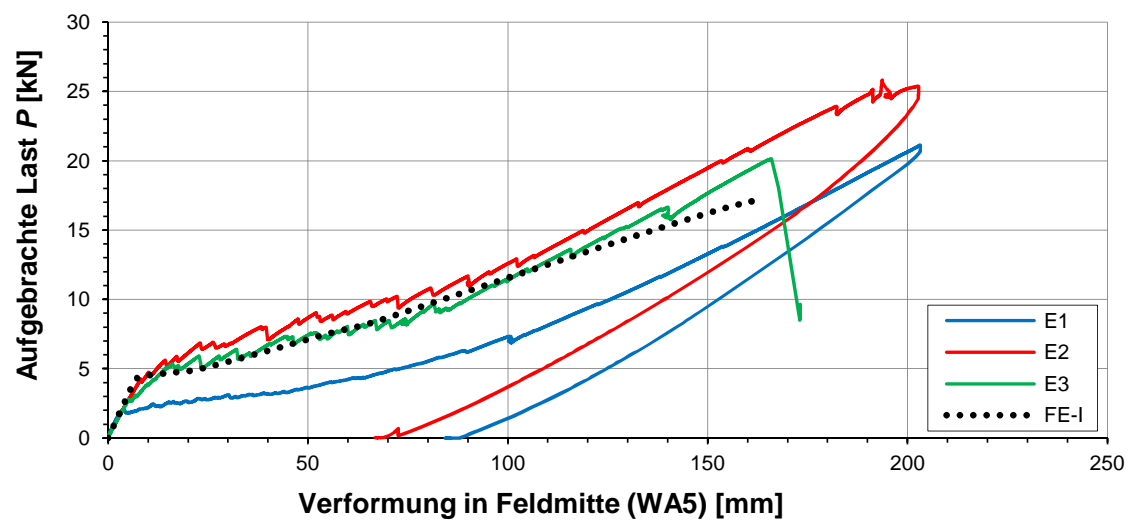

Abb. 6.16: Nachrechnung der Lastverformungskurven der Großbauteilversuche mit Einzellasten

\subsubsection{Großbauteilversuche mit Beanspruchung durch eine Flächenlast}

Die Lastverformungskurven der Modellierung der Versuche mit Flächenbelastung stimmen zu Beginn im Zustand I genau mit den wahren Kurven überein (siehe Abbildung 6.17. Bei den rechnerisch ermittelten Kurven ist der Übergang in den Zustand II gut zu erkennen. Die Kurven flachen deutlich ab und weichen dabei von den experimentell ermittelten Kurven ab. Bei einer im Vergleich zu den Versuchen um etwa $30 \%$ geringeren Belastung von etwa $17 \mathrm{kN} / \mathrm{m}^{2}$ flacht die rechnerisch ermittelte Kurve erneut ab. Grund dafür ist das Überschreiten der Scherfließspannung im Bereich des Mittelauflagers. Die beiden Berechnungen brachen bei einer Verformung von $30 \mathrm{~mm}$ bzw. $35 \mathrm{~mm}$ 
aufgrund des Überschreitens der maximal definierten Zugspannungsdehnungswerte des bewehrten Betons ab.

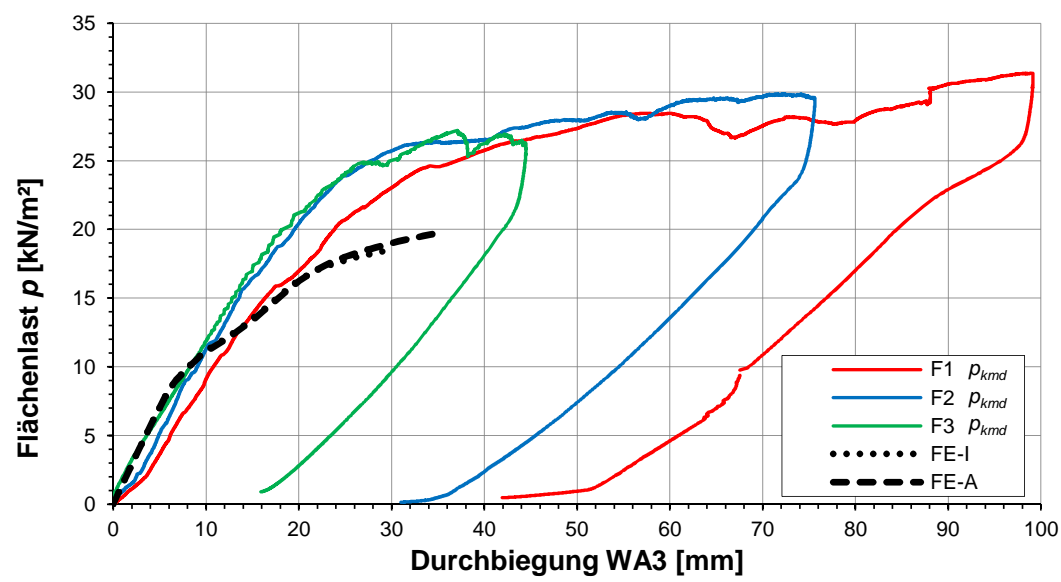

Abb. 6.17: Nachrechnung der Lastverformungskurven der Großbauteilversuche mit Flächenlast

In Abbildung 6.18 sind die rechnerisch und experimentell ermittelten Auflagerkräfte auf der linken Ordinate und die Faktoren $\alpha$ zur Beschreibung der Lasteinzugsfläche der Auflager auf der rechten Ordinate über der Verformung dargestellt. Da die errechnete Flächenlast im Vergleich zu den Versuchen zu gering ist, sind auch die resultierenden Auflagerkräfte im Vergleich zu den Messwerten zu gering.

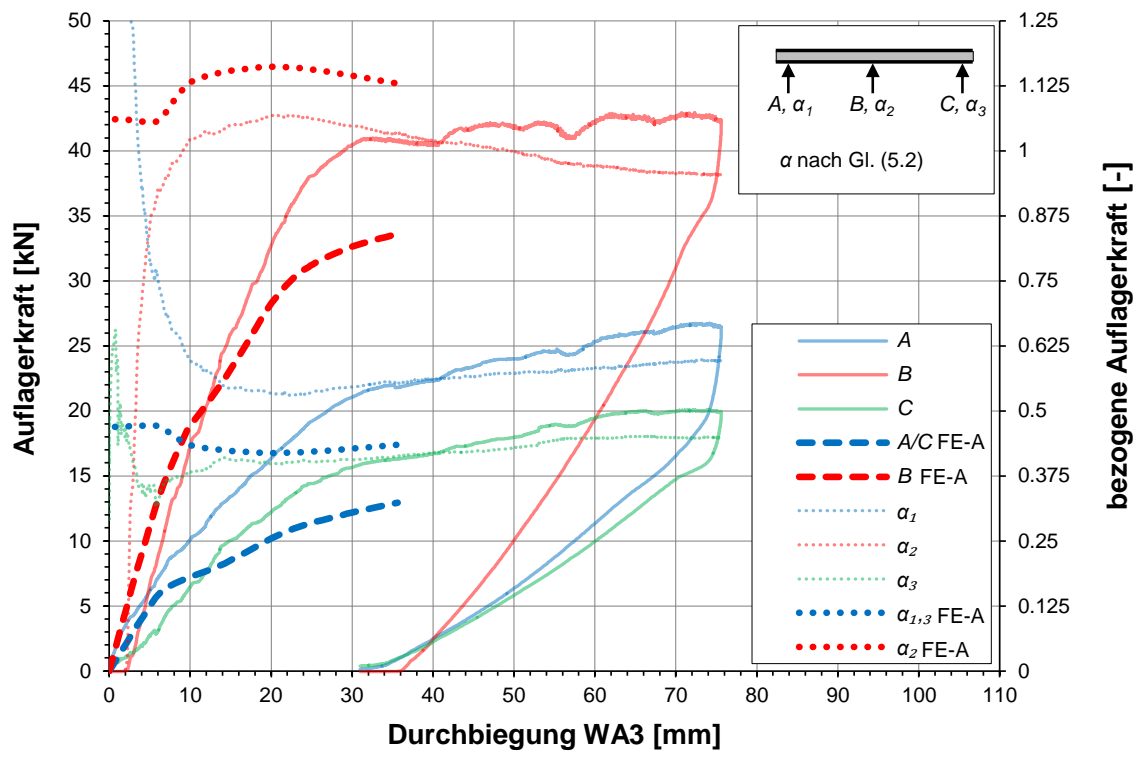

Abb. 6.18: Experimentell und rechnerisch ermittelte Auflagerkräfte und bezogene Auflagerkräfte $\alpha$ über der Verformung der Großbauteilversuche mit Flächenlast 
Die Kurvenverläufe der berechneten $\alpha$-Faktoren stimmen gut mit denen der Versuche überein. Bis zu einer Verformung von etwa $6 \mathrm{~mm}$ sind die $\alpha$-Faktoren etwa konstant. Im Anschluss daran nimmt der $\alpha_{2}$-Faktor zu, während die Faktoren $\alpha_{1}$ und $\alpha_{3}$ abnehmen. Infolge des Reißens der Betondeckschichten verändert sich die Steifigkeit des Bauteils, so dass sich die Lasteinzugsfläche am Mittelauflager vergrößert und die Lasteinzugsflächen an den Endauflagern entsprechend verringern. Dieser Effekt kann auch durch eine Nachrechnung mithilfe der Sandwichtheorie bestätigt werden (siehe Abschnitt 8.2.2.7, Erläuterungen zur Schnittgrößenumlagerung infolge der Rissbildung anhand eines Beispiels' auf Seite 185 ff.). Weiterhin wird durch die Ergebnisse der FEBerechnungen bestätigt, dass die Abnahme des $\alpha_{2}$-Faktors am Mittelauflager bei einer Verformung von etwa $20 \mathrm{~mm}$ durch die Plastizierung des Kerns sowie der Bewehrung im Bereich des Mittelauflagers verursacht wird.

In den nachfolgenden Abbildungen 6.19, 6.20 und 6.21 sind die Scherspannungen im Verbund und im Kern, die Druckspannungen in $y$-Richtung im Kern sowie die Zug- und Druckspannungen in $x$-Richtung bei einer Flächenbelastung von $15 \mathrm{kN} / \mathrm{m}^{2}$ dargestellt. Bei dieser Belastung treten im Kern und im Verbund rechts und links des Mittelauflagers Spannungen auf, die die definierte Fließscherspannung von $150 \mathrm{kN} / \mathrm{m}^{2}$ erreichen (siehe Abbildung 6.19).

Die Spannungen in $y$-Richtung im Kern im Bereich des Mittelauflagers erreichen einen Wert von $-125 \mathrm{kN} / \mathrm{m}^{2}$ und sind somit geringer als die definierte Druckfestigkeit von $480 \mathrm{kN} / \mathrm{m}^{2}$ (siehe Abbildung 6.20). In der Modellierung treten daher keine plastischen Verformungen in $y$-Richtung im Bereich des Mittelauflagers auf, wie sie aufgrund der starken Zusammendrückung in den Versuchen beobachtet wurden.

In Abbildung 6.21 sind die Zug- und Druckspannungen in $x$-Richtung auf den Außenseiten der Deckschichten dargestellt. Auf der oberen Deckschicht treten über den Auflagern Zugspannungen auf, die zum Reißen des Betons führen. In den Feldern stellt sich eine Druckspannung von maximal $-12,5 \mathrm{~N} / \mathrm{mm}^{2}$ ein. Diese entspricht der Spannung, die in den Versuchen aus den gemessenen Dehnungswerten und dem Elastizitätsmodul des Betons von $43.000 \mathrm{~N} / \mathrm{m}^{2}$ berechnet werden konnten (siehe Abbildung 5.68 s. 120 .

An der Unterseite der unteren Deckschicht treten im Bereich der Auflager lokal große Druckspannungen auf. Diese resultieren aus den Druckzonen, die sich infolge der Biegebeanspruchung an den Außenkanten der Lastverteilungsplatten einstellen. In den Feldern wird die untere Deckschicht nahezu vollständig durch Zugspannungen beansprucht. Anhand der Krümmungen in den Spannungslinien und der Spannungswerte, lassen sich die Deckschichten in Bereiche mit den jeweiligen Zuständen I, II a und II b unterteilen. 

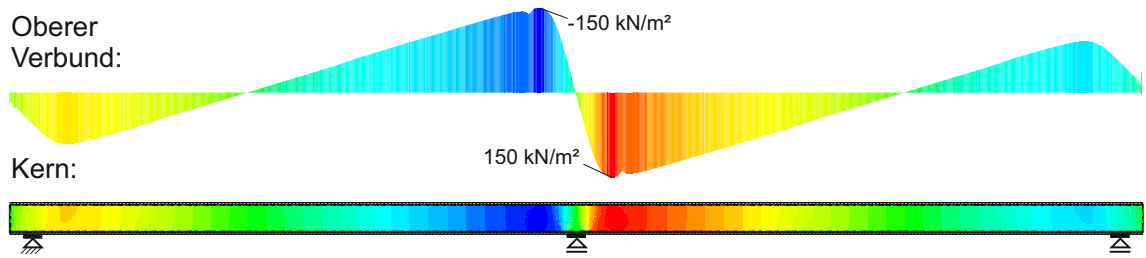

Unterer
Verbund:

$150 \mathrm{kN} / \mathrm{m}^{2}$

Scherspannungen $\left[\mathrm{kN} / \mathrm{m}^{2}\right]$ :

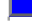

$-75,0$

$-37,5$

0,0

37,5

75,0

112,5

Abb. 6.19: Scherspannungen im oberen und unteren Verbund sowie im Kern bei $p=15 \mathrm{kN} / \mathrm{m}^{2}$

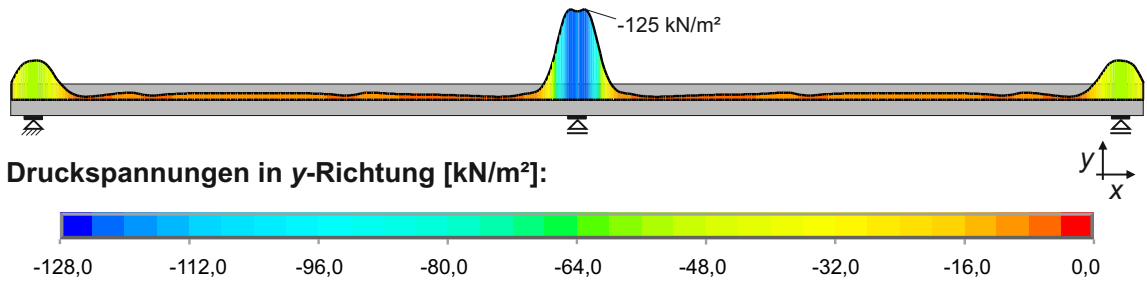

Abb. 6.20: Druckspannungen in $y$-Richtung mittig im Kern bei $p=15 \mathrm{kN} / \mathrm{m}^{2}$

Oberseite obere Deckschicht:
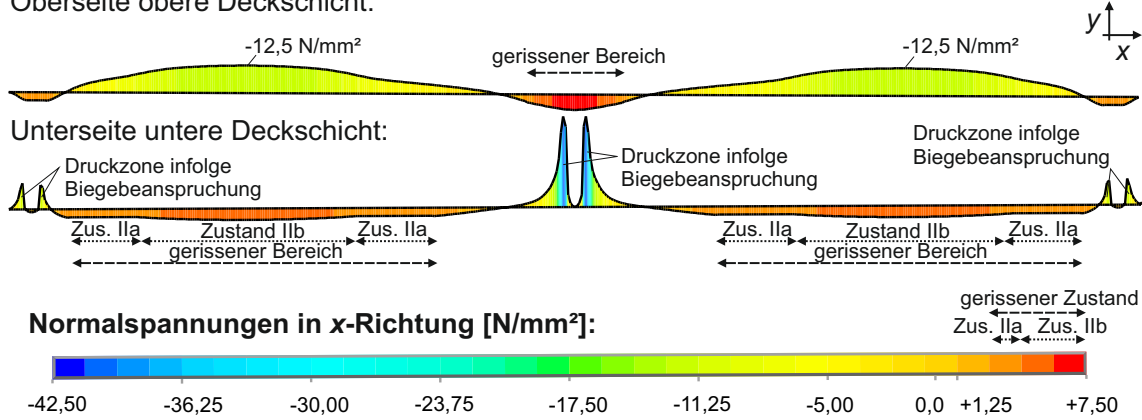

Abb. 6.21: Normalspannungen in $x$-Richtung auf den Außenseiten der Deckschichten bei $p=$ $15 \mathrm{kN} / \mathrm{m}^{2}$ 



\section{$7 \quad$ Zusammenfassung und Diskussion der Ergebnisse}

\subsection{Generelle Beschreibung des Biegetragverhaltens der Sandwichelemente}

Die untersuchten Sandwichelemente weisen ein typisches Sandwichtragverhalten auf, wie es auch bei den Sandwichelementen mit metallischen Deckschichten beobachtet werden kann. Die Betondeckschichten werden überwiegend durch Normalspannungen beansprucht. Diese resultieren aus den Deckschichtmomenten $M_{o}$ und $M_{u}$ sowie aus den Normalkräften $N_{s}$, die sich aus dem Sandwichmoment $M_{s}$ ergeben. Der XPS-Kern der Sandwichelemente wird überwiegend durch Schubspannungen $\tau$ beansprucht, die aus der Querkraft $V_{s}$ resultieren.

Der Elastizitätsmodul des Hochleistungsbetons ist etwa um den Faktor 1.000 größer, als der Elastizitätsmodul des XPS. Aufgrund des Hookeschen Gesetzes, sind die Normalspannungen der Deckschichten dadurch ebenfalls um den Faktor 1.000 größer, als die Normalspannungen im Kern. Die Vernachlässigung der Normalspannungen des Kerns bei der Berechnung der Schnittgrößen mithilfe der Sandwichtheorie ist daher problemlos möglich.

Zur besseren Verdeutlichung des Tragverhaltens der Sandwichelmente sind die Ergebnisse einer FE-Modellierung eines 4-Punkt Biegeversuchs an einem Sandwichstreifen in Abbildung 7.1 dargestellt. Die Hauptzug- und Hauptdruckspannungen des Kerns sind als Pfeile vektoriell dargestellt. Die Bereiche der Deckschichten die durch Zugund Drucknormalspannungen beansprucht werden, sind jeweils in roter und blauer Farbe dargestellt.

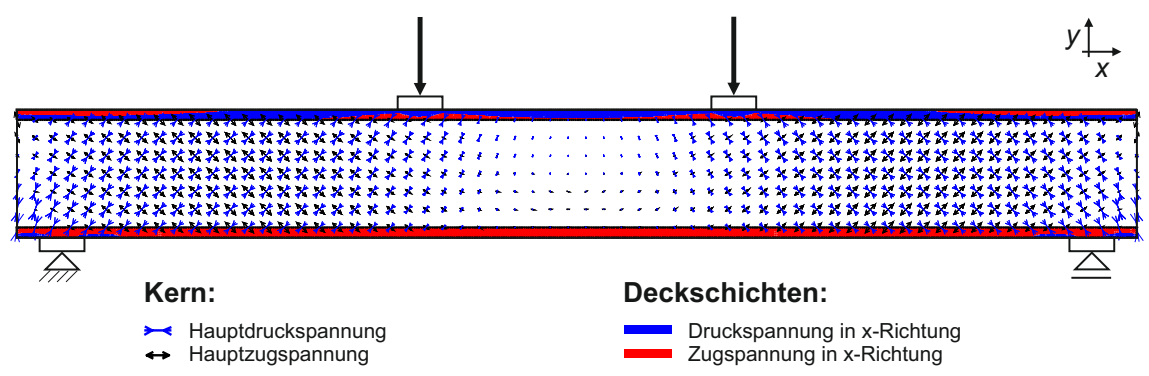

Abb. 7.1: Darstellung der Spannungen im Kern und in den Deckschichten eines 4-Punkt Biegeversuchs (S.X5.S.-10.120.10-FE-A) an der Plastizitätsgrenze 
Die untere Deckschicht wird durch die Zugnormalkraft aus dem Sandwichmoment nahezu vollständig durch Zugspannungen beansprucht. In den Versuchen stellten sich infolge dessen Trennrisse ein. Zum linken und rechten Ende des Sandwichstreifens nimmt das Sandwichmoment ab, während durch die Einleitung der Auflagerkräfte gröBere Deckschichtmomente entstehen. Aus diesem Grund treten in diesem Bereich in den Versuchen zum Teil Biegerisse auf. In der FE-Modellierung treten infolge der Deckschichtmomente Druckspannungen auf.

Die obere Deckschicht wird infolge der Drucknormalkraft aus dem Sandwichmoment überwiegend durch Druckspannungen beansprucht. Im Bereich der Lasteinleitung entstehen durch große lokale Deckschichtmomente Zugspannungen auf der Unterseite der Deckschicht. Die großen Deckschichtmomente und die dadurch resultierenden Zugspannungen werden in den Versuchen zum Teil durch die Bildung von Biegerissen deutlich.

Auf der Oberseite der oberen Deckschicht treten am linken und rechten Ende ebenfalls Zugspannungen auf, welche durch das Auftreten von Rissen in den Versuchen bestätigt werden. In diesem Bereich wird die obere Deckschicht durch die Einleitung der Hauptzugspannungen im Kern einer Biegebeanspruchung ausgesetzt. Es entstehen negative Deckschichtmomente die zu Biegerissen führen.

Im Kern sind im Bereich zwischen den Auflagern und den Lasteinleitungspunkten die Hauptspannungen am größten. In diesem Bereich wird die Querkraft bzw. die daraus resultierenden Schubspannungen in Form von Hauptspannungen übertragen. Dabei verlaufen die Hauptzug- und Hauptdruckspannungen im Winkel von etwa $45^{\circ}$ zueinander und übertragen dabei kontinuierlich die Schubkräfte zwischen oberer- und unterer Deckschicht. Die Schubverformung des Kerns konnte durch eine konstante Neigung der aufgezeichneten Linien zu den Auflagern hin, wahrgenommen werden.

\subsection{Schertragverhalten des XPS}

\subsubsection{Allgemeines}

Das Materialverhalten von XPS unter Schubbeanspruchung ist zu Beginn der Belastung nahezu linear-elastisch und geht bei steigender Beanspruchung in einen plastischen Bereich über. Die Plastizierung des XPS wird durch die Verformung der Zellstruktur des XPS hervorgerufen. Infolge der Scherbeanspruchung bilden sich, wie durch Gibson u. Ashby 2001 beschrieben, an den Knotenpunkten der Zellkanten Fließgelenke aus. Weiterhin beulen und knicken die Zellwände und Zellkanten aus. Durch Computertomographie-Aufnahmen von XPS-Proben, die aus den Probekörpern entnommen wurden, kann dies belegt werden.

Dazu wurde je eine würfelförmige XPS-Probe aus dem Bereich der plastischen Verformungen sowie aus der Mitte des Probekörper S.X5.S-15.120.15-3 entnommen. Mithilfe der Computertomographie wurden Visualisierungen der Zellstruktur erstellt. In der Visualisierung der Probe, die aus dem Bereich mit plastischen Verformungen entnommen wurde (siehe Abbildung 7.3 ist zu erkennen, dass die Zellwände des XPS zum Teil stark verformt bzw. geknittert sind (gekennzeichnet durch schwarze Kreise). Die 

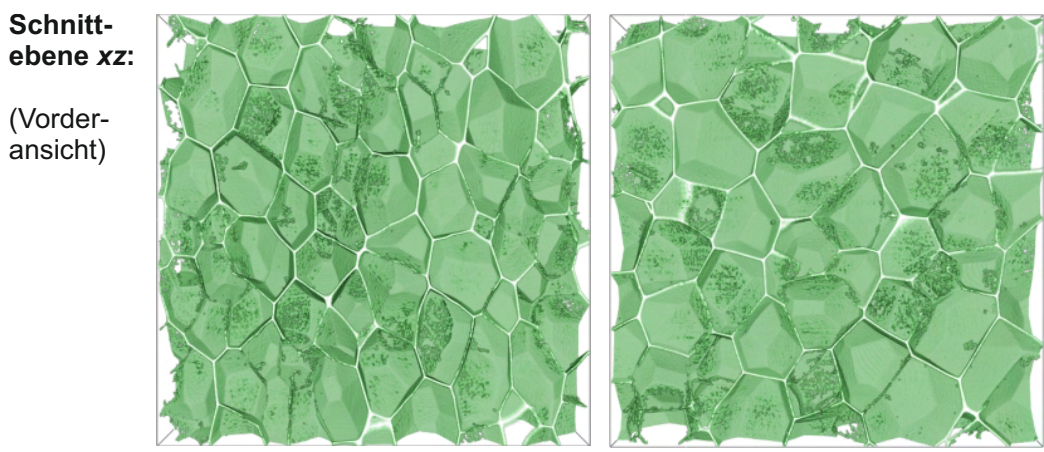

Schnittebene $y z$ :

(Seitenansicht)

Schnittebene $x y$ :

(Draufsicht)
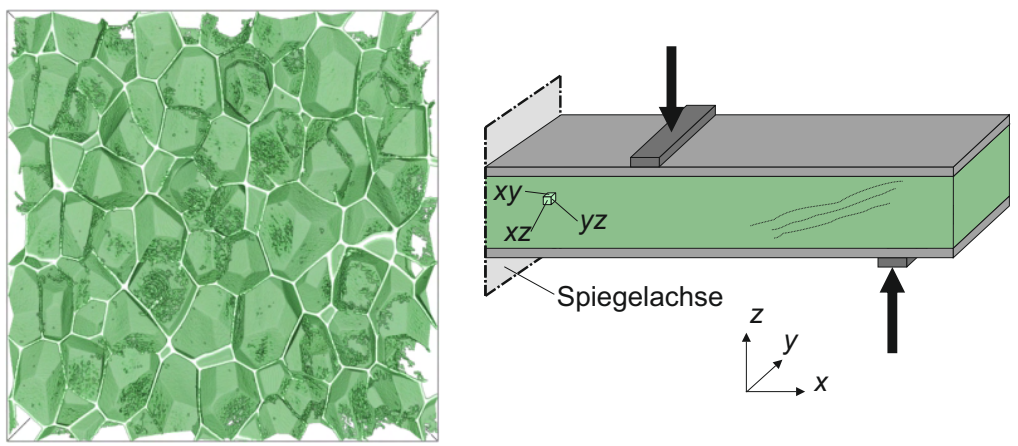

Abb. 7.2: CT-Visualisierung von XPS aus dem ungestörten Bereich (S.X5.S-15.120.15-3)

Schnittebene $x z$ :

(Vorderansicht)
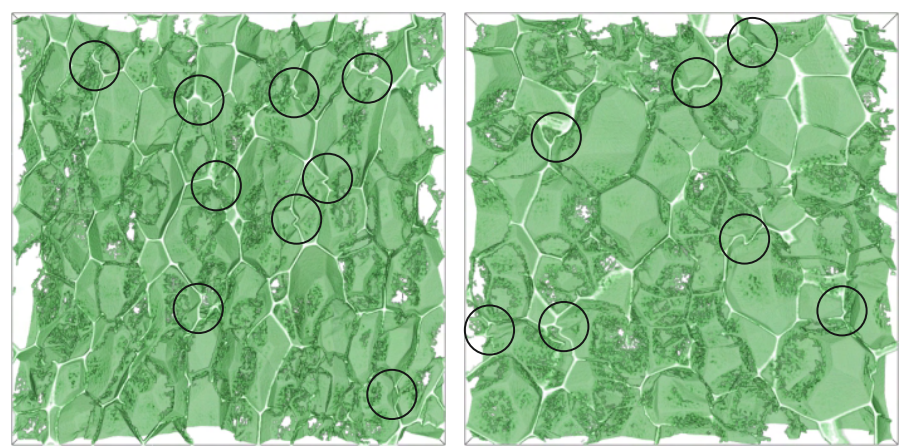

Schnittebene $y z$ :

(Seitenansicht)

Schnittebene $x y$ :
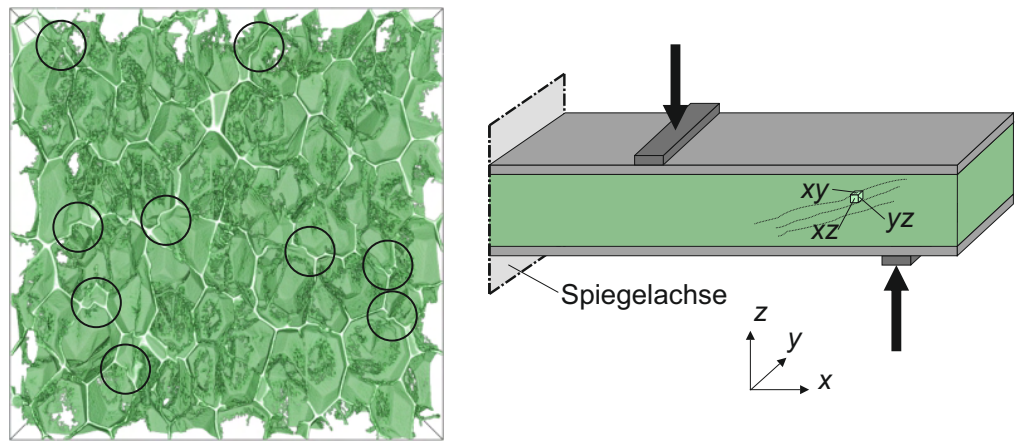

Abb. 7.3: CT-Visualisierung des XPS aus dem Bereich plastischer Verformungen (S.X5.S15.120.15-3) 
Zellstruktur der Probe, die aus der Mitte des Probekörpers entnommen wurde, lässt keine Deformierungen erkennen (siehe Abbildung 7.2).

Sowohl in den Scher- als auch in den Biegeversuchen war durch die Krümmung der aufgezeichneten Linien zu erkennen, dass die plastischen Verformungen im XPS lokal auftraten und dabei meist diagonal, in Richtung der Hauptzugspannungen über dem Kern ausgerichtet waren. In den Scherversuchen verlief diese Diagonale von der linken unteren bis zur rechten oberen Ecke des Probekörpers. In den Biegeversuchen bildete sich ebenfalls ein diagonaler Bereich plastischer Verformungen von der unteren Deckschicht bis zum linken und rechten Ende der oberen Deckschicht aus.

Durch die Verwendung des fotogrammetrischen Messsystems konnten diese Verformungen auch quantifiziert werden. In Abbildung 7.4 sind die Verformungen senkrecht zur Oberfläche des Probekörpers C.X2.C-10.120.10-3 dargestellt. Dabei wurde das XPS im blaueingefärbten Bereich zum Teil über $0,5 \mathrm{~mm}$ eingeschnürt. Über die Messsoftware konnten auch die Scherdehnungen ausgegeben werden (siehe Abbildung 7.5. Diese erreichten im rot eingefärbten Bereich bis zu $8 \%$.
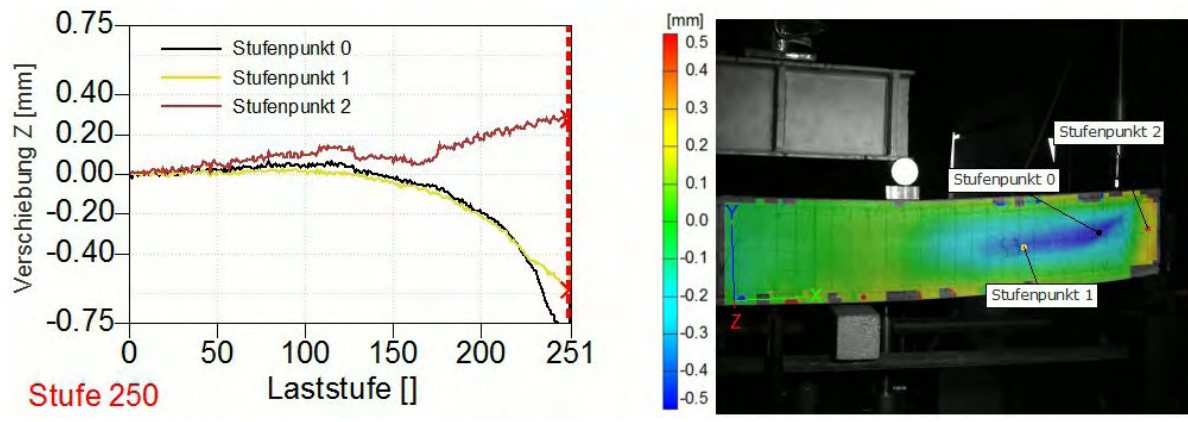

Abb. 7.4: Verschiebung der Oberfläche in z-Richtung
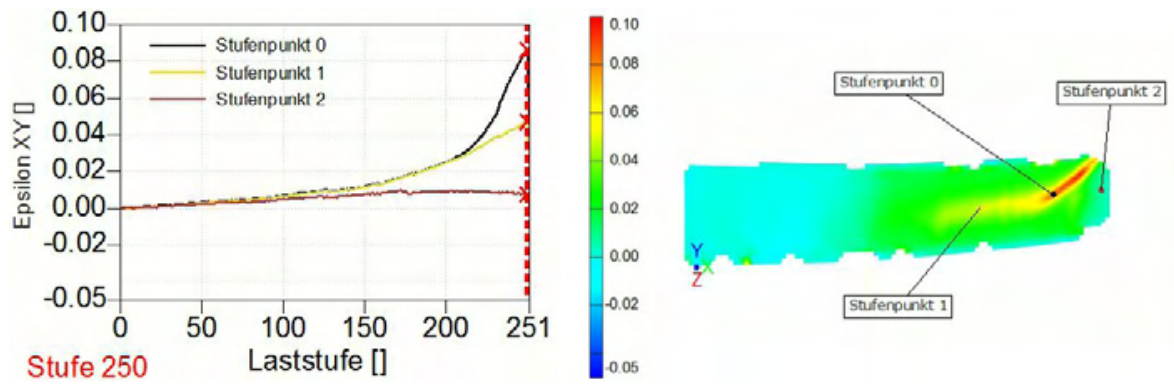

Abb. 7.5: Scherdehnungen

Das Stabilitätsversagen der Zellwände und Zellkanten durch Ausknicken und Ausbeulen stellt eine lokale Schwächung des XPS dar. Bei weiterer Belastung nehmen die Dehnungen daher in diesem geschwächten Bereich lokal weiter zu und zwar in Richtung der Hauptspannungen. Eine Steigerung der aufnehmbaren Spannungen in diesem Bereich ist nur begrenzt möglich. Das Versagen der Zellstruktur bewirkt weiterhin, dass das XPS durch die Zugbeanspruchung eingeschnürt wird. 
Diese lokalen Plastizierungseffekte konnten mit dem erstellten FE-Modell nicht abgebildet werden. Bei der Nachrechnung der Versuche wies das XPS-Materialgesetz im plastischen Bereich einen ansteigenden Ast auf. Dies hatte zur Folge, dass das XPS nicht nur in einem lokalen Bereich, sondern über die gesamte Dicke des Kerns plastizierte. Weiterhin konnte bei den beiden verwendeten Materialgesetzen nur eine konstante Querdehnzahl definiert werden. Zur Simulierung der Plastizierungs- und Einschnürungseffekte scheint es jedoch erforderlich, die Abnahme der Querdehnzahl im plastischen Materialbereich zu berücksichtigen.

\subsubsection{Kritische Betrachtung der Scherversuche}

Für die Scherversuche wurde der einhüftige Aufbau mit Einzelprobekörpern nach DIN EN 12090 verwendet. Es wurden Probekörper mit einer Dicke $d$ von 120 bzw. $140 \mathrm{~mm}$ geprüft. Die Dicke der Probekörper war somit größer, als die in der Norm geforderten Probekörper mit einer Dicke von $50 \mathrm{~mm}$. Es ist zu vermuten, dass das Verhältnis der Länge $l$ und der Dicke $d$ einen Einfluss auf das Tragverhalten der Probekörper hat. Mit dem FE-Modell zur Nachrechnung der Scherprobekörper wurden daher Probekörper mit unterschiedlichem $l / d$-Verhältnis berechnet. Dabei wurde die Dicke mit $120 \mathrm{~mm}$ und die Länge der Probekörper entsprechend mit 120, 250, 600 und $1.200 \mathrm{~mm}$ festgelegt. Die Berechnungen wurden sowohl mit dem BISO als auch mit dem ANISOMaterialgesetz durchgeführt (siehe Abbildung 7.6 und 7.7). Wie bereits in Abschnitt 6.3.1 (siehe S. 135 beschrieben, wurden die Scherspannungen aus den Auflagerkräften bezogen auf die Scherfläche $l \cdot b$ berechnet.

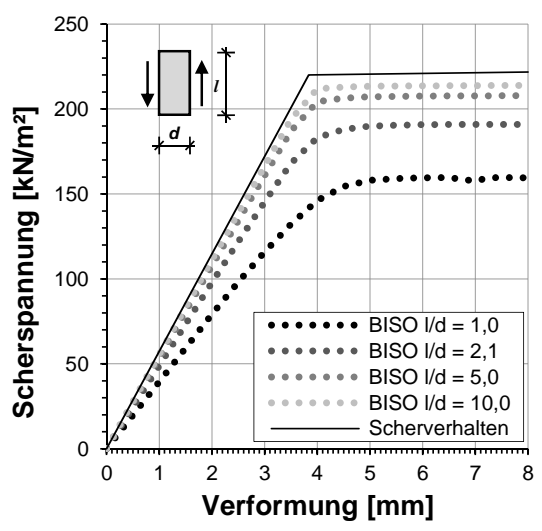

Abb. 7.6: Mit Materialoption BISO berechnete ScherspannungsVerformungs-Kurven bei unterschiedlichem $l / d$-Verhältnis und XPS-Charge X1

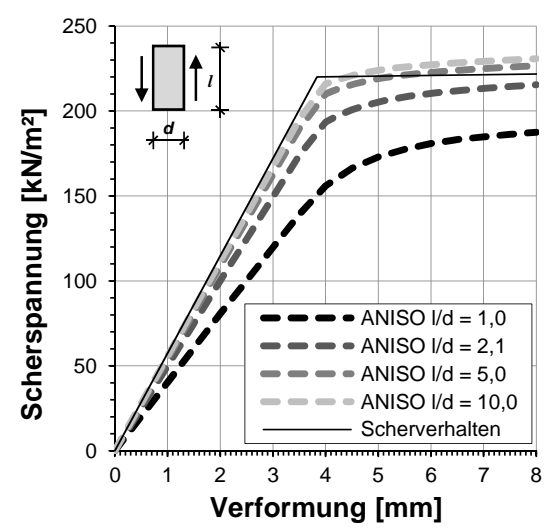

Abb. 7.7: Mit Materialoption ANISO berechnete ScherspannungsVerformungs-Kurven bei unterschiedlichem $l / d$-Verhältnis und XPS-Charge X1

In beiden Diagrammen lässt sich erkennen, dass die Lastverformungskurven der Probekörper mit geringem $l / d$-Verhältnis flacher verlaufen und geringere maximale Scherspannungen erreichen, als die Probekörper mit einem hohen $l / d$-Verhältnis. Generell liegen die mit dem ANISO-Materialgesetz bestimmten Kurven näher an der theoreti- 
schen Kurve, die über die Eingabe der Schereigenschaften definiert wurde (schwarze, durchgezogene Linie).

Die Verläufe der Scherspannungsverformungskurven der Probekörper mit einem $l / d$ Verhältnis von 5,0 und 10,0 sind in etwa gleich und die Fließspannungen unterscheiden sich nur um etwa $2 \%$. Die Fließspannung der Modellierung mit einem $l / d$-Verhältnis von 2,1, das auch die Probekörper in den Versuchen aufwiesen, ist hingegen um rund $10 \%$ geringer, als die der Modellierung mit einem Verhältnis von 10,0.

Die Abschäleffekte in den Eckbereichen der Probekörper wirken sich bei Scherprobekörpern mit geringem $l / d$-Verhältnis stärker auf das Lastverformungsverhalten und die maximal erreichbaren Scherspannungen aus, als bei Probekörpern mit großem $l / d$-Verhältnis. Die Abweichungen der verwendeten Probekörper mit einer Länge und Breite von 250 und $120 \mathrm{~mm}$ liegen noch im tolerierbarem Bereich. Jedoch wird empfohlen, die Länge der Probekörper grundsätzlich auf die fünffache Dicke festzulegen. Somit ist eine bessere Vergleichbarkeit der Prüfergebnisse gegeben.

\subsection{Einfluss der verschiedenen Parameter auf das Biegetragverhalten der Sandwichelemente}

\subsubsection{Herstellungsmethode}

Der Einfluss der beiden Herstellungsvarianten auf das Tragverhalten der Biegeprobekörper war nur gering. Grundsätzlich erzielten die Probekörper bei denen der Kern in den frischen Beton gedrückt wurde zum Teil nur geringfügig niedrigere Versagenslasten. Durch das nachträgliche Aufschneiden der Probekörper und entfernen des XPS an den Deckschichten lies sich jedoch feststellen, dass die Probekörper der Methode „Eindrücken des XPS-Kerns in den Beton“ teilweise erhebliche Fehlstellen im Verbund aufwiesen (siehe Abbildungen im Anhang A.1.1 S. 244). Der Verbund der Probekörper der Methode „Betonieren auf dem Kern“ wies hingegen eine nahezu fehlerlose Beschaffenheit auf.

Die Probekörper der Versuchsreihe „Herstellungsmethode“ waren die ersten Probekörper, die im Rahmen dieser Arbeit hergestellt wurden. In den weiteren Versuchsreihen konnte durch die gewonnenen Erfahrungen die Herstelltechnik weiter optimiert werden, so dass die Verbundqualitäten an die der Variante "Direktes Betonieren auf dem Kern“ heranreichten (siehe Anhang A.1.1, S. 244. Auch bei der Herstellung der Großbauteilprobekörper konnte mit der Variante „Eindrücken des XPS-Kerns in den Beton“ bei Verwendung der Gewichte und des Flächenrüttlers ein sehr guter Verbund erzielt werden, der überwiegend nur kleinere Luftblasen von $d<50 \mathrm{~mm}$ aufwies (siehe Anhang A.5.3 S. 265.

\subsubsection{Bewehrungsart}

Der Bewehrungsgrad und die Art des Bewehrungsmaterials beeinflusst deutlich die Steifigkeit und die Traglast eines Sandwichelements. Der Elastizitätsmodul und die 
Querschnittsfläche der Bewehrung geht indirekt über den Elastizitätsmodul der Deckschichten in die Berechnung der Sandwichbiegesteifigkeit $B_{s}$ und der Eigenbiegesteifigkeiten der Deckschichten $B_{o}$ und $B_{u}$ ein. Die Glasfaser-Textilbewehrung wies den geringsten Elastizitätsmodul und die geringste Querschnittsfläche auf. Folglich war auch die Steifigkeit im Zustand II der Probekörper am geringsten.

Die Probekörper mit Mikro- und Carbonfaserbewehrung wiesen in etwa den gleichen Bewehrungsquerschnitt auf. Auch die Elastizitätsmoduln der reinen Bewehrungsmaterialien, d. h. des Stahldrahts und der Carbonfaser, waren in etwa gleich. Jedoch wiesen die mit Carbonfasergelege bewehrten Biegeprobekörper im Zustand II eine geringere Steifigkeit auf als die mikrobewehrten Probekörper. Wie bei den Dehnkörperversuchen auch, kann die geringe Steifigkeit mit der Welligkeit der Filamente begründet werden. Nach dem Reißen des Betons zogen sich die Filamente gerade. Dies führte zu einer Dehnungszunahme und zu einer Verringerung der Steifigkeit des Geleges.

Zur exakten Berechnung der Spannungen, Verformungen und Schnittgrößen sollten daher die effektiven Elastizitätsmoduln aus Dehnkörperversuchen verwendet werden. Mithilfe der effektiven Elastizitätsmoduln der Mikrobewehrung und der Carbonfaserbewehrung, die in den Dehnkörperversuchen zu etwa $160.000 \mathrm{~N} / \mathrm{mm}^{2}$ bzw. $60.000 \mathrm{~N} / \mathrm{mm}^{2}$ bestimmt wurden, lässt sich der Steifigkeitsunterschied der beiden Materialien im Zustand II begründen.

\subsubsection{Dichte des XPS}

Durch die Dichte des XPS wird das Schubtragverhalten eines Sandwichelements entscheidend beeinflusst. Bei steigender Dichte des XPS nimmt die Zellgröße ab. Somit vergrößern sich folglich auch die Festigkeiten und Steifigkeiten des XPS. Entscheidend für das Sandwichtragverhalten sind dabei der Schubmodul und die Scherfestigkeit des XPS.

Es wurden zwei Parameterstudien auf Basis der Finite-Element-Methode durchgeführt, bei denen diese beiden Parameter variiert wurden. Die Berechnung erfolgte aufbauend auf der Modellierung der Versuche C.X2.C-10.120.10, die als 4-Punkt-Biegeversuche mit Carbonfaser-Bewehrung und der XPS-Charge X2 durchgeführt wurden. Das XPS wurde dabei mit dem ANISo-Materialgesetz modelliert. In der ersten Parameterstudie wurden nur der Schubmodul $G_{x y}$ variiert, während in der zweiten Parameterstudie nur die Scherfließspannung $\tau_{x y}$ verändert wurde. Alle anderen Materialeigenschaften des XPS-Materialgesetzes blieben unverändert.

In den Abbildung 7.8 und 7.9 sind die berechneten Lastverformungskurven der beiden Studien dargestellt. Eine Vergrößerung des Schubmoduls $G$ bewirkt eine Steigerung der Schubsteifigkeit $S$, die nach Gleichung 3.19 (siehe S. 30) berechnet wird. Eine Vergrößerung der Schubsteifigkeit resultiert folglich in einem insgesamt steiferen Bauteilverhalten des Sandwichstreifens. Durch das Diagramm in Abbildung 3.19 wird weiterhin deutlich, dass der Zusammenhang zwischen Schubmodul und Verformung nicht linear ist. Bei abnehmendem Schubmodul nimmt die Verformung überproportional zu. 


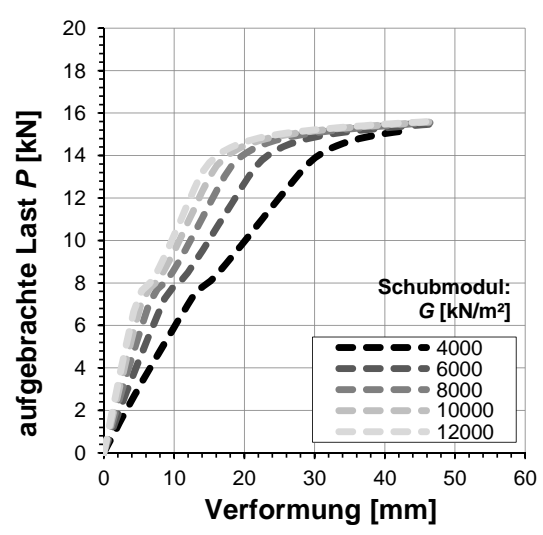

Abb. 7.8: FEM-Parameterstudie zur Untersuchung des Einflusses des Schubmoduls des XPS auf das Lastverformungsverhalten

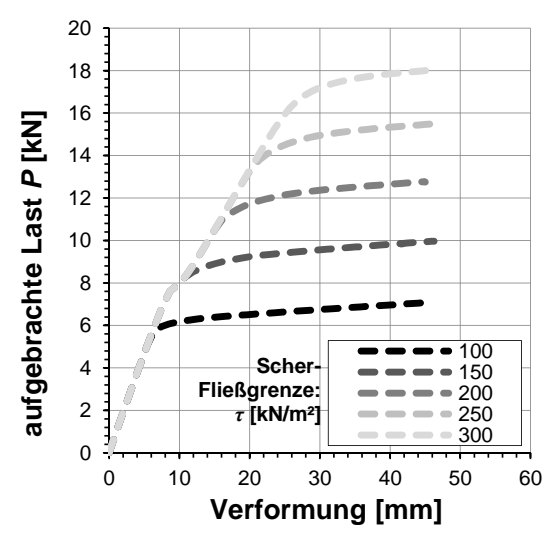

Abb. 7.9: FEM-Parameterstudie zur Untersuchung des Einflusses der Scherfestigkeit des XPS auf das Lastverformungsverhalten

Eine Vergrößerung der Scherfließspannung $\tau_{x y}$ hingegen beeinflusst die Steifigkeit des Bauteils nur in der Hinsicht, dass die Lastverformungskurve erst bei einer höheren aufgebrachten Last $P$ in den plastischen Verformungsbereich übergeht.

\subsubsection{Schichtdicken}

In den Versuchen an Probekörpern mit unterschiedlichem Verhältnis der Schichtdicken konnte der Einfluss der einzelnen Parameter nicht gezielt getrennt voneinander untersucht werden. Anhand von zwei Parameterstudien auf Grundlage der FEModellierung konnte jedoch der Einfluss der Dicke der Deckschichten und der Einfluss der Dicke des Kerns detaillierter untersucht werden. Die Parameterstudien basierten dabei auf den Eingangsparametern der FE-Modellierung S.X5.S-10.120.10-FE (4-Punkt-Biegeversuch mit Mikrobewehrung und XPS-Charge X5). Die Ergebnisse der Modellierung sind in den Abbildungen 7.10 und 7.11 dargstellt.

In der ersten Parameterstudie wurde die Dicke der Deckschichten konstant mit $10 \mathrm{~mm}$ angenommen und die Dicke des Kerns schrittweise von $50 \mathrm{~mm}$ auf $250 \mathrm{~mm}$ vergrößert. Die Kernschichtdicke $d_{s}$ steht in der Gleichung 3.19) zur Berechnung der Schubsteifigkeit im Nenner. Durch Vergrößerung von $d_{s}$ wird somit die Schubsteifigkeit $S$ verringert. Jedoch bewirkt eine Vergrößerung der Kernschichtdicke $d_{s}$ auch eine Vergrößerung des Abstands der Schwerachsen der Deckschichten $e$. In die Gleichungen zur Berechnung der Schubsteifigkeit $S$ sowie der Sandwichbiegesteifigkeit $B_{s}$ geht $e$ im Zähler zum Quadrat ein (siehe Gl. 3.18 und 3.19), S. 30. Daher überwiegt der Einfluss des Parameters $e$ den Einfluss von $d_{s}$. Weiterhin wird mit zunehmendem Abstand der Schwerachsen der Deckschichten $e$ das Kräftepaar $N_{s}$ aus dem Sandwichmoment $M_{s}$ verringert, da gilt $N_{s}=M_{s} / e$. Es kann daher zusammengefasst werden, dass durch eine Vergrößerung der Kernschichtdicke die Steifigkeit und die maximale Tragfähigkeit des Bauteils gesteigert wird. 


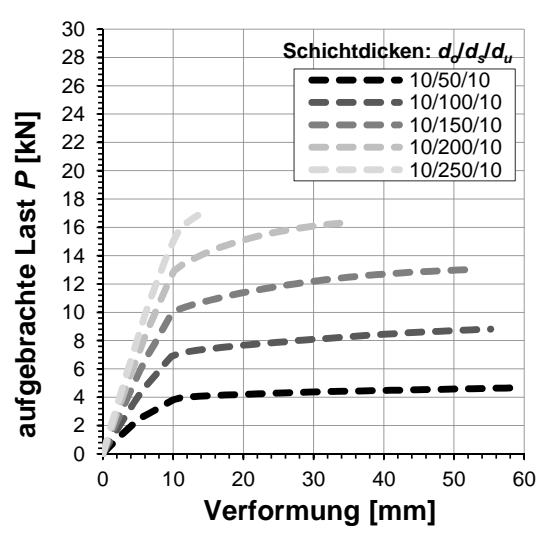

Abb. 7.10: FEM-Parameterstudie zur Untersuchung des Einflusses der Kernschichtdicke auf das Lastverformungsverhalten

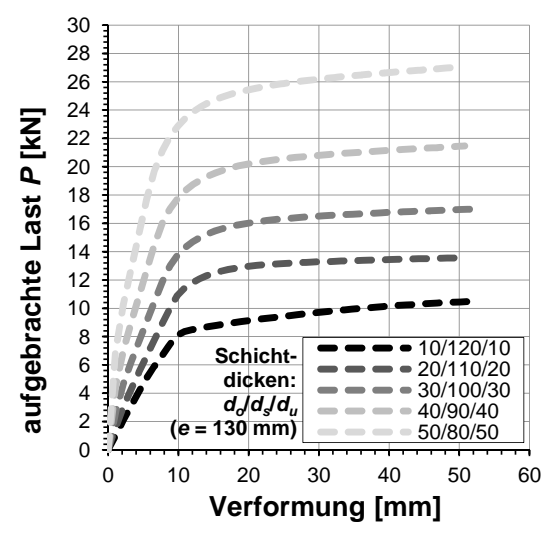

Abb. 7.11: FEM-Parameterstudie zur Untersuchung des Einflusses der Deckschichtdicke auf das Lastverformungsverhalten

In der zweiten Parameterstudie wurde der Abstand zwischen den Schwerachsen der beiden Deckschichten konstant mit $130 \mathrm{~mm}$ angenommen und die Dicke der Deckschichten schrittweise von $10 \mathrm{~mm}$ auf $50 \mathrm{~mm}$ gesteigert. Folglich musste dabei die Dicken der Kerne entsprechend angepasst werden. Daher wurden in dieser Studie weiterhin zwei Parameter variiert: die Dicke der Deckschichten und die Dicke des Kerns. Es zeigte sich, dass durch eine Vergrößerung der Deckschichtdicke die Tragfähigkeit und die Steifigkeit des Bauteils erheblich gesteigert wird. Die Steigerung der Steifigkeit resultiert sowohl aus einer Vergrößerung der Sandwichbiegesteifigkeit $B_{s}$, als auch einer Vergrößerung der Eigenbiegesteifigkeit $B_{o}$ und $B_{u}$. In die Sandwichbiegesteifigkeit $B_{s}$ gehen die Deckschichtdicken $d_{o}$ und $d_{u}$ über die Flächen $A_{o}$ und $A_{u}$ ein und in die Eigenbiegesteifigkeit der Deckschichten über die Trägheitsmomente $I_{o}$ und $I_{u}$. Weiterhin bewirkt die Vergrößerung der Deckschichtdicke eine Reduktion der Spannungen in den Deckschichten, da die Querschnittsflächen und die Widerstandsmomente vergröBert werden (siehe Gl. 3.23 und 3.24).

\subsubsection{Stoßausbildung}

Eine vollflächige Verklebung eines Stoßes im Kern stellt keine Schwächung des Sandwichquerschnitts dar. Sowohl in den Scherversuchen als auch in den Biegeversuchen konnte weder ein Versagen im geklebten Bereich erzielt werden noch eine Reduktion der Steifigkeit festgestellt werden. Ein Einfluss der Klebung auf die Ermüdungsfestigkeit und Dauerhaftigkeit wurde nicht untersucht und sollte in weiteren Versuchen ermittelt werden. 


\subsection{Diskussion der Versagensarten}

\subsubsection{A: ,Zugversagen der Bewehrung“}

Das Zugversagen der Bewehrung stellte in allen Biegeversuchen ein schlagartiges Versagen des Bauteils dar. Bei Verwendung der Mikrobewehrung kündigte sich das Versagen aufgrund des Fließens des Stahldrahtes durch die Vergrößerung der Rissbreiten an. Bei den textilbewehrten Probekörpern war dies nicht der Fall, da die verwendeten Glas- und Carbonfasern keinen Fließbereich aufweisen.

Anhand der Dehnkörperversuche wurden die Zugfestigkeiten der Mikrobewehrung und des Carbonfasergeleges an bewehrten Betonprobekörpern ermittelt. Dabei ereichte die Mikrobewehrung eine mittlere Dehngrenze von $374 \mathrm{~N} / \mathrm{mm}^{2}$ und eine mittlere Zugfestigkeit von $436 \mathrm{~N} / \mathrm{mm}^{2}$. Das Carbonfasergelege erreichte eine mittlere Zugfestigkeit von $752 \mathrm{~N} / \mathrm{mm}^{2}$. Die Versuchsergebnisse der carbonfaserbewehrten Probekörper wiesen jedoch eine deutlich größere Streuung auf als die der mikrobewehrten Probekörper.

Bei Vernachlässigung des Einflusses der Deckschichtmomente kann die Zugspannung der Bewehrung $\sigma_{c a l c}$ rein aus dem Sandwichmoment $M_{s}$ überschlägig ermittelt werden. Das Sandwichmoment entspricht in diesem Fall dem einwirkenden Gesamtmoment $M$ :

$$
\sigma_{c a l c}=\frac{M}{e \cdot A_{f}}
$$

Im Fall der mikrobewehrten und carbonfaserbewehrten Sandwichstreifen liegen die nach Gl. 7.1 errechneten Spannungen der Bewehrung in etwa auf dem Niveau der Dehnkörperversuche. Im Fall der Großbauteilversuche an den carbonfaserbewehrten Sandwichelementen liegen die Spannungen der Bewehrung jedoch in einem Bereich von $970 \mathrm{~N} / \mathrm{mm}^{2}$ und $1.240 \mathrm{~N} / \mathrm{mm}^{2}$ und somit deutlich über denen der Dehnkörperversuche. Es wird davon ausgegangen, dass die Verbundlänge einen starken Einfluss auf die erreichbaren Zugfestigkeiten der Bewehrung hat. Durch die größere Verbundlänge zwischen Beton und Textilbewehrung in den Großbauteilversuchen, kann eine größere Anzahl von Filamenten aktiviert und somit eine höhere Zugfestigkeit erreicht werden.

Die errechneten Spannungen beim Versagen der glasfaserbewehrten Sandwichstreifen betrugen $1.110 \mathrm{~N} / \mathrm{mm}^{2}$ bis $1.470 \mathrm{~N} / \mathrm{mm}^{2}$ und erreichten somit zum Teil größere Werte, als in den Großbauteilversuchen mit carbonfaserbewehrten Probekörpern. Dies kann damit begründet werden, dass durch den größeren Filamentdurchmesser und die geringere Filamentanzahl, der Faserquerschnitt besser aktiviert und die Materialzugfestigkeit besser ausgenutzt wird. Als nachteilig stellte sich in den Biegeversuchen das schlechte Ermüdungsverhalten der Bewehrung heraus, das sich durch vorzeitiges Versagen bei wiederholter Belastung zeigte.

\subsubsection{B: „Druckversagen des Kern an der Lasteinleitungsstelle“}

In den zwei Biegeversuchen C.X1.C-10.120.10-1 und -2 an carbonfaserbewehrten Sandwichstreifen in der Versuchsreihe „Bewehrungsart“ konnte eine starke Zusam- 
mendrückung des Kerns in vertikaler Richtung im Bereich der Lasteinleitung beobachtet werden. Der Grund dafür war die Überschreitung der maximal übertragbaren Druckspannungen des XPS in diesem Bereich.

Durch die FE-Modellierung der beiden Biegeversuche konnte die Druckspannungsverteilung in $y$-Richtung in diesem Bereich berechnet werden. In der linken Hälfte der Abbildung 7.12 ist die Druckspannungsverteilung vor dem Erreichen der Fließdruckspannung dargestellt. In der Verbundfuge zwischen Beton und XPS unter der rechten und linken Kante der steifen Lasteinleitungsplatte bilden sich die maximalen Spannungen. Bei Steigerung der Belastung und Überschreitung der Fließdruckspannung in diesem Bereich plastiziert das XPS und die Spannungen mittig unter der Lasteinleitungsplatte steigen weiter an. Schließlich wird in der Verbundfuge unter der Lasteinleitungsplatte eine gleichmäßige Spannungsverteilung erreicht (siehe Abb. 7.12, rechts).
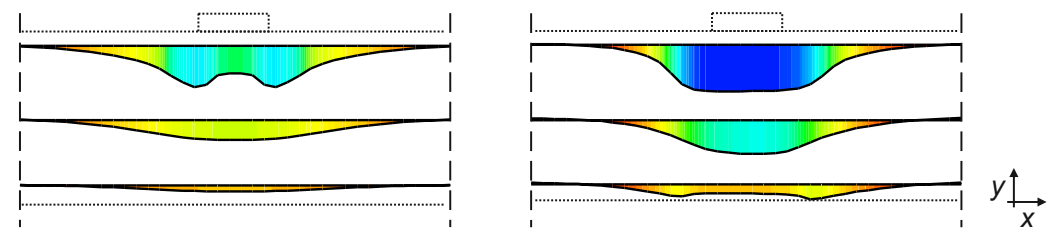

Druckspannungen in $y$-Richtung $\left[\mathrm{N} / \mathrm{mm}^{2}\right]$ :

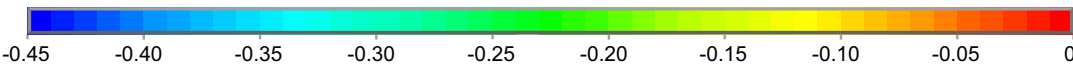

Abb. 7.12: Druckspannungen in $y$-Richtung im Bereich des Lasteinleitungsbereichs bei einer Verformung von $20 \mathrm{~mm}$ (links) und $40 \mathrm{~mm}$ (rechts) (C.X1.C-10.120.10-FE1-I)

In Abbildung 7.13 ist die Verformungsdifferenz zwischen oberer und unterer Deckschicht der Versuche C.X1.C-10.120.10-1 mit einer Breite der Lasteinleitungsplatten von $60 \mathrm{~mm}$ (blau) und C.X1.C-10.120.10-4 mit einer Breite der Lasteinleitungsplatte von $150 \mathrm{~mm}$ (rot) mit den dazugehörigen FE-Nachrechnungen dargestellt. Ab einer Verformung von $20 \mathrm{~mm}$ steigt die Verformungskurve infolge des Plastizierens des XPS im Versuch C.X1.C-10.120.10-1 mit schmaler Lasteinleitungsplatte stark an. Die Verformungskurve des Versuchs C.X1.C-10.120.10-4 hingegen verläuft in etwa linear, so dass von linear-elastischem Materialverhalten im Bereich der Lasteinleitung ausgegangen werden kann.

Die FE-Nachrechnungen weichen relativ stark von den Versuchsergebnissen ab. Jedoch kann eine Zunahme der Verformungen in der Rechnung C.X1.C-10.120.10-FE1-I mit dem isotropem Werkstoffgesetz BISo erzielt werden. Die plastischen Verformungen der FE-Modellierung sind wesentlich geringer als in den Versuchen. Die beiden Werkstoffgesetze des XPS im plastischen Bereich sind mit einer Querdehnzahl von 0,45 nahezu inkompressibel. Es bildet sich daher ein mehraxialer Spannungszustand aus. In den Versuchen hingegen, versagt durch das Überschreiten der Druckfestigkeit die Zellstruktur und das XPS wird stark komprimiert.

Durch das Druckversagen des XPS werden die Momente und Querkräfte der oberen Deckschicht im Bereich der Lasteinleitung vergrößert. Dies führte in den Versuchen zu einem lokalen Biege- oder Querkraftversagen der Deckschicht. 


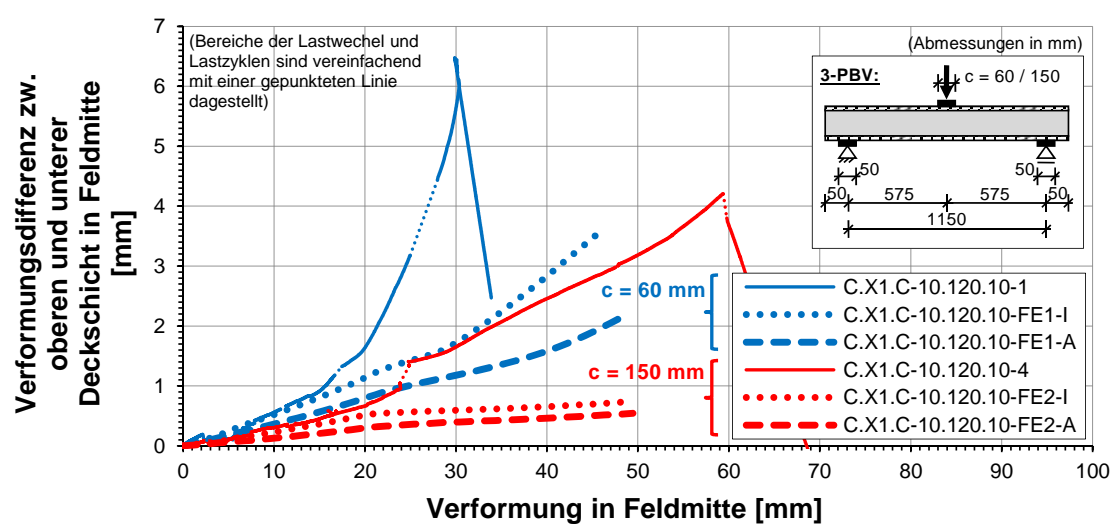

Abb. 7.13: Verformungsdifferenz zwischen oberer und unterer Deckschicht im Bereich der Lasteinleitungsstelle

\subsubsection{C: „Lokales Biegeversagen der Deckschichten“}

Die carbonfaserbewehrten oberen Deckschichten der Probekörper C.X1.C-10.120.101 und -3 der Versuchsreihe „Bewehrungsart“ versagten aufgrund eines lokales Biegeversagens im Bereich der Lasteinleitung. Beim Versagen platzten mehrere Zentimeter große schollenförmige Betonbruchstücke auf der Zugseite der Deckschichten von der Oberfläche ab.

Da die Filamente der Fasern keinen gegenseitigen Verbund aufwiesen, stellte das Gelege eine Schwächung der Betonfläche entlang der Deckschichtachse dar. Diese Trennwirkung wurde verstärkt durch den liegenden ellipsenförmigen Querschnitt der Fasern. Durch die Biegebeanspruchung und die Krümmung des Querschnitts traten Spaltrisse entlang der Deckschichtachse auf (siehe Abbildungen 7.14 und 7.16. Diese konnten auch in den Biegeversuchen an den carbonfaserbewehrten Betonstreifen beobachtet werden (siehe Abbildung 7.15. Schließlich versagte der Beton zwischen der Oberfläche der Deckschichten und der Textilbewehrung in der Deckschichtachse aufgrund des Überschreitens der Betonzugfestigkeit. Hieraus resultierten die beschriebenen Abplatzungen von Betonbruchstücken an den Deckschichtoberflächen.

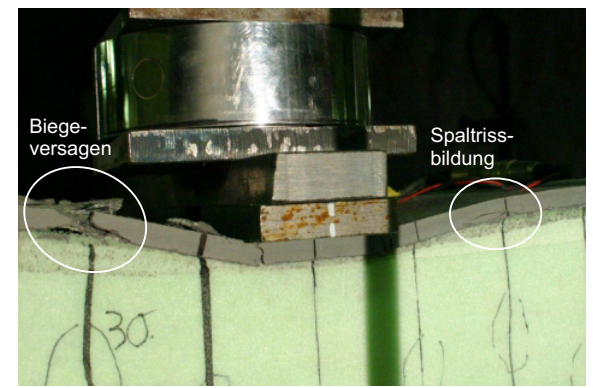

Abb. 7.14: Lokales Biegeversagen und Spaltrissbildung der Deckschicht (C.X1.C-10.120.10-1)

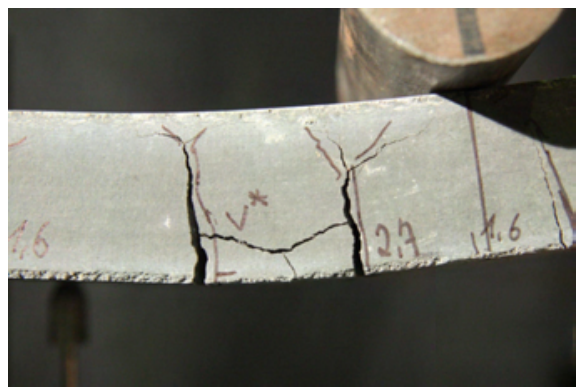

Abb. 7.15: Spaltrissbildung eines carbonbewehrten Betonstreifens in einem Tastversuch 


\subsubsection{D: „Lokales Querkraftversagen der Deckschichten“}

Der carbonfaserbewehrte Sandwichstreifen C.X1.C-10.120.10-2 versagte durch ein Querkraftversagen der oberen Deckschicht im Bereich der Lasteinleitung (siehe Abbildung 7.16). In den Biegeversuchen an den bewehrten Betonstreifen konnte in zwei der Versuchen an carbonfaserbewehrten Probekörpern ebenfalls ein Querkraftversagen festgestellt werden.

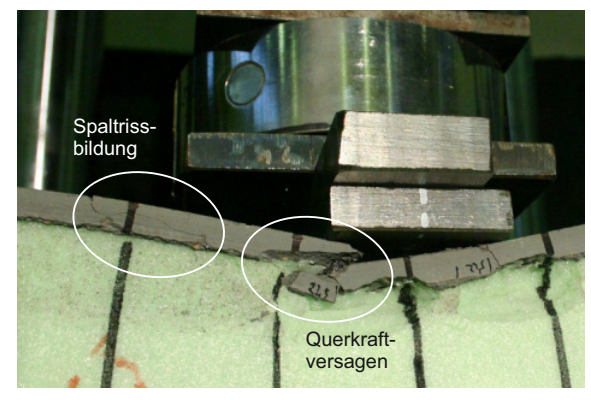

Abb. 7.16: Querkraftversagen und Spaltrissbildung der oberen Deckschicht (C.X1.C-10.120.10-2)

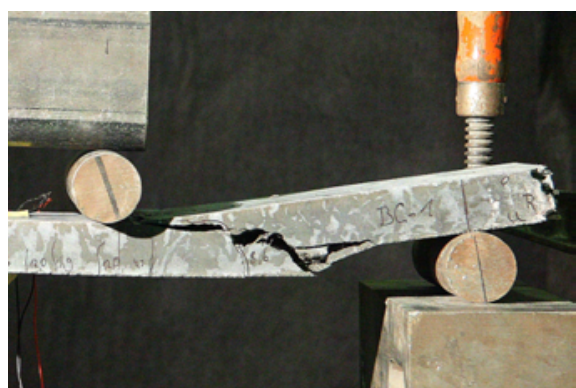

Abb. 7.17: Treppenförmiger Versagensriss beim Querkraftversagen des Probekörpers BC-1

Ein Querkraftversagen konnte somit nur bei der carbonfaserbewehrten Probekörpern beobachtet werden. Aufgrund der hohen Zugfestigkeit des Carbonfasergeleges, wurde ein vorzeitiges Biegeversagen infolge Reißen der Bewehrung ausgeschlossen.

Weiterhin begünstigen die Filamente der Bewehrung ein Querkraftversagen. Wie zuvor beschrieben, weisen die Filamente innerhalb der Faser keinen gegenseitigen Verbund auf. Die Fasern stellen somit bei Belastung senkrecht zur Bauteilachse, durch die Zugspannungen infolge einer Querkraftbeanspruchung, eine Schwächung der Betonfläche und somit der Querkrafttragfähigkeit dar.

Die Annahme der Schwächung des Querschnitts im Bereich der Fasern wird durch die Versagensbilder in den Biegeversuchen an bewehrten Betonstreifen bestätigt. Wie in Abbildung 7.17 zu erkennen ist, verläuft der Versagensriss treppenförmig entlang der drei Lagen der Bewehrung.

\subsubsection{E: „Querkraftversagen des Kerns und Delamination des Kerns und der Deckschichten“}

In 6 von 46 Biegeversuchen an Sandwichstreifen versagten die Probekörper schlagartig durch einen Riss im XPS-Kern und der Delamination der unteren Deckschicht vom Kern. Durch das Entfernen des XPS im Anschluss an die Versuche, konnten bei fünf der sechs Probekörper Fehlstellen in der Verbundfuge zwischen den Deckschichten und dem Kern festgestellt werden (siehe Abbildungen in Anhang A.1.1. S. 244.

Im Versuch C.X2.C-10.120.10-3 kündigte sich das Versagen durch plastische Verformungen und einen ersten, etwa drei Zentimeter langen Riss im XPS im Bereich der Fehlstelle an (siehe Abbildung 7.18). Zur Veranschaulichung des Tragverhaltens 
des Probekörpers mit Fehlstelle in der Verbundfuge, wurde dessen zugehörige FEModellierung C.X2.C-10.120.10-FE-A entsprechend angepasst. Im Bereich der Fehlstelle wurde der starre Verbund gelöst und die Kontaktelemente auf die Option Standard gesetzt, so dass über diese nur noch Druckspannungen übertragen werden konnten. Aufgrund der zweidimensionalen Modellierung wurde somit eine Fehlstelle über die gesamte Breite des modellierten Probekörpers erzeugt.

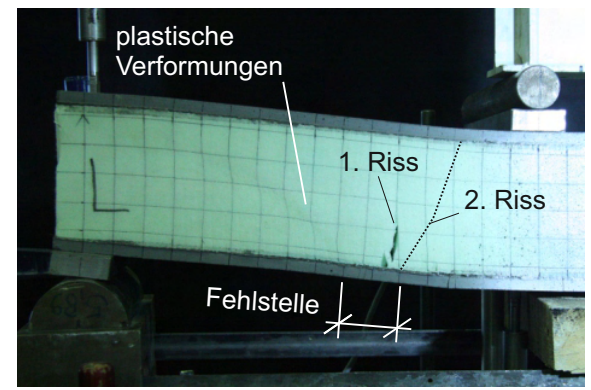

Abb. 7.18: Verformungen im Bereich der Fehlstelle des Probekörpers C.X2.C-10.120.10-3

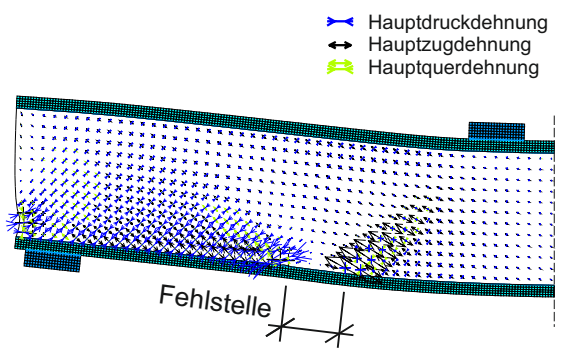

Abb. 7.19: Vektorielle Darstellung der Hauptdehnungen ermittelt durch FE-Modellierung

In der Modellierung treten im Bereich rechts der Fehlstelle große Hauptzugdehnungen auf (siehe Abbildung 7.18). Im Versuch bildeten sich hier zwei Risse im XPS aus. Ein erster Riss der vermutlich durch das vollständige Lösen des Haftverbunds im Bereich der Fehlstelle verursacht wurde und ein zweiter Riss der zum Versagen des Probekörpers führte. Der zweite Riss verlief diagonal von der Fehlstelle bis zum Lasteinleitungspunkt und somit analog zum diagonal verlaufenden Band an Hauptzugspannungen in der Darstellung der Berechnungsergebnisse. Weiterhin lassen sich in der vektoriellen Darstellung der FE-Ergebnisse große Hauptdruckdehnungen und daraus resultierende Hauptdruckspannungen im Bereich links der Fehlstelle feststellen. Diese können auch in den Versuchen durch die Krümmungen der auf dem Kern aufgezeichneten Linien wahrgenommen werden.

Um den Einfluss der Fehlstellengröße auf das Lastverformungsverhalten zu bestimmen, wurden zwei Parameterstudien durchgeführt (siehe Abbildungen 7.20 und 7.21. Unabhängig davon ob die Fehlstelle in der oberen oder unteren Verbundfuge angeordnet wird, ist die Reduzierung der Steifigkeit und der Traglast bei einer Breite $b$ der Fehlstelle von $25 \mathrm{~mm}$ nur äußerst gering; während bei zunehmender Breite der Fehlstelle von $50 \mathrm{~mm}, 100 \mathrm{~mm}$ und $200 \mathrm{~mm}$, insbesondere die Traglast sehr stark abnimmt (um $7 \%, 15 \%$ und $40 \%$ ).

Zusammenfassend kann festgestellt werden, dass durch die Fehlstellen in der Verbundfuge der kontinuierliche Schubfluss zwischen oberer und unterer Deckschicht gestört wird. Durch Spannungsspitzen im Randbereich der Fehlstelle plastiziert das XPS und wird schließlich geschädigt, so dass die Steifigkeit des Bauteils reduziert wird.

Bei den Probekörpern der Biegeversuche traten die Fehlstellen in der Regel nicht über der gesamten Breite, sondern nur über Teilbereichen der Verbundfuge auf. Als konservativer Ansatz auf Grundlage der Parameterstudie wird empfohlen, die Größe dieser meist runden bzw. ovalen Fehlstellen durch die Optimierung der Herstellungsmetho- 


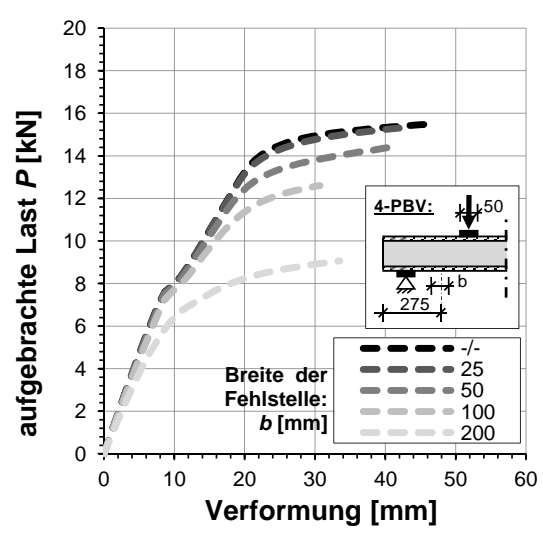

Abb. 7.20: FEM-Parameterstudie zur Untersuchung des Einflusses der Größe einer Fehlstelle in der unteren Verbundfuge

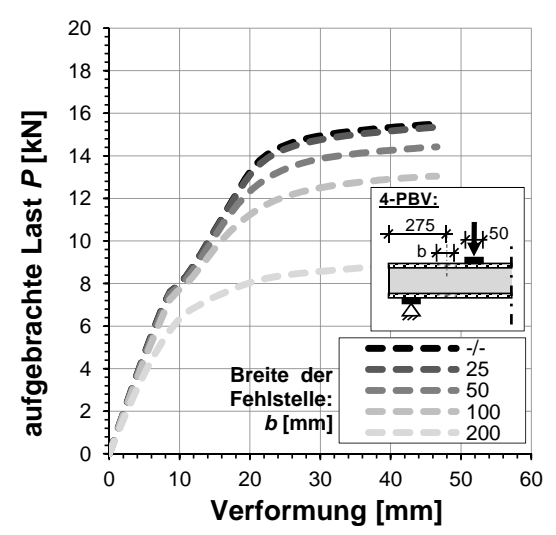

Abb. 7.21: FEM-Parameterstudie zur Untersuchung des Einflusses der Größe einer Fehlstelle in der oberen Verbundfuge

den auf einen mittleren Durchmesser von $50 \mathrm{~mm}$ zu begrenzen. Durch die Versuchsergebnisse und das Aufschneiden der geprüften Probekörper wurde bestätigt, dass bei dieser Fehlstellengröße kein vorzeitiges Versagen eingeleitet wird.

\subsubsection{F: „Schubversagen zwischen Kern- und Deckschicht“}

Ein Schubversagen zwischen Kern- und Deckschicht konnte sowohl in den Scherversuchen, als auch in den Biegeversuchen beobachtet werden. Anhand der Ergebnisse der Scherversuche konnte das Versagen in zwei Arten A und B unterschieden werden.

Die Versagensart A kann als Kohäsionsversagen kategorisiert werden, da das Scherversagen im Bereich des ungestörten XPS auftritt. Die thermisch geprägte Waffelstruktur bleibt dabei im Beton haften. Wie in der CT-Visualisierung in Abbildung 7.22 zu erkennen ist, wird das XPS durch die thermische Prägung in diesem Bereich aufgeschmolzen und verdichtet und weist folglich eine höhere Festigkeit auf, als im ungestörten Bereich. Wenn ein ausreichender Verbund zwischen der Waffelstruktur und dem Beton gewährleistet ist, tritt daher das Versagen im ungestörten XPS auf. Die Scherprobekörper mit Betondeckschichten, die die Versagensart A aufwiesen, erzielten daher Scherfestigkeiten, die sehr nahe an die Scherfestigkeiten der Probekörper ohne Betondeckschichten heranreichten.

Im Fall der Versagensart B tritt ein Adhäsionsversagen zwischen XPS und Beton auf. Dabei löst sich die Waffelstruktur vom Beton ab. Die Oberflächenstruktur des XPS sowie das XPS im ungestörten Bereich bleiben hierbei intakt. Die Scherprobekörper, die diese Versagensart aufwiesen, erreichten grundsätzlich wesentlich geringere Scherfestigkeiten, welche auf einen unzureichenden Verbund zwischen Beton und XPS zurückzuführen sind. Die Gründe hierfür liegen zum einen in der Herstellung der Elemente und zum anderen in der Beschaffenheit der Waffelstruktur begründet. 


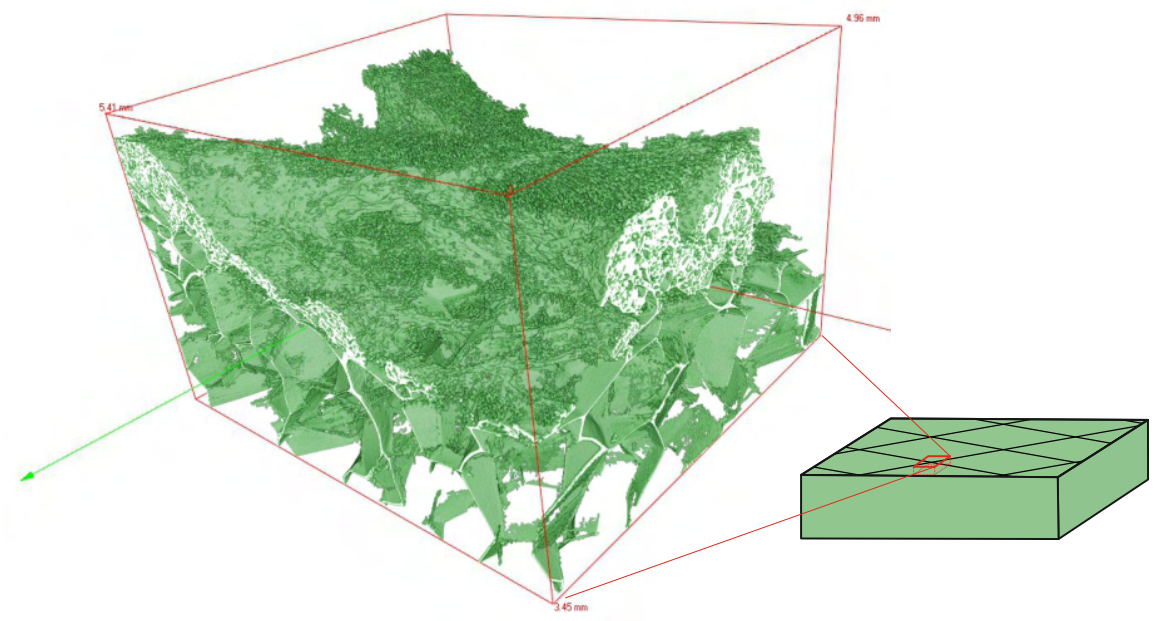

Abb. 7.22: Mit Computertomographie erstellte 3D-Visualisierung der Waffelstruktur des XPS

Indem der XPS-Kern auf den frischen Beton aufgelegt wird, können sich durch das Entlüften des Betons, kleine Luftblasen mit einem Durchmesser von weniger als $1 \mathrm{~mm}$ zwischen Beton und XPS bilden (siehe bspw. Abbildung 5.33, S. 89). Diese reduzieren die Scherfestigkeit des Verbunds. Durch entsprechende Maßnahmen, z. B. das Optimieren des Mischprozesses des Betons, das Einfüllen des Betons über eine Rutsche, das Einhalten von Ruhezeiten vor und nach dem Einfüllen des Betons, kann der negative Einfluss des Entlüftens auf die Scherfestigkeit reduziert werden.

Ein weiterer Grund für den schlechten Verbund ist das schnelle Abbinden des Hochleistungsbetons. Aufgrund dessen bildet sich auf der Oberfläche des Betons schon nach wenigen Minuten eine Schicht mit einer steiferen Konsistenz aus. Durch diese sogenannte „Elefantenhaut“ wird verhindert, dass der Beton die Waffelstruktur vollständig ausfüllt. Die Scherprobekörper wurden grundsätzlich im Anschluss an die Betonage der Biegeprobekörper mit der selben Betoncharge hergestellt. Daher wies der Beton bereits bei der Betonage der Scherprobekörper eine merklich steifere Konsistenz auf, wodurch der schlechtere Verbund mit der Waffelstruktur und die geringere Scherfestigkeit begründet liegen.

Neben der Herstellungsweise wird die Qualität des Verbunds auch durch die Beschaffenheit der Waffelstruktur entscheidend beeinflusst. Grundsätzlich werden die XPSChargen auf die selbe Art und Weise, d. h. durch Einprägen der Waffelstruktur mittels einer heißen Walze, hergestellt. Dabei wird am Fuß der Rippen durch die thermische Prägung die Zellstruktur des XPS freigelegt. Jedoch unterscheidet sich je nach Rohdichte des XPS die Größe der freigelegten Zellen. Durch die Gegenüberstellung der Oberflächenstrukturen dreier XPS-Chargen mit unterschiedlichen Rohdichten in Abbildung 7.23 lässt sich dies erkennen. Durch die größeren Zellen des XPS mit einer geringen Rohdichte wird eine bessere Verzahnung und ein besserer Hinterschnitt erzeugt, als bei einem XPS mit hoher Rohdichte. Dies wurde auch durch die Ergebnisse der Scherversuchsreihen X1, X2 und X3 bestätigt. Obwohl beide XPS-Chargen X2 und X3 eine höhere Rohdichte und Scherfestigkeit aufwiesen, war die Scherfestigkeit der 
Verbundfuge zwischen Beton und XPS geringer, als die der Versuche mit Probekörpern der XPS-Charge X1.
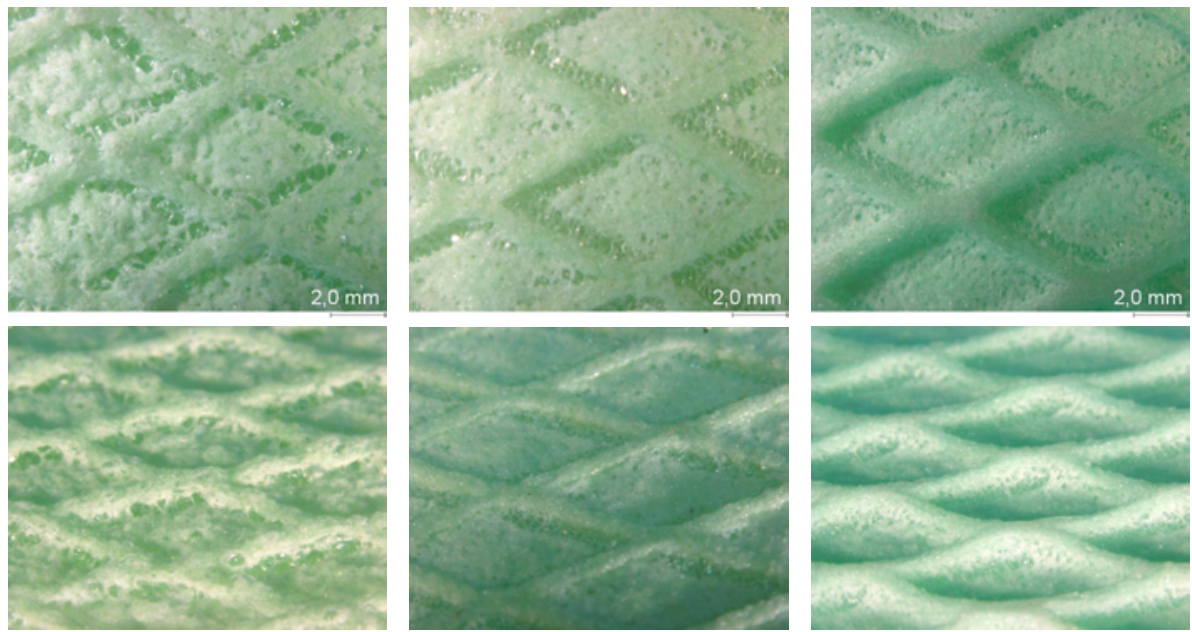

Abb. 7.23: Draufschicht und seitliche Ansicht der Waffelstruktur der XPS-Charge X0 (links), X2 (mitte) und X3 (rechts)

In den Biegeversuchen trat das Schubversagen immer zwischen der oberen Deckschicht und dem Kern auf. In Versuch S.X1.S-10.120.10-2 trat das Schubversagen einmalig über der vollen Länge zwischen dem linken Ende des Probekörpers und der Lasteinleitungsstelle auf. In allen anderen 20 Versuchen in denen diese Versagensart beobachtet werden konnte, löste sich der Verbund nur im Bereich der etwa letzten $5 \mathrm{~cm}$ am rechten oder linken, oder auch beiden Enden des Probekörpers. Das Verbundversagen wird begünstigt durch Zugspannungen im Kern, die auf das linke und rechte Ende der Deckschichten zulaufen (siehe Abbildung 7.24) und zum Teil zum Abschälen des Kerns von der Deckschicht führen, vergleichbar mit dem Versagen der Probekörper in den Scherversuchen.
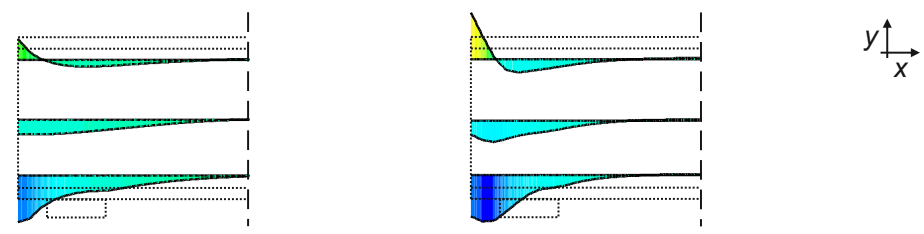

Spannungen in $y$-Richtung $\left[\mathrm{N} / \mathrm{mm}^{2}\right]$ :

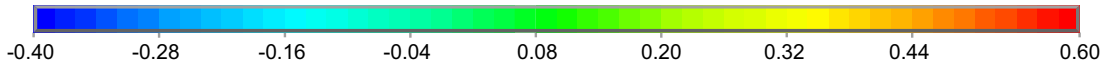

Abb. 7.24: Spannungen in $y$-Richtung im Bereich der Auflagers bei $10 \mathrm{~mm}$ (links) und $50 \mathrm{~mm}$ (rechts) Verformung (S.X0.S-10.120.10-FE2-A) 


\subsubsection{G: "Druckversagen des Kerns an den Auflagern"}

Das Druckversagen des XPS im Bereich der Auflager konnte in den Biegeversuchen an den Sandwichstreifen durch einen charakteristischen Knick am linken oder rechten Ende der Kernschicht festgestellt werden (siehe Abbildung 7.25). Auch bei den FE-Modellierungen der Versuche konnten diese plastischen Verformungen im XPS im Bereich der Auflager wahrgenommen werden (siehe Abbildung 7.26).

Diese Verformungen resultieren aus einer kombinierten Schub- und Druckbeanspruchung des XPS am Auflager. In diesem Bereich verlaufen die Hauptdruckspannungen auf die Enden der Probekörper zu und stützen sich auf die untere Deckschicht ab, wie in der Darstellung der Hauptspannungen in Abbildung 7.1 (siehe S. 151) zu erkennen. In Abbildung 7.24 lässt sich weiterhin erkennen, dass die Druckspannungen in $y$-Richtung im Randbereich ein Maximum erreichen. Die plastischen Verformungen im XPS führen im Gegensatz zu den Verformungen, die an der Lasteinleitung auftreten, nicht zu einer wesentlichen Vergleichmäßigung der Spannungen. Das Maximum der Druckspannungen befindet sich weiterhin am seitlichen Rand der Kernschicht.

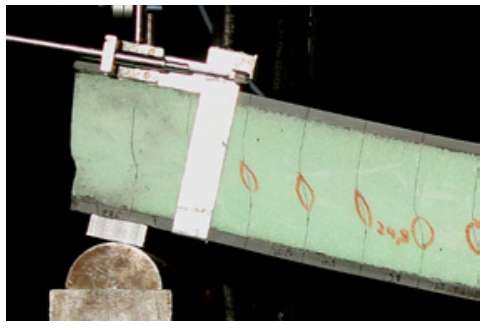

Abb. 7.25: Verformungen im Bereichs des Auflagers (S.X0.S-10.120.10-3)

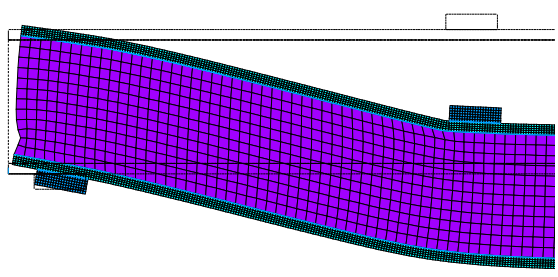

Abb. 7.26: Verformungen ermittelt durch FEM (S.X0.S-10.120.10-F1-A)

\subsection{Parameterstudien an einem Wandelement}

\subsubsection{Allgemeines}

Im Rahmen dieser Arbeit wurde der Einfluss durch eine Temperatur- und Schwindbeanspruchung der Deckschichten experimentell nicht näher untersucht. Um deren Auswirkungen jedoch genauer abschätzen zu können, wurden die Beanspruchungen mithilfe des FE-Modells rechnerisch untersucht. Die Modellierung basierte auf dem Modell zur Nachrechnung der Großbauteilsversuche mit Beanspruchung durch Einzellasten. Die Gesamtlänge von 5,0 m und die Spannweite von 4,5 m, sowie die Materialgesetze des Betons und des XPS wurden übernommen.

\subsubsection{Beanspruchung durch Temperatur}

Im ersten Teil der Modellierung wird die Temperaturverteilung über den Sandwichquerschnitt berechnet. Hierzu wurde der Sommer-Temperaturlastfall nach DIN EN 14509 
für dunkle Oberflächenfarben mit den Oberflächentemperaturen $T_{1}=80^{\circ} \mathrm{C}$ und $T_{2}=$ $25^{\circ} \mathrm{C}$ angenommen. Die Wärmeleitfähigkeit $\lambda$ wurde für den Hochleistungsbeton mit $2,5 \mathrm{~W} / \mathrm{mK}$ und für das XPS mit 0,04 W/mK angenommen. Aufgrund der geringen Dicke und der hohen Leitfähigkeit des Betons, ändert sich die Temperatur über die Deckschichten nur um $0,07^{\circ} \mathrm{C}$. Der Temperaturgradient in den Deckschichten kann daher bei der späteren Bemessung der Sandwichelemente vernachlässigt werden.

Im zweiten Teil der Modellierung werden dann die Verformungen und Spannungen infolge der unterschiedlichen Ausdehnungen der Deckschichten berechnet. Hierzu wurde die Wärmedehnzahl des Betons $\alpha_{T}$ mit 1,17·10 ${ }^{-5}$ angenommen (vgl. Schmidt 2008). In den Abbildungen 7.27 und 7.28 sind jeweils die Verformungen und die Zugspannungen der Deckschichten in Feldmitte über dem Schubmodul $G$ sowie über dem Abstand der Schwerachsen der Deckschichten $e$ aufgetragen.

Der Einfluss des Parameters $G$ ist marginal, während der Einfluss des Parameters $e$ wesentlich größer ist. Der große Einfluss des Achsabstands $e$ lässt sich damit begründen, dass dieser Parameter direkt in die Berechnung der Krümmung aus unterschiedlichen Temperaturdehnungen $\theta$ nach Gl. 3.28) (siehe S. 33) eingeht. Mit abnehmendem Abstand $e$ nimmt die Krümmung $v$ zu und somit folglich auch die Gesamtverformung des Sandwichelements und die Zugspannungen in den Deckschichten.

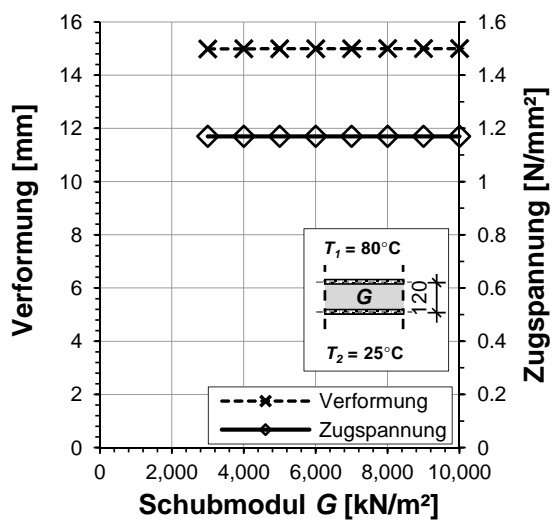

Abb. 7.27: Verformung und max. Zugspannungen in Feldmitte über dem Schubmodul $G$ bei einem Einfeldträger

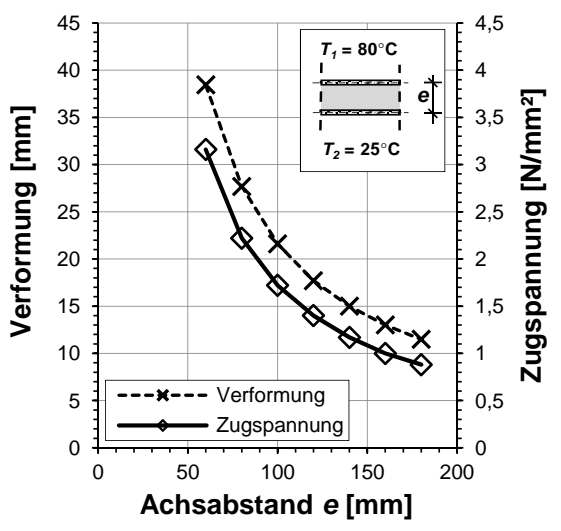

Abb. 7.28: Verformung und max. Zugspannungen in Feldmitte über dem Achsabstands der Deckschichten $e$ bei einem Einfeldträger

Bei der Temperaturbeanspruchung von Einfeld-Sandwichelementen entsteht durch die unterschiedliche Dehnung der Deckschichten eine Krümmung, die zu Zwangspannungen in den Deckschichten und einer Verformung des Sandwichelements führt. Bei Zweifeld-Sandwichelementen wird die Verformung durch das Mittelauflager behindert. Hierdurch wird eine Auflagerreaktion verursacht und erhebliche Spannungen in die Deckschichten induziert. Dieser Fall wird noch anhand einer Beispielrechnung in Abschnitt 8.2.2.7 (siehe S. 185) erläutert. 


\subsubsection{Beanspruchung durch Schwinden der Deckschichten}

Zur Modellierung einer Schwindbeanspruchung eines Sandwichelements wurde die Temperatur der Deckschichten auf $-100^{\circ} \mathrm{C}$ und die Temperatur des Kerns auf $0{ }^{\circ} \mathrm{C}$ definiert. Bei einer Wärmedehnzahl von $\alpha_{T}=1,0 \cdot 10^{-5}$ verursacht diese Temperaturdifferenz von $100^{\circ} \mathrm{K}$ eine Verkürzung der Deckschichten, die einer Schwinddehnung $\epsilon_{s}$ von $1 \mathrm{~mm} / \mathrm{m}$ entspricht. Durch die Schwinddehnung werden Zugspannungen in den Deckschichten und Druckspannungen im Kern hervorgerufen. Die Größe dieser Zwangspannungen ist überwiegend abhängig von den Schichtdicken sowie vom Elastizitätsmodul des Kerns in $x$-Richtung. Wie in Abbildung 7.29 dargestellt ist, nehmen die Spannungen proportional mit steigendem Elastizitätsmodul des XPS in $x$-Richtung zu.

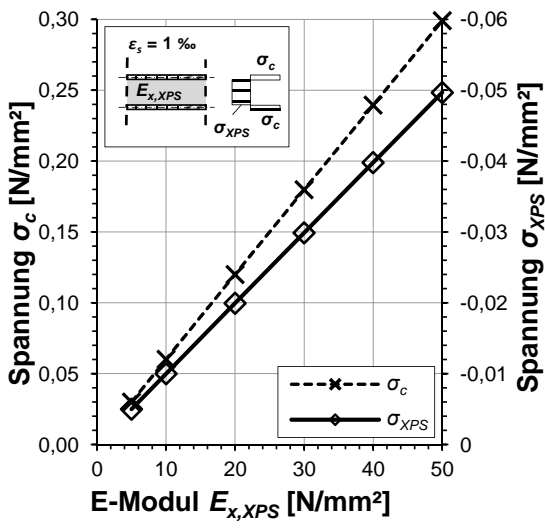

Abb. 7.29: Zugspannungen in den Deckschichten $\sigma_{C}$ und Druckspannungen im Kern $\sigma_{X P S}$ in Feldmitte in Abhängigkeit von $E_{X, X P S}$

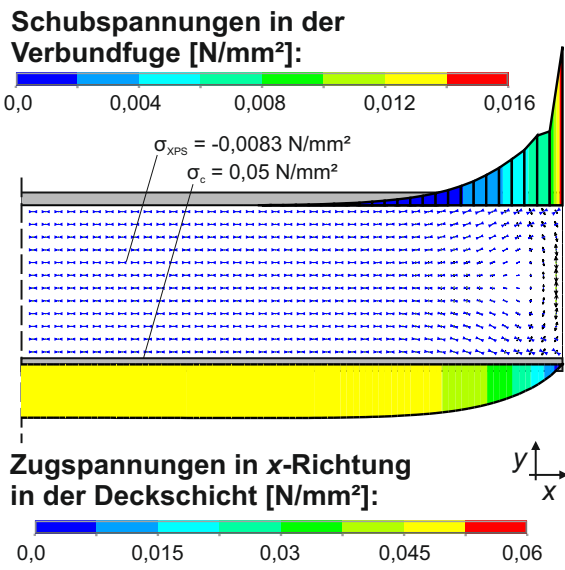

Abb. 7.30: Spannungen in der Verbundfuge und den Deckschichten sowie vektorielle Darstellung der Hauptspannungen bei $\epsilon_{s}=1 \mathrm{~mm} / \mathrm{m}$

In Abbildung 7.30 ist die Spannungsverteilung der Zugspannungen in den Deckschichten und der Schubspannungen in der Verbundfuge sowie der vektorielle Verlauf der Hauptspannungen im Kern am Ende eines Sandwichelements dargestellt. Durch die Schwinddehnung werden die Zwangspannungen in den letzten rund $30 \mathrm{~cm}$ des Sandwichelements eingeleitet. Am äußersten Rand sind die Zugspannungen in den Deckschichten gleich Null und die Verbundspannung maximal. Zur Mitte hin nehmen die Zugspannungen in den Deckschichten und die Druckspannungen im Kern zu und die Verbundspannugen ab, bis sich ein konstanter Spannungsverlauf über das Sandwichelement einstellt. Der Verlauf und die Größe der Spannungen sind unabhängig von der Länge des Elements.

In der vorliegenden Modellierung sind die entstehenden Zwangspannungen sehr gering und würden daher keine Schäden z. B. Rissbildung in den Deckschichten oder Ablösen des Verbunds, hervorrufen. Die hergestellten Biegeprobekörper und Großbauteilprobekörper wiesen ebenfalls keine Schäden durch die Schwinddehnungen auf. 


\section{Bemessungskonzept für die untersuchten Sandwichelemente}

\subsection{Allgemeines}

Im ersten Teil dieses Kapitels wird ein Ansatz zur Ermittlung der Schnittgrößen unter Berücksichtigung der Rissbildung des Betons vorgestellt. Dazu werden die theoretischen Grundlagen und deren Umsetzung im Programm swe2+ erläutert. Die Berechnungsergebnisse werden anhand von Versuchsergebnissen und Nachrechnungen mithilfe von Diagrammen verifiziert.

Im zweiten Teil des Kapitels wird das Konzept zur Bemessung der Sandwichelemente vorgestellt. Die Nachweisführung für die einzelnen Versagensarten erfolgt in Analogie zu DIN EN 14509 durch den Vergleich von Einwirkungen und Widerständen. Durch die Nachrechnung der Versuche wird die Anwendbarkeit des Nachweiskonzepts nachgewiesen. Weiterhin wird ein Vorschlag zur Schwindbemessung der Sandwichelemente vorgestellt. Schließlich werden im letzten Abschnitt beispielhaft ein Wand- sowie ein Dachelement bemessen.

\subsection{Ermittlung der Schnittgrößen}

\subsubsection{Allgemeines}

Die Nachweisführung für Sandwichelemente mit Betondeckschichten unterscheidet sich nicht wesentlich von der Nachweisführung von Elementen mit Metalldeckschichten. Jedoch muss bei der Schnittgrößenermittlung die Steifigkeitsreduzierung durch die Rissbildung in den Betondeckschichten berücksichtigt werden.

Die Schnittgrößen im Zustand I der Sandwichelemente mit Betondeckschichten können problemlos mit den vorhandenen Verfahren der linearen Sandwichtheorie für Sandwichelemente mit biegesteifen Deckschichten ermittelt werden. Überschreiten die auftretenden Normalspannungen jedoch die Zugfestigkeit des Betons, gehen die Deckschichten in diesem Bereich in den Zustand II über. Dabei nimmt die Biege- und Dehnsteifigkeit der Deckschichten im Bereich der Rissbildung ab. Diese Reduzierung der Steifigkeit führt neben einer Vergrößerung der Durchbiegung, auch zu einer Umlagerung der Schnittgrößen über den Querschnitt und die Länge des Sandwichelements. Die Umlagerung der Schnittgrößen resultiert aus der inneren statischen Unbestimmtheit der Sandwichelemente mit biegesteifen Deckschichten und tritt auch im Fall von statisch bestimmt gelagerten Sandwichsystemen auf. 
Eine vereinfachte Berechnung der Durchbiegung und der Schnittgrößenumlagerung von gerissenen Sandwichelementen kann mit dem Lastschrittverfahren nach Horstmann 2010 durchgeführt werden. Dabei wird die reduzierte Biege- und Dehnsteifigkeit im gerissenen Bereich über verschiedene Wichtungsansätze über die volle Länge des Sandwichelements „verschmiert“ (siehe auch Abschnitt 3.3.5.4, IngenieurmodelIe]', S. 44.

Durch die Wahl des entsprechenden Wichtungsansatzes kann die Durchbiegung in Feldmitte des Sandwichelements zutreffend berechnet werden. Jedoch kann die Schnittgrößenumlagerung nicht hinreichend genau ermittelt werden, da sich beispielsweise die Schnittgrößen in Feldmitte des Sandwichelements stärker umlagern als durch den Wichtungsansatz mit der gemittelten Biegesteifigkeit errechnet wird. Daher wird in den folgenden Abschnitten ein weiterer Ansatz zur Ermittlung der Schnittgrößen von Sandwichelementen mit gerissenen Deckschichten vorgestellt.

\subsubsection{Ansatz zur Ermittlung der Schnittgrößen unter Berücksichtigung der Rissbildung in den Deckschichten}

\subsubsection{Theoretische Grundlagen}

Um den Einfluss der Rissbildung exakt abbilden zu können, müssen die Dehn- und Biegesteifigkeiten der Deckschichten in Abhängigkeit ihres Zustands abschnittsweise reduziert werden. Analog zum Ansatz nach Horstmann 2010 werden hierzu drei verschiedene Rissfälle unterschieden (Biegeriss Typ A und B, Trennriss). Je nach auftretendem Rissfall werden die Dehn- und Biegesteifigkeiten entsprechend der nachfolgend beschriebenen Gleichungen für den jeweiligen Teilbereich berechnet.

Im ersten Schritt wird über die auftretenden Normalspannungen nachgewiesen, ob die Deckschichten unter der aufgebrachten Last in dem jeweiligen Teilbereich reißen. Alle folgenden Berechnungen werden dazu auf einen Sandwichstreifen mit einer Breite von einem Meter bezogen.

$$
\sigma_{o \text { bzw. } u}= \pm \frac{6 \cdot\left|M_{d}\right|}{h^{2}}+\frac{N_{d}}{h}
$$

Hierbei entspricht $h$ der Deckschichtdicke. Ist die Spannung auf der Ober- oder Unterseite der Deckschicht $\sigma_{o}$ bzw. $\sigma_{u}$ größer als die Betonzugfestigkeit $f_{c t m}$, reißt der Querschnitt. Im Fall eines gerissenen Querschnitts wird dann weiterhin zwischen Biege- und Trennrissen unterschieden. Zur Unterscheidung wird das auf die Lage der Bewehrung bezogene Moment $M_{E d s}$ verwendet. Ist:

$M_{E d s}>0$, treten Biegerisse auf.

$M_{E d s} \leq 0$, treten Trennrisse auf, da keine Druckzone vorhanden ist.

Dabei errechnet sich das auf die Lage der Bewehrung bezogene Moment $M_{E d s}$ aus $M_{d}$ und $N_{d}$ wie folgt:

$$
M_{E d s}=M_{d}-N_{d} \cdot\left(d-\frac{h}{2}\right)
$$


Dabei ist $d$ die statische Höhe der Bewehrung. Bei zweilagiger Bewehrung muss, in Abhängigkeit des Vorzeichens von $M_{d}$, das Moment auf die obere oder untere Bewehrungslage bezogen werden. Ist das Moment

$$
\begin{aligned}
& M_{d} \geq 0, \text { gilt: } a_{s}=a_{s u} \text { und } d=d_{1} \\
& M_{d}<0, \text { gilt: } M_{d}=\left|M_{d}\right|, a_{s}=a_{s o} \text { und } d=d_{2} .
\end{aligned}
$$

Dabei sind $d_{1}$ und $a_{s u}$ die statische Höhe und der Querschnitt der unteren Bewehrungslage und $d_{2}$ und $a_{s o}$ die statische Höhe und der Querschnitt der oberen Bewehrungslage.

Tritt ein Biegeriss auf, werden weiterhin die beiden Fälle A und B unterschieden. Im Fall B reißt der Querschnitt über die Lage der Bewehrung hinaus auf, so dass diese aktiviert wird. Im Fall A reißt der Querschnitt maximal bis zur Bewehrung auf, so dass diese nicht aktiviert wird und lediglich der Traganteil der Betondruckzone wirkt. In Tabelle 8.1 sind die drei unterschiedlichen Rissarten gegenübergestellt.

Tab. 8.1: Berechnung der Biege- und Dehnsteifigkeiten in Abhängigkeit der Rissart

\section{Unterscheidung der Risse im Zustand II:}

Biegeriss Typ A:

$$
\frac{-N_{d} \cdot d}{M_{E d s}} \geq 1,5
$$

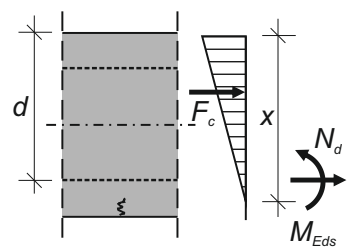

Biegeriss Typ B:

$$
\frac{-N_{d} \cdot d}{M_{E d s}}<1,5
$$

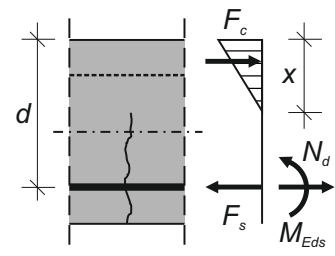

Trennriss:

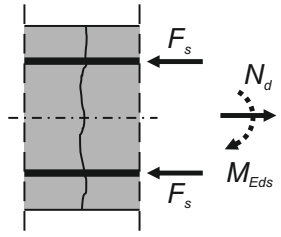

Biegesteifigkeit im Zustand II:

$E I^{\prime \prime}=\frac{\left|M_{d}\right| \cdot x}{\left|\epsilon_{c}\right|}$

siehe GI. 8.3

Annahme:

Dehnsteifigkeit im Zustand II:

$E A^{\prime \prime}=E_{c} A_{c}^{\prime \prime}$

$E A^{\prime \prime}=E_{c} A_{c}^{\prime \prime}+E_{s} a_{s}^{\prime \prime}$

$E A^{\prime \prime}=E_{s} a_{s}^{\prime \prime}$

Dabei bedeuten:
$A_{c}^{\prime \prime} \quad: \quad$ Ungerissener Betonquerschnitt in der Druckzone
$a_{s}^{\prime \prime} \quad$ : Bewehrungsquerschnitt in der gerissenen Zugzone 
Die beiden Fälle $A$ und $B$ können in Abhängigkeit des Koeffizienten $k$ nach Gleichung (8.8) unterschieden werden (Herleitung siehe Anhang C.2 S. 276

$$
k=\frac{-N_{d} \cdot d}{M_{E d s}}
$$

Ist der Koeffizient $k$ kleiner als 1,5, so ist die Druckzonenhöhe $x$ kleiner als die statische Höhe $d$ und ein Biegeriss B tritt auf. Ist der Koeffizient $k$ größer als 1,5, so überschreitet die Druckzonenhöhe die statische Höhe und ein Biegeriss A tritt ein. Bei einem Koeffizienten von $k=d /(d-h / 3)$ entspricht die Druckzonenhöhe der Querschnittshöhe $h$ (siehe Abbildung 8.1.

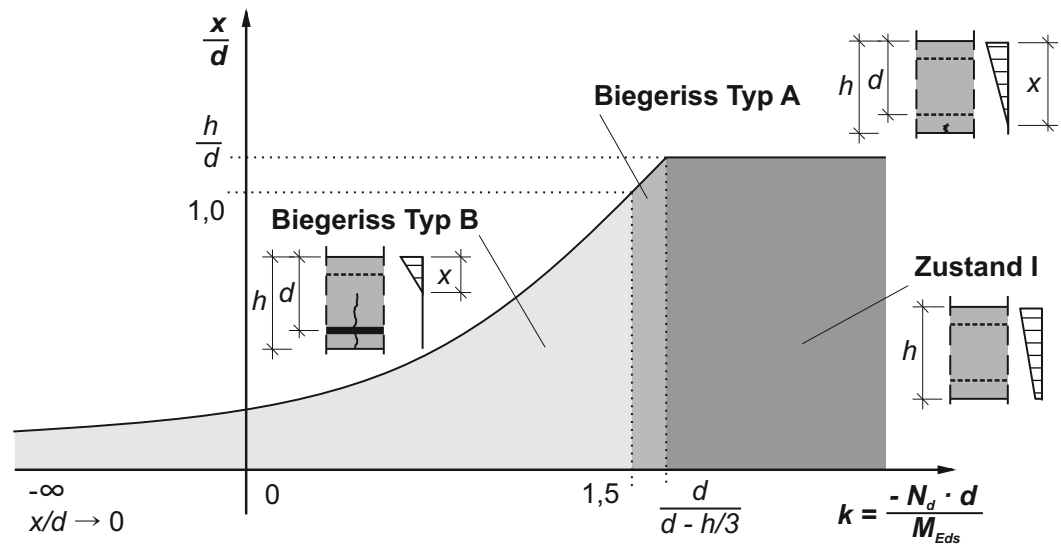

Abb. 8.1: Bezogene Druckzonenhöhe $x / d$ über dem Koeffizient $k$ eines beliebigen Querschnitts

Die in Tabelle 8.1 dargestellten Rissfälle bzw. Zustände können in Abhängigkeit der Schnittgrößen $M_{d}$ und $N_{d}$ sowie der bezogenen Lage der Bewehrung $d / h$ und der Betonzugfestigkeit $f_{c t m}$ in einem Diagramm dargestellt werden (siehe Abbildung 8.2).

Im Fall eines Biegerisses wird die Biegesteifigkeit $E I^{\prime l}$ des Querschnitts über das Moment $M_{d}$ und die Krümmung $k$ ermittelt:

$$
E I^{\prime \prime}=\frac{M_{d}}{K}
$$

Dabei ist die Krümmung:

$$
\kappa=\frac{\left|\epsilon_{c}\right|}{x}
$$

Aus den Gleichungen 8.9 und 8.10 ergibt sich Gleichung 8.3 in Tabelle 8.1 Zur Ermittlung der Biegesteifigkeit wird die Druckzonenhöhe $x$ und die Betondehnung $\epsilon_{c}$ benötigt. Diese müssen über den Dehnungszustand des Querschnitts unter der gegebenen Belastung $M_{d}$ und $N_{d}$ ermittelt werden. Der Dehnungszustand stellt sich so ein, dass die Einwirkungen $M_{d}$ und $N_{d}$ mit den resultierenden Widerständen, d. h. der Betondruckkraft $F_{c}$ und der Zugkraft $F_{s}$ im Gleichgewicht sind. 


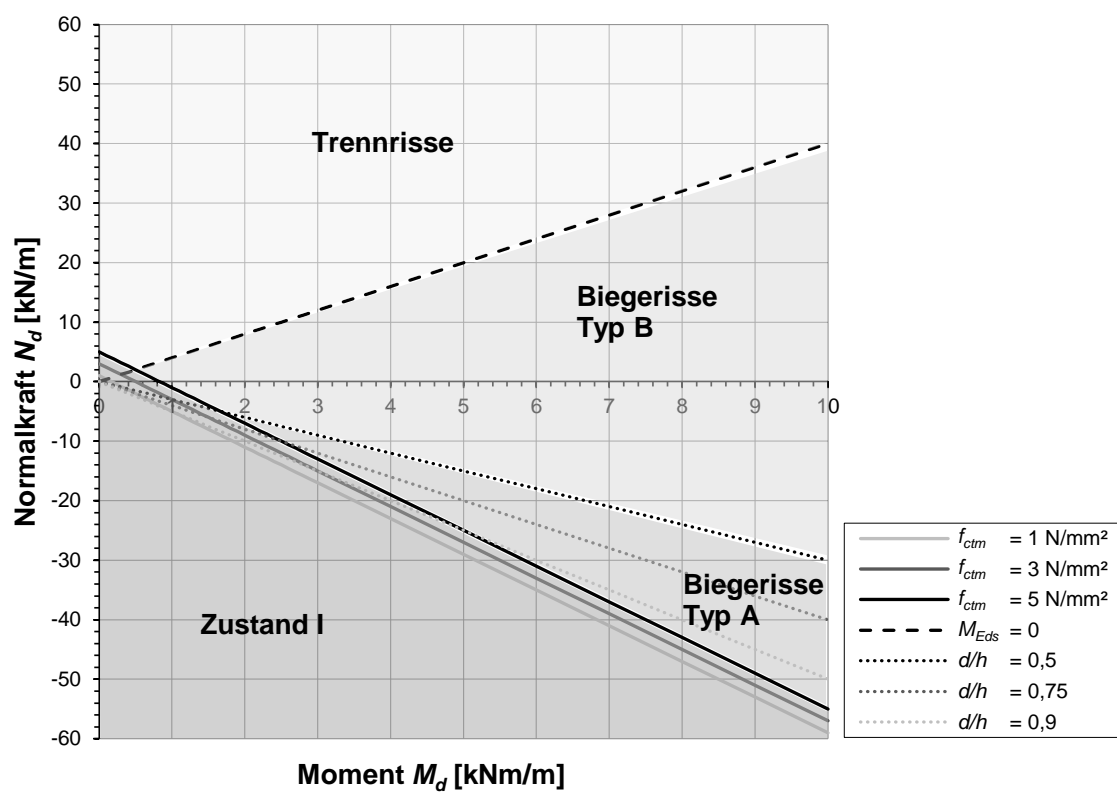

Abb. 8.2: Unterscheidung der Zustände der Deckschichten in Abhängigkeit des Moments $M_{d}$, der Normalkraft $N_{d}$, der Betonzugfestigkeit $f_{c t m}$ und der Lage der Bewehrung $d / h$

Durch Horstmann 2010 wurde die Dehnungsebene mithilfe der Annahme eines Spannungsblocks, also einer konstanten Druckspannung in der Druckzone, ermittelt. Die Annahme eines Spannungsblocks unterstellt bei der Bemessung im Grenzzustand der Tragfähigkeit eine starke Plastizierung der Druckzone. Jedoch kann bei der SchnittgröBenermittlung diese Plastizierung der Druckzone nicht vorausgesetzt werden, da die einzelnen Teilbereiche in den überwiegenden Fällen nicht bis zum Grenzzustand der Tragfähigkeit beansprucht werden.

Die Annahme eines linear-elastischen Werkstoffverhaltens liefert insbesondere bei Verwendung eines Hochleistungsbetons exaktere Werte, da hochfeste Betone bis zu einem Spannungsniveau von $80 \%$ der Druckfestigkeit ein nahezu linear-elastisches Spannungsdehnungsverhalten aufweisen (Schmidt 2008). Durch die Gegenüberstellung der linear-elastischen und der nichtlinearen Spannungsdehnungslinien eines Betons der Festigkeitsklasse C100/115 in Abbildung 8.3 wird dies verdeutlicht.

Unter Annahme eines linear-elastischen Spannungsdehnungsverhaltens des Betons kann die Druckzonenhöhe $x$ mit der folgenden linearen Gleichung dritter Ordnung ermittelt werden (Herleitung siehe Anhang C.1, S. 275. Die Druckzonenhöhe $x$ ist die erste Nullstelle der Gleichung im positiven Bereich von $0<x_{1}<h$.

$$
0=\frac{1}{6} N_{d} E_{c} x^{3}-\frac{1}{2} E_{c}\left(M_{E d s}+N_{d} d\right) x^{2}-E_{s} A_{s} M_{E d s} x+E_{s} A_{s} M_{E d s} d
$$

Die Betondehnung $\epsilon_{c}$ wird über das Hookesche Gesetz aus der Druckspannung $\sigma_{c}$ und dem Elastizitätsmodul $E_{c}$ des Betons berechnet. Dazu berechnet sich die Beton- 
druckspannung wie folgt:

$$
\sigma_{c}=\frac{N_{d} \cdot E_{c} \cdot x}{-\frac{1}{2} \cdot E_{c} \cdot x^{2}+E_{s} \cdot A_{s} \cdot(d-x)}
$$

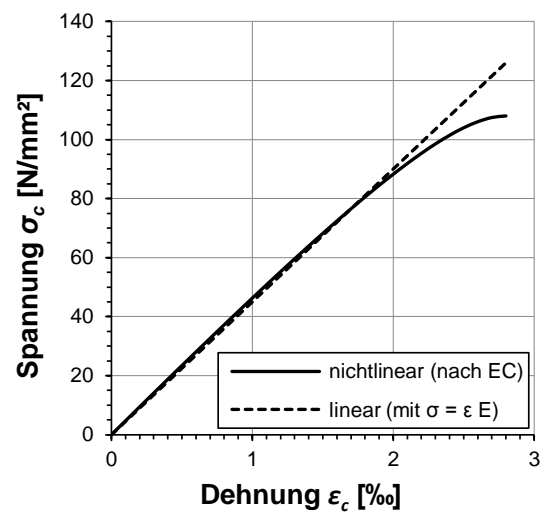

Abb. 8.3: Linear-elastische und nichtlineare Spannungsdehnungslinien eines Betons der Festigkeitsklasse C100/115 nach DIN EN 1992 für "nichtlineare Verfahren der Schnittgrößenermittlung und für Verformungsberechnungen“

\subsubsection{Programmiertechnische Umsetzung des Ansatzes}

Über den Ansatz von Schwarze 1984 und dessen Umsetzung im Programm „swe2“ können bereits abschnittsweise die Schnittgrößen, Spannungen und Verformungen eines Sandwichelements berechnet werden. Um die Steifigkeitsänderung infolge der Rissbildung zu berücksichtigen, wurde das Programm entsprechend umfangreich erweitert und angepasst.

Dies erfolgte unter Anleitung im Rahmen der Studienarbeit von Besch 2014. Das überarbeitete Programm wird im Nachfolgenden nun als „swe2+" bezeichnet und steht auf der Homepage des Fachgebiets „Massivbau und Baukonstruktion“ der TU Kaiserslautern zum Download bereit. Der erweiterte Quellcode sowie die Studienarbeit sind ebenfalls dort verfügbar.

Der Berechnungsalgorithmus des Programms ist schematisch in Abbildung 8.4 dargestellt. Die Berechnung gliedert sich in drei Teile. Wie bereits in Abschnitt 3.3.3.2, Ansatz von Schwarze zur Lösung der Differentialgleichung]' (siehe S. 33) erläutert, wird im ersten Teil zunächst der Lösungsvektor $K$ und dann im zweiten Teil der Lösungsvektor $Z$ berechnet. Abschließend werden im dritten Teil die vollständigen Schnittgrößen und Spannungen über den Querschnitt und die Länge des Sandwichelements berechnet.

Die Berechnung erfolgt iterativ mit einer gewissen Anzahl an Berechnungsschleifen $n$, die in der Eingabemaske entsprechend definiert werden. Als Bestandteil der Berechnung der Lösungsvektoren $K$ und $Z$, werden die erforderlichen Hilfswerte in Abhängigkeit der Rissbildung mit der Funktion f (sigma) (siehe Abbildung 8.5) berechnet. 


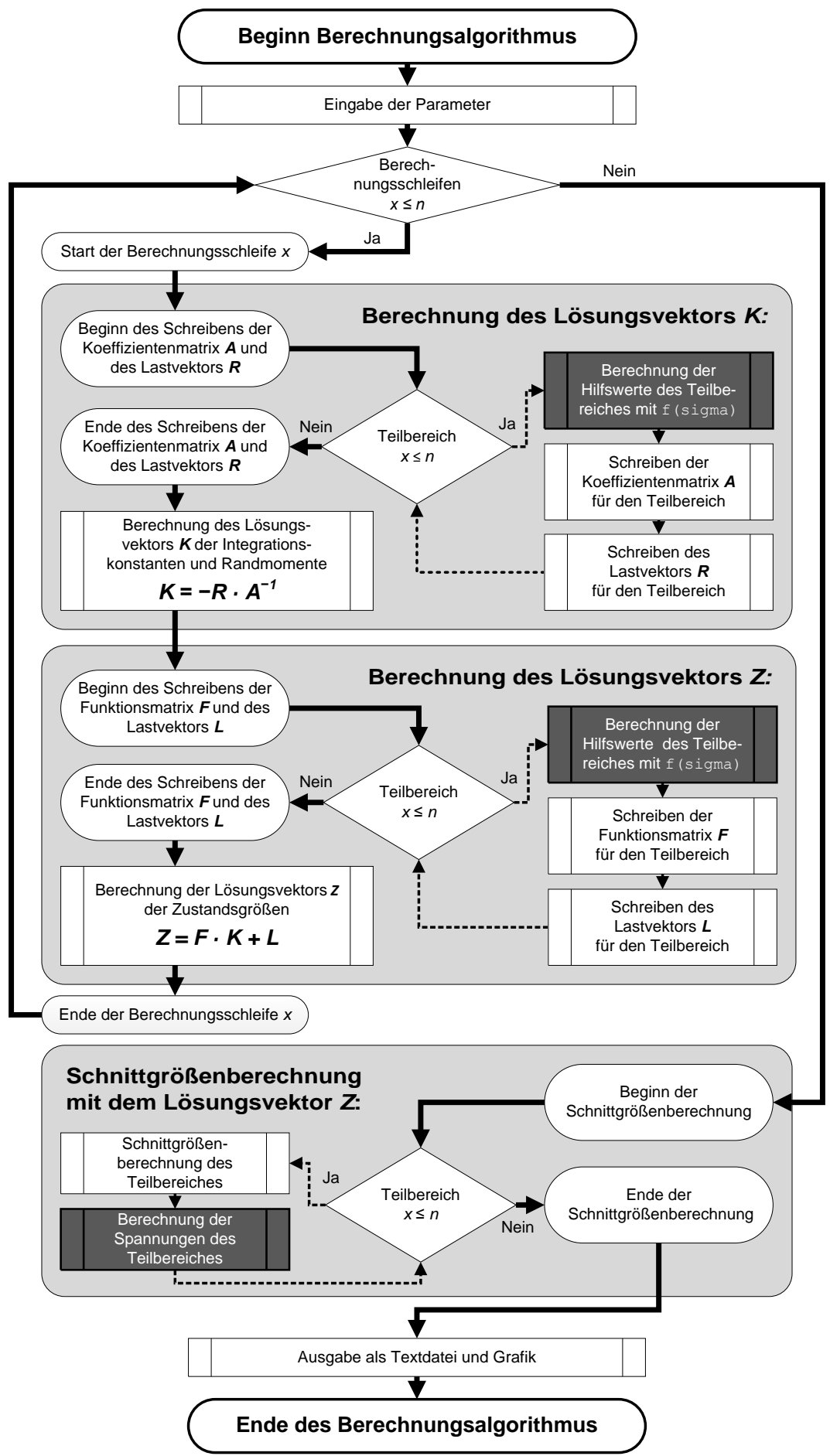

Abb. 8.4: Schematische Darstellung des Berechnungsalgorithmus des Programms swe2+ 
Beim Durchlauf einer jeden Berechnungsschleife wird die Belastung anteilig um $1 / n$ gesteigert. Die Belastungssteigerung ist erforderlich, um die Umlagerung der Schnittgrößen über den Querschnitt und die Länge des Sandwichelements zu berücksichtigen. Insbesondere bei statisch unbestimmt gelagerten Sandwichsystemen treten beispielsweise durch die Rissbildung im Bereich der Mittelauflager Umlagerungen auf, die dann zu weiterer Rissbildung in den Feldern führen können.

Weiterhin wurde bei der Implementierung des Ansatzes festgestellt, dass die Belastungsgeschichte bei der iterativen Berechnung zu berücksichtigten ist. Durch die Rissbildung des Betons und die damit verbundene Schnittgrößenumlagerung, können bereits gerissene Deckschichten wieder „überdrückt“ werden. Dabei darf jedoch die bereits ermittelte reduzierte Biegesteifigkeit der Deckschicht nicht wieder durch einen größeren Wert überschrieben werden, da ansonsten der Zustand der Deckschichten in diesen Fällen fortlaufend zwischen gerissen und ungerissen wechselt und die Berechnung somit nicht konvergiert. Um ein ähnliches Konvergenzproblem zu verhindern, müssen weiterhin die Betonzugfestigkeiten gerissener Bereiche zu Null gesetzt werden.

Der Berechnungsalgorithmus der Funktion $\mathrm{f}$ (sigma) ist in Abbildung 8.5 schematisch dargestellt. Anhand des zuvor beschriebenen Ansatzes wird der Zustand der beiden Deckschichten des jeweiligen Teilbereichs in Abhängigkeit der Momente $M_{s}, M_{o}$ und $M_{u}$ der letzten Berechnungsschleife ermittelt und die Dehn- und Biegesteifigkeit entsprechend berechnet. Abschließend werden der Querschnittszustand (1: Zustand I, 2: Biegeriss A; 3: Biegeriss B, 4: Trennriss), die Biege- und Dehnsteifigkeiten sowie die Betonzugfestigkeit für die nachfolgenden Berechnungsschritte im Querschnittsvektor QV gespeichert.

\subsubsection{Vereinfachungen}

Dem beschriebenen Ansatz zur Ermittlung der Schnittgrößen und der Umsetzung im Programm liegen die folgenden Vereinfachungen zu Grunde:

- Es wird ein linear-elastisches Werkstoffverhalten des Hochleistungsbeton angenommen.

- Der Einfluss von Bewehrungslagen in der Druckzone (Druckbewehrung) wird vernachlässigt.

- Im Fall eines Trennrisses wird die Eigenbiegesteifigkeit der Bewehrungslagen sowie deren Steiner'sche Anteile bei mehreren Bewehrungslagen vernachlässigt.

- Im Fall eines Biegerisses wird nur die Biegesteifigkeit der Druckzone berücksichtigt. Die Eigenbiegesteifigkeit der Bewehrung und deren Steiner'sche Anteile werden vernachlässigt.

- Als Übergangsbedingung zwischen gerissene und ungerissene Teilbereichen wird deren Krümmung stetig angenommen. In Realität bildet sich jedoch ein Knick an einem Riss aus.

Die Auswirkungen dieser Annahmen auf die Ermittlung der Biege- und Dehnsteifigkeit sowie der Schnittgrößen sind gering und können somit vernachlässigt werden. 


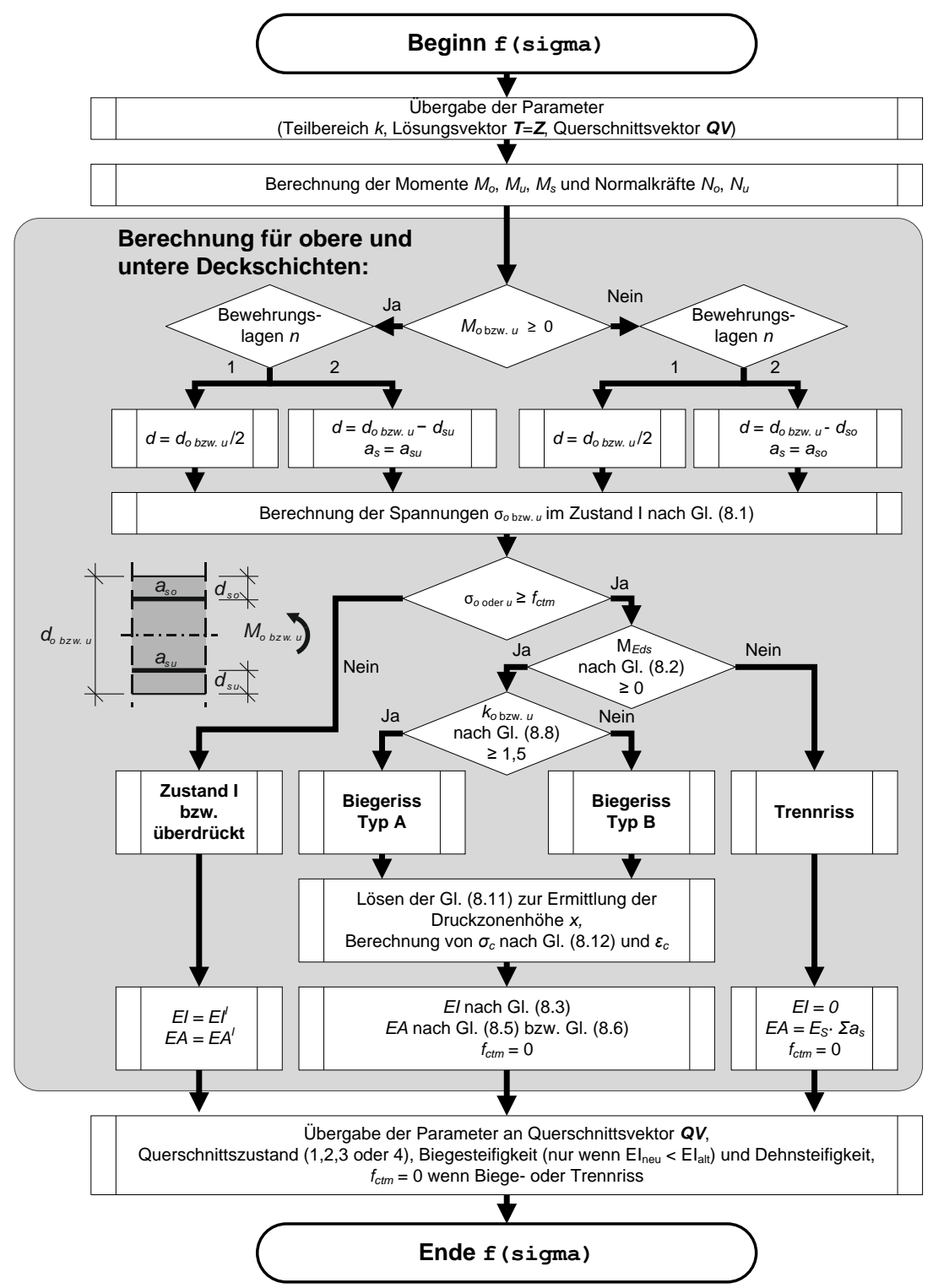

Abb. 8.5: Schematische Darstellung des Berechnungsalgorithmus der Funktion $f$ (sigma)

\subsubsection{Grafische Benutzeroberfläche}

Die bestehende grafische Benutzeroberfläche von swe2 wurde für die Eingabe der Materialeigenschaften von Betondeckschichten entsprechend erweitert. In der Registerkarte „Material“ kann nun über eine Auswahlliste das Material der Deckschichten zwischen „Stahl“ und „Beton“ ausgewählt werden. Wird hier, wie in Abbildung 8.6 dargestellt, „Beton“ ausgewählt, so erscheinen weitere Auswahlmöglichkeiten zur Eingabe der Materialeigenschaften. Neben dem Elastizitätsmodul des Betons und der Beweh- 
rung sowie der Betonzugfestigkeit, muss auch die Anzahl der Berechnungsschleifen angegeben werden. Zusätzlich kann über eine weitere Auswahlliste zwischen einlagiger und zweilagiger Bewehrungsanordnung gewählt werden. Hierzu müssen dann entsprechend die Bewehrungsquerschnitte angegeben werden.
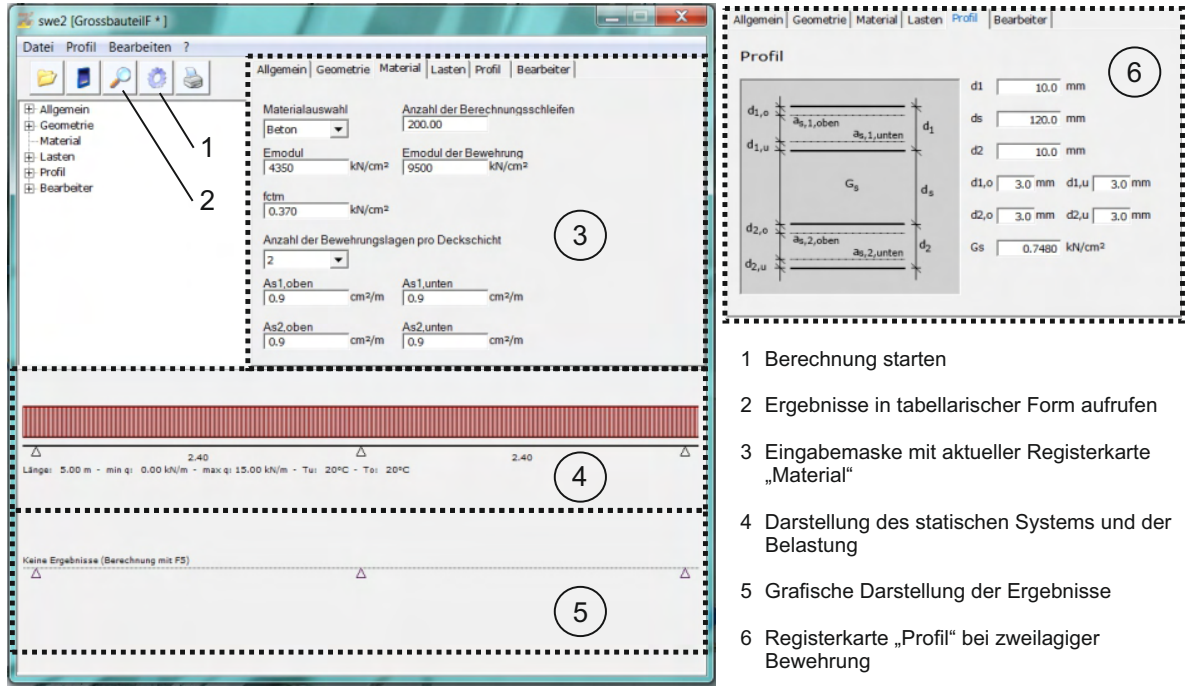

Abb. 8.6: Grafische Benutzeroberfläche von swe2+

In der Registerkarte „Profil“ wird je nach einlagiger oder zweilagiger Bewehrungsanordnung entsprechend ein schematischer Querschnitt des Sandwichelements dargestellt. Über weitere Eingabefelder werden hier die Dicken des Kerns und der Deckschichten, sowie die Lage der einzelnen Bewehrungslagen eingegeben. Weiterhin wird hier der Schubmodul des Kernmaterials definiert.

Nachdem die Schaltfläche „Berechnen“ betätigt wird, können die Ergebnisse entweder grafisch dargestellt oder tabellarisch ausgegeben werden.

\subsubsection{Kontrolle der Berechnungsergebnisse durch Vergleich mit den Versuchsergebnissen}

Zur Kontrolle des Programms wurden die Großbauteilversuche mit Einzel- und Flächenbelastung nachgerechnet. Wie in den Abbildungen 8.7 und 8.8 dargestellt, wurden die statischen Systeme entsprechend der Versuchsaufbauten gewählt. Der Überstand an den Auflagern wurde als Kragarm definiert. Im Fall der Nachrechnung des Zweifeldträgers wurde zur Berücksichtigung der Momentenausrundung am Mittelauflager, ein drittes Feld mit der Länge der Lastverteilungsplatte von $80 \mathrm{~mm}$ angeordnet.

Wie bei den Probekörpern auch, wurden die Schichtdicken des Kerns und der Deckschichten mit $120 \mathrm{~mm}$ und $10 \mathrm{~mm}$ gewählt. Der Schubmodul der XPS-Charge X6 betrug $7.480 \mathrm{kN} / \mathrm{m}^{2}$ (siehe Abbildung 5.28 S. 86 . Der Elastizitätsmodul und die Betonzugfestigkeit wurden wie in den FE-Nachrechnungen mit $43.500 \mathrm{~N} / \mathrm{mm}^{2}$ und $3,7 \mathrm{~N} / \mathrm{mm}^{2}$ (siehe Tabelle 4.3 S. 51) angenommen. Im Gegensatz zu den FE-Modellierungen wur- 


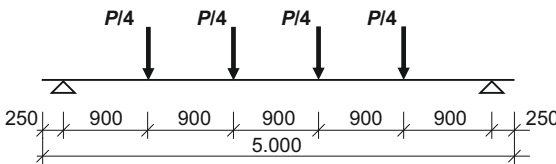

Abb. 8.7: Statisches System zur Nachrechnung der Großbauteilversuche mit Einzellasten

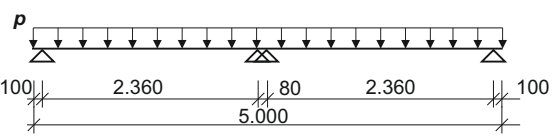

Abb. 8.8: Statisches System zur Nachrechnung der Großbauteilversuche mit Flächenlast

de das Spannungsdehnungsverhalten des Beton im gerissenen Zustand nicht über die Eingabe einer idealisierten Spannungsdehnungslinie, sondern über die Eingabe eines effektiven Elastizitätsmoduls der Bewehrung im Zustand II definiert. Dieser wurde aus den Dehnkörperversuchen übernommen und betrug bei Verwendung des Carbonfasergeleges $E_{o}^{*}=95.000 \mathrm{~N} / \mathrm{mm}^{2}$ (siehe Abbildung 5.41 S. 96). Der Querschnitt des einlagigen Carbonfasergeleges betrug $180 \mathrm{~mm}^{2} / \mathrm{m}$ (siehe Tabelle 4.6 . S. 55 .

Zur Erstellung einer Lastverformungskurve, wurden mit dem Programm swe2+ die Durchbiegungen der Sandwichelemente bei verschiedenen Laststufen berechnet. Dazu wurden unterschiedliche Anzahlen an Iterationsschleifen $n$ gewählt. Die Lastverformungskurven sind zusammen mit den Kurven der Versuche und der FE-Berechnungen in den Abbildungen 8.9 und 8.10 dargestellt.

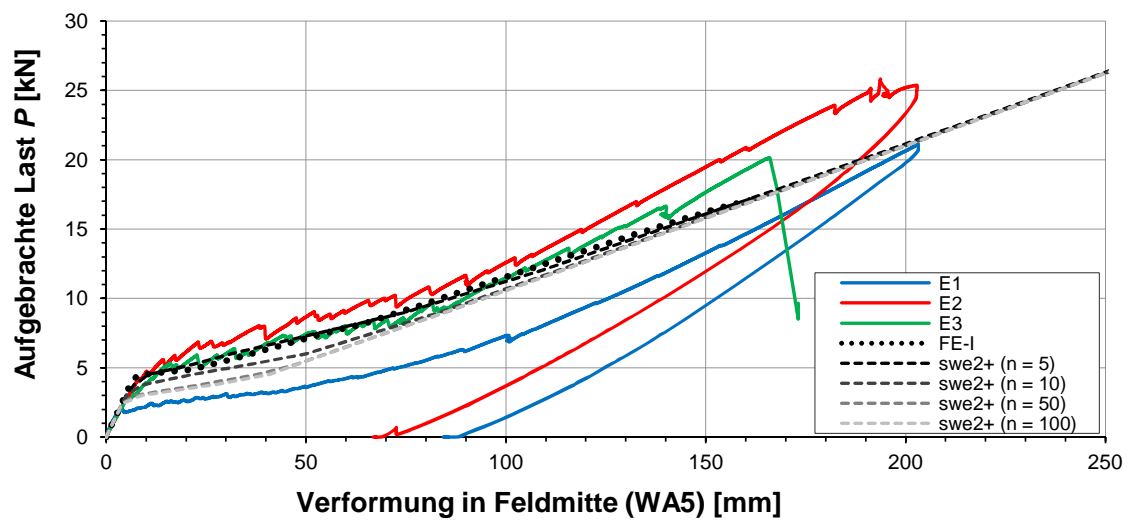

Abb. 8.9: Lastverformungskurven der Großbauteilversuche mit Einzellasten und deren Nachrechnung durch FEM und swe2+

Die Lastverformungskurven, die mit swe2+ ermittelt wurden, weisen generell eine sehr gute Übereinstimmung mit denen der Versuche und der FE-Modellierungen auf. Im Zustand I sind alle Kurven identisch. Im Zustand II weisen die Kurven ebenfalls sehr gute Übereinstimmungen auf. Die mit FEM und swe2+ ermittelten Kurven laufen in etwa parallel zu einander. Wobei die mit swe2+ berechneten Kurven ein etwas geringeres Lastniveau aufweisen. Beide rechnerisch ermittelten Kurven verlaufen im Vergleich zu den experimentell ermittelten Kurven im Bereich des Zustands II b zu flach. Dies bestätigt die These, dass in den Großbauteilversuchen ein größerer Querschnittsanteil der Fasern aktiviert wurde als in den Dehnkörperversuchen. 


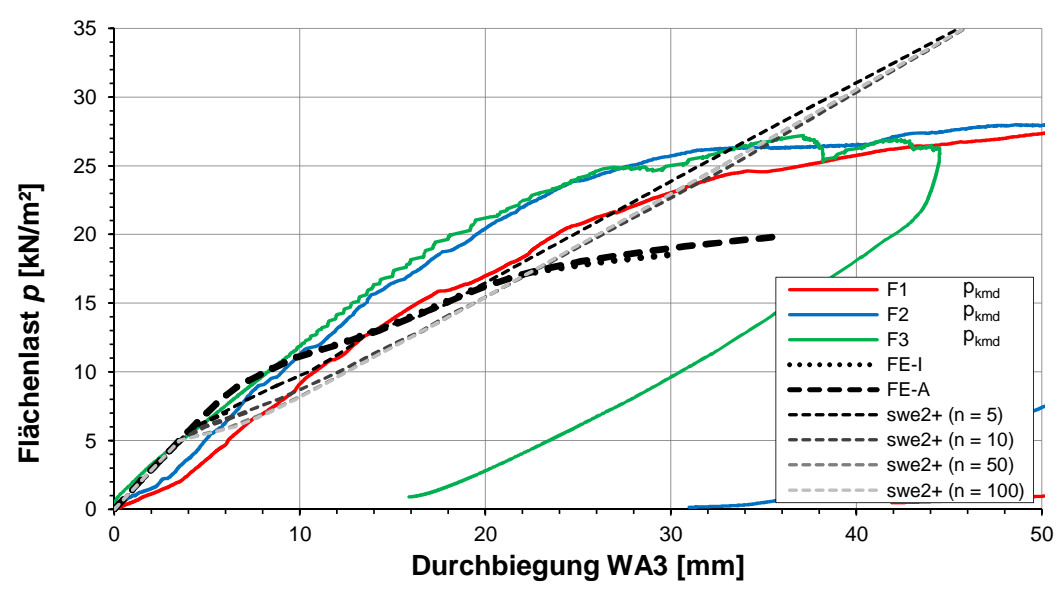

Abb. 8.10: Lastverformungskurven der Großbauteilversuche mit Flächenlast und deren Nachrechnung durch FEM und swe2+

Es besteht weiterhin ein Einfluss der Anzahl der Iterationsschleifen $n$ auf das Lastverformungsverhalten. Dabei unterscheiden sich die berechneten Kurven insbesondere im Zustand II a. Die Kurven, die mit einer hohen Anzahl an Iterationschleifen $n$ erstellt wurden, flachen durch die Rissbildung stärker ab, als die Kurven, die mit einer niedrigeren Anzahl an Schleifen erstellt wurden. In den Berechnungen konnte beobachtet werden, dass sich gerissene Bereich bei den nachfolgenden Iterationsschleifen vergrößern und dadurch die Steifigkeit abfällt. Eine Vergrößerung gerissener Bereiche wird zum einen durch die Umlagerung der Schnittgrößen, zum anderen auch durch die idealisierten Annahmen, die der Berechnung zu Grunde liegen verursacht. Durch die Annahme, dass die Krümmungen zwischen einem gerissenen und einem ungerissenen Bereich identisch sind, wird im ungerissenen Bereich ein Deckschichtmoment induziert, das dann zum Reißen des ungerissenen Bereiches führen kann. Auf diese Weise kann sich ein gerissener Bereich über mehrere Iterationsschleifen hinweg vergrößern.

\subsubsection{Kontrolle der Berechnungsergebnisse durch Vergleichsrechnungen}

Zur weiteren Kontrolle der Berechnungsergebnisse wurden diese überschlägig mithilfe von Diagrammen nachgerechnet. Dazu wurde ein zweifeldriges Sandwichelement mit einer Stützweite von 4,75 m berechnet. Zur Kontrolle der Ergebnisse sollte der Einfluss der biegesteifen Deckschichten möglichst groß sein. Daher wurden in diesem Beispiel, im Vergleich zu den bisher untersuchten Fällen, größere Deckschichtdicken gewählt. Die obere Deckschicht des Sandwichelements wies dabei eine Dicke von $50 \mathrm{~mm}$ und die untere Deckschicht eine Dicke von $25 \mathrm{~mm}$ auf. Die Kerndicke betrug $80 \mathrm{~mm}$.

Das Beispiel wurde sowohl mit ungerissenen als auch mit gerissenen Deckschichten gerechnet. Im Fall der ungerissenen Deckschichten wurde die Zugfestigkeit des Betons auf einen sehr großen Wert gesetzt, so dass dieser in den Berechnungen nicht überschritten wurde. Im Fall der gerissenen Deckschichten wurde die Betonzugfestigkeit 
auf $1 \mathrm{~N} / \mathrm{mm}^{2}$ gesetzt und das Beispiel dann sowohl mit ein- als auch mit zweilagiger Bewehrungsanordnung gerechnet. Alle Eigenschaften des Sandwichelements sind in Tabelle 8.2 zusammengestellt. Als Belastungsfälle wurden eine Gleichstreckenlast von $p=1,5 \mathrm{kN} / \mathrm{m}^{2}$ und $p=3,0 \mathrm{kN} / \mathrm{m}^{2}$ sowie eine Temperaturdifferenz zwischen den Deckschichten von $55^{\circ} \mathrm{K}$ angesetzt.

Tab. 8.2: Eingangsparameter zur Kontrolle der Berechnungsergebnisse mit swe2+ durch eine Vergleichsberechnung

\begin{tabular}{|c|c|c|c|c|c|c|c|}
\hline \multirow{2}{*}{\multicolumn{2}{|c|}{$\begin{array}{l}\text { Eingangs- } \\
\text { parameter }\end{array}$}} & \multirow[b]{3}{*}{50,0} & & \multirow{2}{*}{\multicolumn{2}{|c|}{$\begin{array}{l}\text { Eingangs- } \\
\text { parameter }\end{array}$}} & \multicolumn{2}{|c|}{ Bewehrung } \\
\hline & & & & & & 1-lagig & 2-lagig \\
\hline$d_{o}$ & {$[\mathrm{~mm}]$} & & \multirow{2}{*}{$a_{s 1}$} & oben & {$\left[\mathrm{mm}^{2} / \mathrm{m}\right]$} & \multirow{2}{*}{$1.000,0$} & 500,0 \\
\hline$d_{s}$ & {$[\mathrm{~mm}]$} & 80,0 & & unter & {$\left[\mathrm{mm}^{2} / \mathrm{m}\right]$} & & 500,0 \\
\hline$d_{u}$ & {$[\mathrm{~mm}]$} & 25,0 & \multirow{2}{*}{$a_{s 1}$} & oben & {$\left[\mathrm{mm}^{2} / \mathrm{m}\right]$} & \multirow{2}{*}{500,0} & 250,0 \\
\hline$G$ & {$\left[\mathrm{kN} / \mathrm{m}^{2}\right]$} & 6.000 & & unter & {$\left[\mathrm{mm}^{2} / \mathrm{m}\right]$} & & 250,0 \\
\hline$E_{c}$ & {$\left[\mathrm{~N} / \mathrm{mm}^{2}\right]$} & 30.000 & \multirow{2}{*}{$d_{s 1}$} & oben & {$[\mathrm{mm}]$} & \multirow{2}{*}{25,0} & 5,0 \\
\hline$E_{s}$ & {$\left[\mathrm{~N} / \mathrm{mm}^{2}\right]$} & 160.000 & & unter & {$[\mathrm{mm}]$} & & 5,0 \\
\hline$f_{c t m}$ & {$\left[\mathrm{~N} / \mathrm{mm}^{2}\right]$} & 1,0 & \multirow{2}{*}{$d_{s 2}$} & oben & {$[\mathrm{mm}]$} & \multirow{2}{*}{12,5} & 2,5 \\
\hline$n$ & {$[-]$} & 100,0 & & unter & {$[\mathrm{mm}]$} & & 2,5 \\
\hline
\end{tabular}

Da für die Berechnung der Schnittgrößen von mehrfeldrigen Sandwichelementen mit profilierten Deckschichten keine geschlossenen Gleichungen existieren, erfolgt die Berechnung in der Regel mit EDV-Programmen oder anhand von Diagrammen. Für die folgenden Nachrechnungen wurden die Diagramme aus der Arbeit von Berner 1994 angewendet. In Abhängigkeit des Parameters $k$ nach Gleichung 3.22 (siehe $S$. 30 und des Parameters $B_{D} / B_{S}$ können aus den Diagrammen die Hilfswerte $\beta_{S q}$ und $\beta_{\text {oq }}$ bei Beanspruchung durch eine Gleichstreckenlast, sowie die Hilfswerte $\beta_{S T}$ und $\beta_{O T}$ bei Temperaturbeanspruchung abgelesen werden. Das Diagramm zur Ermittlung von $\beta_{o q}$ für Sandwichelemente mit gleichen Stützweiten wurde bereits in Abbildung 3.16 (S. 31 dargestellt. Für alle weiteren Diagramme sei auf die Arbeit von Berner 1994 verwiesen.

Das Stützmoment $M^{\prime}$ und das Sandwichmoment $M_{S}$ am Mittelauflager errechnen sich aus den Hilfswerten $\beta_{S q}$ und $\beta_{\text {oq }}$ sowie dem Stützmoment $M_{\text {stat }}$ aus der Balkentheorie wie folgt:

$$
\begin{aligned}
M^{\prime} & =\frac{M_{\text {stat }}}{1+\beta_{S q}} \\
M_{S} & =\left(1-\beta_{O q}\right) \cdot M^{\prime}
\end{aligned}
$$

Mit den Hilfswerten $\beta_{S T}$ und $\beta_{O T}$ werden die Sandwich- und Deckschichtmomente am Mittelauflager infolge einer Temperaturbeanspruchung wie folgt berechnet:

$$
\begin{aligned}
& M_{D}=\frac{-\alpha_{T} \cdot\left(T_{u}-T_{o}\right) \cdot B_{D} \cdot \beta_{S T}}{e} \\
& M_{S}=M_{D} \cdot\left(\frac{1}{\beta_{O T}}-1\right)
\end{aligned}
$$

Wie im Programm swe2+ auch, wird zur Berechnung der Wärmeausdehnungskoeffizient $\alpha_{T}$ mit $1,17 \cdot 10^{-5}$ angenommen (vgl. Schmidt 2008). 
Tab. 8.3: Stütz- und Sandwichmomente nach Berner 1994 unter Beanspruchung durch eine Gleichstreckenlast $p$

\begin{tabular}{|c|c|c|c|c|c|c|c|c|c|c|c|}
\hline $\begin{array}{c}p \\
{\left[\mathrm{kN} / \mathrm{m}^{2}\right]}\end{array}$ & $\begin{array}{c}\text { Zu- } \\
\text { stand }\end{array}$ & $\begin{array}{l}\text { Bew.- } \\
\text { Lagen }\end{array}$ & $\begin{array}{c}\boldsymbol{d}_{\mathbf{o}} \\
{[\mathrm{mm}]}\end{array}$ & $\begin{array}{c}k \\
{[-]}\end{array}$ & $\begin{array}{c}B_{D} / B_{s} \\
{[-]}\end{array}$ & $\begin{array}{c}\boldsymbol{\beta}_{o q} \\
{[-]} \\
\end{array}$ & $\begin{array}{c}\boldsymbol{\beta}_{s q} \\
{[-]}\end{array}$ & $\begin{array}{c}\boldsymbol{M}_{\text {stat }} \\
{[\mathrm{kNm} / \mathrm{m}]}\end{array}$ & $\begin{array}{c}\boldsymbol{M}^{\prime} \\
{[\mathrm{kNm} / \mathrm{m}]}\end{array}$ & $\begin{array}{c}M_{\prime \prime}^{\prime} / M_{1}^{\prime}, \\
{[-]}\end{array}$ & $\begin{array}{c}M_{s} \\
{[\mathrm{kNm} / \mathrm{m}]}\end{array}$ \\
\hline \multirow{3}{*}{1,5} & I & - & 50,0 & 0,295 & 0,051 & 0,620 & 0,347 & $-4,23$ & $-3,14$ & - & $-1,19$ \\
\hline & II & 1 & 47,1 & 0,045 & 0,256 & 0,535 & 0,065 & $-4,23$ & $-3,97$ & $26,5 \%$ & $-1,85$ \\
\hline & II & 2 & 35,9 & 0,044 & 0,127 & 0,390 & 0,085 & $-4,23$ & $-3,90$ & $24,1 \%$ & $-2,38$ \\
\hline \multirow{3}{*}{3,0} & I & - & 50,0 & 0,295 & 0,051 & 0,620 & 0,347 & $-8,46$ & $-6,28$ & - & $-2,39$ \\
\hline & II & 1 & 42,9 & 0,045 & 0,202 & 0,490 & 0,070 & $-8,46$ & $-7,91$ & $25,9 \%$ & $-4,03$ \\
\hline & II & 2 & 34,1 & 0,044 & 0,111 & 0,370 & 0,090 & $-8,46$ & $-7,76$ & $23,6 \%$ & $-4,89$ \\
\hline
\end{tabular}

Tab. 8.4: Stütz- und Sandwichmomente nach Berner 1994 unter einer Temperaturbeanspruchung $\Delta T$

\begin{tabular}{|cccccccccccc|}
\hline $\begin{array}{c}\Delta \boldsymbol{T} \\
{\left[{ }^{\circ} \mathrm{K}\right]}\end{array}$ & $\begin{array}{c}\text { Zu- } \\
\text { stand Lew.- }\end{array}$ & $\begin{array}{c}\boldsymbol{d}_{\boldsymbol{o}} \\
{[\mathrm{mm}]}\end{array}$ & $\begin{array}{c}\boldsymbol{k} \\
{[-]}\end{array}$ & $\begin{array}{c}\boldsymbol{B}_{\boldsymbol{D}} / \boldsymbol{B}_{\boldsymbol{s}} \\
{[-]}\end{array}$ & $\begin{array}{c}\boldsymbol{\beta}_{\boldsymbol{s T}} \\
{[-]}\end{array}$ & $\begin{array}{c}\boldsymbol{\beta}_{\text {oT }} \\
{[-]}\end{array}$ & $\begin{array}{c}\boldsymbol{B}_{\boldsymbol{D}} \\
{\left[\mathrm{Nmm}^{2}\right]}\end{array}$ & $\begin{array}{c}\boldsymbol{M}^{\prime} \\
{[\mathrm{kNm} / \mathrm{m}]}\end{array}$ & $\begin{array}{c}\boldsymbol{M}_{, /}^{\prime} / \boldsymbol{M}^{\prime}, \\
{[-]}\end{array}$ & $\begin{array}{c}\boldsymbol{M}_{\boldsymbol{s}} \\
{[\mathrm{kNm} / \mathrm{m}]}\end{array}$ \\
\hline \multirow{3}{*}{55} & I & - & 50,0 & 0,295 & 0,051 & 1,700 & 0,103 & $3,5 \mathrm{E}+11$ & 31,93 & - & 28,66 \\
& II & 1 & 44,1 & 0,045 & 0,217 & 0,750 & 0,112 & $2,2 \mathrm{E}+11$ & 7,92 & $-75,2 \%$ & 7,03 \\
& II & 2 & 42,8 & 0,045 & 0,189 & 0,780 & 0,109 & $2,0 \mathrm{E}+11$ & 7,68 & $-75,9 \%$ & 6,84 \\
\hline
\end{tabular}

Tab. 8.5: Stütz- und Sandwichmomente mit swe2+ unter Beanspruchung durch eine Gleichstreckenlast $p$ und Differenz zu Berner 1994 in Tabelle 8.3

\begin{tabular}{|ccccccccc|}
\hline $\begin{array}{c}p \\
{\left[\mathrm{kN} / \mathrm{m}^{2}\right]}\end{array}$ & $\begin{array}{c}\text { Zu- } \\
\text { stand }\end{array}$ & $\begin{array}{c}\text { Bew.- Auflösung } \\
\text { Lagen }\end{array}$ & {$[-]$} & $\begin{array}{c}\text { Schleifen } \\
{[-]}\end{array}$ & $\begin{array}{c}\boldsymbol{M}^{\prime} \\
{[\mathrm{kNm} / \mathrm{m}]}\end{array}$ & $\begin{array}{c}\text { Differenz } \\
{[-]}\end{array}$ & $\begin{array}{c}\boldsymbol{M}_{\boldsymbol{s}} \\
{[\mathrm{kNm} / \mathrm{m}]}\end{array}$ & $\begin{array}{c}\text { Differenz } \\
{[-]}\end{array}$ \\
\hline \multirow{3}{*}{1,5} & I & - & 5120 & 100 & $-3,17$ & $0,8 \%$ & $-1,19$ & $-0,1 \%$ \\
& II & 1 & 5120 & 100 & $-4,06$ & $2,2 \%$ & $-3,69$ & $99,6 \%$ \\
& II & 2 & 5120 & 100 & $-4,32$ & $10,8 \%$ & $-2,82$ & $18,5 \%$ \\
\multirow{3}{*}{3,0} & I & - & 5120 & 100 & $-6,33$ & $0,8 \%$ & $-2,38$ & $-0,1 \%$ \\
& II & 1 & 5120 & 100 & $-7,94$ & $0,4 \%$ & $-7,01$ & $73,8 \%$ \\
& II & 2 & 5120 & 100 & $-8,56$ & $10,3 \%$ & $-5,48$ & $12,1 \%$ \\
\hline
\end{tabular}

Tab. 8.6: Stütz- und Sandwichmomente mit swe2+ unter einer Temperaturbeanspruchung $\Delta T$ und Differenz zu Berner 1994 in Tabelle 8.4

\begin{tabular}{|ccccccccc|}
\hline $\begin{array}{c}\Delta \boldsymbol{T} \\
{\left[{ }^{\circ} \mathrm{K}\right]}\end{array}$ & $\begin{array}{c}\text { Zu- } \\
\text { stand }\end{array}$ & \multicolumn{2}{c}{ Bew.- Auflösung } & Schleifen & $\boldsymbol{M}^{\prime}$ & Differenz & $\boldsymbol{M}_{\boldsymbol{s}}$ & Differenz \\
\hline \multirow{3}{*}{55} & I & - & 5120 & 100 & 31,60 & $-1,04 \%$ & 28,31 & $-1,21 \%$ \\
& II & 1 & 5120 & 100 & 7,77 & $-1,90 \%$ & 7,02 & $-0,12 \%$ \\
& II & 2 & 5120 & 100 & 7,75 & $0,83 \%$ & 6,91 & $1,02 \%$ \\
\hline
\end{tabular}

Im Zustand I können die Schnittgrößen mithilfe der Diagramme exakt bestimmt werden. Zur Berechnung im Zustand II der Deckschichten müssen Vereinfachungen getroffen werden. Es wird angenommen, dass die untere Deckschicht durch Trennrisse über die volle Länge aufreißt. Die Biegesteifigkeit der unteren Deckschicht wird daher zu Null angesetzt. Die Dehnsteifigkeit der Deckschicht setzt sich dann lediglich aus dem Querschnitt und dem Elastizitätsmodul der Bewehrung zusammen. Die obere Deckschicht wird nur als teilweise gerissen angenommen. Zur Berechnung der Biegesteifigkeit werden die Ergebnisse des Programms swe2+ zu Grunde gelegt. Hierzu wird in Abhängigkeit des Zustands der Deckschicht eine mittlere Deckschichtdicke über die Länge des 
Sandwichelements berechnet. Für ungerissene Bereiche wird die volle Deckschichtdicke, für Bereiche mit Biegerissen die Druckzonenhöhe und für Bereiche mit Trennrissen die Dicke zu Null angenommen. Aus der mittleren Deckschichtdicke wird dann die Biegesteifigkeit und Dehnsteifigkeit der Deckschicht berechnet.

In den Tabellen 8.3 und 8.4 sind die mittleren Deckschichtdicken, die Hilfswerte, das Moment $M_{\text {stat }}$ sowie die Momente $M^{\prime}$ und $M_{S}$ zusammengestellt. Die Ergebnisse der Berechnungen mit swe2+ und die Differenz zu den Nachrechnungen mit den Diagrammen nach Berner 1994 sind in den Tabellen 8.5 und 8.6 dargestellt.

Die Berechnungen mit swe2+ stimmen sehr gut mit den Nachrechnungen nach Berner 1994 überein. Im Zustand I weichen die Ergebnisse nur um rund ein Prozent von den Nachrechnungen ab. Diese Abweichung kann auf das Ablesen der Hilfswerte in den Diagrammen zurückgeführt werden. Im Zustand II weichen die Stützmomente $M^{\prime}$ bei einlagiger Bewehrung und bei einer Beanspruchung durch eine Flächenlast lediglich um maximal zwei Prozent ab. Bei zweilagiger Bewehrung weichen die Momente $M^{\prime}$ um rund zehn Prozent voneinander ab. Durch die Berechnung mit swe2+ wird in diesem Fall das Stützmoment $M^{\prime}$ überschätzt.

Die Sandwichmomente $M_{S}$ am Mittelauflager bei einlagiger Bewehrungsanordnung und flächiger Belastung unterscheiden sich mit bis zu rund $100 \%$ sehr stark von den Nachrechnungen nach Berner 1994 (siehe Tabelle 8.5). Die Nachrechnung bei zweilagiger Bewehrung weist eine geringere Abweichung von bis zu rund $20 \%$ auf. Die Momentenverteilung am Mittelauflager ist stark abhängig vom Querschnittszustand der Deckschichten. Je nach Bewehrungsanordnung treten in den Deckschichten unterschiedliche Zustände und somit unterschiedliche Momentenverteilungen über den Querschnitt auf. Bei der Nachrechnung nachBerner 1994 werden infolge der gemittelten Deckschichtdicke keine lokalen Umlagerungseffekte am Mittelauflager berücksichtigt. Es ist daher davon auszugehen, dass die Berechnung mit swe2+ hier die genaueren Ergebnisse liefert. Bei einer Beanspruchung durch eine Temperaturdifferenz treten, unabhängig von der Anzahl der Bewehrungslagen, sowohl bei dem Moment $M^{\prime}$ als auch bei dem Moment $M_{S}$ Abweichungen von weniger als zwei Prozent auf (siehe Tabelle 8.6.

\subsubsection{Erläuterungen zur Schnittgrößenumlagerung infolge der Rissbildung anhand eines Beispiels}

In den Abbildungen 8.11 und 8.12 sind die Ergebnisse des Berechnungsbeispiels aus dem vorherigen Abschnitt 8.2.2.6 bei einer Flächenlast von $1,5 \mathrm{kN} / \mathrm{m}^{2}$ und einer Temperaturdifferenz von $55^{\circ} \mathrm{K}$ grafisch dargestellt. Zum direkten Vergleich wurden die Ergebnisse der Berechnungen mit gerissenen und ungerissenen Deckschichten in den selben Diagrammen dargestellt. In den Legenden der Diagramme sind die berechneten Schnittgrößen- und Verformungslinien mit ungerissenen Deckschichten mit „”“ und die Kurven der Berechnung mit gerissenen Deckschichten mit „II“ gekennzeichnet.

Im jeweils ersten Diagramm der beiden Abbildungen sind die Querschnittszustände der oberen und unteren Deckschicht im gerissenen Zustand dargestellt. Die Zahlenwerte geben den jeweiligen Zustand des Bereichs wieder. Der Zahlenwert 1 bzw. -1 entspricht dem Zustand I. Die Werte 2 und 3 bzw. -2 und -3 entsprechen den Biegerissen 
$G=6.000 \mathrm{kN} / \mathrm{m}^{2}, E_{c}=30.000 \mathrm{~N} / \mathrm{mm}^{2}, f_{c t m}=1,0 \mathrm{~N} / \mathrm{mm}^{2}, E_{s}=160.000 \mathrm{~N} / \mathrm{mm}^{2}, a_{01} / a_{02}=5,0 \mathrm{~cm} 2 / \mathrm{m}, a_{u 1} / a_{u 2}=2,5 \mathrm{~cm}^{2} / \mathrm{m}$ $p=1.5$
$k N m^{2}$
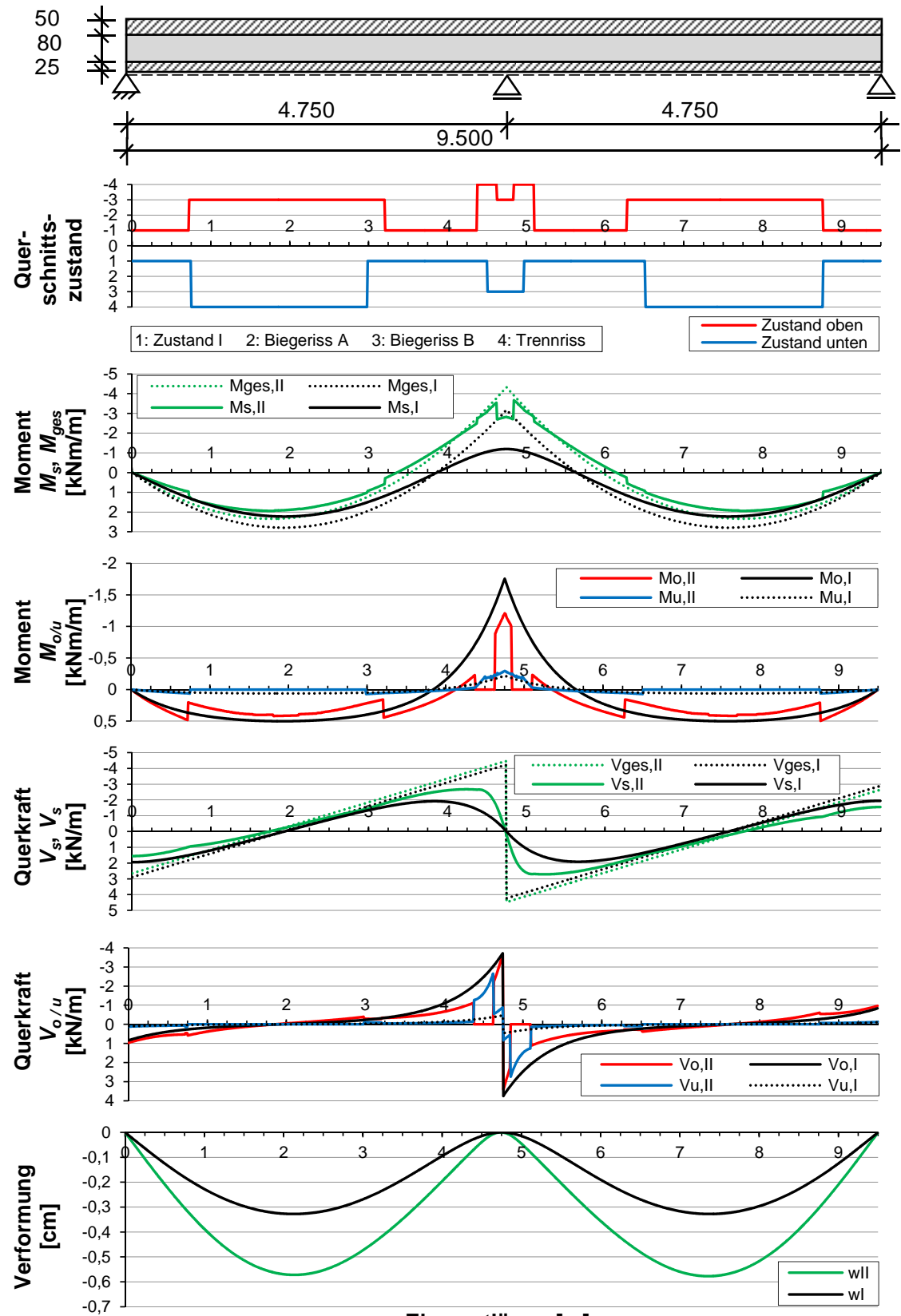

Elementlänge [m]

Abb. 8.11: Querschnittszustand der Deckschichten, Momente, Querkräfte und Verformungen im Zustand I und II über der Länge eines zweifeldrig gelagerten Sandwichelements mit einer Flächenlast von $1,5 \mathrm{kN} / \mathrm{m}^{2}$ 
$G=6.000 \mathrm{kN} / \mathrm{m}^{2}, E_{c}=30.000 \mathrm{~N} / \mathrm{mm}^{2}, f_{c t m}=1,0 \mathrm{~N} / \mathrm{mm}^{2}, E_{s}=160.000 \mathrm{~N} / \mathrm{mm}^{2}, a_{o 1} / a_{o 2}=5,0 \mathrm{~cm}^{2} / \mathrm{m}_{,} a_{u 1} / a_{u 2}=2,5 \mathrm{~cm}{ }^{2} / \mathrm{m}$

$$
T_{o}=80^{\circ} \mathrm{C}, T_{u}=25^{\circ} \mathrm{C} \rightarrow \Delta T=55^{\circ} \mathrm{K}
$$

50
80
25 *
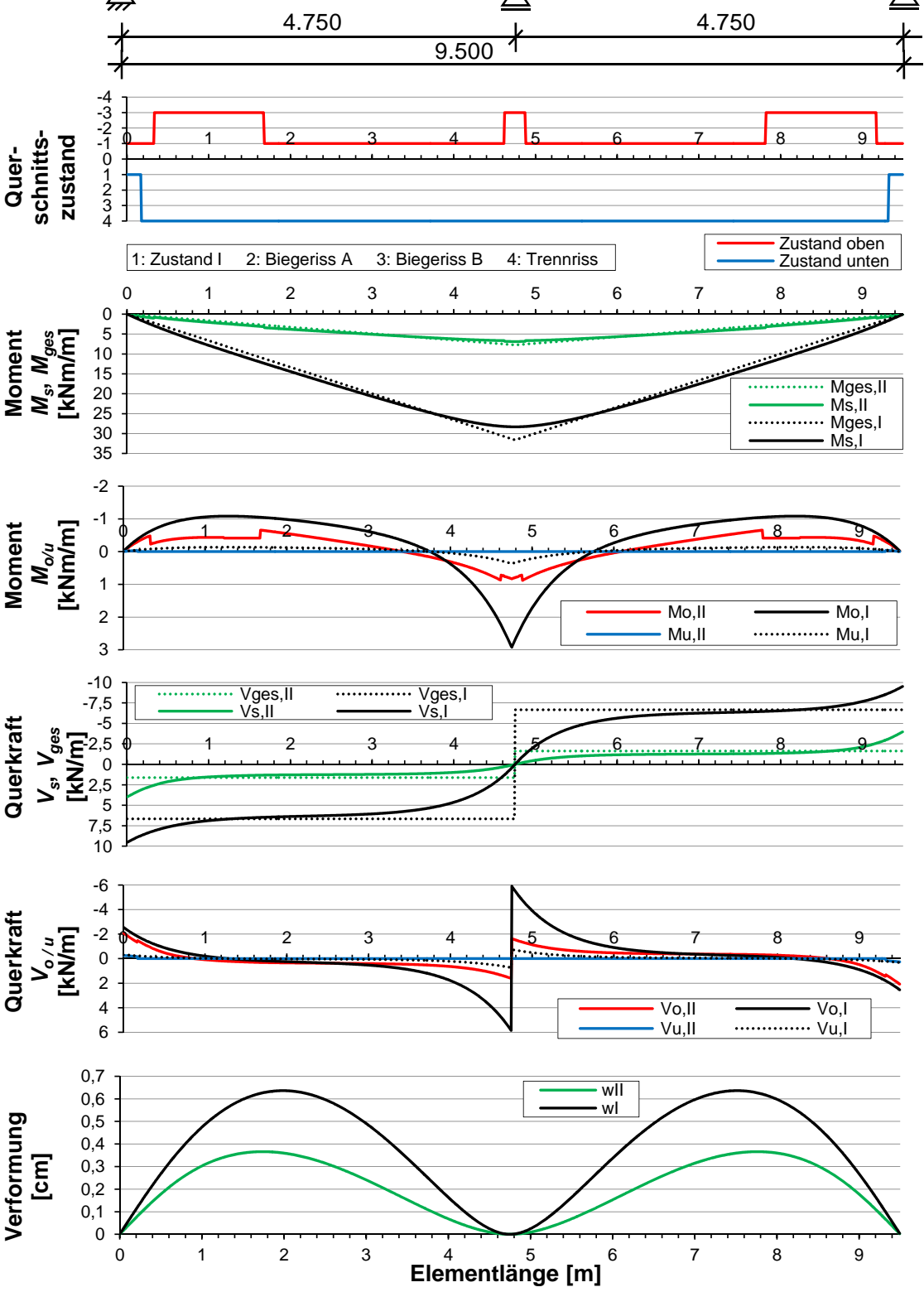

Abb. 8.12: Querschnittszustand der Deckschichten, Momente, Querkräfte und Verformungen im Zustand I und II über der Länge eines zweifeldrig gelagerten Sandwichelements mit einer Beanspruchung durch eine Temperaturdifferenz von $55^{\circ} \mathrm{K}$ 
Typ A und B. Im Fall des Wert 4 bzw. -4 tritt ein Trennriss auf. In den darunterliegend angeordneten Diagrammen sind die Querkraft-, Momenten- und Durchbiegungslinien angeordnet. Durch die Anordnung der Diagramme lässt sich sehr deutlich die Auswirkung der Rissbildung auf die Umlagerung der Schnittgrößen erkennen.

Grundsätzlich reduzieren sich die Deckschichtmomente in den Bereichen in denen sich Biegerisse bilden. Treten Trennrisse auf, wird gar kein Deckschichtmoment übertragen. In den Bereichen, in denen die Deckschichtmomente abnehmen, vergrößert sich entsprechend das Sandwichmoment. An den Übergängen zwischen gerissenen und ungerissenen Bereichen können dabei Sprünge in der Sandwichmomentenlinie festgestellt werden.

Durch den Vergleich der globalen Momente am Mittelauflager lässt sich erkennen, dass das Moment $M_{\text {ges, }}$ mit ungerissenen Deckschichten geringer ist, als das Moment bei gerissenen Deckschichten $M_{\text {ges, } / l}$. Eine Differenz von $25 \%$ zwischen den beiden Momenten $M_{\| /}^{\prime}$ und $M_{l}^{\prime}$ wird auch durch die Nachrechnung mithilfe der Diagramme nach Berner 1994 bestätigt. Die Rissbildung der Deckschichten bewirkt eine Steifigkeitsveränderung die zur Vergrößerung des Stützmoments führt. Dieser Effekt wird durch eine Änderung des Verhältnisses der Biegesteifigkeit des Sandwichquerschnitts $B_{S}$ zur Schubsteifigkeit $S$ hervorgerufen. Geht das Verhältnis von $B_{S} / S$ gegen Null, so ist der Querschnitt verhältnismäßig schubsteif und es stellt sich eine Momentenlinie nach der Balkentheorie ein. Eine Rissbildung in den Deckschichten bewirkt somit eine Reduzierung der Biegesteifigkeit $B_{S}$ bei gleichbleibender Schubsteifigkeit $S$. Dadurch wird das Verhältnis von $B_{S} / S$ kleiner, das Stützmoment nähert sich dem Moment nach Balkentheorie an und wird somit größer. Diese These wird auch durch die Versuchsergebnisse und die FE-Modellierung der Großbauteilversuche mit Flächenbelastung bestätigt. Durch die Änderung der Steifigkeit vergrößert sich die Lasteinzugsfläche am Mittelauflager und die Auflagerkraft nimmt wie in Abbildung 6.17 (siehe S. 147) dargestellt zu.

Das Verhältnis von $B_{S} / S$ wird in den Diagrammen nach Berner 1994 durch den Faktor $k=B_{S} /\left(S \cdot l^{2}\right)$ beschrieben. In Abbildung 3.16 (siehe S. 31) ist der Einfluss des Verhältnisses von $B_{S} / S$ sehr gut grafisch zu erkennen. Bei kleinem $k$-Faktor ist der Querschnitt verhältnismäßig schubsteif und der Abminderungsfaktor des Moments $M_{\text {stat }}$ wird kleiner. Bei steigendem k-Faktor wird der Querschnitt schubweicher und der Abminderungsfaktor nimmt zunächst zu. Steigt der $k$-Faktor weiter an, nehmen schließlich in Abhängigkeit der Biegesteifigkeit des Sandwichquerschnitts $B_{S}$ auch die Biegesteifigkeiten der Deckschichten $B_{D}$ weiter zu, so dass sich das Moment dem der Balkentheorie annähert und somit der Abminderungsfaktor wieder abnimmt.

Aufgrund der Temperaturdifferenz zwischen den beiden Deckschichten entstehen bei dem zweifeldrig gelagerten Sandwichelement große Zwangspannungen. In der Berechnung mit ungerissenen Deckschichten wird ein Sandwichmoment am Mittelauflager von $28,3 \mathrm{kNm} / \mathrm{m}$ hervorgerufen. Durch dieses Sandwichmoment entsteht eine große Zugkraft von $240,9 \mathrm{kN} / \mathrm{m}$ in der unteren Deckschicht. Bei der Berechnung unter Berücksichtigung der Rissbildung, reißt die untere Deckschicht durch die hohe Normalkraftbeanspruchung über die volle Länge des Sandwichelements auf. Weiterhin bilden sich auch Biegerisse in der oberen Deckschicht. Durch die Rissbildung in den Deckschichten werden die Zwangspannungen abgebaut. Hierdurch reduziert sich das 
Sandwichmoment am Mittelauflager um $75 \%$ auf rund $7 \mathrm{kNm} / \mathrm{m}$. Ebenso reduzieren sich auch die Deckschichtmomente und Querkräfte. Die maximale Verformung des Sandwichelements beträgt bei ungerissenen Deckschichten $6,4 \mathrm{~mm}$ und wird infolge der Rissbildung auf $3,7 \mathrm{~mm}$ reduziert.

\subsection{Nachweiskonzept}

\subsubsection{Nachweisführung und Sicherheitskonzept}

Die Nachweisführung erfolgt über ein Vergleich der Bemessungswerte für die Auswirkungen der Einwirkungen $E_{d}$ mit den Bemessungswerten der Widerstände $R_{d}$ bzw. den Kriterien für die Gebrauchstauglichkeit $C_{d}$. Es wird das semiprobabilistische Teilsicherheitssystem des Eurocodes (DIN EN 1990) angewendet.

Dabei gelten die Lastfaktoren $\gamma_{f}$ nach Eurocode 0 und dessen Nationalen Anhang [DIN EN 1990 DIN EN 1990/NA siehe auch Tabelle 3.4 S. 38. Die Kombinationskoeffizienten $\psi_{0}$ und $\psi_{1}$ werden entsprechend DIN EN 14509 übernommnen (siehe Tabelle 3.5). Die Beanspruchungen werden aus Eurocode 1 (DIN EN 1991 DIN EN 1991/NA) übernommen. Weiterhin finden die Temperaturlastfälle aus DIN EN 14509 Anwendung (siehe Tabelle 3.6 S. 40 .

Auf der Widerstandsseite müssen die Materialfaktoren $\gamma_{M}$ und die charakteristischen Materialfestigkeiten $f_{k}$ aus Versuchsergebnissen ermittelt werden. Die Materialfaktoren können mit den Gleichungen nach DIN EN 14509 in Anlehnung an den Eurocode 0 (DIN EN 1990) ermittelt werden (siehe auch GI. 3.51) und 3.52, S. 40). In dieser Arbeit wird der Materialfaktor dabei abweichend von DIN EN 14509 nicht mit der Standardabweichung, sondern mit dem Variationskoeffizient $v_{y}$ der Prüfergebnisse ermittelt. Die Berechnung über den Variationskoeffizient liefert hierbei die mathematisch exakte Lösung.

Die Gleichung zur Berechnung von $\gamma_{M}$ setzt sich wie folgt zusammen:

$$
\gamma_{M}=\alpha \cdot e^{\left(\alpha_{R} \cdot \beta-k_{n}\right) v_{y}}
$$

Dabei bedeuten:

\begin{tabular}{|c|c|c|}
\hline$\alpha$ & : & $\begin{array}{l}\text { Allgemeiner Vorfaktor, nach DIN EN } 14509 \text { ist } \alpha \text { im Zustand } \\
\text { der Tragfähigkeit } 1,05 \text { und im Zustand der Gebrauchstaug- } \\
\text { lichkeit } 1,0\end{array}$ \\
\hline & : & $\begin{array}{l}\text { Wichtungsfaktor für den Widerstand nach der Zuverlässig- } \\
\text { keitsmethode } 1 \text {. Ordnung, } \alpha_{R}=0,8\end{array}$ \\
\hline & : & $\begin{array}{l}\text { Zielwert des Zuverlässigkeitsindex, für einen Bezugszeit- } \\
\text { raum von einem Jahr ist } \beta=4,7 \text { im Zustand der Tragfähigkeit } \\
\text { und } \beta=3,0 \mathrm{im} \text { Zustand der Gebrauchstauglichkeit }\end{array}$ \\
\hline & : & $\begin{array}{l}\text { Fraktilenfaktor, für } 5 \% \text {-Quantilen auf Basis der Grundge- } \\
\text { samtheit ist } k_{n}=1,645\end{array}$ \\
\hline
\end{tabular}


$v_{y} \quad: \quad$ Variationskoeffizient der Prüfergebnisse als Stichprobe, unter Annahme einer logarithmischen Normalverteilung der Ergebnisse

Unter Annahme einer logarithmischen Normalverteilung wird der Variationskoeffizient wie folgt berechnet:

$$
v_{y}=\sqrt{e^{\sigma_{y}^{2}}-1}
$$

Dabei ist $\sigma_{y}$ die Standardabweichung der logarithmierten Prüfergebnisse $\ln (x)$ :

$$
\sigma_{y}=\sqrt{\frac{1}{n-1} \sum_{i=1}^{n}\left(\ln \left(x_{i}\right)-\bar{y}\right)^{2}}
$$

$\bar{y}$ ist der Mittelwert der logarithmierten Prüfergebnisse und $n$ ist die Anzahl der Ergebnisse.

Aus Gleichung 8.17 berechnet sich dann $\gamma_{M}$ für den Grenzzustand der Tragfähigkeit wie folgt:

$$
\gamma_{M}=1,05 \cdot \mathrm{e}^{(0,8 \cdot 4,7-1,645) v_{y}}=1,05 \cdot e^{2,115 v_{y}}
$$

Und für den Zustand der Gebrauchstauglichkeit:

$$
\gamma_{M}=\mathrm{e}^{(0,8 \cdot 3,0-1,645) v_{y}}=e^{0,755 v_{y}}
$$

Die charakteristischen Materialfestigkeiten werden ebenfalls in Anlehnung an den Eurocode 0 (DIN EN 1990) nach DIN EN 14509 als $5 \%$-Fraktilwerte unter Annahme eines $75 \%$-Vertrauensniveaus berechnet.

$$
f_{k}=\mathrm{e}^{\bar{y}-k_{n} \cdot \sigma_{y}}
$$

Der Fraktilenfaktor $k_{n}$ muss entsprechend der Versuchsanzahl $n$ aus Tabelle 8.7]entnommen werden.

Tab. 8.7: $\quad 5 \%$-Fraktilenfaktor $k_{n}$ in Abhängigkeit der Versuchsanzahl $n$ bei einem Vertrauensniveau von $75 \%$ nach DIN ISO 16269-6

\begin{tabular}{|c|cccccccc|}
\hline $\mathbf{n}$ & 3 & 4 & 5 & 6 & 7 & 8 & 9 & 10 \\
$\mathbf{k}_{\mathbf{n}}$ & 3,152 & 2,681 & 2,464 & 2,336 & 2,251 & 2,189 & 2,142 & 2,104 \\
\hline
\end{tabular}

\subsubsection{Nachweise im Grenzzustand der Tragfähigkeit}

\subsubsection{Zugversagen der Bewehrung}

Mit den aus der Schnittgrößenberechnung bestimmten Deckschichtmomenten $M_{d}$ und der aus dem Sandwichmoment $M_{S}$ resultierenden Normalkraft $N_{S}$ kann der Nachweis der Tragfähigkeit der Bewehrung erfolgen. Dabei muss grundsätzlich zwischen einer 
Biegebemessung oder einer reinen Zugbemessung unterschieden werden. Über die Auswertetabelle des Programms swe2+ kann hierzu als Querschnittszustand der entsprechende Risstyp ausgegeben werden.

Der Nachweis der Tragfähigkeit der Bewehrung wurde für alle durchgeführten Biegeversuche an Sandwichstreifen geführt. Dazu wurden die berechneten einwirkenden Zugspannungen $\sigma_{t, m}$ mit den vorhandenen Zugfestigkeiten $f_{t, m}$ verglichen. Die Nachweise wurden ohne Sicherheitsbeiwerte mit Mittelwerten geführt. In allen Fällen wurde die Tragfähigkeit der Bewehrung der unteren Deckschichten in Feldmitte nachgewiesen.

In allen Versuchen traten in der unteren Deckschicht Trennrisse auf, so dass der Momententraganteil der unteren Deckschicht zu Null angenommen wurde. Der Momententraganteil der oberen Deckschicht $M_{o}$ am Gesamtmoment wurde auf Basis von Vergleichsberechnungen mit swe2+ abgeschätzt. Bei einer Deckschichtdicke von $10 \mathrm{~mm}$ betrug der Anteil $\beta_{0}$ etwa $1 \%$, bei einer Dicke von $15 \mathrm{~mm}$ etwa $5 \%$ und bei einer Dicke von $20 \mathrm{~mm}$ etwa $10 \%$. Zur Berechnung von $M_{s}$ wurde der Momententraganteil der oberen Deckschicht vom Gesamtmoment $M$ subtrahiert. Die Nachweise wurden dann rein über die Normalkraft $N_{S}$ aus dem Sandwichmoment $M_{S}$ durchgeführt.

Die einwirkende Zugspannung $\sigma_{t, m}$ berechnet sich damit wie folgt:

$$
\begin{aligned}
\sigma_{t, m} & =\frac{M_{S}}{e \cdot A_{S}} \\
M_{S} & =\left(1-\beta_{0}\right) \cdot M
\end{aligned}
$$

Im Fall der Mikrobewehrung wurden die einwirkenden Zugspannungen am Übergang in den plastischen Bereich $\sigma_{p l}$ und bei Maximallast $\sigma_{\max }$ berechnet. Im Fall der Textilbewehrung wurde die einwirkende Zugspannung immer bei Maximallast berechnet. Für die Mittelwerte der Zugfestigkeiten $f_{t, m}$ der Mikrobewehrung und Carbonfaserbewehrung wurden die Ergebnisse aus den Dehnkörperversuchen verwendet. Für die Mikrobewehrung wurde weiterhin eine mittlere Festigkeit $f_{y, m}$ an der 0,2\%-Dehngrenze aus den Dehnkörperversuchen berechnet. Für die Glasfaserbewehrung wurde eine Zugfestigkeit von $1.000 \mathrm{~N} / \mathrm{mm}^{2}$ angenommen (vgl. Tabelle 4.5. S. 55.

Eine Unterscheidung der Festigkeiten der Mikrobewehrung von $f_{y, m}$ und $f_{t, m}$, erlaubt die Kontrolle der Nachrechnung mit der Festigkeit an der 0,2\%-Dehngrenze sowie mit maximaler Zugfestigkeit. Nach DIN EN 14509 darf sich beim Nachweis im Grenzzustand der Tragfähigkeit durch das Fließen der Deckschichten bei mehrfeldrigen Elementen ein Fließgelenk ausbilden. Bei den hier untersuchten Sandwichelementen kann sich durch das Fließen der Mikrobewehrung ebenfalls ein Fließgelenk ausbilden, jedoch muss mit ausreichender Sicherheit gewährleistet sein, dass die Zugfestigkeit der Bewehrung nicht überschritten wird.

In Abbildung 8.13 ist das Verhältnis der berechneten Zugspannung zu der mittleren Zugfestigkeit $\sigma_{t, m} / f_{t, m}$ für jeden der durchgeführten Biegeversuche berechnet. Für die mikrobewehrten Probekörper sind zwei Verhältnisse angegeben. Die Kreise kennzeichnen dabei das Verhältnis $\sigma_{\max } / f_{t, m}$ und die Rauten kennzeichnen das Verhältnis $\sigma_{p l} / f_{y, m}$. Ist das Verhältnis von $\sigma_{t, m} / f_{t, m}>1,0$ muss theoretisch ein Zugversagen auftreten bzw. das Fließen der Bewehrung einsetzen. Wenn dieser Fall auch in den Versuchen ein- 
getreten war, ist der Markierungspunkt blau dargestellt. Wenn der Probekörper auf eine andere Weise versagte, ist der Markierungspunkt rot eingefärbt. Ist das Verhältnis von $\sigma_{t, m} / f_{t, m} \leq 1,0$ so tritt theoretisch kein Zugversagen auf. Durch die Farbe des Markierungspunktes wird dargestellt, ob diese Aussage durch die Versuchsergebnisse bestätigt wird. Ein blauer Markierungspunkt bedeutet dabei, dass in den Versuchen kein Zugversagen aufgetreten ist. Ein roter Punkt hingegen kennzeichnet, dass ein Zugversagen aufgetreten ist.

Generell lässt sich zusammenfassen, dass durch die roten Markierungspunkte die oberhalb des Verhältnisses von 1,0 liegen, die unkritischen Fälle gekennzeichnet werden, in denen die berechnete Versagensart nicht eingetreten war, da der Probekörper bereits auf eine andere Art versagte. Durch die roten Markierungspunkte, die unterhalb des Verhältnisses von 1,0 liegen, werden hingegen die kritischen Fälle gekennzeichnet in denen die Tragfähigkeit durch das Bemessungskonzept zu günstig abgebildet wurde.

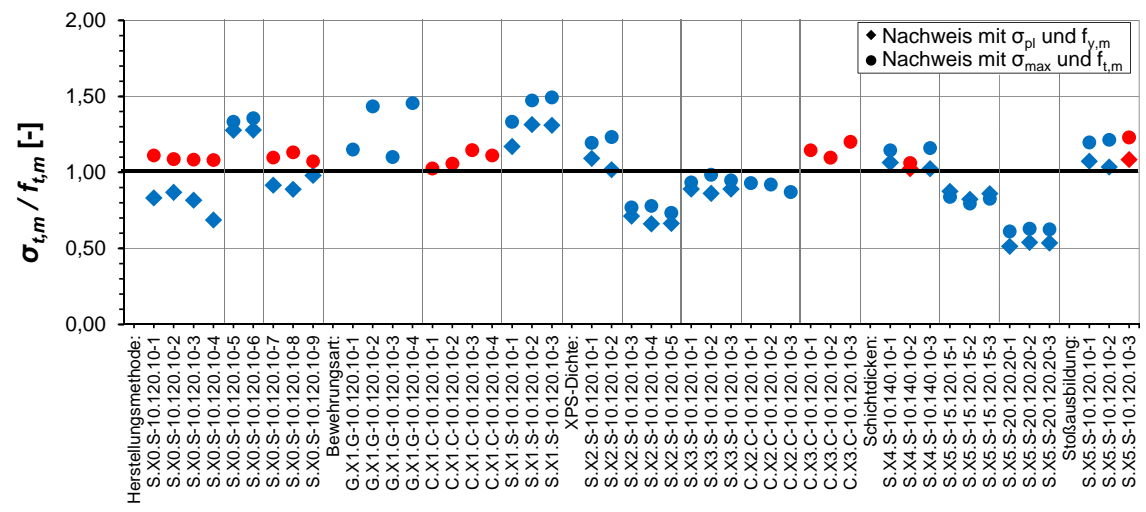

Abb. 8.13: Verhältnis der berechneten Zugspannungen $\sigma_{t, m}$ zu den vorhandenen mittleren Zugfestigkeiten $f_{t, m}$ der Probekörper

Durch die Darstellung des Verhältnisses von $\sigma_{t, m} / f_{t, m}$ zeigt sich, dass durch den Spannungsvergleich das Zugversagen der Bewehrung sehr zielsicher und wirtschaftlich berechnet werden kann. Die berechneten Spannungen sind grundsätzlich größer als die vorhandenen Festigkeiten. Das Auftreten des Bewehrungsversagens wird durch die Berechnung somit nicht unterschätzt. Die berechneten Spannungen sind in Einzelfällen bis zu 50 \% größer als die vorhandenen Festigkeiten. Generell ist jedoch eine wirtschaftliche Bemessung möglich.

Die charakteristischen Zugfestigkeiten sollten für eine praxistaugliche Bemessung für das jeweilig verwendete Bewehrungsmaterial in Versuchen ermittelt werden. Da die Zugfestigkeiten insbesondere bei dem Carbonfasergelege stark von den Verbundeigenschaften zwischen den Fasern und der Betonmatrix abhängen, sollten die Zugfestigkeiten in Dehnkörperversuchen am bewehrtem Beton ermittelt werden. Aus den Mittelwerten und Standardabweichungen der Versuchsergebnisse sind dann die $5 \%$ Fraktilwerte zu ermitteln. 
Die charakteristischen Materialfaktoren und Festigkeiten der hier verwendeten Mikrobewehrung und des Carbonfasergeleges wurden nach Gleichung (8.22) berechnet und sind in den Abbildungen 8.14 und 8.15 dargestellt.

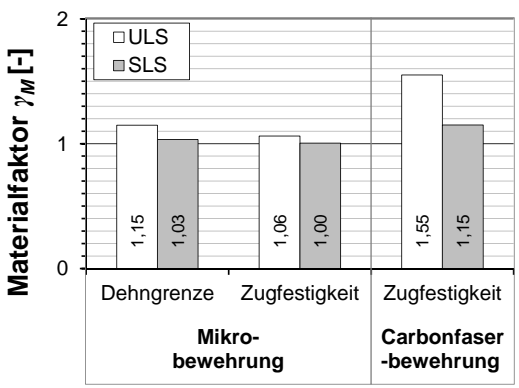

Abb. 8.14: Materialfaktoren $\gamma_{M}$ der Bewehrung auf Basis der Dehnkörperversuche

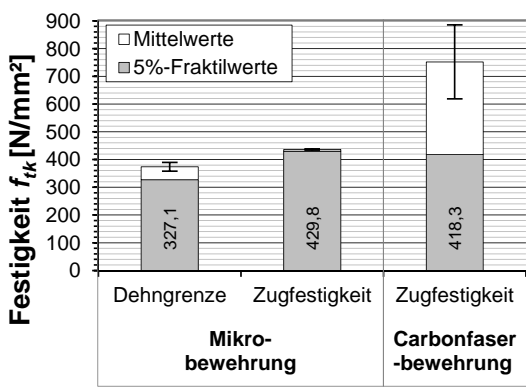

Abb. 8.15: Festigkeiten der Bewehrung als $5 \%$-Fraktilwerte und Mittelwerte mit Standardabweichung

Durch die starke Streuung der Versuchsergebnisse an den carbonfaserbewehrten Dehnkörpern, wird die charakteristische Zugfestigkeit sehr gering und somit kleiner als die Zugfestigkeit der mikrobewehrten Probekörper. Der Materialfaktor liegt mit 1,55 etwas über dem von Hegger $u$. Bornefeld 2012 geforderten Wert von $\gamma_{\text {tex }}=1,5$.

\subsubsection{Scherversagen des Kerns und des Verbunds zwischen Kern und Deckschichten}

Zur Kontrolle des Nachweises wurden die auftretenden Schubspannungen im Kern und in der Verbundfuge zwischen Kern und Deckschicht berechnet und dann mit den Scherfestigkeiten des XPS und des Verbunds zwischen Kern und Deckschicht verglichen. Die Scherspannungen wurden dabei aus der Querkraft des Sandwichquerschnitts $V_{S}$ nach Gleichung 3.25) (siehe S. 32 berechnet. Die Querkraft des Sandwichquerschnitts $V_{S}$ wiederum wurde aus der Gesamtquerkraft $V$ und einem Querkrafttraganteil der Deckschichten $\beta_{0}$ ermittelt:

$$
V_{S}=\left(1-\beta_{0}\right) \cdot V
$$

Die Gesamtquerkraft $V$ wurde beim Übergang in den plastischen Bereich mit $P_{p l} / 2$ angenommen. Der Traganteil der Deckschichten $\beta_{0}$ wurde, wie im vorherigen Abschnitt 8.3.2.1 auch, durch Berechnungen mit swe2+ abgeschätzt. In Abhängigkeit der Deckschichtdicke sowie des Abstands zwischen den Lasteinleitungs- und Auflagerpunkten wurde ein $\beta_{0}$-Faktor im Bereich von $1 \%$ bis $14 \%$ gewählt.

In Abbildung 8.16 sind die Verhältnisse der berechneten Scherspannungen $\tau_{v}$ zu den mittleren Scherfestigkeiten des XPS $f_{X P S, v, m}$ und des Verbunds $f_{X P S, b, m}$ dargestellt. Dabei stellen die Kreise die Verhältniswerte dar, die mit der Scherfestigkeit des XPS (Ergebnisse der Scherversuche ohne Betondeckschichten) ermittelt wurden. Die Rauten stellen die Werte dar, die mit der Scherfestigkeit des Verbunds (Ergebnisse der Scherversuche mit Betondeckschichten) gebildet wurden. 


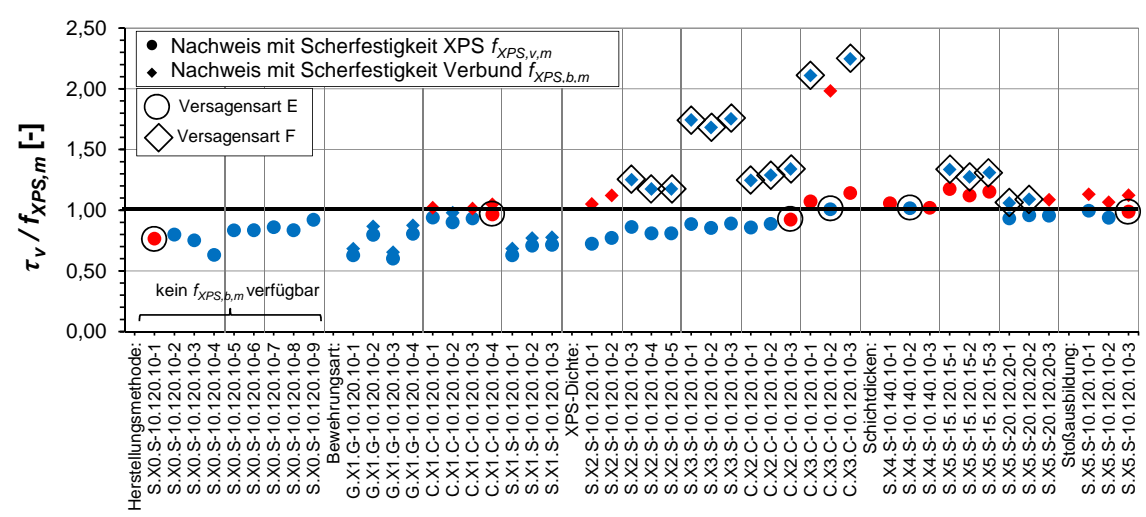

Abb. 8.16: Verhältnis der berechneten Scherspannungen $\tau_{v} z u$ den vorhandenen mittleren Scherfestigkeiten $f_{X P S, v, m}$ des XPS bzW. $f_{X P S, b, m}$ des Verbunds

Das Darstellungsprinzip der Ergebnisse in Abbildung 8.16 ist analog zur Darstellung des Zugversagens der Bewehrung. Zu unterscheiden sind jedoch zwei Versagensarten. Als Versagen des XPS wurde die Versagensart E „Querkraftversagen im Kern und Delamination des Kerns und der Deckschichten" angenommen und als Versagen des Verbunds, die Versagensart F „Schubversagen zwischen Kern und Deckschicht“ (siehe Tabelle 5.3 S. 71. Zum besseren Verständnis sind die Probekörper, die durch eine der beiden Arten versagten, in Abbildung 8.16 gekennzeichnet.

Durch den Vergleich der Spannungen lässt sich erkennen, dass beim überwiegenden Teil der Versuche die Versagensart zutreffend berechnet wird. Beim Versagen der Verbundfuge sind die berechneten Spannungen zum Teil bis zu $130 \%$ größer als die entsprechenden Festigkeiten. Dabei weichen jedoch lediglich die Spannungen der Versuche mit der XPS-Charge X3 stark von den Festigkeiten ab. Da die Abweichungen nur bei dieser Charge auftreten, ist anzunehmen, dass die in den Scherversuchen ermittelte Festigkeit des Verbunds von der Festigkeit der Biegeprobekörper abweicht, bspw. aufgrund der Herstellungsabfolge der Probekörper (siehe Erläuterungen in Abschnitt 7.4.6F: „Schubversagen zwischen Kern- und Deckschicht" S. 165

Das Versagen des Versuchs S.X0.S-10.120.10-1 wird durch den Nachweis um etwa $25 \%$ unterschätzt. Diese Abweichung ist jedoch nicht als kritisch zu bewerten, da der Probekörper im Versuch bei einer Maximallast versagte, die mehr als $50 \%$ über der Last beim Übergang in den plastischen Bereich lag. Weiterhin wird die Abweichung beim Nachweis durch Materialfaktoren abgedeckt.

Die Materialfaktoren $\gamma_{M}$ nach den Gleichungen 8.20 und 8.21) sind für die einzelnen Versuchsreihen der Scherversuche in Abbildung 8.17 dargestellt. Für die Versuchsergebnisse der Probekörper ohne Betondeckschichten liegen die Materialfaktoren im Bereich von:

- $\gamma_{M, U L S}=1,14-1,30 \mathrm{im}$ Zustand der Tragfähigkeit

- $\gamma_{M, S L S}=1,03-1,08 \mathrm{im}$ Zustand der Gebrauchstauglichkeit

Die Materialfaktoren der Probekörper mit Betondeckschichten liegen im Bereich von: 
- $\gamma_{M, U L S}=1,18-1,94 \mathrm{im}$ Zustand der Tragfähigkeit

- $\gamma_{M S L S}=1,04-1,25 \mathrm{im}$ Zustand der Gebrauchstauglichkeit

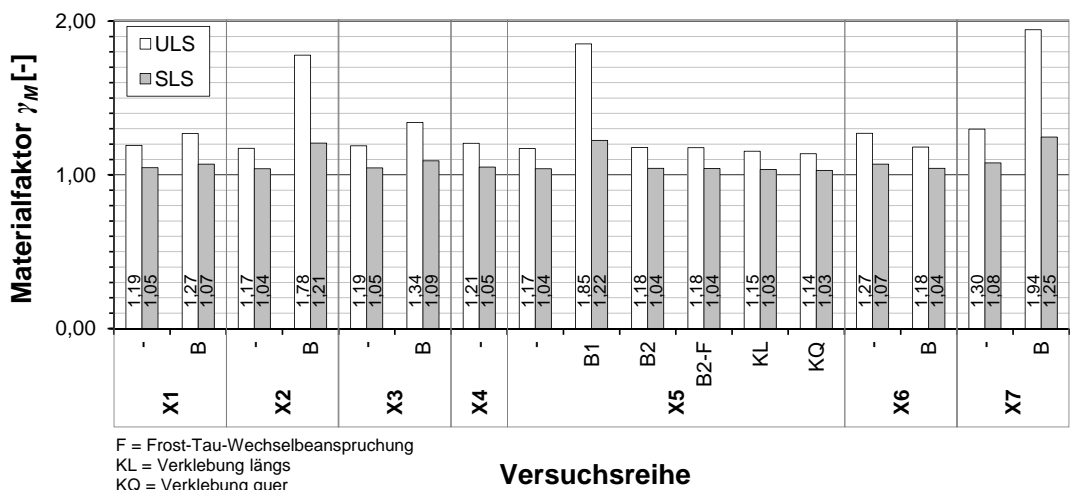

Abb. 8.17: Materialfaktoren $\gamma_{M}$ für den Zustand der Tragfähigkeit (ULS) und den Zustand der Gebrauchstauglichkeit (SLS) auf Basis der Versuchsergebnisse aus den Scherversuchen

Aufgrund der großen Streuungen der Versuchsergebnisse weisen die Versuchsreihen in denen einige Probekörper durch Versagensart $A$ und einige durch die Versagensart $\mathrm{B}$ versagten (bspw. X2 B, X5B1, X7 B) besonders große Materialfaktoren von bis zu rund 2,0 auf. In den Versuchsreihen, in denen nur eine Versagensart festgestellt wurde, betragen die Materialfaktoren im Grenzzustand der Tragfähigkeit weniger als 1,35.

Die charakteristischen Scherfestigkeiten der einzelnen Versuchreihen wurden weiterhin als $5 \%$-Fraktilwerte unter Annahme eines $75 \%$-Vertrauensniveaus nach Gleichung 8.22) berechnet. Die Ergebnisse sind ebenfalls von der Streuung der Versuchsergebnisse abhängig (siehe Abbildung 8.18. In den Versuchsreihen mit besonders großen Streuungen betragen die charakteristischen Scherfestigkeiten nur noch etwa die Hälfte der entsprechenden Mittelwerte.

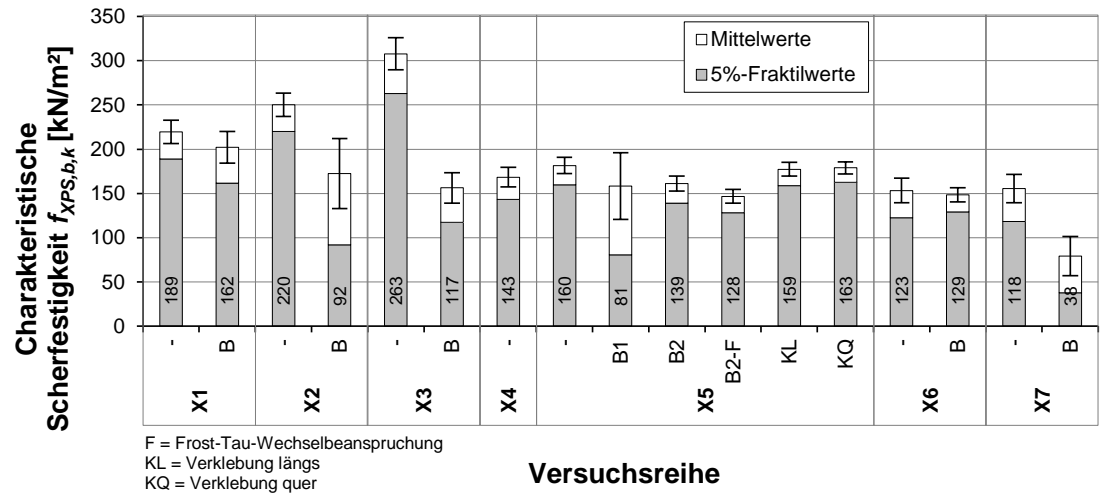

Abb. 8.18: $5 \%$-Fraktilwerte nach Gleichung 8.22 und Mittelwerte mit Darstellung der empirischen Standardabweichung der Scherfestigkeiten aus den Scherversuchen 


\subsubsection{Druckversagen des XPS-Kerns}

Nach DIN EN 14509 wird die aufnehmbare Auflagerkraft an einem Endauflager ohne Überstand oder mit einem Überstand von $<0,6 \cdot e$ nach Gleichung 8.26 ) ermittelt. Ein Endauflager mit einem Überstand von $>0,6 \cdot e$ ist wie ein Mittelauflager nach GI. 8.27 zu bemessen.

$$
\begin{aligned}
& F_{R 1}=B \cdot\left(L_{S}+0,5 \cdot k \cdot e\right) \cdot f_{X P S, c} \quad \text { (Endauflager) } \\
& F_{R 2}=B \cdot\left(L_{S}+k \cdot e\right) \cdot f_{X P S, C} \quad \text { (Mittelauflager) }
\end{aligned}
$$

Dabei sind:

$\begin{array}{lll}B & : & \text { Elementbreite } \\ L_{S} & : & \text { Auflagerbreite } \\ k & : & \text { Lastverteilungsfaktor } \\ e & : & \text { Abstand zwischen den Schwerlinien der Deckschichten } \\ f_{X P S, c} & : & \text { Druckfestigkeit des XPS }\end{array}$

Für die Bemessung wird eine gleichförmige Spannungsverteilung an der Stelle $e / 2$ angenommen (siehe Abbildungen 8.20 und 8.19). Ist $e \geq 100 \mathrm{~mm}$, wird $e$ auf $100 \mathrm{~mm}$ begrenzt und entsprechend in die Gleichungen (8.26) und 8.27) eingesetzt. Die Lastausbreitung wird mit dem Faktor $k$ beschrieben. Dieser muss in Versuchen ermittelt werden oder darf für Kunststoff-Hartschäume mit 0,5 angenommen werden.
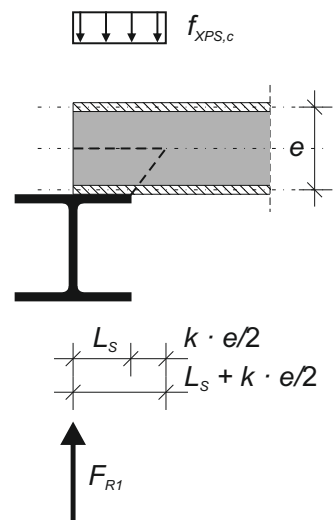

Abb. 8.19: Ermittlung der aufnehmbaren Auflagerkraft am Endauflager
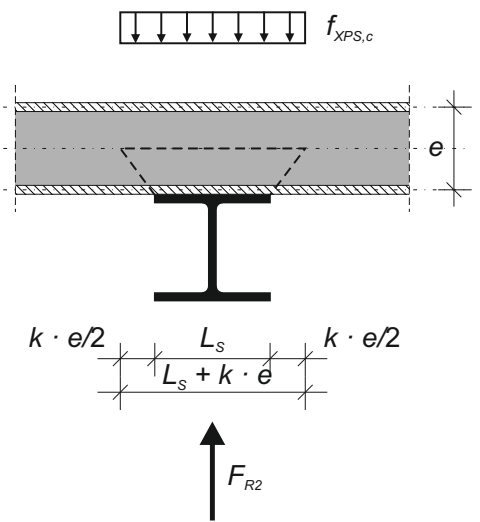

Abb. 8.20: Ermittlung der aufnehmbaren Auflagerkraft am Mittelauflager

Zur Beurteilung des Nachweises im Fall des Druckversagens des XPS wurden ebenfalls die Verhältnisse der berechneten, einwirkenden Druckspannungen zu den den vorhandenen Druckfestigkeiten ermittelt und in Abbildung 8.21 dargestellt. Der Nachweis des Druckversagens wurde hierbei nicht nur an den Auflagern sondern auch an den Lasteinleitungsstellen geführt. Aufgrund der identischen Spannungsverteilung, 
kann der Nachweis an der Lasteinleitungsstelle wie an einem Mittelauflager geführt werden. In Abbildung 8.21 werden durch die Darstellung der Markierungspunkte die beiden Nachweisstellen voneinander unterschieden. Durch die Kreise werden die Verhältnisse der Nachweise am Auflager und durch die Rauten die Verhältnisse der Nachweise an der Lasteinleitungsstelle gekennzeichnet.

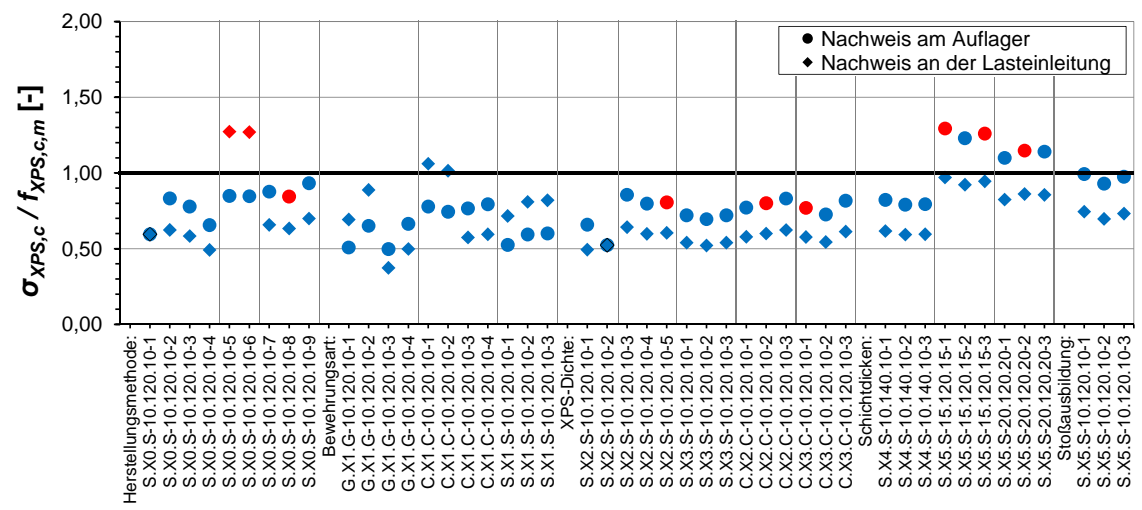

Abb. 8.21: Verhältnis der berechneten Druckspannungen $\sigma_{X P S, m}$ zu vorhandenen mittleren Druckfestigkeiten $f_{X P S, c, m}$ der Probekörper am Auflager und an den Lasteinleitungsstellen

In vier der Fälle wurde durch das Bemessungskonzept die Tragfähigkeit des Kerns am Auflager zu günstig abgebildet, d. h. die Probekörper versagten bereits bei einer rechnerischen Druckspannung, die geringer war als die mittlere Druckfestigkeit des XPS. Wie bereits durch die Ergebnisse der FE-Modellierungenen in Abbildung 7.24 zu erkennen ist, bilden sich infolge der Auflagerkraft sowie der Biege- und Schubbeanspruchung des Sandwichelements am äußersten Rand des Kerns die maximalen Spannungen aus. Diese Spannungsüberschreitungen führen jedoch nur zum Plastizieren des Kerns am äußersten Ende des Kerns und nicht nicht wie in Gleichung 8.26 angenommen über eine größere Breite von $L_{S}+k \cdot e / 2$.

Die Überschreitungen beim Nachweis sind nicht als kritisch zu bewerten, da diese durch die Materialfaktoren und Lastfaktoren abgedeckt werden. Weiterhin traten die Plastizierungen in den Biegeversuchen an den Sandwichstreifen nur bei sehr großen Verformungswerten auf. Aufgrund der Begrenzung der Durchbiegung im Zustand der Gebrauchstauglichkeit, sind diese bei großformatigen Sandwichelementen nicht zu erwarten.

\subsubsection{Lokales Biege- und Querkraftversagen einer Deckschicht}

Das Druckversagen einer vollständig durch Druckspannungen beanspruchten Deckschicht ist aufgrund des großen Querschnitts und der hohen Betondruckfestigkeit nicht zu erwarten. Auch ein Stabilitätsversagen der Deckschicht vergleichbar mit dem Knittern einer metallischen Deckschicht wurde in den Versuchen nicht beobachtet. Jedoch können die Deckschicht infolge großer lokaler Deckschichtmomente beispielsweise an den Auflager oder den Lasteinleitungsstellen versagen. 
Mithilfe der Berechnungsergebnisse des Programms swe2+ können die maßgebenden Stellen, an denen ein lokales Versagen der Deckschichten zu erwarten ist, identifiziert werden. In diesen Bereichen überschreiten die berechneten Spannungen die Materialfestigkeiten des Betons sowie der Bewehrung. Mit den berechneten Schnittgrößen können dann die Nachweise aus den Abschnitten 3.1.2, Mikrobewehrter Hochleistungsbeton' und 3.1.3, Textilbewehrter Beton' (siehe S. 11 und 16ff.) geführt werden.

\subsubsection{Nachweise im Grenzzustand der Gebrauchstauglichkeit}

\subsubsection{Nachweis gegen Rissbildung}

Bei Verwendung der Sandwichelemente als Wandelement, können Risse in den Deckschichten einen optischen Mangel darstellen. In Absprache mit dem Bauherrn sollte vereinbart werden, ob die Sichtbetonoberflächen der Sandwichelemente rissefrei ausgeführt werden sollen. Aufgrund der geringen Rissweite sind die Risse in den Deckschichten lediglich nur aus wenigen Metern Entfernung oder durch die ungleichmäßige Abtrocknung bei direkter Beregnung sichtbar. Gegebenenfalls können durch konstruktive Maßnahmen, z. B. ausreichend große Dachüberstände, Textur auf den Betonoberfläche, diesem optischen Mangel entgegengewirkt werden. Weiterhin ist denkbar, durch die Einstreuung von Kurzschnittfasern die Betonzugfestigkeit zu vergrößern. Werden die Sandwichelemente als Dachelemente ausgeführt, sollten aus Dauerhaftigkeitsgründen keine Risse zugelassen werden, oder eine entsprechende Dachabdichtung vorgesehen werden.

Beim Nachweis gegen Rissbildung in den Deckschichten wird die selten auftretende Lastfallkombination nach Gleichung (3.48) (siehe S. 39] angewendet. Die aus der maßgebenden Lastfallkombination resultierenden Spannungen in den Deckschichten $\sigma_{k}$ werden mit der charakteristischen Betonzugfestikeit $f_{c t k ; 0,05}$ verglichen:

$$
\sigma_{k} \leq f_{c t k ; 0,05}=0,7 \cdot f_{c t m}
$$

\subsubsection{Begrenzung der Durchbiegungen}

Für den Nachweis zur Begrenzung der Durchbiegung gilt die häufig auftretende Lastfallkombination nach Gleichung 3.49) (siehe S. 39). Aus DIN EN 14509 werden die folgenden Durchbiegungsgrenzen übernommen:

- Stützweite $/ / 100$ - Wandelemente und Dachelemente bei Kurzzeitbelastung

- Stützweite //200 - Dachelemente bei Langzeitbelastung

Die Durchbiegung unter Langzeitbeanspruchung muss unter Berücksichtigung des reduzierten Schubmoduls infolge des Kriechens des Kerns $G_{t}$ nach Gleichung 3.50 (siehe S. 40) ermittelt werden. In DIN EN 14509 wird empfohlen, den vorhandenen Schubmodul um den Faktor $1 /\left(1+\varphi_{t}\right)$ abzumindern (siehe Gleichung 3.50, S. 40. Für XPS-Hartschäume wird empfohlen, bei langandauernder Schneebelastung für eine Dauer von $t=2.000 \mathrm{~h} \varphi_{t}=2,4$ zu wählen. Bei dauerhafter Beanspruchung für eine Dauer von $t=100.000 \mathrm{~h}$ sollte $\varphi_{t}=7,0$ gewählt werden. 
Durch eine Nachrechnung der Dauerstandsversuche mithilfe des Programms swe2+ wurden die Kriechfaktoren für die beiden Fällen so gewählt, dass die berechneten Durchbiegungen den Durchbiegungen, die in den Versuchen ermittelt wurden, entsprachen. Auf diese Weise wurde $\varphi_{t}=0,5$ bei $t=2.000 \mathrm{~h}$ und $\varphi_{t}=2,0$ bei $t=100.000 \mathrm{~h}$ ermittelt. Bei der Berechnung der Kriechfaktoren wurde das Kriechen des Betons vernachlässigt, da die Betondruckspannungen bei der höchsten Belastung von $200 \mathrm{~kg}$ lediglich rund $1 \mathrm{~N} / \mathrm{mm}^{2}$ betrugen. Die Ergebnisse der Nachrechnungen weisen sehr gute Übereinstimmungen mit den Versuchsergebnissen auf (siehe Abbildung 8.22. Lediglich im Fall der Probekörper mit einer Belastung von $200 \mathrm{~kg}$ weichen die Berechnungsergebnisse mit zunehmender Belastungsdauer $t$ ab, da hier durch das Programm die Rissbildung in den Deckschichten überschätzt wird.

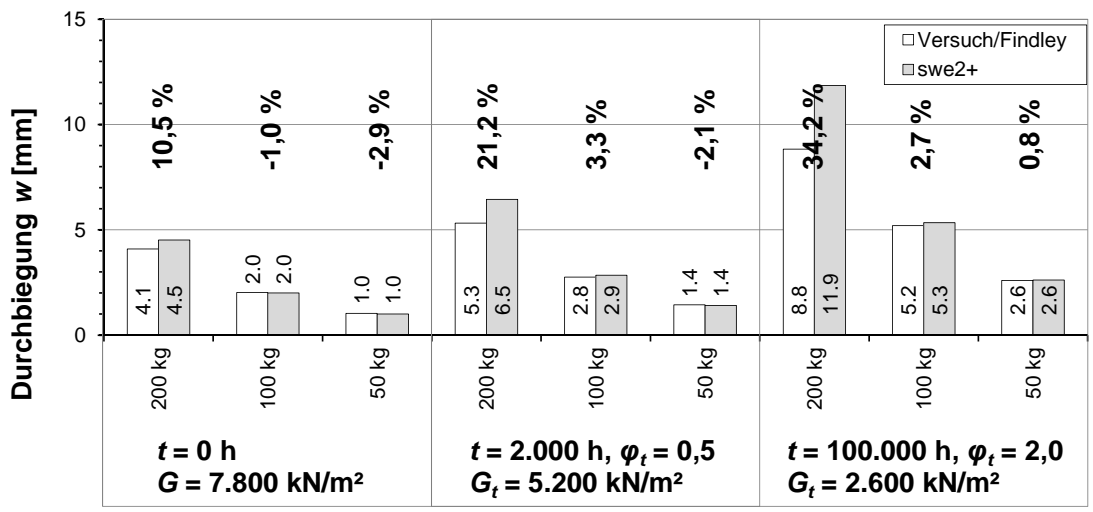

Abb. 8.22: Nachrechnung der Dauerstandsversuche mit swe2+ unter Berücksichtigung des Kriechfaktors $\varphi_{t}$

\subsubsection{Ermüdungsnachweis}

Nach den ergänzenden Angaben des Nationalen Anhangs des Eurocode 2 (DIN EN] 1992/NA) braucht für „Tragwerke des üblichen Hochbaus (...) im Allgemeinen kein Nachweis gegen Ermüdung geführt zu werden“. Wie bereits in Abschnitt 3.3.4.9 (siehe S. 41 beschrieben, wird jedoch nach Anhang B.6 der DIN EN 14509 für Sandwichelemente mit Metalldeckschichten eine Prüfung der Dauerhaftigkeit mit wiederholter Belastung von 5.000 Lastwechseln gefordert. Weiterhin muss die Dauerhaftigkeit der Verbindungsmittel unter einer Beanspruchung einer Temperaturdifferenz nach DIBt 3.93 in einem zyklischen Versuch nachgewiesen werden. Dazu wird die maximal zulässige Schraubenkopfauslenkung $u$ durch eine zyklische Belastung mit einem Lastkollektiv von 20.000 Zyklen mit 4/7 u, 2.000 Zyklen mit 6/7 $u$ und 100 Zyklen mit $7 / 7 u$ bestimmt. Diese Vorgehensweise wurde für die Prüfung im Zulassungsverfahren der Verbindungsmittel von mehrschichtigen Wandtafeln mit Betondeckschichten übernommen (DIBt 1995).

In den Biegeversuchen an Sandwichstreifen, die mit Stahldrahtmatten oder Carbonfasergelege bewehrt waren, konnte bereits gezeigt werden, dass durch eine zyklische Belastung in Anlehnung an DIBt 3.93 bzw. DIBt 1995 kein vorzeitiges Versagen der 
Probekörper herbeigeführt werden konnte. In den Ermüdungsversuchen mit zyklischer Wechselbeanspruchung in Abschnitt 5.5 (siehe S. 102 zeigte sich jedoch, dass eine Ermüdung der Verbundfuge zwischen Kern und Deckschicht auftritt und dadurch ein Versagen des Kerns eingeleitet wird.

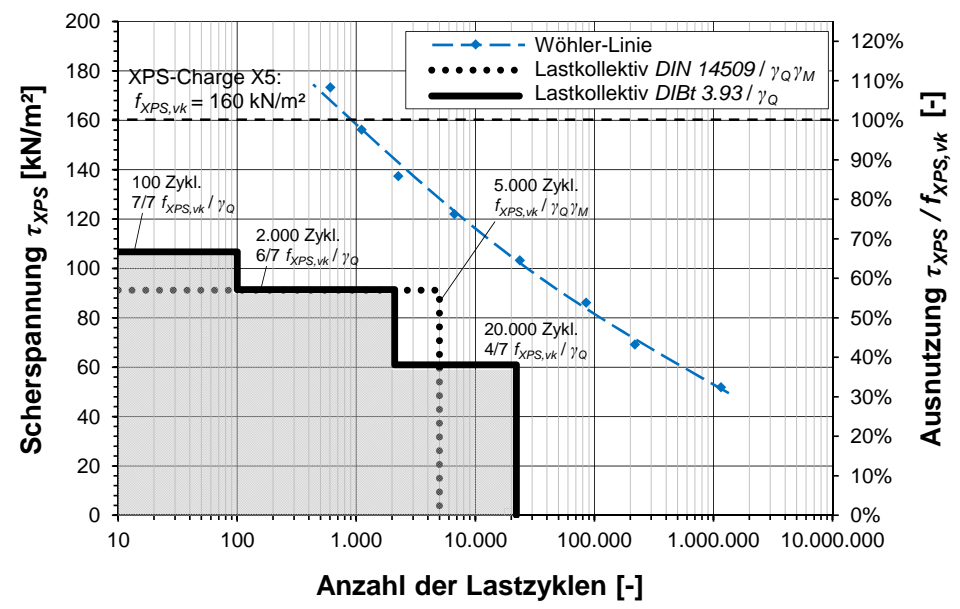

Abb. 8.23: Nachweis gegen Ermüdung anhand der Wöhler-Linie

Zum Nachweis gegen Ermüdung der Sandwichelemente wurden die Lastkollektive aus DIN EN 14509 sowie nach DIBt 3.93 bzw. DIBt 1995 in das Wöhlerdiagramm in Abbildung 8.23 eingetragen. Anstatt der Knitterspannung $\sigma_{w}$ und der Verformung $u$ wurden die Lastkollektive auf die charakteristische Scherfestigkeit der XPS-Charge X5 von $f_{X P S, v k}=160 \mathrm{kN} / \mathrm{m}^{2}$ bezogen. Da die Scherfestigkeiten des Kerns und der Verbundfuge zwischen Kern und Deckschichten unter Temperaturbeanspruchung bereits im Grenzzustand der Tragfähigkeit nachgewiesen werden und der Nachweis der Ermüdung in der Regel auf Gebrauchslastniveau mit $\gamma_{F \text { fat }}=1,0$ geführt wird, kann das Lastniveau der Lastkollektive um $1 / \gamma_{Q}=1 / 1,5$ abgemindert werden. In Anlehnung an DIN EN 14509 wurde das Lastniveau der 5.000 Zyklen zusätzlich um den Materialfaktor der XPS-Charge X5 von $\gamma_{M}=1,17$, d. h. insgesamt um $1 /(1,5 \cdot 1,17)=1 / 1,85$ abgemindert (siehe Abschnitt 3.3.4.9, ,Prüfung der Dauerhaftigkeit von Sandwichelementen:, S. 41.

Anhand Abbildung 8.23 lässt sich erkennen, dass sowohl die gepunktete Linie zur Darstellung des Lastkollektivs in Anlehnung an DIN EN 14509 als auch die durchgezogene Linie zur Darstellung des Lastkollektivs in Anlehnung an DIBt $3.93 \mathrm{bzw}$. DIBt 1995 die Wöhlerlinie der XPS-Charge X5 nicht schneiden und somit der Nachweis der Ermüdungsfestigkeit in beiden Fällen erbracht ist.

Für die praxisgerechte Bemessung der Sandwichelemente scheint der versuchsgestütze Nachweis der Ermüdungsfestigkeit nach dem in Anhang B.2 der DIN EN 14509 beschriebenen Verfahren als ausreichend. Anstatt der Knitterspannung $\sigma_{w}$ sollte jedoch hier das Lastniveau auf die charakteristische Scherfestigkeit $f_{X P S, v k}$ des Kerns im Grenzzustand der Gebrauchstauglichkeit bezogen werden. 


\subsection{Bemessungsbeispiele}

\subsubsection{Allgemeines}

Nachfolgend wird die Bemessung eines Wand- sowie eines Dachelements in den Grenzzuständen der Tragfähigkeit und der Gebrauchstauglichkeit beispielhaft vorgeführt. Aufgrund der großen Anzahl zu untersuchender Lastkombinationen, werden die Nachweise vereinfachend nur jeweils an einer ausgewählten Lastkombination demonstriert.

\subsubsection{Wandelement mit einem Feld}

\subsubsection{System und Abmessungen}

- Abmessungen:

Spannweite: $\quad 5,00 \mathrm{~m}$

Auflagerbreite: $0,10 \mathrm{~m}$

- Querschnitt:

Deckschichtdicke: $10 \mathrm{~mm}$

Kernschichtdicke: $120 \mathrm{~mm}$

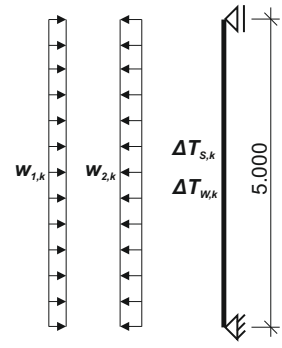

Abb. 8.24: Statisches System des Wandelements

\subsubsection{Materialien und Kennwerte}

- Deckschichten:

Hochleistungsbeton mit 3 Lagen Mikrobewehrung je Deckschicht

\begin{tabular}{|c|c|c|}
\hline Beton & Werte & Verweis \\
\hline Druckfestigkeit: & $f_{c k}=110 \mathrm{~N} / \mathrm{mm}^{2}$ & Gln. 4.1- 4.3, S. 51 \\
\hline Zugfestigkeit: & $f_{c t m}=3,7 \mathrm{~N} / \mathrm{mm}^{2}$ & Tab. 4.3 S. 51 \\
\hline Elastizitätsmodul: & $E_{c}=43.000 \mathrm{~N} / \mathrm{mm}^{2}$ & Tab. 4.3 S. 51 \\
\hline Bewehrung & Werte & Verweis \\
\hline Querschnitt: & $A_{s}=3 \cdot 61,8=185,4 \mathrm{~mm}^{2} / \mathrm{m}$ & \begin{tabular}{ll|l|l|} 
Tab. & 4.4 & S. & 54 \\
\end{tabular} \\
\hline Elastizitätsmodul: & $E_{s}=160.000 \mathrm{~N} / \mathrm{mm}^{2}$ & Abb. 5.41 S. 96 \\
\hline Streckgrenze: & $f_{y k}=325 \mathrm{~N} / \mathrm{mm}^{2}$ & Abb. 8.15 S. 193 \\
\hline
\end{tabular}

- Kernschicht:

Styrodur ${ }^{\circledR} 2800 \mathrm{C}$ - Es werden die Schereigenschaften der Versuchsreihe X5 und X5 B1 angenommen. 


\begin{tabular}{lll} 
XPS & Werte & Verweis \\
\hline Scherfestigkeit XPS: & $f_{X P S, k}=160 \mathrm{kN} / \mathrm{m}^{2}$ & Abb. 8.18, S. 195 \\
Scherfestigkeit Verbund: & $f_{X P S, b, k}=80 \mathrm{kN} / \mathrm{mm}^{2}$ & Abb. 8.18, S. 195 \\
Tang.-Schubmodul: & $G_{X P S, \text { mean }}=7.800 \mathrm{kN} / \mathrm{m}^{2}$ & Abb. 5.28 \\
Sruckfestigkeit: & $f_{X P S, c, k}=330 \mathrm{kN} / \mathrm{m}^{2}$ & Annahme
\end{tabular}

\subsubsection{Beanspruchungen}

\section{Dauerhafte Beanspruchungen $G_{k}$ :}

Unter Annahme, dass beide Deckschichten am Wandfuß aufgelagert sind, treten keine dauerhaften Beanspruchungen aus Eigenlast auf.

\section{Veränderliche Beanspruchungen $Q_{k}$ :}

- Wind: nach DIN EN 1991 und DIN EN 1991/NA

Windlastzone 1, Binnenland

$$
\begin{array}{ll}
q_{k}=0,5 \mathrm{kN} / \mathrm{m}^{2} & \text { bei } h \leq 10 \mathrm{~m} \\
c_{p e, 10}=-1,4 & \text { für vertikale Wände (Bereich } \mathrm{A} \text { ) } \\
c_{p e, 10}=+0,8 & \text { für vertikale Wände (Bereich } \mathrm{D})
\end{array}
$$

Lastfall 1 - Winddruck:

$$
\begin{aligned}
& w_{1, k}=q_{k} \cdot c_{p e} \\
& w_{1, k}=0,5 \mathrm{kN} / \mathrm{m}^{2} \cdot+0,8 \\
& w_{1, k}=+0,4 \mathrm{kN} / \mathrm{m}^{2}
\end{aligned}
$$

Lastfall 2 - Windsog:

$$
\begin{aligned}
& w_{2, k}=q_{k} \cdot c_{p e} \\
& w_{2, k}=0,5 \mathrm{kN} / \mathrm{m}^{2} \cdot-1,4 \\
& w_{2, k}=-0,7 \mathrm{kN} / \mathrm{m}^{2}
\end{aligned}
$$

- Temperatur: nach DIN EN 14509, Farbgruppe III (dunkle Farben)

Lastfall 3 - Sommer im ULS und SLS: $\quad T_{1}=+80^{\circ} \mathrm{C} \quad$ (außen) $T_{2}=+25^{\circ} \mathrm{C} \quad$ (innen)

Lastfall 4 - Winter im ULS und SLS: $\quad T_{1}=-20^{\circ} \mathrm{C} \quad$ (außen) $T_{2}=+20^{\circ} \mathrm{C} \quad$ (innen)

\subsubsection{Maßgebende Lastfallkombinationen}

\section{Grenzzustand der Tragfähigkeit}

Im Grenzzustand der Tragfähigkeit werden die Kombinationen nach Gleichung 3.47 (siehe S. 39) gebildet. Mit den vier vorhandenen Lastfällen können insgesamt zwölf Lastkombinationen gebildet werden (siehe Tabelle 8.8. Die Lastfaktoren der jeweiligen Leiteinwirkungen sind grau schattiert dargestellt. 
Tab. 8.8: Lastkombinationen im Grenzzustand der Tragfähigkeit eines SandwichWandelements

\begin{tabular}{|c|c|c|c|c|c|c|c|c|}
\hline & \multicolumn{2}{|c|}{$\begin{array}{l}\text { LF1 } \\
w_{1, k}\end{array}$} & \multicolumn{2}{|c|}{$\begin{array}{l}\text { LF2 } \\
w_{2, k}\end{array}$} & \multicolumn{2}{|c|}{$\begin{array}{l}\text { LF3 } \\
\Delta T_{\mathrm{s}}\end{array}$} & \multicolumn{2}{|c|}{$\begin{array}{l}\text { LF4 } \\
\Delta T_{\mathrm{w}}\end{array}$} \\
\hline & $\Psi_{0}$ & $\gamma_{Q}$ & $\Psi_{0}$ & $\gamma_{Q}$ & $\Psi_{0}$ & $\gamma_{Q}$ & $\Psi_{0}$ & $\gamma_{Q}$ \\
\hline LK1 & & 1,5 & & & & & & \\
\hline LK2 & & & & 1,5 & & 15 & & \\
\hline LK4 & & & & & & 1,0 & & 1,5 \\
\hline LK5 & & 1,5 & & & 0,6 & 1,5 & & \\
\hline LK6 & & 1,5 & & & & & 0,6 & 1,5 \\
\hline LK7 & & & & 1,5 & 0,6 & 1,5 & & \\
\hline $\begin{array}{l}\text { LK8 } \\
\text { LK9 }\end{array}$ & 06 & 15 & & 1,5 & & & 0,6 & 1,5 \\
\hline LK10 & & & 0,6 & 1,5 & & 1,5 & & \\
\hline LK11 & 0,6 & 1,5 & & & & & & 1,5 \\
\hline LK12 & & & 0,6 & 1,5 & & & & 1,5 \\
\hline
\end{tabular}

\section{Grenzzustand der Gebrauchstauglichkeit}

\section{- Selten auftretende Kombination:}

Die selten auftretende Kombination wird verwendet um nachzuweisen, dass im Grenzzustand der Gebrauchstauglichleit keine sichtbaren Schäden am Element auftreten, z. B. durch Rissbildung in den Deckschichten. Die Kombinationen werden nach Gleichung 3.48 gebildet und entsprechen dabei den Kombinationen im Grenzzustand der Tragfähigkeit. Jedoch werden hierzu die charakteristischen Lasten angesetzt.

\section{- Häufig auftretende Kombination:}

Die häufig auftretende Kombination wird zum Nachweis der Durchbiegungen verwendet. Die Kombinationen werden dazu nach Gleichung 3.49) gebildet (siehe Tabelle 8.9).

Tab. 8.9: Häufig auftretende Lastkombinationen im Grenzzustand der Gebrauchstauglichkeit eines Sandwich-Wandelements

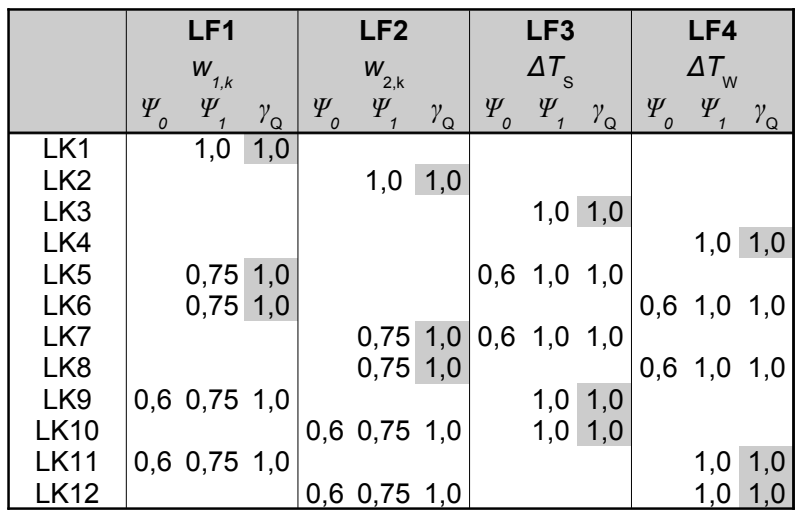




\subsubsection{Schnittgrößenermittlung}

Verbleiben die Deckschichten bei der Berechnung einer Lastkombination im Zustand I, darf das Ergebnis einer Lastkombination durch Superposition aus den Ergebnissen der einzelnen Lastfälle bestimmt werden. Gehen die Deckschichten infolge einer Lastkombination in den Zustand II über, so müssen die Ergebnisse nichtlinear ermittelt werden und dürfen daher nicht überlagert werden.

Die Berechnung einer vollständigen Lastfallkombination ist mit dem Programm swe2+ problemlos möglich, da in der Eingabemaske eine größere Anzahl an Lastfällen eingegeben werden kann. Bei der Eingabe einer Temperaturbeanspruchung muss die Temperaturdifferenz um die Materialfaktoren und Kombinationsbeiwerte entsprechend vergrößert oder verringert werden.

\subsubsection{Nachweise im Grenzzustand der Tragfähigkeit}

\section{Allgemeines:}

Für die Nachweise im Grenzzustand der Tragfähigkeit wurde die Lastfallkombination LK7 (Windsog + Temperaturlastfall Sommer) betrachtet. Der Berechnung mit swe2+ wurde die mittlere Betonzugfestigkeit von $f_{c t m}=3,7 \mathrm{~N} / \mathrm{mm}^{2}$ zu Grunde gelegt.

Lastfallkombination LK7: (Windsog + Temperaturlastfall Sommer)

$$
\begin{aligned}
w_{d} & =\gamma_{Q} \cdot w_{2, k}=1,5 \cdot-0,7 \mathrm{kN} / \mathrm{m}^{2}=-1,05 \mathrm{kN} / \mathrm{m}^{2} \\
\Delta T_{d} & =\gamma_{Q} \cdot \psi_{0} \cdot \Delta T_{S, k}=1,5 \cdot 0,6 \cdot 55^{\circ} \mathrm{K}=49,5^{\circ} \mathrm{K}
\end{aligned}
$$

Nachweis gegen Zugversagen der Bewehrung:

$$
\begin{aligned}
\sigma_{S, d} & \leq f_{y, d} \\
\frac{M_{S, d}}{e \cdot A_{S}} & \leq \frac{f_{y, k}}{\gamma_{M}} \\
\frac{3,24 \mathrm{kNm}}{130 \mathrm{~mm} \cdot 185,4 \mathrm{~N} / \mathrm{mm}^{2}} & \leq \frac{325 \mathrm{~N} / \mathrm{mm}^{2}}{1,15} \\
134,4 \mathrm{~N} / \mathrm{mm}^{2} & \leq 282,6 \mathrm{~N} / \mathrm{mm}^{2} \quad \checkmark
\end{aligned}
$$

Nachweis gegen Schubversagen der Verbundfuge:

$$
\begin{aligned}
& \tau_{V, d} \leq f_{X P S, b, d} \\
& \frac{V_{S, d}}{e \cdot b} \leq \frac{f_{X P S, b, k}}{\gamma_{M}} \\
& \frac{2,39 \mathrm{kN}}{0,13 \mathrm{~m} \cdot 1,00 \mathrm{~m}} \leq \frac{80 \mathrm{kN} / \mathrm{m}^{2}}{2,0} \\
& 18,4 \mathrm{kN} / \mathrm{m}^{2} \leq 40,0 \mathrm{kN} / \mathrm{m}^{2}
\end{aligned}
$$


Der Nachweis gegen das Schubversagen der Verbundfuge ist aufgrund der geringeren Festigkeit maßgebend. Ein Nachweis gegen das Scherversagen des Kerns muss daher nicht geführt werden.

Nachweis gegen Druckversagen des XPS an den Auflagern:

$$
\begin{aligned}
& F_{d} \leq F_{R 1, k} \\
& \frac{w_{2, d} \cdot l \cdot b}{2} \leq \frac{b \cdot\left(L_{S}+0,5 \cdot k \cdot e\right) \cdot f_{X P S, c, k}}{\gamma_{M}} \\
& \frac{1,05 \mathrm{kN} / \mathrm{m}^{2} \cdot 5,0 \mathrm{~m}}{2} \leq \frac{(0,1 \mathrm{~m}+0,5 \cdot 0,5 \cdot 0,1 \mathrm{~m}) \cdot 330 \mathrm{kN} / \mathrm{m}^{2}}{1,5} \\
& 2,63 \mathrm{kN} \leq 41,25 \mathrm{kN}
\end{aligned}
$$

$\gamma_{M}=1,5$ nach

DIN EN 14509

da $e \geq 100 \mathrm{~mm}$, gilt $e=100 \mathrm{~mm}$

\subsubsection{Nachweise im Grenzzustand der Gebrauchstauglichkeit}

\section{Nachweis gegen Rissbildung in den Deckschichten:}

Für den Nachweis gegen Rissbildung in den Deckschichten im Grenzzustand der Gebrauchstauglichkeit wird die selten auftretende Kombination nach Gleichung 3.48 (siehe S. 39 mit der Lastkombination LK7 (Wind Sog + Temperaturlastfall Sommer) gebildet. Diese Kombination gleicht der Lastkombination LK7 im Grenzzustand der Tragfähigkeit. Jedoch werden die Lastfaktoren $\gamma_{f}$ mit 1,0 angesetzt. Die Lastannahmen für die Berechnung mit swe2+ sind daher wie folgt:

Lastfallkombination LK7: (Windsog + Temperaturlastfall Sommer)

$$
\begin{aligned}
& w_{k}=-0,7 \mathrm{kN} / \mathrm{m}^{2} \\
& \Delta T_{k}=\psi_{0} \cdot \Delta T_{S, k}=0,6 \cdot 55^{\circ} \mathrm{K}=33^{\circ} \mathrm{K}
\end{aligned}
$$

Die maximal auftretenden Zugspannungen $\sigma_{c, k}$ infolge der Lastkombination LK7 sind geringer als die charakteristische Betonzugfestigkeit $f_{c t k ; 0,05}$.

$$
\begin{aligned}
\sigma_{c, k} & \leq f_{c t k ; 0,05} \\
\sigma_{c, k} & \leq 0,7 \cdot f_{c t m}
\end{aligned}
$$

$$
2,56 \mathrm{~N} / \mathrm{mm}^{2} \leq 2,59 \mathrm{~N} / \mathrm{mm}^{2}
$$

$f_{c t k ; 0,05}=0,7 \cdot f_{c t m}$ nach Eurocode 2 (DIN EN 1992)

\section{Begrenzung der Verformungen:}

Für den Nachweis der zulässigen Verformungen wird die häufig auftretende Kombination nach Gleichung 3.49) (siehe S. 39) gebildet. Aus der Lastkombination LK10 (Temperaturlastfall Sommer + Windsog) resultieren die größten Verformungen. Die Einwirkungen sind wie folgt:

Lastfallkombination LK10: (Temperaturlastfall Sommer + Windsog)

$$
\begin{aligned}
& \Delta T_{k}=\psi_{0} \cdot \Delta T_{S, k}=1,0 \cdot 55^{\circ} \mathrm{K}=55^{\circ} \mathrm{K} \\
& r_{k}=\psi_{0} \cdot \psi_{0} \cdot w_{2, k}=0,6 \cdot 0,75 \cdot-0,7 \mathrm{kN} / \mathrm{m}^{2}=-0,32 \mathrm{kN} / \mathrm{m}^{2}
\end{aligned}
$$




$$
w_{k} \leq l / 100
$$

$17,0 \mathrm{~mm} \leq 50,0 \mathrm{~mm}$

\subsubsection{Dachelement mit zwei Feldern}

\subsubsection{System und Abmessungen}

- Abmessungen:

Spannweite: $2 \times 4,00 \mathrm{~m}$

Auflagerbreite: $0,10 \mathrm{~m}$
- Querschnitt:

Deckschichtdicke: $10 \mathrm{~mm}$

Kernschichtdicke: $140 \mathrm{~mm}$

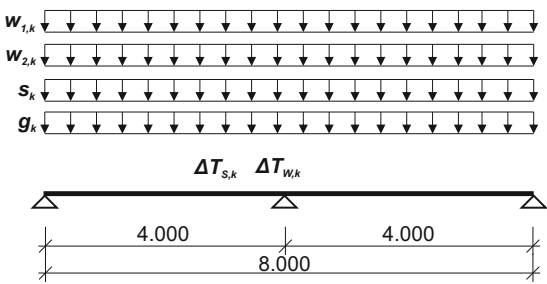

Abb. 8.25: Statisches System des Dachelements

\subsubsection{Materialien und Kennwerte}

- Deckschichten:

Hochleistungsbeton mit 3 Lagen Mikrobewehrung je Deckschicht

\begin{tabular}{|c|c|c|}
\hline Beton & Werte & Verweis \\
\hline Druckfestigkeit: & $f_{c k}=110 \mathrm{~N} / \mathrm{mm}^{2}$ & Gln. 4.1-4.3, S. 51 \\
\hline Zugfestigkeit: & $f_{c t m}=3,7 \mathrm{~N} / \mathrm{mm}^{2}$ & Tab. 4.3 S. 51 \\
\hline Elastizitätsmodul: & $E_{c}=43.000 \mathrm{~N} / \mathrm{mm}^{2}$ & Tab. 4.3 S. 51 \\
\hline Bewehrung & Werte & Verweis \\
\hline Querschnitt: & $A_{s}=3 \cdot 61,8=185,4 \mathrm{~mm}^{2} / \mathrm{m}$ & \begin{tabular}{ll|l|l|} 
Tab. & 4.4 & S. & 54 \\
\end{tabular} \\
\hline Elastizitätsmodul: & $E_{s}=160.000 \mathrm{~N} / \mathrm{mm}^{2}$ & Abb. 5.41 S. 96 \\
\hline Streckgrenze: & $f_{y k}=325 \mathrm{~N} / \mathrm{mm}^{2}$ & Abb. 8.15 S. 193 \\
\hline
\end{tabular}

\section{- Kernschicht:}

Als Materialeigenschaften des XPS wurden hier die Werte der Scherversuchsreihe $\mathrm{X} 4$ angenommen. 


\begin{tabular}{lll} 
XPS & Werte & Verweis \\
\hline Scherfestigkeit XPS: & $f_{X P S, k}=165 \mathrm{kN} / \mathrm{m}^{2}$ & Abb. 8.18, S. 195 \\
Scherfestigkeit Verbund: & $f_{X P S, b, k}=130 \mathrm{kN} / \mathrm{mm}^{2}$ & Annahme \\
Tang.-Schubmodul: & $G_{X P S, \text { mean }}=9.800 \mathrm{kN} / \mathrm{m}^{2}$ & Abb. 5.28 S. 86 \\
Druckfestigkeit: & $f_{X P S, c, k}=465 \mathrm{kN} / \mathrm{m}^{2}$ & Annahme
\end{tabular}

\subsubsection{Beanspruchungen}

\section{Dauerhafte Beanspruchungen $G_{k}$ :}

\section{- Eigenlast:}

Wichte des Betons $\gamma_{C}=25 \mathrm{kN} / \mathrm{m}^{3}$. Die Eigenlast des XPS wird vernachlässigt.

$$
\begin{aligned}
& g_{k}=2 \cdot d \cdot \gamma_{C} \\
& g_{k}=2 \cdot 10 \mathrm{~mm} \cdot 25 \mathrm{kN} / \mathrm{m}^{3}=0,5 \mathrm{kN} / \mathrm{m}^{2}
\end{aligned}
$$

\section{Veränderliche Beanspruchungen $Q_{k}$ :}

- Schnee: nach DIN EN 1991 und DIN EN 1991/NA

Lastfall 2 - Schnee:

$$
\begin{array}{ll}
s_{k}=0,85 \mathrm{kN} / \mathrm{m}^{2} & \begin{array}{l}
\text { Schneelastzone } 2 \\
\text { bei einer Dachneigung } 0^{\circ} \leq \alpha \leq 30^{\circ} \\
\mu_{1}=0,8
\end{array} \\
s_{k}=\mu_{1} \cdot s_{k}=0,8 \cdot 0,85=0,68 \mathrm{kN} / \mathrm{m}^{2}
\end{array}
$$

- Wind: nach DIN EN 1991 und DIN EN 1991/NA

Windlastzone 1, Binnenland

$$
\begin{array}{ll}
q_{k}=0,5 \mathrm{kN} / \mathrm{m}^{2} & \text { bei } h \leq 10 \mathrm{~m} \\
c_{p e, 10}=-1,8 & \text { für Flachdächer mit scharfk. Traufbereich (Bereich F) } \\
c_{p e, 10}=+0,2 & \text { für Flachdächer mit scharfk. Traufbereich (Bereich I) }
\end{array}
$$

Lastfall 3 - Winddruck:

$$
\begin{aligned}
& w_{1, k}=q_{k} \cdot c_{p e} \\
& w_{1, k}=0,5 \mathrm{kN} / \mathrm{m}^{2} \cdot+0,2 \\
& w_{1, k}=+0,25 \mathrm{kN} / \mathrm{m}^{2}
\end{aligned}
$$

Lastfall 4 - Windsog:

$$
\begin{aligned}
& w_{2, k}=q_{k} \cdot c_{p e} \\
& w_{2, k}=0,5 \mathrm{kN} / \mathrm{m}^{2} \cdot-1,8 \\
& w_{2, k}=-0,9 \mathrm{kN} / \mathrm{m}^{2}
\end{aligned}
$$


- Temperatur: nach DIN EN 14509, Farbgruppe III (dunkle Farben)

$\begin{array}{lll}\text { Lastfall } 5 \text { - Sommer im ULS und SLS: } & T_{1}=+80^{\circ} \mathrm{C} & \text { (außen) } \\ & T_{2}=+25^{\circ} \mathrm{C} & \text { (innen) } \\ \text { Lastfall } 6 \text { - Winter im ULS und SLS: } & T_{1}=-20^{\circ} \mathrm{C} & \text { (außen) } \\ & T_{2}=+20^{\circ} \mathrm{C} & \text { (innen) } \\ \text { Lastfall } 7 \text { - Winter im ULS und SLS bei Schnee: } & T_{1}=0^{\circ} \mathrm{C} & \text { (außen) } \\ & T_{2}=+20^{\circ} \mathrm{C} & \text { (innen) }\end{array}$

\subsubsection{Maßgebende Lastfallkombinationen}

\section{Grenzzustand der Tragfähigkeit}

Aus den sieben verschiedenen Lastfällen können im Zustand der Tragfähigkeit 46 mögliche Lastfallkombinationen gebildet werden. Die Tabelle mit der Zusammenstellung der Lastfallkombinationen ist im Anhang C.4, Lastfallkombinationen eines SandwichDachelements " auf Seite 279 dargestellt. Da alle Lastkombinationen auch unter Berücksichtigung eines reduzierten Schubmoduls infolge des Kriechens des Kerns berechnet werden müssen, ergeben sich insgesamt 92 Lastfallkombinationen im Grenzzustand der Tragfähigkeit.

\section{Grenzzustand der Gebrauchstauglichkeit}

Die selten auftretende Kombination im Zustand der Gebrauchstauglichkeit wird analog zu den Lastfallkombinationen LK1 bis LK23 im Grenzzustand der Tragfähigkeit gebildet. Die Lastfaktoren werden hierbei mit 1,0 angesetzt. Für die häufig auftretende Kombination ergeben sich 23 Lastfallkombinationen, die in Tabelle C.1 auf Seite 278 dargestellt sind. Die Berechnung der selten auftretenden und häufig auftretenden Lastfallkombinationen muss jeweils auch unter Berücksichtigung eines reduzierten Schubmoduls infolge des Kriechen des Kerns erfolgen. Daraus ergeben sich jeweils $46 \mathrm{zu}$ berücksichtigende Lastfallkombinationen.

\subsubsection{Schnittgrößenermittlung}

Wie bei der Berechnung der Schnittgrößen des Wandelements können die Lastkombinationen nur dann durch Superposition aus den Berechnungsergebnissen der einzelnen Lastfälle bestimmt werden, wenn die Deckschichten im Zustand I verbleiben. Gehen die Deckschichten in den Zustand II über, müssen die vollständigen Lastfallkombination berechnet werden.

Zur Berücksichtigung des Kriechens des Kerns, werden die Lastfälle „Eigenlast“ und „Schnee“ bei der Bemessung von Sandwichelementen mit metallischen Deckschichten jeweils getrennt mit reduzierten Schubmoduln berechnet und anschließend überlagert. Da dies aufgrund der nichtlinearen Berechnung bei der Berechnung von Sandwichelementen mit gerissenen Betondeckschichten nicht möglich ist, sollte die gesamte Lastfallkombination vereinfachend nur mit einem maßgebenden, reduzierten Schubmodul berechnet werden. 


\subsubsection{Nachweise im Grenzzustand der Tragfähigkeit}

\section{Allgemeines:}

Für die Nachweise im Grenzzustand der Tragfähigkeit wurde die Lastfallkombination LK40 betrachtet. Zur Berechnung mit swe2+ wurde die mittlere Betonzugfestigkeit von $f_{c t m}=3,7 \mathrm{~N} / \mathrm{mm}^{2}$ angesetzt. Die Berechnung erfolgte mit den folgenden Bemessungslasten:

Lastfallkombination LK40:

(Eigenlast (ungünstig) + Temperaturlastfall Sommer + Windsog)

$$
\begin{array}{ll}
\Delta T_{d} & =\gamma_{Q} \cdot \Delta T_{S, k}=1,5 \cdot-55^{\circ} \mathrm{K}=-82.5^{\circ} \mathrm{K} \\
r_{d} & =\gamma_{G} \cdot g_{k}+\gamma_{Q} \cdot s_{k}+\gamma_{Q} \cdot \psi_{0} \cdot w_{2, k} \\
& =1,0 \cdot 0,5 \mathrm{kN} / \mathrm{m}^{2}+1,5 \cdot 0,6 \cdot-0,9 \mathrm{kN} / \mathrm{m}^{2}=0,31 \mathrm{kN} / \mathrm{m}^{2}
\end{array}
$$

\section{Nachweis gegen Zugversagen der Bewehrung:}

Nachweis der Bewehrung der unteren Deckschicht am Mittelauflager (max. positives Moment am Mittelauflager, vgl. Abbildung 8.12, S. 187)

$$
\begin{aligned}
& \sigma_{S, d} \leq f_{y, d} \\
& \frac{M_{S, d}}{e \cdot A_{S}} \leq \frac{f_{y, k}}{\gamma_{M}} \\
& \frac{6,04 \mathrm{kNm}}{150 \mathrm{~mm} \cdot 185,4 \mathrm{~N} / \mathrm{mm}^{2}} \leq \frac{325 \mathrm{~N} / \mathrm{mm}^{2}}{1,15} \\
& 217,2 \mathrm{~N} / \mathrm{mm}^{2} \leq 282,6 \mathrm{~N} / \mathrm{mm}^{2} \quad \checkmark
\end{aligned}
$$

\section{Nachweis gegen Schubversagen der Verbundfuge:}

Nachweis der Verbundfuge links und rechts des Mittelauflagers

$$
\begin{aligned}
\tau_{v, d} & \leq f_{X P S, b, d} \\
\frac{V_{S, d}}{e \cdot b} & \leq \frac{f_{X P S, b, k}}{\gamma_{M}} \\
\frac{2,06 \mathrm{kN}}{150 \mathrm{~mm} \cdot 1000 \mathrm{~mm}} & \leq \frac{130 \mathrm{~N} / \mathrm{mm}^{2}}{2,0} \\
13,7 \mathrm{kN} / \mathrm{m}^{2} & \leq 65 \mathrm{kN} / \mathrm{m}^{2}
\end{aligned}
$$

gewählt:

$\gamma_{M}=2,0$

Der Nachweis gegen das Schubversagen der Verbundfuge ist maßgebend. Ein zusätzlicher Nachweis gegen Scherversagen des Kerns muss daher nicht geführt werden. 


\section{Nachweis gegen Druckversagen des XPS an den Auflagern:}

Nachweis am Mittelauflager

$$
\begin{aligned}
& F_{d} \leq F_{R 2, k} \\
& \left|V_{l, d}\right|+\left|V_{r, d}\right| \leq \frac{b \cdot\left(L_{S}+k \cdot e\right) \cdot f_{X P S, c, k}}{\gamma_{M}} \\
& |2,16 \mathrm{kN}|+|-2,16 \mathrm{kN}| \leq \frac{(0,1 \mathrm{~m}+0,5 \cdot 0,1 \mathrm{~m}) \cdot 465 \mathrm{kN} / \mathrm{m}^{2}}{1,5} \\
& 4,32 \mathrm{kN} \leq 46,5 \mathrm{kN}
\end{aligned}
$$

\subsubsection{Nachweise im Grenzzustand der Gebrauchstauglichkeit}

\section{Nachweis gegen Rissbildung in den Deckschichten:}

Der Nachweis gegen Rissbildung in den Deckschichten im Grenzzustand der Gebrauchstauglichkeit kann nicht erbracht werden. Durch die Temperaturbeanspruchung im Sommer und im Winter werden sehr große Zwangspannungen in den Deckschichten hervorgerufen, die zum Reißen beider Deckschichten führen. In Absprache mit dem Bauherrn können die Sandwichelemente trotz gerissener Deckschichten als Dachelemente eingesetzt werden. Zur Sicherstellung der Dauerhaftigkeit sollte jedoch eine Dachabdichtung vorgesehen werden.

\section{Begrenzung der Verformungen:}

Der Nachweis der zulässigen Verformungen wird sowohl unter kurzzeitiger Belastung als auch unter langzeitiger Belastung unter Berücksichtigung des Kriechens des XPSKerns geführt. Für den Nachweis wird die häufig auftretende Kombination nach Gleichung (3.49) angewendet. Aus der Lastkombination LK20 resultieren die größten Verformungen bei kurzzeitiger Belastung. Die Einwirkungen berechnen sich dabei wie folgt:

- Nachweis unter kurzzeitiger Belastung mit Lastfallkombination LK20:

(Eigenlast + Schnee + Winddruck + Temperaturlastfall Winter bei Schnee $)$

$$
\begin{aligned}
r_{d} & =g_{k}+\psi_{1} \cdot s_{k}+\psi_{0} \cdot \psi_{1} \cdot w_{1, k} \\
& =0,5 \mathrm{kN} / \mathrm{m}^{2}+0,75 \cdot 0,68 \mathrm{kN} / \mathrm{m}^{2}+0,6 \cdot 0,75 \cdot 0,25 \mathrm{kN} / \mathrm{m}^{2} \\
& =1,12 \mathrm{kN} / \mathrm{m}^{2} \\
\Delta T_{k} & =\psi_{0} \cdot \psi_{1} \cdot \Delta T_{W S, k}=1,0 \cdot 1,0 \cdot-20^{\circ} \mathrm{K}=-20^{\circ} \mathrm{K}
\end{aligned}
$$

$$
w_{k} \leq l / 200
$$

\section{$4,1 \mathrm{~mm} \leq 20,0 \mathrm{~mm}$}

- Nachweis unter langzeitiger Belastung mit Lastfallkombination LK2:

(Eigenlast + Schnee)

$$
r_{d}=g_{k}+\psi_{1} \cdot s_{k}=0,5+1,0 \cdot 0,68 \mathrm{kN} / \mathrm{m}^{2}=1,18 \mathrm{kN} / \mathrm{m}^{2}
$$


Die Abminderung des Schubmoduls erfolgt vereinfachend nur mit dem Kriechfaktor $\varphi_{t}=7,0$ zur Berücksichtigung des Kriechens unter einer Eigenlast. Durch diese Annahme wird die Durchbiegung überschätzt. Als ingenieurmäßiger Ansatz könnte hier jedoch auch ein Kriechfaktor zwischen $\varphi_{t}=7,0$ und $\varphi_{t}=2,4$ in Abhängigkeit der Lastverteilung von Eigen- und Schneelast interpoliert werden.

$$
\begin{gathered}
G_{t}=\frac{G}{1+\varphi_{t}}=\frac{9.800 \mathrm{kN} / \mathrm{m}^{2}}{1+7}=1.225 \mathrm{kN} / \mathrm{m}^{2} \\
w_{k} \leq l / 100
\end{gathered}
$$

$$
11,8 \mathrm{~mm} \leq 40,0 \mathrm{~mm}
$$

\subsection{Schwindbemessung der Sandwichelemente}

\subsubsection{Ermittlung der Zwangspannungen}

Wie bereits durch die FE-Berechnungen festgestellt wurde, werden durch das Schwinden der Deckschichten und die gleichzeitige Behinderung dieser Verformung durch den Kern, Zwangspannungen über den Sandwichquerschnitt verursacht. Dabei erfahren die Deckschichten eine Zugbeanspruchung und der Kern eine Druckbeanspruchung. Unter der Annahme, dass beide Deckschichten ungerissen sind, können zur Ermittlung dieser Spannungen leicht die folgenden Beziehungen hergeleitet werden. Wie in den beiden Abbildungen 8.26 und 8.27 dargestellt, muss dabei zwischen Sandwichelementen mit gleichen Deckschichtdicken und Elementen mit unterschiedlichen Deckschichtdicken unterschieden werden.
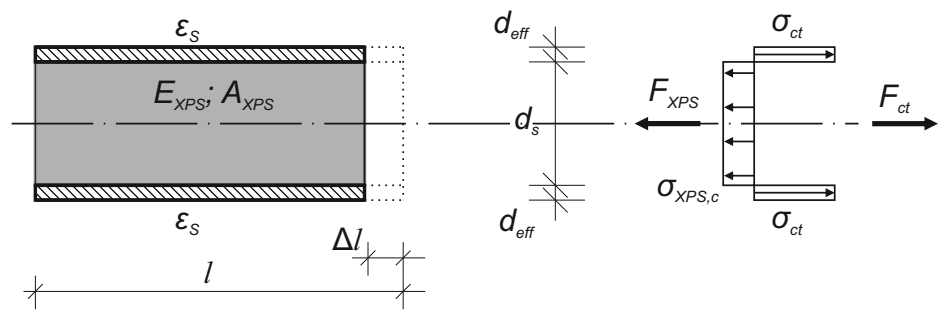

Abb. 8.26: Zwangspannungen infolge einer Schwindbeanspruchung eines Sandwichelements mit Deckschichten gleicher Dicke

Es wird angenommen, dass die Schwinddehnungen $\epsilon_{s}$ beider Deckschichten identisch sind. Die Schwindverkürzung $\Delta l$ errechnet sich aus der Schwinddehnung $\epsilon_{s}$ wie folgt:

$$
\Delta l=\epsilon_{s} \cdot l
$$

Wird das Verbundverhalten als unnachgiebig angenommen, muss sich der Kern ebenfalls um das Maß $\Delta l$ verkürzen.

$$
\Delta l=\frac{F \cdot l}{E_{X P S} \cdot A_{X P S}}
$$




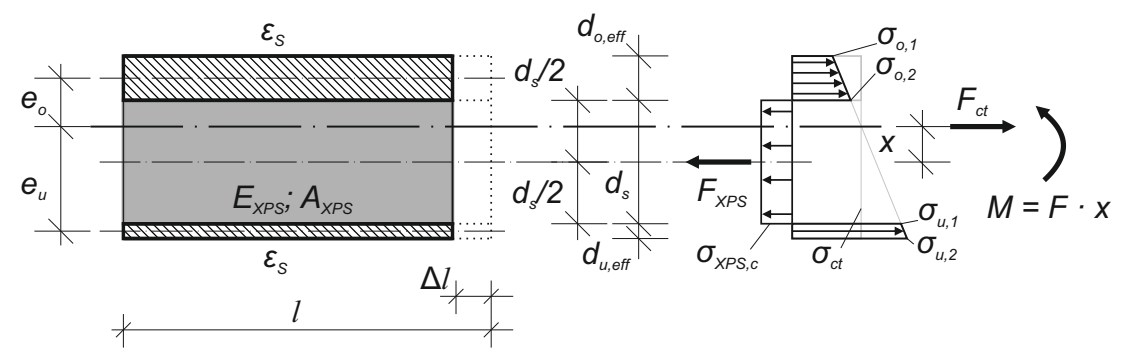

Abb. 8.27: Zwangspannungen infolge einer Schwindbeanspruchung eines Sandwichelements mit Deckschichten unterschiedlicher Dicke

Mit:
$F \quad: \quad$ Resultierende Zugkraft in den Deckschichten $F_{c t}$ oder resul- tierende Druckkraft im Kern $F_{X P S}$. Es gilt: $F=F_{c t}=F_{X P S}$
$E_{X P S} \quad$ : Mittlerer Elastizitätsmodul des XPS
$A_{X P S} \quad: \quad$ Fläche des XPS-Kerns

Durch Gleichsetzen der Gleichungen 8.29 und 8.30 ergibt sich:

$$
\epsilon_{s}=\frac{F}{E_{X P S} \cdot A_{X P S}}
$$

Da $l$ durch das Gleichsetzen der Gleichungen entfällt, sind die Zwangspannungen im Kern und in den Deckschichten unabhängig von der Länge eines Sandwichelements. Dies wurde ebenfalls in den FE-Parameterstudien festgestellt.

Aus Gleichung 8.31 lassen sich die resultierenden Spannungen infolge der Schwinddehnung über den Querschnitt berechnen. Die Druckspannung über den Kern wird wie folgt berechnet:

$$
\sigma_{X P S, C}=\epsilon_{S} \cdot E_{X P S}
$$

Im Fall eines Sandwichelements mit gleicher Dicke der beiden Deckschichten wird die Betonzugspannung wie folgt berechnet:

$$
\sigma_{c t}=\epsilon_{s} \cdot E_{X P S} \cdot \frac{d_{s}}{2 \cdot d_{\text {eff }}}
$$

Bei der Berechnung der Betonzugspannungen sollte eine effektive Deckschichtdicke $d_{\text {eff }}$ angesetzt werden, um eine Querschnittsschwächung infolge von Querfasern und Querdrähten zu berücksichtigen.

Bei Sandwichelementen mit unterschiedlicher Dicke der Deckschichten verschiebt sich die Schwerachse des Querschnitts gegenüber der Schwerachse der Kernschicht. Da die Druckkraft $F_{X P S}$ in der Schwerachse des Kerns und die Zugkraft $F_{c t}$ in der Schwerachse des Querschnitts wirkt, wird infolge des Abstands der beiden Schwerachsen ein Versatzmoment erzeugt (siehe Abbildung 8.27). Neben den Spannungen aus den 
Normalkräften $F_{c t}$ bzw. $F_{X P S}$ müssen daher noch die Biegespannungen aus dem Versatzmoment berücksichtigt werden:

$$
\sigma_{c t}=\epsilon_{s} \cdot E_{X P S} \cdot\left(\frac{d_{s}}{d_{o, \text { eff }}+d_{u, \text { eff }}} \pm \frac{x}{l_{y} / z}\right)
$$

Dabei sind:

$\begin{array}{lll}d_{o, \text { eff }}, d_{u, \text { eff }} & : & \begin{array}{l}\text { Dicke der Deckschichten abzüglich der Querschnittsschwä- } \\ \text { chungen durch Querfasern oder Querdrähte }\end{array} \\ x & : & \text { Abstand der Schwerachsen des Querschnitts und des Kerns } \\ I_{y} & : & \text { Flächenträgheitsmoment des Querschnitts } \\ z & : & \begin{array}{l}\text { Abstand zwischen der Schwerachse des Querschnitts und } \\ \text { der jeweiligen Stelle an der die Spannungen ermittelt werden }\end{array}\end{array}$

Für die einfache Ermittlung der Zwangspannungen bei Sandwichelementen mit gleichen Deckschichtdicken wurden die Gleichungen 8.32 und 8.33) in den Abbildungen 8.28 und 8.29 in Abhängigkeit des Elastiztätsmoduls $E_{X P S}$ aufgetragen.

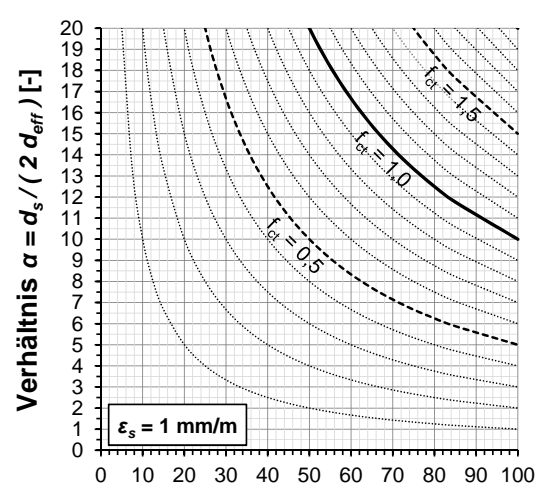

Elastizitätsmodul $E_{X P S}\left[\mathrm{~N} / \mathrm{mm}^{2}\right]$

Abb. 8.28: Betonzugspannungen in Abhängigkeit von $E_{X P S}$ und $\alpha$ bei einer Schwinddehnung von $1,0 \mathrm{~mm} / \mathrm{m}$

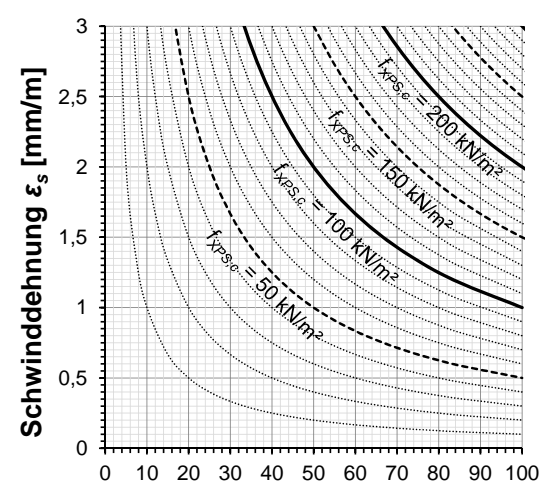

Elastizitätsmodul $E_{X P S}\left[\mathrm{~N} / \mathrm{mm}^{2}\right]$

Abb. 8.29: Druckspannungen im Kern in Abhängigkeit von $E_{X P S}$ und $\epsilon_{s}$

Die Ergebnisse der Herleitungen sind identisch mit den Ergebnissen die in den FEBerechnungen ermittelt wurden (siehe Abbildungen 7.29. S. 170. Auch die Herleitungen zur Berechnung von Sandwichelementen mit unterschiedlicher Deckschichtdicke wurden anhand von FE-Modellierungen kontrolliert. Die Berechnungsergebnisse stimmen auch hier sehr gut überein.

\subsubsection{Einwirkungen und Widerstände}

Die Nachweise zur Schwindbemessung können als Vergleich der Spannungen infolge der Einwirkungen und Widerstände geführt werden. Da die Einwirkungen und Widerstände von verschiedenen, zeitlich veränderlichen Parametern abhängen, müssen 
die Nachweise gegebenenfalls zu verschiedenen Zeitpunkten nach der Betonage der Deckschichten geführt werden.

Die Zwangspannungen aus der Schwinddehnung $\epsilon_{s}$ stellen beim Nachweis die Einwirkung dar. Wie in Abbildung 8.30 zu erkennen ist, tritt ein überwiegender Teil der Schwindverformungen bereits in den ersten Stunden nach dem Erstarrungsende ein. Dabei belaufen sich die Schwinddehnungen bereits auf etwa $1,5 \mathrm{~mm} / \mathrm{m}$. Im Anschluss daran nimmt die Gesamtschwinddehnung insbesondere durch das Trocknungsschwinden noch weiter zu. In den Schwinduntersuchungen an den verwendeten Betonrezepturen betrugen diese zusätzlichen Dehnungen maximal 1,0 $\mathrm{mm} / \mathrm{m}$. Die Gesamtschwinddehnung wird daher mit $2,5 \mathrm{~mm} / \mathrm{m}$ angenommen.

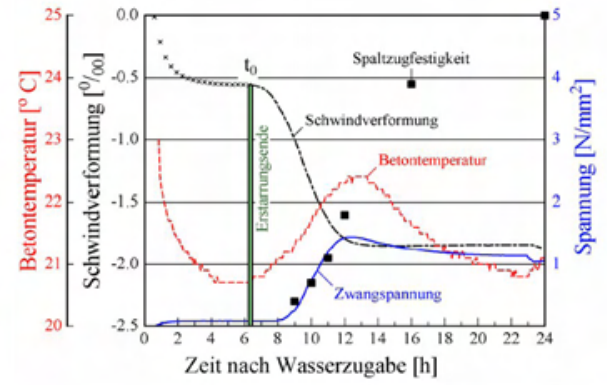

Abb. 8.30: Entwicklung der Betontemperatur, Schwinddehnung, Zwangspannung und Spaltzugfestigkeit eines UHPCs in den ersten 24 Stunden (Schachinger 2007)

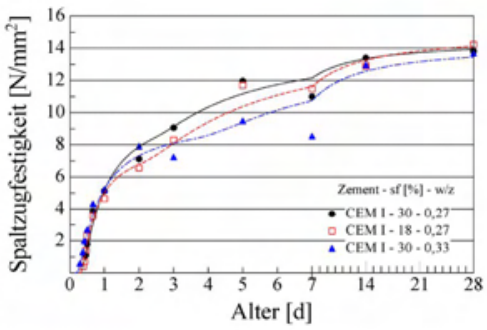

Abb. 8.31: Entwicklung der Spaltzugfestigkeit verschiedener UHPCs in den ersten 28 Tagen Schachinger 2007

Die aus dem Schwinden der Deckschichten resultierenden Zwangspannungen werden durch die Relaxation des XPS und des Betons zum Teil wieder abgebaut. Zum Relaxationsverhalten von XPS liegen keine Angaben in der Literatur vor. Daher wurde die Relaxation in einem Ansatz auf Basis des Kriechverhaltens für zwei XPS-Typen mit unterschiedlicher Rohdichte abgeschätzt (siehe Anhang C.3. Im Fall von Typ 1 reduzierten sich die Zwangspannungen nach einem Tag um rund $15 \%$ und nach 30 Tagen um rund $25 \%$. Bei Typ 2 reduzierten sich die Spannungen in den gleichen Zeiträumen um $5 \%$ und $10 \%$.

Durch die Relaxation des Betons werden die Zwangspannungen insbesondere im jungen Alter des Betons relativ schnell abgebaut. Durch Schachinger 2007 wurde der Anteil der durch Relaxation abgebauten Spannungen eines UHPCs bestimmt. Dazu wurde das Verhältnis der experimentell ermittelten Zwangspannungen zu den theoretisch ermittelten Zwangspannungen gebildet. Nach einem Tag nach Wasserzugabe wurden die Zwangspannungen etwa um 75 bis $93 \%$ und nach 14 Tagen nach der Betonage um 65 bis $87 \%$ durch Relaxation abgebaut.

Die Betonzugfestigkeit stellt beim Nachweis gegen Rissbildung in den Deckschichten den Widerstand dar. Die Zugfestigkeit eines hochfesten Betons steigt schon in den ersten Tagen relativ stark an (vgl. Abbildung 8.31). In den Untersuchungen nach Schachinger 2007 betrug die Spaltzugfestigkeit eines UHPCs mit einem Zement CEM I nach 12 Stunden nach der Wasserzugabe etwa $10 \%$ und nach 24 Stunden bereits et- 
wa $35 \%$ der Spaltzugfestigkeit nach 28 Tagen. Da die Zwangspannungen infolge des Schwinden des Betons in Längs- und in Querrichtung der Deckschichten auftreten, entsteht eine zweiaxiale Zugbeanspruchung der Betondeckschichten. Um diesen Umstand zu berücksichtigen, muss gegebenenfalls die Zugfestigkeit weiter abgemindert werden.

\subsubsection{Nachweis gegen Rissbildung der Deckschichten}

Der Nachweis gegen Rissbildung der Deckschichten wird nach Gleichung [8.35 geführt. Hierbei sind die zeitlich veränderlichen Parameter zum entsprechenden Bemessungszeitpunkt zu verwenden. Da die Rissbildung in den Deckschichten nur einen optischen Mangel darstellt, kann der Nachweis im Grenzzustand der Gebrauchstauglichkeit und somit ohne Sicherheitsbeiwerte geführt werden.

Aufgrund der zeitlichen Entwicklung der beschriebenen Parameter wird empfohlen den Nachweis gegen die Rissbildung der Deckschichten für den Zeitpunkt $t=12$ Stunden nach Wasserzugabe zu führen. Zu diesem Zeitpunkt ist das Verhältnis der Zwangspannungen zur Zugfestigkeit des Betons am größten. Sind größere Schwindverformungen im weiteren Verlauf zu erwarten, so sollte zusätzlich der Nachweis für den Zeitpunkt $t=28$ Tage geführt werden.

$$
k_{r, c,(t)} \cdot \sigma_{c t,(t)} \leq f_{c t k,(t)}
$$

Dabei bedeuten:

$$
\begin{aligned}
& \sigma_{c t,(t)} \quad: \quad \text { Zwangspannungen in den Deckschichten nach den Glei- } \\
& k_{r, c,(t)} \text { : Abminderungsfaktor zur Berücksichtigung der Relaxation }
\end{aligned}
$$

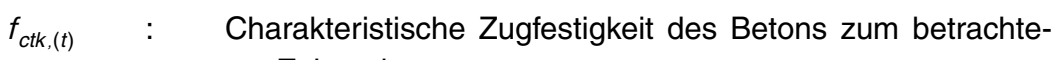

\subsubsection{Nachweis gegen Druckversagen des Kerns}

Der Nachweis der Zwangdruckspannungen im Kern sollte als Nachweis im Grenzzustand der Tragfähigkeit mit den entsprechenden Teilsicherheitsbeiwerten geführt werden:

$$
\begin{aligned}
\sigma_{X P S, c,(t), d} & \leq f_{X P S, c, d} \\
\gamma_{G} \cdot k_{r, X P S,(t)} \cdot \epsilon_{s,(t)} \cdot E_{X P S} & \leq f_{X P S, c, k} / \gamma_{M}
\end{aligned}
$$

Hierbei ist $k_{r, X P S,(t)}$ der Abminderungsfaktor zur Berücksichtigung der Relaxation des XPS. Nach DIN EN 1990 ist das Schwinden des Betons als eine ständige Einwirkung zu kategorisieren. Daher wird $\gamma_{G}=1,35$ angenommen. Der Materialsicherheitsbeiwert $\gamma_{M}$ ist versuchsgestützt nach Gleichung 8.20 (siehe S. 190) zu ermitteln oder kann in Anlehnung an DIN EN 14509 mit 1,4 angenommen werden. 


\subsubsection{Nachweis gegen Versagen des Verbunds}

Die Zugkraft $F_{c t}$ die durch das Schwinden in den beiden Deckschichten hervorgerufen wird, kann anteilig auf die obere und untere Deckschicht bezogen werden. Im Falle eines Sandwichelements mit gleichen Deckschichtdicken sind die beiden Kräfte $F_{c t, o}$ und $F_{c t, u}=F_{c t} / 2$. Bei unterschiedlicher Deckschichtdicke ergeben sich die Kräfte $F_{c t, o}$ und $F_{c t, u}$ aus der Summe der Betonzugspannungen einer jeden Deckschicht.

Die beiden Kräfte $F_{c t, o}$ und $F_{c t, u}$ werden jeweils über die untere und obere Verbundfuge an den beiden äußeren Enden des Sandwichwichelements in den Kern eingeleitet (siehe Abbildung 7.30 S. 170). Die Tragfähigkeit des Verbunds muss daher ebenfalls nachgewiesen werden. Hierzu muss in Abhängigkeit der Steifigkeit des Kerns eine entsprechende Einleitungslänge definiert werden. Weiterhin muss die Schubfestigkeit des Verbunds in Abhängigkeit der Festigkeit des jungen Betons ermittelt werden. Hierzu sind noch weitere Untersuchungen erforderlich.

\subsubsection{Beispiel}

In den nachfolgenden Berechnungen wird beispielhaft die Schwindbemessung eines Sandwichelements demonstiert. Die Dicke beider Deckschichten des Sandwichelements sei $10 \mathrm{~mm}$ und die Dicke des Kerns sei $120 \mathrm{~mm}$. Als Kernmaterial wird die XPSCharge X3 mit einer Rohdichte von $46,9 \mathrm{~kg} / \mathrm{m}^{2}$ vorausgesetzt. Die Elastizitätsmoduln des XPS in $x$ - und $y$-Richtung wurden in Tabelle 6.2 mit rund $15 \mathrm{~N} / \mathrm{mm}^{2}$ bzw. $23 \mathrm{~N} / \mathrm{mm}^{2}$ angenommen. Die Bemessung in $y$-Richtung des Elements wird daher maßgebend. Es wird angenommen, dass die Deckschichten mit jeweils einer Lage des Carbonfasergeleges bewehrt sind. Durch die Schwächung des Betonquerschnitts durch die Querfasern mit einer Dicke von 0,5 mm ergibt sich eine effektive Deckschichtdicke von $d_{\text {eff }}=9,5 \mathrm{~mm}$.

Der Nachweis gegen Rissbildung der Deckschichten wird für den Zeitpunkt $t=12 \mathrm{~h}$ geführt. Auf Grundlage der Werte in Abschnitt 8.5.2, Einwirkungen und Widerstände: wurde die Schwinddehnung $\epsilon_{s,(12 \mathrm{~h})}=1,5 \mathrm{~mm} / \mathrm{m}$, die Betonzugfestigkeit $f_{c t k,(12 \mathrm{~h})}=0,1$. $f_{c t k}$ und der Faktor zur Berücksichtigung der Relaxation des Betons $k_{r, c,(12 \mathrm{~h})}=0,25$ angenommen.

$$
\begin{aligned}
& \sigma_{c t k,(12 \mathrm{~h})} \leq f_{c t k,(12 \mathrm{~h})} \\
& k_{r, c,(12 \mathrm{~h})} \cdot \epsilon_{s,(12 \mathrm{~h})} \cdot E_{X P S} \cdot \frac{d_{s}}{2 \cdot d_{\text {eff }}} \leq f_{c t k,(12 \mathrm{~h})} \\
& 0,25 \cdot 1,5 \% \cdot 23 \mathrm{~N} / \mathrm{mm}^{2} \cdot \frac{120 \mathrm{~mm}}{2 \cdot 9,5 \mathrm{~mm}} \leq 0,1 \cdot 0,7 \cdot 3,7 \mathrm{~N} / \mathrm{mm}^{2} \\
& 0,05 \mathrm{~N} / \mathrm{mm}^{2} \leq 0,2 \mathrm{~N} / \mathrm{mm}^{2}
\end{aligned}
$$

Mit:

$f_{c t k}=0,7 \cdot f_{c t m}$

Bei der Herstellung der Probekörper wurden die Deckschichten an zwei aufeinander folgenden Tagen hergestellt. Nach der Herstellung der ersten Deckschicht wird diese durch das Schwinden des Betons bereits durch Zwangspannungen beansprucht. Wie bei der Bemessung von Sandwichelementen mit unterschiedlicher Deckschichtdicke 
auch müssen in diesem Fall die zusätzlichen Biegespannungen aus einem Versatzmoment berücksichtigt werden.

$$
\begin{aligned}
& \sigma_{c t k,(12 \mathrm{~h}), \text { o. bzw. u. }} \leq f_{c t k,(12 \mathrm{~h})} \\
& k_{r, c,(12 \mathrm{~h})} \cdot \epsilon_{s,(12 \mathrm{~h})} \cdot E_{X P S} \cdot\left(\frac{d_{s}}{d_{\text {eff }}} \pm \frac{d / 2+d_{s} / 2}{\left(d^{3} / 12\right) /(d / 2)}\right) \leq f_{c t k,(12 \mathrm{~h})} \\
& 0,25 \cdot 1,5 \% \cdot 23 \cdot\left(\frac{120}{9,5} \pm \frac{10 / 2+120 / 2}{\left(10^{3} / 12\right) /(10 / 2)}\right) \leq 0,1 \cdot 0,7 \cdot 3,7 \\
& 0,14 \mathrm{~N} / \mathrm{mm}^{2} \leq 0,26 \mathrm{~N} / \mathrm{mm}^{2}
\end{aligned}
$$

Für den Nachweis gegen Druckversagen des XPS wird angenommen, dass der Elastizitätsmodul $E_{X P S}$ dem 75-fachen der Druckfestigkeit $f_{X P S, C}$ entspricht (vgl. Abschnitt 3.2.3.2, Materialverhalten unter Druckbeanspruchung () und das Schwindmaß $\epsilon_{s}=$ $2,5 \mathrm{~mm} / \mathrm{m}$ beträgt. Der Nachweis unter Berücksichtigung der Teilsicherheitsbeiwerte und unter Vernachlässigung der Relaxation des XPS wird grundsätzlich eingehalten:

$$
\begin{aligned}
& \sigma_{X P S, c, d} \leq f_{X P S, c, d} \\
& 1,35 \cdot 2,5 \% \circ \cdot E_{X P S, c} \leq f_{X P S, c, k} / 1,4 \\
& 0,25 \cdot f_{X P S, c} \leq 0,71 \cdot f_{X P S, c} \quad \checkmark
\end{aligned}
$$

Mit:

$E_{X P S, c}=75 \cdot f_{X P S, c}$ 



\section{$9 \quad$ Brandschutz}

\subsection{Klassifizierung der Baustoffe und Bauteile}

\subsubsection{Allgemeines}

Aufgrund der Harmonisierung des Brandschutzes in Europa, gelten in Deutschland zurzeit deutsche und europäische Brandschutznormen parallel. Baustoffe und Bauteile werden sowohl in der deutschen Brandschutznorm DIN 4102 als auch in der europäischen DIN EN 13501 klassifiziert.

Baustoffe werden abhängig von ihrer Brennbarkeit in Klassen unterteilt. In der deutschen Brandschutznorm werden diese Klassen „Baustoffklassen“ und in der europäischen Norm „Euroklassen“ bezeichnet. Die Baustoff- und Euroklassen sind in Tabelle 9.1 gegenübergestellt.

Tab. 9.1: Gegenüberstellung der Baustoffklassen nach MBO 2002 DIN 4102 und DIN EN 13501

\begin{tabular}{|c|c|c|c|}
\hline $\begin{array}{l}\text { Bezeichnung } \\
\text { (nach MBO) }\end{array}$ & $\begin{array}{l}\text { Baustoff- } \\
\text { klasse }^{1}\end{array}$ & $\begin{array}{l}\text { Euro.- } \\
\text { klasse }^{2}\end{array}$ & Anforderungsniveau \\
\hline \multirow{2}{*}{ nichtbrennbar } & A1 & A1 & kein Betrag zum Brand \\
\hline & A2 & $\mathrm{A} 2$ & vernachlässigbarer Beitrag zum Brand \\
\hline schwerentflammbar & B1 & $\begin{array}{l}B \\
C\end{array}$ & $\begin{array}{l}\text { sehr geringer Beitrag zum Brand } \\
\text { geringer Beitraq zum Brand }\end{array}$ \\
\hline normalentflammbar & B2 & $\begin{array}{l}\mathrm{D} \\
\mathrm{E}\end{array}$ & $\begin{array}{l}\text { hinnehmbarer Beitrag zum Brand } \\
\text { hinnehmbares Brandverhalten }\end{array}$ \\
\hline leichtentflammbar & B3 & $\mathrm{F}$ & keine Anforderungen \\
\hline
\end{tabular}

Bauteile hingegen werden in Feuerwiderstandsklassen unterteilt. In der deutschen Norm werden fünf Widerstandsklassen, d. h. F 30, F60, F90, F 120 und F 180 unterschieden. Durch die Klassen wird angegeben, wie lange ein Bauteil einer genormten Brandbeanspruchung widersteht, ohne dass dessen gewünschte Funktion, z. B. dessen Tragfähigkeit, verloren geht. Beispielsweise wird eine Wand mit einer 30-minütigen Feuerwiderstand mit F 30 gekennzeichnet. Im Falle einer nichttragenden Außenwand wird der Buchstabe F durch W ersetzt (siehe Tabelle 9.2).

In der europäischen Norm wird die geforderte Widerstandsdauer ebenfalls in Minuten angegeben. Jedoch wird die Funktion, die durch die Wand erfüllt werden muss durch einen entsprechenden Buchstaben gekennzeichnet. Beispielsweise muss eine Wand mit einer Feuerwiderstandsklasse REI 30 in einer 30-minütigen Brandbeanspruchung die Funktionen R „Résistance - Tragfähigkeit", E „Etanchèitè - Raumabschluss“ und I 
Tab. 9.2: Gegenüberstellung der Feuerwiderstandsklassen für nichttragende Aussenwände nach MBO2002 DIN 4102 und DIN EN 13501

\begin{tabular}{|l|c|c|}
\hline $\begin{array}{c}\text { Bezeichnung } \\
\text { (nach MBO) }\end{array}$ & $\begin{array}{c}\text { Baustoff- }^{\text {klasse }}{ }^{1} \\
\text { Feuerhemmend }\end{array}$ & ' Euroklasse $^{2}$ \\
\hline Hochfeuerhemmend & W 60 & El $30(i \leftrightarrow 0)$ \\
\hline Feuerbeständig & W $60(\mathrm{i} \leftrightarrow 0)$ \\
\hline
\end{tabular}

nach DIN 4102-1

2 nach DIN EN 13501

„Isolation - Wärmedämmung unter Brandbeanspruchung“ erfüllen. Weiterhin wird für nichttragende Wände die Richtung der geforderten Eigenschaft angegeben, d. h. i $\rightarrow 0$ „Von innen nach außen“, $\mathrm{i} \leftarrow$ o „von außen nach innen“. Somit wird eine nichttragende Außenwand mit einer 30-minütigen Feuerwiderstand mit El $30(i \leftrightarrow 0)$ bezeichnet.

\subsubsection{Extrudierter Polystyrol Hartschaum}

Extrudierter Polstyrol Hartschaum beginnt bei einer Temperatur von ungefähr $100^{\circ} \mathrm{C}$ zu erweichen und zu schmelzen. Bei einer Temperatur von über $450^{\circ} \mathrm{C}$ besteht Gefahr von Selbstentzündung. In Deutschland wird daher nur flammgeschütztes FR XPS (engl. flame-retardant XPS) verwendet. FR XPS schrumpft bei Hitze und bewegt sich von der Hitzequelle weg, so dass die Wahrscheinlichkeit einer Entzündung reduziert wird (Antonatus 2010). FR XPS wird nach der deutschen Normung in die Baustoffklasse B1 klassifiziert und gilt somit als schwerentflammbar. Nach europäischer Normung wird FR XPS in die Euroklasse $E$ eingeteilt und gilt somit als normalentflammbarer Baustoff (BASF SE 2011b). Dieser Unterschied in der Baustoffklasse ist auf die unterschiedlichen Prüfverfahren zurückzuführen (Fouad 2006).

\subsubsection{Hochleistungsbeton}

Allgemein wird Beton nach DIN 4102 und DIN EN 13501 als nichtbrennbarer Baustoff der Baustoffklasse bzw. Euroklasse A1 definiert und liefert somit keinen Beitrag zum Brand.

Jedoch weisen hochfeste Betone aufgrund ihres sehr dichten und kapillarporenarmen Gefüges ein ungünstiges Materialverhalten unter einer Brandbeanspruchung auf. Bei einer Temperatur von $105^{\circ} \mathrm{C}$ beginnt das freie und gebundene Wasser im Beton zu verdampfen. Bei hochfesten Betonen kann der Wasserdampfdruck nicht, wie bei einem normalfestem Beton in großen Kapillarporen entspannen oder durch das Gefüge entweichen, so dass durch den Dampfdruck im Beton erhebliche Zugkräfte entstehen. Bereits vor dem Erreichen der Zugfestigkeit in einem Temperaturbereich von 150 bis $300^{\circ} \mathrm{C}$ können durch diese Zugspannungen Mikrorisse im Beton entstehen, die die Tragfähigkeit deutlich reduzieren (König u. a. 2001). Beim Überschreiten der Zugfestigkeit des Betons kommt es dann zu explosionsartigen Abplatzungen (Schmidt 2008).

Durch die Hinzugabe von Polypropylen (PP)-Fasern können die explosionartigen Abplatzungen wirkungsvoll verhindert werden. Durch Pistol u.a. 2011 wurde gezeigt, 
dass sich durch die thermische Zersetzung der PP-Fasern Mikrokanäle bilden, welche sich durch Mikrorisse netzartig zu einem permeablen Transportsystem verbinden. Über das System aus Mikrokanälen und Mikrorissen baut sich der Wasserdampfdruck ab und Zwangspannungen werden vermindert.

\subsubsection{Sandwichelemente}

Zur Klassifizierung der Sandwichelemente in Feuerwiderstandsklassen müssen die entsprechenden Brandversuche durchgeführt werden. Im Rahmen dieser Arbeit wurden das Brandverhalten der Elemente bereits in zwei ersten Tastversuchen untersucht. Diese sind im nachfolgenden Abschnitt 9.3, "Feuerwiderstandsversuche läutert. Jedoch sind zur Klassifizierung weitere umfangreiche Untersuchungen erforderlich.

\subsection{Bauaufsichtliche Anforderungen}

\subsubsection{Allgemeines}

In der Musterbauordnung (MBO 2002) und den sich daran anlehnenden Landesbauordnungen sind die genauen Anforderungen an die Baustoffe und Bauteile für Standardbauaufgaben wie Wohngebäude, kleine Betriebsstätten, Verwaltungs- und Bürogebäude etc. geregelt [Fouad 2006). Die Baustoffe werden hier unterschieden in nichtbrennbare, schwerentflammbare, normalentflammbare und leichtentflammbare Baustoffe. Diese Unterteilung kann den Klassen der deutschen und europäischen Normen direkt gegenübergestellt werden (siehe Tabelle 9.1.

Auch die Anforderungen an den Feuerwiderstand sind in der Musterbauordnung (MBO 2002) angegeben. So müssen „feuerbeständige“ Bauteile einer 90-minütigen, „hochfeuerhemmende“ Bauteile einer 60-minütigen und „feuerhemmende“ Bauteile einer 30minütigen Brandbeanspruchung bei Aufrechterhaltung ihrer Funktion widerstehen. In Tabelle 9.2 ist die Klassifizierung nach $\mathrm{MBO} 2002$ den Klassifizierungen nach den deutschen und europäischen Normen für nichttragende Außenwände gegenübergestellt.

Für Sonderbauten werden die Anforderungen an den Brandschutz in gesonderten Richtlinien und Verordnungen geregelt (z. B. Richtlinie über den baulichen Brandschutz im Industriebau „Industriebaurichtlinie“ IndBauRL|2000 Musterverordnung über den Bau und Betrieb von Verkaufsstätten „Verkaufstättenverordnung“ MVkVO 1995 Musterverordnung über den Bau und Betrieb von Versammlungsstätten „Versammlungsstättenverordnung“ MVStättV2005).

\subsubsection{Sandwich-Wandelemente}

Die selbsttragenden Sandwich-Wandelemente können aufgrund der überwiegenden Beanspruchung durch Horizontallasten als „nichttragende Außenwände“ definiert werden. Nach MBO 2002 § 28 „Außenwände“ müssen nichttragende Außenwände aus 
nichtbrennbaren Baustoffen bestehen. Es sei denn sie sind als raumabschließendes Bauteil feuerhemmend. Da XPS ein brennbarer Baustoff ist, müssen die Sandwichelemente somit den Anforderungen der Feuerwiderstandsklasse W 30 bzw. El 30 entsprechen.

Bei Industriegebäuden mit einer Grundfläche von weniger als $2.000 \mathrm{~m}^{2}$ dürfen nach IndBauRL 2000 die Sandwichelemente als nichttragende Außenwände eingesetzt werden. Übersteigt die Grundfläche $2000 \mathrm{~m}^{2}$ ist der Einsatz von XPS in eingeschossigen Gebäuden ohne selbsttätiger Feuerlöschanlage bzw. mehrgeschossigen Gebäuden mit Feuerlöschanlage nur dann zulässig, wenn das XPS nach der deutschen Brandschutznorm DIN 4102 als „schwerentflammbar“ klassifiziert wird. Da nach der europäischen Norm DIN EN 13501 für XPS nur die Euroklasse E „normalentflammbar“ erreicht wird, ist der Einsatz der Elemente in diesem Fall nicht zulässig. Auch nach MVkVO 1995 dürfen die Außenwände und eingesetzten Dämmstoffe von erdgeschossigen Verkaufsstätten sowie von Verkaufsstätten mit Sprinkleranlage nur aus schwerentflammbaren Baustoffen bestehen. Im Falle von Versammlungsstätten, die mehr als 200 Besucher fassen, dürfen die Außenwände und eingesetzten Dämmstoffe nur aus nichtbrennbaren Baustoffen bestehen (MVStättV 2005).

\subsubsection{Sandwich-Dachelemente}

Nach MBO2002 werden an die Bedachung keine Anforderungen hinsichtlich der Baustoffklasse oder der Feuerwiderstandsklasse gestellt. In § 28 „Dächer“ wird an die Bedachung lediglich eine ausreichend lange Widerstandsfähigkeit gegen Flugfeuer und strahlende Wärme gefordert.

In IndBauRL 2000 wird für die Bedachungen von Industriebauten mit Brandabschnitten und Brandbekämpfungsabschnitten mit einer Dachfläche von mehr als $2.500 \mathrm{~m}^{2}$ gefordert, dass eine Brandausbreitung über das Dach innerhalb eines Abschnittes behindert wird. Dies Forderung wird erfüllt, wenn die tragende Dachschale aus mineralischen Baustoffen, die Bedachung aus nichtbrennbaren Stoffen oder das Dach nach DIN 18234-1,,Baulicher Brandschutz großflächiger Dächer“ ausgeführt wird. Unter gewissen Voraussetzungen werden diese Anforderungen nicht an erdgeschossige Lagerhallen gestellt. Nach MVkVO 1995 müssen die Bedachungen von Dächern, die den oberen Abschluss von Räumen oder Verkaufsstätten bilden aus nichtbrennbaren Baustoffen bestehen. Diese Forderung gilt nach MVStättV 2005 in Analogie auch für Versammlungsstätten. Davon ausgenommen sind Bedachungen über Versammlungsräumen mit einer Grundfläche von nicht mehr als $1.000 \mathrm{~m}^{2}$.

\subsection{Feuerwiderstandsversuche}

\subsubsection{Allgemeines}

Um das in der Literatur beschriebene Brandverhalten der Baustoffe zu bestätigen und das generelle Brandverhalten der Sandwichelemente zu untersuchen, wurden zwei Tastversuche im Brandschutzlabor der BASF durchgeführt. 


\subsubsection{Abmessungen und Herstellung der Probekörper}

Die Probekörper wiesen eine quadratische Grundfläche mit einer Seitenlänge von $560 \mathrm{~mm}$ auf. Die Deckschichtdicke und die Kerndicke der Probekörper betrugen $10 \mathrm{~mm}$ und $120 \mathrm{~mm}$. Die Deckschichten waren jeweils mit drei Drahtgitterlagen bewehrt. Als Beton wurde die Rezeptur HPC-1 verwendet. Im Fall des zweiten Probekörpers wurden zusätzlich $2 \mathrm{~kg} / \mathrm{m}^{3}$ Polypropylen-Fasern zur Mischung hinzugefügt. Die Herstellung der Probekörper erfolgte über „Eindrücken des XPS in den Beton“, wie bereits in Abschnitt 5.2.3, Probekörper und Herstellung' (Seite 64) beschrieben.

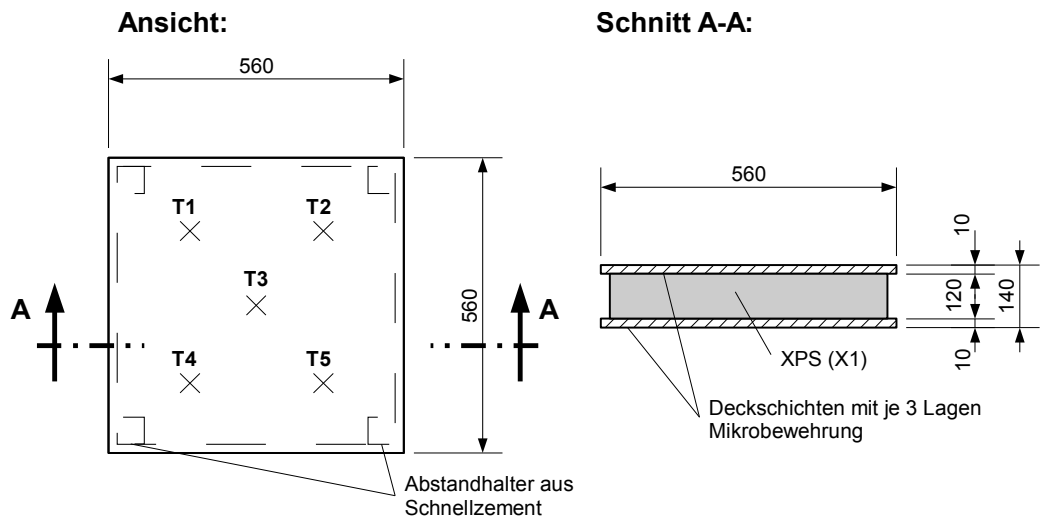

Abb. 9.1: Abmessungen der Probekörper [mm]

\subsubsection{Versuchsdurchführung}

Die Tastversuche wurden in Anlehnung an die entsprechenden Normen DIN 4102 bzw. DIN EN 1363 und DIN EN 1364-1 durchgeführt. Die Brandbeanspruchung wurde nach der Einheitstemperaturkurve (ETK) aufgebracht. Während der Versuche wurde die Oberflächentemperatur der äußeren Deckschicht mittels fünf Temperaturmessfühlern gemessen. Weiterhin wurde die Temperatur im Inneren des Brandofens gemessen.

\subsubsection{Versuchsergebnisse}

Die Messwerte der beiden Versuche sind über der Zeit in den beiden Diagrammen 9.2 und 9.3 dargestellt.

Bereits wenige Minuten nach dem Beginn der Versuche trat aus den oberen Seitenflächen der Probekörper Rauch aus. Wie in Abbildung 9.4 zu erkennen, begann der XPS-Kern hier zu schmelzen und ein größeres Loch bildete sich in der Kernschicht aus. Beim Blick in den Brandofen konnten auf der inneren Deckschicht feine Krakeleerisse mit einem Abstand von etwa 10-20 mm beobachtet werden. Die Risse zeichneten sich dunkel von der Betonfläche ab (siehe Abbildung 9.5). Es wird vermutet, dass diese Dunkelfärbung von der austretenden Feuchtigkeit herrührte. Nach 20 bis $25 \mathrm{Mi}-$ nuten war der XPS-Kern auf der oberen Seitenfläche vollständig weggeschmolzen. Auf 


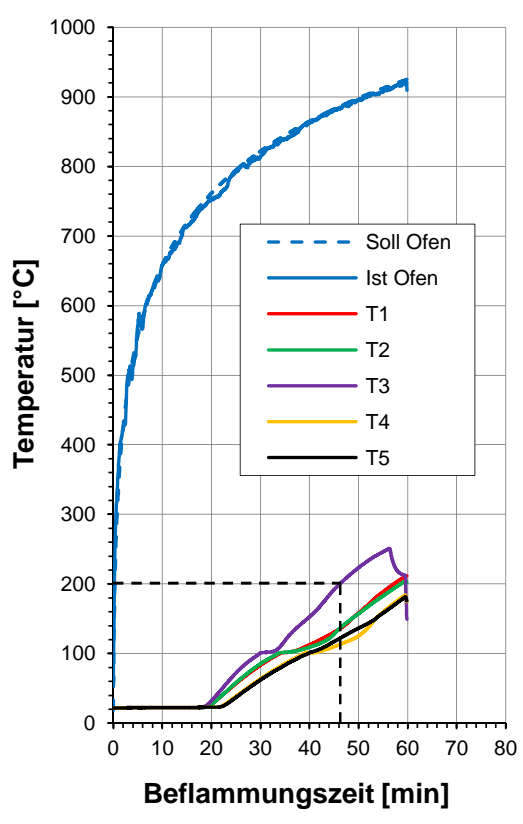

Abb. 9.2: Temperaturkurve Versuch 1

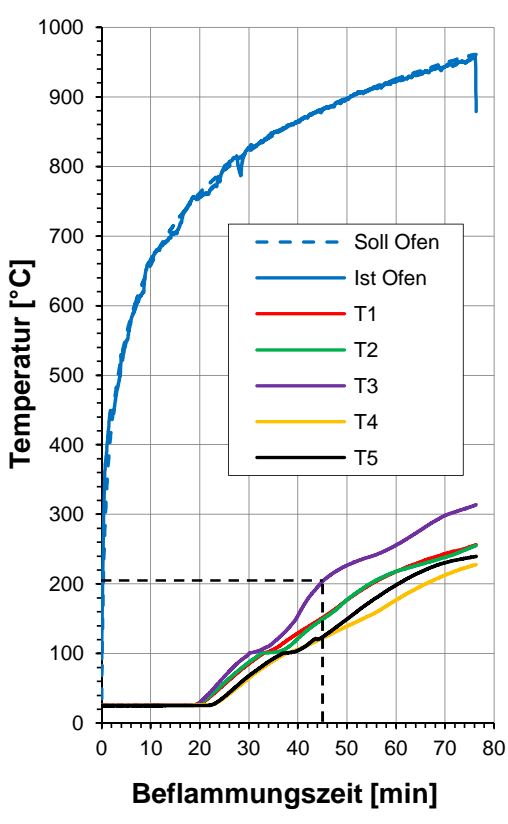

Abb. 9.3: Temperaturkurve Versuch 2

der linken und rechten Seitenfläche konnte ebenfalls das Wegschmelzen des XPS beobachtet werden. Das XPS begann nach einer halben Stunde als flüssige Masse aus der unteren Seitenfläche herauszulaufen.

Das Versagen des ersten Probekörpers, dessen Deckschichten ohne PP-Fasern hergestellt waren, trat nach einer Stunde ein. Dabei kam es zu einer großflächigen, explosionsartigen Abplatzung des Betons an der äußeren, dem Brandofen abgewandten Deckschicht (siehe Abbildung 9.6). Wie bereits zuvor erläutert, war die Ursache hierfür der hohe Wasserdampfdruck im Inneren des gefügedichten Betons. Die innere, dem Brandofen zugewandte Deckschicht war nach dem Versuch noch intakt und wies lediglich eine starke Wölbung von bis zu ca. $17 \mathrm{~mm}$ auf (siche Abbildung 9.7). Durch die Rissbildung der Deckschicht während des Versuchs, konnte der Wasserdampfdruck vorzeitig abgebaut werden. Der zweite Versuch wurde nach 76 Minuten abgebrochen. Das XPS war zu diesem Zeitpunkt nahezu vollständig geschmolzen. Ein Abplatzen des Betons trat aufgrund der Verwendung der PP-Fasern nicht auf. Die beiden Deckschichten des zweiten Versuchskörpers wiesen abgesehen von der Rissbildung und der Wölbung der inneren Deckschicht keine Beschädigungen auf.

Zur Einhaltung des Wärmedämmkriteriums „"“ nach DIN 4102 und DIN EN 13501 darf die mittlere Temperaturerhöhung auf der dem Feuer abgekehrten Seite des Probekörpers nicht mehr als $140^{\circ} \mathrm{C}$ und die maximale Temperaturerhöhung nicht mehr als $180^{\circ} \mathrm{C}$ über der mittleren Ausgangstemperatur liegen. In beiden Versuchen wurde nach 45 Minuten eine Temperatur von $200^{\circ} \mathrm{C}$ an der Stelle des mittig angeordneten Temperaturmessfühlers T3 gemessen und somit das Kriterium einer maximal zulässigen Temperaturerhöhung von $180^{\circ} \mathrm{C}$ gegenüber der Ausgangstemperatur überschritten (siehe gestrichelte Markierung in den Abbildungen 9.2 und 9.3. 


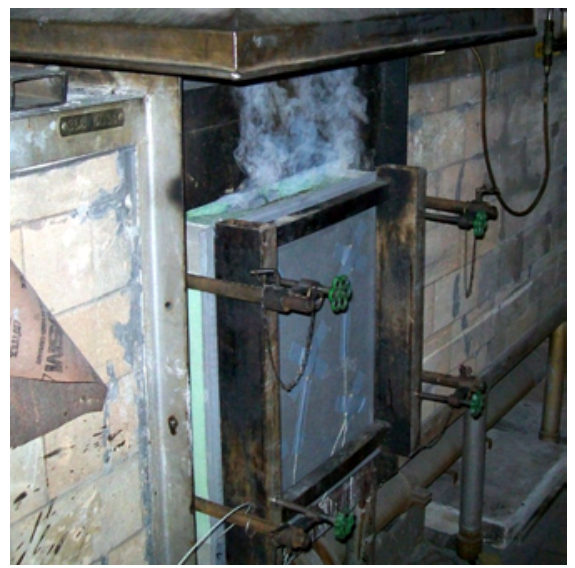

Abb. 9.4: Probekörper 1 nach einer Versuchsdauer von 10 Minuten

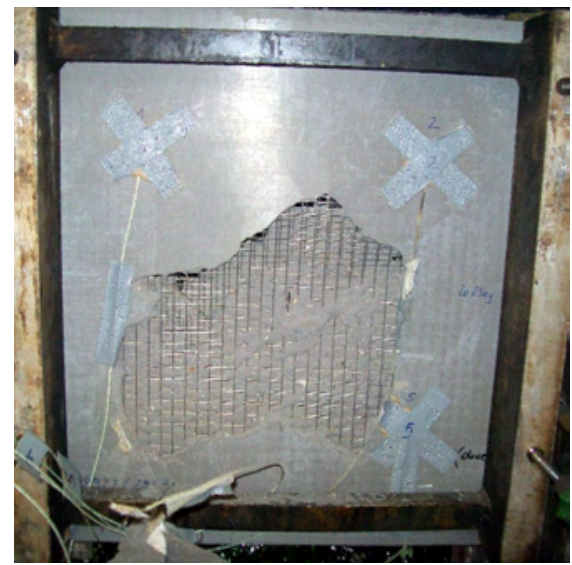

Abb. 9.6: Äußere Deckschicht des ersten Probekörpers nach dem Versuch

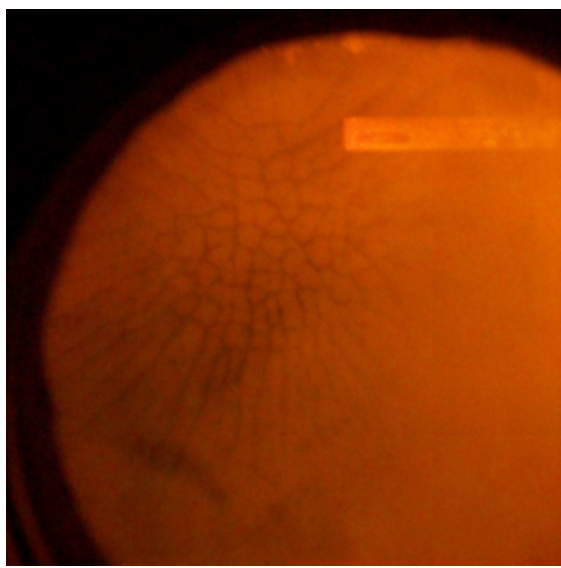

Abb. 9.5: Blick in den Brandofens während des ersten Versuchs

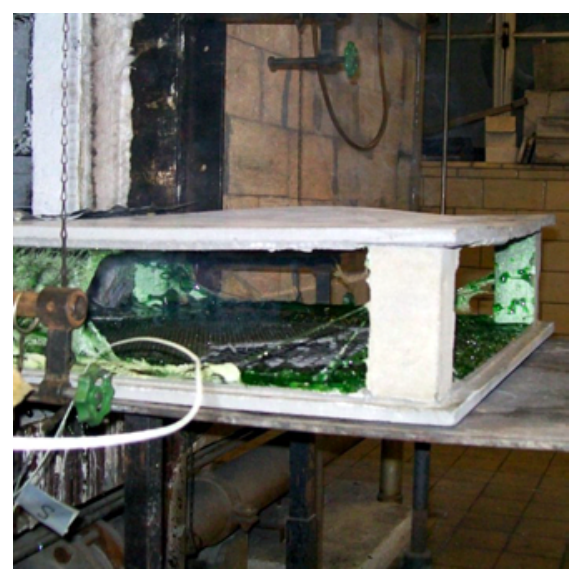

Abb. 9.7: Ausgebauter erster Probekörper nach dem Versuch

Zur Überprüfung des Raumabschlusskriteriums „E“ werden weitere Anforderungen an die Sandwichelemente gestellt. So darf sich ein an die Außenseite des Bauteils gehaltener Wattebausch nicht entzünden. Weiterhin muss bei raumabschließenden Wänden eine Festigkeitsprüfung durchgeführt werden. Dazu wird der Probekörper kurz vor dem Erreichen der Beurteilungszeit an drei verschiedenen Stellen der feuerabgewandten Seite einem Kugelstoß ausgesetzt. Diese Kriterien wurden jedoch in den beiden durchgeführten Tastversuchen nicht überprüft. Diese weitere Untersuchungen sollten an einem großformatigen Sandwichelement durchgeführt werden.

\subsection{Zusammenfassung und Schlussfolgerungen}

Bereits bei einer Temperatur von $100^{\circ} \mathrm{C}$ beginnt das XPS zu schmelzen. Der XPSKern kann somit nicht mehr zur Standfestigkeit des Elements beitragen. Daher müs- 
sen die beiden Deckschichten eine ausreichende Tragfähigkeit und Steifigkeit besitzen, so dass diese den Beanspruchungen in einem Brandfall standhalten. Weiterhin sollten beide Deckschichten an die tragende Unterkonstruktion befestigt werden, um ein Einstürzen der äußeren Deckschicht zu verhindern. Die in der Literatur beschriebenen explosionsartigen Abplatzungen des Hochleistungsbetons konnten in den Versuchen ebenfalls beobachtet werden. Durch die Zugabe von $2 \mathrm{~kg} / \mathrm{m}^{3}$ PolypropylenFasern konnte ein Abplatzen des Betons jedoch verhindert werden.

Durch die Tastversuche wurde gezeigt, dass die in den Brandschutznormen geforderte Wärmedämmfunktion für eine genormte 45-minütige Brandbeanspruchung erfüllt wird. Weitere Versuche an vollständigen Wandelementen mit der entsprechenden Fugenausbildung zwischen den Elementen sollten durchgeführt werden, um das festgestellte Brandverhalten zu bestätigen. Der Schwachpunkt von Sandwichkonstruktionen sind in der Regel die Fugen. Es muss hier verhindert werden, dass die Flammen den Kernwerkstoff erreichen und eventuell entzünden (Fouad 2006. Weiterhin muss in einem solchen Versuch ein Intaktbleiben der Deckschichten nach dem Wegschmelzen des XPS durch eine Stoßbeanspruchung untersucht werden. 


\section{Zusammenfassung und Ausblick}

\subsection{Zusammenfassung}

Im Rahmen dieser Arbeit wurden selbsttragende Sandwichelemente mit Deckschichten aus Hochleistungsbeton und einem Kern aus extrudiertem Polystyrol-Hartschaum (XPS) umfangreich experimentell und rechnerisch untersucht. Es wurde gezeigt, dass die Elemente ohne mechanische Verbindungsmittel ausgeführt werden können. Der nötige Haftverbund zwischen dem Hochleistungsbeton und den XPS-Dämmplatten kann allein über eine thermisch-geprägte, rautenförmige Oberflächenstruktur erzielt werden. In Biegeversuchen an kurzen Sandwichstreifen wurde der Einfluss verschiedener Parameter auf das Tragverhalten und die Versagensarten der Sandwichelemente näher untersucht. Dabei konnten sieben verschiedene Versagensarten festgestellt werden. Generell wiesen die Probekörper ein duktiles Lastverformungsverhalten mit ausgeprägtem plastischen Verformungsvermögen auf.

In Scherversuchen wurde das Schertragverhalten des XPS sowie der Verbundfuge zwischen Beton und XPS bestimmt. Die Scherprobekörper mit Betondeckschichten versagten auf zwei unterschiedliche Arten. Bei der ersten Versagensart A trat ein Versagensriss in unmittelbarer Nähe zur Verbundfuge im XPS auf. Bei der zweiten Versagensart B löste sich aufgrund der schlechteren Qualität des Verbunds, das XPS vom Beton ab. Es konnte jedoch gezeigt werden, dass durch eine sorgfältige Herstellung der Elemente, Schubfestigkeiten der Verbundfuge erzielt werden können, die an die Scherfestigkeiten des ungestörten XPS heranreichen. Auch durch eine intensive Frost-Tau-Wechselbeanspruchung der Scherprobekörper wurde die Scherfestigkeit der Verbundfuge nur geringfügig abgemindert.

Zur weiteren Untersuchung der Dauerhaftigkeit wurden Ermüdungsversuchen mit zyklischer, positiver und negativer Momentenbeanspruchung durchgeführt. Anhand der Versuchsergebnisse wurde eine Wöhlerlinie ermittelt, mit der eine Ermüdungsbemessung mit den Lastkollektiven nach DIN EN 14509 und DIBt 3.93 bzw. DIBt 1995 erfolgte. Es wurde gezeigt, dass durch diese Lastkollektive kein vorzeitiges Versagen der Sandwichelemente herbeigeführt wird.

Neben der Ermittlung der Materialeigenschaften und der Untersuchung des Tragverhaltens wurden Versuche an großmaßstäblichen Sandwichelementen durchgeführt. In drei dieser Versuche wurden über zwei Felder durchlaufende Elemente in einem eigens konstruiertem Unterdruckprüfstand durch eine Flächenlast beansprucht. Auf diese Weise konnte das Tragverhalten unter realitätsnaher Belastung untersucht werden. Der experimentelle Teil dieser Arbeit umfasste weiterhin Dehnkörper- und Biegeversuche an bewehrten Betonstreifen zur Ermittlung des Spannungsdehnungsverhaltens des Betons, Dauerstandsversuche an Sandwichstreifen zur Ermittlung des Kriechver- 
haltens des XPS und die Herstellung von Probewänden zur Untersuchung der Herstellbarkeit und Dauerhaftigkeit von Elementen mit praxisrelevanten Abmessungen.

Neben den experimentellen Untersuchungen wurde das Tragverhalten der Sandwichelemente auch rechnerisch durch FE-Modellierungen untersucht. Dazu wurde die Software ANSYS Classic verwendet. Die Materialgesetze wurden anhand der Ergebnisse aus den Versuchen sowie auf Basis von Angaben aus der Literatur gewählt. Bei der Nachrechnung der Versuche zeigten die Berechnungen sehr gute Übereinstimmungen. Mithilfe der FE-Modellierungen wurden verschiedene Parameterstudien durchgeführt, mit denen neben dem generellen Lastverformungsverhalten auch die Spannungsverteilung über die Länge und den Querschnitt eines Sandwichelements untersucht wurden.

In den Parameterstudien wurde gezeigt, dass Fehlstellen im Verbund über die gesamte Breite des Probekörpers und einer Länge von weniger als $5 \mathrm{~cm}$ die Traglast nur geringfügig abmindern. Daher sollten bei der Herstellung der Elemente, Fehlstellen in der Verbundfuge durch optimierte Herstellungsmethoden auf einen mittleren Durchmesser von $50 \mathrm{~mm}$ begrenzt werden. Weiterhin konnte mit den FE-Modellierungen das Tragverhalten der Sandwichelemente unter einer Temperatur- und einer Schwindbeanspruchung der Deckschichten untersucht werden. Bei einfeldrig-gelagerten Sandwichelementen führen die entstehenden Zwangspannungen infolge einer Temperaturdifferenz der Deckschichten von $55^{\circ} \mathrm{K}$ nicht zum Reißen der Deckschichten. Die Zwangspannungen infolge einer Schwinddehnung der Deckschichten sind ebenfalls sehr gering und nahezu konstant über die Länge des Elements verteilt.

Zur Bemessung von Sandwichelementen mit gerissenen Betondeckschichten müssen die Schnittgrößen zielsicher ermittelt werden. Mit den vorhandenen Rechenverfahren auf Grundlage der Sandwichtheorie ist dies bisher nicht möglich gewesen. Daher wurde das bestehende Programm swe2 um einen Ansatz zur Berücksichtigung der Steifigkeitsänderung infolge der Rissbildung der Deckschichten umfangreich erweitert. Über einen iterativen Berechnungsansatz ist es nun möglich, mit dem neuen Programm swe2+ die Umlagerungen der Schnittgrößen infolge der Rissbildung zu berücksichtigen. Das Programm zeigt im Vergleich zu den Ergebnissen aus den Großbauteilversuchen und überschlägigen Nachrechnungen mithilfe von Diagrammen sehr gute Übereinstimmungen.

Durch das Reißen der Deckschichten lagern sich die Schnittgrößen über den Querschnitt und über die Länge eines Sandwichelements um, so dass ungünstigere Einwirkungskombinationen entstehen können. Reißt eine Deckschicht, reduziert sich deren Biegesteifigkeit. Das Biegemoment der Deckschicht wird dann auf die zweite Deckschicht umgelagert. Weiterhin zeigte sich in den Berechnungsbeispielen an einem Zweifeldelement, dass die Rissbildung und Steifigkeitsänderung zur Vergrößerung des Stützmoments führen kann.

Es wird vorgeschlagen, die Sandwichelemente in Anlehnung an DIN EN 14509 durch den Vergleich von Einwirkungen und Widerständen zu bemessen. Auf der Einwirkungsseite werden dabei die Lastfaktoren $\gamma_{f}$ und Beanspruchungen aus Eurocode 0 und 1 mit den entsprechenden nationalen Anhängen (DIN EN 1990, DIN EN 1991) sowie die Lastfallkombinationen, Kombinationskoeffizienten $\psi$ und Temperaturbeanspruchungen aus DIN EN 14509 übernommen. Auf der Widerstandsseite werden die charakteristi- 
schen Materialfestigkeiten und Materialfaktoren $\gamma_{M}$ auf Grundlage des Eurocodes 0 (DIN EN 1990) aus Versuchsergebnissen ermittelt. Mit dem Nachweiskonzept konnten die Versagensarten der Biegeversuche mit ausreichender Sicherheit und guter Übereinstimmung zielsicher nachgerechnet werden.

Die Materialfaktoren für die Scherfestigkeit des reinen XPS sowie für die Schubfestigkeit der Verbundfuge bei Versagensart $A$ liegen im Bereich von 1,14 bis 1,35. Jedoch konnten in den Versuchsreihen, bei denen die Versagensart B auftrat, höhere Streuungen festgestellt werden, so dass für den Nachweis gegen Versagen der Verbundfuge ein Materialfaktor $\gamma_{M}$ von 2,0 empfohlen wird. Generell sollten in Anlehnung an DIN EN $14509 \mathrm{im}$ Rahmen einer Werkseigenen Produktionskontrolle die Scherfestigkeiten produktionsbegleitend bestimmt und kontrolliert werden. Werden hierbei geringere Streuungen ermittelt, kann der Materialfaktor, wie in DIN EN 14509 empfohlen, auf $\gamma_{M}=1,5$ reduziert werden. Der Materialfaktor zum Nachweis gegen Versagen der Mikrobewehrung kann mit 1,15 angenommen werden. Der Materialfaktor für den Nachweis der Carbonfaser-Textilbewehrung sollte zu 1,5 gewählt werden.

Zum Nachweis gegen Rissbildung der Deckschichten infolge einer Schwindbeanspruchung wurden die entsprechenden Gleichungen hergeleitet. Die Ergebnisse der Bemessungsgleichungen liefern eine sehr gute Übereinstimmung mit den Ergebnissen der FE-Modellierungen. Eine Bildung von Schwindrissen ist besonders in den ersten Tagen nach der Herstellung der Elemente wahrscheinlich. Der Schwindbemessung müssen daher entsprechende Annahmen zur Festigkeit des Betons im jungen Alter zu Grunde gelegt werden.

Abschließend wurde an zwei Beispielrechnungen die Bemessung eines Wand- sowie eines Dachelements demonstriert. Für ein Einfeldelement mit einer Spannweite von $5 \mathrm{~m}$ können die Deckschichten im Grenzzustand der Gebrauchstauglichkeit rissefrei ausgeführt werden. Bei einem Zweifeld-Dachelement ist dies aufgrund der auftretenden Auflagerkräfte und resultierenden Zwangsspannnungen infolge einer Temperaturbeanspruchung nicht möglich. Jedoch können die Anforderungen hinsichtlich der Begrenzung der Verformungen erfüllt werden.

\subsection{Ausblick}

Anhand der Scherversuche mit vorheriger Frost-Tau-Wechselbeanspruchung sowie der Ermüdungsversuche mit einer zyklischen Wechselbeanspruchung wurde die Dauerhaftigkeit der Sandwichelemente bereits experimentell untersucht. Eine weitere Ursache für die Rissbildung der Deckschichten, die im Rahmen dieser Arbeit noch nicht experimentell untersucht wurde, ist ein plötzlicher Temperaturwechsel. Dieser Lastfall tritt beispielsweise bei der direkten Beregnung in einem Gewitter im Sommer auf. Hierzu sollte in einem dafür vorgesehenen Prüfstand in Anlehnung an DIN EN 14509 eine Prüfung mit plötzlichem Temperaturwechsel durchgeführt werden (siehe auch Abschnitt 3.3.4.9 siehe S. 41.

Zum Einsatz der Elemente in der Praxis sind weiterhin noch die konstruktiven Detailpunkte auszuarbeiten. Dazu gehören die Anschlusspunkte an die bestehende Tragkonstruktion sowie die Anschlusspunkte der Sandwichelemente untereinander. Dabei 
müssen die bauphysikalischen Anforderungen hinsichtlich des Wärme-, Feuchte und Brandschutzes erfüllt werden. Aufbauend auf den beschriebenen Tastversuchen, sollte in Feuerwiderstandsversuchen das Brandverhalten von großmaßstäblichen Sandwichelementen untersucht werden.

Die im Rahmen dieser Arbeit untersuchten Sandwichelemente mit dünnen Betondeckschichten bieten noch vielfältige weitere Einsatz- und Optimierungsmöglichkeiten. In weiteren Untersuchungen sollte die Einsatzmöglichkeit der Sandwichelemente als tragende Bauteile untersucht werden. Sandwich-Wandelemente könnten beispielsweise auch zum Abtrag von Vertikallasten herangezogen werden. Analog zu den Sandwichelementen mit metallischen Deckschichten könnten die Sandwichelemente mit Betondeckschichten auch zur Aussteifung des Tragwerks und zur Stabilisierung von Rahmen und Dachpfetten dienen.

Weitere Möglichkeiten zur Optimierung der Sandwichelemente bieten die Betondeckschichten. Durch den Einbau von Kapillarrohrmatten in den Deckschichten könnte die entstehende Wärme abgeführt und zur Klimatisierung des Gebäudes verwendet werden. Durch die Zugabe von Farbpigmenten könnten die Betonflächen dunkler eingefärbt und der Wirkungsgrad dieser Bauteilaktivierung vergrößert werden. Weiterhin bieten die Betondeckschichten auch Möglichkeiten zur Herstellung von freigeformten Sandwichelementen.

Eine weitere Einsatzmöglichkeit der Sandwichelemente wäre beispielsweise die Herstellung von Lärmschutzwänden mit gleichzeitiger Funktion als Ladungsrückhaltesystem. Die Betondeckschichten bieten sehr gute Möglichkeiten zur Oberflächengestaltung, während der Kern aus XPS als Deformationsschicht zur Aufnahme der Anprallenergie dienen könnte (vgl. Thorn 2013). Im Rahmen dieser Arbeit wurde auch der Einsatz der Sandwichelemente als selbstaufschwimmende Hochwasserschutzbarriere untersucht und erfolgreich erprobt (Müller u.a. 2013, Graf 2012). Auch hier sind die Vorteile der Sandwichelemente mit Betondeckschichten die vielseitigen Gestaltungsmöglichkeiten sowie die hohe Oberflächenqualität und Abriebfestigkeit. 


\section{Literaturverzeichnis}

\section{AGEB 2010}

AGEB ; ARBeitsgemeinschaft Energiebilanzen (Hrsg.): Auswertungstabellen zur Energiebilanz Deutschland: Daten für die Jahre 1990 bis 2009. http://www. ag-energiebilanzen.de. Version: 2010

Aicher u. Roth 1987

AICHER, Simon ; ROTH, Walter v.: Ein modifiziertes $\gamma$-Verfahren für das mechanische Analogon: dreischichtiger Sandwichverbund - zweiteiliger verschieblicher Verbund. In: Bautechnik (1987), Nr. 64, S. 21-29

Allen 1969

AlLen, Howard G.: Analysis and Design of Structural Sandwich Panels. Oxford : Pergamon Press, 1969

Antonatus 2010

ANTONATUS, Edith: Vortrag zum Projekttreffen: XPS und Brandverhalten - Prüfung und Klassifizierung: Sicherheit in verschiedenen Anwendungen. Ludwigshafen, 2010-03-22

Ashby 1983

AshBY, Michael F.: The Mechanical Properties of Cellular Solids. In: Metallurgical Transactions $A$ (1983), Nr. 14A, S. 1755-1769

BASF SE 2011a

BASF SE ; BASF SE (Hrsg.): Produktbroschüre: Styrodur@ HT - Die Dämmlösung mit Sonnenschutzfaktor. Ludwigshafen, 2011

\section{BASF SE 2011b}

BASF SE: Produktbroschüre: Europe's green insulation. http://www.styrodur.de Version: 2011-12

\section{Berner 1994}

BERNER, Klaus: Erarbeitung vollständiger Bemessungsgrundlagen im Rahmen bautechnischer Zulassungen für Sandwichbauteile. - Teil 1: Berechnungsgrundlagen in Form von Rechenhilfen für alle relevanten Lastfälle und statischen Systeme: Forschungsbericht. Stuttgart : IRB-Verlag, 1994

\section{Berner 1996}

BERNER, Klaus: Erarbeitung vollständiger Bemessungsgrundlagen im Rahmen bautechnischer Zulassungen für Sandwichbauteile. - Teil 2: Berücksichtigung zusätzlicher Beanspruchungen bei der Bemessung von durchlaufenden Sandwichplatten im Zwischenstützbereich: Forschungsbericht. Stuttgart : IRB-Verlag, 1996

\section{Berner 2009}

BERNER, Klaus: Selbsttragende und aussteifende Sandwichbauteile - Möglichkeiten 
für kleinere und mittlere Gebäude. In: Stahlbau 78 (2009), Nr. 5, S. 298-307

Berner 2012

BERNER, Klaus: Vortrag: Praxisgerechte Verwendung von Sandwichbauteilen für Dacheindeckungen und Wandverkleidungen. Kaiserslautern, 2012-09-26 (Weiterbildung für Tragwerksplaner)

Besch 2014

$\mathrm{BESCH}$, Daniel: Erweiterung der Software "swe2" zur Berechnung von Sandwichelementen mit Deckschichten aus Hochleistungsbeton. Kaiserslautern : TU Kaiserslautern, 2014 (Studienarbeit am Fachgebiet Massivbau und Baukonstruktion der TU Kaiserslautern betreut durch Müller, Frank)

Boy 2005

BoY, Elmar: EPS und XPS im Bauwesen: Eigenschaften und Anwendungen. Ludwigshafen : BASF Aktiengesellschaft, 2005

Chemiereport.at 2008

CHEMIEREPORT.AT: Panoramablick in den Mikrokosmos. http://www. chemiereport.at/panoramablick-den-mikrokosmos Version: 2008

Chopradub 2010

CHOPRADUB, Ake: Zur Tragfähigkeit von punktförmig gestützten filigranen Fassadenplatten aus faserverstärktem Feinkornbeton. Kaiserslautern : TU Kaiserslautern, 2010 (Dissertation)

Curbach 2003

CURBACH, Manfred (Hrsg.): Textile Reinforced Structures. Proceedings of the 2nd Colloquium on textile reinforced structures (CTRS2). Dresden : TU Dresden, 2003

Curbach u. Häußler-Combe 2010

CURBACH, Manfred ; HäUSSLER-COMBE, Ulrich: Jahresbericht 2010: Institut für Massivbau. Dresden : TU Dresden, 2010

Curbach u. Häußler-Combe 2011

CURBACH, Manfred ; HäUSSLER-COMBE, Ulrich: Jahresbericht 2011: Institut für Massivbau. Dresden : TU Dresden, 2011

Curbach $u$. Jesse 2009

CURBACH, Manfred (Hrsg.) ; Jesse, Frank (Hrsg.): Textilbeton - Theorie und Praxis: Tagungsband zum 4. Kolloquium zu textilbewehrten Tragwerken (CTRS4) und zur 1. Anwendertagung. Dresden : TU Dresden, 2009

Di Prisco u. Zani 2012

DI PRISCO, Marco ; ZANI, Giulio: Experimental and numerical analysis of advanced cementitious composites for sustainable roof elements. In: SSCS (Hrsg.): SSCS 2012, 2012

DIBt 1995

DIBT: Grundsätze zur Ermittlung der Temperaturbeanspruchung mehrschichtiger Wandtafeln mit Betondeckschicht. In: DIBt Mitteilungen (1995), Nr. 5, S. 148 


\section{DIBt 2008}

DIBT: Allgemeine bauausichtliche Zulassung Z-15.2-144: TM-Verbundsystem für dreischichtige Stahlbeton-Wandtafeln. Berlin, 2008

\section{DIBt 2014}

DIBT: Allgemeine bauaufsichtliche Zulassung Z-23.5-223: Extrudergeschäumte Polystyrol-Hartschaumplatten "Styrodur 3035 CS", "Styrodur 4000 CS" und "Styrodur 5000 CS" für die Anwendung im Wärmedämmsystem als Perimeterdämmung. Berlin, 2014

\section{DIBt 3.93}

DIBT 3.93: Prüfprogramm für Sandwichkonstruktionen mit einem Stützkern aus Polyurethan (PUR)-Hartschaum frei von bestimmten die Ozonschicht abbauenden Halogenkohlenwasserstoffen zwischen Metalldeckschichten im Zulassungsverfahren: Fassung 3.93

DIN 1045-2 2008

DIN 1045-2: Tragwerke aus Beton, Stahlbeton und Spannbeton - Teil 2: Beton - Festlegung, Eigenschaften, Herstellung und Konformität - Anwendungsregeln zu DIN EN 206-1. 2008-08

DIN 18234-1 2003

DIN 18234-1: Baulicher Brandschutz großflächiger Dächer; Brandbeanspruchung von unten - Teil 1: Begriffe, Anforderungen und Prüfungen; geschlossene Dachflächen. 2003-09

DIN 41021977

DIN 4102: Brandverhalten von Baustoffen und Bauteilen - Teil 1 bis 22. 1977-09 bis 2014-09

DIN EN 120871997

DIN EN 12087: Wärmedämmstoffe für das Bauwesen. Bestimmung der Wasseraufnahme bei langzeitigem Eintauchen. 1997-08

DIN EN 120881997

DIN EN 12088: Wärmedämmstoffe für das Bauwesen. Bestimmung der Wasseraufnahme durch Diffusion. 1997-08

DIN EN 120901997

DIN EN 12090: Wärmedämmstoffe für das Bauwesen. Bestimmung des Verhaltens bei Scherbeanspruchung. 1997-08

DIN EN 120911997

DIN EN 12091: Wärmedämmstoffe für das Bauwesen. Bestimmung des Verhaltens bei Frost-Tau-Wechselbeanspruchung. 1997-08

DIN EN 123502009

DIN EN 12350: Prüfung von Frischbeton - Teil 1 bis 12: DIN EN 12350. 2009-08 bis $2010-12$

DIN EN 123902000

DIN EN 12390: Prüfung von Festbeton - Teil 1 bis 13: DIN EN 12390. 2000-12 bis 2014-06 
DIN EN 12390-2 2009

DIN EN 12390-2: Prüfung von Festbeton - Teil 2: Herstellung und Lagerung von Probekörpern für Festigkeitsprüfungen. 2009-08

DIN EN 12390-2 Ber 12012

DIN EN 12390-2 BER 1: Prüfung von Festbeton - Teil 2: Herstellung und Lagerung von Probekörpern für Festigkeitsprüfungen. Berichtigung zu DIN EN 12390-2:200908. 2012-02

DIN EN 135012010

DIN EN 13501: Klassifizierung von Bauprodukten und Bauarten zu ihrem Brandverhalten - Teil 1 bis 6. 2010-01 bis 2014-07

DIN EN 13632012

DIN EN 1363: Feuerwiderstandsprüfungen -Teil 1 und 2. 2012-10 und 1999-10

DIN EN 1364-1 2011

DIN EN 1364-1: Feuerwiderstandsprüfungen für nichttragende Bauteile; Wände. 2011-11

DIN EN 145092013

DIN EN 14509: Selbsttragende Sandwich-Elemente mit beidseitigen Metalldeckschichten - Werkmäßig hergestellte Produkte - Spezifikationen. 2013-02

DIN EN 16052007

DIN EN 1605: Wärmedämmstoffe für das Bauwesen. Bestimmung der Verformung bei definierter Druck- und Temperaturbeanspruchung. 2007-06

DIN EN 16062007

DIN EN 1606: Wärmedämmstoffe für das Bauwesen. Bestimmung des LangzeitKriechverhaltens bei Druckbeanspruchung. 2007-06

DIN EN 16071997

DIN EN 1607: Wärmedämmstoffe für das Bauwesen. Bestimmung der Zugfestigkeit senkrecht zur Plattenebene. 1997-01

DIN EN 16081997

DIN EN 1608: Wärmedämmstoffe für das Bauwesen. Bestimmung der Zugfestigkeit in Plattenebene. 1997-01

DIN EN 19902010

DIN EN 1990: Eurocode: Grundlagen der Tragwerksplanung. 2010-12

DIN EN 1990/NA 2010

DIN EN 1990/NA: Nationaler Anhang - National festgelegte Parameter - Eurocode: Grundlagen der Tragwerksplanung. 2010-12

DIN EN 19912010

DIN EN 1991: Eurocode 1: Einwirkungen auf Tragwerke. 2010-12

DIN EN 1991/NA 2010

DIN EN 1991/NA: Nationaler Anhang - National festgelegte Parameter - Eurocode

1: Einwirkungen auf Tragwerke. 2010-12 
DIN EN 19922011

DIN EN 1992: Eurocode 2: Bemessung und Konstruktion von Stahlbeton- und Spannbetontragwerken. 2011-01

DIN EN 1992/NA 2011

DIN EN 1992/NA: Nationaler Anhang - National festgelegte Parameter - Eurocode

2: Bemessung und Konstruktion von Stahlbeton- und Spannbetontragwerken. 201101

DIN EN 8261996

DIN EN 826: Wärmedämmstoffe für das Bauwesen. Bestimmung des Verhaltens bei Druckbeanspruchung. 1996-05

DIN ISO 16269-6 2009

DIN ISO 16269-6: Statistische Auswertung von Daten - Teil 6: Ermittlung von statistischen Anteilsbereichen. 2009-10

DUCON 2010

DUCON: Prüfergebnisse von Zugversuchen an normal- und hochfestem Stahldraht mit einem Durchmesser von $1 \mathrm{~mm} .2010$

EU 2002

EU: Richtlinie 2002/91/EG des Europäischen Parlaments und des Rates vom 16. Dezember 2002 über die Gesamtenergieeffizienz von Gebäuden: Richtlinie 2002/91/EG. 2002

\section{Findley 1944}

FINDLEY, William N.: Creep Characteristics of Plastics. In: AMERICAN SOCIETY FOR Testing ANd MATERIALS (Hrsg.): Symposium on Plastics, 1944

Finzel u. Häußler-Combe 2010

FINZEL, Joachim ; HÄUSSLER-COMBE, Ulrich: Textile reinforced concrete sandwich panels. In: BIĆANIĆ, Nenad (Hrsg.) ; BORST, René de (Hrsg.) ; MANG, Herbert (Hrsg.) ; MESCHKE, Günther (Hrsg.): Computational Modelling of Concrete Structures. CRC Press, 2010, S. 789-795

Fontana 2013

FONTANA, Patrick: Poster: Frühes autogenes Schwinden von Hochleistungsbeton (HPC). http://www . bam.de/de/kompetenzen/fachabteilungen/abteilung_7/ fg71/fg71_poster.htm. Version: 2013

\section{Fouad 2006}

FouAD, Nabil A.: Bauphysik Kalender 2006: Brandschutz. Berlin : Ernst \& Sohn, 2006

\section{Fouad 2008}

Fouad, Nabil A. ; Leibniz Universität Hannover (Hrsg.): Prüfbericht: Dämmstoffplatten aus extrudiertem Polystyrol-Hartschaumstoff (XPS) Ermittlung des Langzeitragverhaltens unter Schub- und kombinierter Schub-Druck-Beanspruchung: Abschlussprüfbericht. Hannover, 2008

\section{Fouad 2010a}

FouAD, Nabil A.: Bauphysik Kalender 2010: Energetische Sanierung von Gebäuden. 
Berlin : Ernst \& Sohn, 2010

\section{Fouad $2010 b$}

Fouad, Nabil A. ; Leibniz Universität Hannover (Hrsg.): Prüfbericht: Dämmstoffplatten aus extrudiertem Polystyrol-Hartschaumstoff (XPS) Ermittlung des Kurzzeittragverhaltens unter Schubbeanspruchung. Hannover, 2010

\section{FPX 2012}

FPX ; FPX FachVEREINIGUNG POLYSTYROL-EXtRuderschaumstoff (Hrsg.): XPS-Dämmstoffe: Was ist XPS? http://www.fpx-daemmstoffe.de/was-ist-xps . html Version: 2012

\section{FPX 2013}

FPX ; FPX FachVereinigung POlystyrol-Extruderschaumstoff (Hrsg.): XPS und EPS: Das $X$ macht den Unterschied. http://www .xps-waermedaemmung. de/was-ist-xps/unterschied-xps-zu-eps/ Version: 2013

\section{FSF 2007}

FSF ; Free Software Foundation, Inc (Hrsg.): GNU General Public License (GPL). http://www.gnu.org/licenses/gpl.html. Version: 2007

Gausepohl u. Gellert 1996

Gausepohl, Hermann ; GelleRt, Roland: Kunststoff Handbuch 4: Polystyrol. München : Hanser, 1996

Gibson u. Ashby 2001

GIBSON, Lorna J. ; ASHBY, Michael F.: Cellular Solids: Structure and Properties. Second Edition. Cambridge : Cambridge University Press, 2001

\section{Graf 2012}

GRAF, Christian: Selbstaufschwimmende Hochwasserschutzbarriere als Sandwichelement mit Deckschichten aus Hochleistungsbeton. Kaiserslautern : TU Kaiserslautern, 2012 (Studienarbeit am Fachgebiet Massivbau und Baukonstruktion der TU Kaiserslautern betreut durch Müller, Frank)

\section{Hauser 1999}

HAUSER, Stephan: Hochfester Fasermattenbeton. SIMCON neu / DUCON®. Darmstadt : TU Darmstadt, 1999 (Dissertation)

\section{Hauser $u$. Wörner 1999}

HAUSER, Stephan ; WÖRNER, Johann D.: DUCON, ein innovativer Hochleistungsbeton. In: Beton- und Stahlbetonbau (1999), Nr. 2 + 3, S. 66-75; 141-145

\section{Heese 2014}

HEESE, Christian: Simulation des rheologischen Verhaltens von zementgebundenen Feinkornsystemen. Kaiserslautern : TU Kaiserslautern, 2014 (Dissertation)

\section{Hegger 2001}

HEGGER, Josef (Hrsg.): Textilbeton - 1. Fachkolloquium der Sonderforschungsbereiche 528 und 532. Aachen : RWTH Aachen, 2001

Hegger u. Bornefeld 2012

HEGger, Josef (Hrsg.) ; Bornefeld, Gero (Hrsg.): Abschlussbericht des Son- 
derforschungsbereichs 532 "Textilbewehrter Beton - Grundlagen für die Entwicklung einer neuartigen Technologie". RWTH Aachen, 2012

Hegger u. a. 2006

Hegger, Josef (Hrsg.) ; Brameshuber, Wolfgang (Hrsg.) ; WILL, Norbert (Hrsg.): Textile Reinforced Concrete: RILEM Proceedings pro050. Bagneux : RILEM Publications, 2006

Hegger u. a. 2007

Hegger, Josef ; Horstmann, Michael ; Voss, Stefan ; WILL, Norbert: Textilbewehrter Beton: Tragverhalten, Bemessung und Anwendung. In: Beton- und Stahlbetonbau 102 (2007), Nr. 6, S. 362-370

Hegger u. a. 2009

Hegger, Josef ; WiLl, Norbert ; HorstmanN, Michael: Ergebnisbericht Projekt INSUSHELL: Selbsttragende Sandwichfassade aus Textilbeton und PURHartschaumkernen für den INNOTEX-Neubau des Instituts für Textiltechnik. Aachen : RWTH Aachen, 2009

Hill 1983

HILL, Rodney: The Mathematical Theory of Plasticity. New York : Oxford University Press, 1983

\section{Holmberg u. Plem 1965}

HolmbeRG, Åke ; PlEM, Erik: Behaviour of load-bearing sandwich-type structures.

Stockholm : Statens Institut för Byggnadsforskning, 1965

\section{Horstmann 2010}

HoRstmanN, Michael: Zum Tragverhalten von Sandwichkonstruktionen aus textilbewehrtem Beton. Aachen : RWTH Aachen, 2010 (Dissertation)

IndBauRL 2000

INDBAURL: Richtlinie über den baulichen Brandschutz im Industriebau (Industriebaurichtlinie). 2000

iS-engineering $\mathrm{GmbH} 2007$

IS-ENGINEERING GmBH: SandStat - Programm zur Berechnung von Sandwichbauteilen: Benutzer-Handbuch. 2007

Jesse 2004

JESSE, Frank: Tragverhalten von Filamentgarnen in zementgebundender Matrix. Dresden : TU Dresden, 2004 (Dissertation)

Jesse u. Curbach 2010

JesSE, Frank ; CURBACH, Manfred: Verstärken mit Textilbeton. In: BERGMEISTER, Konrad (Hrsg.) ; FINGERLOOS, Frank (Hrsg.) ; WÖRNER, Johann D. (Hrsg.): Beton Kalender 2010. Berlin : Ernst \& Sohn, 2010, S. 457-565

Joas $u$. Weber 2012

JOAS, Tim ; WEBER, Michael: Experimentelle und rechnerische Untersuchung des Lastverformungsverhaltens von dünnwandigen Bauteilen aus mikro - und textilbewehrtem Hochleistungsbeton unter Zug- und Biegebeanspruchung. Kaiserslautern : TU Kaiserslautern, 2012 (Studienarbeit am Fachgebiet Massivbau und Baukonstruk- 
tion der TU Kaiserslautern betreut durch Müller, Frank)

Jungbluth u. Berner 1986

JUNGBLUth, Otto ; BERNER, Klaus: Verbund- und Sandwichtragwerke. Tragverhalten, Feuerwiderstand, Bauphysik. Berlin : Springer, 1986

Käpplein u. Ummenhofer 2010

KÄPPLEIN, Saskia ; UMmEnHOFER, Thomas: Axial beanspruchte Sandwichelemente in rahmenlosen Konstruktionen. In: Stahlbau 79 (2010), Nr. 10, S. 761-770

Keil u. Schmitt 2010

KEIL, Christian ; SchmiTt, Andreas: Statische und konstruktive Bearbeitung eines Weinberghauses aus Hochleistungsbeton. Kaiserslautern : TU Kaiserslautern, 2010 (Studienarbeit am Fachgebiet Massivbau und Baukonstruktion der TU Kaiserslautern betreut durch Müller, Frank und Kohlmeyer, Christian)

\section{Kohlmeyer 2011}

KOHLMEYER, Christian: Beton - Werkstoff für Höchstleistungen: Eine neue Betongeneration eröffnet eine Vielfalt von Möglichkeiten. In: mb-news (2011), Nr. 1/2011, S. $6-10$

König u. a. 2001

KÖNIG, Gert ; TUE, Nguyen V. ; ZINK, Martin: Hochleistungsbeton: Bemessung, Herstellung und Anwendung. Berlin : Ernst \& Sohn, 2001

\section{Krollmann 1995}

KROLLMANN, Norbert: Langzeitverhlten von extrudiertem Polystyrol-Hartschaum bei konstanter wechselnder Druckbeanspruchung. In: Bauphysik (1995), Nr. 1, S. 11-16

Kulkarni u. a. 2004

KULKARNI, Nitin ; MAHFUZ, Hassan ; JEELANI, Shaik: Fatigue failure mechanism and crack growth in foam core sandwich composites under flexural loading. In: Journal of Reinforced Plastics and Composites (2004), Nr. 23

Lange u. a. 2011

LANGE, Jörg ; BERNER, Klaus ; HöRnEL-METZGER, Beate: Wandscheibentragfähigkeit von Sandwichelementen. In: Stahlbau 80 (2011), Nr. 9, S. 673-677

MBO 2002

MBO: Musterbauordnung. 2002

Molter 2005

MolTER, Matthias: Zum Tragverhalten von textilbewehrtem Beton. Aachen : RWTH Aachen, 2005 (Dissertation)

Müller 2009

MüLLER, Frank: Statische und konstruktive Bearbeitung eines Weinberghauses aus Hochleistungsbeton. Kaiserslautern : TU Kaiserslautern, 2009 (Diplomarbeit am Fachgebiet Massivbau und Baukonstruktion der TU Kaiserslautern sowie an der FH Mainz betreut durch Kohlmeyer, Christian und Kühlen, Rolf)

Müller u. a. 2013

MülleR, Frank ; KoHLmeYeR, Christian ; SchnelL, Jürgen: Self-Erecting Sandwich 
Flood Barrier with Facings of High Performance Concrete. In: The Indian Concrete Journal (ICJ) 87 (2013), Nr. 11, S. 11-16

MVkVO 1995

MVkVO: Musterverordnung über den Bau und betrieb von Verkaufsstätten (MusterVerkaufsstättenverordnung). 1995

MVStättV 2005

MVSTÄTTV: Musterverordnung über den Bau und Betrieb von Versammlungsstätten (Muster-Versammlungsstättenverordnung). 2005

\section{Ngoga 2014}

NGoGA, Fred T.: Finite-Elemente-Berechnungen von Sandwichelementen mit Deckschichten aus Hochleistungsbeton. Kaiserslautern : TU Kaiserslautern, 2014 (Studienarbeit am Fachgebiet Massivbau und Baukonstruktion der TU Kaiserslautern betreut durch Müller, Frank)

NOVACRET 2000a

NOVACRET: Produktdatenblatt cretex® AR310S-800. www.novacret.com Version: 2000

\section{NOVACRET 2000b}

NOVACRET: Produktdatenblatt cretex® AR620S-800. www.novacret.com Version: 2000

NOVACRET 2006

NOVACRET: Produktdatenblatt cretex® M124-10. Bernkastel-Kues, 2006

NOVACRET 2012

NOVACRET: Eigenschaften der cretex® NEG AR-Glasfasern. www novacret .com Version: 2012

\section{Oster 2012}

OSTER, Sebastian: Adhesive Joints for Structural Elements of High Performance Concrete (HPC). In: KARLSRUHE INSTITUTE OF TECHNOLOGY (KIT) (Hrsg.): 9th fib International PhD Symposium in Civil Engineering, 2012, S. 335-340

\section{Pahn 2011}

PAHN, Matthias: Beitrag zur Ermittlung von Schnitt- und Verformungsgrößen bei mehrschichtigen Stahlbetonwandtafeln mit Verbindungsmitteln aus glasfaserverstärktem Kunststoff. Kaiserslautern : TU Kaiserslautern, 2011 (Dissertation)

Pirskawetz u. a. 2011

PIRSKaWetZ, Stephan ; WeIse, Frank ; FontANA, Patrick: Zwangsinduzierte Rissbildung bei erhärtendem Hochleistungsbeton. In: ROGGE, Andreas (Hrsg.) ; MENG, Birgit (Hrsg.): DAfStb-Forschungskolloquium Innovative Forschung für den Betonbau. Berlin : BAM Bundesanstalt für Materialforschung und -prüfung, 2011, S. 126133

Pistol u. a. 2011

PISTOL, Klaus ; Weise, Frank ; MenG, Birgit: Zur Wirkungsweise von PolypropylenFasern in brandbeanspruchten Hochleistungsbetonen. In: RoGGE, Andreas (Hrsg.) ; MENG, Birgit (Hrsg.): DAfStb-Forschungskolloquium Innovative Forschung für den 
Betonbau. Berlin : BAM Bundesanstalt für Materialforschung und -prüfung, 2011, S. 134-140

Pöter 2009

PÖTER, Hans: Metalleichtbaukonstruktionen: Früher und heute. In: Stahlbau 78 (2009), Nr. 5, S. 288-297

Raabe 2003

RAABE, Oliver: Schnittgrößenermittlung bei Sandwichbauteilen mit Hilfe von MSEXCEL® - 1. Teil. http://sandwich.fh-mainz.de/service.html Version: 2003

\section{Ramm 2012}

Ramm, Wieland: Über die Anfänge des Eisenbetonbaus in Deutschland und die Pioniere der ersten Jahre. In: Beton- und Stahlbetonbau (2012), Nr. 5, S. 335-356

\section{Rinde 1970}

RINDE, J. A.: Poisson's Ratio for Rigid Plastic Foams. In: Journal of Applied Polymer Science (1970), Nr. 14, S. 1913-1926

\section{Schachinger 2007}

SCHACHINGER, Axel I.: Maßnahmen zur Herstellung von rissefreien Bauteilen aus ultrahochfestem Beton mit hoher Duktilität. München : TU München, 2007 (Dissertation)

\section{SchießI 1989}

SCHIESSL, Peter: Grundlagen der Neuregelung zur Beschränkung der Rißbreite. In: Deutscher Ausschuss für StAHLBeton (Hrsg.): Heft 400. Erläuterungen zu DIN 1045. Berlin : Beuth, 1989, S. 157-175

\section{Schmidt 2008}

SCHMIDT, Michael: Sachstandsbericht Ultrahochfester Beton. 1. Berlin : Beuth, 2008 (Deutscher Ausschuss für Stahlbeton)

Schneider u. a. 2009

SCHNEIDER, Hartwig N. ; SCHÄTZKE, Christian ; FEgER, Christiane ; HoRstmanN, Michael ; PAK, Daniel: Modulare Bausysteme aus Textilbeton-Sandwichelementen. In: CURBACH, Manfred (Hrsg.) ; JesSE, Frank (Hrsg.): Textile Reinforced Structures, 2009

\section{Schnell 2011}

SCHNELL, Jürgen (Hrsg.): Bauen im Bestand: Grundlagen für die Tragwerksplanung im Bestand: Skript. 2011

\section{Schnell 2013}

SCHNELL, Jürgen: Vorlesungsskript. Beton- und Spannbetonbau 5. Kaiserslautern : TU Kaiserslautern, 2013

\section{Schnell u. a. 2010}

SCHNELL, Jürgen ; SCHLADITZ, Katja ; SCHULER, Frank: Richtungsanalyse von Fasern in Betonen auf Basis der Computer-Tomographie. In: Beton- und Stahlbetonbau (2010), Nr. 2/2010, S. 72-77 
Schnellenbach-Held u. Prager 2011

SCHNellenbaCH-Held, Martina ; Prager, Melanie: Mikrobewehrter Ultrahochleistungsbeton: Trag- und Verformungsverhalten. In: Beton- und Stahlbetonbau (2011), Nr. 11, S. $770-778$

\section{Schwarze 1984}

SCHWARZE, Knut: Numerische Methoden zur Berechnung von Sandwichelementen.

In: Stahlbau (1984), Nr. 12/1984, S. 363-370

SGL Carbon 2012a

SGL CARBON: Composite Materials: Verstärkungsmaterialien aus Carbonfasern für die Bauindustrie. http://www.sglgroup.com/cms/international/products/ product-groups/cm/textile-products/index.html?__locale=de. Version: 2012

SGL Carbon 2012b

SGL CARBON: SIGRAFIL® C: Die Carbonfaser auf der Basis von PAN. http://www.sglgroup.com/cms/international/products/product-groups/cf/ carbon-fibers-for-intake-fittings/index.html?__locale=de Version: 2012

Shaw u. Sata 1966

ShaW, M. C. ; SATA, T.: The Plastic Behavior of Cellular Materials. In: International Journal of Mechanical Sciences (1966), Nr. 7, S. 469-472

Shih $u$. Lee 1978

SHIH, C. F. ; LEE, D.: Further Developments in Anisotropic Plasticity. In: Journal of Engineering Materials and Technology (1978), Nr. 100, S. 294-302

Stamm u. Witte 1974

Stamm, Klaus ; WitTE, Horst: Sandwichkonstruktionen: Berechnung, Fertigung, Ausführung. Wien : Springer-Verlag, 1974

Störi 1979

STÖRI, Fritz: Der Stoff aus dem Schäume sind: Die Geschichte vom Styropor . Ludwigshafen : BASF Aktiengesellschaft, 1979

Teil 2 der LTB 2012

TEIL 2 DER LTB: Teil 2 der Liste der Technischen Baubestimmungen. Anwendungsregelungen für Bauprodukte und Bausätze nach europäischen technischen Zulassungen und harmonisierten Normen nach der Bauproduktenrichtlinie - Ausgabe September 2011. Ausgabe 2. 2012-04

\section{Thorn 2013}

THORN, Kay-Uwe: Neuentwicklung eines Ladungsrückhaltesystems aus textilbewehrten Betonfertigteilen. Darmstadt : TU Darmstadt, 2013 (Dissertation)

\section{Urbanek 2011}

URBANEK, Dirk ; ZBN CIVIL ENGINEers (Hrsg.): swe2: Dokumentation. http: //www.swe2.de/. Version:2011

Voss 2008

Voss, Stefan: Ingenieurmodelle zum Tragverhalten von textilbewehrtem Beton. Aachen : RWTH Aachen, 2008 (Dissertation) 


\section{Wölfel 1987}

WöLFEL, Eilhard: Nachgiebiger Verbund: Eine Näherungslösung und deren Anwendungsmöglichkeiten. In: Stahlbau (1987), Nr. 6/1987, S. 173-180 


\section{A Anhang „Experimentelle Untersuchungen“}

\section{A.1 Zusammenstellung zu den Biegeversuchen an Sandwichstreifen}

Tab. A.1: Eigenschaften der Biegeprobekörper

\begin{tabular}{|c|c|c|c|c|c|c|c|c|}
\hline Probekörper & $\begin{array}{c}\text { Abr } \\
\boldsymbol{d}_{s} \\
{[\mathrm{~mm}]}\end{array}$ & $\begin{array}{c}\text { eessun } \\
d_{o} \\
{[\mathrm{~mm}]}\end{array}$ & $\begin{array}{c}\text { gen } \\
\boldsymbol{d}_{u} \\
{[\mathrm{~mm}]}\end{array}$ & Typ & $\begin{array}{c}\boldsymbol{f}_{c m, \text { cube }} \\
{\left[\mathrm{N} / \mathrm{mm}^{2}\right]}\end{array}$ & $\begin{array}{c}\text { Beton }{ }^{1} \\
E_{c m} \\
{\left[\mathrm{~N} / \mathrm{mm}^{2}\right]}\end{array}$ & $\begin{array}{c}\boldsymbol{f}_{c t m, f l} \\
{\left[\mathrm{~N} / \mathrm{mm}^{2}\right]}\end{array}$ & $\begin{array}{c}\boldsymbol{f}_{c t m, s p} \\
{\left[\mathrm{~N} / \mathrm{mm}^{2}\right]}\end{array}$ \\
\hline $\begin{array}{l}\text { Herstellungsmethode: } \\
\text { S.X0.S-10.120.10-1 } \\
\text { S.X0.S-10.120.10-2 } \\
\text { S.XO.S-10.120.10-3 } \\
\text { S.X0.S-10.120.10-4 } \\
\text { S.XO.S-10.120.10-5 } \\
\text { S.XO.S-10.120.10-6 } \\
\text { S.X0.S-10.120.10-7 } \\
\text { S.XO.S-10.120.10-8 } \\
\text { S.XO.S-10.120.10-9 }\end{array}$ & $\begin{array}{l}\text { n.v. } \\
\text { n.v. } \\
\text { n.v. } \\
\text { n.v. } \\
\text { n.v. } \\
\text { n.v. } \\
\text { n.v. } \\
\text { n.v. } \\
\text { n.v. }\end{array}$ & $\begin{array}{c}10,2 \\
10,5 \\
9,8 \\
10,3 \\
10,2 \\
9,8 \\
11,3 \\
8,7 \\
10,0\end{array}$ & $\begin{array}{c}10,2 \\
10,7 \\
10,0 \\
10,2 \\
10,5 \\
9,8 \\
9,8 \\
10,5 \\
9,5\end{array}$ & $\mathrm{HPC}-1^{*}$ & 133,7 & 36.160 & n.v. & 3,7 \\
\hline $\begin{array}{l}\text { Bewehrungsart: } \\
\text { G.X1.G-10.120.10-1 } \\
\text { G.X1.G-10.120.10-2 } \\
\text { G.X1.G-10.120.10-3 } \\
\text { G.X1.G-10.120.10-4 } \\
\text { C.X1.C-10.120.10-1 } \\
\text { C.X1.C-10.120.10-2 } \\
\text { C.X1.C-10.120.10-3 } \\
\text { C.X1.C-10.120.10-4 } \\
\text { S.X1.S-10.120.10-1 } \\
\text { S.X1.S-10.120.10-2 } \\
\text { S.X1.S-10.120.10-3 }\end{array}$ & $\begin{array}{l}120 \\
120 \\
120 \\
120 \\
120 \\
120 \\
120 \\
120 \\
120 \\
120 \\
120 \\
\end{array}$ & $\begin{array}{c}9,7 \\
8,8 \\
11,5 \\
11,8 \\
11,8 \\
11,1 \\
10,3 \\
10,5 \\
12,5 \\
12,6 \\
14,1\end{array}$ & $\begin{array}{c}6,4 \\
9,9 \\
10,4 \\
9,6 \\
10,7 \\
10,6 \\
9,9 \\
9,9 \\
11,8 \\
12,8 \\
12,3 \\
\end{array}$ & HPC-1 & 121,6 & 34.865 & 6,9 & 3,1 \\
\hline $\begin{array}{l}\text { XPS-Dichte: } \\
\text { S.X2.S-10.120.10-1 } \\
\text { S.X2.S-10.120.10-2 } \\
\text { S.X2.S-10.120.10-3 } \\
\text { S.X2.S-10.120.10-4 } \\
\text { S.X2.S-10.120.10-5 } \\
\text { S.X3.S-10.120.10-1 } \\
\text { S.X3.S-10.120.10-2 } \\
\text { S.X3.S-10.120.10-3 }\end{array}$ & $\begin{array}{l}120 \\
120 \\
120 \\
120 \\
120 \\
119 \\
119 \\
118\end{array}$ & $\begin{array}{c}12,3 \\
11,9 \\
10,9 \\
9,9 \\
11,9 \\
11,1 \\
11,4 \\
11,9\end{array}$ & $\begin{array}{c}11,5 \\
10,4 \\
10,7 \\
9,8 \\
10,2 \\
10,7 \\
10,6 \\
10,6\end{array}$ & HPC-1 & 126,8 & 35.505 & 13,8 & 3,4 \\
\hline $\begin{array}{l}\text { C.X2.C-10.120.10-1 } \\
\text { C.X2.C-10.120.10-2 } \\
\text { C.X2.C-10.120.10-3 } \\
\text { C.X3.C-10.120.10-1 } \\
\text { C.X3.C-10.120.10-2 } \\
\text { C.X3.C-10.120.10-3 }\end{array}$ & $\begin{array}{l}120 \\
120 \\
120 \\
116 \\
116 \\
116 \\
\end{array}$ & $\begin{array}{l}10,7 \\
10,9 \\
11,0 \\
10,3 \\
11,1 \\
10,0 \\
\end{array}$ & $\begin{array}{l}10,3 \\
10,7 \\
10,6 \\
10,6 \\
10,6 \\
10,2 \\
\end{array}$ & HPC-2 & 145,4 & 40.460 & 11,9 & 5,2 \\
\hline
\end{tabular}

${ }^{1}$ Mittelwerte aus den verschiedenen Mischungen einer Prüfserie

$\mathrm{HPC}-1^{\star}=$ Fehler beim Abwiegen der Ausgangsstoffe 
Tab. A.2: Eigenschaften der Biegeprobekörper (Fortsetzung)

\begin{tabular}{|c|c|c|c|c|c|c|c|c|}
\hline Probekörper & $\begin{array}{c}\text { Abr } \\
\boldsymbol{d}_{s} \\
{[\mathrm{~mm}]}\end{array}$ & $\begin{array}{c}\text { iessun } \\
d_{o} \\
{[\mathrm{~mm}]}\end{array}$ & $\begin{array}{l}\text { gen } \\
\boldsymbol{d}_{u} \\
{[\mathrm{~mm}]}\end{array}$ & Typ & $\begin{array}{c}\boldsymbol{f}_{c m . c u b e} \\
{\left[\mathrm{~N} / \mathrm{mm}^{2}\right]}\end{array}$ & $\begin{array}{c}\text { Beton }^{1} \\
E_{c m} \\
{\left[\mathrm{~N} / \mathrm{mm}^{2}\right]}\end{array}$ & $\begin{array}{c}\boldsymbol{f}_{c t m, f l} \\
{\left[\mathrm{~N} / \mathrm{mm}^{2}\right]}\end{array}$ & $\begin{array}{c}\boldsymbol{f}_{c t m . s p} \\
{\left[\mathrm{~N} / \mathrm{mm}^{2}\right]}\end{array}$ \\
\hline \multicolumn{9}{|l|}{ Dicke der Lagen: } \\
\hline S.X4.S-10.140.10-1 & 140 & 11,7 & 10,4 & \multirow{9}{*}{ HPC-1 } & \multirow{9}{*}{122,5} & \multirow{9}{*}{35.420} & \multirow{9}{*}{10,7} & \multirow{9}{*}{3,8} \\
\hline S.X4.S $-10.140 .10-2$ & 140 & 10,7 & 11,8 & & & & & \\
\hline S.X4.S-10.140.10-3 & 140 & 11,0 & 11,6 & & & & & \\
\hline S.X5.S-15.120.15-1 & 120 & 16,8 & 16,9 & & & & & \\
\hline S.X5.S-15.120.15-2 & 120 & 16,7 & 16,1 & & & & & \\
\hline S.X5.S-15.120.15-3 & 120 & 16,2 & 15,6 & & & & & \\
\hline S.X5.S-20.120.20-1 & 120 & 19,4 & 17,7 & & & & & \\
\hline S.X5.S-20.120.20-2 & 120 & 20,1 & 21,0 & & & & & \\
\hline S.X5.S-20.120.20-3 & 120 & 20,3 & 20,2 & & & & & \\
\hline \multicolumn{9}{|l|}{ Stoßausbildung: } \\
\hline S.X5.S-10.120.10-1 & 120 & 12,6 & 11,7 & & & & & \\
\hline S.X5.S-10.120.10-2 & 120 & 11,2 & 11,4 & HPC-2 & 142,9 & 42.710 & 9,5 & 4,1 \\
\hline S.X5.S-10.120.10-3 & 120 & 10,8 & 10,7 & & & & & \\
\hline
\end{tabular}

Mittelwerte aus den verschiedenen Mischungen einer Prüfserie

\section{A.1.1 Zusammenstellung der aufgeschnittenen Biegeprobekörper}

\section{A.1.1.1 Biegeprobekörper der Versuchsreihe „Herstellungsmethode“}

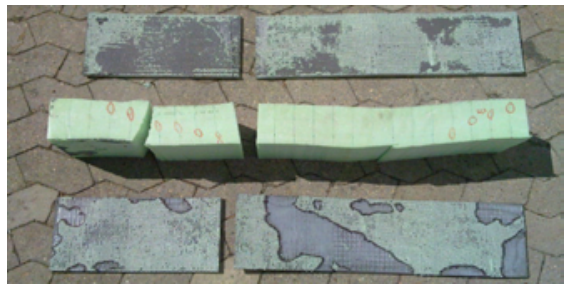

Abb. A.1: $\quad$ S.X0.S-10.120.10-1

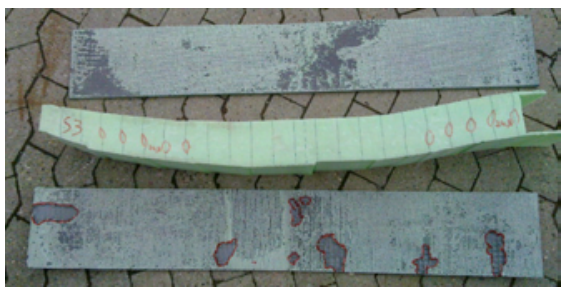

Abb. A.3: S.X0.S-10.120.10-3

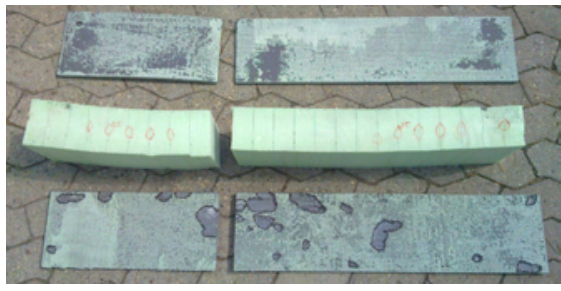

Abb. A.2: S.X0.S-10.120.10-2

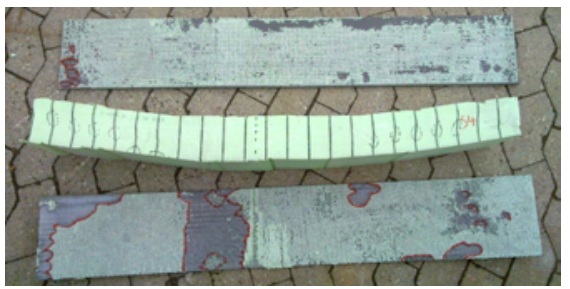

Abb. A.4: S.X0.S-10.120.10-4 


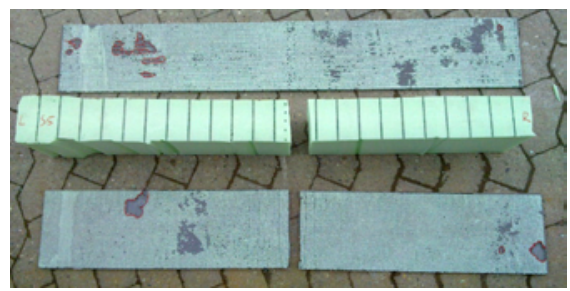

Abb. A.5: S.X0.S-10.120.10-5

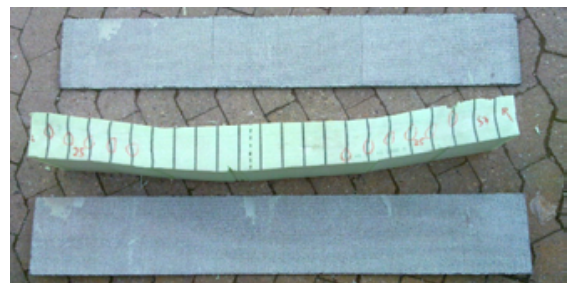

Abb. A.7: $\quad$ S.X0.S-10.120.10-7

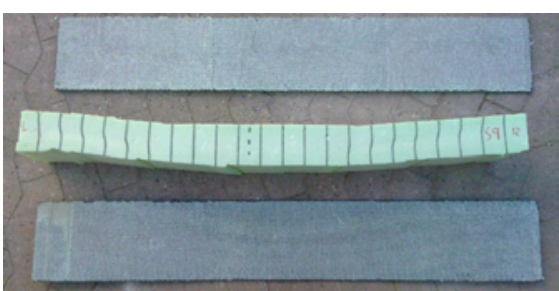

Abb. A.9: S.X0.S-10.120.10-9

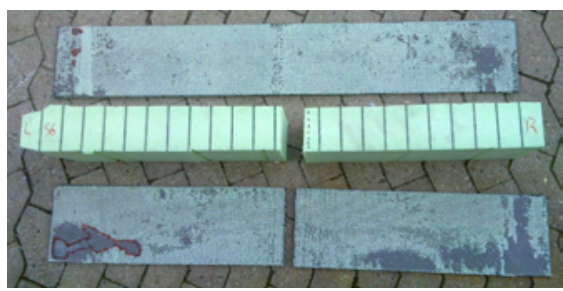

Abb. A.6: S.X0.S-10.120.10-6

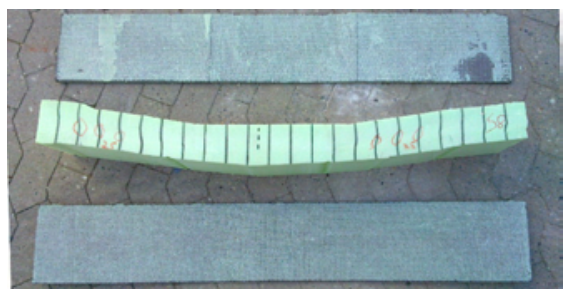

Abb. A.8: S.X0.S-10.120.10-8

\section{A.1.1.2 Biegeprobekörper der Versuchsreihe „Bewehrungsart“}

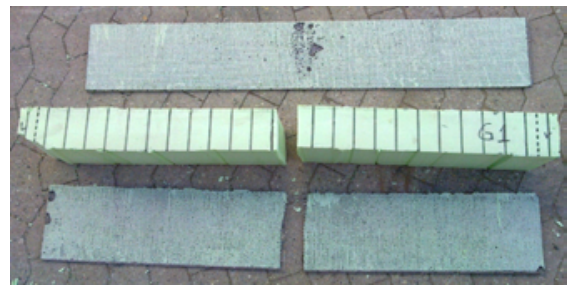

Abb. A.10: G.X1.G-10.120.10-1

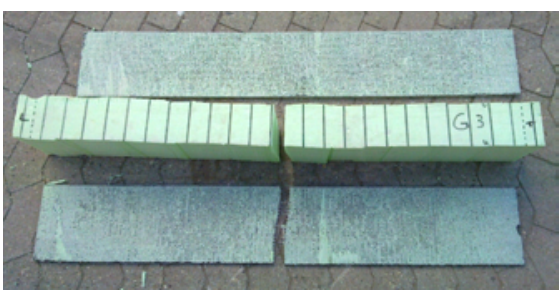

Abb. A.12: G.X1.G-10.120.10-3

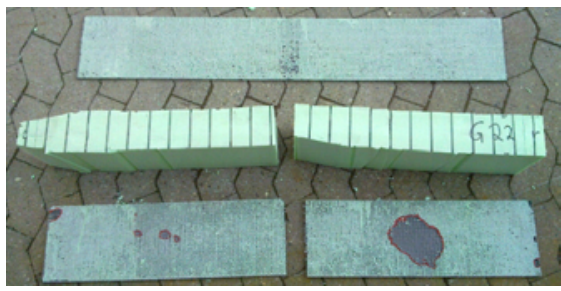

Abb. A.11: G.X1.G-10.120.10-2

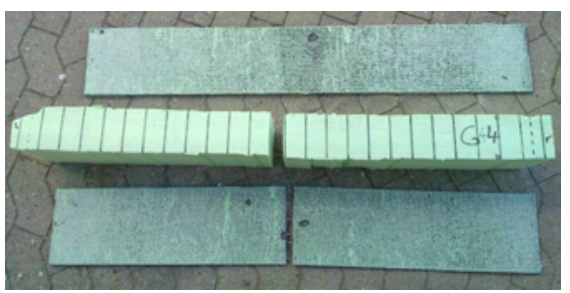

Abb. A.13: G.X1.G-10.120.10-4 


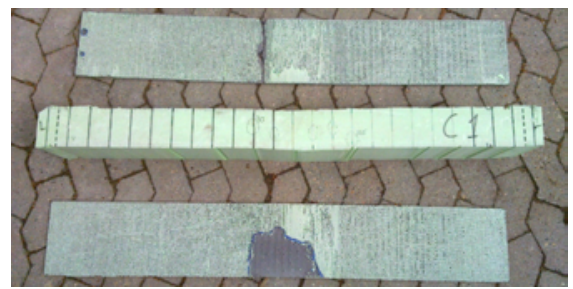

Abb. A.14: C.X1.C-10.120.10-1

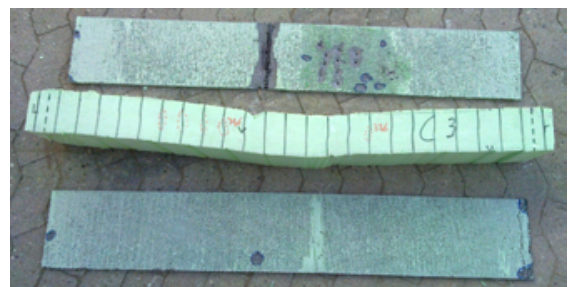

Abb. A.16: C.X1.C-10.120.10-3

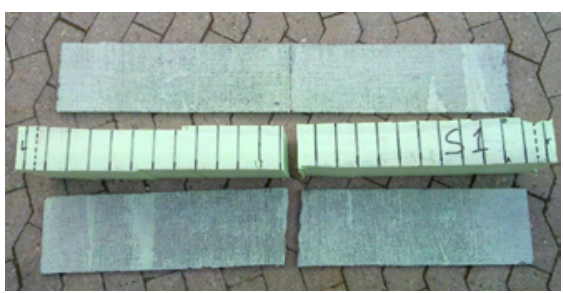

Abb. A.18: S.X1.S-10.120.10-1

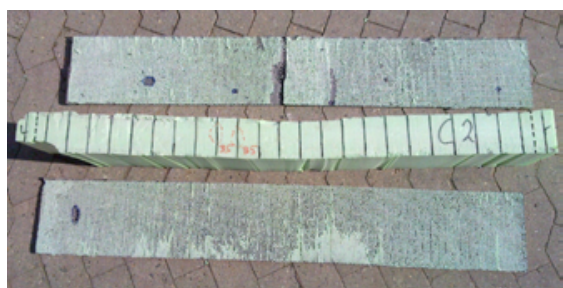

Abb. A.15: C.X1.C-10.120.10-2

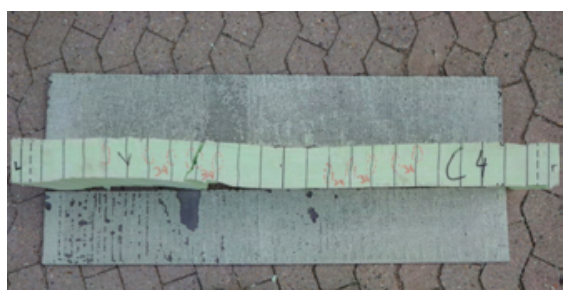

Abb. A.17: C.X1.C-10.120.10-4

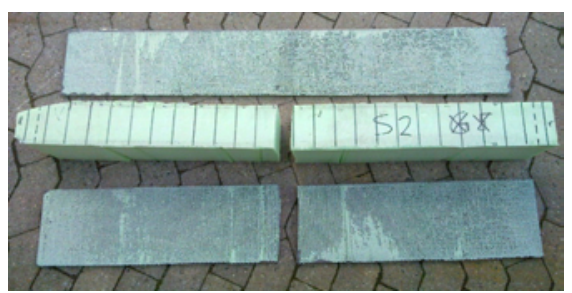

Abb. A.19: S.X1.S-10.120.10-2

\section{A.1.1.3 Biegeprobekörper der Versuchsreihe „XPS-Dichte“}

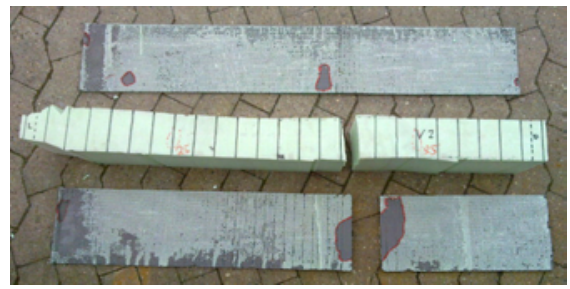

Abb. A.20: S.X2.S-10.120.10-1

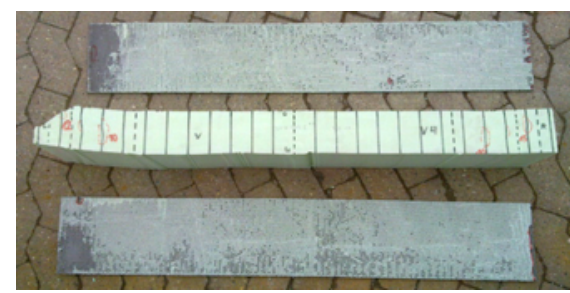

Abb. A.22: S.X2.S-10.120.10-3

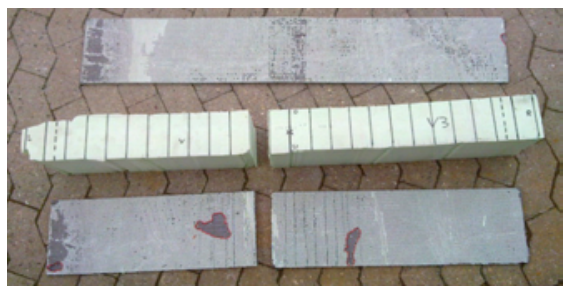

Abb. A.21: S.X2.S-10.120.10-2

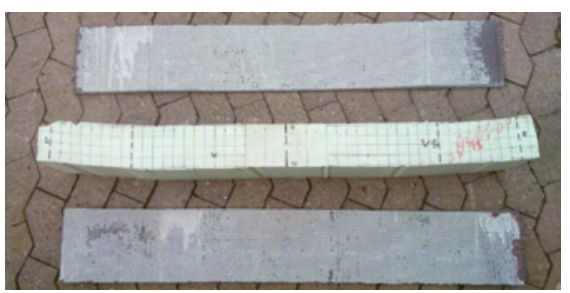

Abb. A.23: S.X2.S-10.120.10-4 


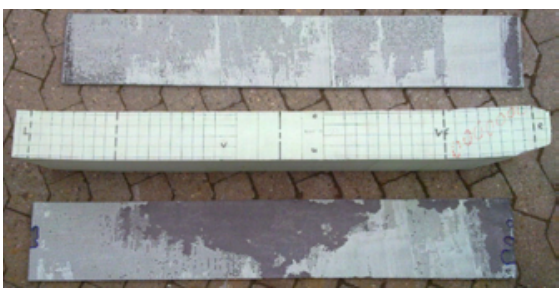

Abb. A.24: S.X2.S-10.120.10-5

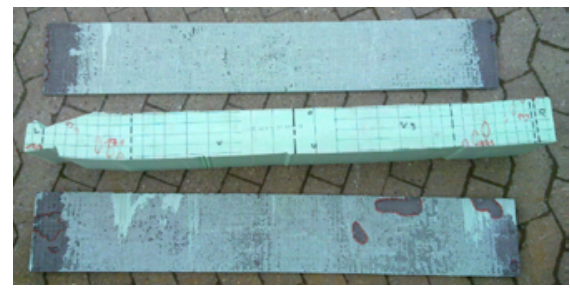

Abb. A.26: S.X3.S-10.120.10-2

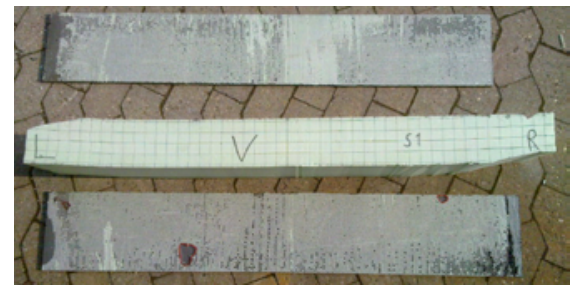

Abb. A.28: C.X2.C-10.120.10-1

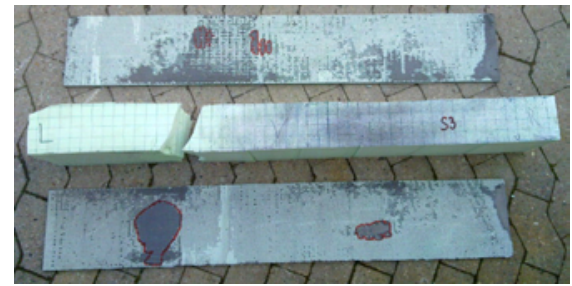

Abb. A.30: C.X2.C-10.120.10-3

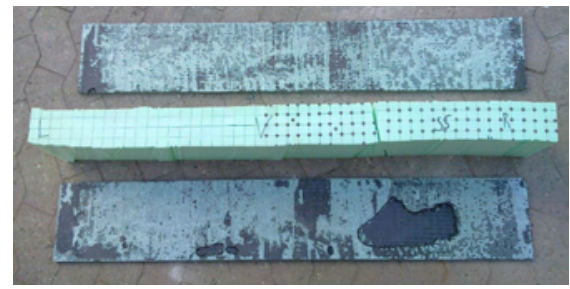

Abb. A.32: C.X3.C-10.120.10-2

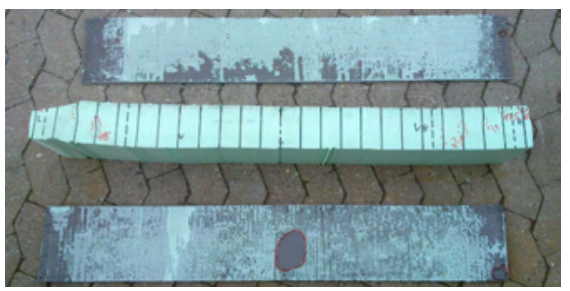

Abb. A.25: S.X3.S-10.120.10-1

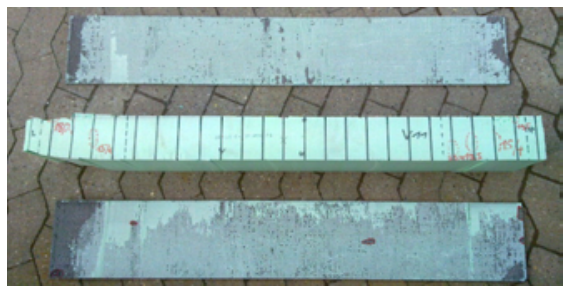

Abb. A.27: S.X3.S-10.120.10-3

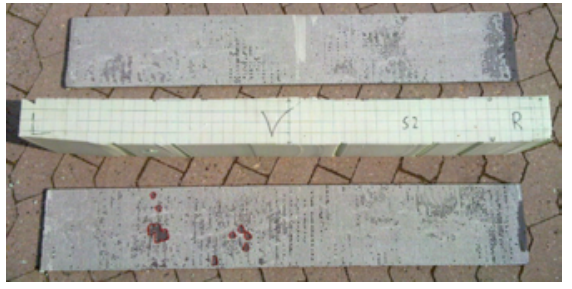

Abb. A.29: C.X2.C-10.120.10-2

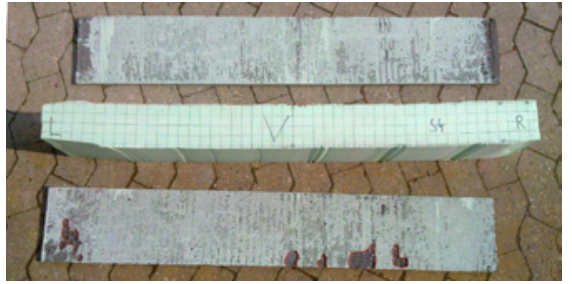

Abb. A.31: C.X3.C-10.120.10-1

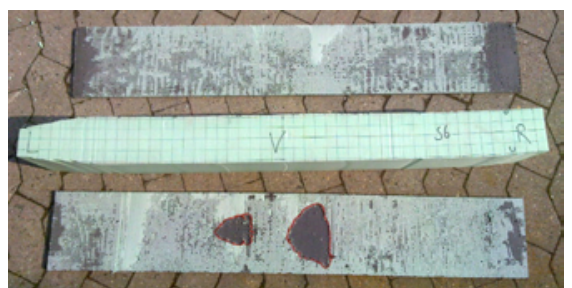

Abb. A.33: C.X3.C-10.120.10-3 


\section{A.1.1.4 Biegeprobekörper der Versuchsreihe „Schichtdicken“}
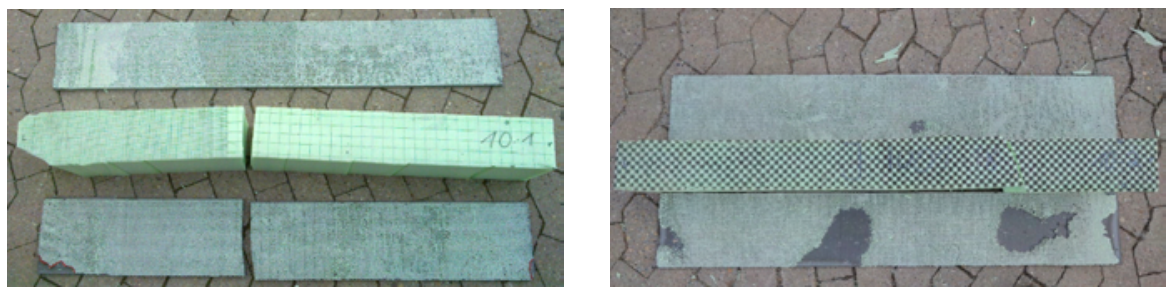

Abb. A.34: S.X4.S-10.140.10-1

Abb. A.35: S.X4.S-10.140.10-2
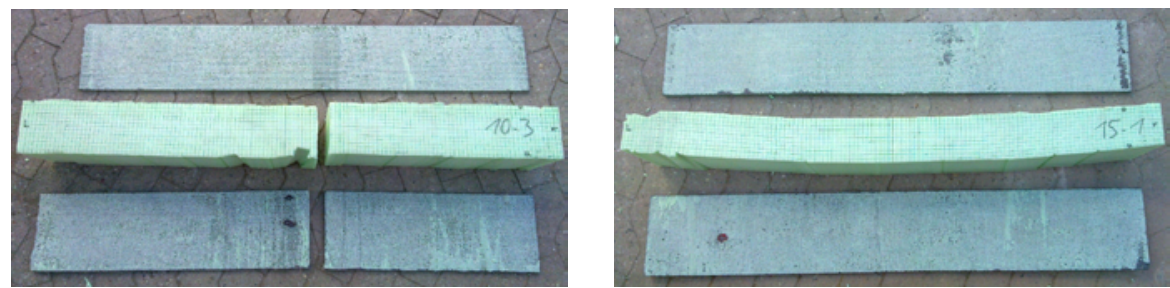

Abb. A.36: S.X4.S-10.140.10-3

Abb. A.37: S.X5.S-15.120.15-1
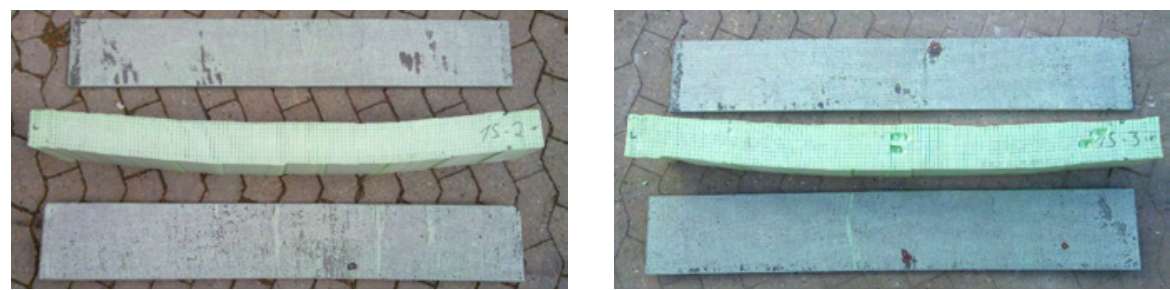

Abb. A.38: S.X5.S-15.120.15-2

Abb. A.39: S.X5.S-15.120.15-3
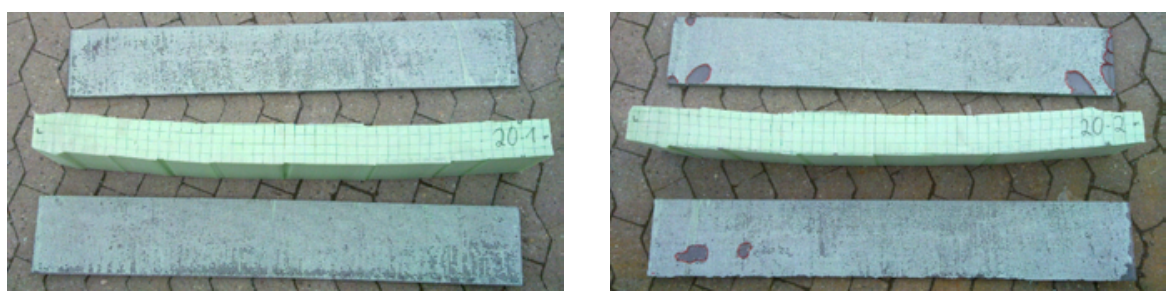

Abb. A.40: S.X5.S-20.120.20-1

Abb. A.41: S.X5.S-20.120.20-2

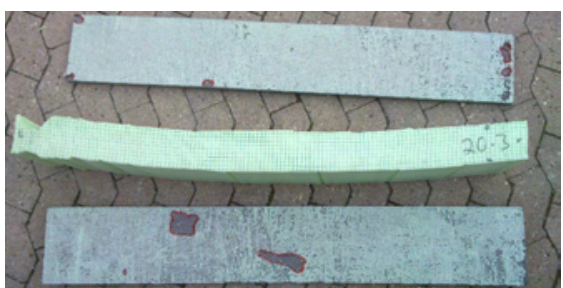

Abb. A.42: S.X5.S-20.120.20-3 


\section{A.1.1.5 Biegeprobekörper der Versuchsreihe „Stoßausbildung“}

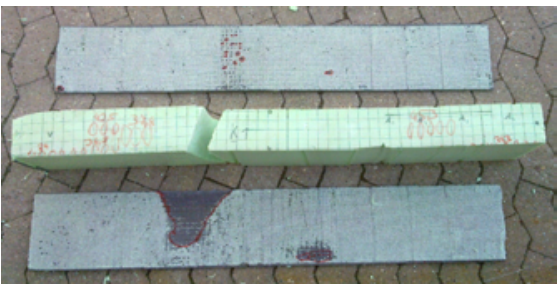

Abb. A.43: S.X5.S-10.120.10-1K

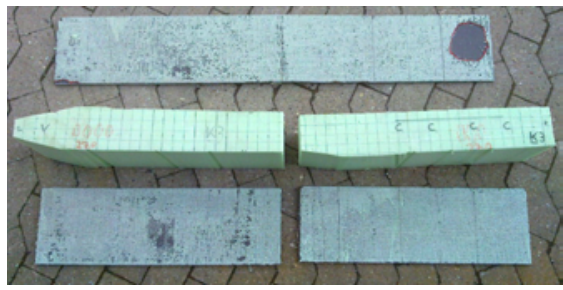

Abb. A.45: S.X5.S-10.120.10-3K

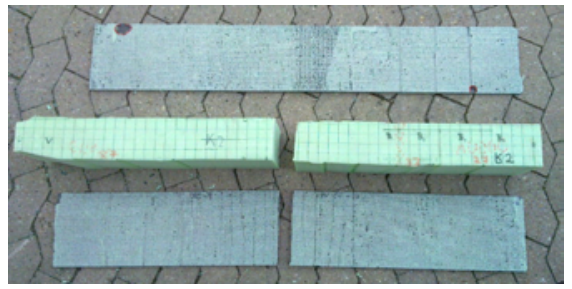

Abb. A.44: S.X5.S-10.120.10-2K 


\section{A.2 Zusammenstellung der Scherversuche}

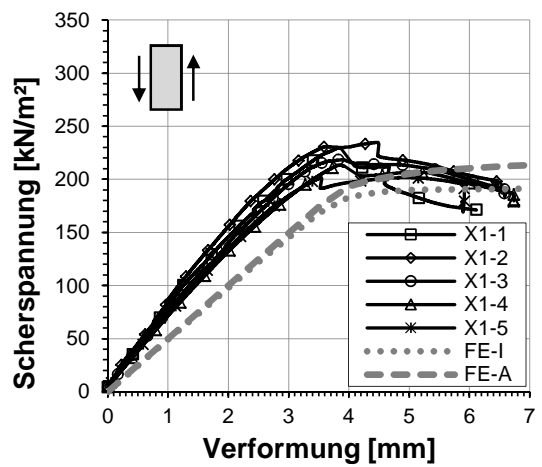

Abb. A.46: Scherspannungs-VerformungsDiagramm der Versuchsreihe $\mathbf{X} \mathbf{1}$ ohne Betondeckschichten

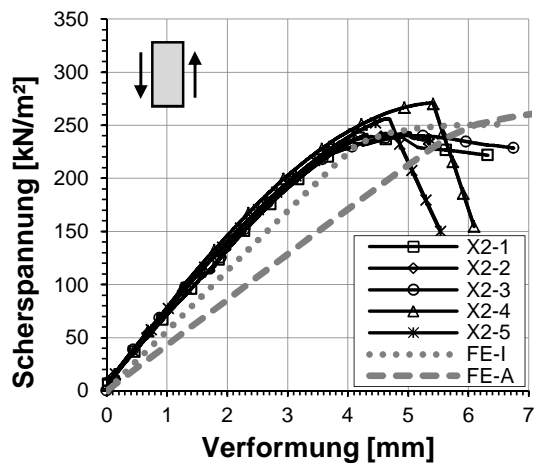

Abb. A.48: Scherspannungs-VerformungsDiagramm der Versuchsreihe $\mathbf{X} 2$ ohne Betondeckschichten

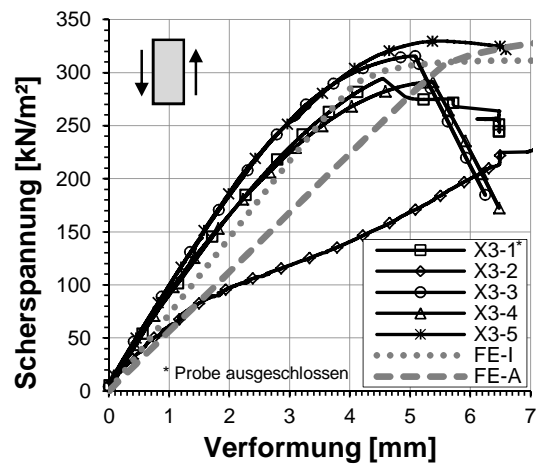

Abb. A.50: Scherspannungs-VerformungsDiagramm der Versuchsreihe $\mathbf{X} \mathbf{3}$ ohne Betondeckschichten

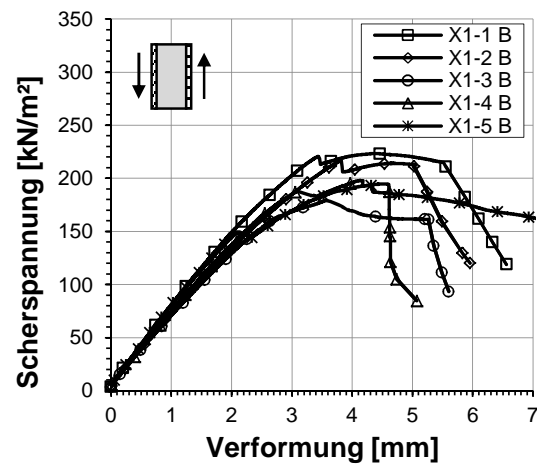

Abb. A.47: Scherspannungs-VerformungsDiagramm der Versuchsreihe X1 B mit Betondeckschichten

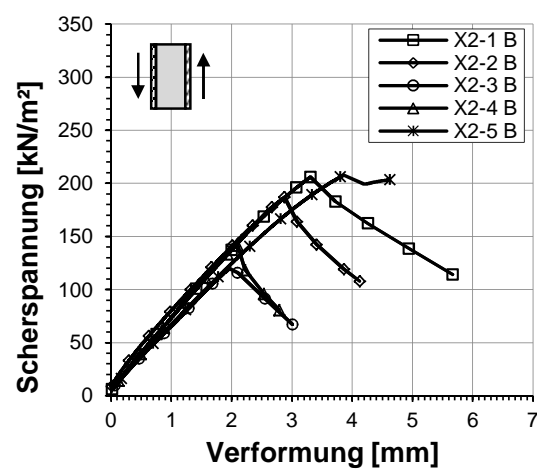

Abb. A.49: Scherspannungs-VerformungsDiagramm der Versuchsreihe X2 B mit Betondeckschichten

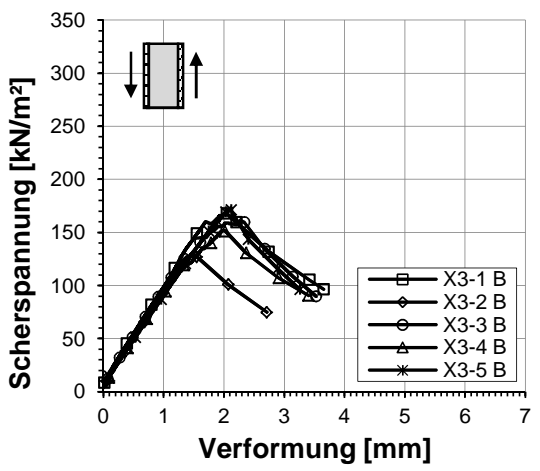

Abb. A.51: Scherspannungs-VerformungsDiagramm der Versuchsreihe X3 B mit Betondeckschichten 


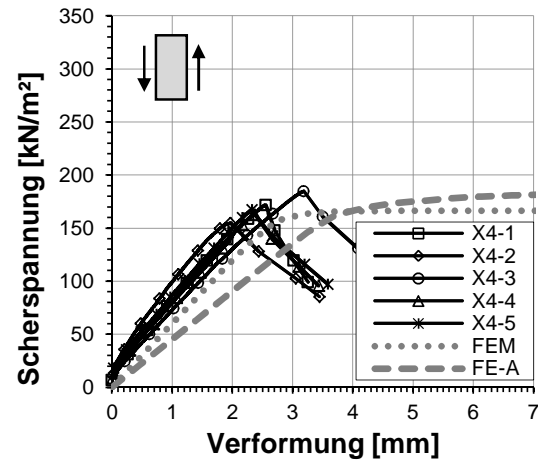

Abb. A.52: Scherspannungs-VerformungsDiagramm der Versuchsreihe $\mathbf{X} 4$ ohne Betondeckschichten

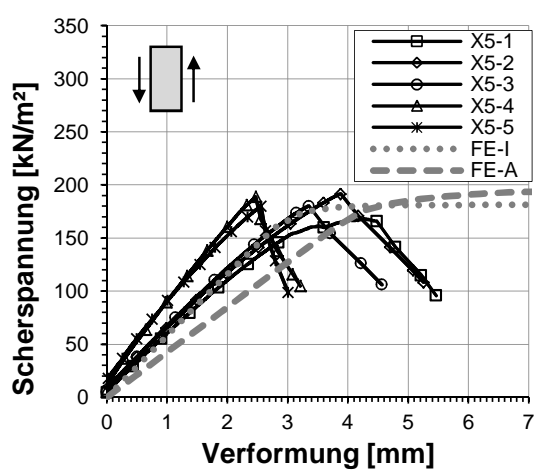

Abb. A.53: Scherspannungs-VerformungsDiagramm der Versuchsreihe $\mathbf{X} 5$ ohne Betondeckschichten

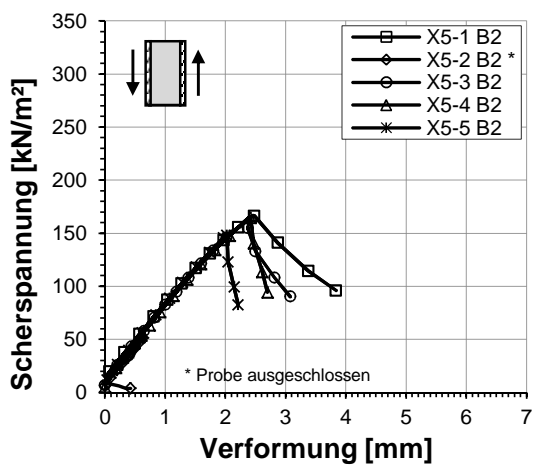

Abb. A.55: Scherspannungs-VerformungsDiagramm der Versuchsreihe X5 B2 mit Betondeckschichten

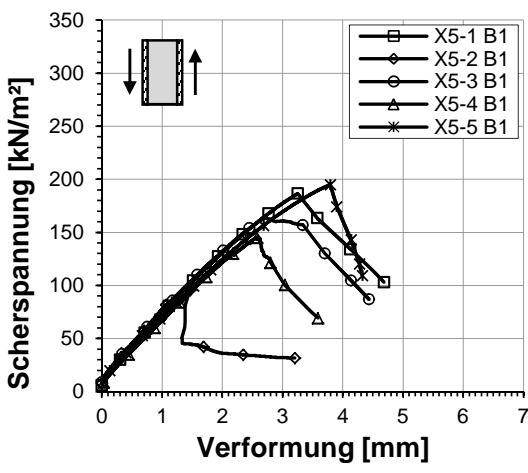

Abb. A.54: Scherspannungs-VerformungsDiagramm der Versuchsreihe X5 B1 mit Betondeckschichten

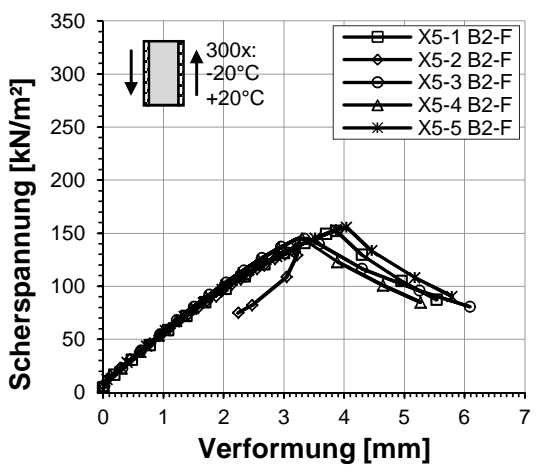

Abb. A.56: Scherspannungs-VerformungsDiagramm der Versuchsreihe X5 B2-F mit Betondeckschichten und Frost-Tau-Beanspruchung 


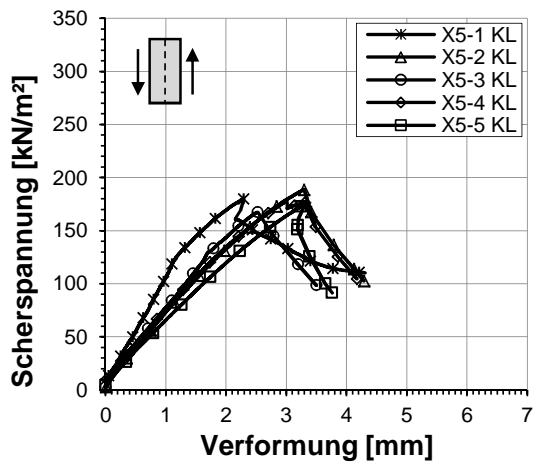

Abb. A.57: Scherspannungs-VerformungsDiagramm der Versuchsreihe X5 KL mit geklebter Längsfuge

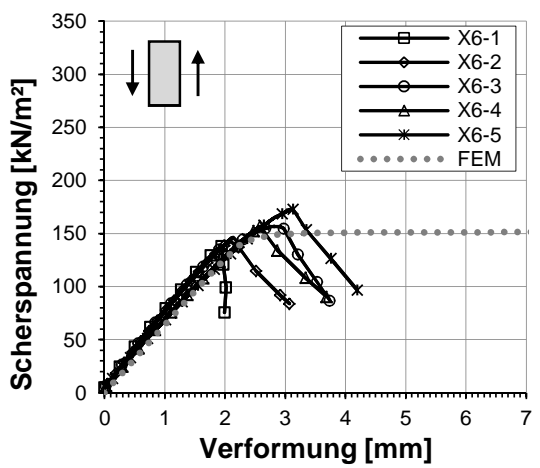

Abb. A.59: Scherspannungs-VerformungsDiagramm der Versuchsreihe $\mathbf{X} \mathbf{6}$ ohne Betondeckschichten

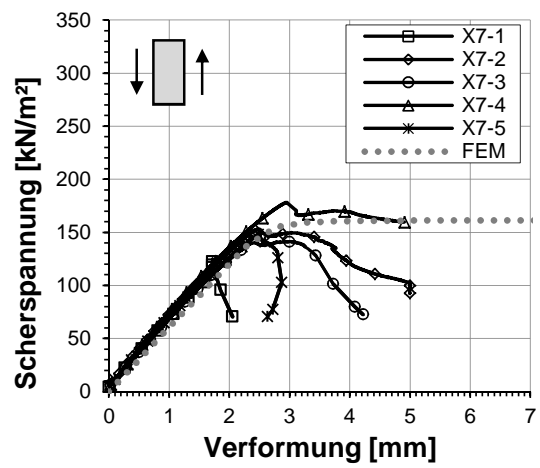

Abb. A.61: Scherspannungs-VerformungsDiagramm der Versuchsreihe $\mathbf{X 7}$ ohne Betondeckschichten

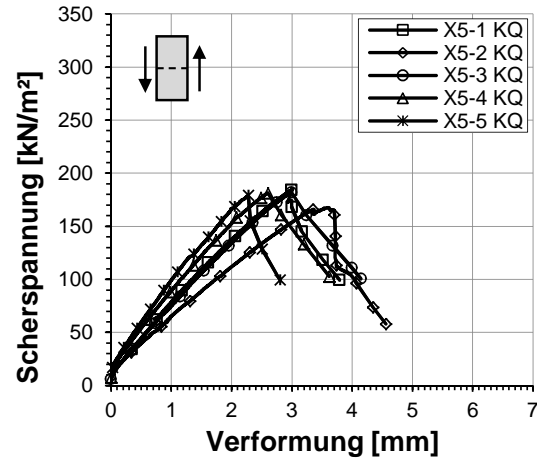

Abb. A.58: Scherspannungs-VerformungsDiagramm der Versuchsreihe X5 KQ mit geklebter Querfuge

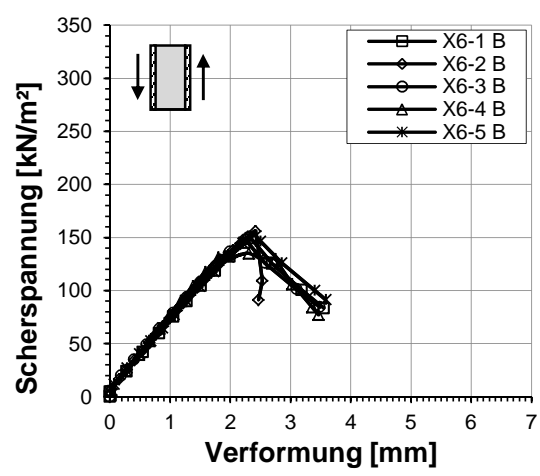

Abb. A.60: Scherspannungs-VerformungsDiagramm der Versuchsreihe X6 B mit Betondeckschichten

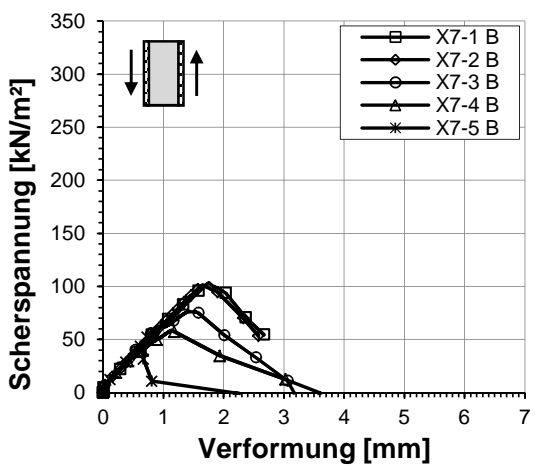

Abb. A.62: Scherspannungs-VerformungsDiagramm der Versuchsreihe X7 B mit Betondeckschichten 


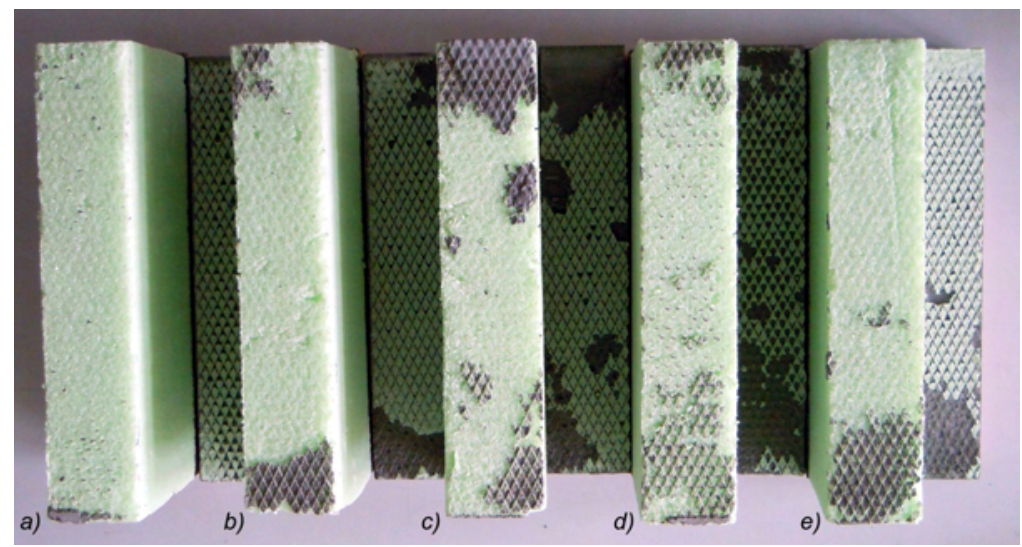

Abb. A.63: Probekörper X1-1 B - X1-5B [a) - e)] nach dem Versagen

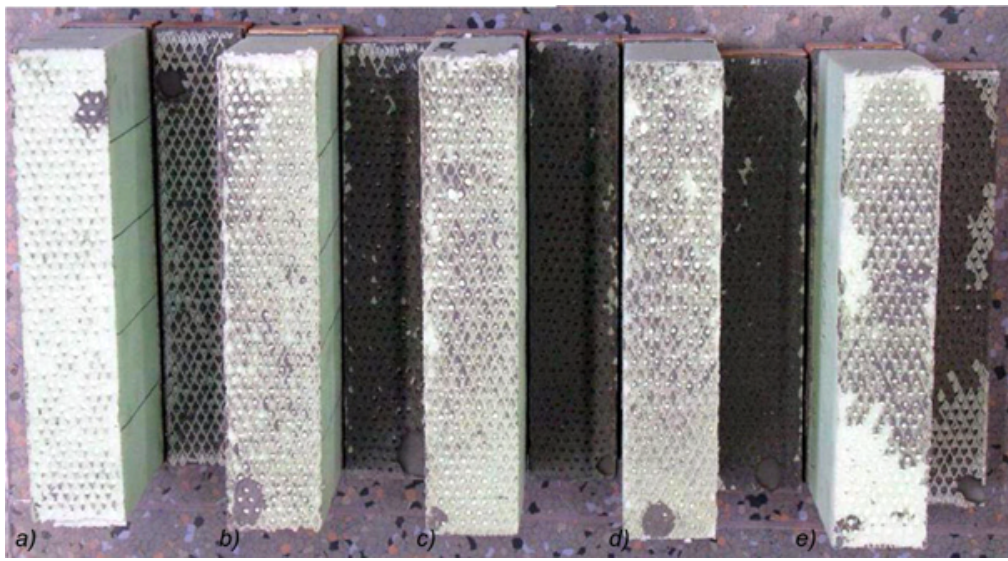

Abb. A.64: Probekörper X2-1 B - X2-5B [a) - e)] nach dem Versagen

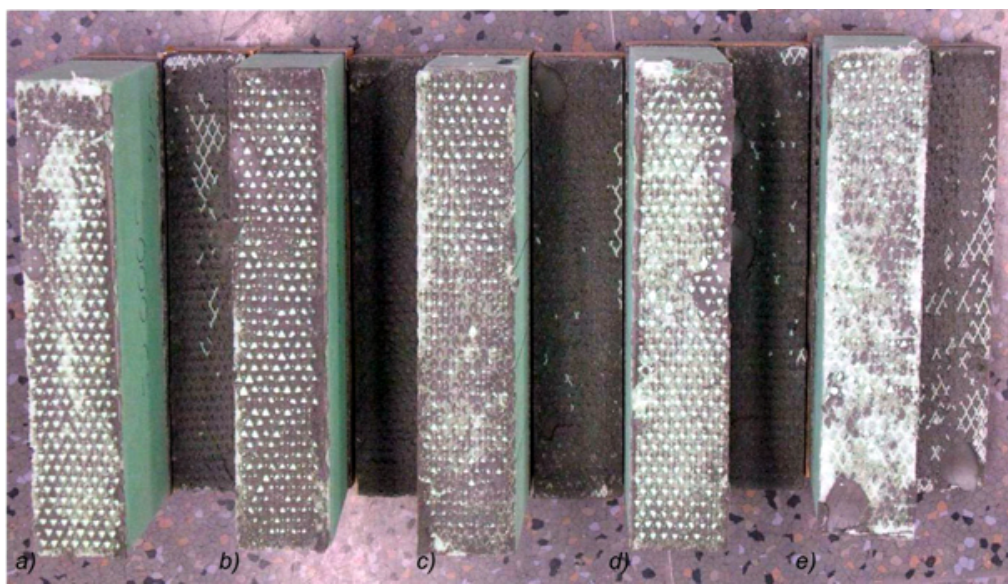

Abb. A.65: Probekörper X3-1 B - X3-5 B [a) - e)] nach dem Versagen 


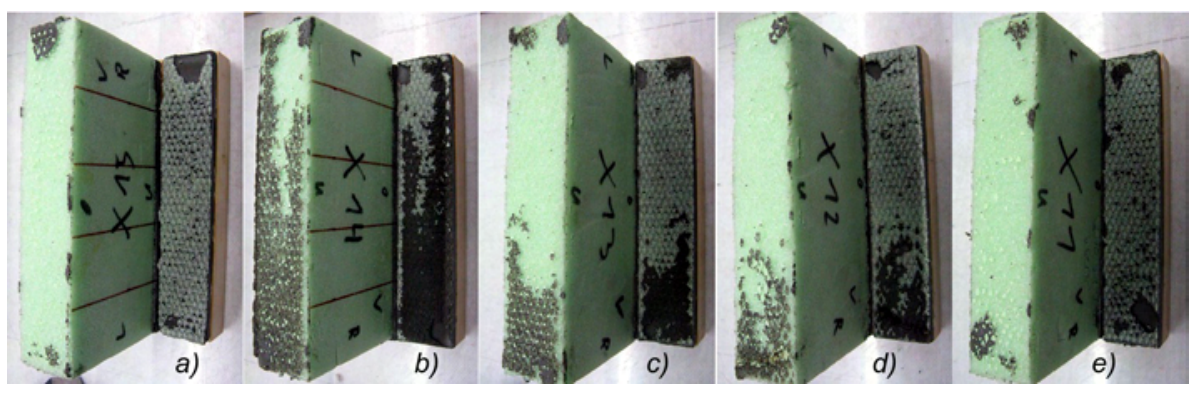

Abb. A.66: Probekörper X5-1 B1 - X5-5 B1 [a) - e)] nach dem Versagen

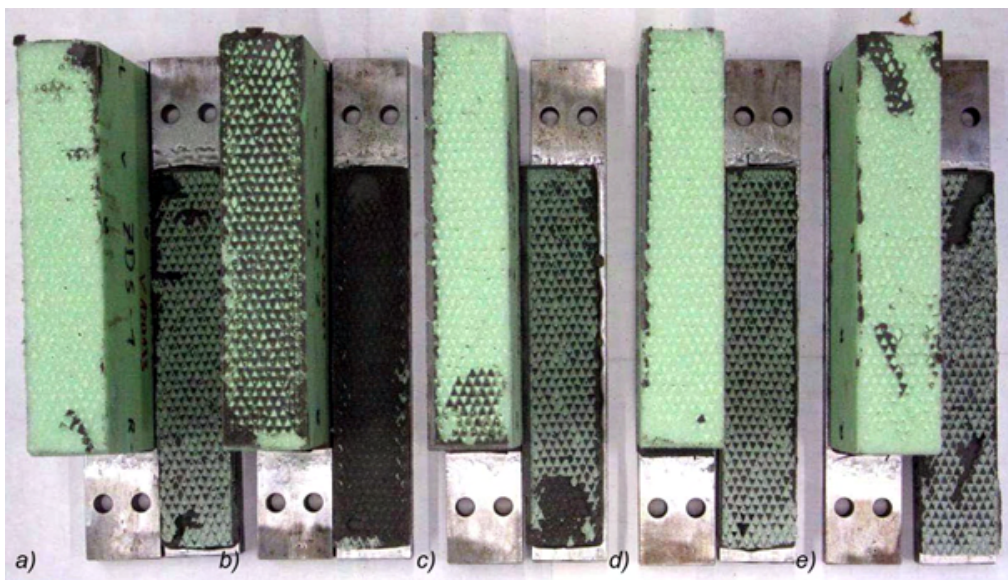

Abb. A.67: Probekörper X5-1 B2 - X5-5 B2 [a) - e)] nach dem Versagen

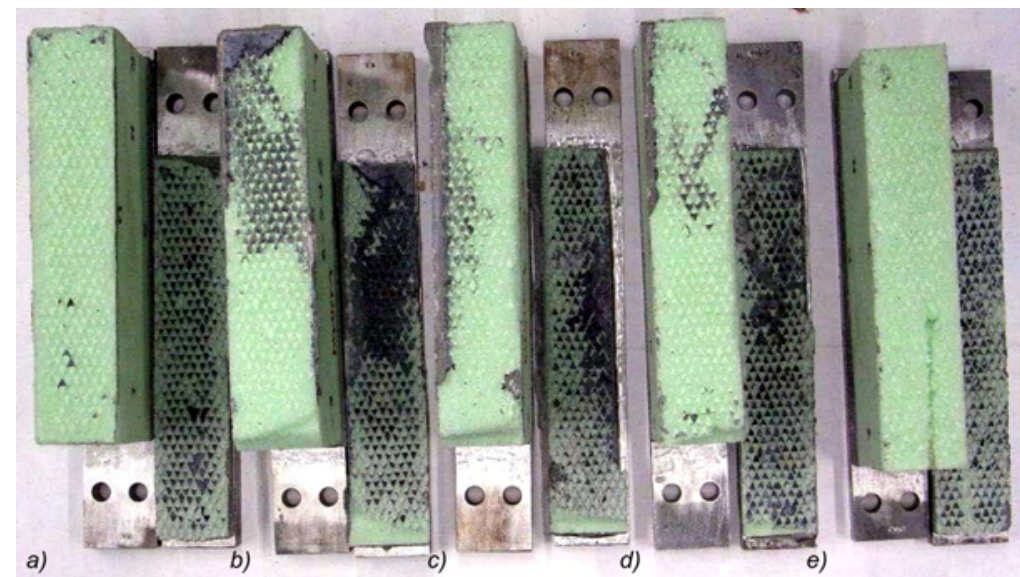

Abb. A.68: Probekörper X5-1 B2-F - X5-5 B2-F [a) - e)] nach dem Versagen 


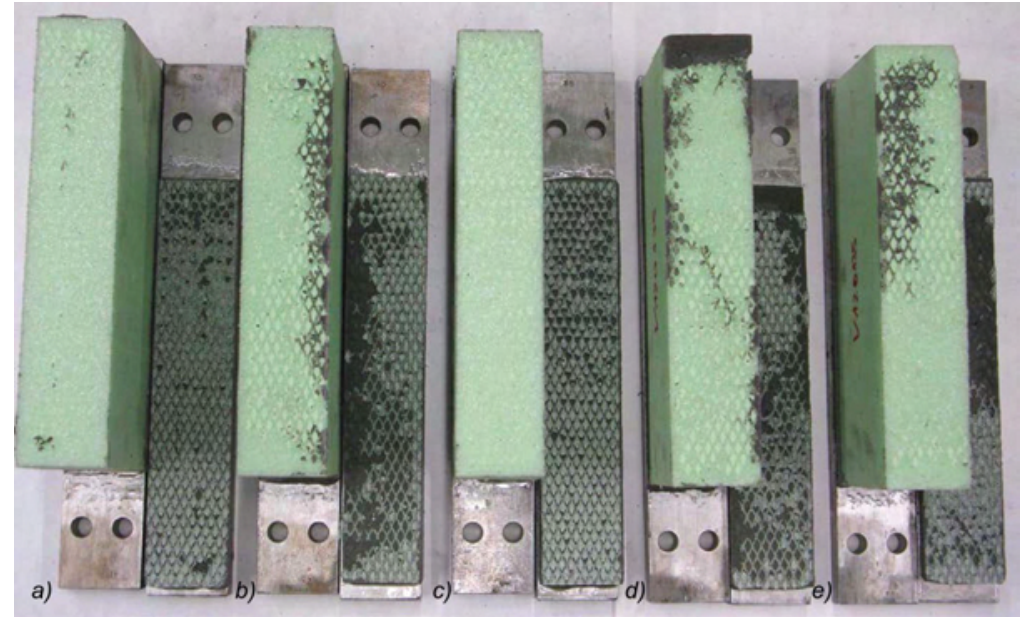

Abb. A.69: Probekörper X6-1 B - X6-5B [a) - e)] nach dem Versagen

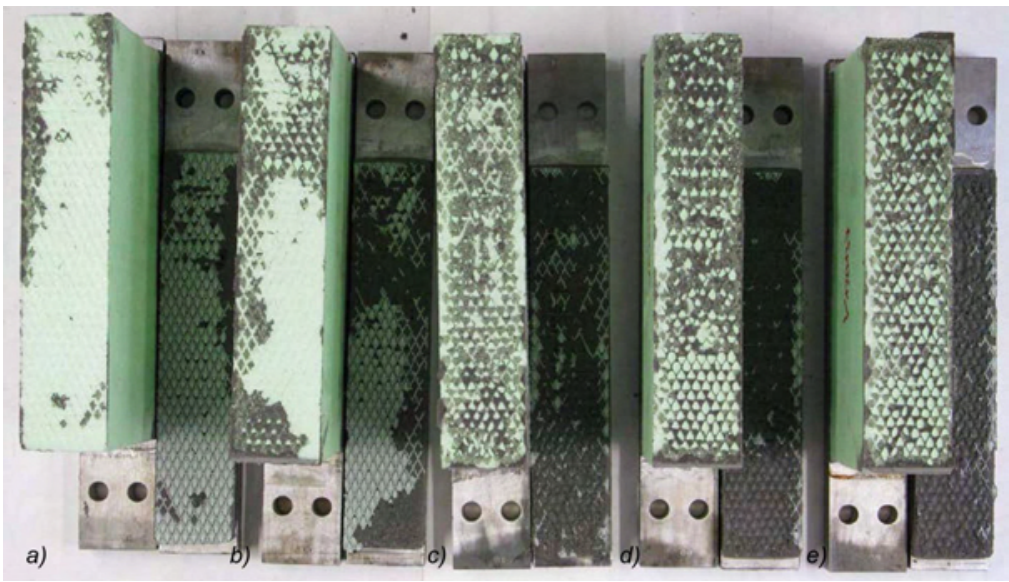

Abb. A.70: Probekörper X7-1 B - X7-5B [a) - e)] nach dem Versagen 


\section{A.3 Zusammenstellung der Biege- und Zugversuche an bewehrtem Beton}

Tab. A.3: Materialeigenschaften der Probekörper zu den Biege- und Zugversuchen

\begin{tabular}{|c|c|c|c|c|c|c|c|c|c|}
\hline \multirow[b]{2}{*}{$\begin{array}{l}\text { Versuchs- } \\
\text { art }\end{array}$} & \multirow[b]{2}{*}{ Nr. } & \multirow{2}{*}{$\begin{array}{c}\text { Dicke } \\
\boldsymbol{d} \\
{[\mathrm{mm}]}\end{array}$} & \multicolumn{2}{|c|}{ Bewehrung } & \multicolumn{5}{|c|}{ Beton } \\
\hline & & & $\begin{array}{c}\text { Lagen } \\
{[-]}\end{array}$ & $\begin{array}{l}n_{f}^{1} \\
{[-]}\end{array}$ & Typ & $\begin{array}{c}f_{c m, c u b e} \\
{\left[\mathrm{~N} / \mathrm{mm}^{2}\right]}\end{array}$ & $\begin{array}{c}E_{c m} \\
{\left[\mathrm{~N} / \mathrm{mm}^{2}\right]}\end{array}$ & $\begin{array}{c}\boldsymbol{f}_{c t m, f f} \\
{\left[\mathrm{~N} / \mathrm{mm}^{2}\right]}\end{array}$ & $\begin{array}{c}\boldsymbol{f}_{c t m, s p} \\
{\left[\mathrm{~N} / \mathrm{mm}^{2}\right]}\end{array}$ \\
\hline \multirow{15}{*}{$\begin{array}{c}\text { Zug- } \\
\text { versuche }\end{array}$} & DS1-I & 10,7 & & & & & & & \\
\hline & DS2-I & 10,7 & 3 & 16 & HPC-3 & 152,8 & 40.870 & 12,1 & 5,6 \\
\hline & DS3-I & 9,5 & & & & & & & \\
\hline & DS1-II & - & & & & & & & \\
\hline & DS2-II & - & 3 & 16 & HPC-3 & 141,0 & 38.620 & 11,8 & 4,0 \\
\hline & DS3-II & - & & & & & & & \\
\hline & DC1-I & 8,4 & & & & & & & \\
\hline & DC2-I & 9,8 & 1 & 18 & HPC-3 & 152,8 & 40.870 & 12,1 & 5,6 \\
\hline & DC3-I & 10,0 & & & & & & & \\
\hline & DC1-II & - & & & & & & & \\
\hline & DC2-II & - & & & & 141,0 & 38.620 & 11,8 & 4,0 \\
\hline & DC3-II & - & 1 & 18 & HPC-3 & & & & \\
\hline & DC4-II & - & 1 & 10 & वге & & & & \\
\hline & DC5-II & - & & & & 130,3 & n.v. & 9,7 & n.v. \\
\hline & DC6-II & - & & & & & & & \\
\hline \multirow{6}{*}{$\begin{array}{l}\text { Biege- } \\
\text { versuche }\end{array}$} & BS1 & 29,7 & & & & & & & \\
\hline & BS2 & 30,6 & 10 & 12 & HPC-3 & 145,8 & 40.440 & 11,8 & 4,2 \\
\hline & BS3 & 29,2 & & & & & & & \\
\hline & $\mathrm{BC} 1$ & 30,0 & & & & & & & \\
\hline & $\mathrm{BC} 2$ & 30,2 & 3 & 13 & HPC-3 & 145,8 & 40.440 & 11,8 & 4,2 \\
\hline & $\mathrm{BC} 3$ & 30,2 & & & & & & & \\
\hline
\end{tabular}

\section{A.4 Versuchsstand für die Ermüdungsversuche}




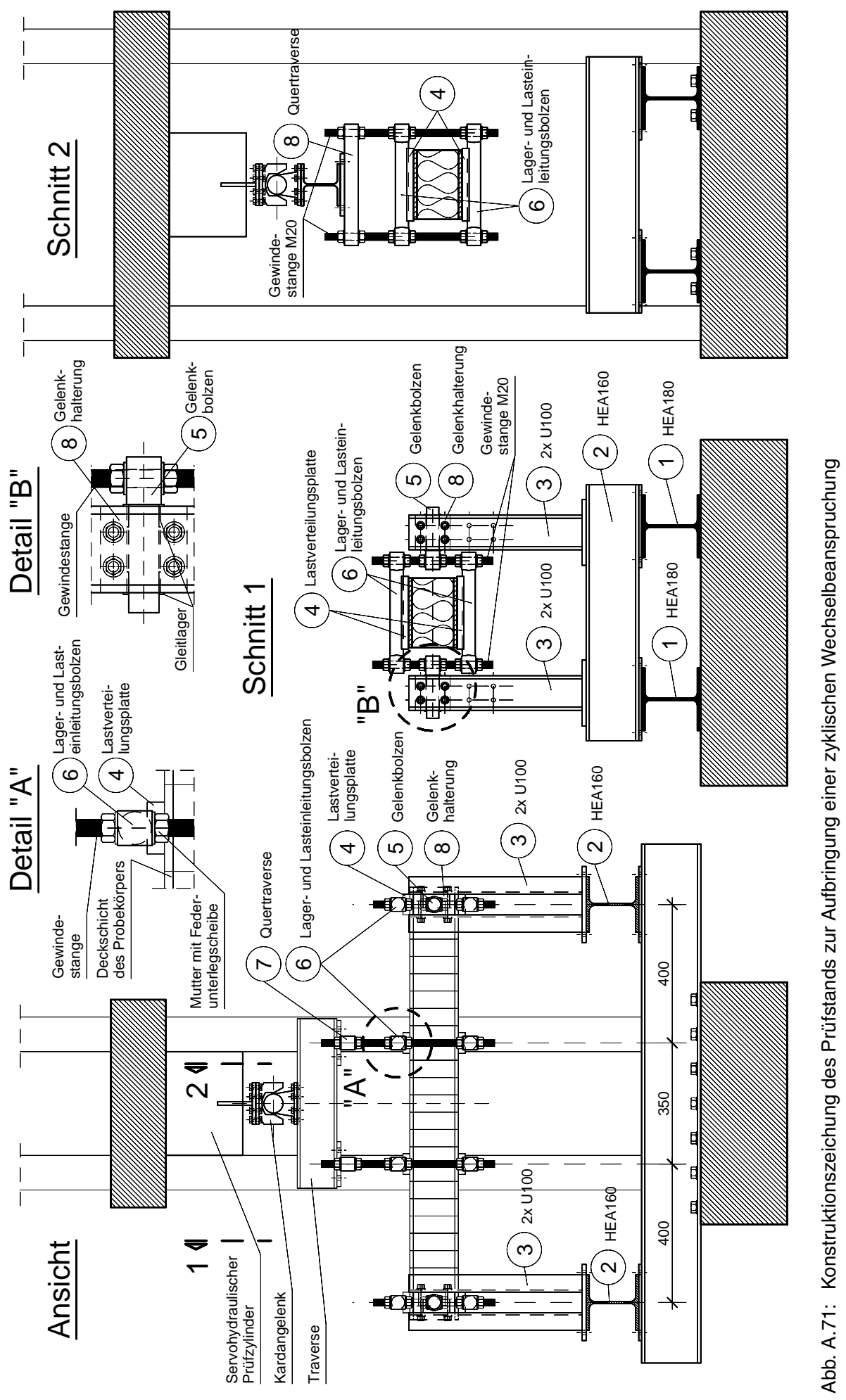




\section{A.4.1 Zusammenstellung der aufgeschnittenen Probekörper}
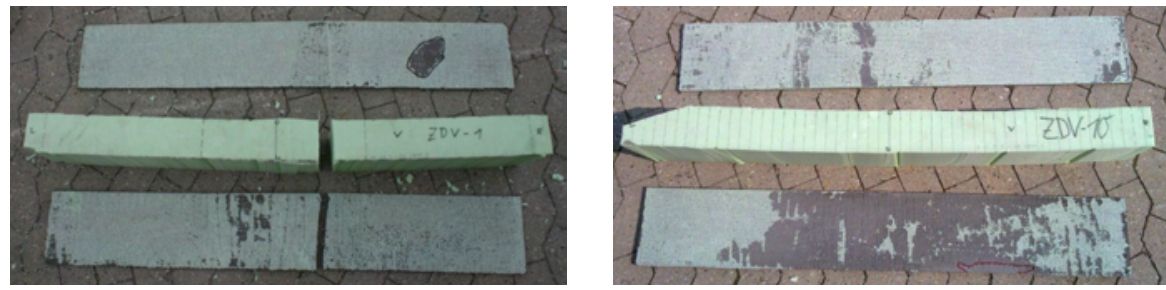

Abb. A.72: S.X5.S-10.120.10-1

Abb. A.73: S.X5.S-10.120.10-2
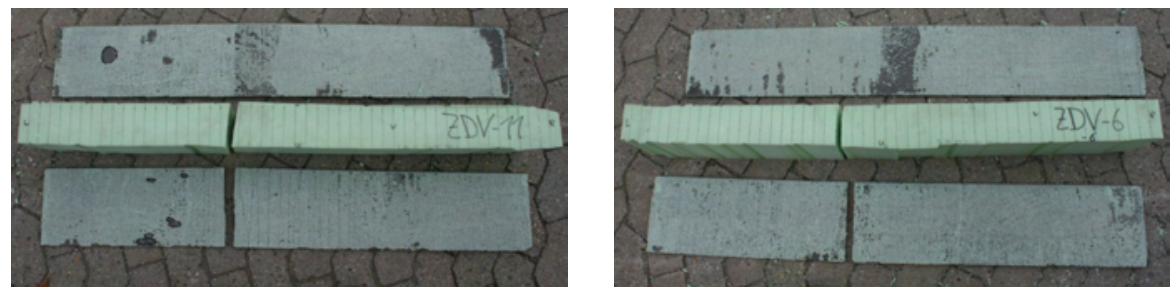

Abb. A.74: S.X5.S-10.120.10-3

Abb. A.75: S.X5.S-10.120.10-4
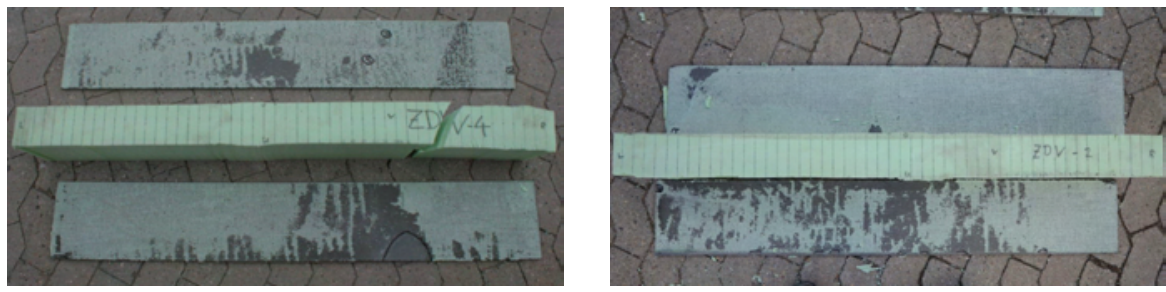

Abb. A.76: S.X5.S-10.120.10-5

Abb. A.77: S.X5.S-10.120.10-6
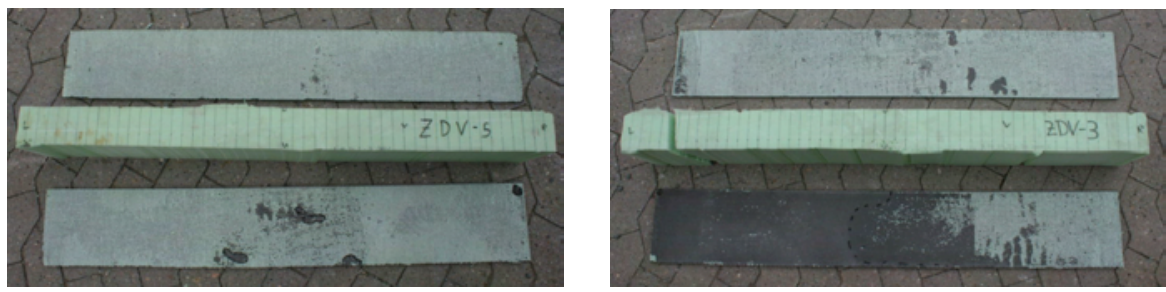

Abb. A.78: S.X0.S-10.120.10-7

Abb. A.79: S.X5.S-10.120.10-8
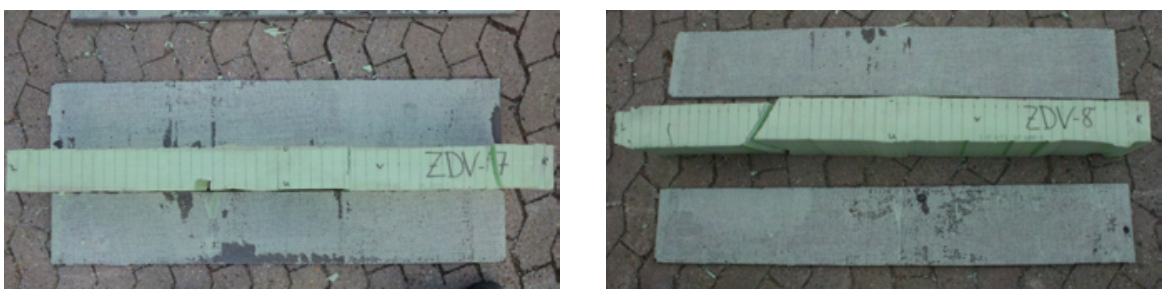

Abb. A.80: S.X5.S-10.120.10-9

Abb. A.81: S.X5.S-10-120.10-10 


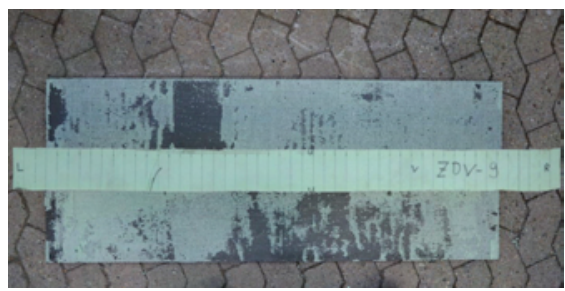

Abb. A.82: S.X5.S-10.120.10-11

\section{A.5 Zusammenstellung der Großbauteilversuche}

\section{A.5.1 Beanspruchung durch Einzellasten}

\section{A.5.1.1 Versuch E2}

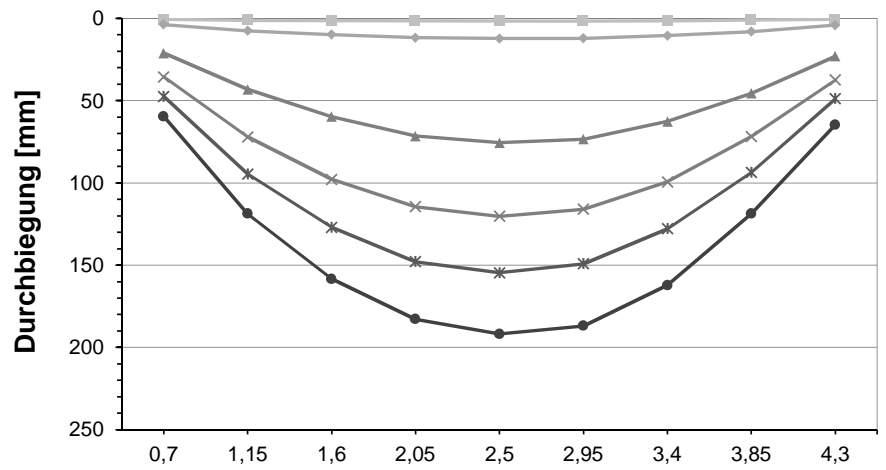

Laststufe

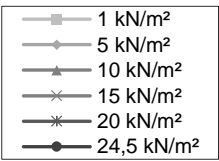

Lage der WA über die Länge des Probekörpers [m]

Abb. A.83: Durchbiegungen der unteren Deckschicht über der Länge des Probekörpers E2

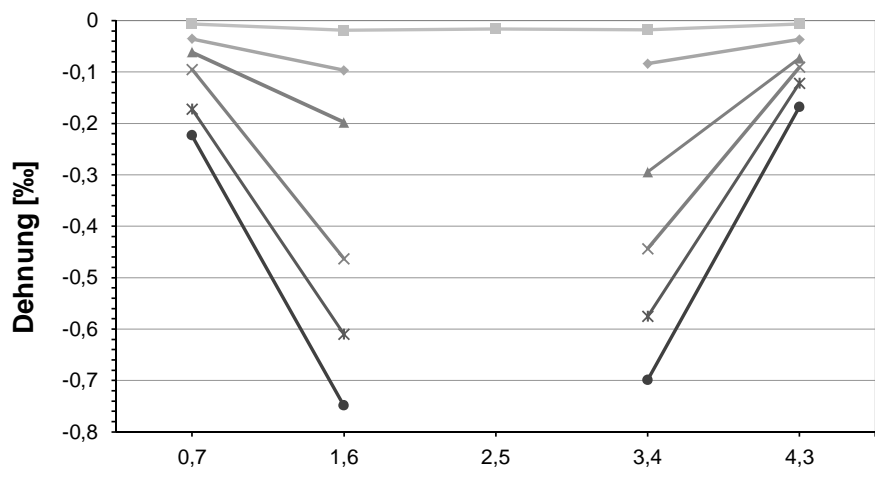

\section{Laststufe:}

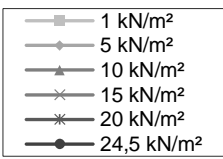

Lage der DMS über die Länge des Probekörpers [m]

Abb. A.84: Dehnungen auf der Oberseite der oberen Deckschicht über der Länge des Probekörpers E2 


\section{A.5.1.2 Versuch E3}

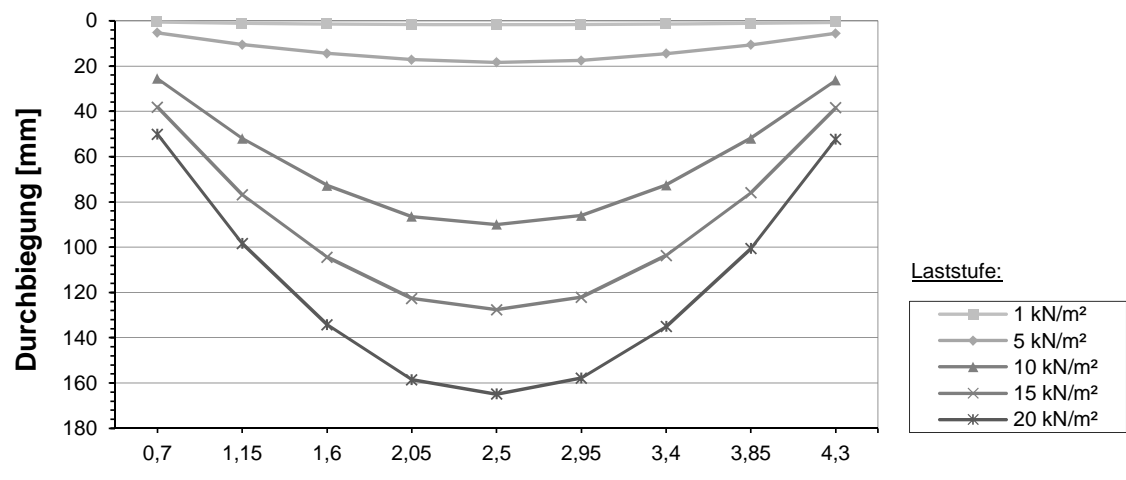

Lage der WA über die Länge des Probekörpers [m]

Abb. A.85: Durchbiegungen der unteren Deckschicht über der Länge des Probekörpers E3

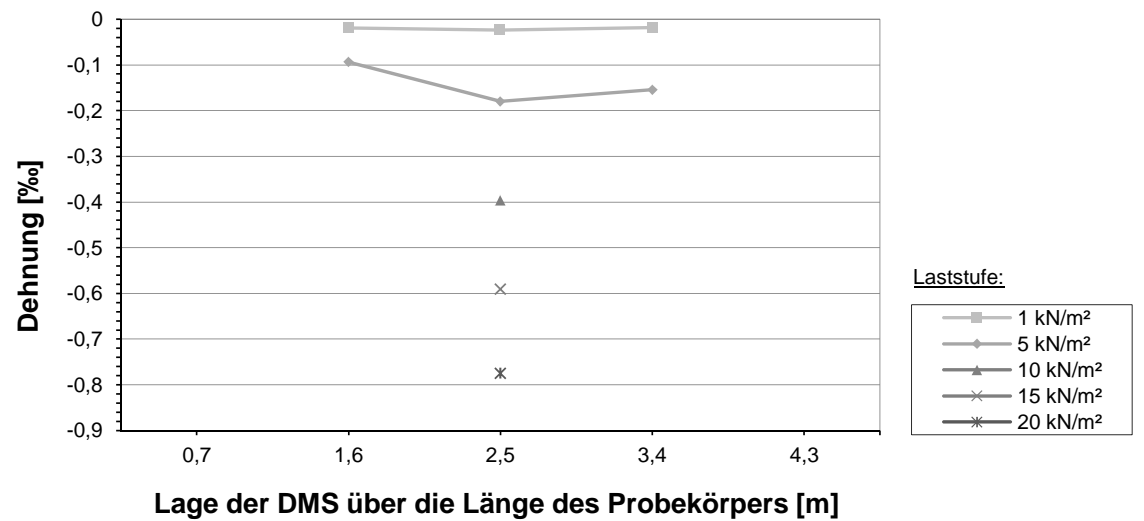

Abb. A.86: Dehnungen auf der Oberseite der oberen Deckschicht über der Länge des Probekörpers E3

\section{A.5.2 Beanspruchung durch Flächenlasten}

\section{A.5.2.1 Versuch F1}




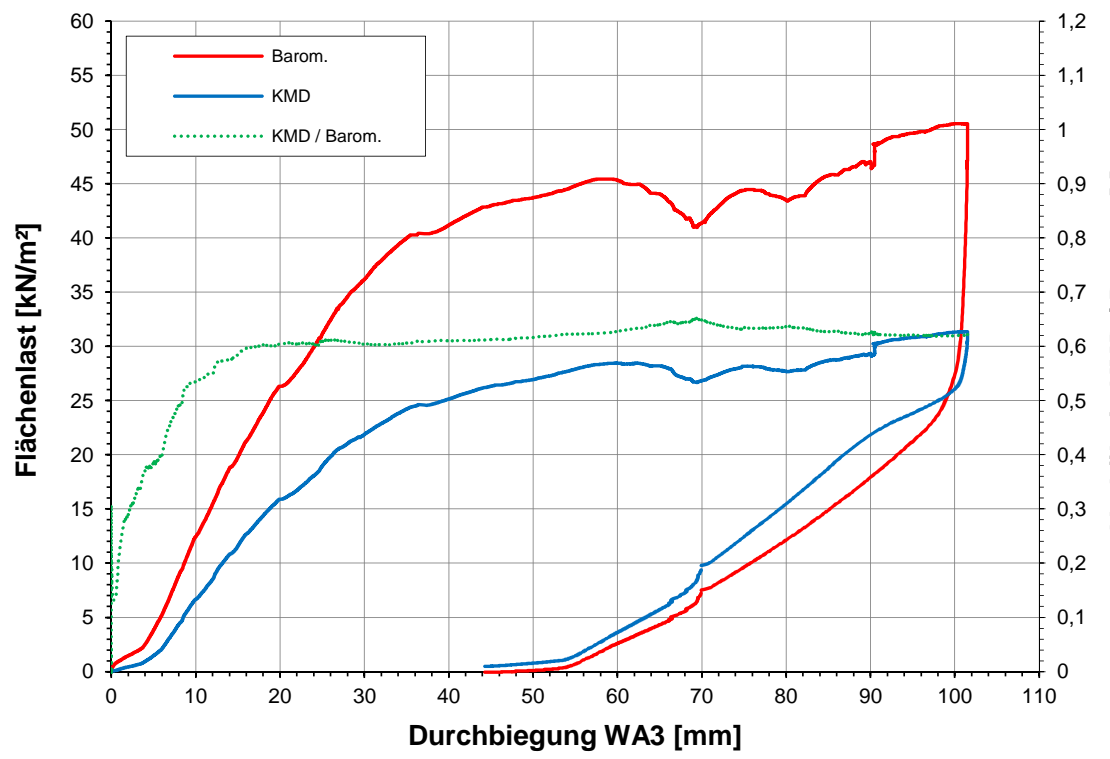

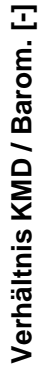

Abb. A.87: Flächenlast-Durchbiegungs-Diagramm des Versuchs F1

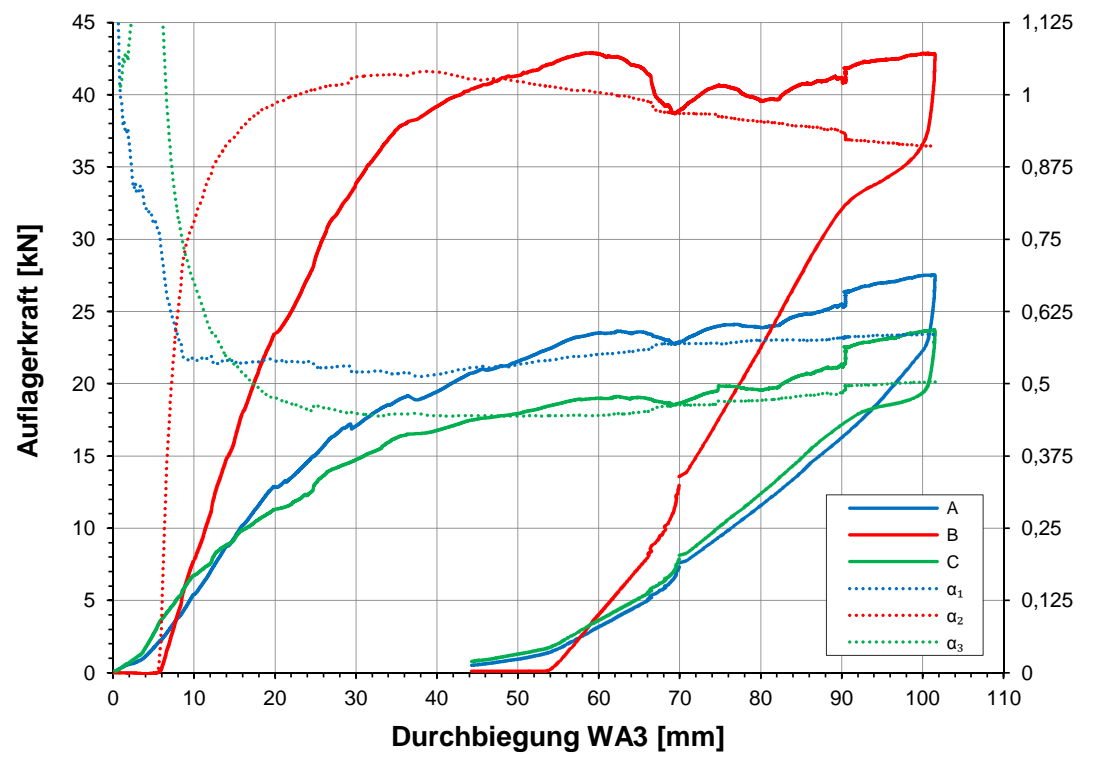

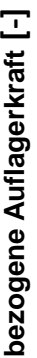

Abb. A.88: Auflagerkräfte-Durchbiegungs-Diagramm des Versuchs F1 


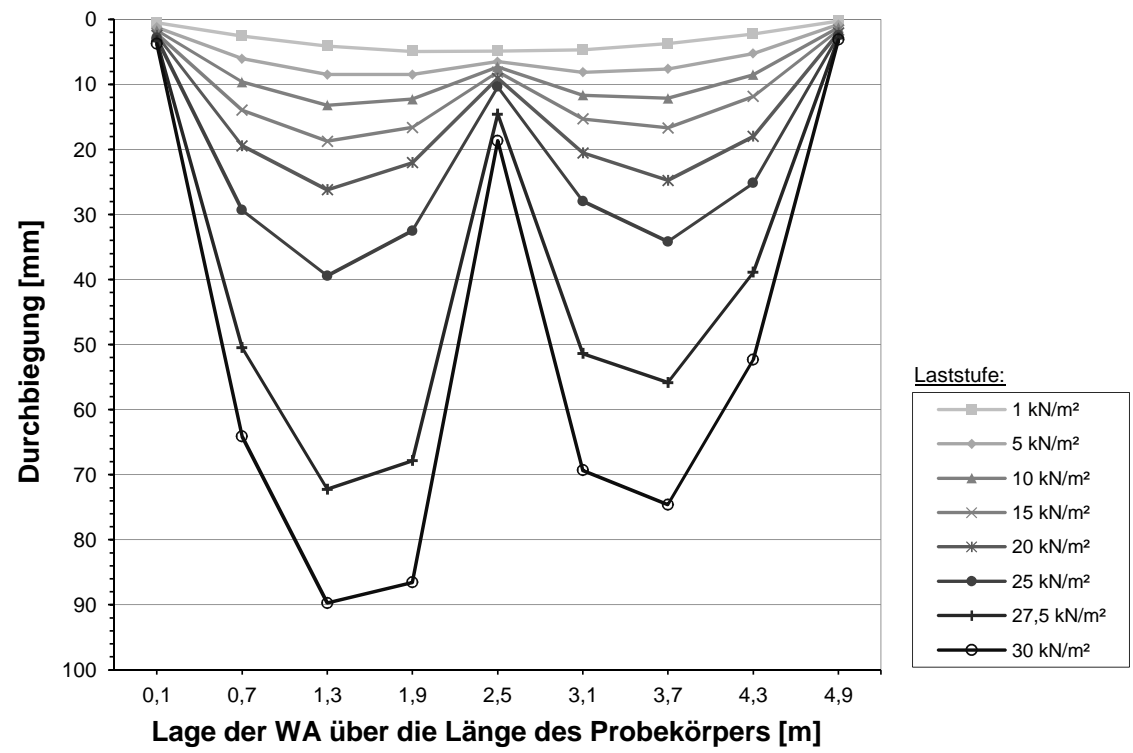

Abb. A.89: Durchbiegungen der oberen Deckschicht über der Länge des Probekörpers F1
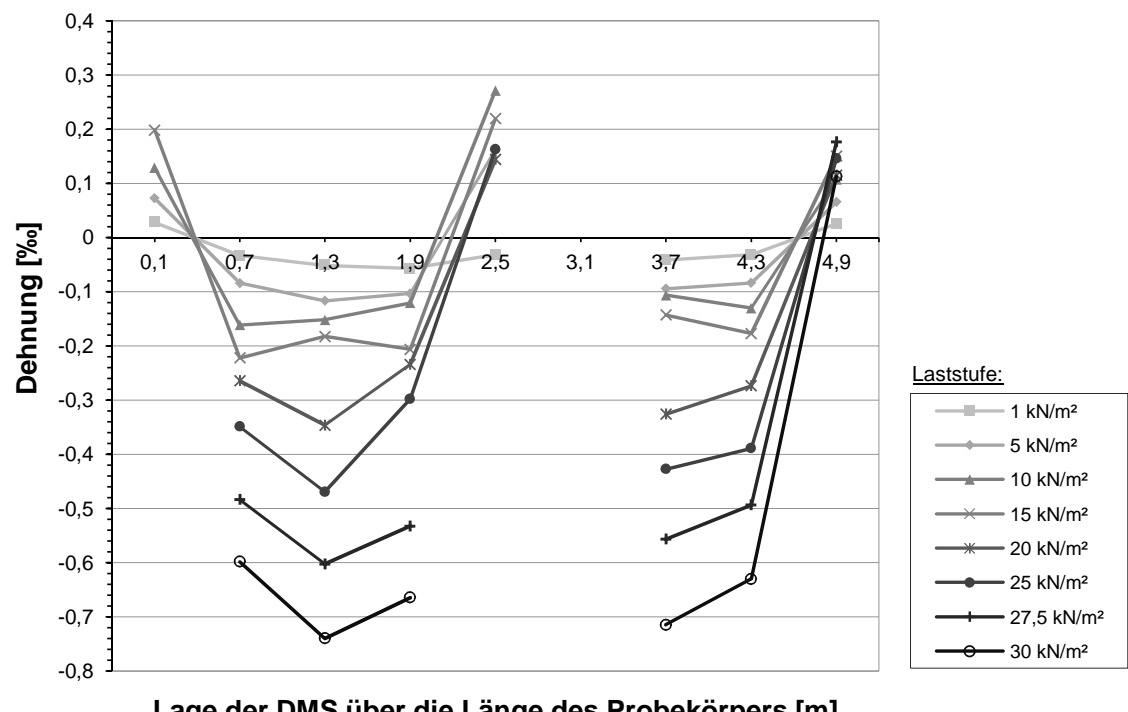

Lage der DMS über die Länge des Probekörpers [m]

Abb. A.90: Dehnungen auf der Oberseite der oberen Deckschicht über der Länge des Probekörpers F1 


\section{A.5.2.2 Versuch F3}

Kommentar: Der Versuch wurde im Unterdruck-Prüfstand mehrfach mit steigender Beanspruchung be- und entlastet. Die folgenden Diagramme zeigen jeweils den letzten Be- und Entlastungszyklus mit der höchsten aufgebrachten Beanspruchung.

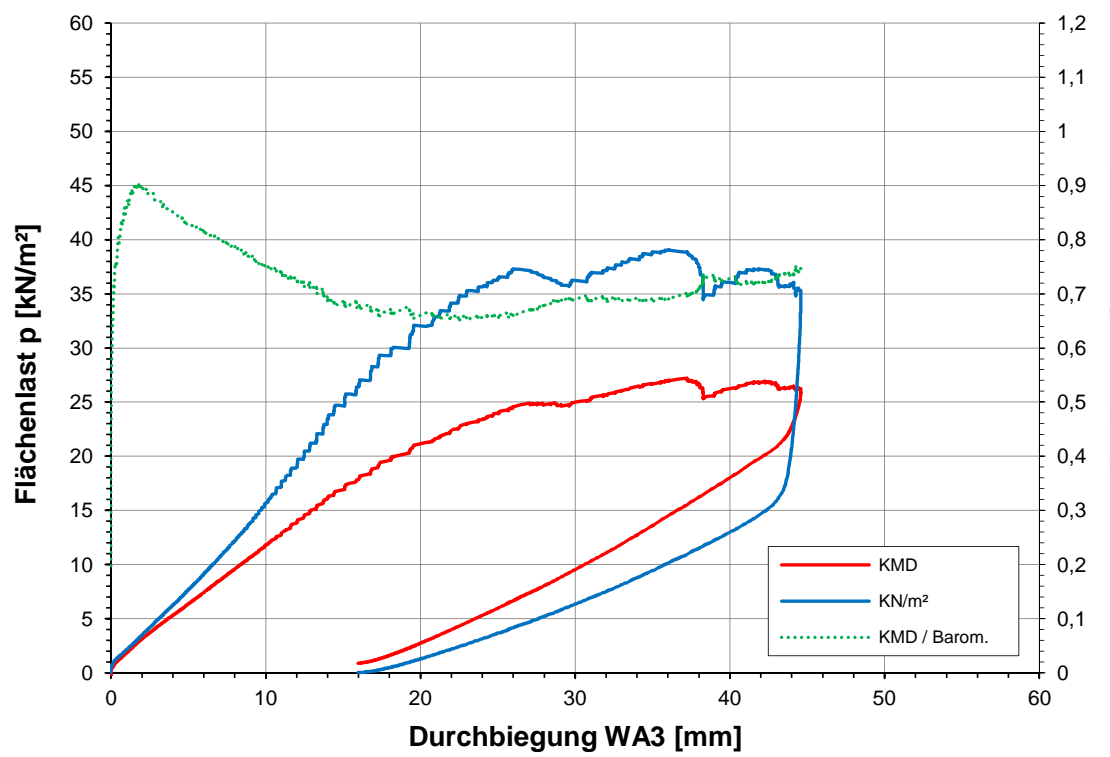

Abb. A.91: Flächenlast-Durchbiegungs-Diagramm des Versuchs F3

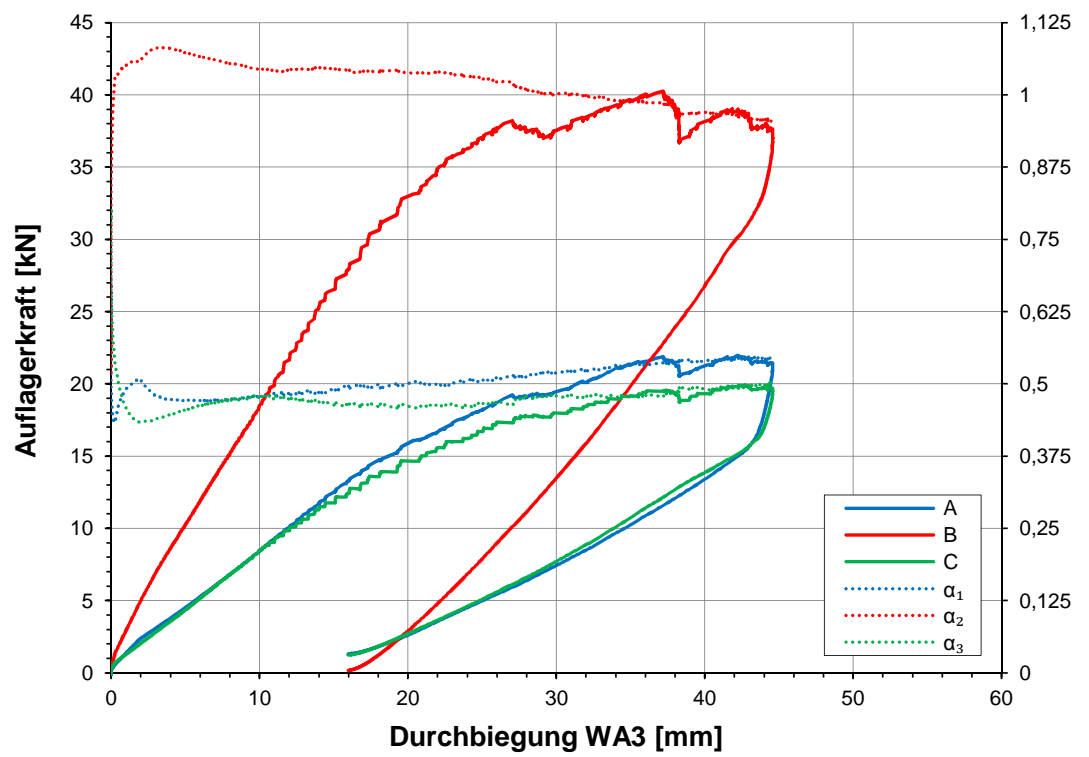

Abb. A.92: Auflagerkräfte-Durchbiegungs-Diagramm des Versuchs F3 


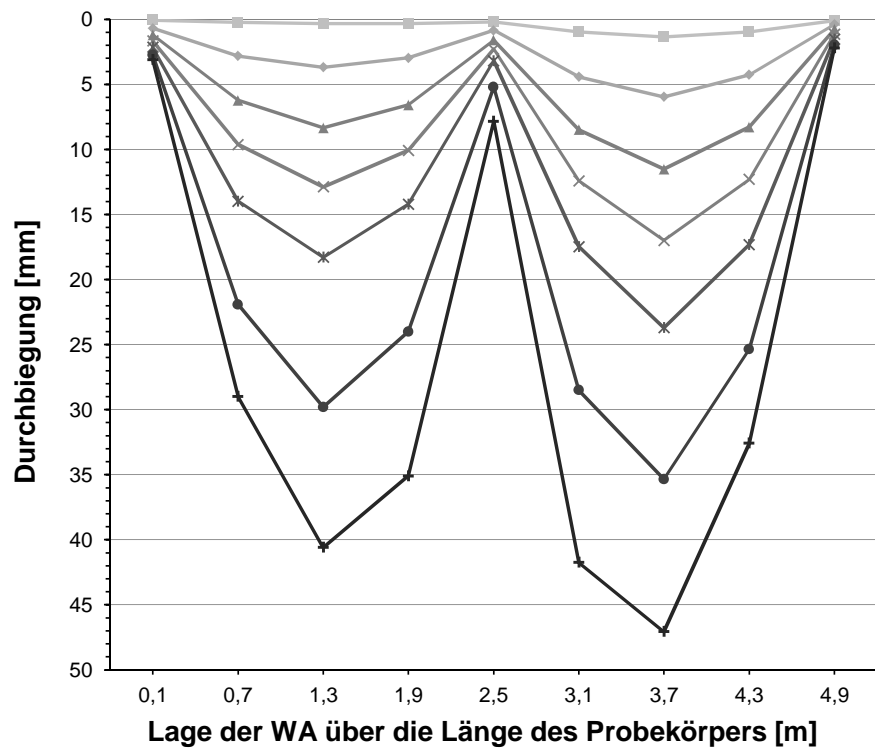

Laststufe:

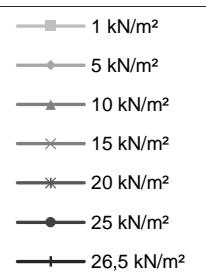

Lage der WA über die Länge des Probekörpers [m]

Abb. A.93: Durchbiegungen der oberen Deckschicht über der Länge des Probekörpers F3
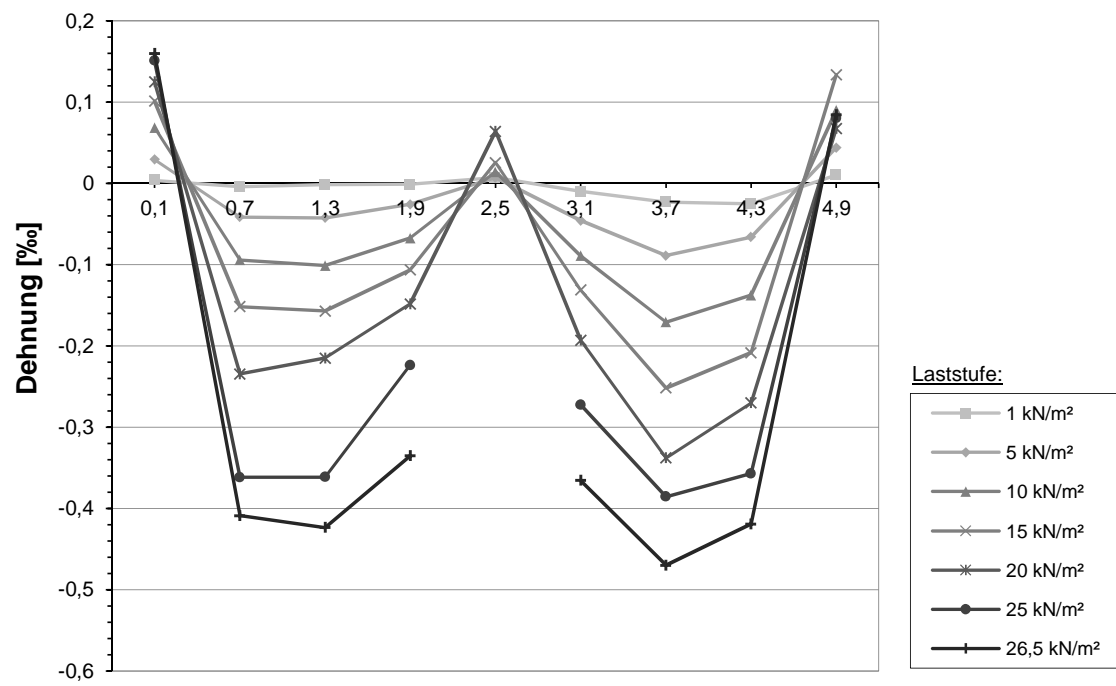

Lage der DMS über die Länge des Probekörpers [m]

Abb. A.94: Dehnungen auf der Oberseite der oberen Deckschicht über der Länge des Probekörpers F3 


\section{A.5.3 Zusammenstellung der aufgeschnittenen Biegeprobekörper}
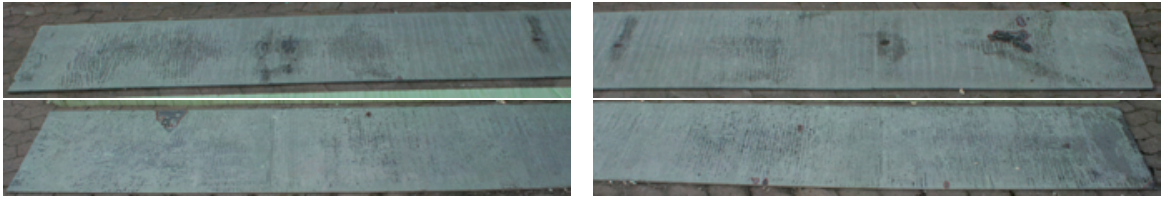

Abb. A.95: obere und untere Deckschicht des Großbauteilprobekörpers E1
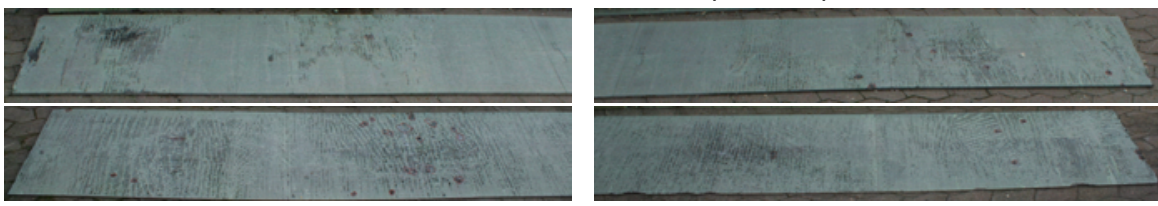

Abb. A.96: obere und untere Deckschicht des Großbauteilprobekörpers E2

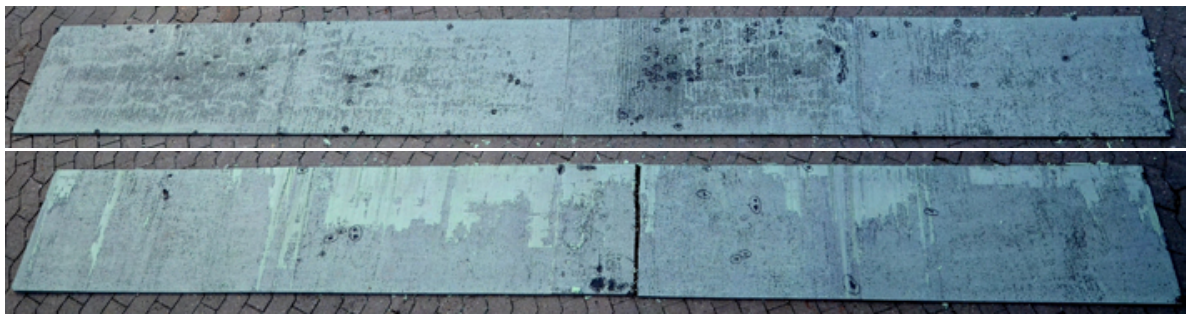

Abb. A.97: obere und untere Deckschicht des Großbauteilprobekörpers E3
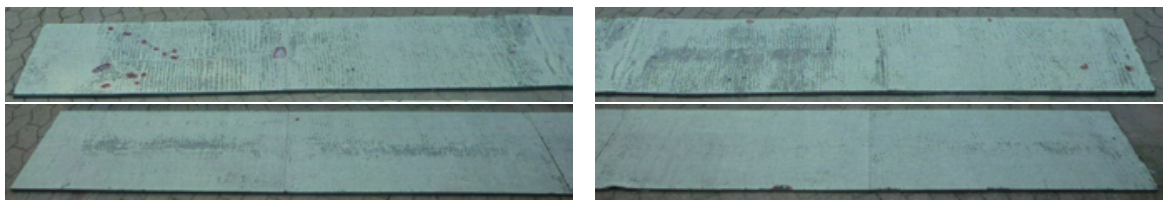

Abb. A.98: obere und untere Deckschicht des Großbauteilprobekörpers F1
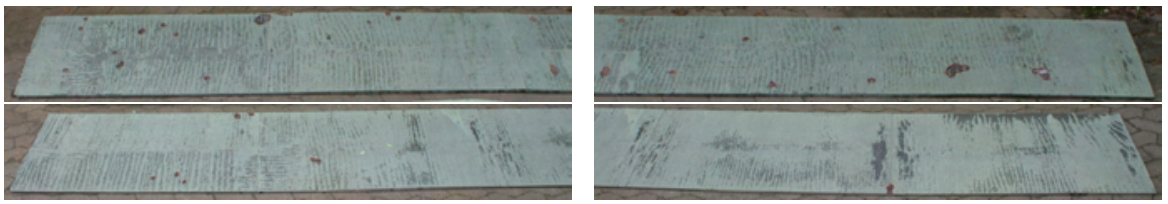

Abb. A.99: obere und untere Deckschicht des Großbauteilprobekörpers F2
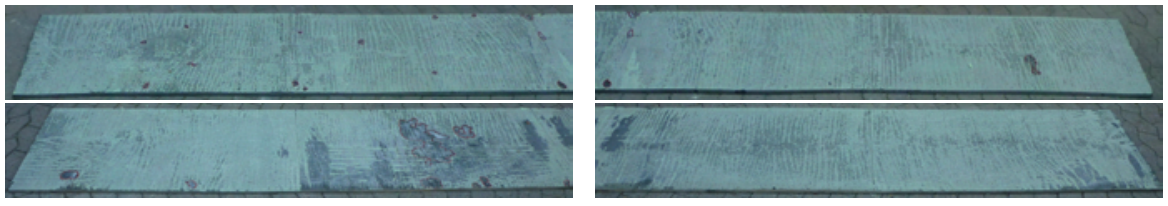

Abb. A.100: obere und untere Deckschicht des Großbauteilprobekörpers F3 



\section{B Anhang „Rechnerische Untersuchungen“}

\section{B.1 Eingabewerte}

Tab. B.1: $\quad$ Eingabewerte zur FE-Berechnung der Biegeversuche an kurzen Sandwichstreifen

\begin{tabular}{|c|c|c|c|c|c|c|c|}
\hline & & 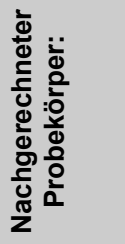 & 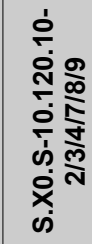 & 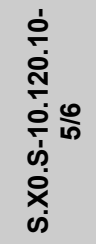 & 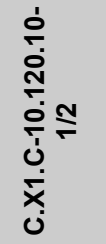 & 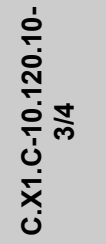 & 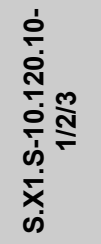 \\
\hline \multirow{5}{*}{$\begin{array}{l}\text { Versuchsreihe: } \\
\text { Versuchsart: } \\
\text { Überstand } \quad \text { (siehe Abb. 5.3) } \\
\text { Lasteinleitung } \quad \text { (siehe Abb. 5.3) } \\
\text { Lasteinl.Platte }\end{array}$} & & & \multicolumn{2}{|c|}{ Herstellung } & \multicolumn{3}{|c|}{ Bewehrung } \\
\hline & & & 4-PBV & 3-PBV & 3-PBV & 3-PBV & 3-PBV \\
\hline & $a$ & {$[\mathrm{~mm}]$} & 50 & 50 & & 50 & \\
\hline & $b$ & {$[\mathrm{~mm}]$} & 400 & 575 & & 575 & \\
\hline & $c$ & {$[\mathrm{~mm}]$} & 50 & 50 & 60 & 150 & 60 \\
\hline \multicolumn{3}{|l|}{ Deckschichten: } & \multirow{2}{*}{\multicolumn{2}{|c|}{36.165}} & & & \\
\hline E-Modul $\quad$ (Messwert) & $E_{m}$ & {$\left[\mathrm{~N} / \mathrm{mm}^{2}\right]$} & & & & 34.865 & \\
\hline Zugfestigkeit (nach GI. 4.4) & $f_{c t m}$ & {$\left[\mathrm{~N} / \mathrm{mm}^{2}\right]$} & \multicolumn{2}{|c|}{3,7} & & 3,1 & \\
\hline Bewehrung: & & & \multicolumn{2}{|c|}{ S } & $\mathrm{C}$ & $\mathrm{C}$ & $S$ \\
\hline Lagen: & & & \multicolumn{2}{|c|}{3} & 1 & 1 & 3 \\
\hline (Messwert) & $d_{0}$ & & \multicolumn{2}{|c|}{10,1} & 11,4 & 10,4 & 13,1 \\
\hline Dicke unten $\quad$ (Messwert) & $d_{u}$ & & \multicolumn{2}{|c|}{10,1} & 10,7 & 9,9 & 12,3 \\
\hline E-Modul (bew.) (nach GI. 6.5) & $E_{c}$ & {$\left[\mathrm{~N} / \mathrm{mm}^{2}\right]$} & \multicolumn{2}{|c|}{34.895} & 36.591 & 36.730 & 33.948 \\
\hline \multicolumn{8}{|l|}{ Punkte $\sigma-\varepsilon-K u r v e:$} \\
\hline Punkt A: & $\begin{array}{l}\sigma_{c, A} \\
\varepsilon_{c, A}\end{array}$ & $\begin{array}{c}{\left[\mathrm{N} / \mathrm{mm}^{2}\right]} \\
{[\% \mathrm{o}]}\end{array}$ & \multicolumn{2}{|c|}{0} & & $\begin{array}{l}0 \\
0\end{array}$ & \\
\hline \multirow{2}{*}{ Punkt B: } & $\sigma_{c, B}$ & {$\left[\mathrm{~N} / \mathrm{mm}^{2}\right]$} & \multicolumn{2}{|c|}{3,5701} & 3,2534 & 3,2658 & 3,0185 \\
\hline & $\varepsilon_{c, B}$ & {$[\% \circ]$} & \multicolumn{2}{|c|}{0,1023} & 0,0889 & 0,0889 & 0,0889 \\
\hline \multirow{2}{*}{ Punkt C: } & $\sigma_{c, c}$ & {$\left[\mathrm{~N} / \mathrm{mm}^{2}\right]$} & \multicolumn{2}{|c|}{4,2841} & 3,4161 & 3,4291 & 3,6222 \\
\hline & $\varepsilon_{c, c}$ & [\%o] & \multicolumn{2}{|c|}{0,9974} & 1,9227 & 1,7857 & 1,0204 \\
\hline \multirow{2}{*}{ Punkt D: } & $\sigma_{c, D}$ & {$\left[\mathrm{~N} / \mathrm{mm}^{2}\right]$} & \multicolumn{2}{|c|}{7,0919} & 13,084 & 14,141 & 5,8234 \\
\hline & $\varepsilon_{c, D}$ & [\%o] & \multicolumn{2}{|c|}{1,9377} & 9,3766 & 9,3968 & 1,9182 \\
\hline \multirow{2}{*}{ Punkt E: } & $\sigma_{c, E}$ & {$\left[\mathrm{~N} / \mathrm{mm}^{2}\right]$} & \multicolumn{2}{|c|}{8,2117} & - & - & 6,7429 \\
\hline & $\varepsilon_{c, E}$ & [\%o] & \multicolumn{2}{|c|}{50} & - & - & 50 \\
\hline \multicolumn{3}{|l|}{ Kern: } & & & & & \\
\hline XPS-Charge: & & & $X 5^{*}$ & $X 5^{*}$ & X1 & X1 & X1 \\
\hline Dicke XPS: & $d_{s}$ & & 120 & 120 & 120,0 & 120,0 & 120,0 \\
\hline
\end{tabular}

*Annahme 
Tab. B.2: Eingabewerte zur FE-Berechnung der Biegeversuche an kurzen Sandwichstreifen (Fortsetzung)

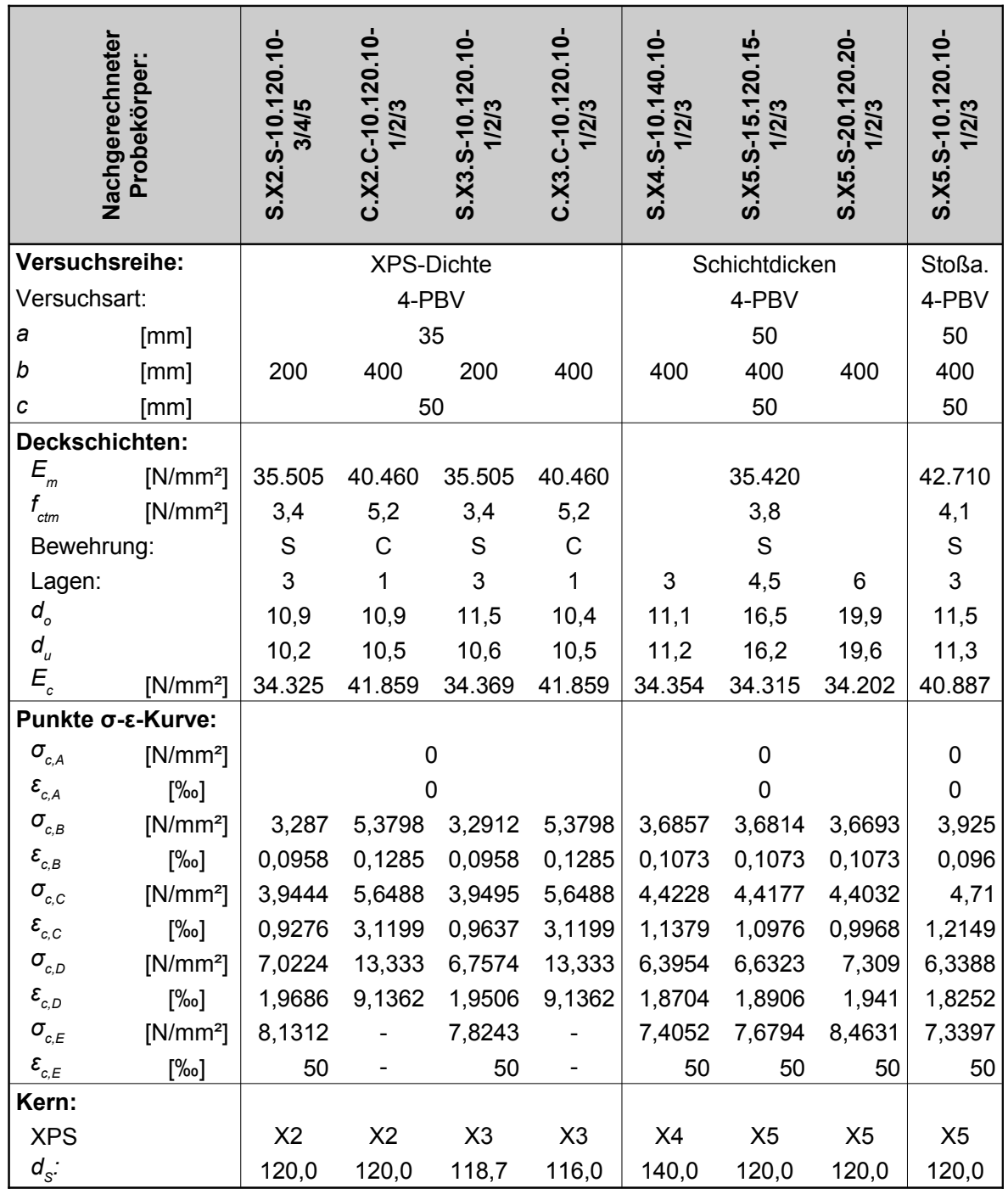

\section{B.2 Commandfile}

Apdl B.1: Beispiel für eine ANSYS-Commandfile eines 3-Punkt-Biegeversuchs

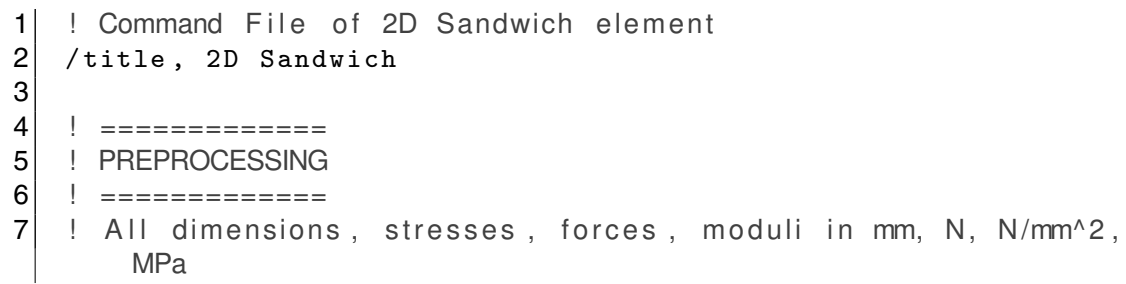




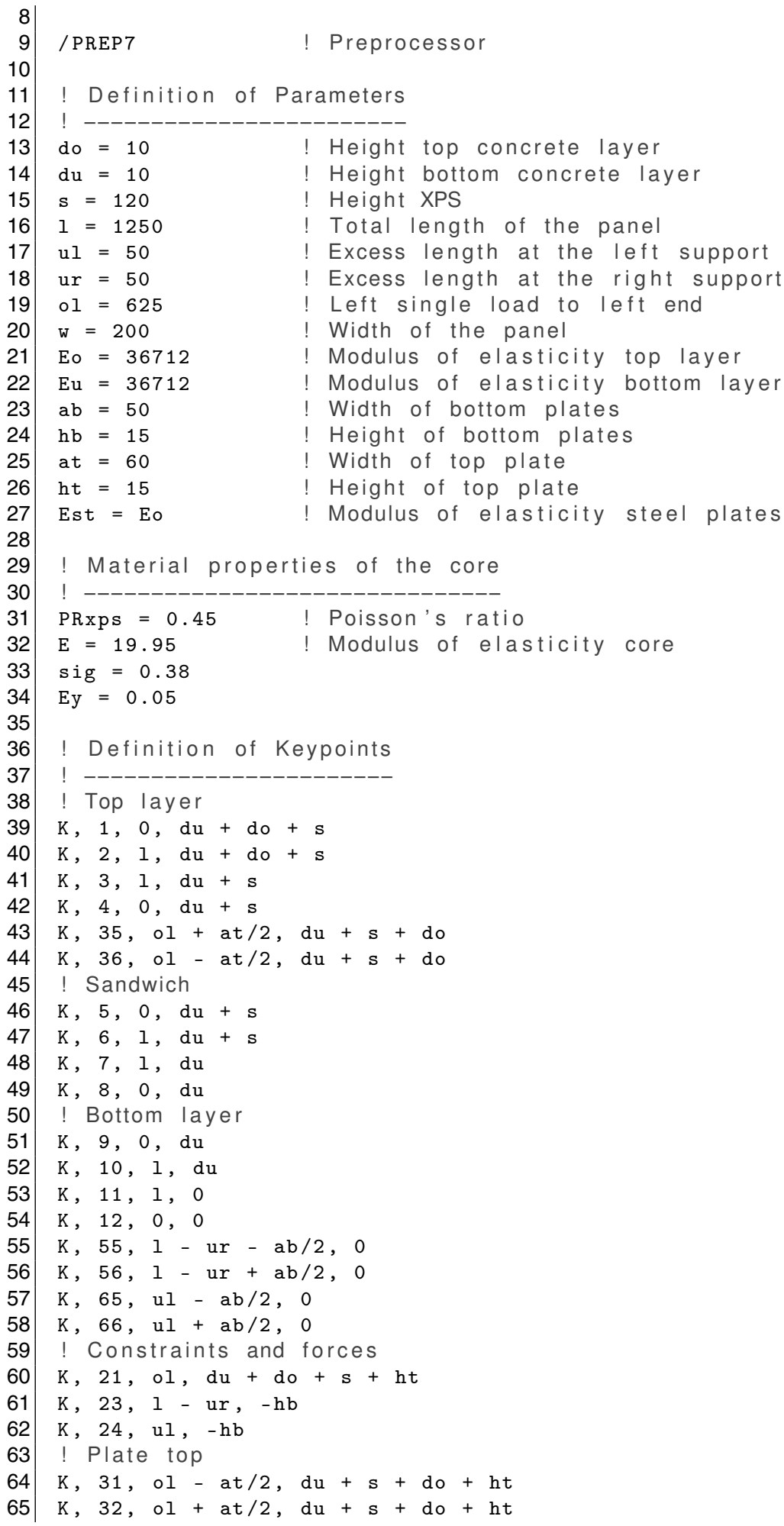




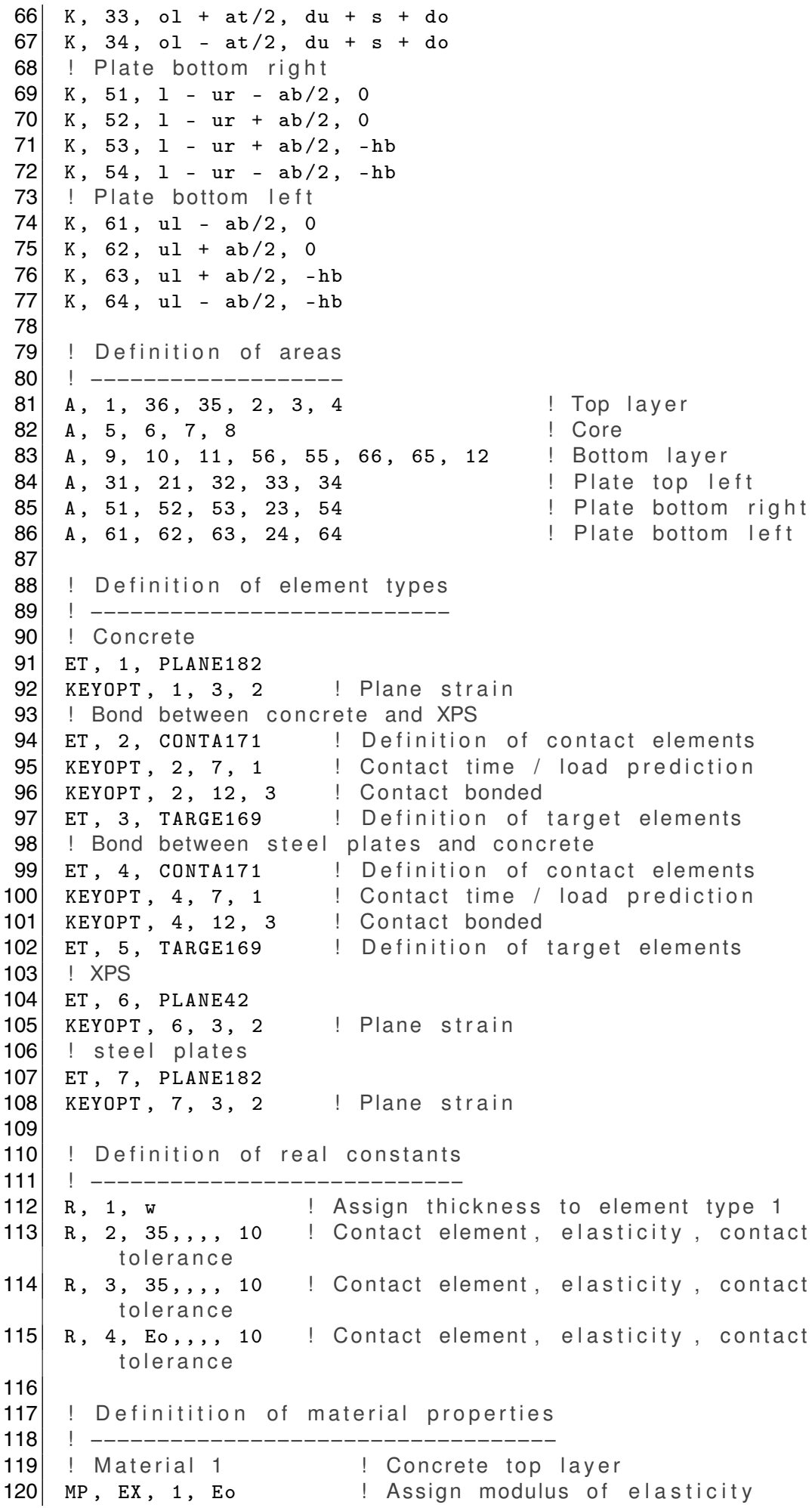




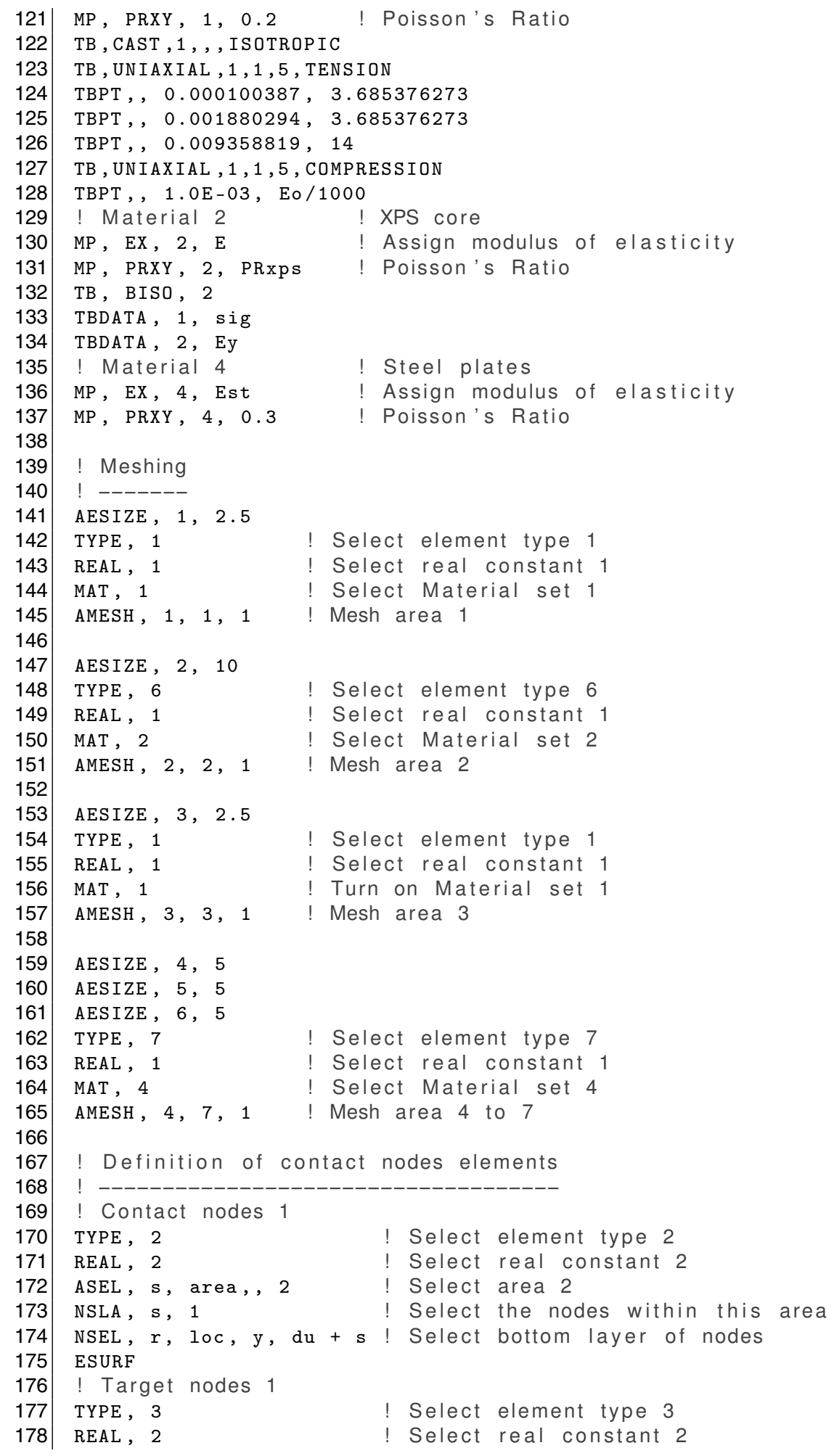




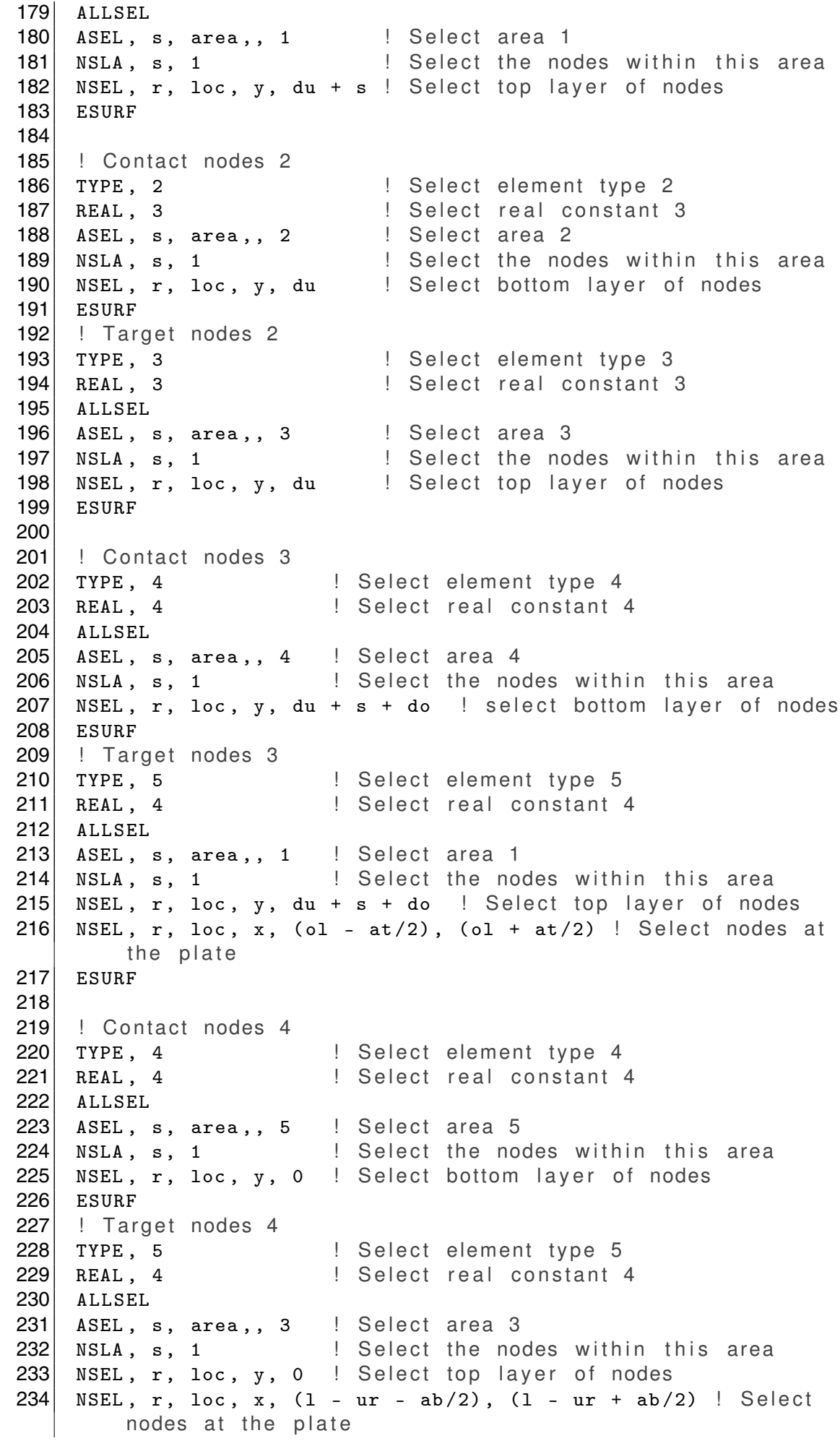




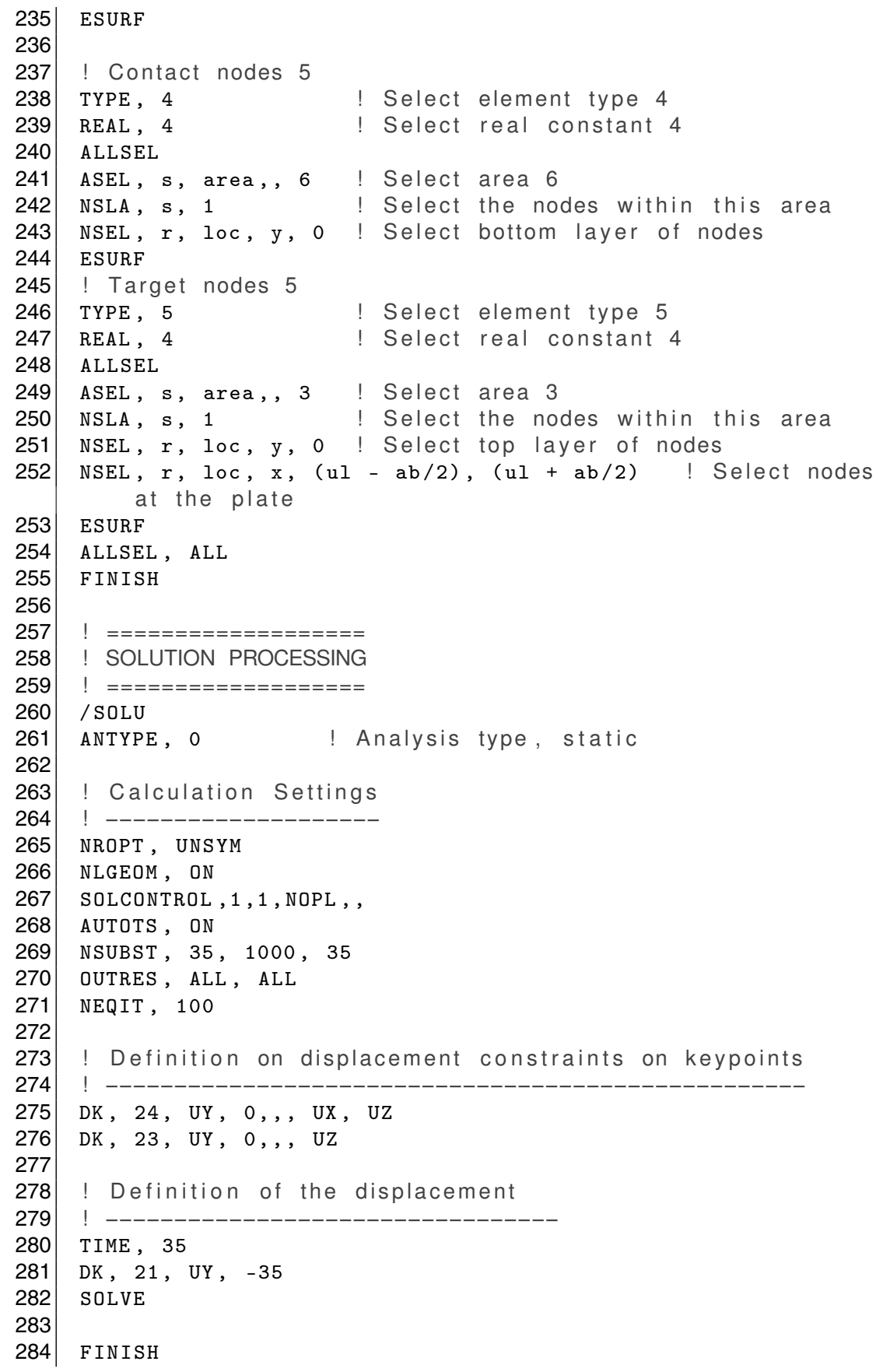


Tab. B.3: Eingabewerte zur FE-Berechnung der Großbauteilversuche

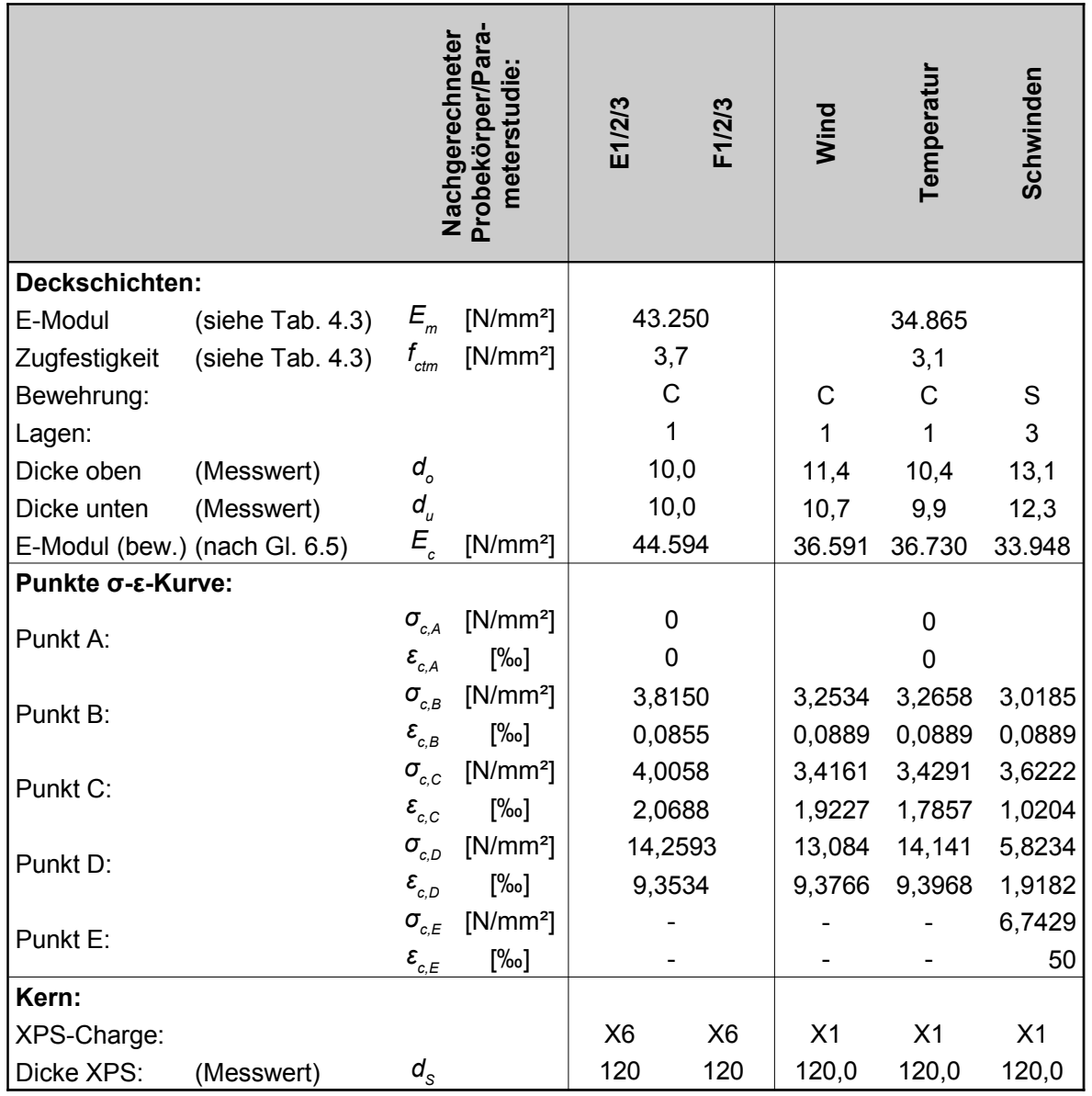




\section{Anhang „Nachweiskonzept“}

\section{C.1 Herleitung der Gleichung zur Bestimmung des Dehnungszustandes}
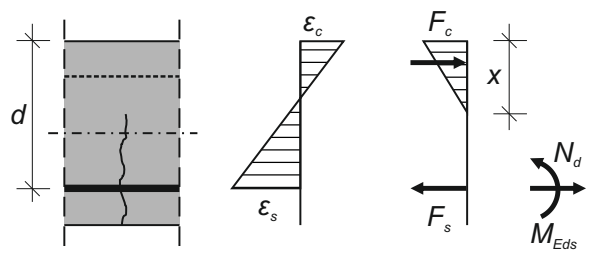

Abb. C.1: Dehnungen, Spannungen und Schnittgrößen am gerissenen Querschnitt

Aus der Summe der Momente ergibt sich:

$$
\begin{aligned}
& 0=M_{E d s}-F_{c} \cdot z \\
& 0=M_{E d s}-\frac{1}{2} \cdot \sigma_{c} \cdot x \cdot\left(d-\frac{x}{3}\right)
\end{aligned}
$$

Aus der Summe der Horizontalkräfte folgt:

$$
\begin{gathered}
F_{s}=F_{c}+N_{d} \\
E_{s} \cdot A_{s} \cdot \epsilon_{s}=\frac{1}{2} \cdot \sigma_{c} \cdot x+N_{d} \\
\epsilon_{s}=\frac{\frac{1}{2} \cdot \sigma_{c} \cdot x+N_{d}}{E_{s} \cdot A_{s}}
\end{gathered}
$$

Über den Zusammenhang zwischen den Dehnungen und der Druckzonenhöhe $x$ kann $\epsilon_{s}$ auch wie folgt beschrieben werden:

$$
\epsilon_{s}=\frac{\epsilon_{c} \cdot(d-x)}{x}
$$

Durch Gleichsetzen von C.5 und C.6 ergibt sich:

$$
\frac{\frac{1}{2} \cdot \sigma_{c} \cdot x+N_{d}}{E_{s} \cdot A_{s}}=\frac{\epsilon_{c} \cdot(d-x)}{x}
$$

Aus dem Hookeschen Gesetz ergibt sich $\epsilon_{c}$ zu:

$$
\epsilon_{c}=\frac{\sigma_{c}}{E_{c}}
$$


Und durch Einsetzen von $\epsilon_{c}$ in Gl. (C.7) resultiert:

$$
\frac{\frac{1}{2} \cdot \sigma_{c} \cdot x+N_{d}}{E_{s} \cdot A_{s}}=\frac{\sigma_{c} \cdot(d-x)}{E_{c} \cdot x}
$$

Daraus folgt $\sigma_{c}$ zu:

$$
\sigma_{c}=\frac{N_{d} \cdot E_{c} \cdot x}{-\frac{1}{2} \cdot E_{c} \cdot x^{2}+E_{s} \cdot A_{s} \cdot(d-x)}
$$

Durch Einsetzen von GI. C.10 in GI. C.2 und kurzem Umformen ergibt sich folgende lineare Gleichung dritten Grades:

$$
0=\frac{1}{6} N_{d} E_{c} \cdot x^{3}-\frac{1}{2} E_{c}\left(M_{E d s}+N_{d} d\right) \cdot x^{2}-E_{s} A_{s} M_{E d s} \cdot x+E_{s} A_{s} M_{E d s} d
$$

\section{C.2 Herleitung des $k$-Faktors}

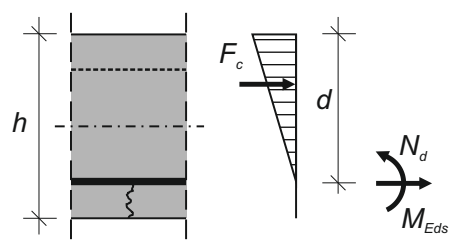

Abb. C.2: Spannungen und Schnittgrößen am gerissenen Querschnitt im Fall von Biegeriss Typ A

Aus der Summe der Momente ergibt sich:

$$
M_{E d s}=F_{c} \cdot d \cdot \frac{2}{3}
$$

Es gilt:

$$
F_{c}=-N_{d}
$$

Daraus folgt:

$$
\begin{aligned}
& M_{E d s}=-N_{d} \cdot d \cdot \frac{2}{3} \\
& k=\frac{-N_{d} \cdot d}{M_{E d s}}=1,5
\end{aligned}
$$




\section{C.3 Abschätzung der Relaxation des XPS}

Beim Kriechen nimmt unter einer konstant aufgebrachten Spannung die Dehnung mit der Zeit ab. Bei der Relaxation hingegen nimmt unter einer konstant aufgebrachten Dehnung die Spannung ab. Im Fall der Schwindverformung und der dadurch aufgebrachten Dehnung reduziert sich die Spannung daher infolge der Relaxation des XPS.

Da keine Angaben zum Relaxationsverhalten des XPS verfügbar waren, wurde die Relaxation mithilfe des folgenden Ansatzes über die Kriecheigenschaften abgeschätzt.

Es werden Zeitinkremente $\Delta t$ mit beliebiger Größe festgelegt. Über die Dauer jedes Zeitinkrements reduziert sich die Zwangspannung infolge der Relaxation welche mit einem reduzierter Elastizitätsmodul $E_{X P S,(\Delta t)}$ berücksichtigt wird.

$$
\sigma_{X P S, c,(\Delta t)}=\epsilon_{s} \cdot E_{X P S,(\Delta t)}
$$

Der reduzierte Elastizitätsmodul $E_{X P S,(\Delta t)}$ wird für jedes Zeitinkrement unter Annahme einer fiktiven Gesamtdehnung $\epsilon_{(\Delta t)}$ neu berechnet.

$$
E_{X P S,(\Delta t)}=\frac{\sigma_{X P S, C}}{\epsilon_{(\Delta t)}}
$$

Dabei berechnet sich die Gesamtdehnung $\epsilon_{(\Delta t)}$ aus dem Schwindanteil $\epsilon_{s}$ und einem Kriechanteils $m \cdot t^{n}$ (siehe auch Abschnitt 3.2.3.5, Materialverhalten unter dauerhafter Beanspruchung') zu:

$$
\epsilon_{(t)}=\epsilon_{s}+m \cdot t^{n}
$$

Die Werkstoffkonstanten $m$ und $n$ des Kriechanteils sind dabei abhängig von der aufgebrachten Spannung $\sigma_{X P S, C}$. Für jedes Zeitinkrement wird hier die Spannung des vorherigen Inkrements $\sigma_{X P S, c, \Delta t-1}$ angesetzt.

Durch Krollmann 1995 wurde die Spannungsabhängigkeit von $m$ und $n$ für zwei XPSTypen mit den folgenden empirischen Formeln beschrieben.

Für XPS-Typ 1:

$$
\begin{aligned}
m & =2,7950 \cdot 10^{-6} \cdot \sigma_{X P S, C}+1,6177 \cdot 10^{-12} \cdot \sigma_{X P S, C}^{3} \\
n & =0,23359+2,1558 \cdot 10^{-5} \cdot \sigma_{X P S, C}
\end{aligned}
$$

Und XPS-Typ 2:

$$
\begin{aligned}
m & =3,9296 \cdot 10^{-7} \cdot \sigma_{X P S, c}+6,0919 \cdot 10^{-12} \cdot \sigma_{X P S, C}^{3} \\
n & =0,25968-3,3955 \cdot 10^{-5} \cdot \sigma_{X P S, C}
\end{aligned}
$$

Mit den Gleichungen C.19 und C.20 bzw. C.21 und C.22 können beispielhaft die Zwangspannungen der beiden XPS-Typen 1 und 2 in Abbildung C.3 über der Zeit $t$ aufgetragen werden. 


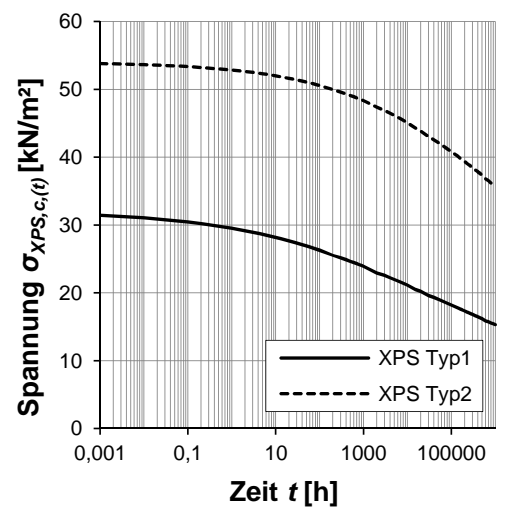

Abb. C.3: Abnahme der Zwangspannungen durch Relaxation

\section{C.4 Lastfallkombinationen eines Sandwich-Dachelements}

Tab. C.1: Häufig auftretende Lastkombinationen im Grenzzustand der Gebrauchstauglichkeit eines Sandwich-Dachelements

\begin{tabular}{|c|c|c|c|c|c|c|c|c|c|c|c|c|c|c|c|c|c|}
\hline & $\begin{array}{c}\text { LF1 } \\
g_{k} \\
\gamma_{G}\end{array}$ & $\Psi_{0}$ & $\begin{array}{c}\text { LF2 } \\
s_{\mathrm{k}} \\
\Psi_{1}\end{array}$ & $\gamma_{Q}$ & $\Psi_{0}$ & $\begin{array}{c}\text { LF3 } \\
w_{1, k} \\
\Psi_{1}\end{array}$ & $\gamma_{Q}$ & $\Psi_{0}$ & $\begin{array}{c}\text { LF4 } \\
w_{2, \mathrm{k}} \\
\Psi_{1}\end{array}$ & $\gamma_{Q}$ & & $\begin{array}{l}\text { LF5 } \\
\Delta T_{\mathrm{s}} \\
\Psi_{1}\end{array}$ & $\gamma_{Q}$ & & $\begin{array}{l}\text { LF6 } \\
\Delta T_{\mathrm{w}} \\
\Psi_{1} \quad \gamma_{\mathrm{Q}}\end{array}$ & $\begin{array}{c}\text { LF7 } \\
\Delta T_{\text {ws }} \\
\Psi_{0} \Psi_{1}\end{array}$ & $\gamma_{Q}$ \\
\hline LK1 & 1,0 & & & & & & & & & & & & & & & & \\
\hline LK2 & 1,0 & & 1,0 & 1,0 & & & & & & & & & & & & & \\
\hline LK3 & 1,0 & & & & & 1,0 & 1,0 & & & & & & & & & & \\
\hline LK4 & 1,0 & & & & & & & & 1,0 & 1,0 & & & & & & & \\
\hline LK5 & 1,0 & & & & & & & & & & & 1,01 & 1,0 & & & & \\
\hline LK6 & 1,0 & & & & & & & & & & & & & & $1,0 \quad 1,0$ & & \\
\hline LK7 & 1,0 & & 0,75 & 1,0 & 0,6 & 0,75 & 1,0 & & & & & & & & & & \\
\hline LK8 & 1,0 & & 0,75 & 1,0 & & & & 0,6 & 0,75 & 1,0 & & & & & & & \\
\hline LK9 & 1,0 & & 0,75 & 1,0 & & & & & & & & & & & & $\begin{array}{lll}1,0 & 1,0 & 1\end{array}$ & 1,0 \\
\hline LK10 & 1,0 & 0,6 & 0,75 & 1,0 & & 0,75 & 1,0 & & & & & & & & & & \\
\hline LK11 & 1,0 & & & & & 0,75 & 1,0 & & & & 0,6 & 1,0 & 1,0 & & & & \\
\hline LK12 & 1,0 & & & & & 0,75 & 1,0 & & & & & & & 0,6 & $1,0 \quad 1,0$ & & \\
\hline LK13 & 1,0 & 0,6 & 0,75 & 1,0 & & & & & 0,75 & 1,0 & & & & & & & \\
\hline LK14 & 1,0 & & & & & & & & 0,75 & 1,0 & 0,6 & 1,0 & 1,0 & & & & \\
\hline LK15 & 1,0 & & & & & & & & 0,75 & 1,0 & & & & 0,6 & $1,0 \quad 1,0$ & & \\
\hline LK16 & 1,0 & & & & 0,6 & 0,75 & 1,0 & & & & & 1,01 & 1,0 & & & & \\
\hline LK17 & 1,0 & & & & & & & 0,6 & 0,75 & 1,0 & & 1,01 & 1,0 & & & & \\
\hline LK18 & 1,0 & & & & 0,6 & 0,75 & 1,0 & & & & & & & & $1,0 \quad 1,0$ & & \\
\hline LK19 & 1,0 & & & & & & & 0,6 & 0,75 & 1,0 & & & & & $1,0 \quad 1,0$ & & \\
\hline LK20 & 1,0 & & 0,75 & 1,0 & 0,6 & 0,75 & 1,0 & & & & & & & & & $1,0 \quad 1,0$ & 1,0 \\
\hline LK21 & 1,0 & & 0,75 & 1,0 & & & & 0,6 & 0,75 & 1,0 & & & & & & $1,0 \quad 1,0$ & 1,0 \\
\hline LK22 & 1,0 & 0,6 & 0,75 & 1,0 & & 0,75 & 1,0 & & & & & & & & & $1,0 \quad 1,0$ & 1,0 \\
\hline LK23 & 1,0 & 0,6 & 0,75 & 1,0 & & & & & 0,75 & 1,0 & & & & & & $1,0 \quad 1,0$ & 1,0 \\
\hline
\end{tabular}


Tab. C.2: Lastkombinationen im Grenzzustand der Tragfähigkeit eines Sandwich-

Dachelements

\begin{tabular}{|c|c|c|c|c|c|c|c|c|c|c|c|c|c|}
\hline & \multirow{2}{*}{$\begin{array}{c}\text { LF1 } \\
g_{k} \\
\gamma_{G}\end{array}$} & \multicolumn{2}{|c|}{$\begin{array}{c}\text { LF2 } \\
s_{k}\end{array}$} & \multicolumn{2}{|c|}{$\begin{array}{l}\text { LF3 } \\
w_{1, k}\end{array}$} & \multicolumn{2}{|c|}{$\begin{array}{l}\text { LF4 } \\
w_{2, \mathrm{k}}\end{array}$} & \multicolumn{2}{|c|}{$\begin{array}{l}\text { LF5 } \\
\Delta T_{\mathrm{s}}\end{array}$} & \multicolumn{2}{|c|}{$\begin{array}{l}\text { LF6 } \\
\Delta T_{\mathrm{w}}\end{array}$} & \multicolumn{2}{|c|}{$\begin{array}{l}\text { LF7 } \\
\Delta T_{\text {ws }}\end{array}$} \\
\hline & & $\Psi_{0}$ & $\gamma_{Q}$ & $\Psi_{0}$ & $\gamma_{Q}$ & & $\gamma_{Q}$ & $\Psi_{0}$ & $\gamma_{Q}$ & $\Psi_{0}$ & $\gamma_{Q}$ & $\Psi_{0}$ & $\gamma_{Q}$ \\
\hline LK1 & 1,35 & & & & & & & & & & & & \\
\hline LK2 & 1,35 & & 1,5 & & & & & & & & & & \\
\hline LK3 & 1,35 & & & & 1,5 & & & & & & & & \\
\hline LK4 & 1,35 & & & & & & 1,5 & & & & & & \\
\hline LK5 & 1,35 & & & & & & & & 1,5 & & & & \\
\hline LK6 & 1,35 & & & & & & & & & & 1,5 & & \\
\hline LK7 & 1,35 & & 1,5 & 0,6 & 1,5 & & & & & & & & \\
\hline LK8 & 1,35 & & 1,5 & & & 0,6 & 1,5 & & & & & & \\
\hline LK9 & 1,35 & & 1,5 & & & & & & & & & 1,0 & 1,5 \\
\hline LK10 & 1,35 & 0,6 & 1,5 & & 1,5 & & & & & & & & \\
\hline LK11 & 1,35 & & & & 1,5 & & & 0,6 & 1,5 & & & & \\
\hline LK12 & 1,35 & & & & 1,5 & & & & & 0,6 & 1,5 & & \\
\hline LK13 & 1,35 & 0,6 & 1,5 & & & & 1,5 & & & & & & \\
\hline LK14 & 1,35 & & & & & & 1,5 & 0,6 & 1,5 & & & & \\
\hline LK15 & 1,35 & & & & & & 1,5 & & & 0,6 & 1,5 & & \\
\hline LK16 & 1,35 & & & 0,6 & 1,5 & & & & 1,5 & & & & \\
\hline LK17 & 1,35 & & & & & 0,6 & 1,5 & & 1,5 & & & & \\
\hline LK18 & 1,35 & & & 0,6 & 1,5 & & & & & & 1,5 & & \\
\hline LK19 & 1,35 & & & & & 0,6 & 1,5 & & & & 1,5 & & \\
\hline LK20 & 1,35 & & 1,5 & 0,6 & 1,5 & & & & & & & 1,0 & 1,5 \\
\hline LK21 & 1,35 & & 1,5 & & & 0,6 & 1,5 & & & & & 1,0 & 1,5 \\
\hline LK22 & 1,35 & 0,6 & 1,5 & & 1,5 & & & & & & & 1,0 & 1,5 \\
\hline LK23 & 1,35 & 0,6 & 1,5 & & & & 1,5 & & & & & 1,0 & 1,5 \\
\hline LK24 & 1,0 & & & & & & & & & & & & \\
\hline LK25 & 1,0 & & 1,5 & & & & & & & & & & \\
\hline LK26 & 1,0 & & & & 1,5 & & & & & & & & \\
\hline LK27 & 1,0 & & & & & & 1,5 & & & & & & \\
\hline LK28 & 1,0 & & & & & & & & 1,5 & & & & \\
\hline LK29 & 1,0 & & & & & & & & & & 1,5 & & \\
\hline LK30 & 1,0 & & 1,5 & 0,6 & 1,5 & & & & & & & & \\
\hline LK31 & 1,0 & & 1,5 & & & 0,6 & 1,5 & & & & & & \\
\hline LK32 & 1,0 & & 1,5 & & & & & & & & & 1,0 & 1,5 \\
\hline LK33 & 1,0 & 0,6 & 1,5 & & 1,5 & & & & & & & & \\
\hline LK34 & 1,0 & & & & 1,5 & & & 0,6 & 1,5 & & & & \\
\hline LK35 & 1,0 & & & & 1,5 & & & & & 0,6 & 1,5 & & \\
\hline LK36 & 1,0 & 0,6 & 1,5 & & & & 1,5 & & & & & & \\
\hline LK37 & 1,0 & & & & & & 1,5 & 0,6 & 1,5 & & & & \\
\hline LK38 & 1,0 & & & & & & 1,5 & & & 0,6 & 1,5 & & \\
\hline LK39 & 1,0 & & & 0,6 & 1,5 & & & & 1,5 & & & & \\
\hline LK40 & 1,0 & & & & & 0,6 & 1,5 & & 1,5 & & & & \\
\hline LK41 & 1,0 & & & 0,6 & 1,5 & & & & & & 1,5 & & \\
\hline LK42 & 1,0 & & & & & 0,6 & 1,5 & & & & 1,5 & & \\
\hline LK43 & 1,0 & & 1,5 & 0,6 & 1,5 & & & & & & & 1,0 & 1,5 \\
\hline LK44 & 1,0 & & 1,5 & & & 0,6 & 1,5 & & & & & 1,0 & 1,5 \\
\hline LK45 & 1,0 & 0,6 & 1,5 & & 1,5 & & & & & & & 1,0 & 1,5 \\
\hline LK46 & 1,0 & 0,6 & 1,5 & & & & 1,5 & & & & & 1,0 & 1,5 \\
\hline
\end{tabular}





\section{Lebenslauf}

\section{Persönliche Daten}

Name: $\quad$ Frank Antonius Müller

Staatsangehörigkeit: deutsch

\section{Beruflicher Werdegang}

seit 03/2014:

11/2009-02/2014:

1/2003 - 3/2003:

$8 / 2000-1 / 2003$
Tragwerksplaner bei Werner Sobek GmbH, Stuttgart

Wissenschaftlicher Mitarbeiter an der TU Kaiserslautern am Fachgebiet Massivbau und Baukonstruktion bei Prof. Dr.-Ing. Jürgen Schnell, im Forschungsgebiet „Konstruieren mit Hochleistungsbeton“ bei Jun.-Prof. Dr.-Ing. Christian Kohlmeyer

Bauzeichner bei R\&P RUFFERT Ingenieurgesellschaft $\mathrm{mbH}$, Limburg (ehem. Ruffert \& Partner Ingenieurgesellschaft $\mathrm{mbH}$ ) Ausbildung als Bauzeichner im Bauingenieurwesen bei R\&P RUFFERT Ingenieurgesellschaft mbH, Limburg

\section{Hochschulausbildung}

9/2007 - 5/2009: $\quad$ Studium „Civil Engineering Design \& Management“ an der University of Dundee, Schottland, Abschluss: M.Eng.

10/2004 - 7/2007: Studium „Internationales Bauingenieurwesen“ an der Fachhochschule Mainz, Schwerpunktmäßiges Studium: Konstruktiver Ingenieurbau, Abschluss: Dipl.-Ing. (FH)

\section{Schulausbildung}

9/2003 - 6/2004: $\quad$ Fachoberschule im Fachbereich Bautechnik an der FriedrichDessauer-Schule, Limburg

8/1994 - 6/2000: $\quad$ Mittlere Reife an der Realschule Rennerod 